Catalogue OF $\mathrm{THF}$

\title{
Scientific Periodicals
}

$4 \mathrm{~N}$

Calcutta Libraries. 


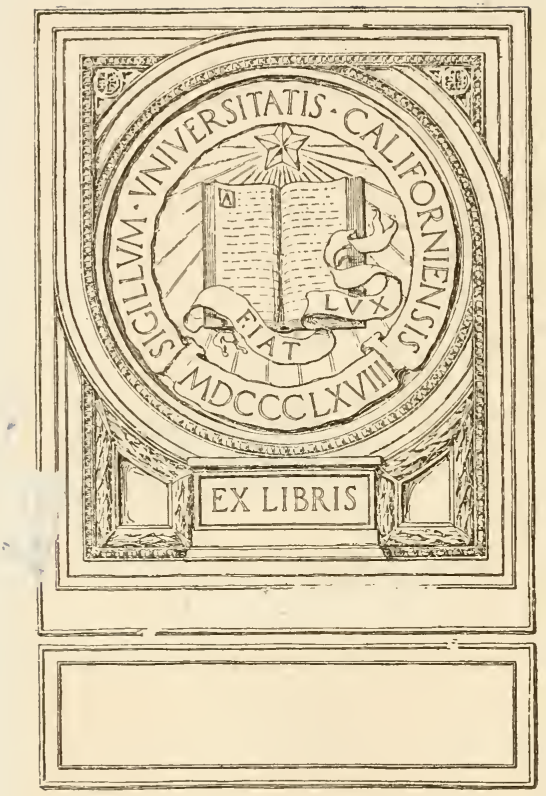




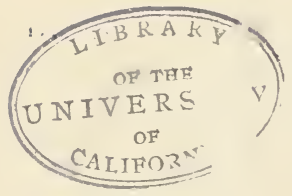





\title{
CATALOGUE
}

OF THE

\section{Scientific Serial Publications}

IN THE

\section{Principal Libraries of Calcutta.}

\author{
Compiled for the \\ ASIATIC SOCIETY OF BENGAL \\ by \\ STANLEY KEMP, B.A., \\ with the assistance of the librarians of the \\ institutions concerned.
}

\section{Calcutta :}

PRINTED AT THE BAPTIST MISSION PRESS AND PUBLISHED BY THE ASIATIC SOCIETY OF BENGAL, I, PARK STREET, CALCUTTA. 
Digitized by the Internet Archive in 2008 with funding from Microsoft Corporation 


\section{PREFACE.}

$\mathrm{T}$

HIS Catalogue is designed for the use of those who have occasion

to consult the scientific periodicals represented in Calcutta libraries, whether belonging to Government or private institutions. In many of these libraries the existing catalogues are either in manuscript or completely out of date; moreover the number of the libraries is large and they are situated at considerable distances apart. Data from twenty-four libraries have been incorporated.

In deciding the difficult question as to what is a scientific serial publication it has seemed best to take as broad a view as possible and include too much rather than too little. A serial publication has been taken to be one whose issues are numbered or dated according to a definite sequence and to which no termination might be anticipated at the time of its initiation. 'The publications of the Ray Society, for instance, are not included as they consist of a series of independent, unnumbered monographs published without any definite sequence; nor are the Reports of the 'Challenger' Expedition, because at their inception a definite end to publication could be foreseen. Serials dealing with the technical applications of science, such as Engineering and Agriculture, have been inserted, together with those concerning such subjects as Archaeology and Economics. When an institution publishes separate serials on both scientific and other subjects, references to all are given: purely administrative reports are, however, omitted.

All parts of periodicals received up to the end of I9I 7 have so far as possible been catalogued; but, owing to the present state of international affairs, it has been found impossible to deal precisely with current scientific literature published in Germany and Austria.

On behalf of the Council of the Asiatic Society of Bengal I wish to express my indebtedness to the heads of the institutions that have supplied information regarding their serials. Had it not been for 
PREFACE.

cordial co-operation on the part of the twenty-four libraries, the preparation of this Catalogue would have been impossible.

Among those who have given assistance in the compilation, iny thanks are first due to Dr. W. A. K. Christie of the Geological Survey of India, who has read through all the proofs and assisted me in many other ways. Owing to his valuable help the work has gained greatly in accuracy and completeness.

I have also to thank Mr. J. Chapman, Librarian of the Imperial Library, who has kindly solved several bibliographical questions that presented unusual difficulty. Dr. G. D. Hope has checked the references to Dutch periodicals and Mr. A. Kolusky those in Russian and allied languages. Babu Balai Lal Dutt, the Society's Librarian, has aided me in many ways.

The cost of publication has been defrayed by a grant made by the Trustees of the Indian Museum, through the kind offices of the Director, Zoological Survey of India.

The Society will be obliged if their attention is drawn to any errors that may be detected. 


\section{CONTENTS.}

PAGE

PREFACE

LIBRARIES CATALOGUED

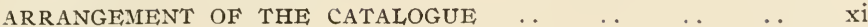

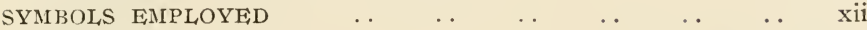

CATALOGUE.

EUROPE.

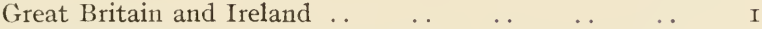

$\begin{array}{llllllll}\text { Austria-Hungary } & \ldots & \ldots & \ldots & \ldots & \ldots & \ldots & 49\end{array}$

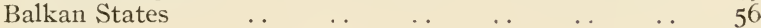

$\begin{array}{lllllllll}\text { Belgium } & \therefore & \ldots & \ldots & \ldots & \ldots & \ldots & \ldots & 57\end{array}$

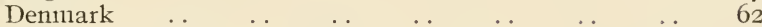

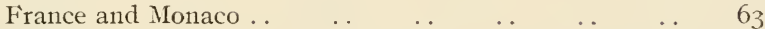

$\begin{array}{lllllllll}\text { Germany } & \ldots & \ldots & \ldots & \ldots & \ldots & \ldots & \ldots & 83\end{array}$

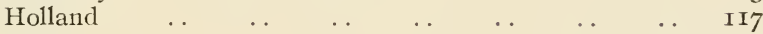

$\begin{array}{lllllllll}\text { Italy } & \ldots & \ldots & \ldots & \ldots & \ldots & \ldots & \ldots & 123\end{array}$

$\begin{array}{lllllllll}\text { Norway } & \ldots & \ldots & \ldots & \ldots & \ldots & \ldots & \ldots & \text { 133 }\end{array}$

$\begin{array}{lllllllll}\text { Russia } & \ldots & \ldots & \ldots & \ldots & \ldots & \ldots & \ldots & \text { I } 35\end{array}$

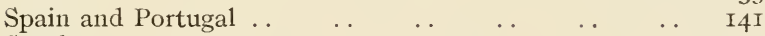

$\begin{array}{lllllllll}\text { Sweden } & \ldots & \ldots & \ldots & \ldots & \ldots & \ldots & \ldots & \text { I4 } 43\end{array}$

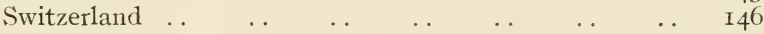

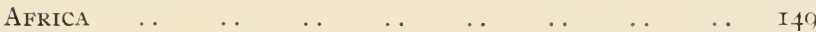

ASIA.

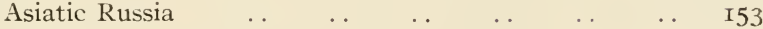

$\begin{array}{lllllllll}\text { Cyprus } & \ldots & \ldots & \ldots & \ldots & \ldots & \ldots & \ldots & \text { I54 }\end{array}$

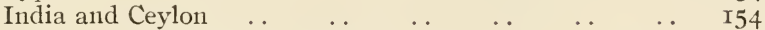

Japan, China and Indo-Chinese countries $\quad \ldots \quad \ldots r$ I83

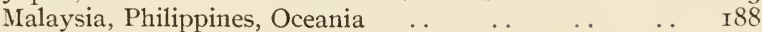

$\begin{array}{llllllllll}\text { Australasia } & \ldots & \ldots & \ldots & \ldots & \ldots & \ldots & \ldots & \text { I95 }\end{array}$

America.

$\begin{array}{lllllllll}\text { Canada } & \ldots & \ldots & \ldots & \ldots & \ldots & \ldots & \ldots & 204\end{array}$

$\begin{array}{llllllll}\text { United States } & \ldots & \ldots & \ldots & \ldots & \ldots & \ldots & 208\end{array}$

South and Central America and West Indies . . $\quad . \quad 246$

INTERNATIONAL

$\begin{array}{lllllllllll}\text { ADDENDA } & \ldots & \ldots & \ldots & \ldots & \ldots & \ldots & \ldots & \ldots & 254\end{array}$

$\begin{array}{lllllllllll}\operatorname{INDEX} & \ldots & \ldots & \ldots & \ldots & \ldots & \ldots & \ldots & \ldots & \ldots & 257\end{array}$ 



\section{LIBRARIES CATALOGUED.}

\section{Archaeological Section, Indian Museum.}

\section{A.s.}

This library is situated in the topmost storey of the new extension of the Indian Aluseum, Chowringhee, and is in the office of the Archaeological Section. It contains books and periodicals devoted mainly to Archaeology. The library is open daily from Io-3o A.M. to 4-30 P.M. (except on Saturday afternoons, Sundays and public holidays), but is intended only for the use of the staff of the Department. For permission to read in the library application should be made to the Officer-inCharge. No books or papers may be removed from the premises.

\section{Asiatic Society of BENGaL.}

\section{A.S.B.}

The library of the Asiatic Society of Bengal is situated in the Society's rooms at no. I, Park Street, and is open daily, except on Sundays, Christmas Day, Good Friday and the King's Birthday, from Io A.M. to 5 P.x. Members are privileged to read in the library and to take books out on loan subject to certain rules. No person who is not a member of the Society is permitted to take away any book from the library without special authority from the Council. or to have access to the library without permission of the President or one of the Secretaries. Members of the Royal Asiatic Society of Great Britain and Ireland, and its branches and Associate Societies, who may be temporarily in Calcutta, are permitted to use the library.

\section{Bishop's COLLEGE.}

\section{B.C.}

Bishop's College Old Library, 224, Lower Circular Road, is open to readers during term time, except on Sundays, Christmas Day and Good Friday, from 7 A.x. to I 2 noon and at other times by appointment. Permission to read in the library may be obtained by personal application to the Principal. No books or papers may be removed from the library.

\section{Botanical, SuRvey OF INDIA.}

\section{B.S.I.}

The library of the Botanical Survey of India, formerly that of the Reporter on Economic Products, is housed in the northern wing of the Indian Museum (entrance in Sudder Street). It is open during office 
hours. Applications for permission to read in the library or to borrow books should be addressed to the Economic Botanist to the Botanical Survey of India, Indian Museum.

\section{Chemical, Examiner's Library.}

\section{C.E.}

This library is situated in the office of the Chemical Examiner at the Medical College. It is open on week-days (gazetted holidays excepted) from II A.M. to 2 P.M., and is intended primarily for the use of the office staff. For permission to read in the library application should be made to the Chemical Examiner. No books or journals may be removed from the premises.

\section{Calcutta University.}

\section{C.U.}

The library of Calcutta University, known as Maharaja Sir Rameswar Singh of Darbhanga University Library, is situated in Pearycharan Sircar Street. It is for the use of resident Fellows of the University, University Professors, Assistant Professors, Readers, Lecturers and resident registered graduates, who alone can take out books from the Library. Facilities are provided to University post-graduate students for reading in the library reading room, but they are not allowed to remove books from the reading room. The latter is open between the hours of 7 A.Mr. and 7 P.M. (Sundays and authorized holidays excepted). Permission may be granted by the Syndicate on the recommendation of the Library Executive Committee to persons who desire to use the library for the purpose of literary or scientific research.

\section{Civil, Engineering College, Sibpur.}

\section{E.G.}

The library of the Civil Engineering College is situated at Sibpur and can be reached from Calcutta by road or by ferry steamer calling at College Ghat. It contains books and periodicals devoted mainly to Engineering and Industries. There are sub-libraries in the various departments of the College, containing the more frequently needed works of reference. During the College session, November to July inclusive, the library is open daily during working hours. Members of the public may be permitted to use the library on written application to the Principal, but books are not lent out of the library except to nembers of the College staff and students.

\section{Geological Survey of India.}

\section{G.S.I.}

On application to the Director, members of the public are permit- 
ted to make use of the Library of the Geological Survey of India between the hours of I0-30 A.M. and 4-30 P.M. on all days except Sundays and gazetted holidays. IVith the exception of certain works of reference. which may not be removed from the premises, books are freely lent to approved persons, who are at liberty to apply either personally or by letter. The library is situated in the offices of the Survey. in the compound of the Indian Museum.

\section{IMPERIAL LIBRARY.}

\section{I.L.}

Metcalfe Hall, I2, Strand Road. A public reference and lending library. The reading rooms are open daily from Io A.M. to 7 P.M., except on Sundays and gazetted holidays, when they are open from 2 P.x. to 5 P.x. Applications for books on loan are received on weekdays, including gazetted holidays, between the hours I0-30 A.M. to 5 P.u. They should be addressed: Imperial Library, Issue Department.

\section{Medical College.}

M.c.

This library, situated in the Medical College, College Street, is available as a day reading-room for students betwcen the hours of I I A.M. and 4 P.M. daily, except on Sundays and College holidays. The library is intended for the use of the Professors and students of the College only, but books will be lent out for research purposes to Medical officers of Government and other approved persons by the Principal, or on the requisition of the Surgeon-General with the Gorernment of Bengal.

\section{Medical College:}

Bacteriological, Biological and Physiological Laboratories.

\section{M.Ba., M.Bi., M.Ph.}

The libraries of these laboratories are situated respectively in the Bacteriological, Biological and Physiological Departments of the Medical College, College Street. They are intended only for the use of the staffs of the Departments and are open, except on gazetted holidays and Sundays, from II A.M. to 4 P.M., and on Saturdays from II A.M. to 2 P.M. Applications for permission to use the libraries should be made to the Principal, Medical College, or to the Professor of the Department concerned.

METEOROLOGICAL OFFICE LibRary.

M.o.

This library is situated in the Meteorological Office at Alipore, and forms a small part of the departmental library, the bulk of which is kept 
at Simla. With the exception of a few works of general reference it contains books on Meteorology only. The question of lending any book or reading it in the Alipore Library must be referred to the Meteorologist in charge.

\section{Presidency Coliege.}

\section{P.C.}

This library is situated in the Presidency College, 86-I, College Street. The library is open daily during term time from Io A.M. to 5 P.Mr., Sundays and authorized holidays excepted. On Saturdays the library closes at 3 P.M. The library is managed by a Committee of three, the Principal and two Professors-one representing the Arts and the other the Science side of the College. The books of the library are for the use of (I) students of the College, (2) the teaching staff, and (3) other persons engaged in studies of an advanced character, especially if they are old students of the Presidency College. In the last case permission must be obtained from the Principal on the recommendation of a member of the Committee, and the privilege is confined to the use of the books in the library. Books of reference and other books confined for special reasons to the library can only be used by readers in the library itself. No periodicals of the current year may, on any account, be taken out of the library. Back numbers of periodicals can be consulted in the library on the same conditions as books. They can be lent out to members of the staff, but, in the case of science periodicals, must be returned on the day of issue.

\section{The Patent OfFice.}

P.o.

The public room of the Patent Office is situated on the ground floor of the Commerce and Industry Building, no. I, Council House Street. It is open from II A.M. to 4 P.M. daily, except on Saturdays, when it is closed at I P.x. Specifications of patents, and patent literature of interest to inventors and others, may be consulted, generally free of charge, but may not be removed from the premises.

\section{Royal Botanic GaRden.}

\section{R.B.G.}

This library is housed in the Herbarium buildings of the Royal Botanic Garden at Sibpur, and can be reached from Calcutta by road or by ferry steamer calling at College or at Garden Ghat. With the exception of some works of general reference it is devoted to books and periodicals on Botanical Science. Persons wishing to consult it may do so at any time during office hours, with the permission of the Librarian. 


\section{L,IBRARIES CATALOGUED.}

The library is primarily a non-lending institution, but the rule is occasionally relaxed for the benefit of recognized institutions and known researchers in India and Burma. Certain irreplaceable books and periodicals are not allowed out on loan.

\section{Scottish Churches College.}

\section{S.c.c.}

The library of the Scottish Churches College is situated at 4, Cornwallis Square and, except on gazetted holidays, is open during the College sessions, from July to April, between the hours of ro-30 A.м. and 4 P.n. The library is intended primarily for the students on the College rolls. Members of the public wishing to make use of the library should apply either personally or by letter to the Principal. but permission to take books out of the library is rarely granted.

\section{Surgeon-General's Librart (Bengal Medical Library).}

\section{S.G.}

This library, which is attached to the office of the Surgeon-General with the Government of Bengal, is situated in Writers' Buildings and is open on working days from II A.M. to 5 P.M., except on Saturdays when it is closed at 2 P.Mr. Medical Officers of all ranks belonging to the Bengal Medical Department are privileged to make use of the library and, subject to certain rules, are permitted to take books out on loan. Students of medical institutions and others wishing to use the library may obtain permission to do so from the Surgeon-General. Bengal, but are not allowed to take books away.

\section{SURVEY OF INDIA.}

\section{S.I.}

This library is situated in the building of the Surveyor General's Office, I3, Wood Street The iibrary, except on Sundays, Saturday afternoons and public holidays, is open between the hours of ro A.M. and I P.M. and between 3 and 4 P.x. Students should apply personally to the Officer-in-charge, Surveyor General's Office, for permission to read in the library. No books or papers may ordinarily be removed from the library.

School of Tropical, Medicine.

\section{S.T $M$.}

This library will be situated in the building of the School of Tropical Medicine, Medical College, College Street. It will be opened after the conclusion of the war; till then the books will be kept in the library of the Asiatic Society of Bengal. The library is not open to the public. 
The Director of the School in special cases will consider applications to use the library.

\section{Telegraph Departuent.}

T.D.

This library is situated in the office of the Chief Electrician at the Telegraph Storeyard, Alipore. It is mainly devoted to books and periodicals on Telegraphy and Telephony. The library is not ordinarily open to the public, but students and others can be given facilities for reading any of the books and periodicals by the Chief Electrician, to whom personal application should be made between the hours of I0-30 A.M. and 4-30 P.M., excepting Sundays, Saturdays and public holidays. No books or papers may be removed from the premises without the Chief Electrician's special permission. The Chief Electrician is willing, in special cases, to consider the question of lending books or papers to institutions outside Calcutta.

\section{St. Xavier's College.}

\section{X.c.}

The St. Xavier's Library and the Goethal's Library are situated in St. Xavier's College, 30, Park Street, and are open daily, except on Sundays, from Io A.x. to 4-30 P.M. Permission to read in the library may be obtained by personal application to the Principal. No books or papers may be removed from the premises.

\section{ZOOLOGICAL, SURVEY OF INDIA.}

\section{Z.S.I}

This library is situated in the main building of the Indian Museum, Chowringhee. With the exception of a few works of general reference, it is mainly devoted to books and periodicals on Zoology and Anthropology. The library, except on Sundays, Saturday afternoons and public holidays, is open between the hours of Io A.M. and 4-30 P.M Students may obtain permission to read in the library by personal application to the Director. No books or papers may ordinarily be removed from the premises, but officers of other departments located in the Museum compound may have them sent to their own library or office on signing a form supplied by the Librarian. The Director is willing, in special cases, to consider the question of lending books or papers to institutions outside Calcutta. 


\section{ARRANGEMENT OF THE CATALOGUE.}

$\mathrm{P}$

UBLICATIONS issued by an institution are entered under the name of that institution, while institutions are listed under the towns in which they are established: independent serials will be found under the town of publication. The names of institutions and the titles of independent publications are arranged together alphabetically under the towns and the towns alphabetically under the countries to which they belong, the order of the latter being shown in the Table of Contents (p. iii). Changes in title, whether of institutions or of publications, have been noted as far as possible and, in the case of independent serials, changes in the town of publication are indicated by crossreferences.

When rolume number and date are followed by a dash, it is to be understood that all subsequent issues are available; but with works published in Germany and Austria, this symbol merely indicates that the publications were being received regularly up to the beginning of the war.

To facilitate reference a copious index has been added. In both index and Catalogue the names of towns are printed in heavy type, those of institutions in roman type and the titles of publications in italics. The numbers in the index, and those used for cross-references, refer to the separate entries and not to the pages. The heavy type capitals, placed after the volume-numbers of individual publications, indicate the libraries in which these volumes will be found.

Except for minor alterations in method, necessitated by the fact that the libraries of a number of different institutions are dealt with, the arrangement of the Catalogue closely follows that of the Catalogue of the Serial Publications in the Library of the Manchester Literary and Philosophical Society, compiled by Mr. A. P. Hunt. Acknowledgment is due to this Socicty and to the Compiler for the provision of so satisfactory a model. 


\section{SYMBOLS EMPLOYED.}

A.S. = Archaeological Section, Indian Museum.

A.S.B. = Asiatic Society of Bengal.

B.C. = Bishop's College.

B.S.I. = Botanical Survey of India.

C.E. = Chemical Examiner to the Govt. of Bengal.

c.u. = Calcutta University.

E.C. = Civil Engineering College, Sibpur.

G.S.I. = Geological Survey of India.

I.L. = Imperial Library.

M.Ba. = Medical College, Bacteriological Laboratory.

M.Bi. = Medical College, Biological Laboratory

M.c. = Medical College (main library).

м.o. = Meteorological Office, Alipore.

M.Ph. = Medical College, Physiological Laboratory.

P.c. = Presidency College.

P.o. = Patent Office.

R.B.G. = Royal Botanic Garden.

S.c.C. $=$ Scottish Churches College.

s.G. = Surgeon General's Library.

s.I. = Survey of India.

S.T.M. = School of Tropical Medicine.

T.D. = Telegraph Department.

X.c. = St. Xavier's College.

z.s.I. = Zoological Survey of India. 


\section{EUROPE.}

\section{GREAT BRITAIN AND IREILAND.}

I. Belfast.-Natural History and Philosophical Society (see also No. 22). Proceedings. I87I/72-. Belfast, I873- (vol. for $1873 / 74$ wanting): G.S.I.

2. Birmingham.-Analytical Laboratories of Southall Bros. and Barclay, Ltd.

Annual Report. Ed. by E. W. Mann. Nos. I9-. Birmingham, IgII-: B.S.I.

Institution of Mechanical Engineers (see No. I80).

3. - Natural History and Philosophical Society.

Proceedings. Vols. 3-, I88I/82-. Birmingham (I883)(vols. 9, if defective) : A.S.B.

Bristol.-Medical Annual and Practitioner's Index (see No. 217).

4. Medico-chirurgical Society.

Bristol Medico-chirurgical Journal. Vols. 26-. Bristol, I908-: S.T.M.

5. — Naturalists' Society.

Proceedings. New ser., vols. I-Io. Bristol, I876-I904 (pts. I, 2, vol. IO, I90I-02, wanting): G.S.I. Fourth ser., vols. I-. Bristol, I907-: G.S.I.

6. Cambridge.-Antiquarian Society.

Proceedings. New ser., vols. 5- (old ser., vols. Ir-), 1903-06-. Cambridge, I907-: A.S.B.

7. Biochemical Journal. Ed. by B. Moore, E. Whitley and others. Vols. I-. Liverpool and Cambridge, I9o6-: M.Ph., P.C. Index to vols. I-25: P.C.

8. - Biometrika: a journal for the statistical study of biological problems. Ed. by W. F. R. Weldon, Karl Pearson and others. Vols. I-. Cambridge, I90I-: P.C., Z.S.I. Index to vols. I-5 :

Z.S.I. 
9. Cambridge.-Journal of Agricultural Science. Ed. by R. H. Biffen, A. D. Hall, T. B. Wood and others. Vols. I-. Cambridge, I905-.

Complete set: B.S.I.

Vols. I-3, I905-07: I.L.

- Journal of Anatomy and Physiology (see No. I9o).

Io. - Journal of Genetics. Ed. by W. Bateson and R. C. Punnett. Vols. I-. Cambridge, IgII-: M.Bi., R.B.G., Z.S.I.

II. - Journal of Hygiene. Vols. I-. Cambridge, London, I9OI-.

Vols. I-, IgoI- (No. 3, vol. 8 \& vol. 9 wanting): S.T.M.

Vols. I-7, IgoI-07 (vol. 5 defective) : S.G.

Voís. 5-, I905- : M.C.

- Journal of Physiology (see No. 195).

I2. - Observatory.

(a) Annual Report. I904/05-. Cambridge, I905- (vols. for I905/06, '06/07, 'IO II wanting): A.S.B.

(b) Astronomical Observations. Vols. I4-23, I842-75. Cambridge, I845-98: A.S.B.

I3. - Parasitology: a supplement to the Journal of Hygiene. Ed. by G. H. F. Nuttall and A. E. Shipley. Vols. I-. Cambridge, I908-: M.Ba., Z.S.I.

I4. — Philosophical Society.

(a) Proceedings. Vols. I-, I843-. London and Cambridge, I866-.

Complete sets: C.U., G.S.I.

Vols. 3, 8-, for $1876 / 80$, I892/95- (vols. 8, 9 defective) : I.L.

Vols. I0-, for I898 $1900-$ : P.C.

Vols. I2-, for I902 04-: A.S.B.

Vols. 14-, for 1906 o8-: Z.S.I.

(b) Transactions. Vols. I- Cambridge, I82I-. Index to vols. I-I2 at end of vol. I2.

Complete sets : C.U., G.S.I.

Vols. I, 20-, I82I, I905-: A.S.B.

Vol. I, I82I : B.C.

Vols. 9-, I856- (vols. 16-18 wanting, vols. 9-13, 15 defective) : I.L.

15. University.

Studies from the Morphological Laboratory. Ed. by F. M. Balfour and (afterwards) A. Sedgwick. Vols. [I]-6. London, I880-96: Z.S.I.

\footnotetext{
A.S. = Archaeological Section. Indian Museum

A.S.B. = Asiatic Society of Bengal.

B.C. = Bishop s College.

B.S.l. = Botanical survey of India

C.E. = Chemical Examner to the Govt. of Bengal.

C.U. = Calcutta Unversity.
}

E.C. = Civil Engineering College, Sibpur.

G.S.1. = Geological Survey ot Inaia.

I.L. = Imperial Liorary.

M.Ba. = Aledical College, Bacteriological Laboratory.

M. Bi. = Medical College. Biological Laboratory.

M.C. = Medical College (mam library) 
I6. Cork.-Cuvierian Society for the Cultivation of the Sciences (see also No. 22).

Report. I849/50-I85o/5I, I854/55. Cork, I85I, I855: G.S.I.

I7. Cullercoats.-Dove Marine Laboratory.

Report. Ed. by A. Meek. New ser., vols. 2-, I9I2/I3-. Newcastle-upon-Tyne, I9I3-: Z.S.I.

I8. Dublin.-Department of Agriculture and Technical Instruction for Ireland.-Fisheries Branch.

Scientific Investigations. I902/03-. Dublin, I905(vol. for Igor wanting) : Z.S.I. Index to vols. for I9OI-05 at end of vol. for I906, I908.

19. - Dublin Journal of Medical Science. Vols. 125-. Dublin, I908-: S.T.M.

20. - Dublin Medical Journal. Vols. I-27. Dublin, I832-45: M.C.

- Geological Society (see Nos. 22 and 27).

- Magazine of Zoology and Botany (see No. 4r).

21. — National Museum of Science and Art.

Museum Bulletin. Nos. I-. Dublin, I9II-: Z.S.I.

22. - Natural History Review: a quarterly journal, including the Transactions of the Belfast Natural History and Philosophical Society, Cork Cuvierian Society, Dublin Natural History Society, Dublin University Zoological Association and the Literary and Scientific Institution of Kilkenny. Vol. I. Dublin, I854: A.S.B., G.S.I., R.B.G. [Continued as :] Nutural History Reviero .... inchuding the Proceedings of the Irish Natural History Societies (and the Journal of the Geological Society of Dublin). Vols. 2, 3. London, I855-56 : A.S.B., G.S.I., R.B.G. [Continued as :] Natural History Review: a quarterly journal of zoology, botany, geology and palaeontology. Ed. by A. H. Haliday and others. Vols. 4-7. London, I857-60 (in vols. 5-7 the sub-title is $A$ quarterly journal of science): A.S.B., G.S.I., R.B.G. [Continued as :] Natural History Reviere: a quarterly journal of biological science. Ed. by G. Busk and others. Vols. I-5. London and Edinburgh, I86I65 : A.S.B., G.S.I., R.B.G., Z.S.I.

23. - Natural History Society (see also No. 22).

Proceedings. Vol. 4, I862-65. Dublin, I865: A.S.B.

24. - Royal Academy of Medicine in Ireland.

Transactions. Vols. 7-. Dublin, I889-(vols. 9, II, I89I, I893 wanting) : C.U.

M.o. = Meteorological Office, Alipore.

M.Ph. = Medical College, Physiological Laboratory.

P.C. = Presidency College.

P.o. = Patent Office.

R.B.G. = Royal Botamc Garden

S.C.C. = Scottish Churcnes College.
S.G. = Surgeon-General's Library.

S.1. = Survey of India.

S.T.M. = scnool of Tropical Medicine.

T.D. = Telegraph Deparment.

X.C. =st. Xavier s College.

z.s.1. = Zoological Survey of India. 
25. Dublin.-Royal Dublin Society.

(a) Economic Proceedings. Vols. I-. Dublin, I899-.

Complete sets: G.S.I., M.O.

Vols. I-, I899- (pt. 2, vol. I wanting) : A.S.B.

(b) Journal. Vols. I-7, I856-78. Dublin, I858-78: G.S.I.

(c) Scientific Proceedings. New ser., vols. I-. Dublin, (I877) I878-. Index to vols. I-8.

Complete set: G.S.I.

Vols. I-, I878- (vols. 3, 4, 9, I defective) and index: A.S.B.

Vols. I2, I3, I9IO-II (rol. 12 defective): P.C.

(d) Scientific Transactions. Ser. 2, vols. I-9. Dublin, I877-I909: A.S.B., G.S.I. Index to vols. I-7 : A.S.B.

26. - Royal Geographical Society of Ireland.

Journal. New ser., vols. I-8. London and Dublin, I86787 (vols. $2,3,7,8$ defective) : A.S.B.

27. — Royal Geological Society of Ireland.

(a) Journal of the Geological Society of Dublin. Vols. I-Io. Dublin, I838-64. [See also No. 22. Continued as (b).]

Vols. I-IO, I838-64 (pt. I, vol. I wanting) : G.S.I.

Vols. 2-6, $1843-56$ : A.S.B.

(b) Journal of the .... Society .... Vols. I-8. Dublin, I867-87 (vol. 7 defective) : G.S.I.

28. - Royal Irish Academy.

(a) Cunningham Memoirs. By J. Casey, D. J. Cunningham and others. Nos. I-Io. Dublin, I880-94.

Nos. I-IO, I 880-94 (nos. 4, 9 wanting) : A.S.B.

Nos. I-7, I880-92 : G.S.I.

No. 7, I 892 : Z.S.I.

(b) Proceedings. Vols. I-Io. Dublin, I84I-70. Index to vols. I-7 at end of vol. 7. [Continued in two parts as $(c)$ and $(d)$.]

Vols. I-IO, I84I-70 (vol. 9 defective) : A.S.B., G.S.I.

(c) Proceedings. Ser. 2, Science. Vols. I-4 (=vols. III4). Dublin, I870-88: A.S.B., G.S.I. [Continued as (e).]

(d) Proceedings. Ser. 2, Polite literature and antiquities. Vols. I, 2 ( $=$ vols. 15, I6). Dublin, I879-88: A.S.B., G.S.I. [Continued as (e).]

(e) Proceedings. Ser. 3. Vols. I-7 (= vols. I7-23). Dublin, I889-I902. [Continued in three parts as $(f),(g)$ and $(h) . j$

\footnotetext{
A.S. = Archaeological Section, Indian Museum A.S B = Asiatic Society of Bengal.

B.C. = Bishop's College.

B.S.1. = Botanical Survey of India

C.E. = Chemical Examiner to the Govt. of Bengal

c.U. = Calcutta University
}

E.C. =Civil Engineering College, Sibpur:

G.S.1. = Geological Survey of India.

I L. = Imperial Library.

M.Ba. = Medical College, Bacteriological Laboratory

M.Ba. = Medical College. Biological Laboratory.

M.C. = Medical College (main library). 
28. Dublin.-Royal Irish Academy (Continued).

Vols. I-6, I889-I902 (vol. 7 wanting) : A.S.B., G.S.I.

Vols. I-7, I889-I902 (vols. I, 2, 6 defective) : Z.S.I.

(f) Proceedings. Ser. 3 , sect. A. Mathematical, astronomical and physical science. Vols. 24-. Dublin, I902- (vol. 28 wanting, other vols. defective): A.S.B.

(g) Proceedings. Ser. 3, sect. B. Biological, geological and chemical science. Vols. 24-. Dublin, I902-.

Complete set : G.S.I.

Vols. 24-, I902- (vols. 25, 26 defective) : A.S.B.

Vols. 24-, I902- (vols. 28, 29 defective) : Z.S.I.

(h) Proceedings. Ser. 3, sect. C. Archaeology, linguistic and literature. Vols. 24-. Dublin, 1902-.

Vols. 24-, I902- (vols. 24,27 defective) : A.S.B.

Vols. 24-, I902- (vols. 28-30 defective) : Z.S.I.

(i) Transactions. Vols. I-3I. Dublin, I787-I9or. [Continued in three parts as $(j),(k)$ and $(l)$.

Vols. I-3I, I787-I9OI (vols. I5, 29 wanting) : A.S.B.

Vols. I-27, I787-I886 (vols. $6-18,20,25,26$ wanting, vol. 24 defective) : G.S.I.

Vols. I-IO, I 787 -I804 : R.B.G.

Vols. 4, 5, I790-9I : B.C.

Vols. 29-3I, I889-I90I (defective) : Z.S.I.

(j) Transactions. Sect. A. Mathematical, astronomical and physical science. Vols. 32-. Dublin, I902-: A.S.B., Z.S.I.

(k) Tranșactions. Sect. B. Biological, geological and chemical science. Vols. 32-. Dublin, I902-.

Complete sets : A.S.B., G.S.I., Z.S.I.

(l) Transactions. Sect. C. Archaeology, linguistic and literature. Vols. 32-. Dublin, I902-: Z.S.I.

(m) Transactions: Irish Manuscript Series. Vol. I, pt. I. Dublin, I880 : G.S.I.

Index to the serial publications from I786-Igo6 (I9I2) : A.S.B., G.S.I.

_- University Zoological and Botanical Association (see No. 22).

29. Dundee.-University College.-Museum of Zoology.

Studies. Ed. by D'Arcy W. Thompson. Vol. I, nos. I-

I2. Dundee, I888-90: Z.S.I.

30. Edinburgh.-Annals of Scottish Natural History. Ed. by J. A. Harvie-Brown, J. W. H. Trail and W. E. Clarke. I892-I9II.

Edinburgh, I892-I9II : R.B.G. [Continued as No. 50.]

M.O. = Meteorological Office, Alipore.

M.Ph. = Medical College, Physiological Laboratory

P.c. = Presidency College.

P.o. = Palent Office

R.B.G $=$ Royal Botanic Garden

S.C.C. $=$ Scottish Churches College.
s.G. =Surgeon-General's Library.

S.I. =Survey of India.

S.T.M. $=$ School of Tropical Medicine.

T.D. = Telegraph Department.

X.C. "=St. Xavier's College. $\cdots$

Z.s.I. =Zoological Survey of India. 
3I. Edinburgh.-Botanical Society.

(a) Transactions. Vols. I-II. Edinburgh, I844-73: R.B.G. [Continued as $(b)$.]

(b) Transactions and Proceedings. Vols. I2-. Edinburgh, I876-.

Complete set: R.B.G.

Vols. I3-I7, I879-87 : A.S.B.

Vols. 22-, I90I-: B.S.I.

32. - Clinical Studies: a quarterly journal of clinical medicine. Vols. I-6. Edinburgh, I903-08: A.S.B.

33. - Edinburgh Journal of Science. Conducted by Brewster. Vols. I-IO. Edinburgh, I824-29: R.B.G.

34. — Edinburgh Medical Journal. Vols. 22-42. Edinburgh, I87696. New ser., vols. I-22. Edinburgh, 1897-1908. New ser. (3rd ser.), vols. I-. Edinburgh, I908-.

Vols. 22-, I 876 - (no. I, vol. 29, I883, wanting) : M.C. Vols. 33-, I888- : S.T.M.

35. - Edinburgh Nere Philosophical Journal. Conducted by $\mathrm{R}$. Jameson. Vols. I-57. Edinburgh, I826-54. New ser., vols. I-I9. Edinburgh, I855-64. [A continuation of No. 36; afterwards incorporated in No. 274.]

Complete set: G.S.I.

Vol. I - new ser., vol. I9, I826-64 (vols. 39, 47-53 wanting): I.L.

Vols. I- 57, I826-54: A.S.B.

Vols. I0, 33-37, 44, I83I, '42-44, '48 : R.B.G.

36. _ Edinburgh Philosophical Journal. Conducted by D. Brewster and R. Jameson. Vols. I-I4. Edinburgh, I8I9-26. [Continued as No 35.]

Complete sets : A.S.B., G.S.I., I.L.

Vols. I-IO, I8I9-24: R.B.G.

37. - Geological Society.

Transactions. Vols. I-, I866-. Edinburgh, I870 (I868) - : G.S.I.

38. - Gypsy Lore Society.

Journal. Vols. I-3. Edinburgh, I888-92 : A.S.B. New ser., vol. I-. Edinburgh, I907-: A.S.B.

39. - Highland (and Agricultural) Society of Scotland.

(a) Prize Essays and Transactions. Ser. I, vols. I-6. Edinburgh, I799-I824: B.S.I. Ser. 2, vols. I-8. Edinburgh, I828-43: B.S.I. [Continued as (b).]

A.S. = Archaeological Section, Indian Museum.

A.S.B. = Asiatic Society of Bengal.

B.C. = Bishop's College.

B.S.I. = Botanical Survey of India.

C.E. = Chemical Examiner to the Govt. of Bengal.

C.U. = Calcutta University.
E.C. = Civil Engineering College. Sibpur.

G.S.I. = Geological Survey of India.

1 L. = Imperial Library.

M. Ba. = Medical Colle£e, Bacteriological Laboratory

M.Bi. = Medical College, Biological Laboratory.

M.C. = Medical College (main library). 
39. Edinburgh.-Highland (and Agricultural) Society .. (Continued).

(b) Transactions. Ser. 3, vols. I-II. Edinburgh, I84365 : B.S.I., R.B.G. Ser. 4, vols. I-20. Edinburgh, I 866-88: B.S.I. Ser. 5, vols. I-I3. Edinburgh, I 889 -I90I : B.S.I.

Index to ser. I-3, I799-I865 : B.S.I.

- Journal of Agriculture (see No. I89).

- Journal of Anatomy and Physiology (see No. I90).

40. - Journal of Pathology and Bacteriology. Ed. by G. S. Woodhead. Vols. I-. Edinburgh, London, I893-.

Vols. I-II, I893-I906 : M.Ba.

Vols. I-6, I893-99: S.G.

Vols. 4-, I897-: M.C.

Vols. 5-7, I898-I90I : A.S.B.

4I. - Magazine of Zoology and Botany. Conducted by W. Jardine, P. J. Selby and Dr. Johnston. Vols. I, 2. Edinburgh, London and Dublin, I837-38: R.B.G. [Afterwards united with No. II9 and continued as No. 79.]

42. - Prescriber: a monthly journal dealing with therapeutics and treatment. Vols. 4-. Edinburgh, I9IO- (vol. 7 wanting, vols. 6, 8-1 I defective): S.T.M.

- Quarterly Journal of Agriculture (see No. 268).

43. - Reviere of Neurology and Psychiatry. Vols. 3-5. Edinburgh, I905-07 : A.S.B.

44. — Royal Botanic Gardens.

Notes. Vols. 2-. Glasgow, I902- : B.S.I.

45. — Royal Observatory.

Astronomical Observations. Vols. I3, I4, I860-77. Edinburgh, I87I-77 : A.S.B.

46. — Royal Physical Society.

Proceedings. Vols. I-, I854-. Edinburgh, I858-.

Vols. I-, I $858-$ (vols. 4-8 wanting) : A.S.B.

Vols. I8一, I909-: Z.S.I.

47. — Royal Scottish Geographical Society.

Scottish Geographical Magazine. Ed. by H. A. Webster,

J. Geikie and others. Vols. I-. Edinburgh, 1885-.

Complete sets : G.S.I., I.L.

Vols. I-6, I 885-90 : A.S.B.

Vols. 4-, I888- (vol. 24 wanting) : S.I.

M o = Meteorological Office, Alipore

M Ph $=$ Medical College. Physiological Laboratory:

P.C. = Presidency College.

Po = Patent Office.

R B. G = Royal Botanic Garden.

S.C.C. $=$ Scottısh Churches College.
S.G. = Surgeon-General's Library.

S.I. = Survey of India

S.T.M. = School of Tropical Medicine.

T.D. = Telegraph Department

X.c. =St. Xavier s college.

Z.s.I. = Zoological Survey of India. 
48. Edinburgh.-Royal Scottish Society of Arts.

(a) Journal. Vols. I7, I8. Edinburgh, I906-08 (vol. I8 defective): G.S.I. [A continuation of $(b)$.]

(b) Transactions (-Proceedings). Vols. I-I6. Edinburgh: I 84I (I835)-I906 (vols. I, I4 defective) : G.S.I. [Continued as $(a)$.]

49. - Royal Society.

(a) Proceedings. Vols. I-, I832-. Edinburgh, I845-.

Vols. I-, I845- (vol. I defective) : A.S.B.

Vols. I-, I845- (vol. 21 wanting) : G.S.I.

Vols. I5-, I889-: R.B.G.

Vols. 24-, I904- : Z.S.I.

(b) Transactions. Vols. I- Edinburgh, I788-. General indexes to vols. I- $34, I_{7} 83-1888$, and to vols. $35-46$, I889-I908.

Vols. I-, I788- (vol. I4 wanting) : A.S.B.

Vols. I-, I788- (vols. 38,39 defective) and indexes: G.S.I.

Vols. I-I2, I788-I8 34 : R.B.G.

Vols. 40-, I900-: P.C., Z.S.I.

- Scottish Geographical Magazine (see No. 47).

50. - Scottish Naturalist. Ed. by W. E. Clarke and others. I9I2. Edinburgh, I9I2: R.B.G. [A continuation of No. 30; see also No. 344.]

5I. - Wernerian Natural History Society.

Memoirs. Vols. I-7. Edinburgh, I8II-38.

Complete set : G.S.I.

Vols. I-5, part I, I 8 II -24 : R.B.G.

52. Glasgow.-Fishery Board for Scotland.

Scientific Investigations. I899-. Glasgow, I900- : Z.S.I.

53. - Geological Society.

Transactions. Vols. I-8. Glasgow, I868-88 : G.S.I.

54. - Glasgore Medical Journal. Vols. 69-. Glasgow, I908-: S.T.M.

55. - Marine Biological Association of the West of Scotland.

(a) Annual Report. I905. Glasgow, I906: Z.S.I.

(b) Communications from the Millport Marine Biological Station. No. I. Glasgow, I900: Z.S.I.

A.S. = Archaeological Section, Indian Museum.

A.S.B. = Asiatic Sociely of Bengal.

B.C. = Bishop s College

B.S.I = Bolanical Survey of India.

C.E. = Chemical Examiner to the Gort. of Bengal.

c. $\mathbf{U}=$ Calcutta Universily.
E.C. = Civil Engineering College, Sibpur.

G.S.I. = Geolosical Survey of India.

l.L. = Imperial L.sbrary.

M.Ba. = Medical College, Bacteriological Laboratory

M.Bl. = Medical College, Biological Laboratory

M.C. = Medical College (main library) 
56. Glasgow.- Practical Mechanic's Journal. Ser. 2, vols. 2-6. Glasgow, I847-[62]: X.C.

57. - (Royal) Philosophical Society.

Proceedings. Vols. I-, I 84 I-. Glasgow, I $844-$ : G.S.I. Index to vols. I-20, I84I-89: G.S.I.

58. Gloucester.-Cotteswold Naturalists' Field Club.

Proceedings. I864, I869. Gloucester [I865, I870] : G.S.I.

59. Greenwich.-Royal Observatory.

(a) Reduction of Greenwich Meteorological Observations. Temperature of the Air. Part III. I84I-90-. Edinburgh, r895-: A.S.B.

(b) Results of the Magnetical and Meteorological Observations, etc. I849-. London. [I850-] I876-.

Complete set: M.O.

Vols. for I883- : A.S.B.

(c) Results of the Photo-heliographic Observations, etc. I901-. Edinburgh, I902-: M.O.

6o. Jersey.-Journal of Marine Zoology and Microscopy. Ed. by J. Hornell. Nos. I-8 (vols. I, 2). Jersey, London, I893-97 (no. 2 wanting): Z.S.I.

6I. - Observatoire St. Louis.

Bulletin des Observations Météorologiques. Ann. I-, I894-. Jersey, St. Hélier, I895- : X.C.

Kew.-Royal Botanic Gardens (see No. 284).

Kilkenny.-Literary and Scientific Institution (see No. 22).

Liverpool.-Annals of Tropical Medicine and Parasitology (see No. $68(a))$.

- Biochemical Journal (see No. 7).

62. Biological Society.

Proceedings and Transactions. -Vols. I2-. Liverpool, I898- : Z.S.I.

63. - Evans' Analytical Notes. I906-. Liverpool and London, I907一 : B.S.I.

64. Geological Society.

(a) Abstract of the Proceedings. Session I-I5, I859-74 [ $=$ vols. I, 2]. Liverpool, I863-74 : G.S.I. [Continued as $(b)$.].

M.o. = Meteorological Office, Alipore.

M.Ph. = Medical College, Physiological Laboratory.

P.C. = Presidency College.

P.o. = Patent Office

R.B.G. = Royal Botanic Garden.

S.C.C. $=$ Scottish Churches College.
S.G. = Surgeon-General's Library.

S.I. = Survey of India.

S.T.M. = School of Tropical Medicine.

T.D. = Telegraph Department.

X.C =St. Xavier's College.

Z.s.I. = Zoological Survey of India 
64. Liverpool.-Geological Society (Continued).

(b) Proceedings. Vols. 3-, I874-. Liverpool, I878 (1875)- (pts. I, 3, vol. 8, wanting) : G.S.I.

65. L Lancashire Sea-Fisheries Laboratory (and Sea-Fish Hatchery at Piel).

Report. I892-. Liverpool, I893-: Z.S.I.

66. — Literary and Philosophical Society.

Proceedings. Vols. I9-, I864/65-. Liverpool, I866-. Index to vols. I-62, I9I2.

Vols. I9-, I866- (vol. 21 wanting), and index: G.S.I.

Vols. 27-, IS73- (vols. 5I, 52 wanting), and index: A.S.B.

67. - Marine Biology Committee and Biological Station, Port Erin.

(a) Annual Report. Nos. I5-. Liverpool, I9OI- (nos I8, 20, 24, 1904, '06, '10 wanting): Z.S.I.

(b) L.M.B.C.Memoirs. Nos. 6, I3, I4, I9. London, I90I-09: Z.S.I.

68. - School of Tropical Medicine.

(a) Annals of Tropical Medicine and Parasitology. Vols. I一. Liverpool, I907-: M.Ba., S.G., S.T.M., Z.S.I.

(b) Bulletin of the Yellow Fever Bureau. Vols. I-. Liverpool, I9II- (no. 6, vol. I, wanting): S.T.M.

69. - University Institute of Commercial Research in the Tropics.

Quarterly Journal. Vols. I-3. Liverpool, I906-I908 (no. I, vol. I, wanting) : B.S.I.

70. London.-Academy: a weekly review of literature, science and art. Vols. 7-67. London, I875-I904 (vol. 41 defective) : A.S.B.

7I. - Acetylene. Vols. 2-8. London, I905-I9II (pts. I-3, vol. 5, wanting): I.L. [Continued as No. 72.]

72. - Acetylene Lighting and Welding Journal. Vol. 9. London, I9I2: I.L. [A continuation of No. 7I.]

73. — American Machinist. Vols. 29-3I. London, I906-08 : E.C.

74. - Analyst. Vols. 4-37. London, I879-r9r2.

Vols. 4-37, I879-I9I2 (vols. 17, 22, 23, 26-36, wanting): C.E.

Vols. 32, 33, 35, 1907-IO : E.C.

75. - Ancient Egypt. Ed. by Flinders Petrie. Vols. I- London and New York, I9I4-: A.S.B.

A.S. Archaeological Section. Indian Museum.

A.S.B. = Asiatic Society of Bengal.

B.C. = Bishop's College.

B.S.I. = Botanical Survey of India

C.E. = Chemical Examiner to the Govt. of Bengal.

c.U. = Calcutta University.
E.C. = Civil Engineering College, Sibpur.

G.S.I. = Geological Survey of India.

I.L. = Imperial Library.

M.Ba. = Medical College, Bacteriological Laboratory

M.BI. = Medical College. Biological Laboratory.

M.c. = Medical College (main library). 
76. London.-Annals and Magazine of Natural History (united with Loudon's Magazine of Natural History [see No. 208]). Vols. 620. London, I84I-47. 2nd ser., vols. I-20. London, I848-

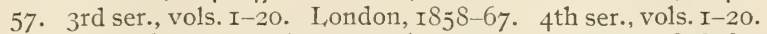
London, I868-77. 5 th ser., vols. I-20. London, I878-87. 6 th ser., vols. I-20. London, I888-97. 7 th ser., vols. I-20. London, I898-I907. Sth ser., vols. I-. London, I908-. [A continuation of No. 79.]

Complete sets : G.S.I., Z.S.I.

Vols. 6-, I84I- (ser. 2, vol. I3, ser. 6, vol. 9 wanting ; ser. 5 , vol. ${ }_{15}$, ser. 6 , vol. 20 , ser. 7 , vol. 20 , ser. 8 , vol. 6 defective) : A.S.B.

Complete set up to 5 th ser., vol. 8, I88I : R.B.G.

77. - Annals of Agriculture. Vols. I-45. London, I790-1808: R.B.G.

78. - Annals of Botany. Ed. by Balfour, Vines, Thiselton-Dyer and others. Vols. I-. Oxford, London, I887-.

Complete set : R.B.G.

Vols. 24-, I9I0-: P.C.

- Annals of Chemistry, Mathematics, etc. (see No. 260).

79. - Annals of Natural History: or magazine of zoology, botany and geology .... By IV. Jardine and others. Vols. I-5. London, I838-40: A.S.B., G.S.I., R.B.G., Z.S.I. [Afterwards combined with No. 208 and continued as No. 76 .]

So. - Annals of Philosophy. By T. Thomson and others. Vols. Ir6. London, I8I3-20: R.B.G. New ser. Vols. I-I2 (=I7-28). London, I82I-26: R.B.G. [United in 1827 with No. 260.]

Anthropological Institute (see No. 2So).

Anthropological Review (see No. SI (a)).

8I. — Anthropological Society.

(a) Anthropological Revierw. Vols. I-7. London, I863-69 (vol. 8 wanting): A.S.B. [Including Transactions, vol. I, and Journal, vols $2-7$.]

(b) Journal of Anthropology. Ed. by Beddoe, Davis and others. Vol. I, nos. I-3. London, I870-7I : A.S.B.

(c) Memoirs. Vol. 3, I86769. London, I870 : A.S.B.

- Archaeologia (see No. $3 \operatorname{II}(a)$ ).

82. - Archives of Surgery. Ed. by J. Hutchinson. Vols. I-9. London, I 889-98: S.G.

M.o. = Meteorological Office, Alipore.

M.Ph. = Medical College. Physiological Laboratory.

P.C. = Presidency College

P.o. = Patent Office.

R.B.G. = Royal Botanic Garden.

S.C.C. = Scottish Churches College.
S.G. = Surgeon-General's Library.

S.I. = Survey of India.

S.T.M. = School of Tropical Medicine

T.D. = Telegraph Department.

X.C. =St. Xavier s College.

z.s.I. =Zoological Survey of India. 
83. London.- Artizan : a monthly record of the progress of civil and mechanical Engineering. Ed. by W. Smith. Vols. 22-26. London, I 864-68 : S.G.

— Asiatick Researches (see No. I048(a-c)).

—_ Astronomical Society (see No. 283).

84. - Athenaeum: journal of literature, science and the fine arts (music and drama). 1834-. London, I834-.

Vols. for $1834-\left(1838,{ }^{\prime} 44,{ }^{\prime} 47,{ }^{\prime} 58, ' 68\right.$ wanting; 1835 defective): I.L.

Vols. for I $840-\left(1843,{ }^{2} 48-49\right.$ wanting; I866, I906, '08 defective) : A.S.B.

Vols. for I 855- March, I906 : G.S.I.

Vols. for $1857-64$ : E.C.

Vols. for $1895^{-1902}$ : X.C.

- Biochemical Journal (see No. 7).

85. - Board of Agriculture.

Journal. Vols. I-. London, I894-. General indexes to vols. I-IO and to vols. II-I7.

Complete sets, with indexes : B.S.I., R.B.G.

Vols. I3-I6, I906-Io : E.C.

86. — Board of Trade.

Journal. Vols. I2-. London, I892- (vol. I2 defective, vols. 22,23 wanting) : B.S.I. General indexes to vols. II4 and to vols. $15^{-20}$ : B.S.I.

87. - Botanical Cabinet: consisting of coloured delineations of plants, from all countries, with a short account of each .... By C. Loddiges and Sons. Vols. I-20. London, I8I7-33: R.B.G.

88. - Botanical Magazine, or Flower-Garden displayed. [By W. Curtis.] Vols. I-I4. London, I777-I80o: R.B.G. [For continuation and index see No. I22.]

89. - Botanical Miscellany: containing figures and descriptions of .... plants .... with occasional botanical notices and infor. mation. By W. J. Hooker. Vols. I-3. London, I830-33: R.B.G. [Continued as No. IgI.]

90. — Botanical Register. By S. Edwards and others. Vols. I-I4. London, I8I5-28: R.B.G. [Vol. I4 is also styled 'New ser., vol. I.' Continued as No. 126.]

9I. - - Botanical Society.

Proceedings. Part i. London, I839: A.S.B.

A.S. = Archaeological Section, Indian Museum

A.S.B. = Astatic Society of Bengal

B.C. = Bishop s College.

B.S.1. = Botanical Survey of India

C.E. = Chemical Examiner to the Govt. of Bengal

c.U. Calcutta University.
E.C. = Civil Engineering College, Sibpur

G.S.I. = Geological Survey of India.

I.L. = Imperial Library.

M.Ba. = Mledical College, Bacteriological Laboratory

M.Bl. = Medical College. Biological Laboratory.

M.C. = Medical College (main library). 
92. London.-Botanist's Repository: comprising colour'd engravings of new and rave plants only, with botanical descriptions ..... By H. Andrews. Vols. I-IO (plates I-664). London, I797 (I799)[I8II] : R.B.G.

93. - Brain: a journal of neurology. Vols. I- London, I878-. Complete set : C.U.

Vols. 28, 29, I905-06 : A.S.B.

- Braithwaite's Retrospect of Medicine (see No. 278).

94. - British and Colonial Druggist. Vols. 37-. London, Igoo-: B.S.I.

95. - British and Foreign Medico-Chirurgical Review and Quarterly Journal of Practical Medicine and Surgery. Vols. I-5I. (=Nos. I-IO2). London, I848-73.

Vols. I-47 (=Nos. I-94), I848-7I (many nos. wanting) : S.G.

Vols. 9-5I (=Nos. I7-IO2), I852-73 (vol. 3 I, = nos. 6r, 62 , wanting): I.L.

96. - British Association for the Advancement of Science.

Report. Nos. I-, I83I-. London, I833-.

Complete sets : C.U., G.S.I., I.L.

Vols. for I $83 \mathrm{I}$ - (vols. for 1840 , ' 53 wanting), index to vols. for I $86 \mathrm{I}-90$ : A.S.B.

Vols. for I842, '48, '5 I, '55, '6I : S.G.

Vols. for I85I-60 and for I9I2- : E.C.

97. — British Astronomical Association.

Journal. Vols. I-. London, I890-.

Complete set : C.U.

Vols. I-I8, I890-I908 : P.C.

98. — British Ecological Society.

Journal of Ecology. Vols. I-. London, I9I3- : B.S.I.

— British Economic Association (see No. 287).

99. - British Flower Garden: containing coloured figures and descriptions of the most ornamental and curious ... plants, etc. By R. Sweet. Vols. I-3. London, I823-38 : R.B.G.

I0o. - British Journal of Ophthalmology. Ed. by S. Stephenson. Vols. I-. London, IgI7- : S.T.M.

Ior. British Journal of Tuberculosis. Ed. by T. N. Kelynack. Vols. I-. London, I907-: S.G.

M.O. = Meteorological Office, Alipore.

M.Ph. = Medical College, Physiological Laboratory.

P.C. = Presidency College.

P.O. = Patent Office.

R.B.G. = Royal Botanic Garden

S.C.C. $\Rightarrow$ Scuttish Churches College.
S.G. = Surgeon-General's Library:

S.I. = Survey of India.

S.T.M. = School of Tropical Medicine

T.D. = Telegraph Department

X.c. =St. Xavier s College.

Z.S.I. = Zoological Survey of India 
I02. London.-British Medical Association.

British Medical Journal. London, I876-. [Two vols. are issued annually.]

Vol. I of 1876 - (vol. I of 1880 , vols. I, 2 of 189 I, ' 92 wanting) : I.L.

Vol. I of I877- (vol. I of 1884 and vols. I, 2 of 1879, '82, '83, '85 wanting): M.C.

Vol. 2 of I880-(vol. I of I883,'87 and vol. 2 of 1888 wanting): S.G.

Vol. 2 of I $88 \mathrm{I}$ - : S.T.M.

Vol. I of I9I4-: B.S.I.

I03. — British Ornithologists' Union.

Ibis. Ed. by Sclater, Newton and Salvin. Vols. I-6. London, I859-64. New ser., vols. I-6. London, I 865-70. Ser. 3, vols. I-6, I87I-76. Ser. 4, vols. I-6. London, I $877-82$. Ser. 5, vols. I-6. London, I883-88. Ser. 6, vols. I-6. London, I889-94. Ser. 7, vols. I-6. London, I895-I90o. Ser. 8, vols. I-6. London, I90I-06. Ser. 9, vols. I-6. London, I907I2. Ser. Io, vols. I-. London, I9I3-.

Complete set : Z.S.I.

Vols. 6, I864- ser. 8, vol. 3, I903: A.S.B.

104. British Pharmaceutical Conference.

Year-Book of Pharmacy and Transactions of the .... Conference. I87I-. London, I87I-: B.S.I. General index to the Year-Book, 1886-I903 : B.S.I.

I05. - Builder : a record of architecture and construction. Vols. I5-. London, 1857- (vols. for I865, '66, '70, '85 wanting): E.C.

- Bulletin of Entomological Research (see No. I72 (a)).

- Carnegie Scholarship Memoirs (see No. I88 (a)).

I06. - Cassier's Engineering Monthly. Vols. 3I-. London, I906-: E.C.

107. Challenger Society.

Memoirs. No. I. London, I909: Z.S.I .

_ Charlesworth's Magazine (see No. 20S).

108. - Chemical News and Journal of Physical Science. Ed. by W. Crookes. Vols. I7-. London, I868-. Index to vols. I-Ioo.

Vols I7-75, I868-97 (many vols. defective) : C.E.

Vols. 33-, 1876- and index: G.S.I.

A.s. = Archaeological Section. Indian Museum.

A.S.B. = Asiatic Society of Bengal

B.C. = Bishop s College.

B.S.I. = Botanical Survey of India.

C.E. = Chemical Examiner to the Govt. of Bengal.

c.U. = Calcutta University.
E.C. =Civil En

G.S I. = Geological Survey of India.

I.L. = Imperial Library.

M. Ba. = Medical College, Bacteriological Laboratory

M.BI. = Medical College. Biological Laboratory.

M.C. = Medical College (main library). 
108. London.-Chemical Nerws ... (Continued).

Vols. 33-, I876-(vols. 57, 66, 89, 99, 102, 105, 106 defective) : A.S.B.

Vols. 50-94, I884-I906 (vols. 75,76 wanting) : B.S.I.

I09. Chemical Society.

(a) Anmual. Report on the Progress of Chemistry. Vols. I-, I904-. London, I905-.

Complete sets : G.S.I., I.L.

Vols. 5-8, for I908-II : E.C.

Vols. 6-, I909 : S.C.C.

(b) Journal. Vols. 15-. London, I862-. [Vols. 16-28 are also styled 'New series, vols. I-I3.' A continuation of (c).]

Coimplete set: P.C.

Vols. I5-28, I862-75 (vol. 22 defective) : A.S.B.

Vols. I5-56, I862-89 (vols. I6-23 and 29-44 wanting): C.E.

Vols. 24-, I87I- : s.c.c.

Vols. 24-, I87I- (vols. $35-38$ and $43-46$ wanting): G.S.I.

Vols. 93-, I908- : E.C.

(c) Quarterly Journal. Vols. I-I4. London, I849-62. [Continued as $(b)$.]

Complete set : P.C.

Vols. II-I4, I859-62 : A.S.B.

Indexes, I84I-I9I2: G.S.I., P.C.

IIo. Chemical World: a monthly journal of chemistry and chemical engineering. Vol. I. London, I9I2: B.S.I.

III. - Chemist. Vols. I, 2. London, I824-25: G.S.I. New ser., vols. 3-5, London, I856-58 : C.E.

II2. - Chemist and Druggist. Vols. 34-. London, I889-.

Vols. 34-, I889- (vols. 4I, 47-49, 5I, 59 wanting) :

R.B.G.

Vols. 36-47, I890-95 (vol. 44 wanting) : C.E.

Vols. 5 [一, I897- (vols. 54,55 wanting) : B.S.I.

Vols. 78, 79, I9II : I.L.

II3. - Cistula Entomologica. Vols. I-3. London, I869-85 (vol. 3 defective) : Z.S.I.

II4. Civil Engineer and Architect's Journal. Vols. I-I9. London, I837-66 : E.C.

II5. Clinical Excerpts. Vols. I3-I5. London, I909-II (defective) : S.T.M.

M.O. = Meteorological Office, Alipore.

M.Ph. = Medical Collere, Physiological Laboratory.

P.C. = Presidency College.

P.O. = Patent O ince.

R.B.G = Royal Botanic Garden.

S.C.C. $=$ Scottish Churches College.
S.G. = Surgeon-General s Library.

s.l. = Survey of India.

S.T.M. = School of Tropical Medicine.

T.D. = Telegraph Department.

X.c. =St. Xavier's College.

Z.S.I. = Zoological Survey of India. 
II6. London.-Clinical Journal. Vols. I4-2I. I,ondon, I899-I903:

S.G. Vols. 3I-. London, I907-: M.C.

II7. Clinical Society.

Transactions. Vols. I-. London, I868-: C.U.

II8. Colliery Guardian and Journal of the Coal and Iron Trades. Vols. 3I-. London, 1876 -.

$$
\begin{aligned}
& \text { Vols. } 31-, \text { I } 876-\text { : G.S.I. } \\
& \text { Vols. } 89-\text {, I905-: I.L. } \\
& \text { Vols. } 92-\text { I I906-: E.C. }
\end{aligned}
$$

II9. Companion to the Botanical Magazine .... By W. J. Hooker, Vols. I, 2. I.ondon, I835-36: R.B.G. [Afterwards united with No. $4 \mathrm{I}$ and continued as No. 79.]

I20. - Conchological Society of Great Britain and Ireland.

Journal of Conchology (with which is incorporated the Proceedings of the .... Society....). Conducted by J. W. Taylor. Vols. 2-7. London, I879-94: A.S.B. [Continued as :] Journal of Conchology (published under the direction of the .... Society ....). Vols. 8-Io. London, I895-I903 : A.S.B. [For vol. 1 see No. 269.]

I2I. - Concrete and Constructional Engineering. Vols. 2-. London, Ino7- : E.C.

- Country Folklore (see No. I50 (a)).

I22. Curtis's Botanical Magazine. Conducted by J. Sims, S. Curtis, W. J. and J. D. Hooker. Vols. I5-. London, I80I- : R.B.G. [Vols. $43-53$ are also styled ' $\mathrm{New}$ ser., vols. I-II'; similarly vols. 54-70 are 'New ser., vols. $1-17$,' vols. $71-130$ are 'Ser. 3 , vols. $1-60$ ' and vols. I3I- are 'Ser. 4, vols. I-.' For earlier vols. see No. 88 and for supplementary vols. see No. I I9.] Subject index to Botanical Magazine, vols. I-I4 and to Curtis's Botanical Magazine, vols. I5-53, I 790-I826: R.B.G. Index to vols. I-IO7, ed. by E. Tonks, I883: R.B.G. Index to Ist, 2nd and 3 rd series (vols. I-I30), by IV. Botting Hemsley, Ig06: R.B.G.

123. - Dissertations and Miscellaneous Pieces relating to the History and Antiquities, the Arts, Sciences and Literature of Asia. By Sir William Jones and others. Vols. I-3. London, I792-96: R.B.G.

I24. - East India Association.

Journal. Vols. I-22. London, I867-93.

Vols. I-22, I 867-93 (defective) : I.L.

Vols. 7-9, I873-75 (vol. 9 defective) : G.S.I.

\footnotetext{
A.S. = Archaeological Section, Indian Museum.

A.S.B. = Asiatic Society of Bengal.

B.C. = Bishop's College.

B.S.1. = Botanical Survey of India.

C.E. = Chemical Examiner to the Govt. of Bengal.

c.U. = Calcutta University.
}

E.C. = Civil Engineering College, Sibpur.

G.S.I = Geological Survey of India.

I.L. = Imperial Library

M.Ba. = Medical College, Bacteriological Laboratory

M.B1. = Medical College, Biological Laboratory.

M.C. = Medical College (main library). 
London.-Economic Journal (see No. 287).

I25. Economic Reviero. Editors, IV. J. H. Campion, J. Carter and others. Vols. I-22. London, I89I-I9I2: C.U.

I26. - Edward's Botanical Register. By J. Lindley. Vols. 15-33. London, I829-47 : R.B.G. [Vols. 15-23 are also styled 'New ser.. vols. 2-10.' For earlier rols, see No. 90.]

I27. - E Egypt Exploration Fund.

Memoirs. Nos. 2-I2. London. I885-94: A.S.B.

I28. - Electrical Engineering. Vols. I-4. London, I907-08: T.D

I29. - Electrical Industrial Supplement. 8 vols. I.ondon, I906-Io : T D.

I30. - Electrical Reviere. Vols. 30-. London, I892-. [A continuation of No. 316.]

Vols. 30-, I892- (vols. 32, 33, 41-53 defective) : I.L.

Vols. 30-7I, I892-I9I2 : T.D.

I3I. — Electrician. Vols. 7-. London, I88I-.

Vols. 7-, I88I-: T.D.

Vols. I6-, I 885 - : P.C.

Vols. 26-, I890- : E.C.

Vols. 55-, I905-: I.L.

132. - Electro-Chemist and Metallurgist and Metallurgical Review. By S. Cowper Coles. Vols. I-4. London, I90I-04 (defective) :

G.S.I. [Continued as No. I46.]

I33. - Engineer. Vols. I-. London, I856-.

Vols. I-, I856- (vols. $18-22,28,32,33,36,100,102$ wanting): E.C.

Vols. I-39, I856-75 : I.L.

Vols. I-I5, I856-63 (vols. 2, 12 wanting) : X.C.

I34. - Engineering. Vols. I-. London, I866-.

Vols. I-60, 79-, I866-95, I905- : I.L.

Vols. 4-, I867-(vols. 6, 8, 13 wanting): E.C.

135. - Engineering Magazine (see No. I437).

136. - Engineering Review. Vols. I-3. London, I893-96 : I.L.

137. - English Mechanic and World of Science. Vols: 22-. London, 1876 -

Yols. 22-, I876- (vols. 22, 23, 24, 26 defective, vols. $45,47,48$ wanting) : X.C.

Vols. 43-58, r886-94: Z.S.I.

Vols. 54-, I892- : E.C.

M.o. = Meteorological Office. Alipore.

M.Ph. = Medical College. Physiological Laboratory.

P.C. = Presidency Collesge.

P.O. = Patent Office.

R.B.G. = Royal Botanic Garden.

S.C.C. $=$ Scottish Churches College.
S.G. =Surgeon-General's Library.

S.1. = Survey of India.

S.T.M. = School of Tropical Medicine

T.D. = Telegraph Department.

X.c. =St. Xavier's College.

Z.S.I. = Zootogical Survey of India. 
I38. London.-Entomological Magazine. [E.d. by E. Newman.] Vols. I-3. London, I833-36: A.S.B.

Entomological Research Committee (see No. I72).

I39. E Entomological Society.

Transactions. Vols. I-5. London, I836-49: Z.S.I. New ser., vols. I-5. London, I850-6I : Z.S.I. Third ser., vols. I-5. I London, I\$62-67: Z.S.I. Fourth ser. I868-. I.ondon, I 868 - : Z.S.I.

I40. - Entomologist. Conducted by E. Newman and others. Vols. 2-. London, I864- (vols. 4, 6, 33 wanting: vols. 34, 46 defective): A.S.B.

I4r. - Entomologist's Annual. Ed. by H. T. Stainton. I862, '63. London, I862, '63: A.S.B.

I42. - Entomologist's Monthly Magazine. Conducted by 'T. Blackburn, H. G. Knaggs and others. Vols. I-. London, I864 — (vol. 47 defective) : A.S.B. [Vols. 26-, I890-, are also styled 'New set., vols. I-'.]

I43. - Entomologist's Record and Journal of Variation. Vols. ILondon, I890- : Z.S.I.

I44. E Epidemiological Society.

Transactions. New ser, vols. 18-26. London, 1898I907: A.S.B. Index for I855-I900, I902: A.S.B. [For earlier series see No. 272.]

I45. Ethnological Society.

(a) Journal. Vols. I-4. Edinburgh, I848-56.

Vols. I-4, I848-56 : Z.S.I.

Vol. I, I848 : I.L.

(b) Transactions. New ser., vols. I-7. London, I86I69 .

Vols. I-7, I 86I-69: 'Z.S.I.

Vols. 2-5, I863-67 : I.L.

- Evans' Analytical. Notes (see No. 63).

146. - Faraday Society.

Transactions. Vols. I-. London, I905-: A.S.B., G.S.I. [A continuation of No. 132.]

147. - Floral Cabinet and Magazine of Exotic Botany. Conducted by G. B. Knowles and F. Westcott. Vols. I, 3. London, I838-40 : R.B.G.

A.S. = Archaeological Section. Indian Museum.

A.S.B. = Asiatic Society of Bengal,

B.C. = Bishop's College.

B.S.l. = Botanical Survey of India

C.E. = Chemical Examiner to the Govt. of Bengal.

c.U. = Calcutta University.
E.C. = Civil Engineering College, Sibpur.

G.S.I. = Geolosical Survey of India.

I.L. = Imperial Library.

M.Ba. = Medical College. Bacteriological Laboratory.

M.BI. = Mledical Cnllege. Biological Laboratory.

M.c. = Medical College (main library). 
I48. London.-Floral Magazine: figures and descriptions of ... new flowers for the garden .... By H. H. Dombrain (and afterwards) IV. G. Smith. New series. 3 rols. (plates 49-I92). London, $1873-75$ : R.B.G.

I49. - Folia Therapeutica. Ed. by A. Baginsky and J. Snowman. Vols. 2, 3. I,ondon, Igo8-00: S.T.M.

I50. — Folklore Society.

(a) Country Folklore. Vols. 5-. London, Igo8-: A.S.B.

(b) Folklore Journal. Tols. I-4. London, I883-86 : I.L.

(c) Folklore Record. Vols. I-5. London, I878-82 : I.L.

(d) Transactions of the ... Socicty: Folklore, a quarterly tradition, etc. Vols. I6-. London, I905- (vol. 23 wanting): A.S.B.

I5I. - Gardener: a magazine of horticulture and floriculture. Ed. by D. Thomson. I872-75. London, $1872-75$ : R.B.G.

I52. - Gardener's Chronicle (and Agricultural Gazette). Ed. by Lindley, Masters and Moore. I84I-73. London, I84I-73: R.B.G. New ser., vols. I-26. London, I874-86: R.B.G. Ser. 3, vols. I-. London, I 887 - : R.B.G.

I53. - Gardener's Magazine. Conducted by J. C. Loudon. Vols. II9. London, I826-43: R.B.G.

\section{- Geographical Journal (see No. 288(a)).}

I54. - Geographical Magazine. Ed. by Clements R. Markham. Vols. I-5. London, I874-78. [A continuation of No. 247.]

Complete sets : A.S.B., G.S.I.

Tols. I-5, I $874-78$ (vols. 4,5 defective) : I.L.

I55. Geological Magazine, with which is incorporated 'The Geologist.' Ed: by 'T. R. Jones and H. Woodward. Vols. I-Io. London, I864-73 Decade 2, vols. I-Io. London, I874-83. Dec. 3, vols. I-IO. London, I884-93. Dec. 4, vols. I-IO. London, I894-I9o3. Dec. 5, vols. I-IO. London, I904-I3. Dec. 6, vols. I-. London, I9I4-. Index for I864-I903, I 905 .

Complete set and index : G.S.I.

Vols. I-, I864- (dec. 4, vol. I and dec. 5, vols. 2-6 wanting) : P.C.

I56. - Geological Record. Ed. by IV. Whitaker and others. I87484. London, I875-89: G.S.I. 
I57. London.-Geological Society.

(a) Proceedings. Vols. I-4. I826-45. London, I834-46: A.S.B., G.S.I.

(b) Quarterly Journal. Vols. I-. London, I845-. Index to vols. I-50, I897.

Complete set and index: G.S.I.

Vols. I-, I845- (vols. $27,48,57,59$ defective) and index: A.S.B.

Vols. I-, I 845 - (vols. 22-24, 39 wanting, vol. 48 defective) and index: I.L.

Vols. 3-, I 847 - (vol. 65 wanting) : P.C.

(c) Transactions. Vols. I-5. London, I8II-2I: A.S.B., G.S.I. 2nd ser., vols. I-7. London, I824-56 : A.S.B., G.S.I.

(d) Geological Literature added to the Geological Society's Library. I894-. London, I894-.

Vols. for I894-- : G.S.I.

Vols. for I8g6- : I.L.

I58. - Geological Survey of Great Britain.

Memoirs of the ... Survey .... and of the Museum of Economic Geology in London. Vols. I-4, pt. I. London, I $846-72$. [Other rols, not serially numbered.]

Complete set: G.S.I.

Vols. I-4, pt. I, I846-72 (vol. 3 wanting): A.S.B.

I59. - Geologist: a popular monthly magazine of genlogy. Ed. by S. T. Mackie. Vols. I-7. London, I858-64: G.S.I. [Afterwards incorporated with No. 155.$]$

I6o. - Geologist: being a record of investigations in geology, mineralogy, etc. for the years I842, '43. Eid. by C. Moxon. 2 vols. London, I $842-43$ : G.S.I.

I6I. - Geologists' Association.

Proceedings. Vols. I-. London, I865-(vols. 7, 21 defective) : G.S.I. Index to vols. I-20, I9IO: G.S.I.

I62. - Glacialists' Association.

Glacialists' Magazine. Ed. by P. F. Kendall. Vols. I-4. London, I893-96 (defective) : G.S.I.

I63. - Grevillea: a monthly (quarterly) record of cryptogamic botany and its literature. E.d. by M. C. Cooke (and afterwards) G. Massee. Vols.1I-22. London, I872-94: R.B.G.

A.S. Archaeological Section, Indian Museum.

A.S B = Asiatic Society of Bengal.

B.C. = Bishop s College

B.S.I. = Eotanical Survey of India

C.E. = Chemical Exammer to the Govt. of Bengal

c. $U$ = Calcutta University.
E.C. = Civil Engineering College, Sibpur. G.S.I. = Geological Survey of India.

i = Imperial Library

L. = Medical College, Bacteriological Laboratory

M.B1. = Medical College. Biological Laboratory.

M.C. = Medical College (main library). 
I64. London.-Guy's Hospital.

Gazette. Vols. 22-27. London, I908-I4 : S.T.M.

I65. - Half-yearly Abstract of the Medical Sciences. Ed. by Ranking. Vols. I-58. London, I845-73 (vol. I3 wanting) : M.C.

I66. — Heart. Vols. I-5. London, I909-I4 : M.C.

I67. - Hooker's Icones Plantarum: or figures, with descriptive characters and remarks, of new and rare plants, selected from the Kerw Herbarium. Ed. by J. D. Hooker [and afterwards (for the Bentham Trustees)] by D. Oliver and W. T. Thiselton Dyer. Vols. II-. London, I867-: R.B.G. [Vols. II-20 are also styled ' 3 rd series, vols. I-IO' and vols. 2 I- ' 4 th series, vols. I-'. For earlier vols. see No. i7o.]

I68. - Hooker's Journal of Botany and Kew Garden Miscellany. Ed. by W. J. Hooker. Vols. I-9. London, I849-57 : R.B.G. [A continuation of No. 207.]

- Horticultural Society (see No. 289).

I69. - Hospital. Vols. 44, 49-5I. Iondon, I908-II (defective): S.T.M.

- Ibis (see No. I03).

I70. - Icones Plantarum: or figures, with brief descriptive characters and remarks, of new or rare plants, selected from the author's herbarium. By W. J. Hooker. Vols. I-IO. I.ondon, I83754: R.B.G. [Vols. 5-10 are also styled 'New Series, vols. I-6'; continued as No. 167.]

I7I. - Illustrated Medical Neres. Vols. I-5. [London], I888-89: A.S.B.

172. - Imperial Bureau of Entomology.

(a) Bulletin of Entomological Research. Issued by the Entomological Research Committee. Vols. I-3. London, I9IO-I2: S.G., Z.S.I. [Continued as:] Bulletin of Entomological Research. Issued by the .... Bureau .... Vols. 4-. London, I9I3-: S.G., Z.S.I.

(b) Reviere of Applied Entomology. Series A, Agricultural. Vols. I-. London, I9I3-: Z.S.I. Series B, Medical and Veterinary. Vols. I-. London, I9I3-: Z.S.I.

I73. — Imperial Institute.

(a) Annual Report, Indian Section, for 1896/97-I906/07. London, I897-I907. B.S.I., G.S.I., I.L.

M o. = Meteorological Office. Alipore

M.Ph. = Medical College. Physiological Laboratory.

P.C. = Presidency College.

P.o. = Patent Oifice.

R.B.G. = Royal Botanic Garden

S.C.C. $=$ Scistish Churches College.
S.G. = Surgeon-General's Library.

S.I. = Survey of India.

S.T.M. = School of Tropıcal Medicine

T.D. = Telegraph Department.

X.c. =St. Xavier s College.

z.s.I. = Zoological Survey of India 
I73. London.-Imperial Institute (Continued).

(b) Bulletin. Vols. I-. London, I903-.

Complete sets : B.S.I., G.S.I.

Vols. II-, I9I3- : E.C.

(c) Colonial Reports-Miscellaneous. Nos. 6o, 79-8I, 83, 85-87. London, I9II-I4: G.S.I.

(d) Imperial Institute Journal. Vols. I-8. London, I895Ig02: B.S.I. [Continued as (b).]

(e) Report. I906-. London, I908- : B.S.I., G.S.I.

I74. — Imperial Institute.-Tropical Diseases Bureau.

Tropical Diseases Bulletin. Vols. I-. London, I9I2-.

Complete sets: M.C., R.B.G., S.G., S.T.M.

Vols. 9-, I9I7-: M.Ba.

- Indian Annals and Magazine of Natural Science (see No. I035).

— Indian Annals of Medical Science (see No. Io69).

I75. - Industries and Iron. Tols. I5-28. London, I893-I90o (vol. 28 defective) : G.S.I.

I76. - Insecta Transwaaliensia: a contribution to a knoweledge of the entomology of South Africa. By W. I. Distant and others. Vol. I. L London, I900-II : Z.S.I.

I77. — Institute of Patent Agents.

Transactions. Vols. I-32. London, I882-I9I4 (vol. 8 wanting) : P.O.

I78. - Institution of Civil Engineers.

(a) Minutes of Proceedings. Session I837-. Vols. I-.

London, I837-. Indexes to vols. I-I70.

Complete set : E.C.

Vols. 3-I53, I844-I903 (vols. 5, I8, 101, 103-106, 108IIO, 132 wanting) : A.S.B.

Vols. 2I-, I862-, and indexes : G.S.I.

Vols. 29-, I870- (vol. 103 wanting), and indexes : I.L.

(b) Transactions. Vols. I-3. London, $1836-42$.

Vols. I-3, I836-42: I.L.

Vol. I, I836 : A.S.B.

I79. - Institution of Electrical Engineers (see also No. 305).

Journal. Vols. I8-. London, I889-. [A continuation of No. 313.]

A.S. = Archaeological Section, Indian Museum.

A.S.B. = Asiatic Society of Benga!

B.C. = Bishop's College.

B.S.I. = Botanical Survey of India

C.E. = Chemical Examiner to the Govt. of Bengal.

c.U. = Calcutta University
E.C. =Civil Engineering College, Sibpur.

G.S.I. = Geological Survey of India

I.L. = Imperial Library.

M.Ba. = Medical College, Bacteriological Lavoratory

M.Bi. = Medical College. Biological Laboratory.

M.C. = Medical College (main library). 
I79. London.-Institution of Electrical Engineers (Continued).

Vols. I 8-4I, I889-I908: X.C.

Vols. I8-40, I889-I908: A.S.B.

Vols. 30-48, I90I-I 2 : C.U.

Vols. $44-$, I9IO- : E.C.

I8o. - Institution of Mechanical Engineers.

Proceedings. 1857 - Birmingham and London, $1857-$ General indexes to vols. for I847-I90o.

Vols. for I857-Igo8 (vol. for I884 defective) and indexes: A.S.B.

Vols. for I884- (vol, for 1905 wanting) : E.C.

I8I. - Institution of Mining and Metallurgy.

Transactions. Vols. I-. London, I892- : E.C., G.S.I. General and personal index to vols. I-I5, I892-I906: G.S.I.

- Institution of Mining Engineers (see No. 336).

I82. — Institution of Petroleum Technologists.

Journal. Ed. by W. H. Dalton. Vols. I- London, I9I5-: G.S.I.

183. - Intellectual Obscrver: a reviewe of natural history, microscopic research and recreative science. Vols. 2-9. London, I863-66: A.S.B.

International Catalogue of Scientific Literature (see No. I59i).

I84. - International Engineering Congress.

Proceedings. rgoI-. London, I902-: I.L.

185. - International Sugar Journal, with which is incorporated the 'Sugar Cane.' Vols. 4-. London, I902-: B.S.I.

I86. — Iron (a newe series of the 'Mechanic's Magazine'). Vols. II-4I. London, I878-93. [A continuation of No. 215, afterwards incorporated in No. 175 .]

Vols. I I-30, I878-87 (vol. I4 wanting) : I.L.

Vols. I3-4I, I879-93 (vols. 3I, 32 wanting) : G.S.I.

I87. - Iron and Coal Trades Review. Vols. 70-. London, I905-.

Vols. 70-, I905-: G.S.I.

Vols. 72-76, 1906-08 (vol. 73 wanting) : E.C.

I88. — Iron and Steel Institute.

(a) Carnegie Scholarship Memoirs. Vols. I-. London, I909-: P.O.

M.O. = Meteorological Office, Alipore.

M.Ph. = Aledical College, Physiological Laboratory

P.C. = Presidency College.

P.O. = Patent Office.

R.B.G = Royal Botanic Garden

S.C.C. $=$ Scottish Churches College.
S.G. = Surgeon-General's Library.

S.I. = Survey of India.

S.T.M. = School of Tropical Medicine

T.D. = Telegraph Department.

X.C. =St. Xavier's College.

Z.S.I. = Zoological Survey of India. 
188. London.-Iron and Steel Institute (Continued).

(b) Journal. I872-. London, I872-. [Vols. 43-, 1893-, bear vol. nos.] Indexes for I869-I9oo.

Vols. for $1872-$ (vol. for 189 I defective) and indexes : G.S.I.

Vols. for $1882-$ : P.O.

Vols. for $\mathrm{r} 89 \mathrm{I}$ - (vols. $85-88$ wanting) : E.C.

(c) Transactions. Vol. I and No. 7. Newcastle-uponTyne, I870 : G.S.I.

I89. - Journal of Agriculture. New ser., vols. I-II. London, Edinburgh, I843-65 : B.S.I., R.B.G. [A continuation of No. 268.]

I9o. - Journal of Anatomy (and Physiology). Conducted by Humphry, Turner, Foster, Cunningham and others. Vols. ILondon. Cambridge, Edinburgh, I867- [vols. 2-9, I868-75, are also styled 'Ser. 2, vols. 1-8,' while vols. 2I-, 1887-, form ' New ser., vols. I-.'] Indexes to vols. I-30.

Complete set and indexes : Z.S.I.

Vols. 25-49, I89I-I9I5 : M.C.

- Journal of Anthropology (see No. EI ( $($ ) )).

191. - Journal of Botany (being a second series of the Botanical Miscellany). By IV. J. Hooker. Vols. I-3. London, I834-4I (vol. 4 wanting): R.B.G. [A continuation of No. 89 ; continued as No. 207.]

192. - Journal of Botany. Ed. by Seeman, Trimen, Britten and others. Vols. I-. London, I863-. [Vols. I0-20, 1872-82, are also styled 'New ser., vols. I-I I.']

Complete set: R.B.G.

Vols. I-4I, I863-I903: B.S.I.

Vols. I4-, I 876 - (vols. 45,46 defective) : A.S.B.

- Journat of Conchology (see No. I20).

- Journal of Ecology (see No. 98).

193. - Journal of Economic Biology. Ed. by W E. Collinge. Vols. I-IO. London, I905-I5: B.S.I., Z.S.I. [Continued as No. 20I.]

194. - Journal of Entomology, descriptive and geographical. Vols. I, 2 London, I 862-66: Z.S.I.

- Journal of Hygiene (see No. II).

- Journal of Marine Zoology and Microscopy (see No. 6o).

- Journal of Mental Science (see No. 225).

- Journal of Pathology and Bacteriology (see No. 40).

A.S. = Archaeological Section. Indian Museum

A.S. B. = Asiatic Society of Bengal.

B.C. = Bishop s College.

B.S.I. = Botanical Survey of India.

C.E. = Chemical Examiner to the Govt. of Bengal.

c.U = Calcutta Unıversity.
E.C. = Civil Engineering College, Sibpur.

G.S.I. = Geological Survey of India.

1.L. = Imperial Library.

M.Ba, = Medical College, Bacteriological Laboratory

M.B1. = Medical College. Biological Laboratory

M.C. = Medical College (main library). 
195. London.-Journal of Physiology. Ed. by M. Foster and J. N. Langley. Vols. I-. London and Cambridge, I878-. Index to vols. I-25.

Vols. I-, I $878-$ (vol. 39 wanting) : P.C.

Vols. I-28, I 878-I902 (vol. 28 defective) and index : S.G.

Vols. 34-, Igo6- : M.Ph.

I96. - Journal of Practical Dietetics and Bacterio-Therapeutics. Vols. I-3. London, I908-II (defective) : S.T.M.

- Journal of Science (see No. 274).

I97. - Journal of Social Science. Ed. by E. Lankester. I855-66. London, I866: S.G.

198. - Journal of Travel and Natural History. Ed. by A. Murray. Vol. I. I,ondon, I868.

Vol. I, I 868 : G.S.I.

Vol. I, nos. 2, 4, 5, IS68: R.B.G.

I99. - Journal of Tropical Medicine and Hvgiene. Ed. by J. Cantlie and others. Vols. IO-. London, I907-.

Vols. IO-, I007- : S.G.

Vols. II-, I908-: S.T.M.

Vols. I8-, I9I5-: M.Ba.

200. - Journal of Vaccine Therapy. Ed. by R. W. Allen. Vols. ILondon, I9I 2- : M.Ba.

20r. Journal of Zoological Research. Ed. by W. E. Collinge. Vols I-. London, I9I6-: B.S.I., Z.S.I. [A continuation of No. I93.]

- Kew Bulletin (see No. 284).

202 . Lancet. I823-. London, I823-. [Two vols. published in each year.?

Vol. I of I823- (vol. I of I855, '60, '71, '73, '83 and vol. 2 of 1882 wanting): M.C.

Vol. I of I834- vol. 2 of I9OI (vol. I of I846, '48, '5I, '53, '68 and vol. 2 of 1841 , '42, '49-51, '54, '63, '68, '69 wanting) : I.L.

Vol. 2 of I877; vol. I of I878; vol. I of I882- : S.T.M.

Vol. I of I880- (vol. I of I885, '91, 1902 and vol. 2 of 1886, '88, 94 wanting): S.G.

Vol. I of I9I4-: B.S.I.

M.o. = Meteorological Office, Alıpore.

M.Ph. = Medical College, Physiological Laboratory.

P.c. = Presidency College.

P.o. = Patent Otfice

R.B.G. = Royal Botanic Garden.

s.c.C. $=$ Scottish Churches College.
S.G = Surgeon-General's Library.

S.I. = Survey of India

S.T.M. = School of Tropical Medicine.

T.D. = Telegraph Department.

X.C =St. Xavier's College.

Z.S.I. = Zoological Survey of India. 
203. London.-Linnean Society.

(a) Journal of the Proceedings of the .... Society. Botany. Vols. I-8. London, I857-65. [Continued as :] Journal of the.... Society. Botany. Vols. 9-. London, I867-. Index to vols. I-20.

Complete sets, and index : A.S.B., B.S.I., G.S.I.

Complete set: R.B.G.

Vols. I-I3, I $857-72$ (vol. 12 wanting, vols. II, I3 defective) : Z.S.I.

Vols. 39-, Igo9- (rol. 39 defective) : P.C.

(b) Journal of the Proceedings of the .... Society. Zoology. Vols. I-7. London, I857-64. [Continued as:] Journal of the .... Society. Zoology. Tols. 8-. London, I 865 -. Index to vols. I-20.

Complete sets, and index : G.S.I., Z.S.I.

Vols. I-, I857- (vol. 3I defectire) and index: A.S.B.

(c) Transactions. Vols. I-30. London, I79I-I875: A.S.B., G.S.I., R.B.G., Z.S.I. Indexes to vols. I-30 : A.S.B., G.S.I., Z.S.I. [Continued in two parts as $(d)$ and $(e)$.]

(d) Transactions. Ser. 2. Botany. Vols. I-. London, (I875) I880- : A.S.B., G.S.I., R.B.G.

(e) Transactions. Ser. 2. Zoology. Vols. I-. London, (I875) I879-: A.S.B., G.S.I., Z.S.I.

204. - Literary Gazette and Journal of Belles Lettres, Science and Art. I855-6I. London, I855-6I : A.S.B.

205. London and Edinburgh Philosophical Magazine and Journal of Science. Conducted by D. Brewster and others. New (3rd) ser., vols. I-37. London, I832-50. [Continued as:] London, Edinburgh and Dublin Philosophical Magazine and Journal of Science. Conducted by D. Brewster and others. 4th ser., vols. I-5o. London, I85I-75. 5th ser. Conducted by R. Kane and others. Vols. I-50. London, I876-I900. 6th ser. Conducted by Lord Kelvin and others. Vols. I- London. IgOI-. [For earlier series see Nos. 33, 260.]

3rd ser., vols. I-, I832- (5th ser., vol. 42 wanting; 6 th ser., vols. $14,21-25$ defective): A.S.B.

3rd ser., vols. I-2I, I832-42 : R.B.G.

3rd ser., vols. I4-, I839- (5th ser., vols. 9, II, 12, 17, 25,26 wanting; a few vols, defective): P.C.

$4^{\text {th }}$ ser., vols. I-I3, I $85 \mathrm{I}-57$ : I.L.

4 th ser., vols. 4 I-, I 87 I- : G.S.I.

4th ser., vol. 4 I- 6 th ser., vol. 32, I87I-I9I6 (4th

A.S. = Archaeological Section. Indian Museum.

A.S.B. = Asiatic Society of Bengal.

B.C. = Bishop s College.

B.S.I. = Botanical Survey of India.

C.E. = Chemical Examiner to the Govt. of Bengal.

c. $U$. = Calcutta University.
E.C. = Civil Engineering College, Sibpur.

G.S I. = Geological Survey of India.

I.L. = Imperial Library.

M. Ba. = Medical College. Bacteriological Laboratory

M.BI. = Medical College. Biological Laboratory.

M.C. = liedical College (main library). 
205. London.-London \& Edinburgh Phil. Mag. .. (Continued).

ser., vols. $43-50,5$ th ser., vols. I, $20,22,25,26$ wanting) : T.D.

5th ser., vols. $33-$, I892- : E.C.

206. - London Geological Journal and Record of Discoveries in British and Foreign Palaeontology. Vol. I, nos. I-3. London, I84647 : G.S.I.

207. L London Journal of Botany : containing figures and descriptions of ... Plants .... with botanical notices ... and occasional portraits and memoirs of eminent Botanists. By W. J. Hooker. Vols. I-7. London, I $842-48$ (vol. 3 wanting): R.B.G. [A continuation of No. I9I ; continued as No. I68.]

— London School of Tropical Medicine (see No. 304).

L Loudon's Magazine (see No. 208).

208. - Magazine of Natural History, and Journal of Zoology, Botany, Mineralogy, Genlogy and Meteorology. [Old ser.] Conducted by J. C. Loudon, vols. I-9. London, I829-36, [Sometimes referred to as 'Loudon's Magazine'.] New ser. Conducted by E. Charlesworth. Vols. I-4. London, I837-40. [Sometimes referred to as 'Charlesworth's Magazine.'] [Afterwards combined with No. 79 and continued as No. 76.]

Complete set : Z.S.I.

Vol. 6, I833- new ser., vol. 4, I840 : A.S.B.

209. - Magazine of Science and School of Arts. Vols. I-9. London, I839-47 (vols. 3-6 wanting) : R.B.G.

- Magazine of Zoology and Botany (see No. 4r).

2ro. - Malacological and Zoological Magazine. Conducted by G. B. Sowerby. Parts I, 2. London, I838-39: Z.S.I.

2Ir. - Malacological Society.

Proceedings. Ed. by B. B. Woodward and E. A. Smith. Vols. I-. London, I895-.

Complete set: G.S.I.

Vols. 9-, I9II- (vol. 9 defective) : Z.S.I.

- Man (see No. $280(b))$.

2I2. Marconigraph. Vol. 2. London, I9I2-I3: T.D. [Continued as No. 321.]

2I3. - Mathematical Gazette. Vols. 6-. London, I9II- : P.C.

\footnotetext{
M O = Meteorological Office, Alipore

$\mathbf{M} \mathbf{P h}=$ Medical College. Physiological Laboratory

P.C. = Presidency College.

PO = Patent Office.

R $\mathbf{B} \mathbf{G}=$ Royal Botanic Garden.

S.C.C. $=$ Scottish Churches College.
}

$$
\begin{aligned}
& \text { S.G. = Surgeon-Generails Library. } \\
& \text { S.l. = Survey of India. } \\
& \text { S.T.M. = School of Tropical Medicine. } \\
& \text { T.D. = Telegraph Department. } \\
& \text { X.C. = St. Xavier S College. } \\
& \text { Z.S.I. = Zoological Survey of India. }
\end{aligned}
$$


2I4. London.-Mathematical Society.

Proceedings. Vols. I-35. London, I865-I903. Ser. 2, vols. I-, London, I903-.

Complete set : P.C.

Ser. 2, vols. 5-IO, I907-I2 : C.U.

215. Mechanic's Magazine. Vols. I-69. London, I824-58. New ser., vols. I-[8]. London, I859-64. [Continued as No. I86.]

Complete set: E.C.

Vols. I-69, I824-58; new ser., vols. I-5, I859-6I : T.D.

Vols. I6-6I, I832-54 (vols. 2I-28 wanting) : X.C.

2I6. Medical and Surgical 'Review of Reviews'. Vol. I. London, I 898 : S.G. [Continued as No. 223.]

217. - Medical Annual and Practitioner's Index. I887-I9Io. London and Bristol, [I888-I9II]: A.S.B.

218. - Medical Annual Synoptical Index. Vols. I-, I887/98-. London and Bristol, [1899]- : A.S.B.

2I9. - Medical Critic and Psychological Journal. Vols. I-3. London, I86I-63: I.L.

220. - Medical Graduates College.

Polyclinic: Journal of the .... College. Vols. I- London, I899-.

Vols. I-7, I899-I903: S.G.

Vols. I2-, Igo8-- S.T.M.

221. - Medical Officer. Vols. 5-. I,ondon, IgII- : S.T.M.

222. - Medical Press and Circular. Vols. I36- (new ser., vols. 85-). London, I908- (vol. I36 defective) : S.T.M.

223. - Medical Review. Vols. 2-. London, I899-. [For vol. I see No. 2 I6.]

Complete set: S.G.

Vols. II-, I908-: S.T.M.

224. - Medical Times and Gazette: a journal of medical science. I864-85. London, I864-85.

Vols. for I $864-85$ : S.G.

Vols. for 187 ( -85 (vols. for I $88 \mathrm{I}-84$ defective) : I.L.

225. Medico-Psychological Association of Gt. Britain and Ireland. Journal of Mental Science. Vols. 26-54. London, I880I908.

A.S. Archaeological Section, Indian Museum. A.S.B. = Astatic Society of Bengal.

B.C. = Bishop s College.

B.S.I. = Botanical Survey of India.

C.E. = Chemical Examiner to the Govt. of Bengal.

c.U. = Calcutta University.
E.C. = Civil Engineering College, Sibpur

G.S.I. = Geological Survey of Indra.

I.L. = Imperial Library.

M.Ba. = Medical College, Bacteriological Laboratory

M.Bi. = Medical College, Biological Laboratory.

M.C. = Medical Collcge (main library). 
225. London.-Medico-Psychological Association .. (Continued).

Vols. 26-54, I $880-$ I 908 (vols. 3 I, $35-43$ wanting) : S.G.

Vols. 39-53, I893-I907 (vols, 4 I, +3 wanting) : A.S.B.

226. - Messenger of Mathematics. Ed. by Whitworth and others. Vols. I-. London and Cambridge, I872-.

Complete set : C.U.

Vols. 7-3I, I878-I902 (vols. 20, 24, 25 defective) : A.S.B.

Vol. 9-, 1879- (vol. 40 defective) : P.C.

Vols. 37-4I, I907-I2 (most vols. defective) : M.S.

- Meteorological Society (see No. 293).

- Meteorological Record (see No. 293).

_ Microscopical Society (see No. 294).

227. - Mineral Industry, its Statistics, Technology and Trade, etc. E, d. by R. P Rothwell and others. Vols. I-. New York and London, I893-.

Complete set: G.S.I.

Vols. 5, II, I2-I4, I896, I902, I903-05: I.L.

Vols. 6-I2, I898-I903: B.S.I.

- Mineralogical Magazine (see No. 228).

228. Mineralogical Society.

Mineralogical Magazine and Journal of the .... Society. Ed. by L. J. Spencer and others. Vols. I-. London and 'Truro, I877-. Index to vols. I-I0, I895.

Complete set, and index: G.S.I.

Vols. I6-, I9II- (vol. I6 defoctive) : P.C.

229. - Mind : a quarterly review of psychology and philosophy. Ed. by G. C. Robertson. Vols. I-I6. London. I876-9I. New Ser. Ed. by G. F. Stout. Vols. I-. London, I892-.

Complete set : C.U.

Vols. 2-I5, I $877-90$ : A.S.B.

Vols. 7-16 and, new ser., vol. I, I882-92 : I.L.

New ser., vols. 9-, I900- : s.c.C.

230. - Mining Journal. Vols. 25-. London, I855-(vol. 25 defective) : G.S.I.

23I. - Mining Magazine. Ed. by T. A. Rickard and others. Vols. I-. London, Ig09- : G.S.I.

232. — Mining Manual. I905-09. London, I905-09: I.L.

M.o. = Meteorological Office, Aipore.

M.Ph = Medical College, Physiological Laboratory

P.C. = Presidencv College.

P O. =Patent Ofíce.

R B.G = Royal Botanic Garder.

S C C $=$ Scottish Churches College.
S.G. = Surgeon-Generai's Library

S.I. = Survey of Inaia.

S.T.M = School of Tropical Medicine

T.D. = Telegraph Departmen:

X.C. =St Xavier s College.

z.s.l. = Zoological Survey of India. 
233. London.-Mining World and Engineering Record. Igo4-II. London, IgO4-II : I.L.

- Monthly Microscopical Journal (see No. $294(b)$ ).

234. - Monthly Numismatic Circular. Vols. I-. London, I893(vol. 20 defective) : A.S.B.

- Museum of Economic Geology (see No. I58).

235. - Museum Rusticum et Commerciale: or select papers on Agriculture, Commerce, Arts and Manufactures. Vols. I-6. London, I764-66: R.B.G.

236. Museums Association.

Museums Journal. Vols. 2-. London, I903-.

Vols. 2-, Igo3- (vol. 2 defective) : Z.S.I.

Vols. I3-, I9I3- : B.S.I.

237. - National Association for the Promotion of Social Science.

Transactions. I857-67. London, I858-68: S.G.

Natural History Review (see No. 22).

238. - Natural Science: a monthly review of scientific progress. Vols. I-I5. London, New York and Eidinburgh, I892-99.

Complete set: G.S.I.

Vols. 4-I3, I894-98: Z.S.I.

239. - - Naturalist's Repository : or miscellany of exotic natural history, etc. By E. Donovan. Tols. I-5. London, [I823]-I834: Z.S.I.

240. - Nature. Vols. I-. London, I870 (I869)-.

Complete sets : G.S.I., I.L., R.B.G.

Vols. I-, I $870-$ (vols. 25,45 , wanting, vols. 88,89 defective) : P.C.

Vols. I-, I 870 - (vols. $45,45,52$ wanting, vols. 77,90 defective) : A.S.B.

Vols. 7-5I, I873-95; 83-, I9IO- : Z.S.I.

Vols. 8, 9, II, I 873-75; vols. 49-, I894- (vol. 6I wanting): X.C.

Vols. 9-89, I $873-$ I9I2 (vols. I3, I4 wanting) : T.D.

Vols. 3I-, I $885-$ : S.I.

Vols. 33 -, I886- : S.C.C.

Vols. 45 -, I 892 - : E.C.

Vols. $45-$, I $892-$ (vols. $45^{-56}$ defective) : B.S.I.

24I. - Nantical Almanac. London, I787, I824-36, I863, I866, I869, I870, I875- : I.L.

A.S. = Archaeological Section, Indian Museum.

A.S.B. = Asiatic Societv of Bengal.

B.C. = Bishop s College.

B.S.I. = Botanical Survev of India.

C.E. = Chemical Examiner to the Govt. of Bengal,

c.U. = Calcutta University.
E. C. Civil Engineering Colleģe, Sibpur.

G.S.1. = Geological Survey of India.

I L. = Imperial Library.

M Ba. = Medical Collere, Bacteriological Laboratory

M.B1. = Medical Collere. Biological Laboratory.

M.C. Medical College (main library). 
242. London.-New Phylologist. Ed. by A. G. Tansley. Vols. ILondon, I902-.

Complete set : R.B.G.

Vols. I4-, IgI5-: B.S.I.

243. - Notes and Queries. Ser. 9, vols. 3-Io. London, I899-I902: A.S.B.

- Novitates Zoologicae (see No. 348).

244. - Numismatic Chronicle. Ed. by J. Y. Akerman. Vols. 4-I2. London, I84I-50 : I.L. [Continued as No. 296.]

245. - Numismatic Journal. Vol. I, 2. London, I837-38: A.S.B. [Continued as No. 244.]

Numismatic Society (see No. 296).

246. - Observatory: a monthly review of astronomy. Ed. by T. Lewis, A. S. Eddington, and others. Vols. 24-. London, IgoI(vols. 3O, 3I defective) : P.C.

247. Ocean Highways. Ed. by Clements R. Markham. Vol.2. London, I872-73. New ser., vol. I. London, I874. [Continued as No. I 54.]

Vol. 2, and new ser., vol. I, I872-74 (nos. I, 6 of vol. 2 wanting) : I.L.

Vol. 2 , nos. 8 , II, I2, and new ser., vol. I, I872-74: G.S.I.

New ser., vol. I. I874: A.S.B.

248. - Ophthalmic Reviere. Vols. 27-. London, [I908]- : S.T.M.

249. - Ophthalmoscope: a monthly review of current ophthalmology. Vols. 6-. London, Igo8- : S.T.M.

250. - Orchid Review: an illustrated monthly journal devoted to orchidology in all its branches. Vols. I-. London, I893- : R.B.G.

25I. Ordnance Survey.

Report of Progress. I892, I900-02, '08, 'II-. London, I893, I9OI-03, 'og, 'I2- : S.I.

252. - Ornithological Miscellany: Ed. by G. D. Rowley. Vols. I-3. London, I875-78: Z.S.I.

253. - Palaeontographical Society.

[Monographs.] Nos. 42-. London, I889- (Earlier vols. do not bear vol. nos.) : G.S.I.

M.O. = Meteorological Office, Alipore.

M.Ph. = Medical College, Physiological Laboratory.

P.C. = Presidency College.

P.o. = Patent O.fice.

R.B.G. = Roval Botanic Garden

S.C.C. $=$ Scottish Churches College.
S.G. = Surgeon-General s Library.

S.I. = Survey of India.

S.T.M. = School of Tropical Medicine.

T.D. = Telesraph Department.

X.c. =St. Xavier s College.

z.s.l. =Zoological Survey of India. 
254. London.-Patent Office.

(a) Illustrated Official Journal. Vols. 8-. London, I880-: P.O.

(b) Official Journal. Vols. I-. London, I884-: P.O.

(c) Reports of Patent Cases. Vols. I-. Itondon, I884- : P.O.

255. - Paxton's Magazine of Botany and Regisler of Flowering Plants. [Ed. by J. Paxton.] Vols. I-I6. London, I836-49: R.B.G.

256. - Perfumery and Essential Oil Record. Ed. by J. C. Umney. Vols. 4-. London, I9I3-: B.S.I.

257. - Petroleum Industrial and Technical Review. Ed. by P. Dvorkovitz. Vols. I-7. London, I899-I902: G.S.I. [Continued as:」 Petroleum Revierw and Mining Nerws. Vols. 8-Io. London, I903-04: G.S.I. [Continued as:] Petroleum Reviere. with which is incorporated "Petroleum." Vols. II-. London, I904-: G.S.I.

259. — Pharmacentical Journal and Pharmacist. Vols. 78-85. London, I907-IO (vols. 82,83 wanting) : E.C.

259. — Pharmaceutical Society.

Pharmacentical Journal (and Transactions). Vols. I-I8. London, I842-59. Ser. 2, vols. I-II. Iondon, I86070. Ser. 3, vols. I-25. London, I87I-95. Ser. 4, vols. I-. London, I895-.

Vols. I-, I 842 (ser. 4, vols. I 2-I5 wanting) : B.S.I.

Vols. I-, I842- (ser. 2, vol. 11, ser. 3, vols. I, 2 wanting) : C.U.

Vols. I- I 842 - (ser. 3, vols. 2, 3, 6-22 wanting) : R.B.G.

Ser. 2, vol. 5- ser. 4, vol. 8, I863-IgO2 (ser. 2. vols. 9-I I wanting; ser. 4 , vol. 5 defective) : C.E.

Ser. 3, vols. IO-I8, I879-88 : S.G.

260. - Philosophical Magazine (and Journal). [Ed.] by A. Tilloch. Vols. I-68. London, [I798]-I826. [Continued as :] Philosoph. ical Magazine, or Annals of Chemistry, Mathematics, Astronomy, Natural History, and General Science. By R. Taylor and others. New ser., vols. I-II, L,ondon, I827-32. [Afterwards amalgamated with No. 33 and continued as No. 205.]

Vols. I-68, I798-I826 (vol. 62 wanting); new ser., vols. I-II, I827-32: A.S.B.

Vols. I-67, I798-I826 (vol. 60 wanting); new ser., vols. I-IO, I $827-3$ I (vol. II wanting): I. L.

\footnotetext{
A.S. = Archaeological Section. Indian Museum.

A.S.B. = Asiatic Society of Benga!

B.C. = Bishop s College.

B.S.I. = Botanical Survey of India

C.E. = Chemical Examiner to the Govt. of Bengal.

c.U. = Calcutta University:
}

E.C. = Civil Engineering College, Sibpur.

G.S.I = Geological Survey of India.

I.L. = Imperial Library.

M.Ba. = Medical College, Bacteriological Laboratory.

M.Bi. = Medical College. Biological Laboratory.

M.C. = Medical College (man library) 
260. London.-Philosophical Magazine (Continued).

Vols. I, 2, I798; new ser., vols. 2-II, I $827-32$ : R.B.G.

26I. - Physical Society (see also No. 305).

Proceedings. Vols. I-, I874-. London, I876- (vol. 23 defective): P.C.

262. - Phytologist: a popular botanical miscellany. Conducted by G. Luxford and E. Newman. Tols. I-3. London, I847-49 (vols. 4,5 and first portions of rols. 2, 3 wanting); R.B.G. [Continued as:] Phytologist: a botanical journul. Ed. by A. Irvine. Vols. I-6. London, I855-63 (vol. 5 wanting) : R.B.G.

Polyclinic (see No. 220).

263. — Post Office Electrical Engineer's Journal. Vols. I-. London, Igo8- : T.D.

264. - Practitioner. Vols. I-79. London, I868-I902. I903-. London, I903-. [Vols. 54-67 are also styled 'New ser., vols. I-I4.']

Vols. I-, I868- (vols. 30, 3I wanting): M.C.

Vols. I-, I 868 - (vols. 30, 3I, 42-47, 5I, 60, 6I, 67 wanting; vols. for 1907, '08, 'I I defective) : S.T.M.

Vols. 24-74, I880-I905 : S.G.

265. - Progressive Medicine. Igo4-. I,ondon, I904- : S.G.

266. - Public Health. Ed. by S. F. Murphy. Vols. 22-. London, Igog- (vol. 27 wanting): S.G.

267. - Quarry. Vols. I3-. I,ondon, Igos- : G.S.I.

268. - Quarterly Journal of Agriculture. Vols. I-I3. London, Edinburgh, I828-43 : B.S.I., R.B.G. [Continued as No. I89.]

269. - Quarterly Journal of Conchology. Vol. I. I,ondon, I874-78 :

A.S.B. [Continued as No. I20.]

270. - Quarterly Journal of Experimental Physiology. Ed. by E. A. Schafer. Vols. I- London, Igo8-.

Complete sets : A.S.B., M.Ph.

Vols. 5-, I9I2- (vol. 5 defective) : P.C.

- Quarterly Journal of Literature, Science and the Arts (see No. 29 I $(d))$.

271. - Quarterly Journal of Microscopical Science. Ed. by E. Lankester and others. Vols. I-8. London, I853-6o. New ser., vols. I-. L,ondon, I86I-. Index to vols. I-7 and new ser., vols. I-28, I $853-88$.

M.o. = Meteorological Otince, Alipore

M.Ph. = Medical College. Physiological Laboratory:

P.C. = Presidency College.

P.o. = Patent Otfice.

R.B.G. = Royal Botanic Garden

S.C.C. $=$ Scottish Churches College.
S.G. = Surseon-General's Library.

S.I. = Survey of India

S.T.M. = School of Tropical Mecicine.

T.D. = Telegraph Department.

X.C. =st. Savier s College.

Z.S.I. = Zoological Survey of India. 
27I. London.-Quarterly Journal of Microscopical Science (Continued).

Vols. I-7, I853-59; new ser., vols. I-29, I86I-89 (vols. II, 12 wanting) : G.S.I.

Vols. I-3, I853-55; new ser., vols. I3-48, I873I 905 (vol, 45 defective) and index : A.S.B.

Vol. I, I853; new ser., vols. I-29, I86I-89 (vols. 4, 9 , I $\overrightarrow{5}, 22-24$ wanting): R.B.G.

New ser., vols. I-, I86I- (vols. 7,8 wanting) and index: Z.S.I.

New ser., vols. 55-57, I9IO-I2 : M.Bi.

272. - Quarterly Journal of Public Health and record of Epidemics and Hygiene, including the Transactions of the Epidemiological Society of London. Ėd. by B. W. Richardson. Nos. I-I6. London, I855-59: S.G.

273. - Quarterly Journal of Pure and Applied Mathematics. Ed. by J. J. Silvester, N. M. Ferrers, J. W. L. Glaisher and others. Vols. I-. I ondon, I 857 -.

Complete set : P.C.

Vols. I-44, I857-I9I2: C.U.

Vols. I6-33, I879-I902 : A.S.B.

274. - Quarterly Journal of Science. Ed. by J. Samuelson and W. Crookes. Vols. I-7. London, I864-70. [Continued as :] Quar. terly Journal of Science and Annals of Mining, etc. Ed. by W. Crookes. New ser., vols. I-8 (=8-I5). London, I87I-78. [Continued as:] Journal of Science and Annals of Biology, etc. Ed. by IV. Crookes. 3rd ser., vols. I-7 (=I6-22). London, I $879-85$.

Complete sets : A.S.B., G.S.I.

New ser., vols. $2-4(=9-\mathrm{II}), \mathrm{IS}_{72-74}$ : C.E.

Vols. I-6, I 864-68 (defective) : S.G.

- Quarterly Journal of Science, Literature and the Arts (see No. 29I $(e, f))$.

275. - Quekett Microscopical Club.

Journal. 2nd ser., vols. I-, London, I882-: Z.S.I.

276. - Recreations in Agriculture, Natural-History, Arts, and Miscellaneous Literature. By J. Anderson. Tols. I-6. London, I799-I802.

277. — Refugium Botanicum: or figures and descriptions . . of little known or new plants of botanical interest. Ed. by IV. IV. Saunders. Vols. I-5. London, I 86.9-73 (vol. 2 wanting) : R.B.G.

278. - Retrospect of Medicine. Ed. by IV. and J. Braithwaite. Vols. 8I-I23. London, I880-I90I (vol. I03 wanting): S.G.

A.S. = Archaeological Section. indian Mruseum.

A.S.B. = Asiatic Society of Bengal.

B C. = Bishop s College

B.S.I. = Botanical Survey of India

C.E. = Chemical Examiner to the Govt. of Bengal.

c.u. = Calcutta University.
E.C. =Civil Engineering College, Sibpur

G.S.I. = Geolosical Survey of India.

I.L. = Imperial Library.

M. Ba. = Medical College, Bacteriological Laboratory

M.Bi. = Medical College. Biological Laboratory.

M.C. = Medical College (main library). 
London.-Review of Applied Entomology (see No. I72(b)).

279. - Royal Agricultural Society of England.

Journal. Vols. I-25. London, I840-64. 2nd ser., vols. $\mathrm{I}-25(=26-5 \mathrm{O})$. London, $\mathrm{r} 865-80$. 3rd ser., vols. I-II $(=5 \mathrm{I}-6 \mathrm{I})$. London, I890-I900. Vols. 62-. I ondon, IgoI-. Indexes to vols. I-IO, I840-55, and to and ser., vols. I-IO, I $865-74$.

Complete set: R.B.G.

Vols. I, I $840-$ 2nd ser., vol. 23 , I887 (2nd ser., vols. 8,16 wanting, vols. $9,15,20,23$ defective) and indexes : I.L.

2nd ser., vols. 3, 6, II, 20, 22, 23; 3rd ser., vols. $\mathrm{I}-3, \mathrm{I} 867-92:$ B.S.I.

280. - Royal Anthropological Institute of Great Britain and Ireland.

(a) Journal. Vols. I-. London, I872- (vols. 28-, I899-, are also styled 'New ser., vols. I-.')

Vols. I-, I872- (vol. 2 defective) : A.S.B.

Vols. I-I8, no. 2 , I872-88 : G.S.I.

(b) Man: a monthly record of anthropological science. Vols. I-. London, IgOI-.

Complete set : I.L.

Vol. I, IgOI, and vols. I3-, IgI3- : A.S.B.

Vols. 2-, IgO2- : Z.S.I.

28I. - Royal Army Medical Corps.

Journal. Vols. I-. [London], I903-.

Vols. I-, I903- (vols. 9-I wanting) : S.T.M.

Vols. 4-, Igo5- : M.C.

Vols. I8-, I9I2- : S.G.

282. - Royal Asiatic Society of Great Britain and Ireland.

(a) Journal. Vols. I-20. London, I834-63. New ser., vols. I-. London, I865-. [Vols, published subsequent to vol. 21, I889, do not bear vol. nos.] Index to vols. I20 and new ser. vols. I-20.

Complete set : I.L.

Vols. I-, I834- (vol. for I908 wanting): A.S.B.

Vol. I, I834- new ser., vol. 20, I888 (vol. 10, new ser., wanting) and index : G.S.I.

Vols. I-7, I834-43 (vol. 6 wanting): R.B.G.

New ser., vols. I-, I865-: C.U.

New ser., vols. I-20, I865-88: M.O.

M.O. = Meteorological Office, Aliporc

M.Ph. = Medical College, Physiological Laboratory.

P.C. = Presidency College.

P.o. = Patent Ofice

R.B.G $\approx$ Royal Botanic Garden.

S.C.C. $=$ Scottish Churches College.
S.G. = Surgeon-General's Library.

S.I. = Survey of India.

S.T.M. = School of Tropical Medicine.

T.D. = Telegraph Department.

X.C. =St. Xavier's College.

Z.S.I. = Zoological Survey of India. 
282. London.-Royal Asiatic Society of Great Britain ... (Continued).

(b) Transactions. Vols. I-3. I,ondon, I827, '30, '35.

Complete sets : A.S.B., G.S.I., I.L.

Vols. 2, 3, I830; '35 : R.B.G.

283. - (Royal) Astronomical Society.

(a) Memoirs of the Astronomical Society. Vols. 2-4. London, I826-3I. [Continued as:] Memoirs of the Royal Astronomical Society. Vols. 5-. London, I833-. General indexes to vols. $1-38,39-40$.

Vols. 2-, I826- (vols. 40,58 wanting, rol. 59 defective) and index : A.S.B.

Vols. I2-, I842- (vol. 13 wanting) and index to vols. 39-6o: I.L.

(b) Monthly Notices of the Astronomical Society. Vol. I, I827-30. London, I83I. [Continued as:] Monthly Notices of the Royal Astronomical Society. Vols. 2--, I8 3 I-. I ondon, I833-. General indexes to vols. I $-39,39-52$ and $53-70$.

Complete set : C.U.

Vols. I-, I $83 \mathrm{I}-$ (vols. 3-5, I9-27 wanting) and indexes: A.S.B.

Vols. 8-, I847- (vols. 16, 19-50 wanting) : P.C.

Vols. 56-62, I896-Ig02 : X.C.

Vols. 69-, I908- (vols. 70,72 defective) : M.S.

284. - Royal Botanic Gardens, Kew.

(a) Bulletin of Miscellaneous Information. Nos. I-. London, I 887 -.

Complete sets : B.S.I., R.B.G.

Nos. I-, I887- (vol. of 1989 wanting, vols. of I9II, 'I4, ' 16 defective): I.L.

(b) Bulletin of Miscellaneous Information. Additional Series. Nos. I--. I,ondon, I $898-$ : B.S.I., R.B.G.

General index, I887-I906: B.S.I., R.B.G.

285. - Royal Botanic Society.

Quarterly Record. Tols. I-6, I880-97. London, I883-98: R.B.G.

286. - Royal Colonial Institute.

(a) Proceedings (Journal). Vols. I-40. London, I870Igo9. Index to vols. I-39. [Continued as $(b)$.]

Complete set and index : G.S.I.

Vols. II-40, I880-I909: A.S.B.

\footnotetext{
A.S. Archaeological Section, Indian Museum.

A.S B = Asiatic Sociely of Benga'.

B.C. = Bishop s College.

B.S.I = Botanical Survey of India.

C.E. = Chemical Examiner to the Govt. of Bengal.

C. $U$ = Calcutta University
}

E C. =Civil Engineering College, Sibpur.

Q.S.1. = Geological Survey of India.

I. L. = Imperial Library

M.Ba = Medical College, Bacteriolosical Laboratory

M.Bi. = Hedical College. Biolosical Iaboratory

M.C. = Medical Colleg 4 (main library). 
286. London.-Royal Colonial Institute (Continued).

(b) United Empire: Proceedings of the ... Institute. New ser., vols. I-. London, I9IO-: A.S.B. [A continuation of $(a)$.]

287. - Royal Eiconomic Society.

Economic Journal: journal of the British Economic Association. Ed. by F. Y. Edgeworth and H. Higgs. Vols. I-I2. I,ondon, I89I-I902 : C.U., S.C.C. [Continued as:] Economic Journal : journal of the .... Society. Vols. I3-. London, I903-: C.U., S.C.C.

288. — Royal Geographical Society.

(a) Geographical Journal (including the Proceedings of the ..... Society). Vols. I-. London, I893-. [A continuation of $(c)$.]

Complete sets : A.S.B., C.U., G.S.I.

Vols. I-, I893- (vol. 10 wanting, vols. 17, I8 defective) : I.L.

Vols. 2-, I893- (vol. 2I wanting) : S.I.

(b) Journal. Vols. I-5o. London, I832-8I. General indexes to vols. I -9 and to I I-20. [Incorporated in (c).]

Complete set and indexes : G.S.I.

Complete set : C.U.

Vols. I -45, I $832-75$, index to vols. I I-20 : A.S.B.

Vols. I-45, I832-75 (vols. $23,24,32,37$ wanting) : I.L.

(c) Proceedings. Vols. I-22, I855-78. London, I857-78.

[Continued as :] Proceedings.... and Monthly Record of Geography. New ser., vols. I-I4. London, I879-92. [Continued as $(a)$.]

Vol. I, I857- new ser., vol. I4, I892 (vol. I, old ser., defective) : G.S.I.

Vol. I, I857- new ser., vol. I4, I892 (vols. 2, I3, old ser., wanting) : A.S.B.

Vol. II, I867- new ser., vol. I4, I892 (vols. 13, I4, 17, 21, 22 and new ser., vols. 1, 5 wanting): I.L.

(d) Supplementary Papers. Vols. I-4. London, I882-90: A.S.B., G.S.I.

289. - (Royal) Horticultural Society.

(a) Journal of the Horticultural Society. Vols. I-9. London, I846-55 : R.B.G. [Vols. for 1856-94 wanting; continued as:] Journal of the Royal .... Society. Vols. I8-. London, I895-: R.B.G.

M.O. = Meteorological Office, Alipore.

M.Ph $=$ Medical College. Physiological Laboratory

P.C. = Presidency College.

PO. = Patent Office.

R B.G = Roval Botanic Garier.

S C C $=$ Scottish Churches College.

\footnotetext{
S.G. =Surgeon-Generai's Library

S.I. = Survey of Incia.

S.T.M $=$ School of Trovical Mecicine.

T.D. = Telegraph Departmen:

X.C. $=$ St Xavier $s$ College

Z.s.I =Zoological Survey of Inciia.
} 
289. London.-(Royal) Horticultural Society (Continued).

(b) Transactions of the Horticultural Society. Vols. I-7. London, I8I2-30. Ser. 2, vols. I-3. London, I835-48. Complete set : R.B.G.

Vols. I-6, I8I5-26 (vols. I, 2 of 2 nd ed.) : A.S.B.

290. — Royal Institute of British Architects.

(a) Joumal. 3rd ser., vols. I-, I893/94-. London, I894- [A continuation of $(b)$.]

Vols. I-, I894- (pt. 2, vol. 3 wanting) : I.L.

Vols. 2-, I895- (vol, I4 wanting) : E.C.

(b) Jounnal of Proceedings. New ser., vols. I-9. London, I885-93 (vols. I-5 wanting): I.L. [A continuation of $(c)$. Continued as $(a)$.

(c) Proceedings. Sess. I880/8I-I883/4. London, I88I84 : I.L. [Continued as $(b)$.]

(d) Transactions. Sess. I880/8I-'83/84. London, I88I84 I.L. New ser., vols. I-8. London, I885-92 : I.L.

291. - Royal Institution of Great Britain.

(a) Journal. Vols. I, 2, I830-3I. London, I83I-[32] : I.L. [For earlier series see $(e),(f),(d),(b)$.]

(b) Journal of Science and the Arts. Vols. I-6. I,ondon, I8I6-I9: G.S.I., I.L., R.B.G. [Continued as $(d),(f),(e),(a)$.]

(c) Notices of the Proceedings Vols.I-. London, I854-. Indexes to vols. I-4 at end of vol. 4 , and to vols. II2 at end of vol. I2.

Complete set: G.S.I.

Vols 2-, I858-: A.S.B.

(d) Quarterly Journal of Literature, Science and the Arts. Vol. 7. London, I8I9: G.S.I., I.L., R.B.G. [A continuation of $(b)$, continued as $(f)$.]

(e) Quarterly Journal of Science, Literature and Art. I827-30. London, I827-30: G.S.I., I.L. [A continuation of $(f)$, continued as $(a)$.]

(f) Quarterly Journal of Science, Literature and the Arts. Vols. 8-22. London, I820-27 : G.S.I., I L., R.B.G. [A continuation of $(d)$, continued as $(e)$.]

292. — Royal Medical and Chirurgical Society.

Medico-chirurgical Transactions. Vols. 4, 28, 33, 34. London, I8I3, '45, '50, '5 I : S.G. Vols. 8I-90. London, I $898-1907$ (vols. $84,88,89$ wanting): A.S.B.

A.S. - Archaeological Section, Inciian Museum.

A.S.B. = Asiatic Societv of Bengal.

B.C. = Bishop s College.

B.S.1. = Botanical Survey of India.

C.E. = Cliemical Examiner to the Govt of Bengal

C.U. = Calcutta University.
E.C. = Civil Engineering College, Sibpur.

G.S.1. = Geological Survey of India.

I L. = Imperial Library.

M Ba. = Medical College. Bacteriological Laboratory

M.Bi. = Medical College. Biological Laboratory.

M.C. = Medical College (main library). 
293. London.-(Royal) Meteorological Society.

(a) Meteorological Record. Vols. I-30. London, I88IIgIo: X.C.

(b) Quarterly Journal of the Meteorological Society. Vols. 3-6. London, I877-80: X.C.

294. - (Royal) Microscopical Society.

(a) Journal of the ... Society, containing its Transactions and Proceedings. Vols. I-3. London, I878-80. Ser. 2, vols. I-. London, I88I-. [Vols. published subsequent to vol. 6 , ser. 2, 1886, do not bear vol. nos.]

Vols. 2, 3, I879-80; ser. 2 , vols. I-6, etc., I88II906 (vol. 2, old ser., and vols. for 1903-06 defective) : A.S.B.

Vols. for I886-93: C.U.

Vols. for I888- : M.C.

Vols. for I894-: Z.S.I.

(b) Monthly Microscopical Journal: Transactions of the Royal Microscopical Socrety, and Record of Histological Research. Vols. I-I8. London, I869-77: R.B.G. [Continued as $(a)$. For earlier series, see (c).]

(c) Transactions of the Microscopical Society. Vols. I-3. London, I844-52. New ser., vols. T-I6. London, I853-68. [Published and sometimes bound up with vols. I -8 and new ser., vols. $1-8$ of No. 27 I. Continued as $(b)$.

Vols. I-3 and new ser., vols. I-I6, I844-68 (vol. 8, new ser., wanting): G.S.I.

Vols. I-3, I844-52 : A.S.B.

New ser., vols. 3-I5, I855-68 : R.B.G.

New ser., vols. 9-I6, I86I-68 : Z.S.I.

295. — Royal Naval Medical Service.

Journal. Ed. by R. C. Munday and IV. L. Martin. Vols. I--. [L,ondon], I9I5- : S.T.M.

296. — Royal Numismatic Society.

Numismatic Chronicle and Journal of the .... Society. New ser., vols. I-20. London, I86I-80. Ser. 3, vols. I20. London, I88I-9o. Ser. 4, vols. I-. London, I9OI-. [For earlier series see No. 244.]

New ser., vols. I, I86I- (new ser., vol. 12 wanting; ser. 4 , vols. 4,5 defective) : A.S.B.

Ser. 4, vols. 5-, IgO5-: I.L.

м o = Meteorological Office, Alipore

$\mathbf{M} \mathbf{P h}=$ Medical College, Physiological Laboratory.

P.C. = Presidency College.

PO = Patent Office.

$\mathbf{R} \mathbf{B} \mathbf{G}=$ Royal Botanic Garden.

S.C.C. $=$ Scottish Churches College.
S.G. = Surgeon-Generails Library

S.I. = Survey of India.

S.T.M. = School of Tropical Medicine

T.D. = Telegraph Department.

X.C. =St. Xavier s college.

z.s.ı. =Zoological Survey of India. 
297. London.-Royal Sanitary Institute.

Journal. Vols. 27-. London, 1907-.

Vols. 27一, I907-: S.G.

Vols. 3I-, I9IO-: E.C.

298. - Royal Society.

(a) Abstracts of the Papers printed in the Philosophical Transactions. Vols. I-4, I800-43. London, I832-43: A.S.B., C.U., G.S.I., P.C. [Continued as :] Abstracts of Papers communicated to the Royal Society. Vols. 5, 6, I843-54. London, I85I-54 : A.S.B., C.U., G.S.I., P.C. [Continued as $(h)$.]

(b) Catalogue of Scientific Papers (I80o-I9oo). Vols. ILondon, I867- [Continued as $(c)$.]

Complete set: G.S.I.

Vols. I-I2 (I $800-83)$. A.S.B., R.B.G.

Vols. I-8 (I800-73) : P.C.

(c) International Catalogue of Scientific Literature (see No. 1597).

(d) Philosophical Transactions. Vols. I-I77. London, I665-I887. Indexes to vols. I-70, 7I-IIO and IIII20. [Many vols. lack rol. nos. Continued in two parts as $(e)$ and $(f)$.

Complete set and indexes (vols. I-9o of original ed.) : A.S.B.

Vols. 9I-I26, I80I-36, index to vols. I I I-I 20 : E.C.

Vols. 9I-97, I80I-07; vols. I02, pt. 2, I03, pt. 2, I8I2-I3; vols. II3, pt. 2-I77, I823-87, and indexes: G.S.I.

Vols. II 8-I77, I828-87 (vols. I54, I864 and I70, I879 defective) : P.C.

Vols. II9-I77, I $829-87$ : R.B.G.

Vols. I2O-I 44, I830-54 (vols. I31-33, I841-43 defectire) : I.L.

(e) Philosophical Transactions. Ser. A. Containing Papers of a Mathematical and Physical Character. Vols. I78-, I887-. I,ondon, I888-.

Complete sets : G.S.I., Z.S.I.

Vols. I78-, I888- (vol. 2 Io defective) : P.C.

Vols. I78-, I888- (vols 199, 200 wanting): A.S.B.

(f) Philosophical Transactions. Ser. B. Containing Papers of a Brologicai Character. Vols. I78-, I887-. London, $x 888$-.

A.S. = Archaeological Section. Indian Museum. A.S B = Asiatic Society of Bengal.

B.C. = Bishop's College.

B.S.1. = Botanical Survey of India

C.E. = Chemical Examiner to the Govt. of Bengat

C.U. Calcutta University.
E.C. =Civil Engneering College, Sihpur.

G.S.I. = Geolosical Survey of Incia.

IL. = Imperial Library

M.Ba. = M edical College, Bacteriological Laboratory

M.BI. = Medical College. Biological Laboratory.

M.C. = Medical College (main library). 
298. London - Royal Society (Continued).

Complete sets : G.S.I., P.C., R.B.G., Z.S.I.

Vols. I78-, I888- (vol. 195 wanting) : A.S.B.

Vols. I97, 204, I905, I9I4 (defective) : I.L.

(g) Philosophical Transactions from 1665-1800, abridged.

I8 vols. London, I8og.

Complete sets : B.C., E.C., S.C.C.

Vols. for I665-I800 (vol. for 1797 wanting) : I.L.

Vol. for $1743-50$. P.C.

(h) Proceedings. Vols. 7-75, I854-I905. London, I856I905. [A continuation of $(a)$. Continued in two parts, as (i) and $(j)$.]

Complete sets : G.S.I., P.C.

Vols. 7-74, I854-I905: C.U.

Vols. 7-74, I854-I905 (vols. 51, 64, 74 defective): A.S.B.

Vols. 8-75, I856-I905: R.B.G.

Vols. 44-75, I888-I905 (vols. 66, i2, 75 defective) : Z.S.I.

Vols. 54-74, I893-1905 : E.C.

(i) Proceedings. Ser. A. Mathematical and Physical Sciences. Vols. 76-, I905-. London, I905-.

Complete sets : A.S.B., C.U., G.S.I., P.C., R.B.G., Z.S.I.

Vols. 76-, I905-(vols. 80-82 wanting): E.C.

(j) Proceedings. Ser. B. Biological Sciences. Vols. 76-, I905-. London, 1905-.

Complete sets : A.S.B., C.U., G.S.I., P.C., R.B.G., Z.S.I.

Vols. $76-84$, I905-II (rols. 80,82 wanting): E.C.

(k) Reports of the Commission on Mediterranean Fever. Parts I-7. Iondon, I905-07.

Complete sets : A.S.B., R.B.G.

Parts 4-7, I906-07: Z.S.I.

(l) Reports of the Sleeping Sickness Commission. Nos. I-. London, I903-.

Nos. I-, I903- (110s. 7-10 wanting): A.S.B.

Nos. I-IO, I903-IO (nos. 7-? wanting) : Z.S.I.

Nos. I-4, I903: S.G.

( $m$ ) Reports to the Evolution Committee. I-5. London, I902-IO: G.S.I., Z.S.I.

(n) Reports to the Malaria Committee. Series [I]-8. I899/I900-I903. London, I900-03.

Complete sets : S.G., Z.S.I.

Ser. I-6, I900-02 : R.B.G.

M o. = Meteorological Office, Alipore.

M.Ph. = Mledical College, Physiological Laboratory

P.C. = Presidency College.

P.o. = Patent Otfice.

R.B.G = Royal Botanic Garden.

S.C.C. = Scuttısh Churches College.
S.G. = Surgeon-Generai s Library:

SI. = Survev of India.

S.T.M. = School of Tropical Medicine

T.D. = Telegraph Department.

X.c. =St. Xavier s College.

Z.s.l. = Zoological Survey of Inclia. 
298. London.-Royal Society (Contimued).

(o) Year-book. I89697-. London, I897-.

Vols. for $1896 / 97$ - (vols. for $1898 / 99$, I900 wanting) : A.S.B.

Vols. for I896/97- (vols. for I898/99, 1900, '04 wanting): Z.S.I.

299. - (Royal) Society of Arts.

(a) Journal of the Society of Arts. Vols. I-55, I852-I907. London, I853-1907. [Continued as:] Journal of the Royal Society of Arts. Vols.56-, I907-. London, I908-.

Complete set: G.S.I.

Vols. I, 2, I853-54 : E.C.

Vols. 23-, I8 75 - (vols. $35-39,4$ I-44, 46 wanting) : I.L. Vols. $23-38$, I $875-90$ : A.S.B.

Vols. $35-58$, I 887 -I 9 Io (many vols. defective) : B.S.I.

Vols. 54-, I906-: R.B.G.

Vols. 64一, I9I6-: S.I.

(b) Papers on Mechanics. Vols. I-4. London, I8Io-43: G.S.I.

(c) Transactions of the Society.... for the Encouragement of Arts, Manufactures and Commerce. Vols. I-55. London, I783-I845. Analytical index to vols. I-25 at end of vol. 26.

Vols. I-55, I783-I845, 3 rd. ed. (vol. 4 wanting): E.C. Vols. I-55, I $783-1845$; vols. I-5 of 2 nd or 3 rd ed. (vols. II, 19, 45 wanting) : A.S.B.

Vols. I-5I, I783-I837 : R.B.G.

300. — Royal Society of Medicine.

Proceedings. Vols. I-, I907/08-. London, I908- : S.T.M.

301. — Royal Statistical Society.

Journal of the Statistical Society of London. Vols. I-49. London, I839-86. [Continued as:] Journal of the Royal Statistical Sociely. Vols. 50-. London, I887-. General indexes to vols. I-50.

Vols. I-, I $830-$ (vols. $17,28,35,50,59$ wanting, vols. $48,56,61$ defective) : I.L.

Vols. I-64, I 839-I9or (vols. 33, 34, 58 defective) and indexes to vols. I-50: A.S.B.

Vols. I-30, I839-67, and indexes to vols. I-25: S.G.

Vols. $49-54$, I 886-92 (rol. 53 wanting) : B.S.I.

A.S. = Archaeological Section, Indian Museunı.

A.S.B. = Asiatic Society of Bengal.

B.C. = Bishop's College.

B.S.I. = Botancal Survey of India

C.E. = Chemical Examiner to the Govt. of Bengal.

c.U. = Calcutta University
E.C. =Civil Engineerng College, Sibpur

G.S.I. = (reological Survey of India

I.L. = Imperial Library.

M.Ba. = Medical College, Bacteriological Laboratory

M.Bi. = Medical College. Biological Laboratory.

M.C. = Medical College (main library) 
302. London.-Saint Thomas's Hospital.

Reports. New series, vols. 25-35, I806-I9o6. London, I $997^{-}$- 908 (vols. 32, 33 wanting) : A.S.B.

303. - Sanitary Record. New series, vols. I- London, I879-.

Vols I-, I879- (vols. 35-4I wanting): S.G.

Vols. 47-49, I9I I-I2 (vol. 49 defective) : S.T.M.

304. - School of Tropical Medicine.

Journal. Vols I-. Itondon, IgII- : S.T.M., M.Ba.

305. - Science Abslracts. Physics and Electrical Engineering. Issued under the direction of the Institution of Electrical Engineers, the Physical Society of London and the American Physical Society. Vols. I-. London I8g8-. [After vol. 5, I902, issued in two separate sections-A (Physics) and B (Engineering).]

Vols. I-, I $8 \mathrm{~g} 8$ - (vol. 12 defective) : P.C.

Vols. I-I4, I8g8-I9II : X.C.

306. - Science Progress: a monthly review of current scientific investigation. Conducted by H. C. Burdett; ed. by J. B. Farmer. Vols. I-6. London, I894-97 : R.B.G. [Vol. 6 is also styled ' New ser., vol. I.']

307. - Science Progress in the Twentieth Century. Ed. by N. H. Alcock and W. G. Freeman. Nos. I-. London, Igo6-.

Complete sets : G.S.I., R.B.G., Z.S.I.

Nos. I-I4, Igo6-Og (no. + wanting) : C.E.

308. - Scientific Memoirs, selected from the Transactions of Foreign Academies of Science and Learned Societies and from Foreign Journals. Ed. by R. Taylor. Vols. I-5. London, I836-52.

Complete set : G.S.I.

Vol. I, I836: A.S.B., R.B.G.

309. - Sleeping Sickness Bureau.

(a) Bulletin. Vols. I.-, Igo8,09-. London, Igo9- : S.T.M.

(b) Kala Azar Bulletin. Vol. I, nos. 1, 2. London, I9III2 : S.T.M.

310. - Society for the Study of Disease in Children.

Report. Vols. I-8. London, I900-08: S.G. Index to vols. I-8: S.G.

Society instituted at London for the encouragement of Arts, Manufactures and Commerce (see No. 299 (c)).

M.O. = Meteorological Office, Alipore

M.Ph. = Medical College, Physiological Laboratory.

P.C. = Presidency College.

P.O. = Patent Otfice.

R.B.G. = Royal Botanic Garden

S.C.C. = Scottish Churches College
S.G. = Surgeon-General s Library.

S.l. = Survey of India.

S.T.M = School of Tropical Medicine

T.D. = Telegraph Department

X.c. =St. Xavier s College.

Z.S.I. = Zoological Survey of India. 
3II. London.-Society of Antiquaries.

(a) Archcoologia: or miscellaneous tracts relating to antiquity. Vols. I-37. London, I773-I857 (vol. 27 wanting; vols. I, I4 of and ed.): A.S.B. Index to vols. I30 : A.S.B.

(b) Proceedings. Vols. 2, 3, I849-56. London, I853-56: A.S.B.

Society of Arts (see No. 299).

312. - Society of Chemical Industry.

Journal. Vols. I-. London, I882-. Indexes to vols. $\mathrm{I}-\mathrm{I} 4$ and $\mathrm{I}_{5}-24$.

Vols. I-, I 882- (vol. 26 wanting). Indexes to vols. $\mathrm{I}-24$ : P.C.

Vols. I-29, I882-I9IO: A.S.B.

Vols. 6-, I887- (vol. 14 wanting). Index to vols. I-I 4 : B.S.I.

Vols. I2, I3, I893-94: C.E.

Vols. I9-, I900-: G.S.I., I.L.

Vols. 25一, Ig06- : E.C.

3I3. - Society of Telegraph Engineers.

Journal. Vols. I-I7. London, I873-88. [Continued as No. 179.]

Complete sets: A.S.B., X.C.

Tols. 7-I7, I878-88 : T.D.

3I4. - Society of Tropical Medicine and Hygiene.

(a) Transactions. Vols. 3-. Iondon, I9IO- : S.T.M.

(b) Year-Book. I9Io II-'I3/I4. I.ondon, IgII-I4: S.T.M.

315. - Spicilegia Zoologica : or original figures and short systematic descriptions of new and unfigured animals. By J. E. Gray. Parts I, 2. London, [I828,'30]: R.B.G., Z.S.I.

- Statistical Society (see No. 30I).

316. - Telegraphic Journal and Electrical Review. Vols. I-29. Lon don, I872-9I. [Continued as No. I30.]

Complete set : T.D.

Vols. $\mathrm{I}-9, \mathrm{r} 872-8 \mathrm{I}: \mathrm{X.C}$.

Vols. 28, 29, I89I : I.L.

317. - Therapist. Vols. I8-. London, I908- (vol. 20 defective) : S.T.M.

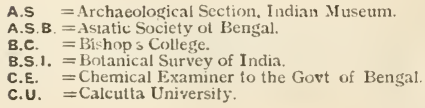

A.S = Archaeological Section. Indian Museum.

A.S.B. = Astatic Society of Bengal.

B.C. = Bísop s College.

B.S.1. = Botanical Survey of India

C.E. = Chemical Examiner to the Govt of Bengal

c. $\boldsymbol{U}$. Calcutta University.

E.C. = Civil Engineering Collese, Sibpur.

G.S 1. = Geolosical Survey of India.

I.L. = Imperial Library.

M.Ba, = Medical College, Bacteriological Laboratory

M.Bi. = Medical College. Bıological Laboratory.

M.C. = Hedical College (matn library). 
3 I 8 London.-Thomson Yates (and Johnston) Laboratories.

Reports. Vol. 3. pt. 2 and vol. 4, pts. I, 2. London, I902: Z.S.I. New series, vol. 5, pts. I, 2 and vol. 6 , pt. I. London, I903-05: Z.S.I.

— Tropical Diseases Bulletin (see No. I74).

3r9. — Tropical Life. Vols. 2-. London, I906- : R.B.G.

320 West India Committee.

(a) Circular. Vols. I-. London, I898- (many vols. de. fective) : B.S.I.

(b) Pamphlet. Nos. I-. London, I905-: B.S.I.

321. Wireless World. Vols. I-. London, I9I3-(vol. 2 wanting) : T.D. [A continuation of No. 2I2.]

322. - Year Book of Wireless Telegraphy and Telephony. Vols. ILondon, I9I3- : T.D.

323. Z Zoological Illustrations, or original figures and descriptions of new, rare, or interesting animals, chiefly selected from the classes of Omithology, Entomology and Conchology. By IT. Swainson. Vols I-3. London, I820-23: A.S.B., Z.S.I. Second series, vols. I-3. London, I829-33 : A.S.B., Z.S.I.

324. Z Zoological Journal. Ed. by 'T. Bell, N. A. Vigors, and others. Vols. I-5. London, I824-35.

Complete sets : G.S.I., Z.S.I.

Vols. I, 2, I825-26: A.S.B.

325. - Zoological Miscellany. By J. E. Gray. [Part I.] London, I83I : Z.S.I.

326. Z Zoological Miscellany. By IV. E. Leach. 3 vols. London, I 8 I4-I7 : A.S.B.

327. Z Zoological Society.

(a) Proceedings of the Committee of Science and Correspondence. Parts I, 2, I830-32. [London, I831-32]. [Continued as:] Proceedings of the .... Society. Parts 3-28. [London], I835-60. [Continued as:] Proceedings of the Scientific Meetings. I86I-90. London, I86I-90. [Continued as:] Proceedings of the General Meetings for Scientific Business. I89I-. London, I89I-. Indexes to vols. for I830-I9IO.

Complete sets and indexes: A.S.B., Z.S.I.

Vols. for I87I-, and indexes for I87I-I9IO : G.S.I.

\footnotetext{
M.o. = Meteorological Office, Alipore.

M.Ph. = Medical College, Physiological Laiboratory:

P.C. = Presidency Collegc.

P.O. = Patent Office

R.B.G. = Royal Botanic Garden

S.C.C. $=$ Scottish Churches College.
}

S.G = Surgeon-General's Lib-ary

S.I. = Survey of India.

S.T.M. = School of Tropical Medicine.

T.D. = Telegraph Depariment.

X.C =St. Xavier's College.

Z.S.I = Zooiogical Survey of India 
327. London.-Zoological Society (Continued).

(b) Record of Zoological Literature. Vols. I-6, I864-69. London, I865-70. [Continued as $(d)$.]

Complete sets : A.S.B., Z.S.I.

Vols. I-5, for $1864-68$ : G.S.I.

(c) Transactions. Vols. I- London, I833-. Index to vols. I-IO.

Complete set: Z.S.I.

Vols. I-, I833- (vol. I6 wanting, vol i7 defective): A.S.B.

Vols. 8-, I874- : G.S.I.

(d) Zoological Record. Vols. 7-, I870-. London, I87I-. [A continuation of $(b)$. From 1906 combined with No. I597, Zoology.]

Complete set : Z.S.I.

Vols. 7-, I870- (vol, for 1906 wanting) : G.S.I.

Vols. 7-34, I870-97 (vol. for 1888 wanting) : A.S.B.

328. - Zoologist. Conducted by E. Newman and others. Vols. I23. London, I843-65. 2nd ser., vols. I-II. London, I86676. 3rd ser. Ed. by J. E. Harting. Vols. I-20. London, 1877-96. 4th ser. Ed. by W. L. Distant. Vols. I-. London, I897-.

Vols. I-I6, I $843-58$ : G.S.I.

Vol. I7, I859, and 2nd ser., vols. I-, I886- : Z.S.I.

329. Manchester:-Chemical Trade Journal. Vols. I-I8. Manchester, I887-96: C.E.

330. - Geological and Mining Society.

Transactions of the Manchester Geological Society. Vols. I-27. Manchester, I84I-I902: G.S.I. [Continued as:] Transactions of the ...... Society. Vols. 28-. Manchester, I905-: G.S.I. Index to vols. I-28: G.S.I.

—_ Geological Society (see No. 330).

331. — John Ryland Library.

Bulletin. Vols. 4-. Manchester, I907-: Z.S.I.

332. L Literary and Philosophical Society.

(a) Memoirs. Vols. I-5 Warrington, Manchester, London, I785-I80z. 2nd ser., vols. I-I5. London, I805-60. 3rd ser., vols. I-Io. London, I862-87. Index to vols. $\mathrm{I}-5$ and ser. 2 , vols. $\mathrm{I}-\mathrm{I} 2$ at end of vol. I2. [Continued as $(b)$.]

A.S. = Archaeological Section. Indian Museum

A.S.B. = Asiatic Society of Bengal

B.C. = Bishop s Collerge.

B.S.I. = Botanical Survey of Indua

C.E. Chemical Examiner to the Govt. of Bengal.

C.U. = Calcutta Unversity.
E.C. =Civil Engineering College, Sibpur.

G.S I. = Geological Survey of India.

M. Ba. = Medical College, Bacteriological Laboratory.

M.Bi. = Medical College. Biological Laboratory.

M.C. = Niedical College (main library). 
332. Manchester.-Literary and Philosophical Society (Continued). Ist, 2nd and 3rd ser., I785-I887 (vol. 3, ist series, wanting) : A.S.B.

Vols. I-5 and 2nd ser., vols. I-4, I785-I824 : R.B.G. 2nd and 3 rd ser. complete, $1805-87$ : G.S.I.

(b) Memoirs and Proceedings. 4th ser., vols. I-Io and [whole series] vols. 4I-. Manchester, I888-. [A continuation of $(a)$.]

Complete set : G.S.I.

Vols. I-IO and 4I-, I888- (vol. 49 defective) : A.S.B.

(c) Proceedings. Vols. I-26, I857-87. Manchester, I862-

87. [Afterwards published with $(b)$.]

Complete set : G.S.I.

Vols. $2-26,1863-87$ : A.S.B.

333. - Medical Chronicle. 4th ser., vols. I4-(=vols 47-). Manchester, I907-- (vols. 14,15 defective) : S.T.M.

334. - Owens College.

Studies in Biology from the Biological Department. Vols. 3, 4. Manchester, I895-99: Z.S.I.

335. — Owens College.-Museum.

Notes from the Manchester Museum. Nos. 5-22. Manchester, 1899-1909 (nos. 7, 9, 14, 15, 17, 18 wanting): Z.S.I.

336. Newcastle-upon-Tyne.--(Federated) Institution of Mining Engineers.

Transactions of the (Federated) Institution of Mining Engineers. Vols. I-. Newcastle-upon-Tyne, I892-. Indexes to vols. I-40.

Complete set and indexes: G.S.I.

Vols. I-, I 892- (vols. 32-37, 40-47 wanting): E.C.

337. - Natural History Society of Northumberland, Durham and Newcastle-upon-Tyne.

Natural History Transactions of Northumberland and Durham. Vols. I-3. I.ondon, I867-70: G.S.I. [For earlier series, see No. 339.]

338. — North of England Institute of Mining (and Mechanical) Engineers.

Transactions. Vols. I, 2 (2nd ed.). Newcastle-uponTyne, I860-63. Vols. 8-. Newcastle-upon-Tyne, I $860-$ (vol. 21 wanting) : G.S.I. Indexes to vols. I-38: G.S.I.

M.o. = Meteorological Office, Alipore.

M.Ph. = Medical College, Physiological Laboratory.

P.C. = Presidency College.

P.o. = Patent Ofíce.

R.B.G. = Royal Botanic Garden

S.C.C. = Scottish Churches College.
S.G. = Surgeon-General's Library.

S.l. = Survey of India.

S.T.M. = School of Troplcal Medicine

T.D. = Telegraph Department.

X.C. =St. Xavier's College.

Z.S.1. Zoological Survey of India. 
339. Newcastle-upon-Tyne.-Tyneside Naturalists' Field Club.

Transactions. Vols. I-6, I846-64. Newcastle-upon-Tyne, I850-54 : G.S.I. [For later series, see No. 337.]

Oxford.-Annals of Botany (see No. 78 ).

340. - Ashmolean Natural History Society of Oxfordshire.

Proceedings and Report. Igo9-. Oxford, I9I0-: G.S.I.

34I. Quarterly Journal of Medicine. Vols. I-. Oxford, I907-: S.T.M.

342. - University.-Hope Department of Zoology.

Hope Reports. Ed. by E. B. Poulton. Vols. I-, I893/97-. Oxford, I897-: Z.S.I.

343. Penzance.-Royal Geological Society of Cornwall.

Transactions. Vols. I-. London and Penzance, I8I8(vols. IO, II detective) : G.S.I.

344. Perth.-Scottish Naturalist. Ed. by J. W. H. Trail. Vols. 7,8 (=new ser., vols. I, 2). Perth, I883-86: Z.S.I.

345. Plymouth.-Devonshire Association for the Advancement of Science, Literature and Art.

Transactions. Vols. I-9. Plymouth, London, I863-77: G.S.I.

346. - Marine Biological Association of the United Kingdom.

Journal. New ser., vols. I-. London and Plymouth, I889- : Z.S.I.

347. Stoneyhurst College.-Observatory.

Results of Meteorological and Magnetical Observations. I869-. Preston, [I870-]: X.C.

348. Tring.-Museum.

Novitates Zoologicae. Ed. by Rothschild, Hartert and Jordan. Vols. I-. London and Aylesbury, I894- : A.S.B., Z.S.I.

349. York.-Yorkshire Philosophical Society.

Annual Report of the Council (Communications to the Monthly Mectings). I825-. York, I826- (Reps. for 1826 and 43 wanting): G.S.I.

A.S. = Archaeological Section, Indian Museun.

A.S.B. = Asiatic Society of Bengal.

B.C. = Bishop's College.

B.S.I. = Botanical Survey of India

C.E. = Chemical Examiner to the Govt. of Bengal.

C.U. = Calcutt $:$ University.
E.C. = Civil Enginecrins Collegc, Sibpur

G.S.I = Geologicit Survey of Indid.

I.L. = Imperial Library.

M. Ba. = Medical College, Bacteriological Laboratory

M.BI. = Medical College. Biolosical Laboratory.

M.C. = M edical College (man library) 


\section{AUSTRIA HUNGARI.}

Budapest.-Académie Nationale Hongroise des Sciences (see No. 355).

- Aquila (see No. 354).

—— Földrajzi Közlemények (see No. 35r).

Königliche Ungarische Geologische Reichsanstalt (see No. 352).

Königliche Ungarische Naturwissenschaftliche Gesellschaft (see No. 356).

350. — Magyar Botanikai Lapok (Ungarische Botanische Blätter). Kiadja degen A Szerkeszti (and others). Evfol. I-. Budapesten, I902-: R.B.G.

35r. — Magyar Földrajzi Társaság.

Földrajzi Kozlemények (Bulletin de la Société Hongroise de Géographie). Köt. I7-. Budapest, I889-(Köt. 23, 25,28 wanting): A.S.B.

352. — Magyar Királyi Földtani Intézet (Königliche Ungarische Geologische Reichsanstalt).

(a) Földtani Közlöny (Geologische Mittheilungen): Zeits. chrift der Ungarischen Geologischen Gesellschaft, zugl. amtliches Organ der $K$. Ungarischen Geologischen Reichsanstalt. Bde. I3-. Budapest, I883-: G.S.I.

(b) Jahresbericht. 1882-. Budapest, r883- : G.S.I. Index, I882-9I : G.S.I.

(c) Mittheilungen aus dem Jahrbuche. Bde. I- Budapest, I87I-: G.S.I.

353. —- Magyar Nemzeti Múzeum.

(a) Annales Historico-naturales Musei Nationalis Hungarici. Szerkeszti Horváth Géza (and others). Köt. I-. Budapest, 1903-. [A continuation of $(b)$.

Complete set: Z.S.I.

Köt. I-8, I903-Io : G.S.I.

(b) Természetrajzi Fiizetek. Szerkeszti Herman Ottó (and others). Köt. I-25. Budapest, I877-I902. [Continu.ed as $(a)$.] Index for $1877-86$.

Köt. I-25 (köt. 2-7, II wanting) and index : G.S.I.

Köt. 22-25, I899-1902 : Z.S.I.

354. - Magyar Ornithologiai Központ.

Aquila ; a Magyar Ornithologiai Központ Folyóirata (Zeits-

M.o. = Meteorological Office, Alipore

M.Ph. = Medical College, Physiological Laboratory.

P.C. = Presidency College.

P.O. = Patent Office.

R.B.G. = Royal Botanic Garden.

S.C.C. = Scottish Churches College.
S.G. = Surgeon-General's Library.

S.I. = Survey of India.

S.T.M. = School of Tropical Medicine.

T.D. = Telegraph Department.

X.C. = St. Xavier's College.

Z.S.I. = Zoological Survey of India. 
354. Budapest.-Magyar Ornithologiai Központ (Continued). chrift für Ornithologie). Jahrg. 4-: Budapest, I897-.

Jahrg. 4-I6, I897-I909: A.S.B.

Jahrg. II-, I904-: Z.S.I.

355. — Magyar Tudományos Akadémia.

(a) Bulletins de l'Académie Nationale Hongroise des Sciences. Nos. I-5. Florence, I884-86: A.S.B.

(b) Ertesitöje. Köt. I-I7 Budapest, I867-93 (köt. 8 wanting; köt. 7,9 defective) : A.S.B.

(c) Nyelvemléktár Régi Magyar Codexek és Nyomtatván. yok. Köt. 4-13. Budapest, I876-85 (köt. 6 wanting) : A.S.B.

(a) Nyelvtudományi Koziemények. Köt. 5-. Budapest, I866- (köt. 9, I0, 12, 27 33, 35 wanting) : A.S.B.

(e) Régi Magyar Költök Tára. Köt. I-5. Budapest, I877-86: A.S.B.

356. - Mathematische und Naturwissenschaftliche Berichte aus Ungarn. Mit Unterstützung der Ungarischen Akademie der Wissenschaften und der K. Ungarischen Naturwissenschaftlichen Gesellschaft. Red. von I. Fröhlich (and others). Bde. I-, I882-. Berlin, Budapest, Leipzig, I884-.

Bde. I-, I884- (Bd. 23 wanting; Bde. 4, 26 defective) : A.S.B.

Bde. I8-26, I903-Io (Bd. 26 defective) : Z.S.I.

- Museum Nationale Hungaricum (see No. 353).

357. - Rovartani Lapok. Havi folyóirat. Különös tekintettel a haznos és káros rovarokra. Szerkesztette és kiadta H. Géza. Köt. 2. Budapest, I885 : Z.S.I.

— Société Hongroise de Géographie (see No. 35I).

— Természetrajzi Fïzetek (see No. 353 (b)).

_- Ungarische Akademie der Wissenschaften (see Nos. 356, 358).

_- Ungarische Botanische Blätter (see No. 350).

— Ungarische Geologische Gesellschaft (see No. 352).

_- Ungarisches National-Museum (see No. 353).

358, Ungarische Revue. Mit Unterstützung der Ungarischen Akademie der Wissenschaften. Herausg. von P. Hunfalvy, G. Heinrich (and others). I88I-95. Budapest, I88I-95 (Bd. for I895 defective) : A.S.B.

A.S. = Archaeological Section, Indian Museum.

A.S.B. = Asiatic Society of Bengal.

B.C. = Bishop's College.

B.S.I. = Botanical Survey of India.

C.E. = Chemical Examiner to the Govt. of Bengal.

c.U. = Calcutta University.
E.C. = Civil Engineering College. Sibpur.

G.S.I. = Geolosical Survey of India.

I.L. = Imperial Library.

M.Ba. = Medical College, Bacteriological Laboratory.

M. Bi. = Medical College. Biological Laboratory.

M.C. = Medical College (nain library). 
359. Graz.-Naturwissenschaftlicher Verein für Steiermark.

Wittheilungen. Bde. [I8]-46, Jalırg. I88I-I909. Graz, I 882 -I9IO.

Bde. [I8]-46, I882-I9Io (Jahrg. 1894/95 wanting): A.S.B.

Bde. 43-45, I907-09: Z.S.I.

360. Zoologisches Institut.

Arbeiten. Bde. 8-. Leipzig, I9o7-(defective) : Z.S.I.

36I. Prag.-Comité für die Naturwissenschaftliche Landesdurchforschung Böhmens.

Archiv der naturwissenschaftlichen Landesdurchforschung von Böhmen. Bde. I-3. Arbeiten der geologischen Section (Abteilung). Prag, 1869-76: G.S.I. Bde. 4-II. Prag, I878-I90I (Geological papers only): G.S.I.

362. - Deutscher Naturwissenschaftlich-IIedicinischer Verein für Böhmen "Lotos."

(a) Abhandlungen. Bde. I, 2. Prag, I898-I90I : Z.S.I.

(b) Jahres-Bericht des Naturhistorischen Vereines "Lotos." Jahrg. 26-28. Prag, $1876-78$ : Z.S.I. [A continuation of (e). Continued as (c).]

(c) "Lotos," Jahrbuch fïr Naturwissenschaft im auftrage des Vercines "Lotos." Neute Folge, Bde. 2-6. Prag, I882-85 (Bd. 5 wanting): Z.S.I. [A continuation of (b). Continued as $(f)$.

(d) "Lotos," Naturwissenschaftliche Zeitschrift herausg. vom .... Verein. Neue Folge, Bde. I- $(=55-)$. Prag, I907-(Bd. 57 warting) : Z.S.I. [A continuation of $(f)$.

(e) "Lotos," Zeitschrift für Naturwissenschaften. Bde. I25. Prag. I85I-75. Register, Bde. I-23. [Continued as $(b)$.]

Bde. I-24, $185 \mathrm{I}-74$, and index : G.S.I.

Bde. 3-25, I853-75 (Bde. 6, 17, 18, 21 wanting): Z.S.I.

(f) Sitzungsberichte des.....Vereines. Neue Folge, Bde. I7-26. Prag, I897-I906: Z.S.I. [A continuation of (c). Continued as $(d)$.

363. - Kaiserlich-Königliche Sternwarte.

(a) Astronomische Beobachtungen. I884-. Prag, I886-:

A.S.B. [A continuation of (b).]

(b) Astronomische, Magnetische und Meteorologische Beo-

м o. = Meteorological Office, Alipore.

M.Ph. = Medical Cinllege. Physiological Laboratory.

P.C. = Presidency College.

P.O. = Patent Office.

R. B.G. = Royal Botanic Garden.

S.C C. $=$ Scuttish Churches College.
S.G. = Surgeon-Generai s Library.

S.I. = Survey of India.

S.T.M. = School of Tropical Medicine

T.D. = Telegraph Department.

X.C. =St. Xavier s College.

Z.S.I. =Zoological Survey of India 
363. Prag.-Kaiserlich-Königliche Sternwarte (Continued). bachtungen. Jahrg. 35-44, I874-83. Prag, I875-84:

A.S.B., M.O. [Continued in two parts as $(a)$ and $(d)$.]

(c) Magnetische und Meteorologische Beobachtungen. Jahrg. 22-34, I86I-73. Prag. I862-74. [Continued as $(b)$.]

Jahrg. 22-34, I 86I-73. I862-74: M.O.

Jahrg. 30-34, I869-73, I870-74: A.S.B.

(d) Magnetische und Meteorologische Beobachtungen. Jahrg. 45-, I884-. Prag, 1885-. [A continuation of (b).]

Complete set: M.O.

Jahrg 45-, I885- $\mathrm{J}$ Jahrg. 67,68 , for 1906-07, wanting) : A.S.B.

— Naturhistorischer Verein "Lotos" (see No. 362).

$3^{6} 4$. Sarajevo.-Bosnisch-Herzegovinisches I,andesmuseum.

Wissenschaftliche Mittheilungen aus Bosnien und der Herzegovina. Red. von M. Hoernes. Bde. 2. Wien, I894: Z.S.I.

365. Trieste.-Mruseo Civico di Storia Naturale Ferdinando Massimiliano.

Atti. Ser. nuova. Tol. $2-4(=$ vol. 8-Io). Trieste, I89oI903.

Vol. $2-4$, I $890-1903:$ A.S.B.
Vol. 4 , I903: Z.S.I.

366. - Società Adriatica di Scienze Naturali.

Bolletino. Vol. I-I5. Trieste, I874-93: A.S.B.

36\%. Vienna.-Anthropologische Gesellschaft.

Wittheilungen. Bde. 2-. Wien, I872- (Bd. 22 defective): A.S.B. [Bde. 11-30, 1882-1900, are also styled "Neue Folge,

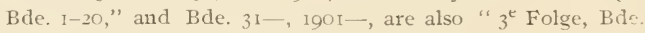
1-.". Indexes to Bde. II-30: A.S.B.

368. — Anthropos: international Zeitschrift für Völker-und Sprachen kunde. Bde. I-. Wien, Igo6-: A.S.B.

369. — Beiträge zur Paläontologie Oesterreich-Ungarns und des Orients. Herausg. von E. ron Mojsisorics, M. Neumayr (and others). Bde. I-. Wien, I882-: G.S.I.

- Geographische Abhandlungen (see No. 599).

370. — Geologische Gesellschaft.

Mitteilungen. Red. von V. Uhlig und C. Diener. Bde. I-. Wien, Ig08- : G.S.I.

A.S. = Archaeological Section. Indian Museun.

A.S B. =Asiatic Society of Bengal.

B.C. = Bishop's College.

B.S.I. = Botanical Survey of India

C.E. =Chemical Examiner to the Govt. of Bengal

c.U. Calcutta University.
E.C. =Civil Engincering College, Stbpur.

G.S.I. = veological Survey of Incia

I L. = Imperial Library.

M.Ba = Medical College, Bacteriologicai Lavorawory

M.BI. = Medical College. Biological Labrratory.

M.C. = IIcdical Colloge (main librarv). 
371. Vienna.-Gesellschaft der Freunde der Naturwissenschaften.

(a) Berichte ïber die Mittheilungen. Herausg. von W. Haidinger. Bde. I-7, I846-50. Wien, I847-5I : A.S.B., G.S.I. Register, Bde. I-7 : G.S.I.

(b) Naturwissenschaftliche Abhandlungen. Herausg. von W. Haidinger. Bde. I-4. IVien, I847-5I : A.S.B., G.S.I.

372. Kaiserliche Akademie der IVissenschaften.

(a) Almanach. Jahrg. 46-. Wien, I896-. Register, Bde. $1-60$.

Jahrg. 46-, I896-( J ahrg. $47,48,53,60$ wanting) and index : G.S.I.

Jahrg. 53-, 1903-, and index: A.S.B.

(b) Archiv für Kunde Oesterreichischer Geschichts-Quellen. Bde. I-. WVien, I848- (Bde. 3I, 42, 80, 93, 95, I02, 104, 106-8 wanting; Bde. 94, 99 defective): A.S.B. Register, Bde. I-IOO: A.S.B.

(c) Denkschriften. Math.-naturwiss. Classe. Bde. IWien, $1850-$. Register, Bde. I-25.

Complete set and index : G.S.I.

Bde. I-, I850-(Bde. 3, 67, 76 wanting): A.S.B.

(d) Denkschriften. Phil.-historisch. Classe. Bde. I-.

Wien, I850-(Bde. 53, 55 defective) : A.S.B. Register, Bde. $36-50$ : A.S.B.

(e) Mittheilungen der Erdbeben-Commission. Neue Folge, Nos. I-. Wien, IgoI-. [Previously published in $(\mathrm{g})$. .]

Nos. I-, IgoI- (nos. 32, 37 wanting): A.S.B.

Nos. I-24, I901-04: G.S.I.

(f) Oesterreichische Geschichts-Quellen (Fontes Rerum Austriacorum). $2^{\mathrm{e}}$ Abtheilung. Diplomataria et Acta. Herausg. von der Historischen Commission der ..... Akademie. Bde. I-. Wien, I849- (Bd. 57 wanting) :

A.S.B. Register, Bde. I-50: A.S.B.

(g) Sitzungsberichte. Math.-naturwiss. Classe. Bde. I-. Wien, I848-. Register, Bde. I-I2O.

Complete set and indexes: G.S.I.

Bde. I-, I848- (Bde. II2, I2I defective); indexes to Bde. 2I-I2O : A.S.B.

(h) Sitzungsberichte. Phil.-historisch. Classe. Bde. I-. Wien, I848-(Bd. $4 \mathrm{I}$ wanting; Bde. 36, I69-172 defective) :

A.S.B.

M. O. = Meteorological Office, Alipore

M.Ph. = Medical College, Physiological Laboratory.

P.C. = Presidency College.

P.O. = Patent Office.

R.B.G = Royal Botanic Garden

S.C.C. $=$ Scottish Churches College
S.G. = Surgeon-General's Library.

S.I. = Survey of India.

S.T.M. = School of Tropical Medicine

T.D. = Telegraph Department.

x.c. =St. Xavier's College.

Z.s.I. =Zoological Survey of Inòia. 
373. Vienna.-Kaiserlich-Königliche Central-Anstalt für Meteorologie und Erdmagnetismus.

Jahrbuicher. Bde. I-8, I848-56. Wien, I854-6I : A.S.B.

374. — Kaiserlich-Königliche Geographische Gesellschaft.

(a) Abhandlungen. Bde. I-4. Wien, I899-I902 : G.S.I.

(b) Mittheilungen. Jahrg. I-45. Wien, I857-I902. [J ahrg. I I-45, I869-1902, are also styled "Neue Folge, Jahrg. I-35."]

Jahrg. I-45, I857-I902 (Jahrg. II, I2, I7 wanting) : G.S.I.

Jahrg. I-9, I857-65 : A.S.B.

375. — Kaiserlich-Königliche Geologische Reichsanstalt.

(a) Abhandlungen. Bde. I-. Wien, I852-.

Complete set: G.S.I.

Bde. I-, I852- (Bde. 7, I3, I5, I7-19 wanting; Bde. 16, 20 defective) : A.S.B.

(b) Jahrbuch. Jahrg. I-. Wien, I850-. General Register, Jahrg. I-50. [From Jahrg. 9 to Jahrg. 33, 1858-83, the Verhandlungen are included in the corresponding Jahrbuch vols.]

Complete set and indexes: G.S.I.

Jahrg. I-, I850-(Jahrg. 38 defective, 4I, 6I wanting) and indexes: A.S.B.

(c) Verhandlungen. I884-. Wien, I884-. [Previously published with $(b)$.]

Complete set : G.S.I.

Vols. for 1884 - (vol. for 1884 defective) : A.S.B.

376. —— Kaiserlich-Königliche Oesterreichische Gesellschaft für Meteorologie.

Meteorologische Zeitschrift. Herausg. von der ...... Gesellschaft .... und der Deutschen Meteorologischen Gesellschaft. Jahrg. I-27. Berlin, Wien, I884I9IO : M.O.

377. — Kaiserlich-Königliches Naturhistorisches Hofmuseum. (See also No. 389.)

Annalen. Red, von F. Steindachner (and others). Bde. I-. Wien, I886-.

Complete sets : G.S.I., R.B.G.

Bde. I-, I886- (Bd. Io defective) : Z.S.I.

Bde. I-, I886- (Bde. 3, 4, 6, II, I3, I5, I9, 25 defective) : A.S.B.

A.S. =Archaeological Section, Indian Museum

A.S.B. = Asiatic Society of Bengal.

B.C. = Bishop s College

B.S.I. = Botanical Survey of India

C.E. = Chemical Examiner to the Guvt. of Bengal.

c. $U$. Calcutta Unversity.
E.C. = Civil Engineering College, Sibpur.

G.S.1. = Geological Survey of India.

L. = Imperial Library.

M.Ba. = Medical College. Bacteriological Laboratory

M.Ba. = Mi. = Iedical College. Biological Laboratory

M.C. = Medical Colle'ige (main library) 
378. Vienna.-Kaiserlich-Königliche Universität.-Embryologisches Institut.

Mitheilungen. Von S. L. Schenk. Bde. I, 2. Wien, I877-83: Z.S.I. Neue Folge, Hfte. $2-5$. Wien, I88792 (Hft. 1 wanting) : Z.S.I.

379. — Kaiserlich Königliche Universität.-Orientalisches Institut.

Tienna Oriental Journal. Vols. I-. Vienna, I887(vols. 12,15 defective) : A.S.B.

380. — Kaiserlich-Königliche Universität.-Zoologisches Institut.

Arbeiten aus dem ...... Instilute und der Zoologischen Station in Triest. Tom. I-. Wien, I878-: Z.S.I. Generalregister, Tom. I-IO : Z.S.I.

38r. — Kaiserlich-Königliche Zoologisch-botanische Gesellschaft.

(a) Abhandlungen. Bde. 4-. Jena, I907-: Z.S.I.

(8) Verhandlungen der Zoologisch-botanischen Vereines. Bde. I-7. Wien, IS52-57. [Each vol. is in two separately paged parts, Sitsungsberichte and Abhandlungen. For continuation and index see $(c)$.]

Bde. I $-7, \mathrm{I} 852-57$ (Bd. + wanting) : A.S.B

Bde. 5-7, I855-57 : Z.S.I.

(c) Verhandlungen. Bde. 8-. Wien, I858-. [A continuation of (b).] Personen-, Orts- und Sach-register, I 85 I -55, I $856-60, I 87$ I-So.

Bde. 8-, I858- (Bde. 41,44 wanting); index, I87 I80 : A.S.B.

Bde. 8-24, I858-74 (Bd. 23 wanting); Bde. 57-, I907- : indexes, I85I-60 : Z.S.I.

- Meteorologische Zeitschrift (see No. 376).

382. - Mineralogische Mittheilungen gesammelt von G. Tschermak. Jahrg. I87I-77. Wien, IS $72-77$ : G.S.I. [Continued as :] Mineralogische und Petrographische Mittheilungen. Herausg. von G. Tschermak. Neue Folge, Bde. I-IO. Wien, I879-87 : G.S.I. [Continued as:] Tschermak's Mineralogische und Petrographische Mittheilungen. Herausg. von F. Becke. Bde. II-. Wien, I888- : G.S.I.

383. - Miscellanea Austriaca ad botanicam, chemiam, et historiam naturalem spectantia, etc. [Ed. by] N. J. Jacquin. Vols. I, 2. Vindobonae, I778, 'SI : Z.S.I.

- Naturwissenschaftliche Abhandlungen (see No. 37 I (b)).

_ Oesterreichische Gesellschaft für Meteorologie (see No. 376).

M o = Meteorological Office, Aipore

M Ph = Medical College. Physiological Laboratory.

P.C. = Presidency College.

PO = Patent Office.

R. B. G $=$ Royal Botanic Garder.

S.C.C. $\approx$ Scottish Churches College.
S.G. = Surgeon-General's Library

S.l. = Survey of India.

S.T.M. = School of Tropical Medicine

T.D. = Telegraph Department.

X.C. =St. Xavier s College.

Z.s.l. = Zoological Survey of India. 
384. Vienna.-Oesterreichischer Alpen-Verein.

Jahrbuch. Red. von E. von Mojsisovics. Neue Folge, Bde. I-5. WVien, I865-69: G.S.I.

385. — Orientalisches Museum.

Oesterreichische Monatsschrift für den Orient. Jahrg. Io, II. Wien, I $884-85$ : A.S.B.

386. - Ornithologischer Verein.

Mittheilungen. Jahrg. I-7. Wien, I877-83: A.S.B.

- Tschermak's Mineralogische und Petrographische. Wittheilungen (see No. 382).

— Vienna Oriental Journal (see No. 379).

387. - Wiener Entomologische Monatschrift. Red. Lederer und Miller. Bde. I-8. Wien, I857-64: Z.S.I.

388. - Wiener Entomologische Zeitung. Herausg. und red. von Ganglbauer, Mik, Hetschko, Reitter (and others). Jahrg. I-. Wien, I882-: Z.S.I.

389. - Wiener Museum der Naturgeschichte. (See also No. 377).

Zoologische Abhandlungen aus den Annalen. Bde. I, 2. Wien, I84I (defective): G.S.I.

Z Zoologisch-botanischer Verein (see No. $38 \mathrm{I}$ ).

Wien see Vienna.

390. Zagreb.-Arkeologičko Družtvo.

Viestnik. Godina I-I4. Zagreb, I879-92: A.S.B. Nove serije. Godina I-Io. Zagreb, I895-I9o9 (Godina 8, 1905, wanting) : A.S.B.

\section{BALKAN STATES.}

39I. Bukharest.-Institutul Geologic al României.

Amuarul. Anul I-. Bucuresti, I908-: G.S.I.

392 - Ministerulŭ I,ucrărilorŭ Publice

Anuarulü Biuroului Geologicŭ. Anulù I-3. I882-85. Anul 5, no. I [I887]. Bucuresci, I884-88: G.S.I. [Continued as .Yo. 393.]

A.S. = Archaeological Section. Indian Museum. A.S.B. = Asiatic Society of Bengal.

B.C. = Bishop's College.

B.S.I. = Botanical Survey of Inciia

C.E. = Chemical Examiner to the Govt. of Bengal.

c.U. = Calcutta University:
E.c. = Civil Engineering College. Sibpur.

G.S.I. = Geological Survey of Incia

I L. = Imperial Library

M.Ba. = Medical Colle đc. Bacteriological Laboratory

M.B1. = Medical College. Biological Laboratory.

M.C. = Medical College (main library). 
393. Bukharest.-Museul de Geologia si de Paleontologia.

Annuarulŭ. Sub direc. G Stefänescu. Anulŭ $1894-96$. Bucuresci, ז895-99: G.S.I. Vol. 4. Bucuresci, I9I0:

G.S.I. [For earlier series see No. 392.]

394. - Revue du Pétrole. Direct. G. M. Murgoci. Ann. I, 2. Bucuresti, I908 : G.S.I.

BEIGIUM.

395. Antwerp.-Académie d'Archéologie de Belgique.

Annales. Tom. 7. Anvers, I850: A.S.B.

396. - Société Royale de Géographie.

Bulletin. Tom. 20-. Anvers, I895- (tom. 20, 21 defective) : I.L.

397. Brussels.-Académie Royale des Sciences et Belles-Lettres de Bruxelles.

(a) Bulletins. Tom. I-I2, I832-45. Bruxelles, I836-45:

G.S.I. [Continued as No. $398(b)$.]

(b) Mémoires. Tom. I-4. Bruxelles, I777-83 (tom. 5, I788, wanting): G.S.I.

(c) Mémoires Couronnés. Tom. 6-I5. Bruxelles, I8274I : G.S.I. [A continuation of $(e)$. Continued as $(d)$.]

(d) Mémoires Couronnés et Mémoires des Savants Etrangers. Tom. I6-I8. Bruxelles, I844-45: G.S.I. [A continuation of $(c)$. Continued as No. $398(j)$.]

(e) Mémoires sur les Questions proposées par l'Académie ... qui ont remportés les Prix de I 774, '76, '77, '78, '79, '80, '82, '83, '85. Bruxelles [I775-86]: G.S.I. [Continued as:] Mémoires sur les Questions proposées par l'Académie .... Tom. I-5. Bruxelles, I8I8-26: G.S.I. [Continued as (c).]

(f) Nouveanx Mémoires. Tom. I-I9. Bruxelles, I82045 : G.S.I. [Continted as No. $398(j)$.

398. — Académie Royale des Sciences, des Lettres et des Beaux-Arts de Belgique.

(a) Annuaire. Ann.2-. Bruxelles, I836-.

Ann. 2-, I 836 - : G.S.I.

Ann. 3I-, I865- (ann. 78, 1912, wauting): A.S.B.

Ann. 75-, I909- : Z.S.I.

M.o. = Meteorologicai Office, Alipore.

M.Ph. = Medical College, Physiological Laboratory.

P.C. = Presidency College.

P.O. = Patent Office

R.B.G. = Royal Botanic Garcien.

S.C.C. $=$ Scottish Churches College.
S.G. = Surgeon-Generai's Libraty

s.1. = Survey of India

S.T.M. = School of Tropical Medicine

T.D. = Telegraph Department.

X.C. =St. Xavier's College.

z.s.l. = Zoological Survey of India. 
398. Brussels.-Académie Royale des Sciences .. (Continued).

(b) Bulletins. Tom. I3-23. Bruxelles, I846-56. $2^{\text {me }}$ sér., tom. I-5o. Bruxelles, I857-80. $3^{\text {me }}$ sér., tom. I-36. Bruxelles, I88I-08. Tables générales: $2^{\text {me }}$ sér., tom. I-20, 2I-50; $3^{\text {me }}$ sér., tom. I-30, 3I-36. [A continuation of No. $397(a)$, continued in two parts as $(c)$ and $(d) .7$

Complete set and indexes: G.S.I.

$2^{\text {me }}$ sér., tom. I9-50, I865-80 ; 3rd sér., tom. I-36, I $88 \mathrm{I}-98$, and indexes: A.S.B.

(c) Bulletin de la Classe des Lettres et des Sciences Morales et Politiques et de la Classe des Beaux-Arts. I899-. Bruxelles, I899-: A.S.B.

(d) Bulletin de la Classe des Sciences. I899-. Bruxelles, I899-.

Complete sets : A.S.B., G.S.I.

Vols. for I907-: Z.S.I.

(e) Mémoires. [Tom. I-3.] Bruxelles, I840-49: G.S.I. [Continued as $(j), 8^{w \prime \prime}$ coll.]

(f) Mémoires. Tom. 20-54. Bruxelles, I847-I904. [Continued in two sections, $(g)$ and $(h)$, each divided into $4^{\text {to }}$ and $8^{\text {ro }}$ collections. A continuation of No. $\left.397(f).\right]$

Complete set : G.S.I.

Tom. 3I-53, I859-98 (tom. 50 wanting): A.S.B.

(g) Mémoires. Classe des Lettres et des Sciences Morales et Politiques et de la classe des Beaux-Arts. Collection in $4^{\text {to }}$. $2^{\text {tue }}$ sér., tom. I-. Bruxelles, I906-: A.S.B. Collection in $8^{\mathrm{w}}$. $2^{\text {me }}$ sér., tom. I--. Bruxelles, I904-: A.S.B.

(h) Mémoires. Classe des Sciences. Collection in $4^{\text {to }} .2^{\text {mac }}$ sér., tom. I- Bruxelles, I907-. Collection in $8^{\text {ro }}$. $2^{\text {mee }}$ sér., tom. I-. Bruxelles, I904-.

Complete set, both collections: G.S.I.

$4^{\text {to }}$ coll., tom. I-, Igo7- (tom. I, 2 defective) ; $8^{\text {vo }}$ coll. complete : A.S.B.

(i) Mémoires Couronnés et autres Mémoires. Collection in 8vi. Tom. 7-66. Bruxelles, I858-I904 [A continuation of $8^{\text {ro }}$ coll. $(j)$. Afterwards merged in $(g)$ and $\left.(h).\right]$

Complete set: G.S.I.

Tom. I0-66, I860-I904 (tom. 48, 62 defective): A.S.B.

(j) Mémoires Couronnés et Mémoires des Savants Étrangers. Collection in $4^{\text {to }}$. [A continuation of No. $397($ d).] Tom. I9-62. Bruxelles, I847-I904. Collection in $8^{\text {ro. }}$ Tom. 4-6. Bruxelles, I850-53. $\quad 44^{\text {to }}$ coll. afterwards

A.s. = Archaeological Seclion, Indian Museum.

A.S.B. = Astatic Society of Bengal.

B.C. = Bishop s College.

B.S I. = Botanical Survey of India.

C.E. = Chemical Examiner to the Grovt of Bengal.

C.U. = Calcutta Universily
E.C. = Ctvil Engineering College. Sibrur.

G.S.I. = Geological Survey of Incha.

l.L. = Imperial Library.

M.Ba. = Medical College, Bacteriological Laboratory

M.Bi. = Medical Collere. Biolosical Laboratory.

M.C. = Medical College (man library). 
398. Brussels.-Académie Royale des Sciences .. (Continued).

merged in $(g)$ and $(h) ; 8^{\mathrm{w}}$ coll. a continuation of $(e)$ and continued as $(i)$.]

Complete set, both collections : G.S.I.

$4^{\text {to }}$ coll., tom. 2S-62, I856-Igo4: A.S.B.

Tables des Mémoires des Membres, des Mémoires Couronnés et de ceux des Savants Étrangers, I8I6-57:

G.S.I. I8j8-78: G.S.I.

Tables générales des Mémoires de l'Académie Royale des Sciences, des Lettres et des Beaux-Arts de Belgique, I772-I 897 : G.S.I.

399. - Annales de Biologie Lacustre. Publ. sous la direct. E. Rousseaı. Tom. I-. Bruxelles, Igo6-: Z.S.I.

Archives de Biologie (see No. 4I5).

400. - Bibliographia Geologica: répertoire des travaux concernant les sciences géologiques dressé d'après la classification décimale de la Bibliographia Universalis. Par M. Mourlon. Sér. A, se rapportant aux publications antérieure à I896. Tom I-9. Bruxelles, I899-Igo6 : G.S.I. Sér. B, se rapportant aux travaux pour à partir du I Janvier, I896. Tom. I-7. Bruxelles, I897-I904: G.S.I. [Tom. I of sér. B lacks title of series and rol. no.]

40I. - Bulletin Agricole du Congo Belge. Tom. 2-. Bruxelles, IgII- (tom. 2 defective) : B.S.I.

402. - Jardin Botanique de l'Etat.

Bulletin. Vol. I. Bruxelles, I902-05: R.B.G.

403. - Musée du Congo.

(a) Annales. Botanique. Sér. I-, I 898 - (Sér. 3 wanting): A.S.B.

(b) Annales. Ethnographie et Anthropologie. Sér. 3-, Ig02- : A.S.B.

(c) Annales. Minéralogie, Géologie, Paléontologie. Sér. I-, I9IO- : A.S.B.

(d) Annales. Zoologie. Sér. I, fasc. 4-, I899-: A.S.B.

404. —- Musée Royal d'Histoire Naturelle de Belgique.

(a) Annales. Tom. I-I4. Bruxelles, I877-96: G.S.I.

(b) Bulletin. Tom. 1-5, no. I. Bruxelles, I882-88: A.S.B., G.S.I., Z.S.I.

(c) Mémoires. Tom. I-. Bruxelles, Igoo-.

Tom. I-, Igoo- (tom. 2 defective) : Z.S.I.

Tom. 3-, IgO4-: A.S.B., G.S.I.

M. . = Meteorological Ofĩce, Aiipore. .

M.Ph = Medical College, Physiological Laboratory

P.C. = Presidency College.

Po. = Patent Ofince.

R B.G = Roval Botanic Garder.

S C C $\mathbf{C}$ Scottish Churches College.
s.G. = Surgeon-Generai's Library

S.I. = Survey of Indis.

S.T.M = School of Trovical Medicine.

T.D. = Telegraph Departmen:

x.c. =St Xaviers College.

Z.S.1. Zooiogical Survey of India. 
405. - Observatoire Royal.

Annales. I868-8I, I885. Paris, I868-8I, I885 : X.C.

— Société Belge de Géographie (see No. 409).

406. - Société (Belge) d'Études Coloniales.

Bulletin. Tom. 8-. Bruxelles, IgoI-(tom. 8, 16, 18 defective): B.S.I.

407. - Société Belge de Geologie, de Paléontologie et d'Hydrologie.

(a) Bulletin (including Mémoires). Tom. I-. Bruxelles, I887- : G.S.I.

(b) Nouveaux Mémoires. No. I-. Bruxelles, I903-: G.S.I.

408. - Société Entomologique de Belgique.

(a) Annales. Tom. I- Bruxelles, I857- Table générale, tom. I-30.

Complete set : Z.S.I.

Tom. I-, I857-(tom. 32, 36, 38, 4I, 45 wanting) and index : A.S.B.

(b) Bulletin ou Comptes-rendus. Tom. 25-27. Bruxelles, I $88 \mathrm{I}-83$ : A.S.B.

(c) Mémoires. Tom. I-. Bruxelles, I892-(tom. io wanting) : Z.S.I.

Société Malacologique de Belgique (see No. 4II).

409. - Société (Royale) Belge de Géographie.

Bulletin. Ann. I-. Bruxelles, I877-.

Ann. I-, I877- (amn. 35, 36 wanting, ann. 22 defective) : G.S.I.

Ann. I8-. I895- (ain, 20, 21, 23 defective): I.L.

Ann. 28-, Igo4- (ann. 29, 30 defective) : A.S.B.

4I0. - Société Royale de Botanique de Belgique.

Bulletin. Tom. I-. Bruxelles, I862-.

Complete set : R.B.G.

Tom. I-39, I862-I 900 : C.U.

'Tom. 40-43, I903-06 : A.S.B.

4I I. - Société (Royale Zoologique et) Malacologique de Belgique.

(a) Annales. Tom. I-. Bruxelles, I863-. [Tom. I I-I5, I 876-80, are also styled "Sér. 2 , tom. I-5"; tom. I6-20, 188I-85 are also "Sér. 3, tom. I-5" and tom. 2I-27, I886-92, are "Sér. 4 , tom. I-7."]

A.S. = Archaeological Section. Inclian Museum

A.S.B. = Asiatic Society of Bengal.

B.C. = Bishop s College.

B.S.I. = Botanical Survey of lnd:a

C.E. = Chemical Examiner to the Govt, of Bengal.

c.U. = Calcutta University
E.C. = Civil Engineering College, Sibpur.

G.S I. = Geological Survey of India.

1.L. = Imperial Library.

M.Ba. = Medical College, Bacteriological Litboratory

M.B1. = Nedical College. Biological Labortutry.

M.c. = licdical College (main library) 
4II. Brussels.-Société (Royale) Malacologique .. (Continued).

Tom. I-, I863- (tom. 35, 36 wanting) : G.S.I.

Tom. I-, I 863 - (tom. 28-30, 34-36 wanting) : A.S.B.

Tom. I5-43, I880-I908 (tom. 28-30, 35, 36, 41, 42 wanting; tom. 31 defective): Z.S.I.

(b) Procés-verbaux des Séances. Tom. II-27. Bruxelles, I $882-98$.

Tom. II-27, I 882-98 (toml. 25, 26 wanting; toll. II, $12,15,20-22,27$ defective) : Z.S.I.

Tom. II-24, I882-95 : A.S.B., G.S.I.

4I2. Société Scientifique.

(a) Annales. Tom. I-. Bruxelles, I877-: X.C. Table analytique, I875-I90I : X.C.

(b) Rerue des Questions Scientifiques. Tom. I-. Louvain, Paris, Bruxelles, I877-: X.C. Table analytique, I877-IgoI : X.C.

4I3. Ghent.-Illustration Horticole: reine mensuelle des plantes les plus remarquables, etc. Directeur, J. Linden. Tom. 36-40 $\left(=5^{\text {me }}\right.$ sér., vol. 3-7). Gand, I889-93 . R.B.G.

4I4. - Tijdschrift over Plantenziekten. Onder red. van J. Ritzema Bos en G. Staes. Jaarg. 2-9. Gent, I8o6-Igo3: Z.S.I.

4I5. Liége.-Archives de Biologie. Par E. van Beneden (and others). Tom. I-. Gand, Leipzig, Liége, Paris, I880- : Z.S.I.

4I6. - Belgique Horticole: journal des jardins, des serres et des vergers. Par C. et E. Morren. Tom. I-I4. Liége, I85I-84: R.B.G. [Continued as:] Belgique Horticole: annales d'horticulture belge et étrangèrc. Par E. Morren. Tom. I5-24. Liége, I865-74: R.B.G. [Continued as:] Belgique Horticole: annales de botanique et d'horticulture. Par E. Morren. Tom. 25. Liége, I875:

R.B.G.

4I7. - Société Géologique de Belgique.

(a) Annales. Tom. I-. Liége, I874-. 'Tables générales' at end of tom. Io and 20 .

'Tom. I--, I874- (tom. I6, 18 defective) : G.S.I.

Tom. I-, I874-(tom. I4, I6, 34, 36, 38-40 defective) :

A.S.B.

(b) Mémoires. Tom. 2-. I.iége, Ig04-: A.S.B., G.S.I. [Tom. 1, 1899-1908, is tom. $25^{\text {bis }}$ of the Annales.]

4I8. - Société Royale des Sciences.

Mémoires. Tom. I-20. Liége. I843-66. Sér. 2, tom. I-20. I,iége, I866-98. Sér. 3, tom. I-. Bruxelles,

M.O. = Meteorological Office, Alinore.

M.Ph. = Medical College, Physiological Laboratory.

P.C. = Presidency College.

P.O. = Patent Ofice.

R.B.G = Roval Iotanic Garden

S.C.C. $=$ Scottish Churches College.
S.G. = Surgeon-General s Library

S.I. = Survey of Incia.

S.T. $M=$ School of Tropical Medicine.

T.D. = Telegraph Department

x.c. =St. Xavier s College.

Z.S.I = Zoological Survey of India. 
4I8. Liége.-Société Royale des Sciences (Continued).

I899--. 'Tables générales' at end of tom. 20, sér. I and tom. 20 , sér. 2.

Tom. I-20, I843-66; sér. 2 , tom. I-8, I866-78 : G.S.I.

Sér. 2, tom. I-20, I866-98 (tom. 6 wanting) : sér. 3, tom. I-, I899- (tom. 8 wanting): A.S.B.

4I9. - Université.-Institut Botanique.

Archives. Tom. I-4. Bruxelles, I897-I907; R.B.G.

420. Louvain.--Université.-Institut Géologique.

Mémoires. Tom. I. Louvain, I9I3: G.S.I.

421. Uccle.-Société d'Études d'Agriculture Tropicale.

Agronomie Tropicale: organe mensuel de la Société ....

Tom. I, 2. Uccle, Igog-IO (tom. 2, no. 2 wanting):

B.S.I.

\section{DENMARK.}

422. Copenhagen.-Conseil Permanent International pour l'Éxploration de la Mer.

Publications de Circonstance. Nos. I-. Copenhague, Ig03- : Z.S.I.

423. - Danish Biologica! Station.

Report of the .... Station to the Board of Agriculture. Nos. I0-. I899/1900-. Copenhagen, I90I-: Z.S.I.

424. — Kongeligt Dansk Videnskabernes Selskab.

(a) Nye Samlıng af det .... Skrifter. Deel I-5. Kiöbenhavn, I7 $8 I-99$ : A.S.B., Z.S.I. [Continued as (d).]

(b) Oversigt over det.... Forhandlinger. I842-. Kjöbenhavn, I843-: G.S.I.

(c) Skrifter. Naturvidenskabelig og mathematisk Afdeling. $5^{\text {te }}$ Raekke, Bd. I-I2. Kjöbenhavn, I849-80: G.S.I. $6^{\text {te }}$ Raekke, Bd. I-I2. Kjöbenhavn, I88o-I904 : G.S.I. $7^{\text {te }}$ Raekke, Bd. I-. Kjöbenhavn, I906-: G.S.I. [For 2nd and 3 rd series see $(a)$ and $(d)$; Ist and 4 th series wanting.]

(d) Skrivter. Bd. I-6. Kjöbenhavn, I802-I8: A.S.B., Z.S.I.

Fortegnelse ... Videnskabelige Arbejder, I742-I89I : G.S.I.

A.S. = Archaeological Section. Indian Museum.

A.S.B. = Asiatic Society of Bengal.

B.C. = Bishop s College.

B.S.1. = Botanical Survey of India.

C.E. = Chemical Examiner to the Govt of Bengal.

c.U. = Calcutta University.
E.C. = Civil Engineering College, Sibpur.

G.S.I = Geological Survey of India.

I.L. = Imperial Library.

M.Ba. = Medical Colleqe, Bacteriological Laboratory

M.B1. = Medical College. Biological Laboratory.

M.C. = Medical College (man Library). 
425. Copenhagen.-Kongeligt Nordisk Oldskrift-Selskab.

(a) Aarböger for Nordisk Oldkyndighed og Historie. Bd. I9-. Kjöbenhavn, I904-: A.S.B.

(b) Mémoires de la Société Royale des Antiquaires du Nord. I836/39-I878/83. Copenhague, [I839-83]. Nouv. sér., I884/89-. Copenhague, [I889]- : A.S.B.

(c) Nordiske Fortidsminder..... Avec des résumés en Français. Hfte. I-6. Kjöbenhavn, I890-I903: A.S.B.

426. - Naturhistorie-Selskab.

Skrivter. Bd. I-6. Kjöbenhavn, I790-I8I0.

Bd. I-5, I790-99: Z.S.I.

Bd. I, 6, I790, I8Io: R.B.G.

427. - Naturhistorisk Forening.

Videnskabelige Meddelelser. Aar I849-. Kjöbenhavn, I849-. 'Alphabetisk Register till Argange I-20,' I849-68, at end of vol. for I868.

Aar I849- (Aar I898, 1904 wanting): Z.S.I.

Aar I849-66, '87-90, I909-II : R.B.G.

428. - Naturhistorisk Tidsschrift. Bd. I-4. Udg. af H. Kröyer. Kjöbenhavn, I837-43: Z.S.I. Raekke II, Bd. I, 2. Kjöbenhavn, I844-49: Z.S.I. Raekke III, Bd. I-I4. Udg. af J. C. Schiödte. Kjöbenhavn, I86I-84: Z.S.I.

- Publications de Circonstance (see No. 422).

429. - Regia Societas Medica Havniensis.

Acta Nova. Vol. I, 2. Havniae, I8I8-2I.

Complete set : Z.S.I.

Vol. I, I8I8: R.B.G.

430. - Skandinavisk Litteratur-selskab.

Skrifter. Bd. I-I9. Kjöbenhavn, I805-23: Z.S.I.

— Société Royale des Antiquaires du Nord (see No. 425).

43I. - Tidsskrift for Naturvidenskaberne. Udg. af H. C. Orsted, J. IV. Hornemann, J. Reinhardt. Bd. I-4. Kjöbenhavn, I822-26 : R.B.G.

\section{FRANCE (AND MONACO).}

432. Amiens.-Société Linnéene du Nord de la France.

Bulletin. Tom. I-6. Amiens, $1872-83$ : Z.S.I.

M.o. = Meteorological Office, Alipore.

M.Ph. = Medical College, Physiological Laboratory.

P.C. = Presidency College.

P.o. = Patent Office

R.B.G. = Royal Botanic Garden.

s.c.C. $=$ Scottish Churches College.
S.G. = Surgeon-General's Library.

S.I. = Survey of India.

S.T.M.=School of Tropical Medicine.

T.D. = Telegraph Department.

X.C $=$ St. Xavier's College.

Z.s.I. Zoological Survey of India 
433. Angers.-Société d’Études Scientifiques.

Bulletin. I881/82-I909. Angers, I882-I909 (vol. for tgos wanting): A.S.B.

Auch.-Société Française de Botanique (see No. 569).

434. Bordeaux.-Académie (Royale, Impériale) des Sciences, Belles. Lettres et Arts.

(a) Actes. Ann. 1-9, I839-47. Bordeaux, Paris, 183047. [A continuation of $(d)$, continned as $(c)$.]

Complete set: G.S.I.

Ann. I, 3, 6, 7 : A.S.B.

(b) Actes. $3^{\text {me }}$ sér., I87 I 72-. Paris. Bordeaux, I872-. (issue of I88I wanting) : A.S.B. [A continuation of (c).]

(c) Recueil des Actes. Ann. IO-I7, I848-55. Bordeaux, Paris, I848-55. [A continuation of $(a)$, continued as (b).] Ann. II, I3-I5. I7 : A.S.B.

Ann. IO, II : G.S.I.

(d) Séance publique. I8I9-36. Bordeaux, [I820]-36 (issue for 1835 wanting): A.S.B. [Continued as $(a)$.]

435. - Société de Géographie Commerciale.

Bulletin. $\quad 2^{\text {me }}$ sér., ann. I-3, I878-80. Bordeaux, I878so : A.S.B.

436. - Société Linnéene.

(a) Actes (Résumé des Travaux). Tom. 4-. Bordeaux, Paris, IS 3 I-. [Tom1. 15-20, $18+7-55$, are also styled ' $2^{e}$ sér., tom. 5-10'; similarly tom. $21-30$, $1856-75$, are ' $3^{\mathrm{e}}$ sér., tom. I-IO,' tom. 31-40, I876-86, are ' $4^{e}$ sér., tom. I-10,' tom. 4150, 1887-96, are ' 5 e sér., tom. $1-10$,' tom. 51-60, 1897-1905, are ' 6 e sér., tom. $1-10$,' and tom. 61 and 62,1906 , '07, are ' $7^{e}$ sér., tom. I, 2.' A continuation of $(b)$.]

(b) Bulletin d'Histoire naturelle. Tom. I-3. Bordeaux. I 830 , I845, I829: G.S.I. [Tom. I and 2 are of the second edition. Continued as $(a)$.

Index to $(b)$ and $(a)$ tom. 4-5I in Catalogue de la Bibliothèque, fasc. 2. Bordeaux, Ig0I : G.S.I.

437. Caen. -Académie des Sciences, Arts et Belles-Lettres.

Rapport général sur les travaux de l'Académie.... jusqu'an ... I8II, I8II-I5. Par P. F. T. Delarivière. 2 vols. Caen, I\&II-(I8I6): A.S.B.

438. - Société Française d'Entomologie.

Revue d'Entomologie. Réd. A. Fauvel. Tom. I-28. Caen, I882-I9II (tom. 26, 28 defective) : Z.S.I.

A.S. = Archaeological Section, Indian Museum A.S.B. = Asiatic Society of Bengal

B.C. = Bishop's College.

B.S.I. = Botanical Survey of India

C.E. = Chemical Examiner to the Govt. of Bengal.

C.U. = Calcutta University
E.C. = Civil Engineering College, Sibpur

G.S.I. = Geological Survey of Incia.

I.L. = Imperial Library.

M.Ba. = Medical College, Bacteriological Laborator y

M.Bl. - Medical College. Biological Laboratory.

M.C. = Medical College (main library) 
439. Caen.-Société Linnéenne du Calvados.

Mémoires. [Tom. I, 2], I824-25. Caen, I824-25: G.S.I.

[Continued as No. $440(b)$.]

440. - Société Linnéenne de Normandie.

(a) Bulletin. Tom. I-10, I855-65. Caen, I856-66. $2^{\text {me }}$ sér., tom. I-IO, I866-76. Caen, I868-76. $3^{\text {me }}$ sér., tom. I-Io, I876-86. Caen, I877-86. $4^{\text {me }}$ sér., tom. I-I0, I886-96. Caen, I887-96. $5^{\text {me }}$ sér., tom, I-IO, I897-1906. Caen, I897-I907. $6^{\text {me }}$ sér., tom. I-, I907--. Caen, I909-.

( $\mathrm{I}^{\text {er }}$ sér.) tom. I一, I856- (tom. 9, sér. 4, defective): G.S.I.

$4^{\text {me }}$ sér., tom. 4-, I890- (toml. 5, 6 sér. 4, wanting): A.S.B.

(b) Mémoires. Tom. 3-, I826-. Caen, Paris, I826-. ['Tom. 17-, 1892-, are also styled sér. 2, tom. I-. For tom. I, 2 see No. 439.]

Complete set : G.S.I.

Tom. I8-, I895- (tom. 21 wanting): A.S.B.

(c) Séance publique. I834-37. Caen, I834-37: G.S.I.

44I. Chateauroux.-Frelon. Journal d'Entomologie. Réd. par J. Desbrochers des Loges. Vol. I, nos. I, 8, I2; vol. 2, nos. 2-II ; vol. 3 , nos. I-I2. Chateauroux, I89I-94: Z.S.I.

442. Cherbourg.- Société (Impériale, Nationale) des Sciences Naturelles et Mathématiques.

Mémoires. Tom. I-. Paris, Cherbourg, I852-. (Tom. $2,7,17$ wanting): A.S.B. Indexes at end of tom. IO, 20 and 30 .

443. Concarneau.-Laboratoire de Zoologie et de Physiologie Maritime.

Travaux Scientifiques. Tom. I-4. Concarneau, I909-I2: Z.S.I.

Courrensan.-Revue de Botanique (see No. 569).

444. Dijon.-Académie des Sciences, Arts et Belles-Lettres.

Mémoires. $2^{\text {me }}$ sér., tom. I-I6, I85I-70. Dijon, I8527 I : G.S.I. $3^{\text {me }}$ sér., tom. I-IO, I87I-87. Dijon, I873-88 : G.S.I. $4^{\text {me }}$ sér., tom. I-; I888-. Dijon, I889- : G.S.I.

445. Grenoble.-Université.-Faculté des Sciences.

Travaux du Laboratoire de Géologie. Tom. 4-. Grenoble, I897-: G.S.I.

M.o. = Meteorological Office, Alipore

M.Ph. = Medical College, Physiological Laboratory:

P.c. = Presidency College

P.o. = Patent Office.

R.B.G. = Royal Botanic Garden

S.C.C. $=$ Scottish Churches College.
S.G. =Suriseon-General's Library.

S.l. = Survey of India.

S.T.M. = Schooi of Tropical Medicine.

T.D. = Telegraph Department.

X.C. =st. Xavier's College.

Z.S.I. $=$ Zoological Survey of India. 
446. Havre.-Société de Géographie Commerciale.

Bulletin. [Ann. I-.] Havre, I884- (ann. 1888, '92 wanting; ann $1884,{ }^{3} 87$, '90, 1902, '06, '07, 'I2 defective): A.S.B.

447. - Société Géologique de Normandie.

Bulletin. Tom. 13. Havre, I89o : G.S.I.

448. Le Mans.-Académie Internationale de Géographie Botanique.

(a) Bulletin. Tom. 8-. Lemans, 1898- (tom. 12, 13, I902-O3 wanting): R.B.G.

(b) Monde des Plantes; Revue mensuelle de Botanique. Tom. I-7. Lemans, I89I-98: R.B.G. [Continued as $(a)$.]

449. Lille.-Révue Biologique du Nord de la France. Par Th. Barrois (and others). Ann. I-7. Lille, I888-95: Z.S.I.

450. - Société Géologique du Nord.

(a) Annales. Tom. I-. Lille, I875-: G.S.I.

(b) Mémoires. Tom. I-. Lille, I876- : G.S.I.

45I. Lyons.-Académie (Royale, Impériale, Nationale) des Sciences, Belles-Lettres et Arts.

(a) Mémoires. Classe des Lettres. Nouv. sér., tom. I-I3. Lyon, I85I-68 (tom. 7,8 wanting): A.S.B.

(b) Mémoires. Classe des Sciences. Nouv. sér., tom. II5. Lyon, I85I-66 (tom. 8 , 9 wanting): A.S.B.

(c) Mémoires. Section des Lettres et Arts. Tom. I, 2. Lyon, I $845-46$

Tom. I, $2, \mathrm{I} 845-46$ : A.S.B.

Tom. 2, I846: R.B.G.

(d) Mémoires. Section des Sciences. Tom. I, 2. Lyon, I845-47 : A.S.B., R.B.G.

Annales des Sciences Physiques et Naturelles, d'Agriculture et de l'Industrie (see No. $457(b)$ ).

452. - Association Lyonnaise des Amis des Sciences Naturelles.

Comptes Rendus. I874, I878-79, I882. Iyon, I875-83: G.S.I.

453. - Commission Départmentale de Météorologie du Rhône.

[Annuaire.] I $2^{\mathrm{me}}-3 \mathrm{I}^{\mathrm{me}}$ ann., [I89o/9I]-I9Io. Lyon, I894-I9I I : X.C.

454. - Laboratoire d'Etudes de la Soie.

Rapport. Ann. I886, r889/90. Lyon, I887, '9I , Z.S.I.

A.S. = Archaeological Section. Indian Museum.

A.S.B. = Asiatic Society of Bengal.

B.C. = Bishop 's College.

B.S.I. = Botanical Survey of India.

C.E. = Chemical Examiner to the Govt. of Bengal.

c.U. = Calcutta University.
E.C. = Civil Enøineering College. Sibpur.

G.S.I. = Geological Survey of India.

I.L. = Imperial Library

M.Ba. = Medical College, Bacteriological Laboratory.

M. Bi. = Medical College. Biological Laboratory.

M.C. = Medical College (main library). 
455. Lyons.-Météorologie Lyonnaise. Ann. I888-I90o. Lyon, I890I 902 : X.C.

456. —— Muséum d'Histoire Naturelle.

(a) Archives. Tom. I-. I,yon, 1872-.

Complete set : G.S.I.

Tom. I一, I872- (tom. 4, Io wanting): A.S.B.

(b) Rapport sur les travaux exécutés pendant l'année 1874 et I880-82. Par L. Lortet. Lyon, I875-83: G.S.I.

457. - Société d'Agriculture, Sciences et Industrie.

(a) Annales. $4^{\text {me }}$ sér., tom. I-Io. Lyon, Paris, I868-77 (tom. 3 wanting): A.S.B. $5^{\text {me }}$ sér., tom. I-IO. Lyon, Paris, I $878-87$ (tom. 5 wanting): A.S.B. $6^{\text {me }}$ sér., tom. I-5. Lyon, Paris, I888-92: A.S.B. $7^{\text {me }}$ sér., tom. I-IO. Lyon, Paris, I893-I902 (tom. 8 wanting): A.S.B. $8^{\text {me }}$ sér., tom. I, 2. Lyon, Paris, I903-04: A.S.B. I905-. Lyon, Paris, I906-: A.S.B. [A continuation of $(b)$.]

(b) Annales des Sciences Physiques et Naturelles, d'Agriculture et de l'Industrie. Tom. I-II. Lyon, Paris, I 838-48 (tom. 4, 5 wanting): A.S.B. 'Table générale,' tom. I-II, in tom. II. $2^{\text {me }}$ sér., tom. 3-8. Lyon, Paris, I851-56: A.S.B. 'Table alphabétique,' tom. I-8, in tom. 8 . $3^{\text {me }}$ sér., tom. I-II. Lyon, Paris, I $857-67$ (tom. 8 wanting): A.S.B. [Continued as $(a)$.]

458. - Société d'Anthropologie.

Bulletin. Tom. I-, I88I 82-. Lyon, Paris, I882(tom. 18,24 wanting) : A.S.B.

459. - Société de Géographie.

Bulletin. 'Tom. 2, 3. I,yon, I878-80 (no. 12, tom. 2 wanting) : A.S.B.

460. - Société Linnéenne.

Annales. I845/46. Lyons, I847: A.S.B. Nouv. sér., tom. I, 2, I852-55. Lyon, I853-55: A.S.B.

46r. - Université.

Annales. Tome. 2, fasc. I-3, I89I-92. Tome. 9, fasc. I, I894. Fasc. 25, 26, 28, 39, I896-98. Paris, Lyon, I89I-98: Z.S.I. Nouv. sér. I. Sciences, Médicine, Fasc. 8, 9, 20, 23, 27-29, 34-35. Lyon, Paris, Igo2I3: Z.S.I.

M.o. = Meteorological Office, Alipore.

M.Ph. = Medical College, Physiological Laboratory.

P.C. = Presidency College.

P.o. = Patent Ofíce.

R.B.G. = Royal Botanic Garden.

S.C.C. $=$ Scottish Churches College.
S.G. = Surgeon-Generai's Library.

S.I. = Survey of India

S.T.M. = School of Tropical Medicine

T.D. = Telegraph Department.

X.c. =St. Xavier s College.

Z.S.I. = Zoological Survey of India 
462. Marseilles.-Faculté des Sciences.

Annales. Tom. I-. Marseille, Paris, I89I-.

Complete set: R.B.G.

Tom. I-, I89I- (tom. 12, 13, 1902-03 wanting) : Z.S.I.

'Tom. 4-, I893- (tom. 4, 6, 14, 1894, '96, 1904 defective, tom. 15 wanting) : G.S.I.

463. - Institut (Botanico-Géologique) Colonial de Marseille.

Annales. Publ. sous la direct. E. Heckel. $\quad \mathrm{I}^{\text {er }}$ sér., tom. I-I8. Paris, Lille, Macon, Marseille, I893-I90I. $2^{\text {me }}$ sér. tom. I-4. Paris, I903-06. [Continued as No. 464 .]

$\mathrm{I}^{\text {er }}$ sér., tom. I- $2^{\text {me }}$ sér., tom. 4: I893-I906 (tom. 3, ${ }^{\text {er }}$ iér., 1897, wanting): R.B.G.

I $^{\text {er }}$ sér., tom. 2- $2^{\text {me }}$ sér., tom. 4, I 895-I906 (tom. 3, ${ }^{\text {Ir }}$ sér., I 897 wanting) : B.S.I.

$\mathrm{I}^{\text {er }}$ sér., tom. 3-9, ISq6-I902 (tom. 4, 6 wanting): $2^{\text {me }}$ sér., tom. I-4, I903-I906 (tom. 3 wanting): G.S.I.

$\mathrm{I}^{\mathrm{er}}$ sér., tom. 3 , I 897 , tom. 5 , fasc. I, I $898 ; 2^{\text {me }}$ sér., tom. 3, I905: Z.S.I.

464. - Musée Colonial de Marseille.

Annales. $2^{\text {me }}$ sér., tom. 5-. Marseille, 1907-. [A continuation of No. 463.]

Complete sets : B.S.I., R.B.G.

Tom. 8, IOIO: Z.S.I.

465. — Musée d'Histoire Naturelle.

Annales. Tom. I-I2. Marseille, I883-I908 (tom. 6-8, 1900-03 wanting): Z.S.I.

466. Monaco.-Institut Océanographique de Monaco (see also No. 502)

Bulletin du Musée Océanographique. Nos. I-87. Monaco, I904-06: Z.S.I. [Continued as:]. Bulletin de l'Institut Océanographique. Nos. 88-I55. Monaco, I9o7-09: Z.S.I. [Continued as:]. Bull. Mus. Océanog. Nos. I56278. Monaco, I9IO-13: Z.S.I. [Continued as:] Bull. Inst. Océanog. Nos. 279-. Monaco, I9I4-: Z.S.I.

467. Nantes.-Société des Sciences Naturelles de l'Ouest de la France.

Bulletin. Tom. I-Io. Nantes, I89I-I90I : A.S.B. $2^{\text {me }}$ sér., tom. I-9. Nantes, I90I-09: A.S.B. Table des matières, I ${ }^{\text {er }}$ sér., tom. I-IO: A.S.B.

468. Paris.-Académie Royale des Inscriptions et Belles-Lettres.

Histoire de l'Académie ... . mémoires de littérature tirez des registres. Tom. I-50. Paris, I736-I808: A.S.B.

\footnotetext{
A.S. = Archaeological Section, Indian Museum

A.S.B. = Asiatic Society of Bengal.

B.C. = Bi:hop s College.

B.S.I. = Botanical Survey of Indıa

C.E. = Chemical Examiner to the Gort. of Bengal.

c. $\mathbf{U}=$ Calcutta Unversity:
}

E.C. = Civil Engineering College, Sibpur.

G.S.I. = Geological Survey of India.

I.L. = Imperial Library

M. Ba. = Medical College, Bacteriological Laboratory

M.Bi. = Medical College, Biological Laboratory

M.C. = Medical College (main library) 
468. Paris.-Académie Royale des Sciences (see No. 501).

469. - Adansonia: recueil périodique d'observations botaniques. Rédigé par H. Baillon. Tom. I-12. Paris, I860-79: R.B.G.

470

- Agriculture Pratique des Pays Chauds (see No. 505).

470. - Annales de Chimie. Par G. de Morveau, A. Lavoisier (and others). Tom. I-95. Paris, I789-I8I5 : G.S.I. 'Tables générales raisonnées, tom. I-96: G.S.I. [Continued as No. 47I.]

471. - Annales de Chimie et de Physique. Par J. Gay-Lussac et Arago, Chevreul, M. Berthelot, Pasteur and others. Tom. I-75. Paris, I8I6-40. $3^{\text {me }}$ sér., tom. I-69. Paris, I84I-63. $4^{\text {me }}$ sér., tom. I-30. Paris, I864-73. $5^{\text {me }}$ sér., tom. I-30. Paris, I87483. $6^{\text {me }}$ sér., tom. I-30. Paris, I884-93. $7^{\text {me }}$ sér., tom. I-30. Paris, I894-I903. $8^{\mathrm{me}}$ sér., tom. I-. Paris, I904-. [A continuation of No. 470.] Tables générales raisonnées, tom. I-30 and $5 \mathrm{I}-75$.

Tom. I- $4^{\text {me }}$ sér., tom. 3, I8I6-64, and index: G.S.I.

Tom. 47, I83I ; $5^{\text {me }}$ sér., tom. $4-8^{\text {me }}$ sér., tom. 6 , I $875^{-I}$ I 95 (6 $6^{\text {me }}$ sér., tom. 2, Io, I9 defective) : A.S.B.

$4^{\text {me }}$ sér., tom. 22- $5^{\text {me }}$ sér.. tom. I2, I87I-77 ( $4^{\text {me }}$ sér., tom. 23 , $5^{\text {me }}$ sér., tom. $4^{-8}$ wanting) : T.D.

$8^{\text {me }}$ sér., tom. 7-, I906- : E.C.

472. - Annales de Géographie. Publ. sous la direct. de P. Vidal de la Blache, L. Gallois et E. de Margerie. Tom. 5, no. 20-. Paris, I896- : G.S.I.

473. - Annales de Malacologie. Sous la direct. de G. Servain. Tom. I. Paris, I870: G.S.I., Z.S.I.

474. - Annales de Paléontologie. Sous la direct. de M. Boule. Tom. I-. Paris, Igo6-: G.S.I.

475. - Annales des Falsifications: bulletin international de la répression des fraudes alimentaires et pharmacentiques. Tom. I-5. Paris, I908-I2 : B.S.I.

476. - Annales des Mines: ou recueil de mémoires sur l'exploitation des mines, et sur les sciences (et les arts) qui s'y rapportent. Tom. I-I3. Paris, I8I7-26: G.S.I. $2^{\text {me }}$ sér., tom. I-8. Paris, I827-30: G.S.I. $3^{\text {me }}$ sér., tom. I-20. Paris, I832-4I : G.S.I. $4^{\text {me }}$ sér., tom. I-20. Paris, I $842-5$ I : G.S.I. $5^{\text {me }}$ sér., tom. I-20. Paris, I852-6I : G.S.I. $6^{\text {me }}$ sér., tom. I-20. Paris, I862-7I : G.S.I. $7^{\text {me }}$ sér., tom. I-20. Paris, I872-8I : G.S.I. $8^{\text {me }}$ sér., tom. I-20. Paris, I882-9I : G.S.I. $9^{\text {me }}$ sér., tom. I-20. Paris, I892-I90I : G.S.I. Io ${ }^{\text {me }}$ sér., tom. I-20. Paris, I902-II : G.S.I. I I ${ }^{\text {me }}$ sér., tom. I-. Paris, I9I2- : G.S.I. Tables alphabétiques et analytiques, sér. I-4, 7-IO : G.S.I.

M O = Meteorolosical Office. Alipore

$\mathbf{M} \mathbf{P h}=$ Míedical College. Physiological Laboratory.

P.C. = Presidency College.

P O Patent Office.

$\mathbf{R} \mathbf{B} \mathbf{G}=$ Royal Botanic Garden.

S.C.C. $=$ Scottish Churches College
S.G. = Surgeon-Generais Libraty.

S.l. = Survey of India.

S.T.M. = School of Tropical Medicine

T.D. = Telegraph Department

X.c. =St. Xavier s College.

z.s.1. = Zoological Survey of India. 
477. Paris-Annales des Sciences Géologiques. Publ. sous la direct. M. Hébert ..... et M. A. Milne-Eidwards. Tom. I-22. Paris, I 869-9I G.S.I.

478. - Annales des Sciences Géologiques ou Archives de Géologie. Par A. Rivière. Ann. I-2, no. 2. Paris, I842-43: G.S.I.

479. - Annales des Sciences Naturelles. Tom. I-30. Paris, I824-33: G.S.I., R.B.G., Z.S.I. [Continued in two parts as $(a)$ and $(b)$.]

(a) Botanique. Par Brogniart (and others). $2^{\text {me }}$ sér., tom. I-20. Paris, I834-43. $3^{\text {me }}$ sér., tom. I-20. Paris, I844-53. $4^{\text {me }}$ sér., tom. I-20. Paris, I854-63. $5^{\text {me }}$ sér., tom. I-20. Paris, I864-74. $6^{\text {me }}$ sér., tom. I-20. Paris, I $875^{-85}$. $7^{\text {me }}$ sér., tom I-20. Paris, I885-95. 8me sér., tom. I-20. Paris, I895-I904. $9^{\text {me }}$ sér., tom. I-. Paris, I905-.

$2^{\text {me }}$ sér., tom1. I-, I834-(tom. I 5-20, sér. 3, wanting): R.B.G.

$2^{\text {me }}$ sér., tom. I- $7^{\text {me }}$ ser., tom. I4, I834-9I : G.S.I. $2^{\text {me }}$ sér., tom. I-II, I834-39 (tom 3, Io wanting) ; $4^{\text {me }}$ sér., tom. 3-20, I855-63: A.S.B.

(b) Zoologie. Par Milne-Edwards (and others). $2^{\text {me }}$ sér., tom. I-20. Paris, $1834-43$. $3^{\text {me }}$ sér., tom. I-20. Paris, I844-53. $4^{\text {me }}$ sér., tom. I-20. Paris, I854-63. $5^{\text {me }}$ sér., tom. I-20. Paris, I864-74. $6^{\text {me }}$ sér., tom. I-20. Paris, I $874-85 . \quad 7^{\text {me }}$ sér., tom. I-20. Paris, I886-95. $8^{\text {me }}$ sér., tom. I-20. Paris, I896-I904. $9^{\text {1ze }}$ sér., tom. I-. Paris, I905-.

$2^{\text {me }}$ sér., tom. I-, I834- (tom. Io, sér. 5 wanting): Z.S.I.

$2^{\text {me }}$ sér., tom. I $-7^{\text {me }}$ sér., tom. 6 , I834-88: G.S.I. $4^{\text {me }}$ sér., tom. 3-18, I $855-62$ : A.S.B.

480. - Annales d'Hygiène Publique et de Médicine Légale. I ${ }^{\text {re }}$ sér., tom. I-50. Paris, I829-53: S.G. $2^{\text {ine }}$ sér., tom. I-50. Paris, I854-78 (tom. 29-32 wanting): S.G. $3^{\text {me }}$ sér., I882-95. Paris, I882-95 (vols. for $1886-89$ defective) : S.G.

48I. - Annales Télégraphiques. Tom. I-25. Paris, I874-89 (tom. 8 wanting): T.D.

482. - Annuaire Géologique Universel. Par Dagincourt, Carez (and others). Tom. I-I4. Paris, I $885-98$ : G.S.I.

483. - Anthropologie. Tom. I-. Paris, I890-: A.S.B.

- Archives de Biologie (see No. 4I5).

484. - Archives de Botanique: ou recueil mensuel de mémoires originaux, etc. Réd. ... sous la direct. de A. J. Guillemin. Tom. I, 2. Paris, I833-34: R.B.G.

A.5. = Arehaeological Section. Induan Museum.

A.S.B. = Asiatic Society of Bengal.

B.C. = Bishop s College.

B.S.I. = Botanical Survey of India

B.S.I. = Chemieal Examiner to the Givt. of Bengal.

c.u. = Calcutta University.
E.C. C Civil Engineering College, Sibpur.

G.S.I. = Geolosical Survey of India

I.L. =Imperial Library.

M.Ba. = Medical College, Bacteriological Laboratory

M.BI. = Medical College. Biological Laboratory.

M.C. = Miedical College (main library) 
485. Paris.-Archives de Parasitologie. Par R. Blanchard. Tom. 8-. Paris, I003-.

$$
\begin{aligned}
& \text { Tom. 8-, I903-: M.Ba. } \\
& \text { Tom. I0-, I905-: Z.S.I. }
\end{aligned}
$$

486. — Archives de Zoologie Expérimentale et Générale. Publ. sous la direct. H. de Lacaze Duthiers (and others). Tom. I-Io. Paris, I872-82: Z.S.I. $2^{\text {me }}$ sér., tom. I-IO. Paris, I883-92: Z.S.I. $3^{\text {me }}$ sér., tom. I-Io. Paris, I893-I902: Z.S.I. $4^{\text {me }}$ sér., tom. I-IO. Paris, I903-09: Z.S.I. $5^{\text {the }}$ sér., tom. I-IO. Paris, I909-I3: Z.S.I. [Whole series.] Tom. 5I-. Paris, I9I2-: Z.S.I.

487. - - Archives Entomologiques, ou recueil contenant des illustrations d'insectes nowveaux ou rares. Par J. Thomson. Tom. I, 2. Paris, $1857-58$ : Z.S.I.

488. - Archives Slaves de Biologie. Dir. par MI. Mendelssohn et C. Richet. Tom. I, fasc. I. Paris, I886: Z.S.I.

489. - Association Française pour 1'Avancement des Sciences. Rapporis Géologiques. I894, '95, '97. Paris, I894-97: G.S.I.

Association Scientifique de France (see No. 489).

490. - Astronomie: revue d'astronomie populaire, de météorologie et physique du globe. Par Flammarion. Ann. I-I2. Paris, I883-94: X.C.

_- Bulletin Astronomique (see No. 530).

— Bulletin de la Carte Géologique de France (see No. 543).

- Bulletin des Sciences Agricoles et Economiques (see No. $563(e)$ ).

_ Bulletin des Sciences Géographiques. Economie publiques, Voyages (see No. $5^{63}(\mathrm{~g})$ )).

- Bulletin des Sciences Historiques, Antiquités et Philologie (see No. $5^{6} 3(h)$ ).

49I. - Bulletin des Sciences Mathématiques. Réd. par G. Darboux, E. Picard (and others). $2^{\text {me }}$ sér., tom. 25-. Paris, I9o I- : P.C.

- Bulletin des Sciences Mathématiques, Astronemiques, Physiques et Chimiques (see No. $5^{6} 3(b)$ ).

— Bulletin des Sciences. Médicales (see No. $563(d)$ ).

_ Bulletin des Sciences Militaires (see No. $5^{6} 3(i)$ ).

Bulletin des Sciences Naturelles et de Géologie (see No. $563(c)$ ).

M.o. = Meteorological Office, Alipore.

M.Ph. = Medical College, Physiological Laboratory

P.C. = Presidency College.

P.O. = Patent Ofice

R.B.G. = Royal Botanic Garden.

s.c.C. $=$ Scottish Churches College
S.G. = Surgeon-General's Library.

S.I. = Survey of India.

S.T.M. = Schuol of Tropical Medicine.

T.D. = Telegraph Department.

X.c =St. Xavier's Collerie.

Z.S.I. Z Zoological Survey of India 
491. Paris.-Bulletin des Sciences Technologiques (see No. $563(f)$ ).

492. - Bulletin d'Hygiène Publique. Tom. I-. Paris, Igog-: S.G.

493. - Bureau Central Météorologique.

(a) Bulletin Mensuel. 1907-. [Paris], I907-: M.0. [A continuation of $(b)$.]

(b) Résumé Général du Temps en Europe. I90I-06. [Paris], Ig0I-06: M.o. [Continued as (a).]

494. — Club Alpin Français.

Anmuaire. Ann. I, I874. Paris, I875 G.S.I.

495. - Comité de l'Asie Française.

Bulletin. I906-. Paris, I906-: G.S.I.

496. - Commission Archéologique de l'Indochine.

Bulletin. I909-. Paris, I909-: A.S.B.

- Cosmos (see No. 523).

497. —_École Polytechnique.

Journal. Cahiers I-II. Paris, An. iii [I795]-I820: A.S.B.

498. — École Supérieure de Pharmacie.

Travaux du Laboratoire de Matière Médicale. Sous la direct. E. Perrot. Ann. I-. Paris, I902-: R.B.G.

499. —— Exposé de l'Electricité. Tom. I-5. Paris, I872-78: T.D.

500. - Flore des Serres et des Jardins de l'Europe. Réd. par M. C. Lemaire (and others). Tom. I-IO. Gand, I845-55: R.B.G. [Continued as:] Flore des Serres.... journal (annales) général d'horticulture. Sous la direct. de J. Decaisne et de L. van Houtte. Tom. II-22. Gand, I856-77 : R.B.G. 'Table générale alphabétique,' tom. I-I5, at end of tom. I5.

— Géographie (see No. $553(\mathrm{c})$ ).

50r. — Institut (National, Impérial des Sciences, Lettres et Arts) de France.-Académie (Royale) des Sciences.

(a) Comptes rendus hebdomadaires des séances. Tom. IParis, I835-. Table générale. tom. 32-6r.

Complete set and index : G.S.I.

Tom. I-I4I, I835-I905 (tom. 23, 123 wanting, tom. 99, I29, 134, 141 defective): A.S.B.

Tom. I-I4O, I835-I905 : C.U.

Tom. I42-, I906-: E.C.

'Tom. I46-, I908-: I.L.

Tom. I50-, I910-: P.C.

A.S. = Archaeological Section, Indian Museum.

A.S.B. = Asiatic Society of Bengal.

B.C. = Bishop's College.

B.S.I. = Botanical Survey of India

C.E. = Chemical Examiner to the Govt. of Bengal

c.U. =Calcutta University
E.C. = Civil Engineering College, Sibpur.

G.S.I. = Geological Survey of India.

I.L. = Imperial Library.

M.Ba. = Medical College, Bacteriological Laboratory

M.Bi. = Iledical College. Biological Laburatory.

M.C. = IIedical College (main hbrary) 
501. Paris.-Institut de France.-Académie des Sciences (Contimued).

(b) Connaissance des Temps: ou movvements célestes à l'usage des Astronomes et des Navigateurs. I759-1842. Paris, I760-I845 (vols. for $1795-1800$, '04, 'O6, '08, '2 I-42 wanting): A.S.B.

(c) Mémoires. Tom. I-45. Paris, I8I8-93. Tables générales, tom. I-40. [Two vols. are entitled Tom. 4I, the second, dated 1879 , and all subsequent volumes being styled 'Deuxieme Série.']

Tom I-45, I8I8-93, and index: G.S.I.

Tom. I-3, I818-20: B.C.

Tom. 27, 31, 35-37, I856, '6o, '66-68 (tom. 27, 37 defective) : Z.S.I.

(d) Mémoires. Litterature et beaux arts. Tom. I-5. Paris. An. vi-xii [I798-I804] (tom. 3 wanting): A.S.B.

(e) Mémoires. Mesur de l'Arc du Méridian entre Dunkerque et Barcelone. Tom. I, 2. Paris, I806-07: A.S.B.

(f) Mémoires presentés ...... par divers Savans. Sciences mathématiques et physiques. [2 ${ }^{\text {me }}$ sér.] Tom. I-34. Paris, I827-1909.

Tom. I-34, I827-I909: Z.S.I.

Tom. I-6, I827-35 (tom. 5 wanting) : I.L.

Tom. I8-32, I 868-I902: G.S.I.

(g) Mémoires. Sciences mathématiques et physiques. Tom. I-6. Paris, an vi [1798]-1805 (tom. 5 wanting): A.S.B. Tom. [9]. Paris, I808: A.S.B. Ann. I810-I5. Paris, I810-I5: B.C.

(h) Mémoires. Sciences morales et politiques. Tom. I-5. Paris, an. vi-xii [I798-I804] (tom. 3 wanting): A.S.B.

(i) Recueil des Pièces qui ont remporté le Prix de l'Académie ...... Tom. 2-7, 1727-61. Paris, 1752-69: A.S.B.

502. — Institut Océanographique.

(a) Annales. Tom. I-. Monaco, Paris, Igro-: Z.S.I.

(b) Bulletin (see No. 466).

503. — Institut Pasteur.

(a) Annales. Tom. I-. Paris, r887-.

Complete set: M.Ba.

Tom. I-2I, I887-I907 : S.G.

Tom. 7-, I893- (tom. 9, IO, I3-I6 wanting) : M.Ba.

Tom. II-24, I897-I9I0 (tol11. I2 wanting, tom. I6 defective) : A.S.B.

M.O. = Meteorological Ofince, Alipore.

M.Ph = Medical College. Physiological Laboratory

P C. = Presidency College.

P O. = Patent Ofrice.

R B.G = Roval Botanic Garder.

S C C $=$ Scottish Churches College.
S.G. = Surgeon-Generai's Library.

S.I. = Survey or Incia.

S.T.M $=$ School of Tropical Medicine

T.T.M = Telegraph Departmen:

..C. =St Xavier s College.

z.s.l. =Zoological Survey of Inciia. 
503. Paris.-Institut Pasteur (Continued).

(b) Bulletin. Tom. I-. Paris, I903-.

'Tom. I-, I903- (tom. 2 defectire) : M.Ba.

Tom. 4-8, I906-Io : Z.S.I.

504. - Intermédiaire des Mathématiciens. Réd. E. Maillet (and others). Tom. I7-. Paris, I9IO- (tom. 20 defective) : P.C.

505. - Jardin Colonial et Jardins d'Essai des Colonies Françaises.

Agriculture Pratique des Pays Chauds. Tom. I4-. Paris, Ig04- : B.S.I.

— Journal Asiatique (see No. 545).

506. - Journal d'Agriculture Tropicale. Publ. par J. Vilbouchevitch. Anu. I- (=nos. I- ). Paris, IgOI-.

Complete set: B.S.I.

Ann. $4-(=$ nos. 3 I- $)$, Ig04- : R.B.G.

507. - Journal de Botanique. Direct. M. L. Morot. Tom. I-. Paris, I887- : R.B.G.

508. - Journal de Botanique, appliquée à l'A Ariculture, à la Pharma. cie, à la Médicine et aux Arts. (Réd. par N. A. Desvaux). Tom. 2, 3. Paris, I8I3-I4: R.B.G.

509. - Journal de Conchyliologie. Réd. par S. Petit de la Saussaye, P. Fischer (and others). Tom. I-. Paris, I850-: G.S.I. [Tom. 5-8 are also styled ' 2 e sér., tom. I-4'; tom. 9-46 are ' 3 e sér., tom. $1-38$ ' and tom. 47 - are ' $f^{e}$ sér., tom. I-'.] Index, tom. I-40 : G.S.I.

5ro. - Journal de l'Anatomie et de la Physiologie normales et pathologiques de l'Homme et des Animaux. Publ. par Robin, Pouchet, Duval (and others). Ann. I-. Paris, I864- : Z.S.I.

5II. - Journal de Mathématiques Pures et Appliquées. Par Liouville (and others). Tom. I-20. Paris, I836-55: C.U., P.C. $\quad 2^{\text {me }}$ sér., tom. I-I9. Paris, I856-74: C.U., P.C. $3^{\text {me }}$ sér., tom. I-IO. Paris, I875-84 : C.U., P.C. $4^{\text {mec }}$ sér., tom. I-IO. Paris, I885-94 : C.U., P.C. $5^{\text {mee }}$ sér., tom. I-Io. Paris, I895-I904: C.U., P.C. $6^{\text {me }}$ sér., tom. I-. Paris, I905- : C.U., P.C.

512. - Journal de Physique. Publ. par J. Ch. D'Almeida. Tom. 7Io. Paris, I878-8I : T.D. $2^{\text {me }}$ sér., tom. I. Paris, I882 : T.D. [Continued as:] Journal de Physique Théorique et Appliqué. Publ. par E. Bouty (and others). $4^{\text {me }}$ sér., tom. I-9. Paris, I902-IO: P.C. $5^{\text {me }}$ sér., tom. I-. Paris, I9I I- (tom. I defective) : P.C.

\footnotetext{
A.S = Archaeological Section. Indian Musem

A.S B. = Asiatic Society of Bengal.

B.C. = Bishop s College.

B.S.I. = Botanical Survey of India.

C.E. = Chemical Exammer to the Govt. of Benga!

C.U. $=$ Calcutta University.
}

E.C. = Civil Engineering College, Sibpur

G.S.I. = icological Survey of Incia.

I L. = Imperıal Library.

M.Ba, = Medical College, Bacteriological Laboratory

M.BI. = Medical College. Biological Laboratory:

M.C. = Medical College (main library). 
5I3. Paris.-Journal de Zoologie, comprenant les differentes branches de cette Science. Par M. P. Gervais. 'Tom. I-6. Paris, I872-77 : Z.S.I.

5I4. - Journal des Economistes: revue (mensuelle) de la science économique et de la statistique. $3^{\text {me }}$ sér., tom. I3-48. Paris, I86977 : C.U. $4^{\text {me }}$ sér., tom. I-48. Paris, I878-89: C.U. $5^{\text {me }}$ ser., tom. I-56 Paris I890-Ig03 (tom. 11, 12, 33-42 wanting): C.U. $6^{\text {me }}$ sér., tom. I-I6. Paris, I904-07 (tom. 5 wanting): C.U. Sme sér., tom. I. Paris, Ig05: C.U.

5I5. - Journal des Savans. I8I6-Igo4. Paris, I8I6-Igo4.

Vols. for I 8 I6-57 : I.L.

Vols. for I837-I 904 (vol. for 1876 wanting) : A.S.B.

516. - Magasin de Conchyliologie. Par F. Guérin. Livr. I-4. Paris, I850 : G.S.I.

517. - Magasin de Zoologie. Publ. par F. E. Guérin-Méneville. Ann. I-8. Paris, I83I-38. [Continued as No. 518.]

Ann. I-8, I83I-38 (defective) : Z.S.I.

Alin. I, I 83 I : A.S.B.

518. - Magasin de Zoologie, d'Anatomie comparée et de Palaeontologie. Par F. E. Guérin-Méneville. Ann. I-6. Paris, I839-44 (defective): Z.S.I. [A cointinuation of No.517. Afterwards combined with No. 550 and continued as No. 540.]

5I0. - Materiaux pour l'Histoive Positive (Primitive) et Philosophique de l'Homme. Par G. de Mortillet. Tom. I-4. Paris, I865-68 (tom. 3 wanting): G.S.I. [Continued as No. 520.]

520. Materiaux pour l'Histoire Primitive et Naturelle de l'Homme. Par E. Trutat et E. Cartailhac. Tom. 5-I7 (=sér. 2, tom. II3). Paris, Toulouse, I869-83 (tom. 6, 10, 13 wanting): G.S.I. [A continuation of No. 519.]

52I. - Matières Grasses. Tom. I-. Paris, Igo8- : B.S.I.

- Mémoires d'Agriculture, etc. (see No. 564).

522. - Mémoires pour servir à une Description géologique de la France. Réd. par O. P. A. Dufrénoy et Elie de Beaumont. Vols. I-4. Paris, I $830-38$ : G.S.I.

523. - Mondes : revue hobdomadaire des sciences et de leurs applications aux arts et à l'industrie. (Also known as Cosmos). Par Moigno. $3^{\text {me }}-$ I9 $9^{\text {me }}$ Ann., tom. 8-56. Paris, I865-8I (tom. 15 wanting): X.C. $3^{\text {e }}$ sér., tom. I-9. Paris, I882-85: X.C.

524 . Musée Guimet.

(a) Annales. Tom. I-. Paris, I88o-: A.S.B.

M.O. = Meteorological Office, Alipore.

M.Ph. = Medical College, Physiological Laboratory.

P.c. = Presidency College.

P.O. = Patent Office.

R.B.G = Royal Botanic Garden.

s.C.C. $=$ Scottish Churches Collcge.
S.G. = Surgeon-General's Library.

S.I. = Survey of India.

S.T.M. = School of Tropical Medicine

T.D. = Telegraph Department.

X.C. =St. Xavier's College.

z.s.ı. = Zoological Survey of India. 
524. Paris.-Musée Guimet (Continued).

(b) Annales.... Bibliothéque d'Etudes. Tom. I-. Paris, I892-: A.S.B.

(c) Annales: Revue de l'Histoire des Religions. Tom. 5-56. Paris, I882-1907 (tom. 11 defective): A.S.B.

(d) Conférences faites au Bibliothèque de Vulgarisation. Tom. 3I-. Paris, I909- (tom. 36 wanting): A.S.B.

525. - Muséum d'Histoire Naturelle.

(a) Annales. Tom. I-20. Paris, I802-27: A.S.B, R.B.G.

(b) Archives. Tom. I-Io Paris, I839-6I : Z.S.I. [A continuation of $(e)$, continued as $(f)$.]

(c) Bulletin. Tom, I-. Paris, I895-.

Complete sets : G.S.I., R.B.G., Z.S.I.

Tom. 2-, I896- (tom 15 wanting) : A.S.B.

(d) Mémoires. Tom. I-20. Paris, I8I5-32: A.S.B., R.B.G. [Continued as $(e)$.]

(e) Nouvelles Annales. Tom. I-4. Paris, I832-35: R.B.G. [A continuation of $(d)$, continued as $(b)$.]

(f) Nouvelles Archives. Tom. I-Io. Paris, I865-74: Z.S.I. $2^{\text {me }}$ sér., tom. I-IO. Paris, I878-88: Z.S.I. $3^{\text {me }}$ sér., tom. I-Io. Paris, I889-98: A.S.B., Z.S.I. $4^{\text {me }}$ sér., tom. I-IO. Paris, I899-I908 : A.S.B., Z.S.I. $5^{\text {me }}$ sér., tom. I-. Paris, I909-: A.S.B. [Tom. I-10, 1865-74, and tom. $6,2^{\text {me }}$ sér., 1883 are each in separately paged parts, entitled 'Mémoires' and 'Bulletins.'] 'Tables générales' at end of last vol. of each series.

526. — Muséum d'Histoire Naturelle.-Herbier.

Notulae Systematicae. Publ. par H. Lecomte. Tom. I-. Paris, I909- (tom. I defective): R.B.G.

527. - Naturaliste: journal des échanges et des nouvelles. Ann. 3-8 (=vols. I-3). Paris, I88I-86 (defective) : Z.S.I. [Continued as:] Naturaliste: revue illustrée des sciences naturelles. Ann. 20, 24, 26, 27 (=sér. 2, ann. I2, I6, I8, I9). Paris, I898, I9o2, '04-05 : Z.S.I.

528. - Nature: revue des sciences et de leurs applications aux arts et l'industrie. I89I-93. Paris, I89I-93: A.S.B.

- Nouveau Journal A siatique (see No. 545).

529. - Nouvelles Annales de Mathématiques. Réd. par M.M. Terquem, Gerono (and others). Tom. I-. Paris, I842-: C.U.

A.S = Archaeological Section, Indian .II useum.

A.S.B. $=$ Aslatic Sociely ot Bengal.

B.C. = Bishop s College.

B.S.1. = Botanical Survey of India

C.E. = Chemical Examiner to the Govt of Bengal.

c.v. = Calcutta University.
E.C. = Civil Engineering College, Sibpur.

G.S.I. = Geologsical Survey of India.

I.L. = Imperial Library.

M.Ba. = Medical College, Bacteriological Laboratory

M.Bi. = Medical College. Biological Laboratory.

M.C. = Medical College (mani library). 
530. Paris.-Observatoire.

Bulletin Astronomique. Tom. 23-. Paris, Igo6- (tom. 24-26 wanting): P.C.

53I. - Opuscules Entomologiques. Par E. Mulsant. Cahiers I-I6. Paris, $1852-75$ : Z.S.I.

532. - Petites Nouvelles Entomologiques. Vols. I, 2 (=nos. I-I86). Paris, I869-77 (nos. 51, 53, 103, 117, 122, 176 wanting) : Z.S.I. [Continued as No. 527.]

533. — Radium, la Radioxctivité, les Radiations : l'ionisation journal de physique théorique et expérimentale. Publ. par J. Danne. Tom. 7-. Paris, I9I0-: P.C.

Recherches Asiatiques (see No. I048 (h)).

534. — Recueil d'Ophtalmologie. I908-II. Paris, I908-II : S.T.M.

535. — Revue Archéologique, ou recueil de documents et de mémoires relatifs à l'étude des monuments, à la numismatique et à la philologie de l'antiquité et du moyen age. Nouv. sér., tom. Ir-4r. Paris, I865-8I : A.S.B.

536. - Revue Critique de Paléozoologie. Publ. sous la direct. MI. Cossmann. Tom. I-. Paris, I897-. Table analytique, tom. I-IO.

Complete set and index: G.S.I.

Tom. I6-, I9I2- : P.C.

- Revue de Botanique (see No. 569).

537. - Revue des Cours Scientifiques de la France et de l'Etranger. Réd. O. Barot (and others). Ann. I-7. Paris, I863-70: A.S.B. [Continued as No. $54^{\mathrm{I}}$.]

538. _ Revue des Cultures Coloniales. Publ. sous la direct. A. MilnePoutingon. Tom. 3-15. Paris, 1898-1904 (tom. 3, 15, defective) : B.S.I.

539. — Revue des Eaux et Foréts. Tom. 2I-25. Paris, I882-86: I.L. Table alphabétique, I882-86 : I.L.

540. - Revne et Magasin de Zoologie Pure et Appliquée. Par F. E. Guérin-Méneville. $2^{\text {me }}$ sér., tom. I-23. Paris, I849-72. $3^{\text {me }}$ sér., tom. I-7. Paris, I873-79. [For earlier series see Nos. 517, $51^{8}$ and 550.$]$

Complete set : Z.S.I.

$2^{\text {me }}$ sér., tom. I-23, $3^{\text {me }}$ sér., tom. I-6, I $849-78$ (tom. $5,3^{\text {me }}$ sér., wanting) : A.S.B.

54I. - Revue Scientifique (de la France et de l'Etranger). Direct. E. Yung, E. Alglave, C. Richet (and others). $2^{\text {me }}$ sér., Ann.

\footnotetext{
M o. = Meteorological Office, Alipore.

M.Ph. = Medical College. Physiological Laboratory.

P.C. = Presidency College.

P. = Patenl Office.

R. B. G = Royal Botanic Garden.

S.C.C. $=$ Scuttish Churches College.
}

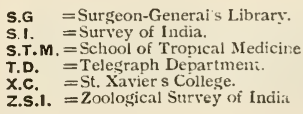

77 ] 
54I. Paris.-Revue Scientifique . . (Continued).

I-Io (=tom. 8-26). Paris, I87I-80: A.S.B. $3^{\text {me }}$ sér., tom. I[26] (=tom. 27-52). Paris, IS8I-93 (tom. 49-5I defective): A.S.B. $4^{\text {me }}$ sér., Ann. 3I-39, tom. I-I8. Paris, I894-I902 (tom. I, 3, I4, I6, I8 defective): A.S.B. [For Ist series see No. 537.]

542. - Revue Universelle des Mines, de la Métallurgie, des Travaux Publics, des Sciences et des Arts appliqués à l'Industrie. Par Ch. de Cuyper. Tom. 2I-28. Paris, Liège, I867-7I : G.S.I.

- Revue Zoologique (see No. 550).

543. - Services de la Carte Géologique de France.

Bulletin. Tom. 5-. Paris, I893- : G.S.I.

544. - Société Académique Indo-Chinoise.

(a) Actes. I877-79. Paris, I879: G.S.I.

(b) Bulletin. Tom. I-2. Paris, I879. $2^{\text {me }}$ sér., tom. I-3. Paris, I 882-9o.

[ I ${ }^{\text {er }}$ sér.,] tom. I-2 and $2^{\text {me }}$ sér., tom. 2 : G.S.I.

$2^{\text {mle }}$ sér., tom. I-3: A.S.B.

545. - Société Asiatique.

Journal Asiatique, ou Recueil de Mémoires, d'Extraits et de Notices relatifs à l'Histoire, à la Philosophie, aux Sciences, à la Littérature et aux Langues des Peuples Orientaux. Tom. I-II. Paris, I822-27. [Continued as :] Nonveau Journal Asiatique, etc. [2 $2^{\text {me }}$ sér.] Tom. I-I6. Paris, I828-35. |Continued as :] Journal Asiatique, etc. $3^{\text {me }}$ sér., tom. I-I4. Paris, I835-42. $4^{\text {me }}$ sér., tom. I-20. Paris, I843-52. $5^{\text {me }}$ sér., tom. I-20. Paris, I853-62 $6^{\text {me }}$ sér, tom. I-20. Paris, I863-72. $7^{\text {me }}$ sér., tom. I-20. Paris, I873-82. $8^{\text {me }}$ sér., tom. I-20. Paris, I883-92. $9^{\text {me }}$ sér., tom. I-20. Paris, I803-I902. Io ${ }^{\text {me }}$ sér., tom. I-20. Paris, I903-I2. I I ${ }^{\text {me }}$ sér., tom. I-. Paris, IgI3-.

Tom. I-, I822- ( $8^{\text {me }}$ sér., tom. I, $20 ; 9^{\text {me }}$ sér., tom. I4, I7, I8, 20; $10^{\text {me }}$ sér., tom. I, wanting: $9^{\text {me }}$ sér., tom. Ir, 1 5, 16, 19; $10^{\text {me }}$ sér., tom. 2, 4, defective); A.S.B.

Tom. I-II, I822-27; $2^{\text {me }}$ sér., tom. I, 3, 5, I 828 , '29, '30: B.C.

546. - Société Astronomique de France.

Bulletin. Ann. 9-22. Paris, I895-I908 : C.U.

547. - Société Botanique de France.

Bulletin. Tom. I-25, I 854-78: R.B.G. $2^{\text {me }}$ sér., tom. II5 (=26-40). Paris, I 879-93: R.B.G. $3^{\text {me }}$ sér., tom.

\footnotetext{
A.S. = Archaeological Section, Indian Museum.

A.S.B. = Asiatic Society of Bengal.

B.C. = Bishop's College.

B.S.I. = Botanical Survey of India.

C.E. = Chemical Examiner to the Govt. of Bengal.

c. $\mathbf{U}$. Calcutla University:
}

E.C. = Civil Engineering College, Sibpur.

G. S.l. = Geological Surver of India

I L. = Imperial Library.

M Ba. = Medical Colleđe, Bacteriological Laboratory

M.BI. = Medical Collere. Biolosical Laboratory.

M.C. = Medical College (main library). 
547. Paris.-Société Botanique de France (Continued).

I-7 $(=4 \mathrm{I}-47)$. Paris, I894-I900 : R.B.G. $4^{\text {mee }}$ sér., tom. I- $(=48-)$. Paris, Igor-: R.B.G. Tables, tom. I-40 : R.B.G.

548. - Société Centrale d'A piculture et d' Insectologie.

Bulletin d'Insectologie agricole, Journal Mensuel de la Société .... Ann. Io-II. Paris, I885-86 : Z.S.I.

549. - Société Chimique de France.

Bulletin. $4^{\text {me }}$ sér., tom. II-. Paris, Igr2-: P.C.

550. - Société Cuvierienne.

Revue Zoologique. Publ. sous la direct. F. E. GuérinMéneville. [Tom. I-II.] Paris, I838-48 : A.S.B., Z.S.I. [Afterwards united with No. 518 and continued as No. 540.]

Société d'Agriculture du Département de la Seine (see No. 564).

55I. - Société d'Anthropologie.

(a) Bulletin. $2^{\text {me }}$ sér., tom. 5-12. Paris, I870-77 (pt. 2, tom. II wanting): A.S.B. $3^{\text {me }}$ sér., tom. I-II. Paris, I878-88: A.S.B. $4^{\text {me }}$ sér., tom. I-Io. Paris, I89o99: A.S.B. [Continued as $(b)$.

(b) Bulletin et Mémoires. $5^{\text {me }}$ sér., tom. I-ro. Paris, 1900-09: A.S.B. $6^{\text {me }}$ sér., tom. I-. Paris, I910-: A.S.B.

(c) Mémoires. $2^{\text {me }}$ sér., tom. 4. Paris, I893: A.S.B. $3^{\text {me }}$ sér., tom. I, 2. Paris, I895-I902 : A.S.B.

Table générale de la Société, I860-79: A.S.B.

552. - Société de Biologie.

Comptes rendus hebdomadaires des Séances et Mémoires. Tom. 60-65. Paris 1906-08 : Z.S.I.

553. — Société de Géographie.

(a) Bulletin. Tom. I-20. Paris, I822-33. $2^{\text {me }}$ sér., tom. I-20. Paris. I834-43. $3^{\text {me }}$ sér., tom. I-I4. Paris, I 844-50. $4^{\text {me }}$ sér., tom. I-20. Paris, I85I-60. $5^{\text {me }}$ sér., tom. I-20. Paris. I86I-70. $6^{\text {me }}$ sér., tom. I-20. Paris, I87I-80. $7^{\text {me }}$ sér., tom. I-20. Paris, I88I-99. Tables des matières, sér. 5-7. [Continued as (c).]

[ $\mathrm{I}^{\mathrm{er}}$ sér.] tom. I- $7^{\text {me }}$ sér. tom. 20, I822-99 (sér. I, tom. 2 ; sér. 4 . tom. $6,7,9,15$; sér. 5 , tom. $5,6,8,11,12$ wanting: sér. 3 , tom. I2 ; sér. 6 , tom. $8,9,15,16$ defective): A.S.B.

M.O. = Meteorological Office, Alipore.

M.Pn. = Medical College, Physiological Laboratory.

P.C. = Presidency College.

P.o. = Patent Oifice.

R.B.G. = Roval Botanic Garden.

S.C.C. = Scottish Churches College.
S.G. = Surgeon-General s Library:

S.G. = Survey of India.

S.T.M. = School of Tropical Medicine.

T.D. = Telegraph Department

X.C. =St. Xavier s College.

Z.S.I. $=$ Zoological Survey of India. 
553. Paris-Société de Géographie (Continued).

[ $\mathrm{I}^{\mathrm{er}}$ sér.] tom. 3- $7^{\text {me }}$ sér., tom. 20, I825-99 (whole of sér. 4 , tom. I- 6 , sér. 5 and tom. 7 , sér. 6 wanting) and index : G.S.I.

(b) Comptes rendus. I882-99. Paris, I883-99.

Vols. for I882-99: G.S.I.

Vols. for I882-98 (vols. for 1883 , '84, '91, '92, '98 defective) : A.S.B.

(c) Géographie. Tom. I-. Paris, I900-.

Complete set: G.S.I.

Tom. I-, I900- (no. 3, tom. I 3 wanting): A.S.B.

Tom. II-, IgO5-: I.L.

(d) Recueil de Voyages et de Mémoires. Tom. 2-8. Paris, I825-66 (tom. 3, 5 wanting): G.S.I.

554. - Société de Pathologie Exotique.

Bulletin. Tom. 8, 9. Y'aris, I9I5-I6: M.Ba.

555. - Société Fintomologique de France.

(a) Annales. Tom. I-II. Paris, I832-42: Z.S.I. $2^{\text {me }}$ sér., tom. I-8. Paris, I $843-52$ : Z.S.I. $3^{\text {me }}$ sér., tom. I-8. Paris, I853-60: Z.S.I. $4^{\text {me }}$ sér., tom. I-IO. Paris, r86r-70: Z.S.I. $5^{\text {me }}$ sér., tom. I-ro. Paris, I87I-80: Z.S.I. $6^{\text {me }}$ sér., tom. I-ro. Paris, I88r-9o: Z.S.I. Ann. I89I-, vol. 40-. Paris, I8gI-(vol. 62 defective): Z.S.I. Tables générales, I832-90: Z.S.I.

(b) Bulletin. Ann. 1832-. Paris, I833-: Z.S.I. [Ann. 1832-97 are bound up with the Annales.]

556. - Société Française de Physique.

Séances. I900-02. Paris, I900-02: X.C.

557. - Société Géologique de France.

(a) Bulletin Tom. I-I4. Paris, I830-43: G.S.I. $2^{\text {me }}$ sér., tom. I-29. Paris, I $844-72$ : G.S.I. $3^{\text {me }}$ ser., tom. I28. Paris, I873-I900: G.S.I. $4^{\text {me }}$ ser., tom. I-. Paris, Igor- : G.S.I. Table générale, $2^{\text {me }}$ sér., tom. I20 : G.S.I.

(b) Mémoires. Tom. I-5. Paris, I833-43: G.S.I. $2^{\text {mee }}$ sér., tom. I-ro. Paris, I844-77 : G.S.I. $3^{\text {me }}$ sér., tom. I-5. Paris, I877-9I : G.S.I.

(c) Mémoires. Paléontologie. Tom. 1-. Paris, I89o-: G.S.I.

558. - Société Linnéene.

Bulletin mensuel. [Tom. I, 2.] Nos. I-I67. Paris, I87497 : R.B.G. 'Table générale' at end of tom. 2.

\footnotetext{
A.S. = Archaeological Section, Indian Muscum.

A.S.B. = Asiatic Society of Bengal.

B.C. = Bishop's College.

B.S.I. = Botanical Survey of India.

C.E. = Chemical Examiner to the Govt of Bengal.

C.U. = Calcutta University.
}

E.C. = Civu Engineering Collesfe, Sibpur.

G.S.I = Geological Survey of India.

I.L. = Imperial Library.

M.Ba. = Medical College, Bacteriological Laboratory.

M.BI. = Medical College. Biological Laboratory.

M.C. = Medical College (mam library). 
559. Paris.-Société Mathématique de France.

Bulletin. Tom. I-. Paris, $1873-$ : C.U.

560. - Société Minéralogique de France.

Bulletin. Tom. I-. Paris, 1878 - : G.S.I. Table des matières, tom. I-IO : G.S.I.

56r. - Société Nationale d'Acclimatation de France.

Revuc des Sciences naturelles appliquées: Bulletin de la Société .... Ann. 48-. Paris, I9OI-(defective): R.B.G.

562. - Société Philomatique.

Rapports généraux des Travaux.... par les Citoyens Riche et Silvestre. I788-99. 4 vols. Paris, [1793]- an. viii [1800]. [Continued as:] Bulletin des Sciences. Tom. I-3, I79I-I805. Paris, I797-I8II. [Continued as:- Nowveau Bulletin des Sciences. Tom. I-3. Paris, I807-I2. [Continued as:] Bulletin des Sciences. Ann. I8I4-24. Paris, I8I4-24. [Continued as:] Nouveau Bulletin des Sciences. Ann. I825-26, I83233. Paris, $1825-33$ [Nothing was published from 182731 or during 1834-35.] [Continued as:」 Extraits des Procés-Verbaux des Séances. I836-63. Paris, I83663. [Continued as:] Bulletin. Sér. 6, tom. I-I3. Paris, I864-77. Sér. 7, tom. I-12, Paris, I877-88. Sér. 8, tom. I-IO. Paris, I889-98. Sér. 9, tom. I-IO. Paris, I899-I908. Sér. Io, tom. I-. Paris, I909-.

Complete set : Z.S.I.

Sér. 8, tom. 6-, I894-(sér. 8, tom. 7 wanting ; sér. 9 , tom. $6,8,10,11$ defective) : A.S.B.

563 - Société pour la Propagation des Connaissances Scientifiques et Industrielles.

(a) Bulletin Général et Universel des Annonces et des Nouvelles Scientifiques. Par de Férussac. 4 vols. Paris, I823: A.S.B. [Continued as:] Bulletin Universel des Sciences et de l'Industrie. [Divided into eight sections as follow:]

(b) Section I.-Bulletin des Sciences mathématiques, astronomiques, physiques el chimiques. Tom. I-I2. Paris, I $824-29$ : A.S.B.

(c) Section II.-Bulletin des Sciences naturelles et de Géologie. Tom. I-27. Paris, 1824-3I.

Complete set : Z.S.I.

Tom. I-I9, I824-29: A.S.B.

M.o. = Meteorological Office. Alipore

M.Ph. = Medical College, Physiological Laboratory.

P.C. = Presidency College.

P.o. = Patent Office.

R.B.G, = Royal Botanic Garden.

s.c.C. $=$ Scottish Churches College.
S.G. = Surgeon-General's Library.

S.I. = Survey of India.

S.T.M. = School of Tropical Medicine.

T.D. = Telegraph Department.

X.C. =st. Xavier's College.

Z.s.I. =Zoological Survey of India. 
563. Paris.-Société Propagation Connaissances Scientifiques (Contd.).

(d) Section III.-Bulletin des Sciences médicales. Tom. I-I9. Paris, I824-29: A.S.B.

(e) Section IV.-Bulletin des Sciences agricoles et économiques. Tom. I-I3. Paris, I824-29: A.S.B.

(f) Section V.-Bulletin des Sciences technologiques. Tom. I-I3. Paris, I824-29: A.S.B.

(g) Section VI.-Bulletin des Sctences géographiques, Economie publique, Voyages. Tom. I-20. Paris, I824-29 (tom. I6 wanting) : A.S.B.

(h) Section VII.-Bulletin des Sciences historiques, Antiquités et Philologie. Tom. I-I3. Paris, I824-29: A.S.B.

(i) Section VIII.-Bulletin des Sciences militaires Tom. I-7. Paris, [I824]-29 (tom. 4 wanting): A.S.B.

564. - Société Royale (et Centrale) d'Agriculture.

Lémoires d'Agriculture, d'Economie rurale et domestique, publ. par la Société Royale d'Agriculture de Paris. Ann. I785-9I. Paris [I786-92]: R.B.G. [Resumed as:] Mémoires d'Agriculture, etc., publ. par la Société d'Agriculture du Département de la Seine. Tom. I-I6. Paris, an ix [ISOI]-I8I8: R.B.G. [Continued as:] Mémoires d'Agriculture, etc., publ. par la Société Royale et Centrale d'Agriculture. Ann. I8I4-34. Paris, I8I4-35 [Ann. I8I7 wanting ?]: R.B.G.

565. - Société Zoologique de France.

(a) Bulletin. Ann. :- Paris, 1876 - : A.S.B.

(b) Mémoires. Tom. I-. Paris, I888- (tom. 24 wanting): A.S.B.

566. Rennes.-Société Scientifique et Médicale de l'Ouest.

Bulletin. Tom. I-. Rennes, I892-(tom. I3, I4 defective): Z.S.I. 'Table générale', tom. I-IO, at end of tom, IO.

567. - Station Entomologique de la Faculté des Sciences.

Insecta: Revue illustrée d'Entomologie. Ann. I-. Rennes, IgII-: Z.S.I.

Toulouse.-Matériaux pour l'Histoire Positive et Philosophique (Primitive et Naturelle) de l'Homme (see Nos. 519, 520).

568. - Musée d'Histoire Naturelle.

Archives. Tom. I. publications I-4. Toulouse, I879-82: Z.S.I.

\footnotetext{
A.S. = Archaeological Section. Indian MItuseum.

A.S.B. = Asiatic Society of Bengal.

B.C. = Bishop's College.

B.S.I. = Botanical Survey of India.

C.E. = Chemical Examiner to the Govt. of Bengal.

c.U. = Calcutta University.
}

E.C. = Civil Engineering College, Sibpur.

G.S.I. = Geolosical Survey of India.

I.L. =I mperial Library

M.Ba. = Medical College. Bacteriological Laboratory.

M.Bi. = Medical College. Biological Laboratory.

M.c. = Medical College (main library) 
569. Toulouse.-Société Française de Botanique.

Revue de Botanique: Bulletin mensuel de la Société.... Tom. I-I3. Auch, Courrensan, Toulouse, I882-95: R.B.G.

570. — Université.-Station de Pisciculture et d'Hydrobiologie.

(a) Bulletin. Nos. I-4. Paris, Toulouse, I904-06: Z.S.I. [Continued as $(b)$.]

(b) Bulletin Populaire (Revue générale, technique et pratique) de la Pisciculture et des Améliorations de la Pêche. Nouv. (2 $\left.2^{\text {me }}\right)$ sér., nos. I-8. Paris, Toulouse, I907-09: Z.S.I.

\section{GERMANY}

57I. Augsberg.-Dinglers Polytechnisches Journal. Bde. 219-243. Augsberg, I876-82: T.D.

572. Berlin.-Annales Mycologici, editi in Notitiam Scientiae Mycologicae Universalis. Herausg. und red. von H. Sydow. Jahrg. I-. Berlin, I903-: R.B.G.

Archiv für Anatome, Physiologie und wissenschaftliche Medi$\operatorname{cin}($ see No. $702(a))$.

573. - Archiv fiur Naturgeschichte. Herausg. von A. Wiegmann, W. Erichson and others. Jahrg. I-. Berlin, I835-. Register, Jahrg. I-25, 25-60.

Complete set and indexes: Z.S.I.

Jahrg. I-54, I835-88 (Hft. I of Bd. 2, Jahrg. 53, 54 wanting) index to Jahrg. I-25: G.S.I.

574. - Archiv für Pathologische Anatomie und Physiologie und für Klinische Medicin. Herausg. von R. Virchow. Bde. I59-. Berlin, I900-. Inhaltsverzeichniss, Bde. I5I-200.

Bde. $159-$, I900-, and index: M.Ba.

Bde. I63-I68, I90I-02 : S.G.

- Archiv fïr Protistenkunde (see No. 687).

- Baessler Institut (see No. 603).

- Berliner Eniomologische Zeitschrift (see No. 584).

- Berlinische Gesellschaft (see No. $588(a, d)$ ).

- Botanische Zeitung (see No. 7 I0). 
575. Berlin.-Botanischer Jahresbericht: systematisch geordnetes Repertorium der Botanischen Literatur aller Länder. Herausg. I. Just. B.S.I., G.S.I., R.B.G. Jahrg. I-IO, I873-82. Berlin, I874-85: [Continued as No. 596.]

576. — Deutsche Chemische Gesellschaft.

Berichte. Jahrg. I-. Berlin, I868-.

Jahrg. I-, I868- (Jahrg. 44 wanting) : P.C.

Jahrg. 33-38, I900-05 : I.L.

577. — Deutsche Entomologische Gesellschaft (see also No.64I).

Deutsche Entomologische Zeitschrift. Jahrg. I88I-. Berlin, I88I- [For Jahrg. 1875-80 see No. 584.]

Jahrg. I88I- : Z.S.I.

Jahrg. I907-- : A.S.B.

578. — Deutsche Geologische Gesellschaft.

Zeitschrift. Bde. I-. Berlin, I849-: G.S.I. Register, Bde. I-50: G.S.I.

579. — Deutsche Gesellschaft für Mechanik und Optik.

(a) Deutsche Mechaniker-Zcitung: Beiblatt zur Zeitschrift für Instrumentenkunde und Organ für die gesamten Glasinstrumenten-Industrie. I90I-. Berlin, I9OI- : P.C.

(b) Zeitschrift für Instrumentenkunde (see No. 613).

— Deutsche Meteorologische Gesellschaft (see No. 376).

580. - Deutscher Apothekerverein.

(a) Archiv der Pharmacie (Pharmazie). Unter Red. E. Schmidt und H. Beckurts. Bde. 234-. Berlin, I896-: C.U.

(b) Jahresbericht der Pharmacie. Nos. 39-. Göttingen, Ig04-: B.S.I.

- Deutsches Archiv für die Physiologie (see No. 702 (e)).

581. - Deutsches Entomologisches Nationalmuseum.

(a) Deutsche Entomologische National-Bibliothek. Bde. I, 2. Berlin, I9IO-II : Z.S.I. [Continued as (b).]

(b) Entomologische Mittheilungen. Bde. I-. Berlin, I9I2- : Z.S.I.

582. —— Elektrotechnischer Verein.

Elektrotechnische Zeitschrift. (Centralblatt fïr Elektrotechnik.) Red. von $\mathrm{E}$ Zetzsche und $\mathrm{R}$. Rühlmann. Jahrg. 6-I9. Berlin, München, I885-I898 (Jahrg. 10, II wanting): C.U.

\footnotetext{
A.S. = Archaeological Section, Indian Museum.

A.S.B. = Asiatic Societv of Bengal.

B.C. = Bishop's College.

B.S.l. = Botanical Survey of India.

C.E. = Chemical Examiner to the Govt of Bengal.

c.U. = Calcutta University.
}

E.C. = Civil Engineering College, Sibpur.

G.S.I. = Geolosical Survey of India

I L. = Imperial Library

M.Ba. = Medical College, Bacleriological Laboratory

M.BI. = Medical College, Biological Laboratory.

M.c. = Medical College (main library). 
Berlin.-Entomologische Wittheilungen (see No. 58 r (b)).

583. - Entomologische Nachrichten. Herausg. von Katter, Karsch. Jahrg. I-26. Putbus, Stettin, Berlin, I875-I900: Z.S.I.

584. — Entomologischer Verein.

Berliner Entomologische Zeitschrift. Bde. I-I8. Berlin, I857-74. [Continued as:]. Deutsche Entomologische Zeitschrift (see also No. 577). Bde. I9-24. Berlin, 1875-80. [Continued as:] Berliner Entomologische Zeitschrift. Bde. 25-. Berlin, I88I-. 'InhaltsVerzeichniss', Jahrg. I- 6 , I857-62, at end of Bd. 6; to Jahrg. 7-I2, I863-68, at end of Bd. I2; to Jahrg. I $3-18,1869-74$, at end of $\mathrm{Bd}$. I8. Index der Arten, Bd. 25-35, I88I-90.

Complete set and indexes: Z.S.I.

Bde. 25一, I88I- (Bd. 39 defective): A.S.B.

585. - Gartenflora : allgemeine Monatsschrift für Deutsche, Russische und Schweizerische Garten- und Blumenkunde. Herausg. von E. Regel (and afterwards) B. Stein. Jahrg. I7-34. Eirlangen, Stuttgart, I852-85: R.B.G. [Continued as:] Gartenflora: Zeitschrift für Garten- und Blumenkunde. Herausg. von B. Stein (and afterwards) L. Wittmack. Jahrg. 35-. Berlin, I886- : R.B.G.

- Geographische Abhandlungen (see No. 599).

— Geographisches Institut (see No. 599).

586. - Gesellschaft für Anthropologie, Ethnologie und Urgeschichte. Zeitschrift für Ethnologie. Bde. I-. Berlin, I869-.

Bde. I, 2, I869-70: G.S.I.

Bde. I9-, I887- (Bde. 21, 23, 35 wanting): A.S.B.

587. - Gesellschaft für Eirdkunde.

(a) Verhandlungen. Bde. I-28. Berlin, I875-I90I : G.S.I. [Afterwards amalgamated with $(b)$.]

(b) Zeitschrift. Bde. I-. Berlin, I866-: G.S.I.

588. — Gesellschaft Naturforschender Freunde.

(a) Beschäftigungen der Berlinischen Gesellschaft. Bde. I-4. Berlin, I775-79: G.S.I., R.B.G. [Continued as $(d)$.]

(b) Magazin. Jahrg. I-8. Berlin, I807-I8: R.B.G.

(c) Neue Schriften. Bde. I-4. Berlin, I795-I803 (Bd. 2 wanting): R.B.G.

(d) Schriften der Berlinischen Gesellschaft. Bde. I-9. Berlin, I780-89: R.B.G. [A continuation of $(a)$.]

M.o. = Meteorological Office, Alipore.

M.Ph. = Medical College, Phystological Laboratory.

P.C. = Presidency College.

P.o. = Patent Office

R. B.G. = Royal Botanic Garden.

S.C.C. $=$ Scottish Churches College.
S.G. = Surgeon-lieneral's Library.

S.I. = Survey of India.

S.T.M. = School of Tropical Meaicinc.

T.D. = Telegraph Department.

X.C =St. Xavier's Cullege.

Z.S.I. = Zoological Survey of India 
588. Berlin.-Gesellschaft Naturforschender Freunde (Continued).

(e) Sitzungs-Berichte. Jahrg. 1860-. Berlin, I860-.

Jahrg. I 860-I908 (Jahrg. 1904 defective) : Z.S.I.

Jahrg. IgOI- (Jahrg. I902 wanting): A.S.B.

- Helios ( $\sec$ No. 656).

589. — Internationale Mitteilungen fïr Bodenkunde. Red. von ( $\dot{ }$. Murgoci, F. Schucht (and others). Bde. I-. Berlin, IgII-: G.S.I.

- Internationale Monatschrift für Anatomie und (Histologie) Physiologie (see No. jiz).

590. - Jahrbuch ïber die Fortschritte der Mathematik. Herausg. von C. Ohrtmann, F. Müller (and others). Bde. I-42, Jahrg I868I9II. Berlin. I87I-I9I4: C.U.

59I. — Jahrbiicher der Gewächskunde. Herausg. von K. Sprengel, A. H. Schrader und H. F. Link. Bde. I. Berlin und Leipzig, (I8I8-) I820 : R.B.G.

592. - Jahresbericht ïber die Fortschritte auf dem Gesamtgebiete der Agrikultur-chemie. Herausg. von A. Hilger und Th. Dietrich.

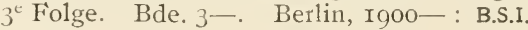

593. - Jahresbericht über die Leistungen und Fortschritte in der gesammten Medicin. Herausg. von R. Virchow. Jahrg. 28-32. Berlin, I893-97: S.G.

594. - Jahresbericht ïber die Nouerungen und Leistungen auf dem Gebiete der Pflanzenkrankheiten. Eirstattet von M. Hollrung. Bd. 7, I904. Berlin, 1905: B.S.I. [Continued as:] Jahresbericht über das Gebiet der Pfanzenkrankheiten. Erstattet von M. Hollrung. Bde. 8-, I905-. Berlin, 1907-: B.S.I.

595. - Journal für die Reine und Angewandte Mathematik. Herausg. von Borchardt, Kronecker (and others). Bde. I-. Berlin, I826-. General indexes to every ro vols.

Complete set : C.U.

Bde. 80-I23, I875-I901 : A.S.B.

596. - Just's Botanischer Jahresbericht. Herausg. E. Koehne (and others). Jahrg. II-. Berlin, I885-. [For earlier vols. see No. 575$]$.

Complete sets : B.S.I., R.B.G.

Jahrg. II-I2, I $885-86$ : G.S.I.

597. — Kaiserliche Marine.

Tabellarische Reiseberich!e nach dem. Meteorologischen Schiffstagebüchern. Bde. I-8, I903-10. Berlin, I904II (Bd.6 wanting): M.O.

A.S. = Archaeological Sectwn, indian Museun

A.S.B. = Asiatic Society of Benga!

B.C. = Bishop's College

B.S.I. = Botanical Survey of India

C.E. = Chemical Examiner to the Govt. of Benestl.

C.U. = Calcutta University
E.C. = Civil Engineering College, Sibpur

G.S.I. = Geological Survey of Incia.

I.L. = Imperial Library

M.Ba. = Medical Collese, Bacteriological Lavoratory

M.Bi. = Medical College. Biological Laboratory.

M.C. = Iledical Collegie (main library). 
598. Berlin.-Kolonial Wirtschaftliche Komitee.

(a) Beihefte zum Tropenpflanzer. Wissenschaftliche und praktische Abhandlungen über tropische Landwirtschaft. Bde. I-. Berlin, I900-- : B.S.I.

(b) Tropenpflanzer. Zeitschrift für tropische Landwirtschaft. Herausg. von O. IVarburg und F. Wohltmann. Jahrg. I-. Berlin, I897-: B.S.I., R.B.G.

599. — Königliche Friedrich-Wilhelm-Universität.-Geographisches Institut.

Geographische Abhandlungen. Herausg. von A. Penck. Bde. I-9. Vienna, Leipzig, I886-I9Io : G.S.I. Neue Folge. Veröffentlichungen des .... Instituts. Bde. I0-. Leipzig, Berlin, I9I2- : G.S.I.

6oo. - Königliche (Preussische) Akademie der Wissenschaften.

(a) Abhandlungen. I804-1907. Berlin, 1815-1907. [Each vol. is divided into several separately paged sections entitled 'Abhanalungen der physikalischen, mathematischen, historischphilologischen, historisch-philosophischen Filasse' or, 'Physikalische, mathematische, historisch-philologische, historischphilosophische Abhandluneen.' Continued in two parts as (b) and (c).] Verzeichniss, I7IO-I870. Inhaltsverzeichniss, $1822-72$.

Complete set and indexes: G.S.I.

Vols. for I822-I907 (vols for $1845-53,1905$ wanting): A.S.B.

(b) Abhandlungen. Philosophisch-historische Classe. I908-. Berlin, I908-: A.S.B. [A continuation of $(a)$.]

(c) Abhandlungen. Physikalisch-mathematische Classe. I908-. Berlin, I908-: A.S.B., G.S.I., Z.S.I. [A continuation of $(a)$.]

(d) Monatsberichte. I856-8I. Berlin, I856-82. Register. I $836-58, ' 59-73, ' 74-8 \mathrm{I}$. [Continued as (e).]

Complete set and indexes for $1836-73$ : A.S.B.

Complete set and index for $1859-73$ : G.S.I.

Vols. for $1865-8 \mathrm{I}$ and indexes for $\mathrm{I} 859-8 \mathrm{I}$ : Z.S.I.

(e) Sitzungsberichte. I882-. Berlin, I882-. [A continuation of $(d)$.]

Complete set : G.S.I.

Vols. for I 882 - (rol. for 1885 defective): A.S.B.

Vols. for I882- (vol. for 1889 defective): Z.S.I.

6or. — Königliche Preussische Geologische Landesanstalt.

(a) Abbildungen und Beschreibsngen Fossiler Pflanzen-Reste.

Von H. Potonié. Lief. I-. Berlin, I903- : G.S.I.

M.O. = Meteorologicai Office, Alipore.

M.Ph. = Medical College, Physiological Laboratory.

P.C. = Presidency College.

P.o. = Patent Office

R.B.G. = Royal Botanic Garden

S.C.C. $=$ Scottish Churches College.
S.G. = Surgeon-Generai's Library.

s.1. = Survey of India.

S.T.M. = School of Tropical Medicine

T.D. = Telegraph Department.

X.C. =St. Xivier's Collese

Z.s.l. =Zoological Survey of Incia. 
6ог. Berlin.-Königliche Preuss. Landesanstalt Geologische (Contd.).

(b) Abhandlungen der.... Landesanstalt. Neue Folge. Hfte. I-. Berlin, I889-(Hfte. 7, 8, 20, 57, 64, 65 wanting, Hft. 9 defective): G.S.I. [A continuation of (c).]

(c) Abhandlungen zur geologischen Specialkarte von Preussen und der Thüringischen Staaten. Bde. I-Io. Berlin, I872-89: G.S.I. [Continued as $(b)$.]

(d) Beiträge zur geologischen Erforschung der Deutschen Schutzgebiete. Hfte. 2-4. Berlin, I9II-I2: G.S.I.

(e) Jahrbuch. I880-. Berlin, I88I-: G.S.I. Register, Jahrg. I-20, I880-99: G.S.I.

602.

Königlicher Botanischer Garten.

(a) Jahrbuch des .... Gartens und des botanischen Museums $z u$ Berlin. Herausg. von A. W. Eichler (and others). Bde. I-5. Berlin, I88I-89: R.B.G.

(b) Notizblatt des .... Gartens und Museums zu Berlin. Bde. I-. Berlin, I897-: R.B.G.

603. — Königliches Museum für Völkerkunde.-Baessler Institut.

Baessler-Archiv. Beitrage zur Völkerkunde, herausg. aus Mitteln des Baessler-Instituts unter Mitwirkung der Direktoren der Ethnologischen Abteilungen des .... Museums. Bde. 2-. Berlin, Leipzig, I9I I- : A.S.B.

604. - Linnaea: ein Journal fïr die Botanik in ıhrem ganzen Umfange (oder Beiträge zur Pflanzenkunde). Herausg. von D. F. L. Schlechtendal (and afterwards) A. Garcke. Jahrg. I-43. Berlin, Halle, I826-82: R.B.G. [Afterwards merged in No. $502(a)$.]

Linnea Entomologica (see No. 76r (b)).

Meteorologische Zeitschrift (see No. 376).

605. — Museum für Naturkunde.

Mitteilungen aus der Zoologischen Sammlung. Bde. I. Berlin, I899-I900: Z.S.I. [Continued as No. 6I6.]

606 - Museumskunde. Zeitschrift für Verwaltung und Technik offentlicher und privater Sammlungen. Bde. I-5. Berlin, I905-09: Z.S.I.

- Palaeontologische Abhandlungen (see No.694).

607 - Prometheus. Illustrirte Wochenschrift über die Fortschritte in Gewerbe, Industrie und Wissenschaft. Herausg. von O. N. Witt. Jahrg. I3-20. Berlin, I902-09: Z.S.I.

A.S. = Archaeological Section. Indian Museum

A.S B. = Asiatic Society of Bengal.

B.C. = Bishop s College.

B.S.I. = Botanical Survey of India

C.E. = Chemical Examiner to the Govt. of Bengat

c.U. = Calcutta University.
E.C. = Civil Engineering College. Sibpur.

G.S.I. = Geological Survey of Inciit.

I.L. = imperial Library.

M.Ba. = Medical College, Bacteriological Laiboratory.

M. Bi. = Medical College. Biological Laboratory.

M.C. = Medical College (main library). 
6o8. Berlin.-Repertorium des Chemischen Theils der Mineralogie. Von C. F. Rammelsberg. Hfte. I-5. Berlin, I843-53: G.S.I. [Supplemente zu dem Handwörterbuch des chemischen Theils der Minerologie.]

\section{Semi-annual Report of Schimmel and Co. (see No. 747)}

6o9. - Spicilegia Zoologica quibus novae imprimis et obscurae animalium species iconibus, descriptionibus atque commentariis illustrantur. Cura P. S. Pallas. Fasc. I-I4. Berolini, I767-80: G.S.I., Z.S.I.

6ro. - Symbolae Antillanae: seu fundamenta florae indiae occidentalis. Edidit I. Urban. Vol. I-. Perolini, Lipsiae, etc., I898-: R.B.G.

- Tropenpflanzer (see No. $59^{8}(b)$ ).

- Virchow's Archiv für Pathologische Anatomie und Physiologie und fïr Klinische Medicin (see No. 574).

6II. — Zeitschrift fïr den Physikalischen und Chemischen Unterricht. Herausg. von F. Poske. Jahrg. 8-I9. Berlin, I895-I906 : C.U. Z Zeitschrift für die gesammten Naturwissenschaften (see No. $673(c))$.

- Zeitschrift fïr Ethnologie (see No. 586).

6r2. Z Zeitschrift für Gletscherkunde. Herausg. von E. Brückner. Organ der Internationalen Gletschercommission. Bde. IBerlin, I907- : G.S.I.

613. — Zeitschrift für Instrumentenkunde. Organ für. Witteilungen aus dem gesamten Gebiete der wissenschajtlichen Technik. Herausg. von Abbé, Foerster, Merz (and others). Bde. 5-. Berlin, I885-. [See also No. 579.]

Bde. 5-26, 1885-1906 (Bde. 9-13, 19, 22 wanting) : C.U. Bde. 2 I-, I90I-: P.C.

- Zeitschrift für Naturwissenschaften (see No. $673(c)$ ).

6r4. — Zeitschrift für Praktische Geologie. Herausg. von M. Krahmann. Jahrg. [I] - I893- Berlin, I893-: G.S.I. Fortschritte der Praktischen Geologie, zugleich General-Register, Bde. I-IO, I893-I,02: G.S.I.

6I5. — Zeitschrift für Wissenschaftliche Insektenbiologie. Bde. 5,6 (=Erste Folge, Bde. I4, I5). Berlin, I909-Io (defective): Z.S.I.

_ Zentralblatt fïr Zoologie Allgemeine und Experimentelle Biologie (see No. 740).

Z Zoologischer Jahresbericht (see No. 845 (c)).

M.o. = Meteorological Office, Aliporc.

M.Ph. = Medical College, Physiological Laboratory.

P.C. = Presidency College

P.O. = Patent Office

R.B.G = Royal Botanic Garden.

S.C.C. = Scottish Churches College.
S.G. = Surgeon-General's Library.

S.l. = Survey of India.

S.T.M. = School of Tropical Medicine

T.D. = Telegraph Department.

X.c. =St. Xavier's College.

Z.S.I. = Zoological Survey of India. 
6I6. Berlin.-Zoologisches Museum.

Mittcilungen. Bde. 2-. Berlin, IgoI-: Z.S.I. [For Bd. I see No. 605.$]$

61\%. Bonn.-Archiv für die gesammte Physiologie des. Menschen und der Thiere. Herausg. von E. F. W. Pflüger. Bde. I-I3I. Bonn, I868-I9IO (Bd. 93 defective) : Z.S.I. [Continued as No. 622.] Register, Bde. $3 I-70$ : Z.S.I.

6r8. - Archiv fur Mikroskopische Anatomie. Herausg. von Schultze, Hertwig (and others). Bde. I-. Bonn, I865- : Z.S.I. Namen- und Sachregister, Bde. I-60 : Z.S.I.

6I9. — Botanische Abhanallungen aus dem Gebiet der Morphologie und Physiolngie. Herausg. von J. Hanstein. Bde. I-4. Bonn, I870-82: R.B.G.

620. — Naturhistorischer Verein der Preussischen Rheinlande (Westfalens und des Reg.-Bezirks Osnabrück).

(a) Correspondenzblatt. I849-94. Bonn. I849-94. [Afterwards incorporated in $(b)$.]

(b) Verhandlungen. Jahrg. I-63. Bonn, I844-I907: G.S.I. [With Jahrg. II-51 wee published the vols. for $1851-94$ of No. 621.$]$

621. - Niederrheinische Gesellschaft für Natur- und Heilkunde.

Sitzungsberichte. I895-1906. Bonn, I895-I907: G.S.I.

[The vols. for $185 \mathrm{I}-94$ were published with Jahrg. II $-5 \mathrm{I}$ of No. $620(b)$.

- Nova Acta Physico-medica (see No. $669(e)$ ).

622. — Pfuger's Archiv fïr die gesammte Physiologie des Menschen und der Thiere. Herausg, ron M. Verworn. Bde. I32-. Bonn. I9IO-: Z.S.I. [For earlier vols. see No. 517.] Register, Bde. 7I-I40: Z.S.I.

Zeitschrift für die kunde des Morganländes (see No. 667).

623. Breslau.-Beiträge zur Biologie der Pfanzen. Herausg. von F. Cohn. Bde. I-. Breslau. I87o-: R.B.G.

- Nova Acla Physico-medica (see No. $669($ e)).

624. - Schlesische Gesellschaft für Vaterländische Cultur.

(a) Abhandlungen. Abtheilung für Naturwissenschaften und Medicin. I86I-73. Breslat, I86I-73 (defective): G.S.I.

(b) Abhandlungen. Philosophisch-historische Abtheilung. I86I-74. Breslau. I86I-74 (defective) : G.S.I.

A.S. = Archaeological Section, Indian Museum.

A.S.B. = Asiatic Society of Bengal.

B.C. = Bishop's College

B.S.1. = Botanical Survey of India

C.E. = Chemical Examiner to the Govt of Bengal.

C.U. = Calcutta University.
E.C. = Civil Engineering College, Sibpur.

G.S.I = Geological Survey of India.

I.L. = Imperial Library.

M.Ba. = Medical College, Bacteriological Laboratory

M.Bi. = Mledical College. Biological Laboratory.

M.C. A Aledical College (man library) 
624. Breslau.-Schlesische Gesellschaft.. (Continued).

(c) Jahresbericht. Nos. 28-. I850-. Breslau, [I $85 \mathrm{I}]$ 一. [A continuation of $(d)$.]

Nos. 28-, IS50- : G.S.I.

Nos. 82 -, IgO4-(No. 83 wanting): A.S.B.

(d) Uebersicht der Arbeiten und Veränderungen. Jahre I 847, '49 [Nos. 25, 27]. Breslau, I848, '50: G.S.I. [Continued as $(c)$.]

Verzeichniss der in den Schriften der ... Gesellschaft ... enthaltenen Aufsätze, ISo4-63: G.S.I.

General-Sachregister der in den Schriften der ... Gesellschaft ... enthaltenen Aufsätze, I804-76: G.S.I.

Fortsetzung des Verzeichnisses der in den Schriften der... Gesellschaft. . . enthaltenen Aufsätze, I864-76: G.S.I.

625. - Verein für Schlesische Insektenkunde.

(a) Jahresheft des T'ereins .... Hfte. [I]_. Breslau, Igo8- : Z.S.I. [Hft. [1] is styled 'Neue Folge, Heft 33' in continuation of $(b)$.]

(b) Zeitschrift für Entomologie. Jahrg. I-6, 8-I5. [Jahrg. 7 not published.7 Breslau, I847-6I: Z.S.I. Neue Folge, Hfte. I-32. Breslau, I870-I907: Z.S.I. [Continued as $(a)$.]

626. - Zoologische Beiträge. Herausg. von A. Schneider (and afterwards) E. Rolide. Bde. I-3. Breslau, I885-92: Z.S.I.

627. Brunswick.-Jahrbuch der Chemie. Bde. I4-22. Braunschweig, Ig05-II : C.E.

628. — Jahresbericht ïber die Fortschritte der Chemie und verwandter Theile anderer Wissenschaften. Herausg. von A. Naumann. I873-. Giessen, Braunschweig, I875-: A.S.B. Register, I867-Ig04: A.S.B.

629. - Magazin für Insektenkunde. Herausg. von K. Illiger. Bde. I-6. Braunschweig, I802-07: Z.S.I.

630. - Naturwissenschaftliche Rundschan. Wochentliche Berichte ïber die Fortschritte auf dem Gesammtgebiete der Naturwissenschaften. Herausg. von IV.Sklarek. Jahrg. 6-9. Braunschweig, I89I94 : Z.S.I.

63r. — Verein für Naturwissenschaft.

Jahresbericht. Nos. [2]-I6. Braunschweig, I880-I90I (No. 15 wanting): A.S.B.

Casse1.-Bibliotheca Zoologica (see No. 762).

M.o. = Meteorological Ofince, Aiipore.

M.Ph = Medical College. Physiological Laboratory

P C. = Presidencv College.

po. = Patent Oince

R B.G = Royal Botanic Garcier.

S C C $=$ Scottish Churches College.
S.G. = Surceon-Generai's Library

S.1. = Survey of India.

S.T. M $=$ School of Trosical Medicine.

T.D = Telegravh Desartmen:

X.C. =St Xiavier S Collese.

Z.S.l. Zoological Survey of indit. 
632. Casse1.-Botanisches Centralblatt : Referirendes Organ für das Gesammtgebiet der Botanik des In- und Auslands. Herausg. von O. Uhlworm (and others).

(a) Beihefte zum Botanischen Centralblatt. Bde. I-. Cassel, Jena, Leipzig, Dresden, I89I-.

Complete set: R.B.G.

Bde. I-I9, I89I-I906 (Bd. 2 wanting): A.S.B.

Bde. I-9, I89I-I 900 : B.S.I.

(b) Botanisches Centralblatt. Bde. I- Cassel, Jena, I $880-$. General-register, Bde. I-6o.

Complete sets: B.S.I., R.B.G.

Bde. I-99, I880-I905 (Bde. 9I, 94, 97 wanting; Bde. 48, $89,98,99$ defective) and index: A.S.B.

Bde. I-32, I880-87 (Bde. 3, 4 wanting): G.S.I.

633. - Conchologische Mittheilungen, als Forsetzung der Novitates Conchologicae. Herausg. von E. von Martens. Bde. I-3. Cassel, I880-86 (Bd. 3 defective) : Z.S.I.

- Internationale Monatsschrift fïr Anatomie und (Histologie) Physiologie (see No. 7I7).

634. - Journal fïr Ornithologie. Herausg. von J. Cabanis (and others. Jahrg. I-. Cassel, Leipzig, I853-: Z.S.I. GeneralIndex, $1853-67$, '68-93: Z.S.I.

635. - Malakologische Blätter : als Forsetzung der Zeitschrift für Mal. akozoologie. Herausg. von K. T. Menke und L. Pfeiffer. Bde. I-25. Cassel, I854-78: Z.S.I. Neue Folge. Herausg. von S. Clessin. Bde. I-II. Cassel, I879-9I: Z.S.I. [A continuation of No. 685.]

636. — Palaeontographica. Beiträge zur Naturgeschichte der Vorwelt. Herausg. von W. Dunker, K. A. Zittel (and others). Bde. I-. Cassel, I85I-: G.S.I. Supplement Bde. I-. Cassel, Stuttgart, I866-: G.S.I.

637 - Verein für Naturkunde.

(a) Abhandlungen und Bericht. Nos.40-. Kassel, I895-. [A continuation of $(b)$.]

Nos. 40-, I $895-$ (No. 44 wanting): G.S.I.

Nos. 40-, I895- (Nos. 44, 49, 52 wanting): A.S.B.

(b) Bericht. Nos. 26-39. Cassel, I880-94. [Continued as $(a)$.]

Nos. 26-39, I880-94: A.S.B.

Nos. $28-39$, I 88 I-94 : G.S.I.

A.S. = Archaeological Section, inchan Museum.

A.S.B. = Asiatic Society of Bengal.

B.C. = Bishop s College.

B.S.I. = Botanical Survey of Indra.

C.E. = Chemical Examiner to the Govt. of Bengal.

c.U. = Calcutta University.
E.C. =Civil Engineering College, Sibpur. G.S I. = Geological Survey of Incia.

I.L. = Imperai Library.

M.Ba. = Medical Collese, Bacteriological Laboratory.

M.Bi. = Medical College. Biological Laboratory.

M.C. = Niedical College (main library). 
638. Dantzig. - Naturforschende Gesellschaft.

Schriften. Neue Folge, Bde. 7-. Danzig, I888-(Bd. 8 and No. I, Bd. Io wanting): A.S.B.

639. Darmstadt.-Merck's Annual Report of Recent Advances in Pharmacentical Chemistry and Therapeutics. Vols. 7- Darmstadt, I893-: B.S.I.

Dessau.-Naumannia (see No. 77I).

Dresden.-Entomologischer Verein 'Iris' (see No. 64I).

- Etudes Entomologiques (see No. 888).

640. —- Gesellschaft für Botanik und Zoologie.

Nunquam Otiosus. Zoologische Mittheilungen von L. W. Schaufuss, zugleich Organ der Gesellschaft..... Bde. I, 2. Dresden, I870-72: Z.S.I.

64I. - Gesellschaft 'Iris.

(a) Correspondenz-Blatt des Entomologischen Vereines 'Iris'. Bde. I. Dresden, I884-88: A.S.B. [Continued as (b).]

(b).Iris. Deutsche Entomologische Zeitschrift. Herausg. von der Gesellschaft 'Iris'.... in Verbindung mit der Deutschen Entomologischen Gesellschaft zu Berlin. Lepidopterologische Hette. Red. O. Staudinger. Bde. 2-25. Dresden, I889-I9I0. [For Bd. I see (a).]

Bde. 2-25, I889-I9IO : A.S.B.

Bde. 2-5, I889-92 : Z.S.I.

642. - Hedwigia: Organ für Kryptogamenkunde und Phytopathologie, nebst Repertorium fïr Literatur. Red. von G. Hieronymus (and afterwards) P. Hennings. Bde. 43-. Dresden, I904- : R.B.G. General-register, Bde. I-50: R.B.G.

643. - Kolloid-Zeitschrift.

(a) Kolloid-Chemische Beihefte: Ergänzungshefte zur Kolloid-Zeitschrift. Herausg. von Wo. Ostwald. Bde. 2-. Dresden, I9I0-: G.S.I.

(b) Kolloid-Zeitschrift. Herausg. von Wo. Ostwald. Bde. I3-. Dresden, I9I3-: G.S.I. [A continuation of No. 6.8.]

644. — Königliches Mineralogisch-Geologisches und Prähistorisches Museum.

Mittheilungen. Jahrg. I868-75. Dresden, I868-75: G.S.I. Hfte. I-I4. Cassel, Leipzig, I876-98 (Hft. 8 wanting): G.S.I.

M.O. = Meteorological Office, Alipore.

M.Ph. = Medical Collese. Physiological Laboratory

P.C. = Presidency College.

P.O. = Patent Office.

R.B.G. = Royal Botanic Garden.

S.C.C. = Scottish Churches College
S.G. = Surgeon-General s Library

S.I. = Survey of India.

S.T.M. = School of Tropica! Medicine

T.D. = Telegraph Deparlment

X.c. =St. Xavier's College.

Z.S.I. Zoological Survey of India. 
645. Dresden.-Königliches Zoologisches und Anthropologisch-Ethnographisches Museum.

(a) Abhandlungen und Berichte. Herausg. von A. B. Meyer. Bde. [I]-, I886/87一. Berlin, I887-: Z.S.I.

(b) Mittheilungen aus dem Kgl. Zoologischen Museum. Herausg. von A. B. Meyer. Hfte. I-3. Dresden, I877 (I875)-78: A.S.B., Z.S.I.

(c) Publikationen aus dem Kgl. Ethnographischen Museum. Herausg. von A. B. Meyer. Bde. I-I4. Leipzig, Dresden, I88I-I903.

Bde. I-I 4, I88I-I903: A.S.B.

Bde. I-I3, I88I-I900: Z.S.I.

L Leopoldina (see No. $669(\mathrm{c})$ ).

646. — Naturwissenschaftliche Gesellschaft 'Isis.'

(a) Sitzungs-Berichte. Jahrg. I86I-80. Dresden, I862-8I. [Continued as $(b)$.]

Jahrg. I86I-80 (Jahrg. 1862 wanting): G.S.I.

Jahrg. I 870-80 (Jahrg. 1870, '71, '73, '79 defective, Jahrg. 1874-78 wanting): A.S.B.

(b) Sitzungsberichte und Abhandlungen. Jahrg. I88I-. Dresden, I882-.

Jahrg. I88I-I9IO: G.S.I.

Jahrg. I900-: R.B.G.

- Nunquam Otiosus (see No. 640).

— Oekonomische Societät (see No. 730).

647. — Verein für Eirdkunde.

Jahresbericht. Hfte. 8-I6. Dresden, I872-79 (Hfte. Io, II, It wanting): A.S.B.

645. Z Zeitschrift für Chemie und Industrie der Kolloid. Herausg. von IWo. Ostwald. Bde. 8-I2. Dresden, I9II-I3: G.S.I. [Continued as . No. $643(b)$.]

649. Dusseldorf.-Stuhl und Eisen. Zeitschrift fïr das Deutsche Eisenhüttenresen. Jahrg. 20-. Düsseldorf, I900- : G.S.I.

Erlangen.-Biologisches Centralblatt (see No. 708).

- Gartenflora (see No. 585).

— Nova Acta Physico-medica (see No. $669(e)$ ).

650. - Physikalisch-medizinische Societät.

Sitzungsberichte. Hfte.37一. Erlangen, I905-: A.S.B.

A.S. = Archaeological Section, Indian Museum. .

A.S. B, = Asiatic Society of Bengai.

B.C. = Bishop s College

B.S.I. = Botanical Survey of India.

C.E. = Chemical Examiner to the Gort. of Bengal.

c.u. = Calcutta University.
E.c. = Civil Engineering College, Sibpur.

G.S.I. = Geolosical Survey of India.

I.L. = Imperial Library.

M.Ba. = M edical College, Bacteriological Laboratory

M.Bi. = Iredical College. Biological Laboratory

M.c. = Medical College (main library). 
65I. Frankfort on the Main.-Deutsche Malakozoologische Gesellschaft.

(a) Jahrbiicher. Red. von W. Kobelt. Jahrg. I-I4. Frankfurt a. M., I874-87 : Z.S.I. 'Register,' Bde. I. $\mathrm{I} 4$, at end of Bd. I4.

(b) Nachrichtsblatt. Red. von W. Kobelt. Jahrg. I-40. Frankfurt a. M., I869-1908 (Jahrg. 7 wanting, Jahrg. 33, 34 defective) : Z.S.I.

_- Entomologische Zeitschrift (see No. 653).

- Ephemerides (see No. $669(b)$ ).

652. — Gemeinnïtzige Blätter zur Beförderung des Bergbanes und Hüttenbstriebes. Herausg. von A. von Klipstein. Hfte. I, 2 . Frankfurt, Giessen, I849, '59: G.S.I.

653. - Internationaler Entomologischer Verein.

Entomologische Zeitschrift. Zentral-organ des .... Vereines. Jahrg. 24-. Frankfurt a. M., Leipzig, I9I0-(Jahrg. 26 defective): Z.S.I.

- Miscellanea Curiosa Medico-physica (see No. $669(d))$.

654. — Neue Zoologische Gesellschaft.

(a) Znologische Garten. Bde. I-46. Frankfurt a. M., I86oI905: Z.S.I. [Continued as (b).] Inhalts-verzeichniss, Jahrg. I-20 ; Sachregister, Jahrg. 2I-40 : Z.S.I.

(b) Zoologischer Beobachter. Bde. 47-5I. Frankfurt a. M., I9o6-Io: Z.S.I. [A continuation of $(a)$.]

655. - Senckenbergische Naturforschende Gesellschaft.

(a) Abhandlungen. Bde. I-. Frankfurt a. M., I854-.

Complete set: G.S.I.

Bde. 10-, I876- (Bde. 17, 27 wanting; Bde. $14,20,25$. 26 defective) : A.S.B.

Bde. I3-, I884- (Bd. I7 wanting): Z.S.I.

(b) Bericht. 1874/75一. Frankfurt a. M., I876-.

Vols. for $1875 / 76-$ : Z.S.I.

Vols. for 187475 -I902: A.S.B.

(c) Museum Senckenbergianum. Abhandlungen aus dem Gebiete der bescreibenden Naturgeschichte von Mitgliedern der..... Gesellschaft. Bde. I-3. Frankfurt a. M., I834-45 : G.S.I., Z.S.I.

—_ Zoologische Garten (see No. $654(a)$ ).

- Zoologische Gesellschaft (see No. 654).

\% o. = Meteorological Office, Alinore.

M.Ph. = Medical College. Physiological Laboratory.

P.C. $=$ Presidency College.

P.o. = Patent Oifice.

R.B.G. = Royal Botanic Garden.

s.c.c. $=$ Scuttish Churches College.
S.G. = Surgeon-Generai's Library.

SI. = Survey of India.

S.T.M. $=$ School of Tropical Medicine

T.D. = Telegraph Departmeni.

X.c. =St. Xavier s College.

Z.S.I. =Zoolosical Survey of Inciia 
656. Frankfort on the Oder.-Naturwissenschaftlicher Verein des Regierungsbezirks Frankfurt.

Helios. Abhandlungen und monatliche Mittheilungen aus dem Gesammtgebiete der Naturwissenschaften. Bde. 9-. Berlin, I892- (Bde. 20, 24, 25, 27-30 wanting): A.S.B.

Freiberg.-Archiv für Practische Geologie (see No. I6oI).

657. - Beiträge zur geognostischen Kenntniss des Erzgebirges. Herausg. aus dem Ganguntersuchungsarchiv zu Freiberg. Hfte. I-3. Freiberg, I885-67 : G.S.I.

658. — Jahrbuch für das Berg- und Hüttenwesen im Königreiche Sachsen. Herausg. von C. Menzel. Jahrg. I908-. Freiberg, I 908 - : G.S.I.

659. Freiburg in Breisgau.-Naturforschende Gesellschaft.

(a) Berichte. Bde. I-. Freiburg i. B., I886-: G.S.I. [A continuation of $(b)$.]

(b) Berichte über die Verhandlungen der (....) Gesellschaft (für Beförderung der Naturwissenschaften). Bde. I-8. Freiburg i. B., I855-85: G.S.I. [Continued as (a).]

Giessen.-Gemeinnützige Blätter zur Beförderung des Bergbautes und Hiittenbetriebes (see No. 652).

- Jahresbericht über die Fortschritte der Chemic und verwandte Theile anderer Wissenschaften (see No. 628).

660. Oberhessische Gesellschaft für Natur- und Heilkunde.

(a) Bericht. Nos. 7-34. Giessen, 1859-1905 (No. 8 wanting): A.S.B. [Continued in two parts as $(b)$ and $(c)$.]

(b) Bericht. Neue Folge. Medizinische Abteilung. Bde. I-. Giessen, I906- (Bde. 2-5 wanting): A.S.B.

(c) Bericht. Ntue Folge. Naturwissenschaftliche Abteil. ung. Bde. I (I904-06)-. Giessen, I907-(Bde. 2, 3 wanting): A.S.B.

661. Gotha.-Geographischer Anzeiger. Jahrg. I-3. Gotha, I900-02: G.S.I.

662. - Geographisches Jarbuch. Herausg. von E. Behm. Bd. 3. Gotha, I870: G.S.I.

663 - Justus Perthes' Geographische Anstalt.

(a) Mittheilungen aus......Anstalt über wichtige nene Erforschungen auf dem Gesainmtgebiete der Geographie. Von A. Petermann. Bde. I-24. Gotha, I855-78. Inhaltsverzeichniss, I $865-78$. [Continued as $(c)$.]

\footnotetext{
A.S = Archaeological Section, Indian Museum

A.S.B. = Istatic Society of Benga!

B.C. = Bishop s Colleg.e.

B.S I. = Botanical Survey of India

C.E. Chemical Examiner to the Govt. of Bengal.

c.U. = Calcutta University
}

E.C. = Civil Engineering College, Sibpur

G.S.I. = Geological Survey of India.

I.L. = Imperial Library.

M.Ba. = Medical Collese, Bacteriological Laboratory

M.Bi. = Medical Collese. Biolosical Laboraiory.

M.C. = Iedical College (mani library) 
663. Gotha.-Justus Perthes' Geographische Anstalt (Continued).

Complete sets and index : C.U., G.S.I.

Bde. 2 I-24, I875-78 : I.L.

(b) Mittheilungen ...... Ergänzungsband. Bde. I-I2. Gotha, I860-78: G.S.I., C.U. [Continued as $(d)$.]

(c) Petermann's Mittheilungen .... Herausg. von E. Behm, Lindemann and others. Bde. 25-. Gotha, I879-. Inhaltsverzeichniss, $1879-84$. [A continuation of $(a)$.]

Complete sets and index : C.U., G.S.I.

Bde. 25-37, I879-9I (Bde. 32-35 wanting, Bd. 36 defective) ; I.L.

Bde. $3 \mathrm{I}-$, I885- (Bd. 56 wanting): S.I.

(d) Petermann's Mittheilungen ..... Ergänzungsband. Bde. I3-. Gotha, I880- [A continuation of $(b)$.

Complete set: C.U.

Bde. I3- (Bd. 25 wanting, Bd. 24 defective) : G.S.I.

664. Gottingen.-Abbildungen Naturhistorischer Gegenstände. Herausg. von J. F. Blumenbach. [Hfte. I-IO, =plates $\mathrm{I}-\mathrm{IOO}]$. Göttingen, [1796]-I8Io: Z.S.I.

- Deutscher Apothekerverein (see No. 580 ).

665. - Journal für die Botanik. Herausg. von Medicinalrath Schrader. I799-I80I. Göttingen, I799-I803: R.B.G.

666. — Königliche Gesellschaft der Wissenschaften.

(a) Abhandlungen. Bde. I-22. Göttingen, I843-77: G.S.I. [Continued as $(b)$.]

(b) Abhandlungen. Mathematisch-naturwissenschafliche Klasse. Bde. 23-40. Göttingen, 1878-96 (defective) : G.S.I.

(c) Abhandlungen. Mathematisch-physikalische Klasse. Neue Folge. Bde. I-. Berlin, I90o- (Bd. 6 defective): G.S.I.

(d) Göttingische gelehrte Anzeigen. I875-I906. Göttingen, I875-I906 (vols. for 1892 , 1898 defective) : A.S.B.

(e) Nachrichten von der ... Gesellschaft ... und der Georg. A ugusts-Universität. I867-93. Göttingen, I867-93. [Continued in three parts as $(f),(g)$ and $(h)$.]

Vols. for $1867-93$ (vols. for 1878 , '79 wanting) : G.S.I.

Vols. for $1875-93$ (vol. for 1883 defective) : A.S.B.

(f) Nachrichten von der... Gesellschaft... Geschaftliche Mittheilungen. I894-. Göttingen, Berlin, I895-.

Vols. for $1894-$ (vol. for $189+$ defective) : A.S.B.

Vols. for I894-I908 : G.S.I.

M.o. = Meteorological Ottice, Alipore

M.Ph. = Medical College, Physiological Laboratory.

P.c. = Presidency College

P.O. = Patent Ottice

R.B.G. = Royal Botanic Garden.

S.C.C. $=$ Scottish Churches College

\footnotetext{
S.G. = Surgeon-General's Library:

s.l. = Survey of India.

S.T.M. = School of Tropical Miedicine

T.D. = Telegraph Department.

X.C. =St. Xavier's College.

z.s.I. = Zoological Surver of India
} 
666. Gottingen.-Königliche Gesellschaft der Wissenschaften (Contd.).

(g) Nachrichten von der... Gesellschaft ... Mathematischphysikalische Klasse. 1894-. Göttingen, I895-.

Complete set: G.S.I.

Vols. for IS94- (vol. for 1912 defective): A.S.B.

(h) Nachrichten von der... Gesellschaft ... Philologischhistorische Klasse. I894-. Göttingen, I895-(vols. for 1908, 191 I defective): A.S.B.

667. - Zeitschrift für die Kunde des Morganländes. Herausg. von C. Lassen (and others). Bde. I-7. Göttingen, Bonn, I837-50: A.S.B.

Halle.-Academia Caesarea Leopoldino-Carolina Germanica Naturae Curiosorum (see No. 669).

- Annalen der Physik (see No. 7 or (a)).

668. _ Deutsche Bunsen Gesellschaft für angewandte Physikalische Chemie.

Zeitschrift für Elektrochemie. Organ der Deutschen Elektrochemischen Gesellschaft. Jahrg. 2-7. Halle, I895I90I. [Continued as:1 Zeitschrift für Elektrochemie. Herausg. von .... Bunsen Gesellschaft ... Jahrg. 8-. Halle, I902-. [For Jahrg. I, see No. 674.]

Jahrg. 2-, I895- (Jahrg. 17 wanting) : P.C. Jahrg. 7-II, I900-05: C.U.

- Deutsche Elektrochemische Gesellschaft (see No. 668).

- Deutsches Archiv für die Physiologie (see No. $702(e)$ ).

669. Kaiserliche Leopoldino-Carolinische Deutsche Akademie der Naturforscher.

(a) Acta Physico-medica Academiae Caesareae LeopoldinoCarolinae Naturac Curiosorum, exhibentia ephemerides, sive observationes, historias, et experimenta a celeberrimis viris habita, singulari studio collecta. Vols. I-IO. Norimbergae, I727-54: A.S.B., G.S.I. Index, vols. IIO: G.S.I. [A continuation of $(b)$; continued as (e).]

(b) Ephemerides, sive observationum medico-physicarum. Cent I-IO. Norimbergae, Francofurti, Lipsiae, I7I 222 : G.S.I. [A continuation of $(d)$; continued as $(a)$.]

(c) Leopoldina. Hfte. 4-37. Halle, Dresden, I863-I9or.

Hfte. 4-37, I863-I90I (defective) : A.S.B.

Hfte. $7-37,1872-$ I9OI : G.S.I.

(d) Miscellanea Curiosa Medico-physica, sive ephemeridum medico-physicarum germanicarum curiosarum. Dec.

\footnotetext{
A.S. = Archaeological Section. Indian Museum.

A.S.B. = Asiatic Society of Bengal.

B.C. = Bishop's College

B.S.I. = Botanical Survey of India

C.E. = Chemical Examiner to the Govt. of Bengal.

c.U. = Calcutta University.
}

E.C. = Civil Fugineering College, Sibpur.

G.S.I = Geological Survey of India.

I.L. =1mperial Library.

M.Ba. = Medical College, Bacteriological Laboratory.

M.Bl. = Medical College. Biological Laboratory.

M.C. = Medical College (main library). 
669. Halle.-Kaiserliche Leop.-Carolin. Deutsche Akademie .. (Contd.). I, Ann. I-IO. Lipsiae, Francofurti, I670-80: G.S.I. Dec. 2, Ann. I-Io. Norimbergae, I683-92: G.S.I. Dec. 3. Ann. I-IO. Norimbergae, Francofurti, Lipsiae, I694-I706: G.S.I. Indexes, Dec. I-3 : G.S.I. [Continued as $(b)$.]

(e) Nova Acta Physico-medica. Tom. I-8. Norimbergae, I757-9I. [Continued as:] Nova Acta Physico-medica (Verhandlungen der...Akademie). Tom. 9-. Erlangae, Bonnae, Breslau, Halle, etc., I8I8- Index, tom. I-63. [A continuation of $(a)$.]

Tom. I-79, I757-I90I and index: G.S.I.

Tom. I-79, I757-I90I (tom. $25-29,73$ wanting) : A.S.B.

Tom. IO-33, 65-, I82I-67, I895- (tom. II-15, 67, $68,75,76,78,80,83,87,88$ wanting; many defective): Z.S.I.

Tom. I5-I9, I83I-39: R.B.G.

670. — Kali: Zeitschrift für Gewinnung, Verarbeitung und V'erwertung der Kalisalze. Herausg. vom Verein der Kaliinteressenten. Bd. I-. Halle, I907-(Bd. 1 defective) : G.S.I.

- Limnaea (see No. 604).

671. - Magazin der Entomologie. Herausg. von E. F. Germar. Jahrg. I-4. Halle, I8I3-2I : Z.S.I.

672. - Naturforschende Gesellschaft.

(a) Abhandlungen (Bericht). Bde. I-25. Halle, I854I906. (Bde. IO, 11, 13 wanting, Bde. 12, 14, 19 defective): Neue Folge. No. I-. Halle, I9I2-: G.S.I.

(b) Abhandlungen der Hallischen .... Gesellschaft. Bd. I. Dessau und Leipzig, I783: R.B.G.

(c) Bericht über Sitzungen. I880-92. Halle, I88o-92 : G.S.I.

(d) Mitteilungen. Bde. I-. Halle, I9I2-: G.S.I.

(e) Neue Schriften. [Bd. I.] Hfte. I-6. Halle, I809[I8Io ?] : R.B.G.

673. - Naturwissenschaftlicher Verein für Sachsen und Thüringen.

(a) Abhandlungen. Bde. I-2. Berlin, I856-6I : G.S.I.

(b) Jahresbericht. Jahrg. I85I-52. Berlin, I852-53 (defective) : G.S.I.

(c) Zeitschrift für die gesammten Wissenschaften. Bde. I-34. Halle, Berlin, I853-69. 'Register' to Bde. I-20 at end of Bd. 2o. Bde. 35-54 (=Neue Folge, Bde. I-I4; $3^{\mathrm{e}}$ Folge, Bde. I-6). Berlin, I870-8I. [Continued as:]

\footnotetext{
M O. = Meteorological Office, Alipore.

M.Ph. = Medical College, Physiological Laboratory:

P.C. = Presidency College.

P.O. = Patent Otfice.

R.B.G. = Royal Botanic Garden.

S.C C. $=$ Scistish Churches College.
}

S.G = Surgeon-General's Library:

S.1. = Survey ot India.

S.T.M. = School of Tropical Medicine

T.D. = Telegraph Departmeni.

X.C. =St. Xavier s College.

Z.S.I. = Zoological Sturvey of Inciia 
673. Halle-Naturwiss. Verein für Sachsen .. (Continued).

Zeitschrift für Naturwissenschaften. Bde. 55- $\left(=4^{2}\right.$

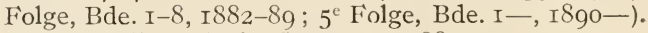
Berlin, Halle, Leipzig, Stuttgart, I882-.

Complete set: Z.S.I.

Bde. I-6I, I853-88: G.S.I.

674. Z Zeitschrift fïr Elektrotechnik und Elektrochemie. Herausg. von A. Wilke und W. Borchers. Jahrg. I. Halle, I894-95:

P.C. [Continued as No. 668.]

Z Zeitschrift für Naturwissenschaften (see No. $673(c)$ ).

675. Hamburg.-Botanisches Staatsinstitut.

(a) Jahresberichte. I905-II. Hamburg, I906-I2: R.B.G.

(b) Mitteilungen. I909-. Hamburg, I9I0- : R.B.G.

[Issued as "Beihct zum Jahrbuch der Hamburgischen wissenschaftlichen Anstaltcn," Bde. 27-.]

676. - Chemisches Central-blatt. $3^{\text {tc }}$ Folge, Jahrg. I6-69. Hamburg und Leipsig, I885-88: C.U. $4^{\text {te }}$ Folge. Red. von R. Arendt. Jahrg. I-8. Hamburg und Leipsig, I889-96: C.U. $5^{\text {te }}$ Folge. Jahrg. II-. Berlin, I907-: G.S.I.

677. — Garten- und Blumenzeitung: Zeitschrift für Garten-und Blumenfreunde, fïr Kunst- und Handelsgärtner. Herausg. von E. Otto. Bde. (Jahrg.) 6-3I. Hamburg, I850-75 : R.B.G.

678. - Mineralogisch-geologisches Institut.

Mitteilungen. Beihefte zum Jahrbuch der Hamburgischen Wissenschaftichen Anstalten. Beihft. 4, Bd. 29 and Beihft. 6, Bd. 30. Hamburg, I9I2, I3: G.S.I.

679. Museum Goddefroy.

Journal. Geographische, ethnographische und naturwissenschaftliche Mittheilungen. Herausg. unter Mitwirkung von Bergh, Günther, A. M.-Edwards, Semper, Spengel, Virchow (and others). Hfte. I-. Hamburg, I873- : Z.S.I.

680. - Naturhistorisches Museum.

Mittheilungen. Jahrg. 6-, I888-. Hamburg, I889-. Jahrg. 6-, I889-: A.S.B.

Jahrg. II-, I894-: Z.S.I.

68I. - Naturwissenschaftlicher Verein.

(a) Abhandlungen aus dem Gebiete der Naturwissenschaften. Bde. 9-. Hamburg, 1886-: A.S.B.

A.S. = Archaenlogical Section. Incuan Museum. A.S.B. $=$ Asiatic Society of Bengal.

B.C. = Bishop s College.

B.S.I. = Botanical Survey of India

C.E. = Chemical Examiner to the Govt. of Bengal.

C.U. Calcutta Üniversity
E.C. = Civil Engineering College, Sibpur. G.S t. = Geological Survey of India.

I.L. = Impertal Library.

M.Ba. = Medical College, Bacteriological Laboratory

M.BI. = Medical College. Biological Laboratory.

M.c. = Miedical College (main library). 
68r. Hamburg. - Naturwissenschaftlicher Verein (Continued).

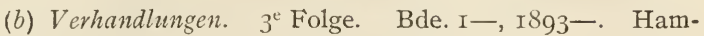
burg, I894-. (Bde. 9, I2 wanting): A.S.B.

682. - Verein für Naturwissenschaftliche Unterhaltung.

Verhandlungen (Sitzungsberichte, Abhandlungen). Bde. 3-5, I876-82. Hamburg, $\mathrm{x} 878-83$ : A.S.B.

683. - Zeitschrift fïr Anorganische Chemie. Herausg. von G. Krüss, G. Tammann (and others). Bde. I- Hamburg und Leipzig, I892--. Register, Bde. I-5o.

Bde. I-, I892- (Bde. 63-72 wanting) and index : P.C. Bde. 52-, I907-: G.S.I.

684. Hanover.-Bonplandia : Zeitschrift fïr die gesammte Botanik. Herausg. von W. E. G. und B. Seemann. Bde. I-Io. Hannover, I853-62 : R.B.G.

685. Z Zeitschrift für Malakozoologie. Herausg. von K. T. Menke und L. Pfeiffer. Jahrg. I-IO. Hannover, I844-53: Z.S.I. [Continued as No. 635.]

Heidelberg.-Berichte über Land- und Forstwirtschaft in DeutschOstafrika (see No. 980).

686. - Jahrbuch fïr Mineralogie, Geognosie, Geologie und Petrefactenkunde. Herausg. von K. C. v. Leonhard und H. G. Bronn. Jahrg. I-3. Heidelberg, I830-32 : G.S.I. [Continued as No. 772.]

687. Jena.-Archiv für Protistenkunde. Begründet von Schauduin, herausg. von Hartmann und Prowazek. Bde. I-, Jena, Ig02-: M.Bi., Z.S.I.

688. - Association Internationale des Botanistes.

Progressus Rei Botanicae. Herausg. von der Association ... Red. von J. P. Lotsy. Bde. I-. Jena, I9o7-: R.B.G.

689. Botanische Mittheilungen aus den Tropen. Herausg. von A. F. W. Schimper. Hfte. I-9. Jena, I888-I90I : R.B.G.

- Botanisches Centralblatt (see No. 632).

$690-$ Centralblatt fïr Bakteriologie, Parasitenkunde und Infektionskrankheiten. Von O. Uhlworm und A. IVebber.

Abteilung I.-Originale. Bde. I9-. Jena, I896-.

Bde. I9-3I, I896-I902: S.G.

Bde. 33-, I903-; M.Ba.

Abteilung I.-Referate. Bde. 33-. Jena, I903- : M.Ba. Abteilung II. Bde. IO-I5. Jena, I903-06: M.Ba.

\footnotetext{
м o = Meteorolosical Office, Alipore

M Ph = Medical College, Physiological Laboratory.

P.C. = Presidency College.

P o = Patent Office.

R. B.G = Royal Botanic Garden

S.C.C. $=$ Scottısh Churches College.
}

s.G. = Surgeon-General's Library:

s.I. = Survey of India.

S.T.M. = School of Tropical Medicine.

T.D. = Telegraph Departnient

X.c. = St. Xavier s College.

Z.S.I. = Zoological Survey of India. 
69I Jena.-Deutsche Mineralogische Gesellschaft.

Fortschritte der Mineralogie, Krystallographie und Petrographie. Herausg. von G. Linck. Bde. I-. Jena, IgII- : G.S.I.

- Isis (see No. 7I0).

692. - Jahresberichte über die Fortschritte der Anatomie und Entwicklungsgeschichte. Herausg von G. Schwalbe. Neue Folge, Bde. I-. I892-. Jena, I897-: Z.S.I.

693. - Medicinisch-naturwissenschaftliche Gesellschaft.

(a) Denkschriften. Bde. 4-. Jena, I893-: Z.S.I. [Consisting of Semon's Zoologische Forschungsreisen, Bde. I-.]

(b) Jenaische Zeitschrift fur (Medicin und) Naturwissenschaft. Bde. I-7. Leipzig, I864-73: Z.S.I. Neue Folge, Bde. I- (=Bde. 8-). Jena, I874-: Z.S.I. Namen und Sachregister, Bde. I-30: Z.S.I.

(c) Sitzungsherichte. I878-86. Jena, I879-87: Z.S.I. [Subsequently issued with $(b)$.]

694. - Palaeontologische Abhandlungen. Herausg. von W. Dames, E. Kayser (and others). Bde. I-4. Berlin, I882-89: G.S.I. Neue Folge, Bde. I-4 (=Bde. 5-8). Jena, I880-I90I : G.S.I. [Continued as:] Geologische und Palaeontologische Abhandlungen. Neue Folge. Herausg, von E. Koken. Bde. 5- (=Bde. 9-). Jena, I9OI- (Bd. 7 (II) wanting) : G.S.I.

_ Progressus Rei Botanicae (see No. 688).

695. - Zeitschrift für Parasitenkunde. Herausg. von E. Hallier und F. A. Zürn. Bde. I-4. Jena, I869-75 : R.B.G.

696. — Zoologische Jahrbïcher. Herausg. von J. W. Spengel.

(a) Zoologische Jahrbiicher. Zeitschrift für Systematik. Geographie und Biologie der Thiere. Bde. I, 2. Jena, I886-87 : Z.S.I. [Continued in three parts as $(b),(c)$ and $(d)$.

(b) - Abtheilung für allgemeine Zoologie und Physiologie der Tiere. Bde. 3I-34. Jena, I9I2-I4 : Z.S.I.

(c) Abtheilung für Anatomie und Ontogenie der Thiere. Bde. 3-. Jena, I888-: Z.S.I.

(d) - Abtheilung für Systematik, Geographie und Biologie der Thiere. Bde. 3-. Jena, I887-: Z.S.I.

(e) Supplement-Band. I-. Jena, I898-: Z.S.I.

General-register, Bde. I-20: Z.S.I.

Kassel.--See Cassel.

A.S. Archaeological Section, Indian Museum.

A.S.B. = Asiatic Society of Eensal.

B.C. = Bishop's College.

B.S.I. = Botanical Survey of Indiat

C.E. = Chemical Examiner to the Govt. of Bengal.

C.U. = Calcutta University.
E.c. = Civil Engineering College, Sibpur

G.S.I. = Geological Survey of India

I L. = Imperial Library.

M Ba. = Medical College, Bacteriological Laloratory

M.Bi. = Medical College. Biological Laboratory.

M.c. = Ifedical College (main library). 
697. Kie1.-Astronomische Nachrichten. Herausg. von A. Krueger und H. Kreutz. Bde. I40-I48. Kiel, I896-99: M.O.

698. - Kommission zur Wissenschaftlichen Untersuchungen der Deutschen Meere.

Wissenschaftliche Heeresuntersuchungen herausg. von der Kommission .... und der Biologischen Anstalt auf Helgoland. Neue Folge. Abtcilung Helgoland. Bde. I-. Kiel, Leipzig, I894-. (Bd. I defective): Z.S.I.

699. - Zoologisches Magazin. Herausg. von C. R. W. Wiedemann. Bd. I, Nos. I-3; Bd. 2, No. I. Kiel, I8I7-I9, I823: Z.S.I.

700. Konigsberg.--(Königliche) Physikalisch-ökonomische Gesellschaft.

(a) Beiträge zur Naturkunde Preussens. No. I-7. Königsberg, I868-90 : G.S.I.

(b) Schriften. Bde. I-, I860-. Königsberg, I86I- : A.S.B., G.S.I. General-register, Jahrg. 26-50, I885I909: A.S.B., G.S.I.

Leipzig.-Annalen der Chemie (und Pharmacie) (see No. $726(a, b))$. 701. - Annalen der Physik (und Chemie).

(a) Annalen der Physik. Herausg. von L. IV. Gilbert. Bde. I-30. Halle, I799-I808: G.S.I. Neue Folge. Bde. I-30 (=Bde. 3I-60). Leipzig, I809-I9: G.S.I. [Continued as (c).]

(b) Annalen der Physik. Herausg. von P. Drude. $4^{\text {e Folge. }}$ Bde. I- $(=$ Bde. $306-)$. Leipzig, I900- $[$ A continuation of $(d)$. 1

Bde. I- (306-), I900- $[$ Bd. II (316) defective]: A.S.B. Bde. I- (306-), I $900-[$ 'Bd. 33 (338) defective] : P.C.

Bde. I-2I (306-326), I900-06 [Bd. 3 (309) wanting] : C.U.

Bde. I-I2 (306-3I7), I900-03: G.S.I.

(c) Annalen der Physik und (der physikalischen) Chemie. Herausg. von L. W. Gilbert and J. C. Poggendorff. Neueste Folge. Bde. I-I6 (=Bde. 6I-76). Leipzig, I8I9-24. Bde. I-30 (=Bde. 77-I06). Leipzig, I82436. $2^{\mathrm{c}}$ Reihe, Bde. I-30 [= Bde. 3I-60 (I07-I36)].

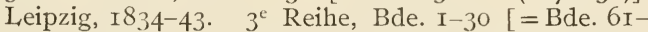
90 (I 37-I66)]. Leipzig, I844-53. $4^{e}$ Reihe, Bde. I-30 [=Bde. 9I-I20 (I67-I96)]. Leipzig, I854-63. $5^{\mathrm{e}}$ Reihe, Bde. I-30 [= Bde. I2I-I50 (I97-226)]. Leipzig, I864-73. $6^{\text {e }}$ Reihe, Bde. I-Io [= Bde. I5II60 $(227-236)]$. Leipzig, I $874-77$. [A continuation of $(a)$; continued as $(d)$.

Complete set : G.S.I.

M.O. = Mleteorological Office, Alinore

M.Ph. = Medical College, Physiological Laboratory.

P.C. = Presidency College.

P.O. = Patent Ofice.

R.B.G. = Roval Botanic Garden.

S.C.C. = Scottish Churches College.
S.G. = Surgeon-General s L.1brary

S.I. = Suriey of India

S.T.M. = School of Tropical Medicine

T.D. = Telegraph Department

X.c. =St. Xavier s College.

Z.s.I. = Zoological Survey of India. 
70I. Leipzig.-Annalen der Physik (und Chemie) (Continued).

$3^{\text {e }}$ Reihe, Bd. $22-4^{\text {e }}$ Reihe, Bd. 24 [= 82-II4 (I58I00)], I85I-6I : X.C.

$5^{\text {e }}$ Reihe, Bde. 4-6 [=I24-I26 (200-202)], r 865 (Bd.

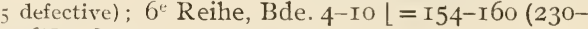
236)], I875-77: A.S.B.

$6^{\text {e }}$ Reihe, Bde. 2-Io [= I52-16o (228-236)], I87477 : T.D.

(d) Annalen der Physik und Chemie. Herausg. von G und E. Wiedemann. Unter Mitwirkung der Physicalischen Gesellschaft in Berlin. Neue Folge, Bde. $\mathrm{I}-69$ [ $\left[=237^{-}\right.$ 305). Leipzig, I877-99. [A continuation of $(c)$; continued as (b).]

Complete sets : A.S.B., G.S.I.

Bde. I-24 (237-260), I877-85 : T.D.

Bde. I8-69 (254-305), r883-99: C.U.

Bde. 54-69 (290-305), I895-99 : P.C.

(e) Beiblätter zu den Annalen der Physik und Chemie. Herausg. von J. C. Poggendorf (und G. und E. Wiedemann). Bde. I-. Leipzig, I877-.

Complete set: A.S.B.

Bde. I-27, I877-I903: G.S.I.

Bde. 9-30, I885-I906 : C.U.

(f) Ergänzungsband. Bde. I-8. Leipzig, I842-7S.

Bde. I-4, I842-54 : G.S.I.

Bd. 8, I 878 : A.S.B., T.D.

'Namen- und Sach-Register' to Bde. I-76, I799-I824: G.S.I.

'Sachregister' to Poggendorf's Annalen, Bde. $\mathrm{I}-\mathrm{r60}(=77-$ 236), Ergänzungsbanden $I-8$ and Jubelband, r824-77 : A.S.B., G.S.I.

'Namenregister' to Poggendorf's Annalen, Bde. I5I-r6o $\left(=227-23^{6}\right)$, Ergänzungsband 7,8 and Wiedemann's Annalen, Bde. I-5o (=237-286), I874-93: A.S.B.

'Namenregister' to Wiedemann's Annalen, Bde. I-35 (=237-27I), I877-88: A.S.B.

'Sachregister' to Wiedemann's Annalen, Bde. I-50 $(=237-286)$, I $877-93$ : A.S.B., G.S.I.

'Register' to Wiedemanu's Annalen, Bde. 5I-69 (=287305) and Drude's Annalen, Bde. I-3o (=306-336), I894-r909: A.S.B., P.C.

'Namenregister.' Beiblätter, Bde. I-I5, I877-9I : A.S.B., G.S.I.

'Register.' Beiblätter, Bde. I6-30, I892-I906: A.S.B.

\footnotetext{
A.S. = Archaeological Sectıon, indian Museum

A.S.B. = Asiatic Soctety of Bengal

B.C. = Bishop's College.

B.S. I. = Botancal Survey of India

C.E. = Chemical Examiner to the Govt, of Benga!

c.u. = Calcutta University
}

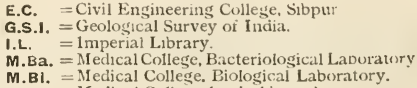


702. Leipzig.-Archiv für Anatomie und Physiologie.

(a) Archiv für Anatomie, Physiologie und Wissenschaftliche Medicin. Herausg. von J. Müller, C. B. Reichert (and others). Jahrg. I834-76. Berlin, I,eipzig [I834-76] (Jahrg. 1863, '64 wanting): Z.S.I. [A continuation of $(c)$; continued in two parts as $(b)$ and $(d)$.]

(b) Archiv für Anatomie und Entwicklungsgeschichte. Herausg. von IV. His, W. Braune (and others). Jahrg. I877-, and supplementary vols. Leipzig, I877-: Z.S.I.

(c) Archiv für Anatomie und Physiologie. Herausg. von J. F. Meckel. Jahrg. I826-32. Leipzig [I826-32]: Z.S.I. [A continuation of $(c)$; continted as $(a)$.]

(d) Archiv für Physiologie. Herausg. von E. du Bois-Reymond (and others). Jahrg. I877-, and supplementary vols. Leipzig, I $877-$ : Z.S.I.

(e) Deutsches Archiv fïr die Physiologie. Herausg. von J. F. Meckel. Bde. I-8. Halle, Berlin, I8I5-23: Z.S.I. [Continued as (c).]

703 - Archiv für die Botanik. Herausg. von J. J. Römer. Bde. I-3. Leipsig, I796-I805: R.B.G.

704 - Archiv für die Systematische Naturgeschichte. Herausg. von F. Weber und D. M. H. Mohr. Bd. I, Stück I. I,eipsig, I804: R.B.G.

705. - Archiv für Schiffs- und Tropen-Hygiene. Herausg. von C. Mense. Bde. I2-. Leipzig, I908-: S.T.M.

Beiträge zur Geophysik (see No. 764).

706. - Beiträge zur Wissenschaftlichen Botanik. Von C. Nägeli. Hfte. I-4. I,eipzig, I858-68: R.B.G.

707. - Bibliographia Zoologica. Ed. J. V. Carus (and afterwards) H. H. Field. Lipsiae, Zurich, I 896 - : Z.S.I. IVols. I-24 were issued as an appendix to No. 741 ; vols. 25 - published by the Concilium Bibliographicum, Zurich.

708. - Biologisches Centralblatt. Herausg. von J. Rosenthal. Bde. I-. Erlangen, I,eipzig, I88I-: Z.S.I.

709. - Botanische Jahrbücher fïr Systematik, Pflanzengeschichte und Pflanzengeographie. Herausg. von A. Engler. Bde. I-. Leipzig, I88I-: R.B.G.

- Botanisches Centralblatt (see No. 632).

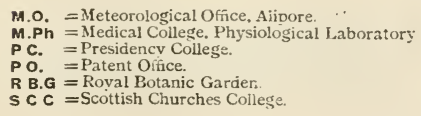

R B.G = Royal Botanic Garder.
S C C $=$ Scottish Churches College.

s.G. =Surqeon-Generai's Library

s.l. = Survev of Incia

S.T.M = School of Tropical Medicine.

T.D. =Telegraph Departmen:

X.C. =St Xavier s College. 
710. Leipzig.-Botanische Zeiinng. Bde. I-68. Herausg. von H. Moh1, D. F. L. von Schlechtendal (and others). Berlin, Leipzig, I843IgIO: R.B.G.

Chemisches Central-blatt (see No. 676).

7II. — Deutsche Mathematiker Vereinigung. Jahresbericht. Bd. 22. Leipzig, I9I3: C.U.

712. - Deutsche Morgenländische Gesellschaft.

(a) Abhandlungen für die Kunde des Morgenländes. Bde. I-. Leipzig, I859--(No. I, Bd. 12 wanting): A.S.B.

(b) Wissenschaftlicher Jahresbericht über die Morgenländischen Studien. I862-80. Leipzig, I87 I-83 (issues for I $868-75$ wanting) : A.S.B.

(c) Zeitschrift. Bde. I-. Leipzig, I847-. Register, Bde. I-6o.

Complete set: I.L.

Bde. I-, I847- (Bde. 1 I , 17,48 wanting) and index: A.S.B.

Bde. I-62, I847-I908 : C.U.

713. - Deutsche Zoologische Gesellschaft.

Verhandlungen. Jahrg. I-. Leipzig, I89I-: Z.S.I.

—Deutsches Archiv für die Physiologie (see No. $702(e)$ ).

- Drude's Annalen der Physik (see No. 70I (b)).

- Entomologische Zeitschrift (see No. 653).

- Ephemerides (see No. 669 (b)).

—_ Gegenbaur's Morphologisches Jahrbuch (see No. 728).

— Geographische Abhandlungen (see No. 599).

7I4. - Geologisches Centralblatt. Herausg. von K. Keilhack. Bde. I-. Leipzig, I90I-: G.S.I.

715. - Gesellschaft für Errdkunde.

(a) Mittheilungen. I910-. Leipzig, I9II-: M.O. [A continuation of $(b)$.]

(b) Mittheilungen des Vereins fïr Erdkunde. I883-I909. Leipzig, I884-I9Io. [Continued as $(a)$.]

Complete set: M.o.

Vols. for $1883-1902$ (vol, for 1899 wanting) : G.S.I.

(c) Wissenschaftliche Veröffentlichungen des Vereins für Erdkunde. Bde. I-6. Leipzig, I89I-I904: G.S.I.

\footnotetext{
A.S. = Archaeological Section, Indian Museum.

A.S.B. = Aslatic Society of Bengal.

B.C. = Bishop S College.

B.S.I. = Botanical Survey of India

C.E. = Chemical Examiner to the Govt of Bengal

c.U. = Calcutta University
}

E.C. = Civil Engineering College, Sibptur.

G.S.I. = Geological Survey of India.

l.L. = Imperial Library:

M.Ba. = Afedical College, Bacteriological Laboratory

M.Bi. = Medical College, Biological Labisatory.

M.C. = Medical College (main library). 
716. Leipzig.-Insekten-Borse: Internationales Wochenblatt der Ento. mologie. Jahrg. I8, 20, 2I. Leipzig, Igor, '03, '04: Z.S.I.

7I\%. - Internationale Monatsschrifl für Anatomie und Histologie. Herausg, von E. A. Schafer, L. Testut (and others). Bde. I-3. Paris, London, Cassel, Leipzig, Berlin, I884-86: Z.S.I. [Continued as:] Internationale Monatsschrift für Anatomie und Physiologie. Bde. 4-. Cassel, Leipzig, Berlin, I887-: Z.S.I. Index to Bde. I-Io: Z.S.I.

7I8. - Internationale Revue des gesamten Hydrobiologie und Hydrographie. Red. R. Wolterek. Bde. I-, with supplements. Leipzig, Igo8- : Z.S.I.

719. - Isis (oder Encyclopädische Zeitung). Von Oken. Jahrg. I8I748. Jena, Leipzig [I8I7]-I848 : Z.S.I.

720. - Jahrbuch der Astronomie und Geophysik. Herausg. von H. J. Klein. Bde. I-. Leipzig, I89I-: G.S.I.

72I. — Jahrbuch der Radioaktivität und Electronik. Herausg. von J. Stark. Bde. I-, Leipzig, Igo4- : P.C.

- Jahrbiücher der Gewächskunde (see No. 59I).

722. - Jahresberichte über die Fortschritte der Anatomie und Physiologie. Herausg. von Fr. Hofmann, G. Schwalbe (and others). Bde. I-20. Leipzig, I873-92 : Z.S.I. General-register, Bde. I-20: Z.S.I.

723. - Jahresbericht über die Fortschritte in der Lehre von Pathogenen Mikroorganismen. Herausg. von P. von Baumgarten (and others). Jahrg. I6, I7, I900, 'OI. Leipzig, I902, '03: M.Ba.

- Just's Botanische Jahresbericht (see No. 596).

— Justus Perthes' Geographische Anstalt (see No. 663).

724. K Königliche Sächsische Gesellschaft der Wissenschaften.

(a) Abhandlungen der mathematisch-physischen Classe. Bde. I-. I,eipzig, I852-.

Complete set: G.S.I.

Bde. I3-, I887-: Z.S.I.

Bde. I8-, I893-: A.S.B.

(b) Abhandlungen der philologisch-historischen Classe. Bde. I-5. Leipzig, I850-70: G.S.I. Bde. I3-. Leipzig, I893- (no. 5, Bd. 24 wanting): A.S.B.

$[(a)$ and $(b)$ also bear volume numbers as a combined series of Abhandungen.]

M.o. = Meteorologicai Office, Alipore

M.Ph. = Medical College. Physiological Laboratory.

P.C. = Presidency College

P.o. = Patent Office.

R.B.G. = Royal Botanic Garden.

S.C.C. $=$ Scottish Churches College.
S.G. = Surgeon-General's Library:

S.G. = Surgeon-General's

S.T.M. = School of Tropical Medicine

T.D. = Telegraph Department.

X.c. =St. Xavier's College.

z.s.1. = Zoological Survey of Incia. 
724. Leipzig.-Königliche Sächsische Gesellschaft .. (Continued).

(c) Berichte ïber die Verhandlungen .... Mathematischphysische Classe. Bde. 22-. Leipzig, 1870-.

Bde. 22-, I870-: G.S.I.

Bde. 35 -, I 883 - : Z.S.I.

Bde. 44一, I892-: A.S.B.

(d) Berichte ïber die V'erhandlungen .... Philologischhistorische Classe. Bde. 44-. Leipzig, I892-: A.S.B.

Register der Abhandlungen und Berichte, I $846-85$ : Z.S.I. ; I846-95: G.S.I., Z.S.I.

725. — Kosmos: Zeitschrift für einheitliche Weltanschaunng auf Grund der Entwicklungslehre. Herausg. von O. Caspari (and others). Jahrg. I-4, Bde. I-8. Leipzig, I877-8I : Z.S.I. [Continued as :] Kosmos: Zeitschrift für Entwicklungslehre und einheitliche Weltanschaming. Herausg. von E. Krause und B. Vetter. Jahrg. 5-7, Bde. 9-I3. Stuttgart, I88I-83: Z.S.I. [Continued as:] Kosmos Zeilschrift für die gesamte Entwicklungslehre. Herausg. von B. Vetter. [Jahrg. I4-I9] : I884-86. Stuttgart, I884-86: Z.S.I.

726. - Liebig's Annalen der Chemie (und Pharmacie).

(a) Annalen der Chemie und Pharmacie. Herausg. von F. Wohler und J. Liebig. Bde. 33-76. Leipzig, Heidelberg, I840-50. Neue Reihe, Bde. I-92 (=77-I68). Leipzig, Heidelberg, I85I-73. Supplement-Bde. I-8, Leipzig, Heidelberg, I86I-72. [A continuation of (b): continued as $(d)$.]

Complete set: P.C.

Neue Reihe, Bde. 25-32 (=IOI-IO8) (Bd. 30 wanting): A.S.B.

(b) Annalen der Pharmacie. Herausg. von R. Brandes, P. L. Geiger und J. Liebig. Bde. I-32. Leipzig, Heidelberg, I832-39: P.C. [Continued as $(a)$.]

(c) Justus Liebig's Annalen der Chemie. Herausg. von F. Wöhler, H. Kopp (and others). Bde. I73-. Leipzig, Heidelberg, I874-(Bde. 371-384, I909-II, wanting): P.C. [A continuation of $(d)$.]

(d) Justus Liebig's Annalen der Chemie und Pharmacie. Herausg. von F. Wöhler, H. Kopp (and others). Neue Reihe, Bde. 93-96 (= I69-I72). Leipzig, Heidelberg, I873-74 : P.C. [A continuation of $(a)$; continued as $(c)$.]

Autoren- und Sach-Register, Bde. I-I64, I832-72 : P.C.

General-Register, Bde. I65-328, I873-I903: P.C.

A.S. = Archaeological Seclion, Indian Museum.

A.S.B. = Asialic Society of Bengal.

B.C. = Bishop s College.

B.S.I = Botanical Survey of India

C.E. = Chemical Examiner to the Govt of Bengal.

c.U = Calcutta Unversity.
E.C. =Civil Engineering College, Sibnur.

G.S.1. = Geolosical Survey of India.

I L. = Imperial Library.

M. Ba. = Medical College, Bacteriolorical Laboratory

M.Bi. = Medical Collese. Biological Laboratory

M.C. = Medical College (main library). 
727. Leipzig.-Mathematische Annalen. Herausg. von A. Clebsch, C. Neumann (and others). Bde. I-. Leipzig, I869-.

Complete set: C.U.

Bde. 68-, I9I0-: P.C.

_- Miscellanea Curiosa Medico-physica (see No. 669(d)). Monthly International Journal of Anatomy and Histology (see No. 717 ).

728. - Morphologisches Jahrbuch. Eine Zeitschrift für Anatomie und Entwicklungsgeschichte. Herausg. von C. Gegenbaur. Bde. I-30. Leipzig, I876-I902: Z.S.I. [Continued as:] Gegenbaur's Morphologisches Jahrbuch. Herausg. von G. Ruge. Bde. 3I-. Leipzig, I903-: Z.S.I. Namen- und Sach-Register, Bde. I40 : Z.S.I.

729. - Nene Entdeckungen im ganzen Umfang der Pflanzenkunde. Herausg. von K. Sprengel. Bde. I-3. Leipzig, I820-22 : R.B.G.

730. — Oekonomische Societät.

(a) Neure und Grössere Schriften. Bd. I. Dresden, I80I : R.B.G.

(b) Schriften. Bde. I-S. Dresden, I77I-90: R.B.G.

73I. — Orientalisches Archiv: illustrierte Zeitschrift fur Kunst, Kulturgeschichte und Völkerkunde der Länder des Ostens. Herausg. von H. Grothe. Bd. I. Leipzig, I9IO-II : A.S.B.

732. — Physikalische Zeitschrift. Herausg. von E. Riecke und H. T. Simon. Bde. I-. Leipzig, I899- (Bd. I3 defective) : P.C.

— Poggendorf's Annalen der Physik und Chemie (see No. $70 \mathrm{r}(c)$ ).

- Repertorium für Experimental-Physik, etc. (see No. 752).

733. - Städtisches Museum für Völkerkunde.

(a) Jahrbuch. Bde. I-. L Leipzig, I907-.

Bde. I-4, I907-II : Z.S.I.

Bde. 2-, I908-: A.S.B.

(b) I'eröffentlichungen. Hfte. I-4. I,eipzig, I907-I2: Z.S.I.

__ Verein für Erdkunde (see No. 7I5).

-Wiedemann's Annalen der Physik und Chemie (see No. 7or $(d)$ ).

- Wissenschaftliche Meeresuntersuchungen (see No. 698).

_ Zeitschrift für Anorganische Chemie (see No. 683).

734. — Zeitschrift für die Entomologie. Herausg. von E. F. Germar. Bde. I-5. Leipzig, I839-44 : Z.S.I.

M.O. = Meteorological Office, Alipore.

M.Ph. = Medical Collese, Physiologicąl Laboratory.

P.c. = Presidency College.

P.o. = Patent Office

R. B. G = Royal Botanic Garden

S.C.C. = Scottish Churches Codlege.
S.G. = Surgeon-General's Libtary

S.I. = Survey of India.

S.T.M. = School of Tropical Medicine.

T.D. = Telegraph Department.

X.c =St. Xavier's College.

Z.S.1. Zoological Survey of India. 
735. Leipzig.-Zeitschrift fïr Hygiene und Infektionskrankheiten. Herausg. von R. Koch und C. Flugge (and others). Bde. 2I-. Leipzig, I896-. Register, Bde. I-6o.

Bde. 2I-40, I896-I902 (Bd. 40 defective) : S.G.

Bde. 42-, I903-, and index: M.Ba.

736. — Zeitschrift für Krystallographie und Mineralogie. Herausg. von P. Groth. Bde. I-. Leipzig, I877-: G.S.I. Sachregister, Bde. I-50 : G.S.I. Autoren-Register, Bde. I-50 : G.S.I.

—_ Zeitschrift für Naturwissenschaften (see No. $673(c)$ ).

737. - Zeitschrift für Physikalische Chemie, Stoechiometrie und Verwandtschaftslehre. Herausg, von W. Ostwald und J. H. van't Hoff. Bde. I-. Leipzig, I887- (Bde. 58, 59, 70 wanting): P.C. Namen- und Sach-Register, Bde. 25-50 : P.C.

738. — Zeitschrift für IVissenschaftliche Mikroskopie und für Mikroskopische Technik. Begrundet von W. J. Behrens, herausg. von E. Küster. Bde. 23-. Leipzig, I906-: Z.S.I.

739. — Zeitschrift fïr Wissenschaftliche Zoologie. Herausg. von C. T. von Siebold, A. Kölliker (and others). Bde. I-. Leipzig, I 849 - : Z.S.I. Namen- und Sach-register, Bde. I-75 : Z.S.I.

740. - Zentralblatt fïr Zoologie Allgemeine und Experimentelle Biologie. Herausg. von A. Schuberg und H. Poli. Bde. ILeipzig, Berlin, I9I2-: M.Bi., Z.S.I. [A continuation of No. 742.]

74I. Z Zoologischer Anzeiger. Herausg. von J. V. Carus und E. Korschelt. Bde. (Jahrg.) I-. Leipzig, I878-: Z.S.I. Register, Jahrg. I-35: Z.S.I.

742. — Zoologisches Centralblatt (Zentralblatt). Herausg. von'A. Schuberg. Bde. (Jahrg.) I-I8. Leipzig, I895-I9II: Z.S.I. [Continued as No. 740.]

- Zoologisches Jahresbericht (see No. $845(c))$.

743. Lubeck.-Geographische Gesellschaft und Naturhistorisches Museum.

Mitteilungen. $2^{\text {te }}$ Reihe, Hfte. I8-26. Lübeck, I904-I3: Z.S.I.

744. Magdeburg.-Museum für Natur- und Hiemat-kunde.

Abhandlungen und Berichte des Museums ....... und dem Naturwissenschaftlichen Verein. Bde. 2-. Magdeburg, I909-: A.S.B.

A.S. = Archaeological Section. Indian Museum A.S B. = Asiatıc Society of Bengal.

B.C. = Bishop s Collerge.

B.S.I. = Botanical Survey of India

C.E. = Chemical Examiner to the Govt. of Bengat

C.U. = Calcutta Unniversity.
E.C. = Civil Engineering Colleđe. Sibpur. G.S.l. = Geolosical Survey of incia.

G. = Imperial Library

M.Ba. = Medical College, Bacteriological Laboratory.

M.B1. = Medical College. Biological Laboratory.

M.C. = Medical College (main library). 
745. Marburg.-Gesellschaft zur Beförderung der gesammten Naturwissenschaften.

Sitzungsberichte. Jahrg. I866-8I. Marburg, I866-8I : Z.S.I. Inhalts-T'erzeichniss, I866 77: Z.S.I.

746. Metz.-Académie Royale.

Mémoires. Ann. I7-27, I $835 / 36-1845 / 46$. Metz, Paris, I $836-46$ : A.S.B.

747. Miltitz.-Schimmel and Co. (Fritzsche Bros.).

Semi-annual Report. I900-I2. Miltitz, I900-I2: B.S.I. [Continued as:] Semi-annual Report on Essential Oils and Synthetic Perfumes. I9I3-. Miltitz, I9I3- : B.S.I.

Munich.-Baierische Akademie (see No. 750).

—_Elektrotechnische Zeitschrift (see No. 582).

748. - Geognostisch-paläontologische Beiträge. Herausg. von E. W. Benecke. Vol. I, 2. München, I866-76: G.S.I.

749. - Geographische Gesellschaft.

Mitteilungen. Bde. I-7. München, I904-I2: M.O.

750. - Konigliche Bayerische Akademie der Wissenschaften.

(a) Abhandlungen der historischen Classe. Bde. I-. München, I833-. (Bd. 9 wanting, Bd. 23 defective): A.S.B.

(b) Abhandlungen der matheinatisch-physikalischen Classe. Bde. I-. München, I832-.

Complete set: G.S.I.

Bde. I-, I832- (Bde. 9, Io wanting, Bd. 21 defective): A.S.B.

Bde. 25-, I9I2-: Z.S.I.

(c) Abhandlungen der philosophisch-philologischen Classe. Bde. I-. München, I835-. (Bd. 9 wanting, Bd. 23 defective): A.S.B.

(d) Bulletin. Jahrg. I843-53. München, I843-53: A.S.B.

(e) Denkschriften. Bde. I-9, I8o8-24. München, I8o925 (Bde. 5, 8 wanting): A.S.B. [Continued in three sections, $(a),(b)$ and $(c)$.

(f) Gelehrte Anzeigen. Bde. I-49. München, I835-59: A.S.B.

(g) Jahrbuch. I9I2-. München, I9I3-: A.S.B.

(h) Nene philosophische Abhandlungen. Bde. I-7. München, I778-97 : G.S.I. [Continued as (e).]

M.O. = Mcteorological Office, Alipors.

M.Ph. = Medical College. Physiological Laboratory

P.C. = Presidency College.

P.o. = Patent Olfice.

R.B.G = Royal Botanic Garden.

S.C.C. = Scottish Churches College.
S.G. = Surgeon-General's Library.

S.I. = Survey of India.

S.T.M. = School of Tropical Medicine.

T.D. = Telegraph Department

X.C. =St. Xavier's College.

Z.S.I. '=Zoological Survey of India. 
750. Munich-Kön. Bayer. Akad. Wissenschaften (Continued).

(i) Sitzungsberichte. Jahrg. I860-70. München, 1860-70: G.S.I. [Continued in two parts as $(j)$ and $(k)$.]

(j) Sitzungsberichte der mathematisch-physikalischen Classe. Bde. I-. München, I $87 \mathrm{I}-$. Inhaltsverzeichniss, Jahrg. I87I-85, I886-99, I860-I9I0.

Complete set and indexes: G.S.I.

Bde. I-, I87 I- (Bde. 5, 27, 32, 33 wanting; Bde. 35 . $36,38,39$ defective) and indexes: A.S.B.

Bde. 40-, I9I0-: Z.S.I.

Bde. 4I-, I9II-: R.B.G.

(k) Sitzungsberichte der philosophisch-philologischen und historischen Classe. Bde. I-, Jahrg. I87I-. München, I87I-(Vols. 3, 4, 1875, vol. I of I88I, vol. 2 of 1893 , vol. 1 of 1897 wanting; vols. for 1906 , 'o9 defective): A.S.B. Inhaltsverzeichniss, Jahrg. I871-99: A.S.B.

Register zu den Abhandlungen, Denkschrifte11 und Reden, I807-I9I3: A.S.B., G.S.I.

Register zu den ersten 5o Jahrg. der Sitzungsberichte der mathematisch-physikalischen Klasse, I860-I9I0: G.S.I.

751. — Königliche Sternwarte.

(a) Annalen. Herausg. von J. Lamont. Bde. 8-2I. München, I855-76. [Continued as (b).]

Bde. $8-2 \mathrm{I}, \mathrm{I} 855-76$ (Bd. 22 wanting): G.S.I.

Bde. 9-I4, I857-65: A.S.B.

(b) Meteorologische und Magnetische Beobachtungen. Jahrg. I876-82. München, I $877-83$ : G.S.I. [A continuation of $(a)$; continued as $(c)$.]

(c) Neme Annalen. Bde. I-4. München, r890-I909: G.S.I. [A continuation of $(b)$.]

(d) Supplementbände zu den Annalen. Bde. 2-I4. München, $1857-84$ (Bd. 12 wanting): G.S.I.

752. - Repertorium für Experimental-physik, für physikalische Technik, mathematische und astronomische Instrumentenkunde. Herausg. von Ph. Carl. Bde. I6-I8. München, Leipzig, I880-82: A.S.B. [Continued as:] Repertorium der Physik. Herausg. von F. Exner. Bde. I9-20. München, Iseipzig, I883-84 : A.S.B.

753. Neubrandenburg.-Verein der Freunde der Naturgeschichte in Meklenburg.

Archiv. Herausg. von E. Boll. Hfte. (Jahrg.) I-I9. Neubrandenburg, $1847-65$ : G.S.I.

A.S. = Archaeological Section, Indian Museun.

A.S.B. = Asiatic Society of Bengal.

B.C. = Bishop's College.

B.S.I. = Botanical Survey of India

C.E. = Chemical lixaminer to the Govt. of Bengal.

C.U. = Calcutta University.
E.C. = Civil Engineering College, Sibpur.

G.S.I = Geolosical Survey of India.

I.L. = Imperial Library

M.Ba. = Medical College, Bacteriological Laboratrory

M.BI. = Ml edical College. Biological Laboratory.

M.C. = Medical College (man hbrary) 
Neubrandenburg.-Zeitschrift fïr systematische Hymenopterologie und Diptcrologic (see No. 776 ).

Nuremburg.-Acta Physico-medica, etc. (see No. 669 $(a)$ ).

__ Botanische Literatur-Blätter (see No. $760(a)$ ).

754. Commercium litterarium ad rei medicae et scientiae naturalis incrementum institutum quo quicquid novissime observatum, agitatum, scriptum vel peractum est, succincte dilucideque exponitur. Vol. I-I5. Norimbergae, I73I-45: G.S.I.

- Ephemerides (see No. $669(b)$ ).

755. - Faunae Insectorum Germanicae Initia .... Herausg. von D. G. W. F. Panzer. Hfte. I-I Io. Nürnberg. [I792-] I793I 809 [-23]: Z.S.I. [Continued as No. 759.]

L Literaturblätter für Botanik (see No. $760(e))$.

—. Miscellanea Curiosa Medico-physica (see No. $760(d))$.

756. — Monatlich-herausgegebene Insecten-Belustigung. Von A. J. Rösel von Rosenhof. Theile I-4. Nürnberg, [I746-6I] : A.S.B.

757. - Naturhistorische Gesellschaft.

Abhandlungen. Bde. II-. Nürnberg, I 898 -

Bde. II-, I808-: A.S.B.

Bd. I3, I900: Z.S.I.

758. — Romisch Kaiserliche Akademie der Naturforscher.

Auserlesene medicinisch-chirurgisch-anatomisch-chymisch und botanische Abhandlungen: aus dem lateinischen Bde. I-20. Nürnberg, I755-7I : G.S.I.

750. Ratisbon.-Deutschlands Insecten .... Fortgesetzt von G. A. IT. Herrick-Schäffer (and in part by) C. L. Koch. Hfte. III-Igo. Regensburg. (I829-44) : Z.S.I. [A continuation of No. 755.]

760. — Königlich-Bayerische Botanische Gesellschaft in Regensburg.

(a) Botanische Literatur-Blätter zur periodischen Darstellung der Fortschritte der Pflanzenkunte, etc. Bd. 2. Nürnberg, I829: R.B.G. [A continuation of (e).]

(b) Botanische Zeitung walche Recensionen. Abhandlungen .... und Nachrichten die Botanik betreffend enthält. Jahrg. I-3. Regensburg, I802-04: R.B.G.

(c) Denkschriften. Bd. [I], Abth. I, 2. Regensburg, I I5 $_{5}$ I8: R.B.G.

(d) Flora, oder (allgemeine) botanische Zeitung. Herausg. von der.... Gesellschaft (and afterwards by) D. H.

M.o. = Meteorological Otice, Alipore

M.Ph. = Medical College, Physiological Laiooratory:

P.C. = Presidency College

P.o. = Patent Otice

R.B.G. = Royal Botanic Garden

S.C.C. $=$ Scottish Churches College.
S.G. = Surgeon-General's Library.

S.I. = Survey of India.

S.T.M. $=$ School of Tropical Medicine.

T.D. = Telegraph Department.

X.C. =st. Xavier's College.

Z.S.l. = Zoological Surrey of India. 
760. Ratisbon.--Kön.-Bayer. Botanische (Gesellschaft (Continued).

Hoppe, A. E. Fürnrohr, G. A. IV. Herrick-Schäffer (and others). Jahrg. I-, Regensburg, I8I8- : R.B.G. LJahrg. 26-75 are also styled 'Neue Reihe, Jahrg. I-50'; the numeration is then carried on as Bde. 76 -, while Bde. IOIare also styled 'Neue Folge, Bde. I-'.]

(e) Literaturblätter fïr reine und angewandte Botanik. Bd. I. Nürnberg, I828: R.B.G. [Continued as $(a)$.]

Regensburg see Ratisbon.

Stettin.-Entomologische Nachrichten (see No. 583).

761. - E Entomologischer Verein.

(a) Entomologische Zeitung. Jahrg. I-. Stettin, I840-. Jahrg. I-, I840-- (Hft. I, Jahrg. 74 wanting): A.S.B. Jahrg. 68一, I907-: Z.S.I.

(b) Linnea Entomologica. Zeitschrift herausg. von dem .... Vereine in Stettin. Bde. I-I3. Berlin, I846-59: Z.S.I.

762. Strasburg.-Bibliotheca Zoologica: Original-Abhandlungen aus dem Gesammtgebiete der Zoologie. Herausg. von R. Leuckart und C. Chun. Bde. I-8 (=Hfte. I-2I). Cassel, Stuttgart, I887-98: Z.S.I. [Continned as No. 775.]

Bureau des Königlichen Oberpräsidiums.

Abhandlungen zur geologischen Specialkarte von ElsassLothringen (see No. 765 (a)).

763. - Internationale Kommission für Wissenschaftliche Luftschiffahrt. (Commission International pour l'Aérostation Scientifique).

(a) Beobachtungen mit bemannten, unbemmannten Ballons und Drachen sowie auf Berg- und Wolkenstationen (Observations des Ascensions Internationales simultanées et des Stations de Montagne et de Nuages. I9OI-. Strassburg, I903- (vols. for 1902, 'o4 wanting) : M.O.

(b) Veröffentlichungen. Herausg. von H. Hergesell. Jahrg. r905-. Strassburg, I907-: A.S.B.

764. — Kaiser-Wilhelms-Universität.

Beiträge zur Geophysik. Abhandlungen aus dem geographischen Seminar der Universität. Bde. I. Stuttgart, I887 : G.S.I. [Continued as:] Beiträge zur Geophysik. Zeitschrift für physikalische Erdkunde. Herausg. von G. Gerland. Bde. 2-. Stuttgart, Leipzig, I895-:

A.S. = Archaeological Section. Indian Museum.

A.S.B. = Asiatic Society of Bengal.

B.C. = Bishop's College.

B.S.I. = Botanical Survey of India.

C.E. = Chemical Examiner to the Govt. of Bengat.

C.U. = Calcutta University.
E.C. = Civil Engineering College. Sibpur.

G.S.1. = Geological Survey of India.

I.L. = Imperial Library

M.Ba. = Medical College, Bacteriological Laboratory.

M.BI. = Medical College. Biological Laboratory.

M.C. = Medical College (main library). 
764. Strasburg.-Kaiser-Wilhelms-Universität (Continued).

G.S.I. Erganzungsbanden I, 2. Leipzig, I902, '04: G.S.I.

765. - Kommission für die Geologische Landesuntersuchung von Elsass-Lothringen.

(a) Abhandlungen zur geologischen Specialkarte... Bd. I-5. Strassburg, I875-97 : G.S.I. Neue Folge. Hfte. I-6. Strassburg, I898-I905: G.S.I.

(b) Mittheilungen. Bde. I-. Strassburg, I888-: G.S.I.

766. - Société des Sciences Naturelles.

Mémoires de la Société (du Museum) d'Histoire Naturelle.

Tom. I-4. Paris, Strasbourg, I830-53: G.S.I. [Continued as:] Mémoires de la Société des Sciences Naturelles. Tom. 5, 6. Paris, Strasbourg, $1858-70$ : G.S.I.

Société du Museum d'Histoire Naturelle (see No. 766).

767. Stuttgart.-A usland: Ueberschau der neuesten Forschungen auf dem Gebiete der Natur-, Erd- und Völkerkunde. Herausg. von F. von Hellwald. Jahrg. 53-54. Stuttgart, I88o-8I : G.S.I. [Continued as:] Ausland: Wochenschrift fïr Länder- und Völkerkunde. Jahrg. 55-6o. Stuttgait, r882-87 : G.S.I.

— Beiträge zur Geophysik (see No. 764).

768. - Centralblatt iir IIneralogie, Geologie und Palacontologie, in Verbindung mit dem Nenen Jahrbuch fïr Mineralogie, etc. Herausg. von IIs Bauer, E. Koken (and others). I9oo-. Stuttgart, I900- : G.S.I.

- Deutsche Ornithologen-Gesellschaft (-Verein) (see No. 77I).

769. - Entomologische Rundschau. Herausg. von C. Schaufuss. I909-IO. Stuttgart, I909-IO: Z.S.I.

- Gartenflora (see No. $5^{85}$ ).

770. - Humboldt: Monatsschrift fïr die gesamten Naturwissenschaften. Herausg. von O. Dammer. Jahrg. 6-9. Stuttgart, I887-90: Z.S.I.

Kosmos (see No. 725).

77r. - Naumannia : Archiv fïr die Ornithologie vorzugsweise Europa's. Organ der Deutschen Ornithologen-Verein. Herausg. von E. Baldamus. Bd. I, 2 and Jahrg. I853, '54. Stuttgart, I85I-54: Z.S.I. [Continued as:] Naumannia: Journal für die Ornithologie vorzugsweise Europa's. Organ der Deutschen OrnithologenGesellschaft. Rer. von E. Baldamus. Jahrg. I855-58. Dessau, L.eipzig, I855-58: Z.S.I. [Afterwards merged in No. 634.]

M.o. = Meteorological Office, Alipore.

M.Ph. = Medical College, Physiological Laboratory.

P.C. $=$ Presidency College.

P.o. = Patent Office

R.B.G. = RoyaI Botanic Garden.

S.C.C. $=$ Scottish Churches College.
S.G. = Surgeon-General's Library.

S.I. = Survey of India.

S.T.M. $=$ School of Tropical Medicine.

T.D. = Telegraph Department.

X.C =St. Xavier's College.

Z.S.I. = Zoological Survey of India. 
772. Stuttgart.-Nenes Jahrbuch für Mineralogie, Geognosie, Geologie und Petrefaktenkunde. Herausg. von K. C. v. Leonhard und H. Bronn. Jahrg. I833-62. Stuttgart, I833-62: G.S.I. [A con tinuation of No. 686. Continned as:] Neues Jahrbuch für Mineralogie, Geologie und Paläontologie. Herausg. von G. Leonhard, H. B. Geinitz (and others). Jahrg. I863-. Stuttgart, I863-: G.S.I. Beilage-Bände. Bde. I-. Stuttgart, I88I-: G.S.I. Repertorium, I830-89, I895-99: G.S.I.

773. — Verein für Vaterländische Naturkunde in Württemberg.

Jahreshefte. Jahrg. I-. Stuttgart, I845-.

Complete set: G.S.I.

Jahrg. 30-, I874-: A.S.B.

774. - Zeitschrift für Morphologie und Anthropologie. Herausg. von G. Schwalbe. Bde. I-. Stuttgart, I $899-$ : Z.S.I. Sonderhefte [I], 2, I906, 'I2: Z.S.I.

— Zeitschrift für Natureissenschaften (see No. 673).

775. - Zoologica: Original-Abhandlungen aus dem Gesammtgebiete der Zoologie. Herausg. von R. Leuckart und C. Chun. Bde. 9(=Hfte. 22-). Stuttgart, I897-: Z.S.I. [For Bde. I-8 see No. 762.$]$

776. Teschendorf.-Zeitschrift für systematische Hymenopterologie und Dipterologie. Herausg. von F. W. Konow. Jahrg. I-8, heft 2. Teschendorf bei Stargard in Mecklenburg, I90I-08: Z.S.I. [Afterwards merged in No. 577.]

777. Weimar.-Teutschland, Geognostisch-geologisch Dargestellt: eine Zeitschrift herausg. von C. Keferstein. Bde. I-7. Weimar. I 82 I-3I : G.S.I.

778. Wiesbaden.-Ergebnisse der Anatomie und Entwickelungsgeschichte. Herausg, von Fr. Merkel und R. Bonnet. Bde. I-. Wiesbaden, I $892-$ : Z.S.I.

779. — Nassauischer Verein für Naturkunde.

(a) Jahrbiicher. Hfte. I9-. Wiesbaden, I864-. [A continuation of $(b)$.]

Complete set: Z.S.I.

Hfte. I9-24, I864-70 : G.S.I.

(b) Jahrbücher des I'ereins jür Naturkunde im Herzogthum Nassau. Hfte. I-I8. Wiesbaden, I844-63. [For continnation see $(a)$. I

Complete set : G.S.I.

Hfte. $3-18,1846-63$ (Hfte. 9,12 wanting): Z.S.I.

A.S. Archaeological Section, Indian Museum. A.S.B. = Astatic Society of Benga!

B.C. = Bishop s College.

B.S.I. = Botanical Survey of India

C.E. Chemical Examiner to the Guvt. of Bengal.

C. $U$. Calcutta Universily:
E.C. = Civil Engineering Colleyse, Sibpur

G.S.I. = Geological Survey of India.

I.L. = Imperial Library.

M.Ba. = Medical Collese, Bacleriological Laboratory

M.BI. = Medicat Collese. Biological Laburatory;

M.C. = Medical College (masn library). 
780. Stuttgart.-Zeitschrift fuir Analytische Chemie. Herausg. von $\mathrm{H}$. Fresenius (and others). Jahrg. 46-. Wiesbaden, 1907-: G.S.I.

78I. Wurzburg.-Königliche Julius-Maximilians-Universität.

(a) Arbeiten aus dem Zoologisch-zootomischen Institut. Herausg. von C. Semper und A. Schuberg. Bde. IIo. Würzburg, I $874-95$ : Z.S.I.

(b) Arbeiten des Botanischen Instituts. Herausg. von J. Sachs. Bde. I-3. Leipzig, I87I-88: R.B.G.

782. — Physikalisch-Medicinische Gesellschaft.

Wïrburger Naturwissenschaftliche Zeitschrift (Sitzungsberichte). Bde. I-6. IVürzburg, I86o-67: G.S.I., Z.S.I.

783. - Zoologische Annalen: Zeitschrift für Geschichte der Zoologie. Herausg. von M. Braun. Bde. I-. Würzburg, I904-: Z.S.I.

\section{HOLLAND.}

784. Amsterdam.-Jaarboek van het Iijnwesen in Nederlandsch OostIndië. Jaarg. I-, I872-. Amsterdam, Bataria, I872-. Register, I8 $72-8 \mathrm{I}$. [The volumes are frequently in two parts, variously styled 'Wetenschappelijk Gedeelte', 'Technisch en Administratief redeelte', 'Algemeen Gedeelte' and 'Terhandelingen '.]

Jahrg. I-, for $1872-($ Jaarg. 5-7, $14-30,35$ wanting, Jaarg. 2, 4,13 defective) and index: G.S.I.

Jahrg. $38-43$, for I909-I4 (Jaarg 38, 43 defective) : A.S.B.

785. - Joumal de Botanique Néerlandaise. Réd. par F. A. IV. Miquel. Tom. I. Amsterdam, L'trecht, I86I : R.B.G.

786. — Koloniaal Instituut.

(a) Mededeeling. Afdeeling Handelsmuseum. Nos. IAmsterdam, I9I4-: R.B.G.

(b) Mededeeling. Afdeeling Tropische Hygiene. Nos. I-. Amsterdam, I9I4-: Z.S.I.

(c) Vereeniging. Jaarverslag 2-. Amsterdam, I9I3-.

Jaarverslag 2-, I9I3-: R.B.G.

Jaarverslag 4-, I9I 4-: Z.S.I.

Koloniaal Museum (sce No. 799).

M.o. = Meteorological Office, Alipore.

M.Ph. = Iedical College, Physiological Laboratory.

P.C. = Presidency College.

P.o. = Patent Office.

R.B.G. = Royal Botanic Garden

S C.C. $=$ Scottish Churches College.

\author{
S.G. = Surgeon-Generai's Library. \\ S.l. = Survey of India. \\ S.T.M. = School of Tropical Medicine \\ T.D. = Telegraph Department. \\ X.C. =St. Xavier's College. \\ Z.s.1. = Zoological Survey of India.
}


787. Amsterdam.-Koninklijk Nederlandsch Instituut van Wetenschappen, Letterkunde en Schoone Kunsten.

(a) Nieuwe T'erhandelingen der Eerste Krlasse. Deel 3-7. Amsterdam, I83I-38: A.S.B.

(b) Verhandelingen der Eerste Klasse. $3^{\mathrm{e}}$ Reeks, Deel I-5. Amsterdam, I849-52: G.S.I.

788. — Koninklijk Zoölogisch Genootschap "Natura Artis Magistra."

(a) Bijdragen tot de Dierkunde. Aflev. I-. Amsterdam, I 848-(defective): A.S.B.

(b) Nederlandsch Tijdschrift voor de Dierkunde. Red. van P. Bleeker (and others). Jaarg. I-4. Amsterdam, I $863-72$ : A.S.B.

789. - Koninklijke Akademie van Wetenschappen.

(a) Jaarboek. I885-. Amsterdam, I885-: A.S.B.

(b) Proceedings of the Section of Sciences. Vols. I-. Amsterdam, I899-: A.S.B., R.B.G. [Translated from (i).]

(c) T'erhandelingen ......Afdeeling Letterkunde. Nieuwe Reeks, Deel I-. Amsterdam, I896 (1892)-(Deel 5 wanting): A.S.B.

(d) Terhandelingen. [Afdeeling Natuurkunde.] Deel 3-9. Amsterdam, I856-6I: G.S.I.

(e) - I $\mathrm{I}^{\text {te }}$ sectie. Deel I-. Amsterdam, I893 (I892) (Deel 4,12 wanting): A.S.B.

(f) $-2^{\text {de }}$ sectie. Deel I-. Amsterdam, I893 (I892)(Deel 8,13 wanting): A.S.B.

(g) Verslagen en Mededeelingen .... Afdeeling Letterkunde.

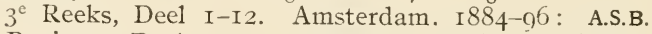
Register, Deel I-I2: A.S.B. $4^{\text {e }}$ Reeks, Deel I-. Amsterdam, I897-: A.S.B.

(h) Terslagen en Mededeelingen...... Afdeeling Natuur-

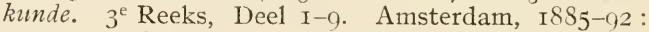
A.S.B. Register, Deel I-9: A.S.B. [Continued as $(j)$.]

(i) Verslagen van de gerwone T'ergaderingen der Wis-en Natuurkundige Afdeeling. Deel 4-, I89596-. Amsterdam, I896-. [A continuation of $(j)$.]

Deel 4-, I 896-(D1. 4 defective): A.S.B.

Deel 5-13, I897-I905 (D1. I1, I2 wanting): R.B.G.

(j) Terslagen van de Zittingen van de Wis-en Natuur kundige Afdeeling. Deel I-3, I892-95. Amsterdam, I893-95 : A.S.B., R.B.G. [Continued as $(i)$.]

\footnotetext{
A.5. Archaeological Section, Indian Museum.

A.S.B. = Asiatic Society of Bengal.

B.C. = Bishop's College.

B.S.I. = Botanical Survey of India

C.E. = Chemical Examiner to the Guvt of Bengal.

c.U. = Calcutta University.
}

E.C. = Civil Engineering College, Sibpur

G.S.I. = Geolosical Survey of India.

1 L. = Imperial Library.

M. Ba. = Medical College, Bacteriological Laboratory

M. BI. = Medical College, Biological Laboratory.

M.C. = Medical College (main library). 
Amsterdam.-Natuurkundig Tijdschrift voor Nederlandsch-Indië (see No. II94).

- Nederlandsch Kruidkundig Archief (see No. 8Io).

- Nederlandsch Tijdschrift voor de Dierkunde (see No. 788 (b)).

790. - Revue Coloniale Internationale. Tom. I-5. Amsterdam, I $885-87$ : R.B.G.

79I. — Tijdschrift voor Natuurlijke Geschiedenis en Physiologie. Uitg. door J. van der Hoeven en IV. H. de Vriese. Deel I-I2. Amsterdam, Leiden, I834-45: Z.S.I.

792. Apeldoorn.-Commission Internationale de Magnétisme Terrestre. Caractère Magnétique de chaque jour des Mois. Igo6-. Apeldoorn, De Bilt, I906-: M.O.

793. Delft.—Ecole Polytechnique

Annales. Tom. I-7. Leide, I885-97 : G.S.I.

794. Gröningen.-Academia Groningana.

Annales (Acta saecularia). I8I5-37. Groningae, I8I7-39: G.S.I.

795. Haarlem.-Commissie belast met het Vervaardigen eener geologische Beschrijving en Kaart van Nederland.

Verhandelingen. Deel I, 2. Haarlem, I853-54 : G.S.I.

796. — Hollandsche Maatschappij der Wetenschappen.

Archives Néerlandaises des Sciences Exactes et Naturelles. Publiées par la Société Hollandaise des Sciences. Tom. I-30. La Haye, Haarlem, I866-97 (tom. I9 defective): Z.S.I. Sér. 2, tom. I-6. La Haye, I897-I90I : Z.S.I.

_- Koloniaal Museum (see No. 799).

797. —usée Teyler.

Archives. Vol. I-3. Haarlem, I875, '69. '70: A.S.B. [Vol. I is of the znd edition.]

798. - Nederlandsche Maatschappij ter Bevordering von Nijverheid.

Tijdschrift. [Ser. 5.] I889-92. Haarlem, 's Gravenhage, I889-92 : Z.S.I. Nieuwe Reeks, Deel I-3. Leiden, I897-99 (defective) : Z.S.I.

799. - Nederlandsche Maatschappij ter Bevordering van Nijverheid.-Koloniaal IIuseum.

(a) Bulletin. Nos. I-52. Amsterdam, I893-I9r3. Generaal Register, nos. I-52.

Complete sets and index: B.S.I., R.B.G.

Nos. I-45, I892-I9I9 (no. 2 wanting): Z.S.I.

M o = Meteorological Olfice, Alipore

$\mathbf{M} \mathbf{P h}=$ Medical College. Physiological Laboratory.

P.C. = Presidency Collegre.

PO = Patent Office.

R.B.G = Royal Botanic Garden.

S.C.C. $=$ Scottish Churches College
S.G. = Surgeon-General's Library

S.I. = Survey of India

S.T.M. = School of Tropical Medicine.

T.D. = Telegraph Department.

X.C. $=$ St. Savier s college.

Z.s.I. = Zoological Survey of India. 
799. Haarlem.-Nederlandsche Maatschappij .. (Continued).

(b) Extra Bulletin. Aflev. I-7. Amsterdam, 1894-1902 (Aflev. 6 wanting): Z.S.I.

— Niederländisches Archiv fïr Zoologie (see No. So4).

Société Hollandaise des Sciences (see No. 796).

Hague, The.-Archives Néerlandaises des Sciences Exactes et Naturelles (see No. 796).

- Entomologische Berichten (see No. 802 (a)).

800. _ Geologisch-mijnbouwkundig Genootschap voor Nederland en Koloniën.

(a) Verhandelingen. Geologische Serie. Deel I-. 's Gravenhage, IgI2- (defective): G.S.I.

(b) Verhandelingen. Mijnbouwkundige Serie. Deel I-. 's Gravenhage, I9I6- (defective) : G.S.I.

Sor. — Koninklijk Instituut voor de Taal-, Land-, en Volkenkunde van Nederlandsch-Indië.

Biịdragen tot de Taal-, Land-, en I'olkenkunde van Nederlandsch-Indië: Tijdschrift van het .... Institunt .... Deel I-4. 's Gravenhage, I853-56: A.S.B. Nieuwe Tolgreeks, Deel I-8. 's Gravenhage, I856-64 (D1. 3 wanting, D1. 4,7 defective) : A.S.B. $3^{\text {de }}$ Volgreeks, Deel I-II. 's Gravenhage, I866-76 (Dl. Io wanting): A.S.B. $4^{\text {de }}$ Volgreeks, Deel I-Io. 's Gravenhage, I877-85: A.S.B. $5^{\text {de }}$ Volgreeks, Deel I-Io (=whole ser., D1. 3544). 's Gravenhage, I 886-94 (D1. 7 defective): A.S.B. $6^{\text {dic }}$ Volgreeks, Deel I- Io $\left(=45^{-54}\right)$. 's Gravenhage, I895-I902 (D1. 7 wanting): A.S.B. $7^{\text {de }}$ Volgreeks, Deel I-IO $(=55-64)$. 's Gravenhage, Igo3-Io (D1. 7 defective): A.S.B. [Whole series], Deel 65-.' 's Gravenhage, I9II-: A.S.B.

802 - Nederlandsche Entomologische Vereeniging.

(a) Entomologische Berichten. Deel I-, no. I-, I90I-. 's Gravenhage, IgoI- : A.S.B., Z.S.I.

(b) Mémoires d'Entomologie publiés par la Société Entom-' ologique des Pays-Bas. Sous la direct. J. van der Hoeven (and others). Tom. I. La Haye, 1858 : Z.S.I. [Continued as (c).]

(c) Tijdschrift voor Entomologie. Red. ran J. van der Hoeven, J. A. Herklots (and others). Deel 2-. La Haye, Haarlem, 's Gravenhage, I859-. [Deel 9-16, $1866-73$, are also styled " $2{ }^{\mathrm{e}}$ Reeks, Decl 1-8." For Deel I see (b).] Repertorium, Deel I 7-24.

A.S. = Archaeological Section. Indian Mluseum.

A.S B $=$ Asiatic Society of Bentral.

B.C. = Bishops College

B.S.I. = Botanical Survey of India

C.E. = Chemical Examiner to the Guvt. of Bensa!

c.U = Calcutta University.
E.C. = Civil Engineering College, Slppur.

G.S.I, = vieolosical Surve; of Inciat.

I.S. = lmperial Library

M. Ba. = Medical College, Bacteriological Laboratory.

M.Bl. = Medical College. Biological Laboratory.

M.C. = Medical College (main library). 
802. Hague, The.-Nederl. Entomol. Vereeniging (Continued).

Deel 2-, I859- (Deel 49 defective) and index: Z.S.I.

Deel 35-, I89I-(Deel 52, 53 wanting): A.S.B.

— Société Entomologique des Pays-Bas (see No. $802(b)$ ).

— Tijdschrift voor Entomologie (see No. $802(c)$ ).

Leyden.--Geologisches Reichs-Museum (see No. So6).

803. - Internationales Archiv für Ethnographie. Red. J. D. E. Schmeltz. Bde. I-I5. Leiden, I888-I902. Suppl. Bde. I-I3. Leiden, I895-I900.

Bde. I-I5, I888-I902 (Bde. 3,12 defective) : A.S.B.

Bde. I-I3, I888-I900: I.L. Suppl. Bde. I-I3, I895-I900 (Bde. 2, 6, 8, IO, I2 wanting): I.L.

- Musée Royale d'Ethnographie (see No. 805).

_- Museum (see No. 807).

- Nederlandsch Kruidkundig Archief (see No. Si (a)).

—_ Nederlandsche Dierkundige Vereeniging (see No. 8II).

So4. - Niederländisches Archiv für Zoologie. Herausg. von E. Selenka (and afterwards) C. K. Hoffmann. Bde. I-5. Haarlem, Leipzig. Leiden, I87I-82: Z.S.I. Suppl. Bd. I. Leiden, Leipzig, I88I-82: Z.S.I.

- Notes from the Leyden. Museum (see No. $\$ 07(a))$.

So5. — Rijks Ethnographisch Museum.

(a) Notices Anthropologiques publiées par la .Musée Royal d'Ethnographie de Leyde. Tom. I-. Leyde, I904-: I.L.

(b) Terslag van den Directeur. I899 I900-. 's Gravenhage, I900- (issues of $1900 \mathrm{OI}$, 'O1 02, '13.14 wanting): Z.S.I.

806. — Rijks Geologisch II _ - Reum (Geologisches Reichs-IIuseum).

(a) Sammlungen. I $\mathrm{I}^{\text {te }}$ Serie. Beiträge zur Geologie OstAsiens und Australiens. Bde. I-- Leiden, I88I-: G.S.I.

(b) $-2^{\text {te }}$ Serie. Beiträge zur Geologie von Niederländisch-Indien ... Bde. I, 2. Leiden, I887-I902: G.S.I.

(c) Neue Folge. Herausg. von K. Martin. Bde. I-. Leiden, I895- (Bd. I defective): G.S.I.

M.O. = Meteorological Office. Aipore

M.Ph. = Medical College, Physiological Laboratory.

P.C. = Presidency College.

P.O. = Patent Oince.

R.B.G. = Royal Botanic Garden.

S.C.C. = Scottish Churches College.
S.G. = Surgeon-General s Library.

S.1. = Survey of India.

S.T.M. = School of Tropical Medicine

T.D. = Telesraph Department

X.C. =St. Xavier's College.

z.S.I Z Zoological Survey of India. 
807. Leyden.-Rijks Museum van Natuurlijke Historie.

(a) Notes from the Leyden Museum. Vols. 2-26. Leyden, I8S0-I9I4: Z.S.I. Index, I879-99: Z.S.I. [For vol. I see (b). Continued as $(c)$.]

(b) Notes from the Royal Zoological Musenm of the Netherlands at Leyden. Vol. I. Leyden, I879: Z.S.I. [Continued as $(a)$.]

(c) Zoologische Mededeelingen. Deel I-. Leiden, I915-: Z.S.I. [A continuation of $(a)$.]

808. — Rijks-Universiteit.-IIuseum Botanicum.

Annales Musei Botanici Lugduno-Batavi. Edidit F. A. G. Miquel. Vol. I-4. Amstelodami, Traiecti ad Rhenum. I863-69: A.S.B.

_- Royal Zoological Museum of the Netherlands (see No. $807(b)$ ).

_- Tijdschrift voor Entomologie (see No. 802).

8og. — T'oung Pao: ou Archives concernant l'Histoire, les Langues, la Géographie et l'Ethnographie de l'Asie Orientale. Dir. par H. Cordier et E. Chavannes. Vols. II-. Leide, I9IO-(vols. I2, 13 wanting; vol. 14 defective): A.S.B.

_ Zoologische Mededeelingen (see No. 807 (c)).

8Io. Nimeguen.-Nederlandsche Botanische Vereeniging.

(a) Nederlandsch Kruidkundig Archief. Uitgeg. door W. H. de Vriese, W. F. R. Suringar (and others). Deel I-5. Leyden, Amsterdam, 1846-70: R.B.G. [Continued as $(c)$.]

(b) Recueil des Travaux Botaniques Néerlandais. Nos. INimégue, I904-: R.B.G.

(c) Verslagen en Mededeelingen der...... Vereeniging. Ser. 2, Deel, I-6. Nijmegen, I87I-95: R.B.G. Ser. 3, Deel I, 2. Nijmegen, IS96-I904: R.B.G. Nos. I-. Nijmegen, I904-: R.B.G. [A continuation of $(a)$.]

Société Botanique Néerlandaise (see No. 8Io).

8I I. Rotterdam.-Nederlandsche Dierkundige Vereeniging.

Tijdschrijt. Ser. 2, Deel Io-. Leiden, I908-: Z.S.I.

Register, ser. I, D1. I- ser. 2, D1. I0, I875-I908 : Z.S.I.

's Gravenhage see Hague.

A.S. = Archaeological Section, Indian Mluseum.

A.S.B. = Asiatic Society of Bengal

B.C. = Bishop s College.

B.S.I. = Botanical Survey of India.

C.E. = Chemical Examiner to the Govt, of Bengal.

c.u. Calcutta University.
E.C. = Civil Engineering College, Sibpur.

G.S I. = Geological Survey of India

I.L. = Imperial Library

M.Ba. = Medical College, Bacteriological Laboratory

M.Bi. = Medical College. Biological Laboratory.

M.C. = Medical College (main library). 


\section{I'TALY.}

812. Acireale.-(Reale) Accademia di Scienze, Lettere ed Arti degli Zelanti.

(a) Atti e Rendiconti. Memoire della Classe di Scienze. Nuova Serie, vol. 8-Io, I896-Igoo. Acireale, I898I90I : G.S.I. [Continued as $(d)$.]

(b) - - Rendiconti. Nuova Serie, vol. Io, I898Igoo. Acireale, Ig02: G.S.I. [Continued as $(e)$.

(c) Rendiconti e Memorie. Memorie della Classe di Lettere. Serie $3^{\mathrm{a}}$, vol. 6-, Igo7 o8-. Acireale IgIo-(vol. 7 wanting): G.S.I.

(d) - Memorie della Classe di Scienze. Serie $3^{\mathrm{a}}$, vol. I-, I901/02 - . Acireale, I903- (vol. 5 wanting): G.S.I. [A continuation of $(a)$.]

(e) $\begin{aligned} & \text { I906- Rendiconti. Serie } 3^{\mathrm{a}} \text {, vol. I-. Acireale, } \\ & \text { - G.S.I. [A continuation of }(b) .]\end{aligned}$

Bologna.-Accademia delle Scienze dell' Istituto (see No. 8I5).

813. - Annali di Storia Naturale. Ed. Jacopo Marsigli. Tom. I-4. Bologna, I829-30: G.S.I. [Afterwards revived as No. 814.]

8I4. - Nuovi Annali delle Scienze Naturali. Pubbl. dai A. Alessandrini, A. Bertolini (and others). Tom. I-Io. Bologna, I838-43: G.S.I. [Continued as :] Nuovi Annali delle Scienze Naturali e Rendiconto delle sessioni della Società A graria, e dell' Accademia delle Scienze dell' Istituto di Bologna. Pubbl. dai A. Alessandrini, A. Bertolini (and others). Ser. $2^{\text {a }}$, tom. I-IO. Bologna, I84448: G.S.I. [Continued as:] Nuovi Annali delle Scienze Naturali e Rendiconto dei Lavori dell' Acuademia delle Scienze dell' Istituto e della Società Agraria di Bologna. Pubbl. sotto la direz. A. Alessandrini, A. Bertolini and (others). Ser. $3^{\text {a }}$, tom. I-Io. Bologna, I850-54: G.S.I. 'Indice' to Ist and 2nd series at end of tom. 2, ser. 3. 'Indice' to 3 rd ser. at end of tom. Io.

8I5. - (Reale) Accademia delle Scienze dell' Istituto.

(a) De Bononiensi Scientiarum et Artium Instituto atque Academia Commentarii. Tom. I-7. Bononiae, I73I9I : G.S.I. [Afterwards resumed as $(f)$.

(b) Memorie. Tom. I-I2. Bologna, I850-6I. u Ser. 2a, tom. I-Io. Bologna, I862-70. Ser. $3^{\text {a }}$, tom. I-Io. Bologna, I87I-79. Ser. $4^{\text {it }}$, tom. I-IO. Bologna, I880-90. Ser. 5 ${ }^{\text {a }}$, tom. I-Io. Bologna, I8go-Igo4. Ser. 6a', tom, I-4. Bologna, I904-07. 'Indici Gener-

M.o. = Meteorological Oifice, Alipore.

M.Ph. = Mledical College, Physiological Laboratory.

P.C. = Presidency College.

P.o. = Patent Office.

R.B.G = Royal Botanic Garden.

S.C.C. = Scottish Churches College.
S.G. = Surgeon-General's Library.

S.1. = Survey of India.

S.T.M. = School of Tropical Medicine

T.D. = Telegraph Department.

X.c. '=St. Xavier's College

z.s.i. =Zoological Survey of India. 
SI5. Bologna.-(Reale) Accad. delle Scienze dell' Instituto (Continued) ale' in last vol. of each series. [Continued as (c).]

Complete set: G.S.I.

Ser. 6", tom. 4, I907: Z.S.I.

(c) Memorie. Classe di Scienze Fisiche. Ser. $6^{\mathrm{a}}$, tom. 5-. Bologna, Igo8-: G.S.I., Z.S.I. [A continuation of $(b)$.

(d) Memorie. Classe di Scienze Morali: Sezione di Scienze Giuridiche. Tom. I-. Bologna, Igo8-: A.S.B.

(e) Memorie. Classe di Scienze Morali : Sezione di Scienze Storico-Filologiche. Tom. I-. Bologna, I908-: A.S.B.

(f) Novi Commentarii Academiae Scientiartm Instituti Bononiensis. Tom. I-Io. Bononiae, I834-49: G.S.I.

'Indices Generales', tom. I-IO, at end of tom. Io.

(g) Rendiconto delle Sessioni. Nuova ser., vol. I-II. Bologna, I897-I907. [Continued as $(h)$.]

Complete set: G.S.I.

Vol. II, I907: Z.S.I.

(h) Rendiconto delle Sessioni. Classe di Scienze Fisiche. Nuova ser., vol. I2-. Bologna, I908-: G.S.I., Z.S.I. [A continuation of $(g)$. $]$

(i) Rendiconto delle Sessioni. Classe di Scienze Morali. Ser. $\mathrm{I}^{\mathrm{a}}$, vol. I-. Bologna, I907-: A.S.B.

8I6. — Repertorium Italicum complectens Zoologiam, Mineralogiam, Geologiam ct Palaeontologiam. Cura J. J. Bianconi. Ann. I 853, '54. Bononiae, I 853, '54: G.S.I.

817. - Rivista Italiana di Ornitologia. Ann. I, num. I, 2. Bologna, IOII : Z.S.I.

— Rivista Italiana di Paleontologia (see No. \$55).

8IS. - Scientia. Vol. I-. Bologna, Igo8-.

Complete set: I.L.

Vol. I9-, I916-: G.S.I.

8I9. Catania.-Accademia Gioenia di Scienze Naturali.

Atti. Tom. I-7. Catania, I825-33: G.S.I. Ser. $2^{a}$, tom. I-I6. Catania, I844-60: G.S.I.

820. —-Malpighia: Rassegna mensile di Botanica. Red. L. Buscalioni. Ann. 24-. Catania, I9I2- (Ann. 24 defective): R.B.G.

Florence.-Agricoltura Coloniale (see No. 822).

—_Archivio per l'Antropologia e la Etnologia (see No. 830).

A.S. = Archaeological Section, Indian Museum.

A.S.B. = Asiatic Society of Bengal.

B.C. $=$ Bishop s College.

B.S.I. = Botanical Survey of Inclua

C.E. = Chemical Examincr to the Govt of Bengal

c. $U$ = Calcuta Eniversity
E.C. = Civil Engineering College. Sibnur.

G.S.I. = Geotogical Survey of India.

I L. = Imperial Library.

M. Ba. = Medicul College, Bacteriological Laboratory

M.Bi. = Medical College, Biological Laboratory

M.C. =.Ifedical College (main library) 
Florence.-Archivio per la Zoologia, l'Anatomia e la Fisiologia (see No. $83 \mathrm{I})$.

821. Giornale Botanico Italiano. Compilato per cura della Sezione Botanica dei Congressi Scientifici Italiani da F. Parlatore. Ann. 2. Firenze, I846-5I ; R.B.G. [Continued as No. 823.]

822. - Istituto Agricolo Coloniale Italiano.

Agricoltura Coloniale: Organo dell' Istituto ..... e dei Sir. vizi Agrari dell' Eritrea e della Somalia Italiana. Anno I-. Firenze, Novara, I907-: B.S.I.

823. - Nuovo Giornale Botanico Italiano. Pubbl. da O. Beccari (and afterwards) T. Caruel. Vol. I-I9. Firenze, I869-87. [Continned as:] Nuovo Giornale .... e Bulletino della Società Botanica Italiana. Vol. 20-25. Firenze, I888-93. [Continued as:] Nuovo Giornale ....e Memorie della Società Botanica Italiana. Nuova Serie, vol. I-. Firenze, I894-.

Complete set: R.B.G.

Tom. I- nuov. ser., vol. I2, I869-I905 : C.U.

824. - Real Orto Botanico Fiorentino.

Lavori eseguiti nel R. Orto .... Fasc. I-5. Firenze, IgoI05: R.B.G.

- Reale Comitato Geologico d'Italia (see No. 865).

825. - Reale Stazione di Entomologia Agraria.

'Redia'. Giornale di Entomologia. Vol. 5-. Firenze, Igo8- : Z.S.I.

Scienziati Italiani (see No. 858$)$.

S20. - Società Africana d'Italia: sezione Fiorentina (see also No. 844).

Bulletino. Tom. I-IO. Firenze, I885-94: A.S.B.

827. - Società Asiatica Italiana.

Giornale. Tom. I-6. Firenze, IS87-92: I.L.

828. - Società Botanica Italiana (see also No. 823 ).

Bullettino. Ann. I892-I907. Firenze, IS92-I907. [Prexiously published with vol. $20-25$ of No. $823 . \mathrm{j}$

Ann. I892-I907 : R.B.G.

Ann. I892-I903: C.U.

829. - Società Entomologica Italiana.

Bullettino. Ann. I-. Firenze, I869-: Z.S.I. Indice, ann. II-20: Z.S.I.

M O. = Meteorological Ofrice. Alipore.

M.Ph. = Medical College. Physiological Laboratory.

P.c. = Presidency College.

P.O. = Patent Office.

R.B.G, = Royal Botanic Garder:

S.C.C. = Scuttish Churches College.
S.G. = Surgeon-Generai s Library

S.I. = Survey of Incia.

S.T.M. = School of Tropical Medicine

T.D. = Telegraph Departmeni.

X.c. =St. Xavier s College.

Z.s.l. = Zoological Survey of Indi: 
830. Florence.-Società Italiana di Antropologia e di Ettnologia.

Archivio per l'Antropologia e la Etnologia. Tom. 3-. Firenze, 1874-(tom. 7, 10, 15, 30, 37, 39 defective) : A.S.B.

83I. Genoa.-Archivio per la Zoologia, l'Anatomia e la Fisiologia. Pubbl. per cura di G. Canestrini (and others). Vol. I-4, fasc. I. Genova, Modena, I86I-66: Z.S.I. Ser. 2, tom. I, 2, fasc. I. Torino, Firenze, 1869-70 : Z.S.I.

832. - Museo Civico di Storia Naturale.

Annali. Pubbl. per cura di G. Doria (and others). Vo1. I-20. Genova, I870-84. Ser. 2, vol. I-20 $(=2 \mathrm{I}-40)$. Genova, I884-99. Ser. 3, vol. I- (=4I-). Genova, I904-. Indice generale, vol. I-40.

Vol. I-, I870- (ser. I, vol. 7, I6 wanting) and index, vol. I-40 : A.S.B.

Vol. 2-, I872-: Z.S.I.

833. — Regia Università degli Studi.

Bolletino dei Musei di Zoologia e Anatomia Comparata. Nos. 22-I32. Genova, I894-I905: Z.S.I. [Continued as:] Bollettino del Museo di Zoologia. Nos. I-. Genova, Igo6- (nos. $8-42$ wanting): Z.S.I.

Lucca.-Scienziati Italiani (see No. 858).

834. Milan.-Annali di Matematica pura ed applicata. Già direcc. F. Brioschi. Serie 3, tom. 20-22. Milano, I9I3-I4 : C.U.

835. — Fondazione Scientifica Cagnola.

Atti della Fondazione.... dalla sua Istituzione in Poi. Vol. 23-. Milano, I9I3-: Z.S.I.

836. - Imperiale Regio Istituto del Regno Lombardo-Veneto.

Memnrie. Vol. I-5, I8I2-35. Milano, I8I9-38: G.S.I.

— Imperiale Regio Istituto Lombardo di Scienze, I,ettere ed Arti (see No. 837).

_- Museo Civico di Storia Naturale (see No. 838).

837. — Reale Istituto Lombardo di Scienze, Lettere ed Arti.

(a) Giornale dell' I.R. Istituto .... e Biblioteca Italiana. [Nuova serie.] Tom. I-9. Milano, I847-56: G.S.I.

(b) Memorie. Vol. I-9. Milano, I843-63: G.S.I. [Vol. 8, 9 are also styled ' $2^{\mathrm{a}}$ serie, vol. $2,3$. ']

(c) Memorie: Classe di Scienze Matematiche e Naturali. Vol. Io-I2 ( $=$ ser. $3^{\text {a }}$, vol. I-3). Milano, I867-73: G.S.I. Vol. 20, 2I $\left(=\right.$ ser. $3^{\mathrm{a}}$, vols. II-I2). Milano, Igo8-09 (defective) : Z.S.I. [For earlier vols. see (b).]

A.S. = Archaeological Section. Indian Museum

A.S.B. = Asiatic Society of Bengal

B.C. = Bishop's College.

B.S.I. = Botanical Survey of India

C.E. = Chemical Examincr to the Govt. of Bengal

c.U. = Calcutta University
E.C. = Civil Engineering College, Sibpur

G.S.I. = Geological Survey of India.

I.L. = Imperial Library.

M. Ba. = Medical College, Bacteriological Laboratory

M.Bi. = Medical College. Biological Laboratory.

M.C. = Medica! College (main library) 
837. Milan.-Reale Istituto Lombardo di Scienze .. (Continued).

(d) Rendiconti. Ser. $2^{a}$, vol. I3-I8. Milano, Napoli, Pisa, I88o-85 (vol. I5 wanting): G.S.I. Vol. 4I-. Milano, I908- (vol. 4 I, $+3,44$ defective) : Z.S.I.

Scienziati Italiani (see No. 858 ).

Società Geologica (see No. $838(a))$.

838. - Società Italiana di Scienze Naturali.

(a) Atti della Società Geologica residente in Milano. Vol. x. Milano, I859. [Continued as:] Atti della Società Italiana ... Vol. 2-35. Milano, I860-95. [Continued as:] Atti della Socieià ....e del Museo Civico di Storia Naturale in Milano. Vol.36-. Milano, I896-.

Complete set: G.S.I.

Vol. 5I-, I9I2-: A.S.B.

(b) Memorie della Società .... Tom. I-3. Milano, I86573. [Continued as:] Memorie della Società ...e e del Museo Civico di Storia Naturale di Milano. Tom. 5-. Nilano, I895-. (Tom. 5 is also styled 'I della Nuova Serie').

Tom. I-, I865- (tom. 2 , 4 wanting): G.S.I.

Tom. 7一, I9ro-: A.S.B.

'Indice Generale' to $(a)$ and $(b)$, I859-I906: G.S.I.

Modena.-Archivio per la Zoologia, l'Anatomia e la Fisiologia (see No. 83I).

839. - Società Sismologica Italiana.

Bollettino. Vol. I-. Modena, Roma, I895- : G.S.I.

Naples.-Museo Zoologico (see No. 842).

Orto Botanico (see No. 843).

840. - (Reale) Accademia delle Scienze Fisiche e Matematiche.

(a) Atti. Vol. I-9. Napoli, I863-82 : G.S.I. Ser. $2^{2}$, vol. I-. Napoli, I888-: G.S.I.

(b) Rendiconto. Ann. I-25. Napoli, 1862-86: G.S.I. Ser. $2^{a}$, vol. I-8 (An11. 26-33). Napoli, I887-94: G.S.I. Ser. $3^{a}$, vol. I- (Ann. 34-). Napoli, I895- (vol. I7, I8 wanting): G.S.I.

'Indice Generale' $(a)$ and (b), I862-I903: G.S.I.

84I. - Reale Istituto d'Incoraggiamento.

Atti. [Ser. 6.] Tom. 6o-63. Napoli, 1909-I2: G.S.I.

M.o. = Meteorological Office. Alipore.

M.Ph = Medical College. Physiological Laboratory

P C. = Presidency College.

P o. = Patent Ofice.

R B.G = Royal Botanic Garden

S C C $=$ Scottish Churches College.
s.G. =Surgeon-Generai's Library

s.l. = Survey of India.

S.T. $M=$ School of Trodical Medicine

T.D. $=$ Telegraph Dedartmeni.

X.C. =St Xavier s College.

z.s.t. = Zoological Survey of Incia. 
842. Naples.-Regia Università degli Studi.-Museo Zoologico. Annuario. Nuova Serie, vol. I-. Napoli, IgoI-: Z.S.I.

843. — Regia Università degli Studi.-Orto Botanico. Bullettino. Tom, I-. Napoli, I899-: R.B.G.

Scienziati Italiani (see No. 858).

844. - Società Africana d'Italia.

Bollettino. Ann. 6-. Napoli, IS87- (An11, ${ }_{13-20}, 28-3$. wanting; remainder defective): A.S.B.

_- Società Reale di Napoli (see No. 840).

845. Z Zoologische Station.

(a) Fauna und Flora des Golfes von Neapel u. der angrenzenden Meeres-Abschnitte. Monog. I-.. Leipzig, Berlin, I880-: Z.S.I. [Each monograph is complete in itself and is paged separately.]

(b) Mittheilungen. Bde. I-. Leipzig, Berlin, I879-: Z.S.I.

(c) Zoologischer Jahresbericht. 1879-. Leipzig, Berlin, I880-: Z.S.I. Register, I886-I900 : Z.S.I.

846. Padua.-Bollettino Mensile di Bachicoltura. Dirett. E. Quajat, E. Verson. Ser. 2, Ann 8-12. Padova, I890-94: Z.S.I. Ser. 3, Ann. I-4. Padova, I895-98: Z.S.I. [Continued as No. 847.]

— Istituto Geologico (see No. 848).

847. - Reale Stazione Bacologica.

Anmuario. Vol. 27--. Padova, I899-: Z.S.I.

845. — Regia Università.-Istituto Geologico.

Memorie. Vol. I-. Padova, I912-- : G.S.I.

849. - Revista di Mineralogia e Cristallografia Italiana. Dir. R. Panebianco. Vol. 39. Padova, I909: G.S.I.

- Scienziati Italiani (see No. 858).

850. Palermo.-Annales de Géologie et de Paléontologie. Publ. sous la direct. du Marquis Antoine de Gregorio. Livr. I-29. Palerme, Turin, I886-I900: G.S.I.

851. Circolo Matematico.

Rendiconti. Vol. 27-34. Palermo, Igog-I2: C.U.

852. — Reale Orto Botanico.

Bollettino del R. Orto ... (e Giardino Coloniale di Palermo).

Ann. I-II. Palermo, I899-I9I3: R.B.G. Nuova serie, vol. I-. Palermo, I9I4-: R.B.G.

A.S. = Archaeological Section, Indian Museum.

A.S.B. = Asiatic Society of Bengral.

B.C. = Bishop's College.

B.S.I. = Botanical Survey of India.

C.E. = Chemical Examiner to the Guvt of Bengal.

c.U. = Calcutta University.
E.C. = Civis Engineering College. Sibpur.

G.S.I = Geolosical Survey of India.

I.L. = Imperial Library

M.Ba. = Medical Collese, Bacteriological Laboralory

M.Bl. = Medical College. Biological Laboratory:

M.C. = Medical College (man lıbrary). 
Palermo.-Real Osservatorio (see No. 854).

— Regio Istituto Tecnico (see No. 854).

853. - Società degli Spettroscopisti Italiani.

Memorie. Vol. 4-35. Palermo, Roma, Catania, I875I906 (vols. I6, 21 wanting, rol. 34 defective): A.S.B. [Preriously published with rols. 7 -10 of 1 o. 854 ; rol. 4 is, in part, a reprint of a section of vol. is of No. 854.]

854. - Società di Scienze Naturali ed Economiche (Regio Istituto Tecnico).

Giomale di Scienze Naturali ed Economiche (Bullettino Meteorologico del R. Osservatorio). Vol. I-6. Palermo, I866-7I: G.S.I. [Continued as:] Giornale.... (Bullettino ....) [and] Memorie della Societi degli Spettroscopisti Italiani. Vol. 7-II. Palermo, I87I75: G.S.I. [Continued as:] Giornale .... (Bullettino ....). Vol. I2-. Palermo, I877- (vol. 20 defective) : G.S.I.

855. Parma.-Rivista Italiana di Paleontologia. Red. C. Fornasini (and others). Vol. I-. Bologna, Perugia, Parma, I895-: G.S.I.

Perugia.-Rivista Italiana di Paleontologia (see No. 855).

856. Pisa.-Reale Università.

(q) Palaeontographia Italica. Pubbl. per cura del M. Canavari. Vol. I-. Pisa, I896-: G.S.I.

(b) Studi e Ricerche di Chimica Agraria. Fasc. I-. Pisa, I882- (Fasc. 6,7 , II wanting) : B.S.I.

$85 \%$ - - Regia Università degli Studi.-Istituto Botanico.

Ricerche e Lav'ori eseguiti nell' Istituto ... . Fasc. I, 2, I88287. Pisa, I886-88: R.B.G.

858. - Scienziati Italiani.

Atti della .... Riunione. Riun. I-7. Pisa, Torino, Firenze, Padova, Lucca, Milano, Napoli, I840-46 : G.S.I.

859. - Società Toscana di Scienze Naturali.

(a) Atti .... Memorie. Tol. I-. Pisa, I875-: A.S.B., G.S.I.

(b) Atti .... Processi Verbali. Vol. I-. Pisa, I878-.

Complete set: G.S.I.

Vol. I-, I878-(rol. 3, 4, 6, 7, I3, I4, 16 defective): A.S.B.

M.o. = Meteorological Oftice, Alipore.

M.Ph. = Medical College, Physiological Laboratory.

P.C. = Presidency College

P.O. = Patent Office.

R.B.G. = Royal Botanic Garden.

S.C.C. $=$ Scottish Churches College.
S.G. = Surgeon-General's Library:

S.I. = Survey of India.

S.T.M. = School of Tropical Mecicine

T.D. = Telegraph Deparment.

X.C. =St. Xaver s College.

Z.S.I. = Zoological Surver of India. 
86o. Portici.--Regia Scuola Superiore d'Agricoltura.

(a) Annali. Ser. 2, vol. 7-. Portici, I907-.

Vol. 7-, I907- (vol. 8, 12 wanting): R.B.G.

Vol. 8, I908: A.S.B.

(b) Bollettino del Laboratorio di Zoologia Generale e Agraria. Vol. I-. Portici, I907-.

Complete set: A.S.B.

Vol. I-, I907- (vol. 3 wanting): Z.S.I.

861. Rome.-Annali di Botanica. Pubbl. dal Prof. R. Pirotta. Vol. I-. Roma, I904-: R.B.G.

_ Collegio Romano.-Osservatorio (see No. 863).

Comitato Talassographico (see No. $868(b)$ ).

862. - International Institute of Agriculture.

(a) Bulletin of the Bureau of Agricultural Intelligence and of Plant Diseases. Nos. 2-. Rome, rgro-.

Nos. 2-, I9I0-: I.L.

Nos. 4-6, I9I2: A.S.B.

(b) Bulletin of the Bureau of Economic and Social Intelligence. Nos. I-3. Rome, I9I2: A.S.B.

(c) Monographs on Agricultural Cooperation in various Countries. Nos. I-. Rome, I9II-: I.L.

863. — Pontificia Università Gregoriana.

Bullettino Meteorologico dell' Osservatorio del Collegio Romano, con Corrispondenza e Bibliografia. Vol. I827. Roma, I879-88: X.C.

864. — Reale Accademia dei Lincei.

(a) Atti ... Memorie della Classe di Scienze Fisiche. Matematiche e Naturali. Ser. 3a, vol. I-I9. Roma, I87784: G.S.I. Index to vol. I-Io in vol. ro. Ser. $4^{\mathrm{a}}$, vol. I-7. Roma, I885-94: G.S.I. Ser. 5 ${ }^{\text {, }}$, vol. I, 2. Roma, I $895-98$ : G.S.I.

(b) Atti ... Rendiconti. Ser. $4^{\mathrm{a}}$, vol. I-7. Roma, I885-9I: G.S.I. [A continuation of $(d)$, continued in two parts as $(c)$ and $(e)$.

(c) Atti ... Rendiconti ... Classe di Scienze Fisiche, Matematiche e Naturali. Ser. 5", vol. I-. Roma, I892-. [For earlier series see $(b)$ and $(d)$.]

Vol. I-, I892- (vol. 22 defective) : G.S.I.

Vol. 7-, I898- (defective): A.S.B.

A.S. = Archaeological Section. Indian Museum.

A.S.B. = Asiatic Society of Bengal.

B.C. = Bishop's College.

B.S.l. = Botanical Survey of India.

C.E. = Chemical Examiner to the Govt. of Bengal.

C.U. = Calcutta University.
E.C. =Civil Engineering College, Sibpur.

G.S.I. = Geological Survey of India.

I.L. = Imperial Library

M. Ba $_{\text {I }}$ Iedical College, Bacteriological Laboratory.

M.Bi. = Medical College. Biological Laboratory.

M.C. = Medical College (main library). 
864. Rome.-Reale Accademia dei Lincei (Continued).

(d) Atti ... Transunti. Ser. $3^{\mathrm{a}}$, vol. I-8. Roma, I877-84. [Continued as (b).]

Complete set: G.S.I.

Vol. I $-7,1877-83$ (vol. 4,5 wanting): A.S.B.

(e) Rendiconti ... Classe di Scienze Morali, Storiche e Filologiche. Ser. ${ }^{\text {a }}$, vol. 7-9. Roma, 1898-1900 (defective) : A.S.B.

S65. — Reale Comitato Geologico d'Italia.

(a) Bollettino. Vol. I-. Firenze, Roma, I870-(vol. 19-2I wanting): G.S.I.

(b) Memorie per servire alla Descrizione della Carta Geologica d'Italia. Vol. I-. Firenze, Roma, I87I-: G.S.I.

866. —— Reale Ufficio Geologico.

Memorie descrittive della Carta Geologica d'Italia. Vol. I-. Roma, I886- (vol. io defective ?): G.S.I.

867. - Società Geologica Italiana.

Bollettino. Vol. 5-. Roma, I886-(vol. 30 defective): G.S.I. Indice, vol. I-20 : G.S.I.

868. - Società Italiana per il Progresso delle Scienze.

(a) Atti. Vol. I-, I907-. Roma, I908-: A.S.B.

(b) Bollettino del Comitato Talassografico. Num. I, I909Io. Roma, I9I0: A.S.B. [For continuation see No. 876.]

869. - Società Romana di Antropologia.

Atti (Rivista di Antropologia). Vol. 8-, I901/02-. Roma, I902-(Vol. 13, 15 defective): A.S.B.

Turin.-Académie (Royale) des Sciences (see No. 87 I $(d)$ ).

Archivio per la Zoologia l'Anatomia e la Fisiologia (see No. 831).

870. - Cosmos: Comunicazioni sui Progressi più recenti e notevoli della Geografia e Scienze affini di Guido Cora. Vol. I-2. Torino, I873-74: G.S.I.

_ Mélanges de Philosophie et de Mathématique (see No. 87I (c)).

_- Museo Zoologico (see No. 875).

- Osservatorio Astronomico (see No. 872).

871. —— Reale Accademia delle Scienze.

(a) Annuario. Anno I. Torino, I877 : A.S.B., G.S.I.

M.O. = Meteorological Office, Alipore.

M.Ph. = Medical College, Physiological Laboratory.

P.C. = Presidency College.

P.o. = Patent Office.

R.B.G = Royal Botanic Garden

S.C.C $=$ Scottish Churches College.
S.G. = Surgeon-Generai's Library.

S.G. = Surgeon-Generais

S.T.M. = School of Tropical Medicine.

T.D. = Telegraph Department.

X.C. =St. Xavier's College.

Z.S.I. =Zoological Survey of Incia. 
871. Turin.-Reale Accademia delle Scienze (Continued).

(b) Atti. Vol. I-, I86566-. Torino, I866-. Indice generale, vol. I-50.

Complete set and index: G.S.I.

Vol. I-, I866- (vol. I4 wanting): A.S.B.

(c) Mélanges de Philosophie et de Mathématique. Tom. $2-5,1760-73$. Turin $[1762-76$ ?] : G.S.I. [For tom. 1, see $(f)$; for index and later vols. see $(d)$.

(d) Mémoires de l'Académie (Royale) des Sciences (Littérature et Beaux-Arts). Tom. I-6 [ = tom. 6-I I of whole series], I-84-I800. Turin, I786-I80I : A.S.B., G.S.I. [Continued as:] Mémoires.... Ann. x-1812 [10 vols., $=$ tom. $12-21$ of whole series]. Turin, I803-I3: A.S.B., G.S.I. [Divided into two series, each of 5 vols., entitled 'Littérature et BeauX-Avts' and 'Scionces Physiques et Mathematiques.'] [Continued as:] Mémoires .... Tom. 22, I8r3-I4. Turin, I8I6: A.S.B., G.S.I. [For tom. I-5 of whole series see $(c)$ and $(f)$; for continuation see $(e)$.] 'Indice,' I759-I8I4, at end of tom. 22.

(e) Memorie della ... Accademia ... Tom 23-40. Torino, I8I8-38: A.S.B., G.S.I. $2^{\text {a }}$ Serie, Tom. I-. Torino, I839-: A.S.B., G.S.I. [A continuation of (d). Decennial general indexes issued with each tenth volume.]

(f) Miscellanea Philosophico-mathematica Societatis privatae Taurinensis. Tom. I. Augustae-Taurinorum, I759: G.S.I. [Continued as (c).]

872. — Reale Accademia delle Scienze.-(R.) Osservatorio Astronomico.

(a) Bollettino Meteorologico dell' Osservatorio Astronomico. Anno 3-22. Torino, I868-87. [Continued as (b).]

Complete set: G.S.I.

Ann. 3-22 (ann. 7 wanting): A.S.B.

(b) Osservazioni Meteorologichi di R. Accad. d. Sci., all' Osservatorio della R. Università. Anno I866-. Torino, [1867]-.

Anno I866-98, [1867]-99: X.C.

Anno I888-, I890-: A.S.B.

873. — Reale Istituto Botanico.

Annuario. Red. R. Pirotta. Ann. I-Io. Roma, Nilano, I 885 -I908 : R.B.G.

874. — Reale Museo Commerciale.

Bulletino (della Camera di Commercio e Industria e) del ..... Museo ... Vol. 7-. Torino, 1908-: B.S.I.

A.S. = Archaeological Section, Indian Museum

A.S. B = Asiatic Society of Bengal.

B.C. = Bishop s College.

B.S.I. = Botanical Survey of India.

C.E. = Chemical Examiner to the Govt. of Bengal.

c. $\mathbf{U}=$ Calcutta Unversity:
E.C. = Civil Engincering College, Sibpur.

G.S.I. = Geological Survey of India.

I.L. = Imperial Library.

M.Ba. = Medical College, Bacteriolosical Laboratory

M.Bi. = Iledical College. Biological Laboratory

M.C. = Medical College (main library). 
875. Turin.--Regia Università degli Studi.-Museo Zoologico.

Bollettino dei Musei di Zoologia ed Anatomia comparata dell R. Università. Vol. I-. Torino, I886-: Z.S.I.

'Indice afabetico', I886-95: Z.S.I.

Regia Università degli Studi.-Osservatorio Astronomico (see No. 872).

- Scienziati Italiani (see No. 858).

- Societas Privata Taurinensis (see No. $87 \mathrm{I}(f)$ ).

Venice.-Imperiale Regio Istituto Veneto di Scienze, Littere ed Arti (see No. 877).

876. — Reale Comitato Talassografico Italiano.

Bollettino Bimestrale. Vol. 2-, I9Io/2-. Venezia, I9I3-: A.S.B. [For vol. I see No. $868(b)$.$] .$

877. — Reale Istituto Veneto di Scienze, Lettere ed Arti.

(a) Atti delle Adunanze $I[m p e r i a l e] R[$ egio] Istituto Veneto. Tom. I-7. Venezia, I84I-48: G.S.I. Ser. $2^{\text {a }}$, tom. I-6. Venezia, I850-55 : G.S.I. [Continued as:] Atti dell' I.R. Ist. Ven. Ser. $3^{\text {a }}$, tom. I-II. Venezia, I858-66: G.S.I. [Continued as:] Atti del Regio Istituto Veneto. Tom. I2-I6. Venezia, I866-7I : G.S.I. Ser. $4^{\mathrm{a}}$, tom. I-3. Venezia, I87I-74: G.S.I. [Continued as:] Atti del .... Istituto ... Ser. $5^{\mathrm{a}}$, tom. I-8. Venezia, I87482 : G.S.I. Ser. 6 , tom. I-7. Venezia, I882-89: G.S.I. Ser. $7^{\text {a }}$, tom. I-IO. Venezia, I889-99 (defective) : G.S.I. Ser. $8^{a}$, tom. I-II. Venezia, I899-I909 (tom. I-4 defective): G.S.I. [Tom. 3-10, ser. 7, are also styled 'Tom. 50$57^{\prime}$ '; similarly tom. I-I I, ser. 8, are also 'Tom. 58-68.' Tom. 1 and 2 , ser. 7 , are each erroneously styled 'Tom. 38. .]

(b) Memorie dell' I.R. Istituto I'eneto. Tom. I-I3. Venezia, I843-67 : G.S.I. [Continued as :] Memorie del ..... Istituto ... Tom. I4-25. Venezia, I868-96 : G.S.I.

Indice generale, I840-94: G.S.I.

878. Bergen.-Museum.

\section{NORWAY.}

(a) Aarbog. I892-. Bergen, I893-(Pt. 2 of vol. for 1904 wanting): Z.S.I.

(b) Aarsberetning. I890-9I. Bergen, I89I-92 : Z.S.I. [Continued as $(a)$.1

M.o. = Neteorological Oifice, Alinore.

M.Ph. = Medical College, Physiological Laboratory.

P.C. = Presidency College.

P.o. = Patent Office.

R.B.G. = Royal Botanic Garden.

S.C.C. $=$ Scottish Churches College.
S.G. = Surgeon-General s Library

S.I. = Survey of India.

S.T.M. = School of Tropical Medicine

T.D. = Telegraph Department

X.c. = St. Xavier's College

Z.S.I Z Zoological Survey of India. 
878. Bergen.--Museum (Contimied).

(c) Report on Norwegian Marine Investigations. By J. Hiort, O. Nordgaard and H. H. Gran. I vol., I89597. Bergen, I899: Z.S.I. [Continued as No. 879.]

879. — Report on Norwegian Fishery and Marine Investigations. Ed. by J. Hjort. Vols. I, 2. Kristiania, Bergen, I900-09: Z.S.I. [A continuation of No. $878(c)$.

880. Christiania.-Archiv for Mathematik og Naturvidenskab. Udg. af S. Lie, Worm-Muiller, G. O. Sars (and others). Bd. I-. Kristiania, I8;6-: Z.S.I.

88r. - Gaea Norvegica. Herausg. von B. M. Keilhau. Hfte. I-3. Christiania, I838-50.

Complete set: G.S.I.

Hfte. I, 2, I838-44: A.S.B.

882. — Norsk Gradmaalingskommission (Norwegische Commission der Europäischen Gradmessung).

(a) Geodätische Arbeiten. Hfte. I-7. Christiania, I88290: G.S.I.

(b) Vandstandsobservationer. Hfe. I-6. Christiania, I882I904 : G.S.I.

883. - Norsk Meteorologisk Institut.

(a) Jahrbuch des Norwegischen Meteorologischen Instituts. I874-81. Christiania, I877-82: A.S.B.

(b) Meteorologisk Aarbog. Annales de l' Institut Météorologique de Norvége. Aargang 2, I866, Christiania. [I867]: A.S.B. [Continued as (c).]

(c) Norsk Meteorologisk Aarbog. Aargang [I], 2, 5, 7, I867, '68, '71, '74. Christiania, I868-74: A.S.B.

- Norwegische Commission der Europäischen Gradmessung (see No. 882).

— Nyt Magazin for Naturvidenskaberne (see No. 884).

884. — Physiographisk Forening.

Nyt Magazin for Naturvidenskaberne. Bd. I-. Christiania, I838-(Bd. 9, II, If wanting): A.S.B. [The Society was dissolved at the end of 1873 and from vol. 20 and onwards the magazine was edited by Sars, Kjerulf, Hiortdah1 and others.]

Report on Norwegian Fishery and Marine Investigations (see No. 879 ).

\footnotetext{
A.S. = Archaeological Section, Indian Museum

A.S.B. = Asiatic Society of Benga!

B.C. = Bishop's College.

B.S.I. = Botanical Survey of Indiat

C.E. = Chemical Examiner to the Govt. of Bengal.

c. $U$. Calcutta University
}

E.C. = Civil Engineering College, Sibpur

G.S.I. = Geological Survey of India

I.L. = Imperial Library.

M.Ba. = Medical College, Bacteriological Laboratory

M. Bi. = Medical College. Biological Laboratory.

M.C. = Medical College (main Itbrary) 
885. Christiania.-Videnskabs Selskabet.

(a) Forhandlinger. Aar I858-. Christiania, 1859- (Aar I854, '83, '98, 1904 wanting; Aar 1893 defective): A.S.B. Registre, I868-77 : A.S.B.

(b) Skrifter. I. Mathematisk-naturvidenskabelig Klasse. I894. Kristiania, I895: A.S.B.

(c) Skrifter. II. Historisk-filosofisk Klasse. I894-. Kristiania, I894- (Vols. for 1896 , I904, 'O5 wanting): A.S.B.

886. Trondhjem.-Kongeligt Norske Videnskabers-Selskab. Skrifter. I905-. Trondhjem, I906-: Z.S.I.

POR'TUGAL see SPAIN.

\section{RUSSIA.}

887. Dorpat.-Naturforscher Gesellschaft.

(a) Archiv fïr die Naturkunde Liv-, Ehst-und Kurlands. $\mathrm{I}^{\text {te }}$ Serie. Mineralogische IVissenschaften nebst Chemie. Bd. 9. Dorpat, I882-87 : A.S.B. $2^{\text {te }}$ Serie. Biologische Naturkunde. Bde. 8-I3. Dorpat, I882-I905 (Bde. 8, II, I2 defective) : A.S.B.

(b) Schriften. No. II-. Dorpat, I902-(No. 17, 19 wanting) : A.S.B.

(c) Sitzungsberichte. Bde.6-. Dorpat, I884-: A.S.B.

Helsingfors.-Commission Géologique de la Finlande (see No. 892).

888. - Etudes Entomologiqiles. Red. par V. de Moltschulsky. Ann. I-I I. Helsingfors, Dresde. I852-62 : Z.S.I.

Fennia (see No. 894).

889. — Finska Vetenskaps-Societet.

(a) Acta Societatis Scientiarum Fennicae. Tom. I2-. Helsingforsiae, I883-(Tom. I9, 25, 28-31 wanting; tom. 4 I, 43,44 defective): A.S.B.

(b) Bidrag till Kännendom om Finlands, Natur och Folk. Häft 63-. Helsingfors, I905- (Hft. 74 wanting; Hft. 70 defective): A.S.B.

(c) Ofversigt (-Meddelanden) af .... Societetens Förhandlingar. Vol. 23-. Helsingfors, I88I- (Vols. 30, 35, 44,45 wanting) : . A.S.B.

\footnotetext{
Mo = Meteorological Office, Alipore

M Ph = Medical College, Physiological Laboratory.

P.C. = Presidency College.

PO = Patent Office

R. B.G. = Royal Botanic Garden.

S.C.C. $=$ Scottish Churches College
}

S.G. = Surgeon-General's Library

S.1. = Survey of India.

S.T.M. = School of Tropical Medicine.

T.D. = Telegraph Department.

X.C. =St. Xavier s College.

z.S.I. = Zoological Survey of India. 
89o. Helsingfors.-Finska Vetenskaps-Societet.--Meteorologischer Zentralanstalt.

Meteorologisches Jahrbuch fïr Finland. Bde. 7-, 1907-. Helsingfors, Kuopio. I9I2-: A.S.B.

891. — Geografiska Förening i Finland.

(a) Meddelanden. [Vol.] 4, 9, I897 98, I9I0-I2. Helsingfors, I899, I9I 2 : G.S.I.

(b) Vetenskapliga Meddelanden. [Vol.] I-3, I892-96. Helsingfors, I $892-96$ : G.S.I.

892. - Geologiska Kommission i Finland.

(a) Bulletin de la Commission Géologique de la Finlande. Tom. I.-, No. I-. Helsingfors, I895-: A.S.B., G.S.I.

(b) Geotekniska Meddelanden. No. I-. Helsingfors, Igo6- : G.S.I.

89.3. - Societas pro Fauna et Flora Fennica.

(a) Acta. Vol. I-. Helsingforsiae, I875- (Vol. 3, 4, I2, $2 \mathrm{I}-23,25,35$ wanting): A.S.B.

(b) Meddelanden. Häft I-. Helsingfors, I876-(Hft. 14, 27-29 wanting): A.S.B.

Societas Scientiarum Fennica (see No. $889(a)$ ).

894. — Société de Géographie de Finlande.

Fennia. Bulletin. Tom. I-. Helsingfors, I889-: G.S.I.

Société Finlandaise de Géographie (see No. 891).

895. - Société Finno-Ongrienne.

(a) Journal. Tom. I2-. Helsingissä, I894- (Tom. I3, I4 wanting): A.S.B.

(b) Mémoires. Tom. 3-. Helsingfors, I892-(Tom. 4-8, IO wanting): A.S.B.

Jurjew see Dorpat.

896. Kiev.-Obshchestvo Estestvoīspuitateleĭ (Sociét' des Naturalistes). Zapisski (Mémoires). Tom. Io-. Kiev, I899- (Tom. I I wanting; tom. 12, 14 defective): A.S.B.

A.S = Archaeological Section, Indian Museum A.S.B. = Asiatic Society ot Lengal.

B.C. = Bishop s Colloge.

B.S.I. = Bolanical Survey of India.

C.E. = Chemical Examiner to the Govt of Bengal.

C.U. = Calcutta University:
E.C. = Civil Engineering College, Sibpur.

G.S.I. = Geological Survey of India.

I. = Imperial Library

M.Ba. = Medtcal Collesse, Bacteriological Laboratory

M.Bi. = Medical College. Btological Laboratory.

M.C. = Mledical College (nam library). 
897. Moscow.-Société Impériale des Naturalistes.

(a) Bulletin. Tom. (Ann.) I-62. Moscou, I829-86. Nouv; sér., tom. I--. Moscou, I887-. 'Table des matières,' tom. I-56, I 829-8I.

Tom. I-, I829- (tom. 8, I835 wanting) and index : G.S.I.

Tom. I- nouv. sér., tom. 6, I829-93 (tom. 42 wanting) : R.B.G.

Tom. 43-, I870- (defective) : A.S.B.

(b) Mémoires. Tom. 6. Moscou, I823: R.B.G.

(c) Nouveaux Mémoires. Tom. I-. Moscou, I829-. ['Tom. I-13 are also styled 'Tom. 7-19' in continuation of $(b)$; Tom. I4, 15 are each also styled 'Tom. 20.' Tom. 16- are also 'Tom. $2 \mathrm{I}-$ '.]

Tom. I-, I829- : G.S.I.

Tom. I6, I7, I898-1910 (tom. 17 defective) : A.S.B.

898. Odessa.-Novorossiǔskoe Obshchestvo Esstestvoīspuitateler̆ (Société des Naturalistes de la Nouvelle Russie).

Zapiskī (Mémoires). Tom. 9-II. Odessa, I884-87 : A.S.B.

899. Orenburg.-Uchenaya Arkhivnaya Komissi (Commission Scientifique des Archives).

Trudui (Travaux). Tom. 2-. Orenburg, I897- : A.S.B.

Petrograd.-Academia Scientiarum Imperialis Petropolitana (see No. 900).

900. — Académie Impériale des Sciences.

(a) Acta Academiae Scientianum Imperialis Petropolitanae. I777-82. Petropoli, I778-86: G.S.I. [A continuation of $(h)$; continued as $(g)$.

(b) Beiträge zur Kenntniss des Russischen Reiches und der angränzenden Länder Asiens. Herausg. von K. E. Baer, G. v. Helmersen, L. v. Schrenck (and others). Bde. I-26. St. Petersburg, I839-7I : G.S.I. $2^{\mathrm{e}}$ Folge,

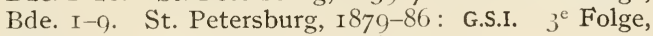
Bde. I-7. St. Petersburg, I886-90: G.S.I.

(c) Bulletin. Tom. I-36. St. Pétersbourg, I860-94 [Tom. 33-36 are also styled "Nouv. sér., tom. I-4."] [Continued as:] İzvyestiva Imperatorskoü Akademī Nauk.... Bulletin. $\quad 5^{\text {e }}$ sér., tom. I- 25. St. Pétersbourg, I8941906. $6^{e}$ sér., tom. I-. St. Pétersbourg, I907-.

M o. = Meteorological Office, Alipore.

M.Ph. = Medical College, Physiological Laboratory.

P.C. = Presıdency Collese.

P.O. = Patent Ofifice.

R.B.G, $=$ Royal Botanic Garden.

S.C.C. $=$ Scuttish Churches College.
S.G. = Surgeon-Generai's Library.

S.I. = Survey of India.

S.T.M. = School of Tropical Medicine

T.D. = Telegraph Department.

X.c. =St. Xavier $\mathrm{s}$ Collese.

Z.s.I. = Zoological Survey of India 
900. Petrograd.-Académie Impériale des Sciences (Continued).

[For earlier series see $(d)$.]

Complete set: R.B.G.

Tom. I-, I860-(tom. 17, 33-35 and sér. 5, toin. I wanting; sér. 5, tom. 15,25 and sér. 6 , tom. 6 defective): A.S.B.

Tom. I-, I860- (tom. I3, I4, 33-35 wanting, tom. 32, 36 and sér. 5, tom. 1, 15 defective): G.S.I.

(d) Bulletin de la Classe Physico-mathématique. Tom. I-I6. St. Pétersbourg, I $843^{-5}$ (tom. I7 wanting): G.S.I. [For later series see $(c)$.]

(e) Commentarii Academiae Scientiarum Imperialis Petropolitanae. Tom. I-I4. Petropoli; I728-5I : G.S.I. [Continued as $(h)$.]

(f) Mémoires. Tom. I-II. St. Pétersbourg, I809-30.

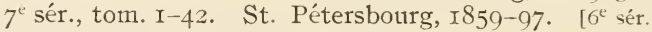
wanting. Continued in two parts as $(i)$ and $(j)$.

Tom. I-I I, I809-30, and sér. 7, tom. I-42, I850-05 (tom. 27,41 wanting, tom. 40,42 defective): G.S.I.

Sér. 7, tom. I-42,11859-97 : A.S.B.

(g) Nova Acta Academiae Scientiarum Imperialis Petropolitanae. Tom. I-I5. Petropoli, I787-I806: G.S.I. [A continuation of $(a)$.]

(h) Novi Commentarii Academiae Scientiarum Imperialis Petropolitanae. Tom. I-20. Petropoli, I750-76: G.S.I. [A continuation of $(e)$; continued as $(a)$.]

(i) Zapiskì İmperatorskoü Akademiï Nauk po Fizīkomatematīcheskomu Otdyeleniyu. Mémoires ... Classe

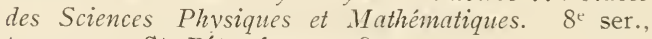
tom. I-. St. Pétersbourg, I894-.

Tom. I-, IS94- (tom. I8 defective): A.S.B.

Tom. I-20, I894-I907 (defective) : G.S.I.

(j) Zapiski İmp. Akad. Nauk po Istoriko-filologhïcheskomu Otdyeleniyu. Mémoires .... Classe des Sciences Historico-philologiques. $8^{\mathrm{e}}$ ser., tom. I-. St. Pétersbourg, I $895^{-}$: A.S.B.

Tableau générale to $(a),(c-h)$, I728-I870: G.S.I.

90 I — Académie Impériale des Sciences.-Commission (Centrale) Sismique Permanente.

(a) Bulletin. Ann. I907, '08, 'II. S.-Peterburgh, [I908]I9I2 : G.S.I.

(b) Comptes rendus des Séances. Tom. I, livr. 2-. St. Pétersbourg, I903- (tom. I, 2 defective): G.S.I.

\footnotetext{
A.S. = Archaeological Section, Indian Museum.

A.S.B. = Asiatic Society of Bengal.

B.C. = Bishop's College.

B.S.1. = Botanical Survey of Inciia

C.E. = Chemical Examiner to the Govt of Bengal

C.U. = Calcutta University.
}

E.C. = Civil Engineering College, Sibnur

G.S.I. = Geological Survey of India.

i L. = Imperial Libiary.

M Ba. = Medical College, Bacteriological Labora lorv

M.Bi. = Medical College. Biological Laboratory.

M.C. = Mcdical College (main library). 
902. Petrograd.-Académie Impériale des Sciences.-Musée Botanique. Travaux. Tom. I-. St. Pétersbourg, I902-: R.B.G.

903. — Académie Impériale des Sciences.—Musée d'Anthropologie et d'Ethnographie.

Sbornik Muzeya (Publications). No. I-3. S.-Peterburgh, I900-OI : A.S.B.

904. —- Académie Impériale des Sciences.-Musée Géologique Pierre le Grand.

Travaux. Tom. I-. S.-Peterburgh, I907-: G.S.I.

905. — Académie Impériale des Sciences.—Musée Zoologique.

(a) Anmuaire. Tom. I- S.-Peterburgh, I896- (Tom. I5 defective): A.S.B.

(b) Faune de la Russie et des Pays limitrophes. Tom. I-. St. Pétersbourg, I9I I- : A.S.B.

Beiträge zur Kenntniss des Russischen Reiches und der angränzenden Länder Asiens (see No. $900(b)$ ).

906. - Cabinet de sa Majesté.

Travaux de la Séction Géologique (Trudui Gheologhīcheskoì Chasti). Tom. I-7. S.-Peterburgh, I895-I909 (tom. 6 defective): G.S.I.

_ Comité Géologique (see No. 907).

- Commission (Centrale) Sismique Permanente (see No. 9or).

_ Faune de la Russie et des Pays limitrophes (see No. 905 (b)).

_- Gesellschaft für die gesammte Mineralogie (see No. 9I0).

907. —- Gheologīcheskiı Komitet (Comité Géologique).

(a) Bulletin. Tom. I-. St. Pétersbourg, I883-.

Complete set: G.S.I.

Tom. 7-, I889- (tom. 12, 13 wanting; tom. 23 defective): A.S.B.

(b) Trudui (Mémoires). Tom. I-20. St. Pétersbourg, I883-I902. Nouv. sér., livr. I-. St. Pétersbourg, I903-.

Tom. I-. I883- (tom. 12 defective, nouv. sér., livr. 39 wanting): G.S.I.

Tom. I-, I883-(tom. 6, II, 12 defective): A.S.B.

_- Hortus Petropolitanus (see No. 908).

__ Imperatorskaya Akademia Nauk (see No. 900).

M.o. = Meteorological Office. Alipore.

M.Ph. = Medical Collese, Physıological Laboratory

P.C. = Presidency College.

P.O. = Patent Office

R.B.G. = Royal Botanic Garden.

S.C.C. $=$ Scottish Churches College.
S.G. = Surgeon-General's Library

S.I. = Survey of India.

S.T.M. = School of Tropical Meaicine.

T.D. = Telegraph Department

X.C =St. Xavier's College.

Z.S.I. = Zoological Survey of India 
908. Petrograd.-Imperatorskiı Sankt-Peterburghskiı Botanīcheskiı Sad.

Acta Horti Petropolitan. Trudui ... Tom. I-. S.-Peterburgh, I87I-.

Complete set: R.B.G.

Tom. 4-, I876-(tom. 29, 30 defective) : A.S.B.

909. - Imperatorskoe Russkoe Geograficheskoe Obshchestvo.

(a) İvyestiya. Tom. I9-. S.-Peterburgh, I883-.

Tom. I9-, I 883 - : A.S.B.

Tom. 28-, I892- (tom. 48, I9 I2, wanting): M.O.

(b) Otchet. r883-. S.-Peterburgh, $188_{4}$ - : A.S.B.

Imperatorskoe Sankt-Peterburghskoe Mïneraloghïcheskoe Obshchestvo (see No. 910).

- Imperatorskoe Sankt - Peterburghskoe Obshchestvo Estestvoispuitatelě̆ (see No. 9I2).

- Materialien zur Geologie Russlands (see No. 910 (a)).

- Musée Botanique (see No. 902).

—- Musée d'Anthropologie et d'Ethnographie (see No. 903).

- Musée Géologique Pierre le Grand (see No. 904).

- Musée Zoologique (see No. 905).

910. - (Russisch-)Kaiserliche Mineralogische Gesellschaft (Gesellschaft für die gesammte Mineralogie: Imperatorskoe SanktPeterburghskoe Mineraloghicheskoe Obshchestvo).

(a) Materialien zur Geologie Russlands (Materialui dlya Gheologhī Rossī). Bde. I-. St. Petersburg, I869-.

Complete set: G.S.I.

Bde. I6-20, I893-1909: A.S.B.

(b) Schriften (Geschichte und Wissenschaftliche Beschäftigungen von $\mathrm{I} 8 \mathrm{I} 7$ bis $\mathrm{I} 842$ ). Bd. I. St. Petersburg, I 842 : G.S.I.

(c) Verhandlungen (Zapiskī). I842-63. St. Petersburg, I842-64. $2^{\mathrm{e}}$ Ser., Bde. I-. St. Petersburg, I866-.

Complete set: G.S.I.

$2^{\mathrm{e}}$ Ser., Bde. $28-$, I $89 \mathrm{I}-$ : A.S.B.

Systematisches Sach- und Namen-register zu der $2^{\text {en }}$ serie der Verhandlungen und den Materialien zur Geologie Russlands, I866-95 : G.S.I. ; I885-95 : A.S.B.

_- Revue Russe d'Entomologic (see No. 9I I (b)).

— Section Géologique du Cabinet de sa Majesté (sce No. 9o6).

A.S. =Archaeological Section, indian II useum,

A.S B $=$ Asiatic Society of Bengal.

B.c. = Bishop's College.

B.S.I. = Botanical Survey of India

C.E. Chemical Exammer to the Govt. of Bengat

C.U = Calcutta University.
E.C. = Civil Engineering College, Sibpur.

G.S.I. = Geological Survey of Incia.

I $L$. = Imperial Library.

M.Ba. =Nedical College, Bacterioiogical Laboratory.

M.Bi. = Nedical College. Biological Laboratory.

M.C. = II clical College (main library). 
9II. Petrograd.-Société Entomologique Russe (Russkoe Entomologhīcheskoe Obshchestvo).

(a) Horae Societatis Entomologicae Rossicae. (Trudui ...) Tom. I-. S.-Peterburgh, I86I- : Z.S.I.

(b) Revue Russe d'Entomologie (Russkoe Entomologhīcheskoe Obozryenie). Tom. I-. Taroslav1. S.-Peterburgh, IgOI-: Z.S.I. [Tom. I-5 were edited by D. Glasounow, A. Jakowlew and others, the remainder by the Society.]

912. - Société Impériale des Naturalistes (Imperatorskoe SanktPeterburghskoe Obshchestvo Estestvoispuitateleı̌).

Travaux (Trudui). Tom. 34-. S.-Peterburgh, I903-: Z.S.I.

9I3. Saratow.-Volzhskaya Biologhicheskaya Stantziya (Biologische Station am Wolga-Fluss).

Rabotui (Arbeiten). Bde. 3-. Saratow, I907-(Bd. 3 defective): Z.S.I.

9I4. Warsaw.-Ezheghodnīk po Gheologhī̄ ì Mineraloghī Rossī̄ (Annnaire Géologique et Minéralogique de la Russie). Réd. par N. Krischtafowitsch. Tom. (Vol.) I, 2, I5. Varshava, NovoAleksandriya, I896-97, I9I3 (defective): G.S.I.

9I5. Yalta.-Imperatorskiı Nikitskiı Sad.

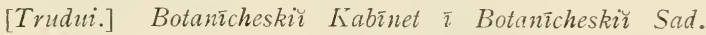

No. I-. Yalta, IgI6-: R.B.G.

\section{SPAIN AND PORTUGAL.}

9I6. Barcelona.-Club Montanyenc.

Butlleti. No. I-io. Barcelona, I9I2: Z.S.I.

917. - Junta de Ciències Naturals.

(a) Amuri. IgI6. Barcelona, [IgI6]: G.S.I.

(b) Publicacions. Serie Botànica. Yo. I-. Barcelona, IgI7-: Z.S.I.

(c) - Serie Zoologica. No. 2-. Barcelona, I9I7(nos. 3-6 wanting): Z.S.I.

(d) - Serie Biològico Oceanogràphica. No. I- Barcelona, I9I7- : Z.S.I.

9I8. Lisbon.-Academia Real das Sciencias.

(a) Historia e Memorias. (Memorias). $2^{2}$ ser., tom. I-3. Lisboa, I843-56 : G.S.I. [Continued as (c).]

M.o. = Meteorologicai Otfice, Alipore.

M.Ph. = Medical College, Physiological Laboratory.

P.C. = Presidency College.

P.o. = Patent Office

R.B.G. = Royal Botanic Garder.

S.C.C. $=$ Scottish Churches College.
S.G. = Surgeon-General's Library.

s.l. = Survey of India.

S.T.M. = School of Tropical Medicine.

T.D. = Telegraph Department.

X.C. =St. Xavier's Collese.

Z.s.t. = Zoological Survey of India. 
9I8. Lisbon.-Academia Real das Sciencias (Continued).

(b) Journal de Sciencas Mathematicas, Physicas e Naturaes. Tom. I-I2. Lisboa, I866-88. $2^{\mathrm{a}}$ ser., tom. I-3. Lisboa, I889-94.

Tom. I- $2^{i}$ ser., tom. 3, I866-94 (defective) : Z.S.I.

Tom. 5,1876 : A.S.B.

(c) Memorias.... Classe de Sciencias Mathematicas, Physicas e Naturaes. Nova ser, tom. I-3. Lisboa, I85465: G.S.I. [A continuation of $(a)$.]

919. - Commissão do Serviço Geologico de Portugal.

Communicaçoes da Commissão dos Trabalhos Geologicos de Portugal. Tom. I, 2. Lisboa, I883-92. G.S.I. [Continued as:] Comm. da Direç̧ão Trab. Geol. Port. Tom. 3. Lisboa, I895-98: G.S.I. [Continued as :] Comm. $d a$ Direç̧. dos Serviços Geol. Port. Tom. 4. Lisboa, I9oooI : G.S.I. [Continued as:] Comm. da Commissão do Serviço Geol. Port. Tom. 5-. Lisboa, 1904-: G.S.I.

920. - Sociedade de Geographia de Lisboa.

Boletim. $2^{\text {a }}$ Ser., No.4-6. Lisboa, I88I : G.S.I.

921. Société Portugaise des Sciences Naturelles.

(a) Bulletin. Vol. I-. Lisbonne, I908-: Z.S.I.

(b) Mémoires. No. I, pt. 2-. Lisbonne, I9I3- (no. 2 wanting): Z.S.I.

922. Madrid.-Real Academia de Ciencias (Exactas, Físicas y Naturales).

(a) Memorias. Tom. I-. Madrid, I850- (tom. 7-Io wanting) : G.S.I.

(b) Revista. Tom. I-. Madrid, I904-: G.S.I.

923. - Revisia de los Progressos de las Ciencias Exactas, Físicas y Naturales. Tom. 21, 22. Madrid, I886-89 (defective): G.S.I.

924. - (Real) Sociedad Geográfica.

(a) Boletin. Tom. I-. Madrid, I876-: G.S.I. 'Indice', tom. I-2O, at end of tom. $2 \mathrm{I}$.

(b) Revista di Geografía Colonial y Mercantil. Tom. IMadrid, I897- : G.S.I.

Repertorio de Publicaciones y Tareas, I90I-Io: G.S.I.

925. Ona.-Colegio Maximo de la Compañia de Jesus.

Observaciones Meleorologicas hechas en el Colegio .....en Oña, Provincia de Burgos. I903-Io. Oña, I903-II : X.C.

A.S. = Archaeological Section. Indian Museum

A.S.B. = Asiatic Society of Bengal.

B.C. = Bishop s College.

B.S.l. = Botanical Survey of Ind!a.

C.E. = Chemical Examiner to the Govt. of Bengal

C.U. = Calcutta University.
E.c. = Civil Engineering College, Sibpur.

G.S.I. = Geological Survey of India.

L. = Imperial Library

M.Ba. = Medical College, Bacteriological Laboratory

M.Bl. = Medical College. Biological Laboratory.

M.C. = liedical College (main library). 
926. Oporto.-Academia Polytechnica.

Annaes Scientificos. Vol. I-. Coimbra, Ig06- (vol. I defective) : G.S.I.

927. Tortosa.-Observatoire de 1'Ebre.

Bulletin. Tom. I-3. Tortosa, I910-12: X.C.

\section{SIVEDEN.}

928. Lund.-Botaniska Notiser. Utg. af C. F. O. Nordstedt (and others). Ar I897-. Lund, I897- : R.B.G.

Stockholm.-Académie Royale des Sciences (see No. 932).

929. — Acta Mathematica. Herausg. von (Réd. par) G. Mittag-Leffler. Bde. (Tom.) I-36. Uppsala, Stockholm, I882-I9I3: C.U.

930. — Entomologiska Förening.

Entomologisk Tidskrift (Journal Entomologique). Årg. I-. Stockholm, I880-. Alfabetiskt register, Årg. I-IO, IS80-89, ̊․․ II-30, I890-I909.

Årg. I-, I880- (Árg. 29, 30 wanting, .̊rg. 19 defective) and indexes: Z.S.I. Àrg. I-30, I880-I909: A.S.B.

931. - Geologiska Förening.

Förhandlingar. Bd. 22-. Stockholm, I900-: G.S.I. Generalregister, Bd.22-3I, I900-09: G.S.I.

932. — Kongliga (Kungl.) Svenska Vetenskaps-Akademi.

(a) Arkiv för Botanik. Bd. I-. Stockholm, I903-: A.S.B., B.S.I., G.S.I., R.B.G. [For earlier series see $(f)$.]

(b) Arkiv för Kemi, Mineralogi och Geologi. Bd. I-. Stockholm, I903-. A.S.B., G.S.I. (For earlier series see $(k)$.]

(c) Arkiv för Matematik, Astronomi och Fysik. Bd. I-. Stockholm, I903-. [For earlier series see $(k)$.

Complete set: G.S.I.

Bd. I-, I903- (Bd. I, 2 defective): A.S.B.

(d) Arkiv för Zoologi. Bd. I-. Stockholm, I903-: A.S.B., G.S.I., Z.S.I. [For earlier series see $(f)$.]

M.O. = Meteorological Ofíce, Aisoore.

M.Ph $=$ Medical College. Physiological Laboratory

P C. = Presidency College.

P O. = Patent Office

R B.G = Roval Botanic Gardier.

S C C $=$ Scotlish Churches College.

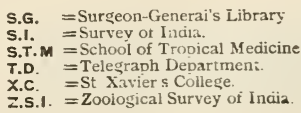

=Surgeon-Generai's Library

S.I. $=$ Survey of India.

T.D. = Telegraph Departmen

Z.S.1. = Zoological Survey of Inciia 
932. Stockholm.-Kongliga Svenska Vetenskaps-Akad. (Continued).

(e) Arsbok. I903-. Stockholm, I903-.

Complete set: G.S.I.

Vols. for I903- (vols. for I910, '12, '13 wanting): A.S.B.

(f) Bihang till Handlingar. Bd. I-28. Stockholm, I872I903. [Continued as $(a)$ and $(d)$.]

Complete sets : A.S.B., G.S.I.

Bd. 6-28, I880-I903 (botanical papers only): R.B.G.

Bd. I2-28, I887-I903: Z.S.I.

(g) Handlingar. Vol. I-40, I739-79. Stockholm, I74I79. Vol. I-33, I780-I8I2. Stockholm, I780-I8I2. I8I3-36. Stockholm, I8I3-38. Ny Följd, Bd. I-, I855-. Stockholm, I858-. Register, I739-I8I2.

Vol. I-, I74I- (vols. for I837-54 wanting) and index : G.S.I.

Ny Följd, Bd. I-, I858- : C.U., Z.S.I.

Ny Följd, Bd. 5-, I864- (Bd. 38 wanting, Bd. 36 defective): A.S.B.

(h) Jahresbericht der Kön. Schwedischen Akademie der Wissenchaften über die Fortschritte der Botanik. Uebersetzt ..... von C. T. Beilschmied. Jahre I828, '32-34. Bresla1, I835-36: R.B.G.

(i) Lefnadsteckningar..... Bd. I - Stockholm, Uppsala, I869-.

Complete set: G.S.I.

Bd. 4-, I899-: A.S.B.

(j) Meteorologiska Iakttagelser i Sverige (Observations Météorologiques Suédoises). Bd. I-, I859-. Stockholm, I860-. [Bd. I5- are also styled ' $2^{\mathrm{a}}$ ser., Bd. I-'.]

Complete set: G.S.I.

Bd. 7-, I866- (defective): A.S.B.

(k) Öfversigt af .... Förhandlingar. Ärg. I-59, I 844-I902. Stockholn1, I845-I903. [Continued as $(b)$ and $(c)$.]

Complete set : G.S.I.

Arg. 22-59, I865-I903 : A.S.B.

Arg. 44-5I, I887-94 (botanical papers only): R.B.G.

Förteckning öfver ... Skrifter, I826-83 : G.S.I.

933. — Kongliga Svenska Vetenskaps-Akademi.-Nobel Institut.

(a) Les Prix Nobel. I90I-. Stockholm, I904-A.S.B.

(b) Meddetanden. Bd. I-. Uppsala, Stockholm, Igo5-. Complete set: G.S.I.

Bd. I-, I907- (Bd. I defective): A.S.B.

\footnotetext{
A.S. = Archacological Section, Indian Museum

A.S.B. = Asiatic Society of Bengal

B.C. = Bishop's College.

B.S.1. = Botanical Survey of Incia

C.E. = Chemical Examiner to the Govt. of Bengal.

c.u. = Calcutta University.
}

E.C. = Cavis Engineerins College, Silopur

G.S.I = Geological Survey of 1ndia.

I.L. = Imperial Library.

M.Ba. = Medical College, Bacteriolosical Laboratory

M.BI. = Medical College. Brological Laboratory.

M.C. = Medical College (main library) 
934. Stockholm. - Kongliga Vitterhets Historie och Antikvitets Akademi.

Fornvännen. Meddelanden fran ..... Akademien. I906-. Stockholm, I906-: A.S.B.

935. - Sveriges Geologiska Undersökning.

(a) Afhandlingar och Uppsatser. Ser. C. No. I-203. Stockholn, I868-Ig06 (nos, 2, 3, 23, I60 wanting) : G.S.I. [Afterwards incorporated in (b).] Ser. Ca. No. I-. Stockholm, I900- : G.S.I.

(b) Arsbok Lincluding (a), Ser. c]. I907-. Stockholm, I907一: G.S.I.

(c) Kartblad med beskrifningar. Ser. A a. No. I-. Stockholm, I862- (nos. I29, 136, I39, 142-145, I48 wanting): G.S.I. Ser. A b. No. I-I5. Stockholm, I $877-93$ : G.S.I. Ser. A c. No. I-8. Stockholm, I902-04 (no. 7 wanting): G.S.I. Ser. B a. No. 4-. Stockholm, I884- (no. 8 wanting): G.S.I. Ser. B b. No. I-9. Stockholm, I88I[I902] : G.S.I.

Systematisk Forteckning öfver offentliggjorda Arbeten, I 862-93: G.S.I.

Upsala.-Acta Mathematica (see No. 929).

_- Geological Institution (see No. 938).

936. — Kongliga Humanistiska Vetenskaps-Samfundet.

Skrifter. Bd. 7-. Uppsala, Leipzig, I900- (Bd. 9 wanting): A.S.B.

937. — Kongliga Vetenskaps-Societet.

Nova Acta Regiae Societatis Upsaliensis. Vol. I-I4. Upsaliae, I773-I850: R.B.G. Ser. 3, vol. I-5. Upsaliae, I $855-65$ : G.S.I.

938. — Kongliga (Kung1.) U niversitet.

(a) Arsskrift. I886-. Uppsala, I886-.

Vols. for I 886 - : R.B.G.

Vols. for I903-: A.S.B.

(b) Bulletin of the Geological Institution. Ed. by $\mathrm{H}$. Sjögren. Vols. I-. Upsala, I893-. Index to vols. I-IO at end of vol. Io.

Complete set: G.S.I.

Vols. I-, I893- (vol. 3 wanting) : A.S.B.

Vols. 3-6, I 898-1905 (vols. 3, 5 defective): Z.S.I.

Vols. 8-II, I906-I2: I.L.

M.o. = Meteorological Ofífice. Alipore.

M.Ph. = Medical College, Physiological Laiooratory.

P.C. = Presidency College.

P.O. = Patent Office

R.B.G. = Royal Botanic Garden.

S.C.C. = Scottish Churches College.
S.G. = Surgeon-General's Library:

S.1. = Survey of India.

S.T.M. = School of Tropical Medicine.

T.D. = Telegraph Department.

X.c. =St. Xavier's College.

Z.s.1. = Zoological Survey of India 


\section{SWITZERLAND.}

939. Basle.-Naturforschende Gesellschaft.

(a) Bericht ïber die Verhandlungen. Thle. I-Io. Basel, I835-52: G.S.I. [Continued as (b).] 'Register', Thle. IIo, at end of Thl. Io.

(b) Verhandlungen. Thle. (Bde.) I-. Basel, I857-. Namenverzeichnis und Sachregister, Bde. 6-I2, I875I900. Autorenregister, Bde. $\mathrm{I}-2 \mathrm{O}$ at end of $\mathrm{Bd} .20$.

Complete set and index : G.S.I.

Bde. I3-, I902-, and index : Z.S.I.

Bde. I6-, I903-: A.S.B.

- Schweizerische Paläontologische Gesellschaft (see No. 952).

Berne.-Beiträge zur Geologischen Karte der Schweiz (see No. 958).

—_Eclogae Geologicae Helvetiae (see No. 943).

940. — Journal Télégraphique. Tom. 27-37. Berne, I903-I9I3 (tom. 30-33 wanting): T.D.

941. - Schweizer Alpenclub.

Jahrbuch. Jahrg. 2-6. Bern, I865-70: G.S.I.

942. — Schweizerische Entomologische Gesellschaft (Société Entomologique Suisse).

Mittheilungen (Bulletin). Bde.4一. Schaffhausen, Bern, I873-.

Bde. 4-IO, I873-I903 (Bde. +8 defective): A.S.B.

Bde. 6-, I884 : Z.S.I.

943. - Schweizerische Geologische Gesellschaft (Société Géologique Suisse).

Eclogae Geologicae Helvetiae (Recueil periodique: Mittheilungen). Vols. I-. Lausanne, I888- : G.S.I.

944. Fribourg.-Société Fribourgeoise des Sciences Naturelles.

Bulletin (Compte-Rendu). Ann. 8-II, I887-90. Fribourg, I 89 I : R.B.G.

Geneva.-Archives des Sciences Physiques et Naturelles (see No. $945(b))$.

—_ Archives Suisses d'Anthropologie Générale (see No. 948).

945. — Bibliothèque Universelle.

(a) Bibliothèque Universelle et Revue Suisse. Ann. 79-83, nouv. pér., tom. $4^{8-64}$. Lausanne, I874-78: G.S.I.

A.S. = Archaeological Section. Indian Museum

A.S.B. = Asiatic Society of Bengal.

B.C. = Bishop's College.

B.S.I. = Botancal Survey of India.

C.E. = Chemical Examiner to the Govt. of Bengal.

c.U. = Calcutta University.
E.C. = Civil Engineering College, Sibpur.

G.S.I. = Geolosical Survey of India.

I.L. = Imperal Library

M.Ba. = Medical College, Bacteriological Laboratory

M.BI. = Aedical College. Biological Laboratory.

M.C. = Medical College (main library). 
945. Geneva.-Bibliothèque Universelle (Continued).

Ann. 84-92, $3^{\text {mx }}$ pér., tom. I-36. Lausanne, I879-87: G.S.I.

(b) Bibliothèque Universelle (et Revue Suisse). Archives des Sciences Physiques et Naturelles. Nouv. pér., tom. 4964. Genève, I874-78. $3^{\text {nue }}$ pér., tom. I-34. Genève, I $878-95 . \quad 4^{\text {me }}$ pér., tom. I-. Genève, r 896 -.

Nouv. pér. tom. $49-3^{\text {me }}$ pér., tom. I8, I874-87: G.S.I.

$3^{\text {the }}$ pér., tom. I-, I $878-$ A.S.B.

946. - Herbier Boissier.

Bulletin. Tom. I-7. Genève, I893-99: R.B.G. $2^{\text {me }}$ sér.,

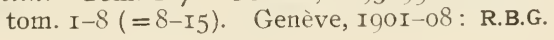

947. — Institut National Genèvois.

Mémoires. Tom. I-I8. Genève, I 854-I900 : Z.S.I.

948. — Institut Suisse d'Anthropologie Générale.

Archives Suisses d'Anthropologie Générale. Tom. I-. Genève, IgI4- : A.S.B., Z.S.I.

949. - Jardin Botanique.

Annuaire du Conservatoire et du Jardin... Tom. I-. Genève, I877-: R.B.G.

950. - Matériaux pour la Paléontologie Suisse. Publ. par F J. Pictet. Sér. I-6. Genève, I854-73 : G.S.I.

95I. - Musée d' Histoire Naturelle.

Revue Suisse de Zoologie et Annales du Musée.... Tom. I-. Genève, I893- : Z.S.I.

952. - Schweizerische Paläontologische Gessellschaft (Société Paléontologique Suisse).

Abhandlungen (Mémoires). Vol. I-. Basel, Genf, etc., I874- : G.S.I.

953. - Société de Physique et d'Histoire Naturelle.

(a) Compte Rendu des Séances. [Nos.] I-, I884-. Genève, I 885 -.

Nos. I-, I885- (nos. 17-27 wanting): A.S.B.

Nos. 27-, I9IO- (no. 32 wanting): G.S.I.

(b) Mémoires. Tom. I- Genève, I82I-.

Complete set: G.S.I.

Tom. 5, 9, I 832, ' $4 \mathrm{I}-42$ : R.B.G.

Tom. 9-, I $84 \mathrm{I}$ - (tom. 22, 23 wanting; tom. 33 defec. tive) : A.S.B.

M.o. = Meteorological Office, Alipore.

M.Ph. = Medical College. Physiological Laboratory.

P.C. = Presidency College.

P.O. = Patent O.fice.

R.B.G. = Royal Botanic Garden.

S.C.C. = Scottish Churches College.
S.G. = Surgeon-General s Library.

S.I. = Survey of India.

S.T.M. = School of Tropical Medicine.

T.D. = Telegraph Department

X.C. =St. Xavier $\mathrm{s}$ College.

Z.S.I. = Zoological Survey of India. 
953. Geneva.-Société Paléontologique Suisse (see No. 952).

954. Lausanne.-Bibliothèque Universelle et Revue Suisse (see No. 945). _- Eclogae Geologicae Helvetiae (see No. 943).

955. - - Société Vaudoise des Sciences Naturelles.

Bulletin (Procès-Verbaux). Vol. 5-. Lausanne, 1858-: G.S.I. 'Tables', vol. I-IO, at end of vol. IO; vol. 2 I30 , at end of vol. 40.

Neuchatel.-Allgemeine Schweizerische Gesellschaft für die gesamten Naturwissenschaften (see No. 957).

956. - Société (Neuchâteloise) des Sciences Naturelles.

(a) Bulletin. Tom. 2-, I846-. Neuchatel, 1848- (tom. 4, 17-20 wanting): G.S.I.

(b) Mémoires. Tom. I-4. Neuchatel, I835-74: G.S.I.

'Table des Matières', tom. I-25 of $(a)$ and tom. I-4 of $(b)$, I $832-97$ : G.S.I.

Schaffhausen.-Schweizerische Entomologische Gesellschaft (see No. 942).

957. Zurich.-Allgemeine Schweizerische Gesellschaft für die gesamten Naturwissenschaften (Société Helvétique des Sciences Naturelles).

(a) Denkschriften. Bd. I. Zürich, I829: G.S.I. [Continued as $(b)$.]

(b) Neue Denkschriften (Nouveaux Mémoires). Bde. I-27: Abth. I. Neuchâtel, Zürich, I837-76: G.S.I. [Also disided into decades.]

958. - Allgemeine Schweizerische Gesellschaft für die gesamten Naturwissenschaften.-Geologische Kommission.

Beiträge zur Geologischen Karte der Schweiz (Matériaux. pour la Carte Géologique de la Suissee). Lief. I-37. Bern, etc., I862-97 (defective): G.S.I.

959. - Annalen der Botanick. Herausg. von P. Usteri. Stück I24. Zürich, I79I-I800: R.B.G [Stück 7-24 are also entitled Neue Annalen der Botanick.]

96o. — Botanisches Magazin (Magazin für die Botanik). Herausg. von J. J. Römer und P. Usteri. Bde. I-4 (=Stück I-I2). Zürich, $\mathrm{I}_{7} 87-90$ (Bd. 3 wanting): R.B.G.

Concilium Bibliographicum.

Bibliographia Zoologica (see No. 707).

_- Geologische Kommission (see No. 958).

A.S. =Archaeological Section, Indian Museum.

A.S.B. = Asiatic Society of Bengal.

B.C. = Bishop's College.

B.S.I. = Botanical Survey of India

C.E. = Chemical Examiner to the Govt. of Bengal.

c.u. = Calcutta University
E.c. = Civil Engineermg College, Stbpur

G.S.I. = Geolosical Survey of India.

I.L. = Imperial Library.

M.Ba. = Medical College, Bacteriological Laboratory

M.Bi. = Mledical College, Biological Laboratory:

M.c. = Iledical College (main library) 
961. Zurich.-Naturforschende Gesellschaft.

(a) Abhandlungen. Bde. I-3. Zürich, I76I-66: G.S.I., R.B.G.

(b) In die Zürcherische Jugend ... Jahr. I799-1870, Stück I-72. [Zürich, I799]-I870: G.S.I. [Continued as $(e)$.]

(c) Bericht über die Verhandlungen. I825-37. Zürich, I $826-38$ : G.S.I.

(d) Mittheilungen. Bde. I-4. Zürich, I849-56 : G.S.I.

(e) Nenjahrsblatt. [Stück] 73-I0I, I87 I-99. Zürich, I 87 I- [99] (Stück 87, 91, 93, 99 wanting): G.S.I.

(f) Vierteljahrsschrift. Jahrg. I-. Zürich, I856-.

Complete set: G.S.I.

Jahrg. 36-, I89I- (Jahrg. 42 defective) : A.S.B.

Generalregister der Publikationen, I799-I892 : G.S.I.

962. - Neues Magazin für die Botanik in ihrem ganzen Umfang. Herausg. von J. J. Römer. Bd. I. Zïrich, I794: R.B.G.

\section{AFRICA.}

963. Antananarivo.-Académie Malgache.

Bulletin. Tom. 8-, I9Io-. Tananarive, I9II- : A.S.B.

964. - Gouvernement Général, Colonie de Madagascar et Dépendances.

Bulletin Economique. Ann. 2-. Tananarive, r902-:

\section{B.S.I.}

965. - - Observatoire Royal de Madagascar.

[Observations.] Tom. 4, 5, I892-93. Tananarive, I893: X.c.

966. Beira.-Companhia de Moçambique.

Agricultural Journal of the Mozambique Company. Vols. I-. Beira, I9II-: B.S.I.

967. Bulawayo.-Rhodesia Museum.

(a) Annual Report. Nos I-, I902-. Bulawayo, [1902]- (nos. 3, 5, 6 wanting) : Z.S.I.

(b) Special Report. No. 2. Bulawayo, I904: Z.S.I.

Cairo.-Egypt Exploration Fund (see No. I27).

M.O. = Meteorological Office, Alipore.

M.Ph. = Medical College, Physiological Laboratory.

P.C. = Presidency College.

P.o. = Patent Office.

R.B.G = Royal Botanic Garden

s.C.C. $=$ Scottish Churches College.
S.G. = Surgeon-Generai's Library:

S.l. = Survey of India.

S.T.M. = School of Tropical Medicine.

T.D. = Telegraph Department.

X.c. =St. Xavier's College.

z.s.I. =Zoological Survey of India. 
Cairo.-Geological Survey of Egypt (see No. 97r).

968. — Institut Egyptien.

Bulletin. $4^{\text {me }}$ sér., no. 2, I90I. Le Caire, I902 : G.S.I.

969. - Khedivial Agricultural Society.

Yearbook. I905-. Cairo, 1906- (vols. for 1907, 'o8 wanting): B.S.I.

970. - Survey Department, Esypt.

Survey Department Paper. Nos. I, I4, I6, I8-20, 25, 29, 30. Cairo, I907-I3: G.S.I.

971. - Survey Department.-Geological Survey of Egypt.

[Publications.] Palaeontological Series. Nos. 2-. Cairo, I9I4-: G.S.I.

972. Cape Town.-Department of Agriculture, Cape of Good Hope.

(a) Agricultural Journal of the Cape of Good Hope. Vols. I-37. Cape Town, I889-I9Io (vols. I, 2 defective): B.S.I. [Afterwards combined with Nos. $988(a)$ and 993 and continued as No. 994.]

(b) Marine Investigations in South Atrica. Vols. I-4. Cape Town, I902-08: Z.S.I.

973. — Department of Agriculture.-Geological Commission.

Annual Report. Nos.[I]-I6, I896-IgII. Cape Town, I897-I9I2. Index to Reps. I-8, I896-I903.

Complete set and index : G.S.I.

Reps. for I896-I9II (Reps. for I904, 'o5, 'og wanting): A.S.B.

Reps. for I898, '99 : Z.S.I.

- Marine Biological Report (see No. 979).

- Marine Investigations in South Africa (see No. $972(b))$.

974. — Royal Observatory.

Observations made at the Magnetical and Meleorological Observatory at the Cape of Good Hope. I vol., I 84I-46. London, I85I : A.S.B.

975. — Royal Society of South Africa.

Transactions. Vols. I-, Igo9-. Cape Town, I909-: A.S.B. [A continuation of No. 978.]

976. — South African Central Locust Bureau.

Report of the Committee of Control. Nos. I-, I907-. Cape Town, I908-.

A.S. = Archaeological Section. Indian Museum.

A.S $\mathbf{B}=$. Isiatic Society of Bengal.

B.C. = Bishop s College.

B.S.I. = Botanical Survey of India.

C.E. = Chemical Examiner to the Govt. of Bengat

c.U $=$ Calcutta University.
E.C. = Civil Engmeering College. Sibpur. G.S.I = Geological Survey of India.

I.L. = Imperial Library.

M.Ba. = Medical College, Bacteriological Laboratory

M.BI. = Medical College. Biological Laboratury:

M.C. = Medical College (main library). 
976. Cape Town.-South African Central Locust Bureau (Continued).

Complete set: B.S.I.

Nos. 2-4, for I908-IO: Z.S.I.

977. — South African Museum.

Annals. Vols. I-. London, I898-.

Complete sets : G.S.I., Z.S.I.

Vols. I-, I898- (no. 4 , vol. Io wanting): A.S.B.

978. - South African Philosophical Society.

Transactions. Vols. I-I8, I877-I909. Cape Town, I880

(I878)-I909. [Continued as No. 975.]

Vols. I-I8, I880-I 909 (vols. I3, I5, I7 defective) : A.S.B.

Vols. 3-7, I885-96 (vol. 5 wanting; vols. 3,4 , 6 defective) : Z.S.I.

979. Union of South Africa.-Province of Cape of Good Hope.

Marine Biological Report. Nos. I-. I912/13-. Cape Town, I9I3- : Z.S.I.

980. Dar-es-Salam.-Kaiserliche Gouvernement ron Deutsch-OstAfrika.

(a) Berichte ïber Land- und Forstwistsckaft im DeutschOst-Afrika. Bde. I-3. Hoidelberg, I900-I I.

Bde. I-3, I900-II : B.S.I.

OF Tाप

Bde. I-3, Hft. 4, I900-d 8 : N Z. S.I. E R S I T

Bde. I, 2, I900-06: R.B.G

(b) Pflanzer: Ratgeber fiur tropische Landwirtschaft, unter Mitwirkung des (herausg. von) Biologisch-Landwirtschaftlichen Instituts Amani. Jahrg. I-6. Tanga, Daressalam, I905-Io (Jahrg. I defective): B.S.I. [Continued as :] Pflanzer: Zeitschrift fïr Land- und Forstwirtschaft in Deutsch-Ost-Afrika. Jahrg. 7-. Tanga, Daressalam, I9I I- : B.S.I., R.B.G.

981. Durban.-Medical Journal of South Africa. Vols. 9-. Durban, I9I3- : S.T.M. [A continuation of No. 983.]

982. — Museum.

Annals. Vols. I-. Durban, I9I4-: Z.S.I.

983. - Transvaal Medical Journal. Vols. I-8. Durban, I905-13:

S.T.M. [Continued as No. 981.] Index to vols. I-7: S.T.M.

984. Grahamstown.-Albany Museum.

Records. Vols. I-. Grahamstown, I903-: Z.S.I.

M.O. = Meteorological Office, Alipore.

M.Ph. = Medical College, Physiological Laboratory.

P.C. = Presidency College.

P.o. = Patent Office.

R.B.G $=$ Royal Botanic Garden.

S.C.C. $=$ Scottish Churches College.
S.G. = Surgeon-General's Library.

S.I. = Survey of India.

S.T.M. $=$ School of Tropical Medicine.

T.D. = Telegraph Department.

X.c. =St. Xavier's Collese.

Z.s.I. =Zoological Survey of India. 
985. Johannesburg.-Geological Society of South Africa.

Transactions (and Proceedings). Vols. I-. Johannesburg, I896- (vol. 5 defective): G.S.I. Index, vols. II3, I 896-I9Io : G.S.I.

986. Khartoum.-Gordon Memorial College.-Wellcome Research Laboratories.

Report. Nos I-. London. I903- : S.T.M., Z.S.I.

Leopoldville.-Bulletin Agricole du Congo Belge (see No. 40r).

$9^{\circledR} 7$. Nairobi.-Agricultural Department, British East Africa.

(a) Agricultural Journal of British East Africa. Vols. I-. Mombasa and Nairobi, I908- : B.S.I.

(b) Annual Report. r907/08-. Nairobi, I908- : B.S.I.

988. Pietermaritzburg.-Department of Agriculture, Natal.

(a) Natal Agricultural Journal (and Mining Record). Vols. 3-I5. Pietermaritzburg, I900-IO. [Afterwards combined with Nos. 972 and 993 and continued as No. 994.]

Vols. 3-I5, I900-IO (vols. 3-5 defective): B.S.I.

Vols. 8-I5, I905-IO : R.B.G.

(b) Report of the Government Entomologist. I-, I899/ I900-. Pietermaritzburg, I9OI-: B.S.I.

989. - Geological Survey of Natal and Zululand.

Report. Nos. I-3. Pietermaritzburg, I90I-07: G.S.I.

990. - Natal Government Museum.

Annals. Ed. by Warren. Vols. I-. London, Igo6--.

Complete set: Z.S.I.

Vol. I, I906-08 : G.S.I.

991. Port Louis.—Revue Agricole de Maurice, la Réunion et Madagascar. Publ. sous la direct. de M. A. Daruty de Grandpré. Ann. III. Port Louis, I887-97 (ann. 1 defective, ann. $+-5,8$ wanting): R.B.G.

992. - Station Agronomique, Colonie de Mauritius.

(a) Bulletin. Nos. [3]-. Maurice, I9oI-: B.S.I.

(b) Rapport Annuel. I895-I9oo. Maurice, I896-I9oI :

B.S.I. [Afterwards published with $(a)$.]

993. Pretoria.-Department of Agriculture, Transvaal.

Transvaal Agricultural Journal. Vols. I-9. Pretoria, I902-IO (vol. I defective): B.S.I. [Afterwards combined with Nos. 972 and $988(a)$ and continued as No. 994.]

A.S. = Archaeological Section, Indian Museum.,

A.S.B. = Asiatic Society of Bengal.

B.C. = Bishop's College.

B.S.I. = Botanical Survey of India.

C.E. = Chemical Examiner to the Govt. of Bengal

c.U. = Calcutta University.
E.C. = Civil Engineering College, Sibpur.

G.S.I. = Geological Survey of India

I L. = Imperial Library

M.Ba. = Medical College, Bacteriological Laboratory

M.Bi. = Medical College. Biological Laboratory.

M.C. Medical College (main library). 
994. Pretoria.-Department of Agriculture, Union of South Africa.

Agricultural Journal of the Union of South Africa. Vols. I-. Pretoria, I9II-: B.S.I. [A continuation of Nos. 972, $988(a)$ and 993 in combination.]

_ Geological Survey of South Africa (see No. 995).

_ Geological Survey of the Transvaal (see No. 997).

995. - Mines Department, Union of South Africa.-Geological Survey.

Annual Reports. I9I0-. Pretoria, I9II- : G.S.I. [A continuation of No. $997(b)$.

996. - Report of the State Mining Engineer to the Government of the South African Republic. I896-98. Pretoria, I897-99: G.S.I.

997. — Transvaal Mining Department.-Geological Survey.

(a) Memoir. Nos. I-5. Pretoria, I905-Io: G.S.I.

(b) Report. I903-09. Pretoria, I904-IO : G.S.I. [Continued as No. 995.]

998. - Transvaal Museum.

Annals (Mededzelingen). Vols. I-. Pretoria, I9II-: Z.S.I.

999. St. Helena.-Magnetical and Meteorological Observatory.

Observations made at the .... Observatory. Vol. 2, I840-49.

London, I860: A.S.B.

Iooo. Salisbury.-Department of Agriculture, Rhodesia.

Rhodesia Agricultural Journal. Vols. 7-. Salisbury, I909- (vol. 7 defective) : B.S.I.

\section{ASIA.}

\section{ASIATIC RUSSIA.}

I00I. Tiflis.-Musée du Caucase (Kaukasisches Museum).

(a) Bulletin. Vols. 8-. Tiflis, I9I4-: Z.S.I. [A continuation of $(c)$.]

(b) Mémoires. Sér. A., nos. I-. Tiflis, I915-: Z.S.I.

(c) Milteilungen. Bde. I-7. Tiflis, I897-I9I3: Z.S.I. [Continued as $(a)$.]

м о = Meteorological Office, Alipore

$\mathbf{M}$ Ph $=$ Medical College, Physiological Laboratory.

P.C. = Presidency College.

P o = Patent Office.

$\mathbf{R} \mathbf{B} \mathbf{G}=$ Roval Botanic Garden

s.c.C. = Scottish Churches College.

20
S.G. = Surgeon-General's Library.

S.I. = Survey of India.

S.T.M. $=$ School of Tropical Medicine.

T.D. = Telegraph Department.

X.C. $=$ St. Xavier s College.
Z.S.I. $=$ Zoological Survey of India. 
I002. Tiflis.-Tïlissskiı Botanīcheskiı̌ Sad (Jardin Botanique de Tiflis). Trudui (Moniteur). Vuip. (Livr.) I-. Tiflis, Yuryer (Dorpat), I895-: R.B.G.

I003. Vladivostok.-Vostochny̌r Institut (Oriental Institute). İvyestiya (Bulletin). Tom. I一, I899/I900-. Wladiwostok, I900- (tom. I3, I8-20, 29, 42, 44, 47 wanting): A.S.B.

\section{CYPRUS.}

I004. Nicosia.-Cyprus Journal, a quarterly review of the agriculture and industries (and archaeology) of Cyprus. Vols. I-. Nicosia, I904-: B.S.I.

\section{INDIA AND CEYLON.}

Allahabad.-Archaeological Survey of India, Northern Circle (see (No. III3).

1005. - Brief sketch of the Meteorology of the United Provinces and adjacent parts of Rajputana and the Punjab. I896-I909. Allahabad, I897-I9Io: M.O.

1006. - Department of Land Records and Agriculture, United Provinces of Agra and Oudh.

Bulletin. Nos. I-. Allahabad, I895-: B.S.I., I.L.

I007. - Government of the North-Western Provinces.

(a) Selections from Public Correspondence... of the Government, North-Western Provinces. Parts I-43Agra, I849-67. 2nd ed., vols. I-4. Agra, I855-56. [Continued as $(b)$.]

Pts. $1-43,1849-67$ (pts. I, 2, 12 wanting); 2nd ed., vols. I, 2, I 855 : I.L.

and ed., vols. 2-4, I $855-56$ : G.S.I.

(b) Selections from the Records of Government, NorthWestern Provinces. New ser., vols. I-5 Allahabad, I864-69: G.S.I, I.L. 2nd ser., vols. I-6. Allahabad, I869-74: G.S.I., I.L.

roo8. - Indian Forester. Vols. I--. Mussoorie, Roorkee, Allahabad. Calcutta, I876-. [Numerous separately paged supplements in vols. I8-30, I892-1904.]

Complete sets : B.S.I., R.B.G., I.L.

Vols. I-, I 876 - (vol. 38 defective): A.S.B.

A.S. = Archaeological Section, Indian Mluseum.

A.S.B. = Asiatic Society of Bengal.

B.C. = Bishop's College.

B.S.l. = Botanical Survey of India

C.E. = Chemical Examiner to the Govt of Bengal.

C.U. a Calcutta University:
E.C. = Cuvir Engineering College, Sibpur. G.S.I = Geological Survey of Indaa.

I.L. = Imperial Library.

M.Ea. = Medical College, Bacteriolosical Laboratory M.Bi. = Medical Collese. Biological Laboratory. M.C. = Medical College (man library). 
roo8. Allahabad.-Indian Forester (Continued).

Vols. I4-, I888- (vol. 3I defective): Z.S.I.

Vols. 3I-, I905- : E.C.

Vols. 32-37, Igo6-I I : G.S.I.

- Indian Journal of Economics (see No. IOI5).

Ioog. - Indian Medical Journal. Ed. by C. W. S. Deakin. Vols. 3-9. Allahabad, Lahore, I884-89 (vols. 3, 4, 7 defective, vol. 5 wanting): I.L.

I0I0. - Indian Notes and Queries. Ed. by R. C. Temple. Vol. 4. [Allahabad]. I886-87 : I.L. [A continuation of No. Ior3; resumed as No. Ior2.]

IoII. - Indian Telegraphist. Vols. I-2I. Allahabad, I893-I9I3: T.D.

ror2. - North Indian Notes and Quevies. Ed. by W. Crooke. Vols. I-5. Allahabad, I89I-95. [For earlier series see Nos. IoIo and IOI3.]

Complete set : I.L.

Vols. 2-5, I892-95 : A.S.B., Z.S.I.

Ior3. - Punjab Notes and Queries. Eid. by R. C. Temple. Vols. I3. Allahabad, 1883-86. [Continued as No. 1010.]

Complete set: I.L.

Vols. I, I883: A.S.B.

IOI4. United Provinces Historical Society.

Journal Vols. I-. Calcutta, I9I7-: I.L.

IOI5. - University - Department of Eiconomics.

Indian Journal of Economics. Vols. I-. Allahabad, I9I6-: I.L.

IoI6. Bangalore.-Archaeological Department, Mysore State.

(a) Anmual Report of the ... Department. I904/05I909/IO. Bangalore, I905-IO. [A continuation of (b), continued as $(d)$.]

Complete set: I.L.

Reps. for 1908/09, 'og/10: A.S.B.

(b) Annual Report on the Archaeological Survey of Mysore. I903/04. Bangalore, I904: I.L. [Continued as $(a)$.]

(c) Epigraphia Carnatica. Ed. by B. L. Rice. Vols. [I]I2. Bangalore, I886-I905. [Vols. I-3 lack vol. nos.]

Complete set: I.L.

Vols. 5-I2, I9OI-05: A.S.

Vols. 5-II, I9OI-O5 (vol. 9 wanting): A.S.B.

м o. = Meteorological Office, Alipore.

M.Ph. = Medical College. Physiological Laboratory

P.C. = Presidency College.

P.o. = Patent Oifice.

R.B.G. = Royal Botanic Garden.

S.C.C. $=$ Scisttish Churches College.
S.G. = Surgeon-Generai s Library:

S I. = Survey of India.

S.T.M. = School of Tropical Medicine

T.D. = Telegraph Department

X.c. =St. Xavier s Collerse.

Z.S.1. = Zoological Survey of India 
Ior6. Bangalore.-Archaeological Department .. (Continued).

(d) Report on the working of the ...... Department. I9Io/II-. Bangalore, I9II- : A.S.B., I.L.

_- Archaeological Survey of Mysore (see No. Ior6 (b)).

ror7. - Department of Agriculture, Mysore State.

(a) Bulletin. Nos. I-. Bangalore, I902-: B.S.I.

(b) Bulletin. Entomological Series. Nos. I, 2. Bangalore, IgII : Z.S.I.

(c) Bulletin. Mycological Series. Nos. I- Bangalore, Igog-: B.S.I.

— Epigraphia Carnatica (see No. Ior6 (c)).

IOI8. - Ethnological Survey of Mysore.

[Report.] By H. V. Nanjundayya. Nos. I-. Bangalore, I906-: I.L.

Ior9. - Geological Department, Mysore State.

(a) Bulletin. Nos. I-. Bangalore, Igo4-: G.S.I.

(b) Memoirs. Vols. I-. Bangalore, [IgOI]- : I.L., G.S.I.

(c) Records. Vols. I-. I894/97-. Bangalore, [I900]-.

Complete set: G.S I.

Vols. I-, for I894/97- (vols. 5, 7, II wanting): A.S.B.

Vols. 5-, for I903/04-: I.L.

I020. Meteorological Department, Mysore State.

(a) Meteorology in Mysore: being results of observations at Bangalore, Mysore, Hassan and Chitaldrug. I893-. Bangalore, I894-.

Vols. for I893-I9II : M.O.

Vols. for I895-: I.L.

Vols. for I906- (vol. for 1908 wanting): A.S.B.

(b) Mysore Meteorological Memoirs. Nos. I- Bangalore, IgOI- : I.L.

(c) Report on Rainfall Registration in Mysore. I893-. Bangalore, I894-.

Vols. for I893-I9II : M.O.

Vols. for I 897 - (vols. for I905-IO wanting): A.S.B.

I02I, - Mysore Economic Conference.

Mysore Economic Journal. Vols. I-. Bangalore, I9I5(vol. I, no. 2 wanting): I.L.

- Mysore Meteorological Memoirs (see No. I020 (b)).

A.S. = Archaeological Section. Indian Museum.

A.S.B. = Asiatic Society of Bengal.

B.C. = Bishop s College.

B.S.I. = Botanical Survey of India.

C.E. = Chemical Examiner to the Govt. of Bengat.

c.U. = Calcutta University.
E.C. = Civil Engineering College, Sibpur.

G.S I. = Geological Survey of India.

I.L. = Imperial Library.

M.Ba. = Medical College, Bacteriological Laboratory

M.Bi. = Medical College. Biological Laboratory.

M.C. = Medical College (main library). 
I022. Bangalore.-Mythic Society.

Quarterly Journal. Vols. I-. Madras, [1909]-.

Complete set: I.L.

Vols. 6-, I9I5:I6-: A.S.B.

I023. - Planter's Chronicle. Vols. I-. Bangalore, I906-: B.S.I.

1024. - Quarterly Journal of Veterinary Science in India and Army Animal Management. Ed. by C. Steel and others. Nos. I-29. Bangalore, I882-90 (nos. 2-5 wanting) : I.L.

1025. Bankipore.-Bihar and Orissa Research Society.

Journal. Vols. I-. Bankipore, I9I5-: A.S.B., I.L.

1026. Bombay.-Agricultural and Horticultural Society of Western India.

Transactions. I839-42. Bombay, I842: I.L.

I027. - Agri-Horticultural Society.

Reports. I863, '64, '65, '68. Bombay, I864-69: I.L.

I028. - Anthropological Society.

Journal. Vols. I-. Bombay, [I886]-: A.S.B., I.L.

I029. - Archaeological Survey of India, Western Circle.

Progress Report. 1905'06-. Bombay, [1906]-. [A continuation of No. $\operatorname{LOzO}(a)$.]

Complete sets : A.S., I.L.

Reps. for 1905 06- (Reps. for 1907-08, 'o8-09 wanting):

A.S.B.

1030. - Archaeological Survey of Western India.

(a) Progress Report. I889 90-I904 05. Bombay, [I89o1905]. [Continued as No. 1029.]

Complete set : I.L.

Reps. for I889/90-I904 05 (Reps. for 1891/92, '96/97 wanting) : A.S.B.

Reps. for I889.90-I904/05 (Reps. 1890/9I, '98/991900 or wanting): A.S.

(b) Report. [Old series.] Nos. [I]-8, [8A], 9-II, IIA, I2. Bombay, I874-9I. [A revised edition of no. II, 1885 , was issued in 1897.]

Complete set: I.L.

Nos I-I2, I 874-9I (nos, 5, $7-9$ wanting): A.S.

Nos. $2,3,5-7,9$, I2, I875-9I : A.S.B.

(c) Report. [New series.] Vols. [I]-. London, Calcutta, Bombay, 1874- [Vols. I, 2, lack vol. nos. Vols. I-5 are subsequently referred to officially as vols. $I-5$ of the New

M.o. = Ireteorological Office. Alupore

M.Ph. = Medical College. Physiological Laboratory.

P.C. $=$ Presidency College.

P.o. = Patent Office

R. B.G. = Royal Botanic Garden.

S.C.C. $=$ Scottish Churches College.
S.G. = Surgeon-General's Library.

S.I. = Survey of India.

S.T.M. = School of Tropical Medicine.

T.D. = Telegraph Department.

X.C =St. Xavier's College.

z.s.l. Zoological Survey of India 
I030. Bombay.-Archaeological Survey of Western India (Continued).

Imperial Series (see No. Ira7 $(c)$ ); vols. $6,7,8$ are respectively vols. 23,24 and 16 of that series.]

Complete sets : A.S., I.L.

Vols. 2-, I876-: A.S.B.

I03I. - Department of (Land Records and) Agriculture, Bombay. Bulletin. Nos. I-. Bombay: I885-.

Nos. I-, I885- (nos. 2, 3 wanting): B.S.I.

Nos. $8-$, I 887 - (nos. I2, I3, 48 wanting): I.L.

I032. - Geographical Society.

Transactions. Vols. I-I9. Bombay, I844-74. Index to vols. I-I7, I $844-66$.

Complete sets : G.S.I., I.L.

Vols. I-I8, I844-68, and index: A.S.B.

I033. - Government of Bombay.

Selections from the Records of the Bombay Government.

Nos. I-I5. Bombay, I852-55. New ser., nos. I-.

Bombay, I854-. [No. 23 I not published.]

Nos. I-, I $852-$ (a few nos. wanting) : I.L.

New ser., nos. I-, I854- (a few nos. wanting): G.S.I.

I034. - Grant College Medical Society.

Transactions. No. I. Bombay, 1865 : A.S.B.

I035. - Indian Annals and Magazine of Natural Science. Conducted by J. A. Murray. Vol. I, nos. I-3. Bombay and London, I887 : I.L., Z.S.I.

I036. - Indian Antiquary: a journal of oriental research in archacology, epigraphy, ethnology, geography, history, folklore, languages, literature, numismatics, philosophy, religion, etc. Ed. by J. Burgess and others. Vols. I-. Bombay, I872-.

Complete sets : A.S.B., C.U., I.L.

Vols. I-, I872- (vols. 3I, 33 defective) : A.S.

I037. - Indian Textile Journal. Vols. I-. Bombay, I89I-

Vols. I-. I89I- (vol. I defective) : I.L.

Vols. 2 I-, I9IO- : B.S.I.

I038. Literary Society.

Transactions. Vols. I-3. London, I8I9-23: A.S.B., G.S.I.,

I.L. [For index see No. 1043.]

_ Marine Survey of India (sce No. Io84).

A.S. =Archaeological Section, Indian Museum.

A.S.B. $=$ Asiatic Society of Bengal.

B.c. = Bishop s College.

B.S.I. = Botanical Survey of India.

C.E. = Chemical Examiner to the Govt of Benga!

c.u. = Calcutta Unversity.
E.c. = Civil Engineering College, Sibpur.

G.S.I. = Geolosical Survey of Indta.

I L. = Imperial Library.

M.Ba. = Medical College, Bacteriological Laboratory

M. Bi. = Medical College. Biological Laboratory

M.C. = Medical College (main library). 
I039. Bombay.-Medical and Physical Society.

Transactions. Nos. I-Io. Bombay, 1838-5I. New series, nos. I-I2. Bombay, I85I-76. New series, nos. I-I2, Bombay, I882-89.

Nos. I-IO, I838-5I (no. 8 wanting); new ser., nos. I-I $2, I 85 I-76$ (no. 4 wanting); new ser., nos. I-I2, I882-89 (no. 2 wanting): I.L.

Nos. 2-IO, I839-5I; new ser., nos. I-II, I85I-7I : A.S.B.

I040. - Natural History Society.

Journal. Vols. I-. Bombay, I886-.

Complete sets: B.S.I., G.S.I., R.B.G., Z.S.I.

Vols. I-, I886- (vols. 7, 9-14 defective) : A.S.B.

Vols. 2-, I887- (vols. 4,6 defective): I.L.

104I. - Observatories (Colaba and Alibag).

Observations made at the Magnetical and Meteorological Observatory at Bombay. I845-47. Bombay, I846-5I. [Continued as:] Magnetical and Meteorological Observations made at the Hon. E. I. Company's Observatory, Bombay. I848-70. Bombay, I85I-72. [Continued as:] Magnetical and Meteorological (and Seismological) Observations made at the Government Observatory, Bombay. I87I/78-I9or. Bombay, I88I-I903. [Continned as:] Magnetical, Meteorological and Seismological Observations made at the Government Observatories, Bombay and Alibag. 1902-. Bombay, I903-.

Yols. for 1845 - (vols. for 1849, '59, '60, 62-64, '79-82, '8, '87, '91 wanting): M.O.

Vols. for I845-I9OI (vols. for $1883-85$ wanting): A.S.B.

Vols. for I87I-83: X.C.

Vols. for I87I-I905 (vols. for 1891, '92 wanting) : G.S.I. Vols. for I898-: I.L.

1042. - Orientalist, a journal of oriental literature, arts and sciences, folklore, etc. Ed. by W. Goonetilleke. Vols. I-4, pt. 8 . Bombay, I884-92: I.L.

In43. - Royal Asiatic Society, Bombay branch.

Journal. Vols. I-. Bombay, I84I-. Index to Trans. Lit. Soc. Bombay, vols. I-3, and to Journal, vols. I-I 7 .

Complete sets and index: A.S.B., C.U., G.S.I.

Vols. I-, I844- (vol. 7, 1863, wanting) : I.L.

Vols. I-4, I2, I3, I844-52, '76-77 : R.B.G.

Taprobanian (see No. I 107$)$.

M.O. = Meteorological Office, Ainpore

M.Ph = Medical College. Physiological Laboratory

P C. = Presidency College.

Po. = Patent Ofince.

R B.G = Roval Botanic Garder.

S C C $=$ Scottish Churches College.

\footnotetext{
s.G. = Surgeon-Generai's Library

S.t. = Survey or India.

S.T. $m=$ School of Trovical Meaicine.

T.D = Telegraph Debartmen:

X.C. =St Xaviers College.

z.s.l. = Zoological Survey of India.
} 
I044. Calcutta.-Agricultural and Horticultural Society of India.

(a) Journal. [Old series.] Vols. I-I4. Calcutta, I84267. Indexes to vols. I-9, I857, and to vols. I-I4, I867. [A continuation of $(c)$, continued as $(b)$.]

Complete set and index to vols. I-9 : G.S.I.

Complete set: B.S.I.

Vols. I-I 4, I $842-67$ (vol. 6 wanting): index to vols. I-I 4 : I.L.

Vols. I-I3, I842-65: A.S.B.

(b) Journal (and Proceedings). New series, vols. I-. Calcutta, 1867 -. [A continuation of $(a)$.]

Complete sets : B.S.I., G.S.I.

Vols. I-, I867- (rols. 3, 4 defective) : A.S.B.

Vols. I-II, I867-I 901 : G.S.I.

Vols. I-II, I807-97 (vols. 8, IO, 1 I defective) : I.L.

(c) Transactions. Vols. I-8. Serampore, Calcutta, I8294I. [Continued as $(a)$.]

Complete sets: B.S.I., I.L.

Vols. I-8, I829-4I (vol. 7 wanting): G.S.I.

Vols. I-3, I829-37; vol. 8 , I84I : A.S.B.

- Agricultural Journal of India (see No. II4I (a)).

I045. - Agricultural Ledger. Ed. by the Reporter on Economic Products to the Govt. of India. Vols. [I]-I7, I892-I9II/I2. Calcutta, I892-I9I3. [Divided into numerous separately paged series.] General indexes to issues of I892-99 in no. I3 of I899; to issues of $1900-05$ in no. I2 of I905; to issues of I906-I2 in no. 6 of IgII/I2.

Complete sets: B.S.I., G.S.I., R.B.G.

Vols. for I892-I9II I2 (vols. for 1894, '95, I9or, '07 defective): A.S.B.

Vols. for I892-I9II/I2 (vols. for 1896, '98 defective): I.L.

Vols. for I892-I907: S.G.

Agricultural Research Institute (see No. II4I).

I046. - Archaeological Survey of India.

(a) Corpus Inscriptionum Indicarum. Vols. I and 3 . Calcutta, I877, I888. [Vol. 2 not published.]

Vols. I, 3, 1877 , '88 : A.S.B., I.L.

Vol. I, I877 : A.S., G.S.I.

(b) Epigraphia Indica : a collection of inscriptions supplementary to the Corpus Inscriptionum Indicarum. Ed.

A.S = Archaeological Section. Indian Museum

A.S.B. = Asiatic Society of Bengal.

B.C. = Bíshop s College.

B.S.I. = Botanical Survey of India.

C.E. = Chemical Examiner to the Govt of Bengal

c.u. =Calcutta University
E.c. = Civil Engineering College, Sibpur.

G.S.I. = Geological Survey of India.

I.L. = Imperial Library.

M.Ba. = Medical College, Bacteriological Laboratory

M.BI. = Medical College, Biological Laboraiory.

M.C. = Medical College (man library) 
1046. Calcutta.-Archaeological Survey of India (Continued).

by J. Burgess and others. Vols. I-. Calcutta, I $89_{2}$-. [Vol. I is subsequently referred to as vol. I 3 of the New Imperial Series (No. I $147(c)$ ) and rol. 2 is vol. I4 of that series. Vol. 3, I $894 / 95$, was issued as a supplement to the Indian Antiquary (No. 1036).]

Complete sets : A.S., C.U., I.L.

Vols. I-, I892- (vol. 9, pt. 4 wanting): A.S.B.

Vols. I, 2, I892-94 (vol. 2 defective) : G.S.I.

(c) Epigraphia Indo-Moslemica: Supplementary to Epigra. phia Indica. Ed. by E. D. Ross and (afterwards) J. Horovitz. Vols. I-. Calcutta, I907-: A.S., A.S.B. I.L.

(d) Reports. By A. Cunningham. Vols. I-23, I86 I 62I $884 / 85$. Calcutta, Simla, I87I-87. Index to vols. I-23.

Complete sets and index : A.S., A.S.B., I.L.

Complete set: G.S.I.

Vols. I-23, I $87 \mathrm{I}-87$ (vol. 5 wanting), and index: C.U.

(e) Reports. New Imperial Series (see No. I I47 (c)).

I047. - Archaeological Survey of India, Eastern (Bengal) Circle.

Annual Report. I900/o I-. Calcutta, [I90I]-.

Complete sets: A.S., I.L.

Reps. for I900/OI-(Rep. for 1903/04 wanting): A.S.B.

I048. — Asiatic Society of Bengal.

(a) Aszatick Researchis, or iransactions of the Society, instituted in Bengal, for inquiring into the history and antiquities, the arts, sciences and literature of Asia. Vols. I-20. Calcutta, I788-I836. Index to vols. I-I8. [For index to vols. 19,20 see $(d)$ ].

Complete sets and index : A.S.B., R.B.G., Z.S.I.

Vols. I-20, I7 $88-I 836$ (plates of vol. I9 wanting) and index : I.L.

Vols. I-I9, I7 $88-1836$, and index: B.C.

Vols. I2-20, I8I6-I836 (vol. 15 wanting) and index: G.S.I.

(b) Asiatick Researches ..... Vols. I-I2. London, I798I8I8. [An unauthorised reprint.]

Complete set: I.L.

Vols. I-I2, I806-I 8 (mostly of 3 rd, $4^{\text {th }}$ and 5 th editions) : A.S.B.

Vols. I-II, I798-I8I2 : G.S.I.

M.o. = Meteorological Office, Alipore.

M.Ph. = Medical College, Physiological Laboratory.

P.C. = Presidency College.

P.o. = Patent Office.

R.B.G. = Royal Botanic Garden

S.C.C. $=$ Scottish Churches College.

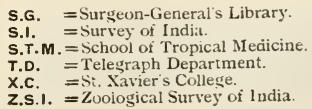

S.G. = Surgeon-General's Library

S.I. = Survey of India.

S.T.M. = School of Tropical Medicine.

T.D. = Telegraph Department.

X.C. =Si. Xavier's College.

Z.s.l. = Zuoiogical Survey of India 
I048. Calcutta.-Asiatic Society of Bengal (Continued).

(c) Asiatick Researches. Popular edition. Vols. I, 2. Calcutta, I884-[85]: A.S.B., I.L.

(d) Journal. Vols. I-75. Calcutta, I832-I904-. [Vols. 74, 75 contain "Materials for a Flora of the Malayan Peninsula ", still in progress. Vols. 34-73 are divided into two or three separately paged sections, according to subject, viz. "History, antiquities, etc.," "Natural history" and "Anthropology." The series commenced as No. 1062. After 1904 united with $(g)$ and continued as $(e)$.] Index to vols. I920 of the Asiatick Researches and to vols. I-23 of the Journal.

Complete sets and index: A.S.B., G.S.I., I.L.

Complete sets : C.U., R.B.G.

Vols. I-I9, I832-50 (vols. 3, 4 wanting, vol. 7 defective) : B.C.

Vols. 7-75, I838-I904-(vols. 22, 23, 29 wanting): Z.S.I.

Vols. 25-73, I857-I904 (vols. 3I, 33, 36, 37, 42 wanting): E.C.

(e) Journal and Proceedings. New series, vols. I-. Calcutta, I905-: A.S.B., C.U., E.C., G.S.I., I.L., R.B.G., Z.S.I.

(f) Memoirs. Vols. I-. Calcutta, I905-: A.S.B., G.S.I., I.L., R.B.G., Z.S.I.

(g) Proceedings. I865-I904. Calcutta, I866-I905. [Subsequently united with $(d)$ to form $(e)$.]

Complete sets: A.S.B., C.U., G.S.I., I.L., R.B.G., Z.S.I. Issues of I 866, '67, '7I-87, '92-I90I, '03, '04 : E.C.

(h) Recherches Asiatiques, ou mémoires de la société........ traduis de l'anglois par A. Labaume: revus et augmentés de notes. 2 tom. Paris, an. xiv-I805: I.L. [A French edition of $(a)$.]

I049. - Astronomical Society of India.

Journal. Vols. I-. Calcutta, I9IO-: I.L., P.C.

I050. - Bengal Economic Association.

Bengal Economic Journal. Ed. by C. J. Hamilton and J. C. Coyajee. Vols. I- Calcutta, IgI6-: I.L., S.C.C.

- Board of Scientific Advice (see No. I I48).

ro5r. - Botanical Survey of India.

Records. Vols. I-. Calcutta, I893-.

Complete sets : A.S.B., B.S.I., I.L., R.B.G., Z.S.I.

Vols. I-5, I893-I9I2 (vols. I, 5 defective): G.S.I.

A.S. = Archaeological Section, Indian Museum

A.S.B. = Asiatic Society of Bengal.

B.C. = Bishop's College.

B.S.1. = Botanical Survey of India

C.E. = Chemical Examiner to the Govt. of Bengal.

c.U. = Calcutta University.
E.C. = Civil Engineering College, Sibpur. G.S.I = Geolosical Survey of India.

i.L. = Imperial Library

M.Ba. = Medical College, Bacteriological Laboratory

M.Bi. = Medical College. Biological Laboratory.

M.C. = Medical College (main library) 
I052. Calcutta.-Calcutta Journal of Medicine. Ed. by M. L. Sircar and (afterwards) A. L. Sircar. Vols. I-. Calcutta, I868一.

Vols. I-, I 868 - (vol. 9 wanting) : I.L.

Vols. I-8, I868-76 (vols. 4, 5 defective); vols. 29-, IgII- : A.S.B.

I053. - Calcutta Journal of Natural History (and miscellany of the arts and sciences in India). Conducted by J. M'Cleliand and others. Vols. I-8. Calcutta, I84I-47. General index, vols. I-8, at end of vol. 8

Complete sets: A.S.B., G.S.I., I.L., R.B.G.

Vols. I -8 , I 84 I -47 (vol. 7 wanting): Z.S.I.

Calcutta Medical Journal (see No. I087).

I054. - Calcutta Medical Neres. Vol. I, nos. I-8. Calcutta, I880: I.L.

1055. - Civil Veterinary Department of India.

(a) Journal of Tropical Veterinary Science. Ed. by H. F. Pease. Vols. I-7, no. I. Calcutta, I906-I2.

Complete sets: I.L., Z.S.I.

Vols. I-6, Igo6-II : S.T.M.

(b) Memoirs. Nos. I-3. Calcutta [I909-II]. [Continued as No. 114 I (c), Veterinary Series.]

Complete set : I.L.

Nos. I, 2, I909-IO: A.S.B.

- Corpus Inscriptionum Indicarum (see No. I046 (a)).

I056. - Department of (Land Records and) Agriculture, Bengal.

(a) Bulletin. Agricultural series. Nos. I-8. Calcutta, I896-I906: I.L. [Continued as (c).]

(b) Bulletin. V'eterinary series. No. I. Calcutta, 1896 : I.L.

(c) Quarterly Journal. Vols. I-. Calcutta, Igo8-.

Complete sets : B.S.I., R.B.G.

Vols. I-, I908- (vol. 3 defective): I.L.

- Department of Agriculture, Government of India (see No. II4I).

1057. — Department of Fisheries, Bengal (Bihar and Orissa).

Bulletin. Nos. I-. Calcutta, I9I3-: I.L., Z.S.I.

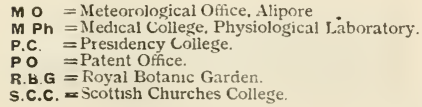

S.G. = Surgeon-General's Library:

S.I. = Survey of India.

S.T.M. = School of Tropical Medicine.

T.D. = Telegraph Department.

X.c. $=$ St. Xavier s college

Z.S.1. = Zoological Survey of India. 
I058. Calcutta.-Department of Mines, India.

Report of the Inspection of Mines in India. 1893/94-1900. Calcutta, I894-IgoI : G.S.I. [Continued as:] Report of the Chief Inspector of Mines in India. IgOI-. Calcutta, I902-: G.S.I., I.L.

I059. - Engineer's Journal. Vols. I-I2. Calcutta, I858-69.

Vols. I-I2, I858-69 (vol. 4, I86I wanting): E.C.

Vols. I-9, I $858-66$ : G.S.I.

Vols. 3-II, I860-68: I.L.

_- Epigraphia Indica (see No. $1046(b)$ ).

_- Epigraphia Indo-Moslemica (see No. $1046(c))$.

_ Forest Research Institute (see No. IIOg).

I060. - Gardener's Magazine. Vols. I-I3. Calcutta, I899-I9II (vols. I, 8, IO-I3 defective) : I.L.

I061. - Geological Survey of India.

(a) Annual Report. I858,59-67. Calcutta, I859-68: G.S.I., I.L. [Afterwards incorporated in $(f)$.]

(b) General Report. I897/98-I902/03. Calcutta, I898I903. [Published during a period when the issue of the Records was suspended.]

Complete sets: G.S.I., I.L., Z.S.I.

Reps. for 1899/1900-1902/03: A.S.B.

(c) Palaeontologia Indica: being figures and descriptions of the organic remains procured during the progress of the ... Survey ... Calcutta, I86I-I9I2. [Divided into I6 series as under:]

Series I, 3, 5, 6, 8. Cretaceous Fauna of Southern India. Vols. I-4. I86I-73.

Series 2, II, I2. Fossil Flora of the Gondwana System. Vols. I-4. I863-86.

Series 4. Indian Pretertiary I'ertebrata. Vol. I. I $865-85$.

Series 7, I4. Tertiary and Upper Cretaceous Fauna of Western India. Vol. I. I87I-85.

Series 9. Jurassic Fauna of Kach. Vols. I-3. I873I903.

Series Io. Indian Tertiary and Post-tertiary Vertebrata. Vols. I-4. I $874-87$.

Series I3. Salt-Range Fossils. Vols. I, 2, 4. I879$9 \mathrm{I}$.

Series I5. Himalayan Fossils. Vols. I-7. I895I9I 2.

A.S. =Archaeological Section, Indian Museum.

A.S.B. = Asiatic Society of Bengal.

B.C. = Bishop s College.

B.S.l. = Botanical Survey of India.

C.E. = Chemical Examiner to the Govt. of Bengal.

c. $\mathrm{U}$. Calcutta University.
E.c. = Civil Engineering College, Sibpur.

G.S.I. = Geological Survey of India.

I.L. = Imperial Library.

M.Ba. = Medical College, Bacteriological Laboratory

M.Bi. = Medical College. Biological Laboratory

M.C. = Medical College (main library) 
I06I. Calcutta.-Geological Survey of India (Conlinued).

Series r6. Baluchistan Fossils. Vol. I. I895-97.

Complete sets: A.S.B., C.U., G.S.I., I.L., P.C., R.B.G., Z.S.I. [Continued as:]

New series, vols. I-. Calcutta, I899-: A.S.B., C.U., G.S.I., I.L., P.C., R.B.G., Z.S.I.

Index to the genera and species described up to I89I, I892: A.S.B., C.U., G.S.I., I.L., P.C., R.B.G., Z.S.I.

(d) Memoirs. Vols. I-. Calcutta, I859-. Indexes to vols. I-20, I892; to vols. 2 I-35, I9I6.

Complete sets and indexes: E.C., G.S.I., I.L., R.B.G., Z.S.I.

Vols. I-, I 859- (vol. 3 defective) and indexes : A.S.B.

Vols. I-, I 859- (vol. 18 defective) and indexes: P.C.

Vols. I-4I, I859-I9I3 (many vols. defective): M.O.

(e) Quarterly Notes. I895-I906. Calcutta, I895-I906.

Complete set : G.S.I.

Parts for I895-1903 O4 (Pts. for 1895, 1902 03, 1903/04 defective): I.L.

(f) Records. Vols. I-. Calcutta, I870-. [See note under

(b).] Indexes to vols. I-20, I89o; to vols. $2 \mathrm{I}-30$, I898.

Complete sets and indexes: A.S.B., C.E., C.U., G.S.I., I.L., R.B.G., Z.S.I.

Vols. I-43, I870-I9I3 (vol. 43 defective): M.O.

Vols. 3-, I870- (rols. 6, I4 defective): P.C.

Vols. 7-, IS74- (vols. I4, 29, 30, 42 defective): M.C.

I062. _- Gleanings in Science. [Ed. by J. D. Herbert and J. Prinsep.] Vols. I-3. Calcutta, IS29-3I: A.S.B., G.S.I., I.L., R.B.G. [Continued as No. $\mathrm{IO}_{4} 8(d)$.]

I063. - Government of Bengal.

Selections from the Records of the Bengal Government.

Nos. I-46. Calcutta, I85I-73.

Nos. I-46, I $85 \mathrm{I}-73$ (no. 44 wanting) : G.S.I.

Nos. I-43 A, I85I-69: I.L.

_- Great Trigonometrical Survey (see No. Iogs $(b, d, k))$.

Illustrations of the Zoology of the Royal Indian Marine Sur. vey Ship 'Investigator' (see No. Io80 (a)).

I064. - India Journal of Medical and Physical Science. Ed. by F. Corbyn. New Ser., vol. I. Calcutta, I836: A.S.B. [For earlier series see No. 1065.]

\footnotetext{
M.o. = Meteorological Otice, Alipore

M.Ph. = Medical College, Physiological Laboratory.

P.C. = Presidency College.

P.O. = Patent Office.

R.B. $\mathbf{G}=$ Royal Botanic Garden

S.C.C. $=$ Scottish Churches College.
}

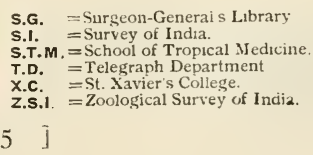

S.G. = Surgeon-Generais Library

.I. = Survey of India.

T.D. = Telegraph Department

x. $=$ St Xavier's College. 
1065. Calcutta.-India Journal of Medical Science. Ed. by J. Grant and J. T. Pearson. Tols. I, 2. Calcutta, I834-35: A.S.B. [Continued as No. 1064.]

I066. - India Review and Journal of Foreign Science and the Arts. Ed. by F. Corbyn. Vols. I-8. Calcutta, I837-47.

Complete set: I.L.

Vols. I -7, I $837-43$ : G.S.I.

Vols. I-6, I $837-42$ (vol. 5 wanting) : A.S.B.

_- India Weather Review (see No. II5I $(c)$ ).

1067. - Indian Agriculturist: a monthly journal of Indian agriculture, mineralogy and statistics Vols. I-. Calcutta, I876-.

Complete set: B.S.I.

Vols. I-I6, I876-91 : I.L.

ro68. — Indian and Eastern Engineer. Vols. 25-28. Calcutta, I89597. New series, vols. I-. Calcutta, I897- [For earlier vols, see No. 1072.]

Complete sets: G.S.I., I.L.

Vols. 25-28 and new ser., vols. I-27, I895-I9Io (vols. 5, 15, 24, 25 wanting): E.C.

New ser., vols. I-25, I897-I909: B.S.I.

New ser., vols. 3-, I898- (vols. 17,20 , 30 wanting, others defective) : A.S.B.

I069. - Indian Annals of Medical Science. Vols. I-[I9], = Nos. [I]37. Calcutta, London, I853-77. [Each volume, except the last, consists of two numbers.]

Nos. I-37, I853-77 (nos. 17, 28, 33-35 wanting): M.C.

Nos. I-32, I853-74 (nos. 17, 28 wanting): A.S.B.

Nos. I-29, I $853-72$ (no. 28 wanting) : G.S.I.

Nos. I-25, I853-69; no. 37, IS77 : I.L.

Nos. 2-2I, I854-67 : R.B.G.

Nos. 27, 35-37, I87I-77: S.G.

I070. - Indian Association for the Cultivation of Science.

(a) Bulletin. Nos.3-. Calcutta, I9II-.

Nos. 3-, I9II-: I.L.

Nos. 6-, I9I2-: A.S.B.

(b) Proceedings. Vols. I-. Calcutta, I9I7-: A.S.B., I.L.

(c) Report. 1897-. Calcutta, I897-.

Reps. for I897- (Reps. for 1903-05, 1908, 1910-12 wanting) : I.L.

Reps. for I9I3- : A.S.B.

A.S. = Archaeological Section. Indian Museum. A.S.B. = Asiatic Society of Bengal.

B.C. = Bishop s College.

B.S.I. = Botanical Survey of India.

C.E. = Chemical Examiner to the Govt. of Bengal.

C.U. = Calculta University.
E.C. = Civil Enrineering College, Sibpur.

G.S I. = Geological Survey of India.

I.L. = Imperial Library.

M.Ba. = Medical College, Bacteriological Laboratory

M.Bi. = Medical College, Biological Laboratory.

M.C. = Medical College (main library). 
I07I. Calcutta.-Indian Economist: a monthly journal devoted to economic and statistical enquiries concerning India. E.d. by R. Knight. Vols. I-6. Calcutta, 1870-75 : G.S.I., I.L.

I072. - Indian Engineer. Vols. I-23. Calcutta, I886-95. [For con. tinuation see No. I068.]

Complete sets: E.C., G.S.I., I.L.

Vols. I-3, I886-87: A.S.B.

I073. - Indian Engineering. Vols. I-. Calcutta, I887-.

Complete set : I.L.

Vols. I-, I 887 - (vol. 49 defective) : A.S.B.

Vols. I-, I887- (vol. I3, I4 wanting) : E.C.

Vols. I-I6, no. 7, I887-94: G.S.I.

Vols. 3-, I888-: M.O.

I074. Indian Engineers' Association.

Transactions. Vol. I. Calcutta, I872: A.S.B.

—_Indian Forest Memoirs (see No. IIog (e)).

- Indian Forest Records (see No. IIog $(f)$ ).

_- Indian Forester (see No. I008).

1075. - Indian Gardening. Ed. by H. St. John Jackson. Vols. I-5. Calcutta, I897-99. [Continued as No. ro8I.]

Complete set: R.B.G.

Vol. I, nos. I-I5; vol. 2 , no. I : I897-98 : I.L.

Vols. 3-5, I898-99: A.S.B.

- Indian Journal of Medical Research (see No. I079(a)).

ro76. —-Indian Lancet. Ed. by L. Fernandez. Vol. 6, no. 9-vol. 3I, no. I7. Calcutta, I895-I908. [For earlier vols. see No. I09o.]

Complete set : I.L.

Vols. 6-30, I897-rgo7 (vols. I6-19, 23, 24, 29 defective): S.G.

Vols. 7-3I, I896-I908 (many vols. defective) : A.S.B.

1077. - Indian Medical Gazette. Vols. I- Calcutta, I866-.

Vols. I-, I866- (vols. II wanting) : M.C.

Vols. I-, I866- (vols. 4-9, I I-13, I5, I6 wanting, vols. I, 2, IO, 21, 25, 27, 29, 36 defective) : I.L.

Vols. 2-, I867- (vol. 23 wanting) : A.S.B.

Vols. $15-$, 1880- (vols. $17-2 n, 23,26$ wanting) : S.G.

Vols. $25,26,37,39,4$ I, 42, I900-07 : R.B.G.

Vols. 29-, I894-: B.S.I.

Vols. 42-, I907- (vols. 42, 44, 45 defective) : Z.S.I.

M.o. = Meteorological Office, Alipore.

M.Ph. = Medical College, Physiological Laboratory.

P.C. = Presidency College.

P.O. = Patent Office

R.B.G $=$ Royal Botanic Garden

S.C.C. $=$ Scottish Churches College.
S.G. =Surgeon-General's Library.

s.l. = Survey of India.

S.T.M. = School of Tropical Medicine

T.D. = Telegraph Department.

X.C. '=St. Xavier's College.

Z.S.t. EZoological Survey of India. 
I078. Calcutta.-Indian Medical Record. Vols. 2-. Calcutta, I89I-. [For vol. I see No. 1089 .]

$$
\begin{aligned}
& \text { Vols. } 2-\text {, I } 89 \text { I- (vols. } 27-30,32 \text { defective) : I.L. } \\
& \text { Vols. } 4-\text {, I } 893-(\text { vol. } 5 \text { wanting) : S.G. } \\
& \text { Vols. } 30-\text {, I9IO-: A.S.B. }
\end{aligned}
$$

I079. — Indian Medical Service.

(a) Indian Journal of Medical Research. Vols. I- Calcutta, I9I2- : B.S.I., I.L., M.Ba., M.C., R.B.G., S.G., Z.S.I.

(b) Scientific Memoirs by Medical Officers of the Army of India Parts I-I2. Calcutta, I885-I901. [Continued as $(c)$.]

Complete sets : A.S.B., B.S.I., I.L., S.G., Z.S.I.

Parts I-6, I 885-9I : G.S.I.

(c) Scientific Memoirs by Officers of the Medical and Sanitary Departments of the Government of India. New series, nos. I-60. Calcutta, I902-I3.

Complete sets : A.S.B., B.S.I., I.L., S.G.

Nos. 2-60, I902-I3 : Z.S.I.

—_ Indian Meteorological Memoirs (see No. II5I (b)).

I080. Indian Museum.

(a) Illustrations of the Zoology of the Royal Indian Marine Survey Ship 'Investigator.' Crustacea Entomostraca. Parts I, 2. Calcutta, 1907-08: Z.S.I. Crustacea Malacostraca. Parts I-I2 Calcutta, I892-I907 : Z.S.I. Echinoderma. Parts I, 2. Calcutta, I894-95: Z.S.I. Fish. Parts I-9. Calcutta, I892-I908: Z.S.I. Mollusca. Parts I-6. Calcutta, I897-I909: Z.S.I. [Plates subsequently published in this series are issued and bound with o. $1099(a)$.]

(b) Indian Museum Notes. Vols. I-6, no. I. Calcutta, I889-I903: A.S.B., B.S.I., I.L., Z.S.I. [A continuation of $(d)$.]

(c) Memoirs (see No. $1099(a)$ ).

(d) Notes on Economic Entomology. Nos. I, 2. Calcutta, I 888 : A.S.B., I.L., Z.S.I. [Continued as (b).]

(e) Records (see No. Iog9 (b)).

1081. - Indian Planting and Gardening. Ed. by H. St. John Jackson. Vols. 6-. Calcutta, I900-: R.B.G. [A continuation of No. 1075.]

1082. - Indian Tea Association.

Quarterly Journal. Nos. I- Calcutta, I90I-: Z.S.I.

\footnotetext{
A.S. ${ }^{-}=$Archaeological Section, Indian Museum.

A.S.B. = Asiatic Society of Bengal.

B.C. = Bishop's College.

B.S.I. = Botanical Survey of India.

C.E. $x$ Chemical Examiner to the Govt of Bengal.

c.U. $\rightarrow$ Calcutta University.
}

E.C. = Clvil Engineering College, Sibpur.

G.S.I = Geological Survey of India.

l.L. = Imperial Library.

M.Ba. = Medical College, Bacteriological Laboratory

M.Bi. = Mfedical College. Biological Liboratory.

M.c. = Medical College (man library). 
I083. Calcutta.-Inland (Imperial) Customs Department.

Report on the Administration of the .... Department with appendices). I867/68-8o/8I. Calcutta, Agra, Allahabad, I868-8I : G.S.I.

- Journal of Tropical Veterinary Sience (see No. I055 (a)).

I084. — Marine Survey of India.

(a) Administration Report of the ... Survey... I883/84-. Poona, Bombay, I884-. [A continuation of $(b)$, ]

Reps. for I883/84- (Reps. for $1884 / 85^{-}$'86/87, I905/06 wanting) : Z.S.I.

Reps. for I883/84- I900/OI (Rep. for $1892 / 93$ wanting) : R.B.G.

Reps. for I886/87-I905/06 : G.S.I.

(b) General Report on the Operations of the ... Survey. I $874-80 / 81$. Calcutta, I876-82: Z.S.I. [Continued as $(a)$.]

1085. Mathematical Society.

Bulletin. Nos. I-. Calcutta, Igog-: C.U., I.L.

I086. - Medical and Physical Society.

Transactions. Vols. I -9. Calcutta, I825-45.

Complete sets : G.S.I., I.L.

Vols. I-7, I825-35: A.S.B.

Vols. 2-8, I826-36 : R.B.G.

I087. - Medical Club.

Calcutta Medical Journal. Vols. I- Calcutta, I906-.

Complete sets : C.U., I.L., M.C.

Vols. I-, Igo6- (vol. 4 wanting) : A.S.B.

I089. - Medical Record. Ed. by J. R. Wallace. Vol. I. Calcutta, I890: I.L. [Continued as No. 1078.]

Iogo. - Medical Reporter. Ed. by L. Fernandez. Vols. I-6, no. 8. Calcutta, I892-95. [For continuation see No. 1076.]

Complete set : I.L.

Vols. I-6, I892-95 (vols. 2,5 wanting) : S.G.

Vols. 3-6, I $894-95$ : A.S.B.

Iogr. Medico-Surgical Journal of the Tropics. Vols.2-. Calcutta, I909-: I.L.

Meteorological Department, Government of India (see No. II5I).

M.o. = Meteorological Office, Aiipore

M.Ph $=$ Medical College. Physiological Laboratory

PC. = Presidency College.

P O. = Patent Office.

R B.G = Royal Botanic Garden

S C C $=$ Scottish Churches College.
S.G. = Surgeon-Generai's Library:

S.I. = Survey of India.

S.T. $\mathbf{M}=$ School of Tropical Mecicins

T.D. $=$ Telegraph Departmeni.

X.C. $=$ St Xavier s College.

Z.S.I. $=$ Zoological Survey of India. 
I092. Calcutta.-Meteorological Reporter to the Government of Bengal. Report. I $86768-74$. Calcutta, I868-75: G.S.I.

I093. - Microscopical Society.

Bulletin. Vols. I-6. [Calcutta], I80I-97 (defective) : A.S.B.

I094. - Mining and Geological Institute of India.

Transactions. Vols. I-. Calcutta, Igo6-: A.S.B., G.S.I.

I095. - Oriental Miscellany: consisting of original productions and translations. Vol. I. Calcutta, I798: G.S.I.

- Palaeontologia Indica (see No. Io6I (c)).

—_ Paludism (see No. II 49).

- Rainfall of India (see No. II5I $(e)$ ).

Iog6. - Royal Botanic Garden.

Annals. Vols. I-. Calcutta and London, I887- : A.S.B., B.S.I., C.U., G.S.I., I.L., R.B.G., Z.S.I.

I097. - Stray Feathers, a journal of ornithology for India and its dependancies. Ed. by A. Hume. Vols. I-II. Calcutta, I873[88].

Complete set: I.L.

Vols. I-II, I873-88 (vol. 11 defective) : Z.S.I.

Vols. I-IO, I873-87 : A.S.B.

Iog8. - Survey of India.

(a) Abstract of the Results of the Hourly Meteorological Observations taken at the Surveyor General's Office. I 853-77. [Calcutta, I853-77]: A.S.B., M.O., S.I. [A continuation of $(h)$.]

(b) Account of the Operations of the Great Trigonometrical Survey of India. Vols. I-. Dehra Dun, Calcutta, I 870 -.

Complete sets : C.U., G.S.I., I.L., S.I.

Vol. I-IQ, I870-IQIO : Z.S.I.

Vols. I-4I, I870-90 (vols. I3, 20-34, 36-40 wanting) :

A.S.B.

(c) Extracts from the Narrative Reports of Officers of the Survey of India. Seasons I900/0I-I908/09. Calcutta, I903-II. [Continued as $(j)$.]

Complete sets : I.L., S.I.

Seasons I902/03-'08/09, I905-II : G.S.I.

(d) General Report on the Operations of the Great Trigonometrical Survey of India. I861/66-I876/77. Dehra

A.S. = Archaeological Section, Indian Museum.

A.S.B. = Aslatic Society of Bengal.

B.C. = Bishop s College.

B.S.I. = Botanical Survey of India.

B.S.I. = Botanical Examiner to the Govt. of Bengal.
C.U. = Calcutta University.
E.C. = Civil Engineering College, Sibp̣ur.

G.S.I. = Geolosical Survey of India.

I.L. = Imperial Lıbrary.

M.Ba. = Medical College, Bacteriological Laboratory

M.BI. = Medical College. Biological Laboraiory.

M.C. = Medical College (mani library). 
Iog8. Calcutta.-Survey of India (Continued).

Dun, Roorkee, Calcutta, I869-78. [Afterwards conbined with $(f)$ and $(g)$ to form $(e)$.]

Complete set : S.I.

Reps. for $186364-1876 / 77$ : I.L.

Reps. for I866 67-I876/77 : G.S.I.

(e) General Report on the Operations of the Survey of India. I877 78 -. Calcutta, I879-. [A continuation of $(d)$, $(f)$ and $(g)$ in combination.]

Complete sets : G.S.I., I.L., S.I.

Reps. for I880/8I-I9IO/II (Reps. for I887/88, '9o' 9 I, '94/95, '98/99, 1903/04, '06/o7 wanting): M.O.

(f) General Report on the Revenue Survey Operations. I860/6I-I876/77. Calcutta, I863-78. [Afterwards combined with $(d)$ and $(g)$ to form $(e)$.]

Complete set: S.I.

Reps. for $1864 / 65-76 / 77$ : G.S.I.

(g) General Report on the Topographical Survey Operations. I860/6I-I876/77. Calcutta, Dehra Dun, I863-78:

S.I. [Afterwards combined with $(d)$ and $(f)$ to form $(e)$.]

(h) Meteorological Register kept at the Surveyor General's Office I840-52. Calcutta, I840-52: A.S.B., M.O. [Continued as $(a)$.]

(i) Professional Paper. Nos. I-. Dehra Dun, Calcutta, I899-.

Complete sets : I.L., S.I.

Nos. I-, I899- (nos. 3, 4 wanting) : G.S.I.

Nos. I2, I3, I9I2: Z.S.I.

(j) Records. Vols. I- Calcutta, I9I2- : A.S.B., G.S.I., I.L., S.I. [A continuation of $(c)$.]

(k) Synopsis of the Results of the Operations of the Great Trigonometrical Survey of India. Tols. I-. Dehra Dun, I874-.

Complete set : S.I.

Vols. I-, I 874- (vol. 22 wanting): I.L.

Vols. I-35, I 874-Igog (vol. 22 wanting): G.S.I.

Vols. I-34, I874-94 (vol. 22 wanting) : Z.S.I.

1099. Zoological Survey of India.

(a) Memoirs of the Indian Museum. Vols. IIg07- : A.S.B., B.S.I., G.S.I., I.L., Z.S.I.

(b) Records of the Indian Museum. Vols. I-. Calcutta, I907-: A.S.B., B.S.I., G.S.I., I.L., M.Bi., Z.S.I.

\footnotetext{
M. o. = Meteorologicai Office, Alipore.

M.Ph. = Medical College, Physiological Laboratory.

P.c. = Presidency College.

P. = Patent Office

R.B.G. = Royal Botanic Garden

S.C.C. $=$ Scottish Churches College.
}

S.G. = Surgeon-General's Library

S.I. = Survey of India.

S.T.M. = School of Tropical Medicine

T.D. = Telegraph Department.

X.c. = St. Xavier's College.

Z.S.I. = Zoological Survey of India.

Calcutta, 
II0o. Colombo.-Archaeological Survey of Ceylon.

(a) Annual Report of the ..... Survey.... North-Central (and Sabaragamuwa) Provinces. I890-I907. Colombo [I89I-I908]: I.L.

(b) Epigraphia Zeylanica, being lithic and other inscriptions of Ceylon. Vols. I-. London, I904- : I.L.

I Iог. - Ceylon Antiquary and Literary Register. Ed. by H. C. P. Bell and J. M. Senaveratne. Vols. I-. Colombo, I9I5-: A.S.B., I.L.

-_Epigraphia Zeylanica (see No. I Ioo $(b))$.

II02. - Government of Ceylon.

(a) Ceylon Administration Reports.-Mineral(ogical) Survey. I903-08. [Colombo], I903-08 (Rep. for 1905 wanting): G.S.I.

(b) Ceylon Administration Reports,-Report of the Marine Biologist. I9Io/II-. [Colombo, I9II-] : Z.S.I.

I103. - Marine Biological Laboratory.

(a) Ceylon Marine Biological Reports. Parts 2-6. Colombo, I906-I2: Z.S.I. [For part I see (b).]

(b) Report. No. I. Colombo, I905: Z.S.I.

I 104. - Meteorological Office and Observatory.

(a) Meteorology of Ceylon. I88I-84. Colombo, I88I-84: M.O. [Continued as (c).]

(b) Report on the Colombo Observatory and the Meteorology of Ceylon. I908--. Colombo, I908-: M.O. [A continuation of $(c)$.]

(c) Report on the Meteorology of Ceylon. r885-1907. Colombo, I885-I907: M.O. [A continuation of $(a)$, continued as $(b)$.]

— Mineralogical Survey, Ceylon (see No. I I02 (a)).

II05. - Museum.

(a) Memoirs. Vols. I-. Colombo, IgI4-: I.L.

(b) Spolia Zeylanica. Parts I-. Colombo, I904-.

Complete set : Z.S.I.

Parts I-, I904- (pts. 24, 25, 27, 28, 34 wanting):

A.S.B.

- Observatory (see No. II04).

A.S. = Archaeological Section, Indian Mnseum.

A.S $\mathbf{B}=$ Asiatic Society of Bengal.

B.C. = Bishop s College.

B.S.I. = Botanical Survey of India

C.E. = Chemical Examiner to the Govt. of Benga1

c. $U$ = Calcutta University.
E.C. = Civil Engineering College, Stbpur.

G.S.I. = Geolosical Survey of Incia.

I L. = Imperial Library.

M.Ba. = Ml edical College. Bacteriological Laboratory.

M.BI. = Medical College. Biological Laboratory.

M.c. = Iledical College (main library). 
I 106. Colombo.-Royal Asiatic Society, Ceylon Branch.

(a) Journal. Vols. [I] -, I845-. Colombo, [1846]-. [Vols. I-6 lack vol. nos. and consist of nos. I-22 with independent pagination.]

Vols. I-, I $845-$ (vols. $3,8,9$, wanting, vols. 2 , 4, Io defertive) : Z.S.I.

Vols. I-2I, I845-I909 (nos. 8, 10, 21 wanting) : G.S.I.

Vols. I-, I845-(vols. 8,9 wanting, vols. I- 6 defective): A.S.B.

Vols. I-9, I $845-85$ : R.B.G.

Vol. I (2 parts), I $845-47$ : I.L.

(b) Proceedings. I875-9o. Colombo, [1875-90] (vols. for 1884-86 wanting): A.S.B., Z.S.I.

Index to the Journals and Proceedings, I845-90, I895: Z.S.I., G.S.I.

_ Royal Botanic Gardens (see No. II37).

- Spolia Zeylanica (see No. II05 (b)).

II07. - Taprobanian, a dravidian journal of oriental studies in and around Ceylon, in natural history, archaeology, philology, history, etc. Vols. I-3. Bombay, I887-88 (vol. 3, pt. 4 wanting): Z.S.I.

Tropical Agriculturist (see No. II37 (c)).

Iro8. Coonoor.-Pasteur Institute of Southern India.

Bulletin. Nos. I-. Madras, I908-: I.L.

II09. Dehra Dun.-Forest Research Institute.

(a) Forest Bulletin. [Old series.] Nos. I-II. Calcutta, I905-07 : B.S.I., I.L.

(b) Forest Bulletin, New series, nos. I- Calcutta, I9I I-.

Complete sets : B.S.I., I.L.

Nos. 4-, I9II-: A.S.B.

(c) Forest Leaflets. Nos I-5. Calcutta. I908-IO : B.S.I., I.L., Z.S.I.

(d) Forest Pamphlets :-

Botany series. Nos. I-. - Calcutta, I9I0-: A.S.B., B.S.I., I.L., Z.S.I.

Chemistry series. Nos. I-. Calcutta, I908- : A.S.B., B.S.I., I.L.

Forest Economy series. Nos. I-. Calcutta, I909-: A.S.B., B.S.I., I.L., Z.S.I.

Forest Zoology series. Nos. I-. Calcutta, I908-: A.S.B., B.S.I., I.L., Z.S.I.

M.o. = Meteorological Office, Alipore.

M.Ph. = Medical College, Physiological Laboratory.

P.C. = Presidency Collese.

P.O. = Patent Office.

R.B.G. = Royal Botanic Garder.

S.C.C. $=$ Scottish Churches College.
S.G. $=$ Surgeon-General's Library.

S.I. = Survey of India.

S.T.M. = School of Tropical Medicine

T.D. = Telegraph Department.

X.c. =St. Xavier s College.

Z.S.I. = Zoological Survey of India 
II00. Dehra Dun.-Forest Research Institute (Continued).

Sylvicultural series. Nos. I-. Calcutta, I909-:

A.S.B., B.S.I., I.L., Z.S.I.

Working plan series. Nos. I-. Calcutta, I908- : A.S.B., B.S.I., I.L., Z.S.I.

(e) Indian Forest Memoirs :-

Chemistry series. Vols. I-. Calcutta, I908- : A.S.B., B.S.I., I.L., Z.S.I.

Economic Products series. Vols. I-. Calcutta, Igo9-: A.S.B., B.S.I., I.L., R.B.G., Z.S.I.

Forest Botany series. Vols. I-. Calcutta, I9II- : A.S.B., B.S.I., Z.S.I.

Forest Zoology series. Vols. I- Calcutta, Igo8- . A.S.B., B.S.I., I.L., Z.S.I.

Sylviculture series. Vols. I-. Calcutta, Igr6- : A.S.B., B.S.I., I.L., Z.S.I.

(f) Indian Forest Records. Vols. I-. Calcutta, I908- : A.S.B., B.S.I., I.L., R.B.G., Z.S.I.

- Indian Forester (see No. Io08).

- Survey of India (see No. Iog8).

I I Io. Goa.-Commissao Archeologica de India Portugueza.

Revista. Vols. I-3. Nova Goa, I904-06: A.S.B.

IIII. Hyderabad.-Archaeological Department, Hyderabad State.

(a) Annual Report. I9r4/I5-. Calcutta, I9I6- : A.S.B., I.L.

(b) [Report.] Hyderabad Archaeological Series. I9I5-. Hyderabad, IgI5- : I.L.

I I 2. - Archaeological Society.

Journal. IgI6-. Bombay, I9I6- : A.S.B., I.L.

Kodaikanal.-Observatory (see No. IJ 30 ).

I I 3. Lahore.-Archaeological Survey of India, Northern Circle (formerly North-Western Provinces and Oudh, or Punjab and United Provinces).

(a) Annual Progress Report. I892/93-I904/05. Roorkee. Lucknow, Lahore, [I893-I905.] [A continuation of $(f)$, continued in two parts as $(b)$ and $(c)$.]

Complete sets : A.S., A.S.B.

Reps. for I893/94-I904/05 : I.L.

(b) Annual Progress Report of the Archaeological Surveyor. I905/06-I909/I0. Lahore, Allahabad, [I906-Io]: A.S., A.S.B., I.L. [Continued as (e).]

A.S. = Archaeological Section, İndian Museum.

A.S.B. = Asiatic Society of Bengal.

B.C. = Bishop's College.

B.S.I. = Botanical Survey of India

C.E. = Chemical Examiner to the Govt. of Bengal.

c.U. = Calcutta University
E.C. = Civil Engineering College, Sibpur G.S.I. = Geological Survey of India.

I.L. = Imperial Library.

M.Ba. = Medical College, Bacteriological La ooratory M.Bi. = Medical College. Biological Laboratory. M.C. = Medical College (main library) 
III3. Lahore.-Archaeological Survey of India, N. Circle (Continued).

(c) Annual Progress Report of the Superintendent. I905/06I909/10. Lahore, [I909--IO]. [Continued as (d).]

(d) Annual Progress Report of the Superintendent, Hindu and Buddhist Momments. I9IO/II-. Lahore, [I9II]- : A.S., A.S.B., I.L.

(e) Anmual Progress Report of the Superintendent, Muhammadan and British Monuments. I9Io/II-. Allahabad, [I9II]-.

Complete sets : A.S.B., I.L.

Reps. for I9II/I2-: A.S.

(f) Progress Reports of the Epigraphical and Architectural Branches. I890/9I-I89I/92. Roorkee, [I89I-92]: I.L. [Continued as $(a)$.]

(g) Reports. [Archaeological Survey of Northern India.] Vols. I-. Calcutta, Allahabad, I889-. [Vols. I and 2 are subsequently referred to officially as vols. II and 12 of the New Imperial Series (No. $1147(c)$ ); vols. 3, 4 and 5 are respectively vols. 18,20 and 26 of that series.]

Complete sets : A.S., I.L.

Vols. I-, I889- (vol. 4 wanting) : A.S.B.

III4. Government of the Punjab.

(a) Selections from the Public ${ }^{\circ}$ Correspondence of the Punjab Administration. Vols. I-6. Lahere, 1853-68. [Continued as $(b)$.]

Complete set : I.L.

Vols. I-3, I 853-57 (vols. 2, 3 defective) : G.S.I.

(b) Selections from the Records of the Government of the Punjab and its dependencies. New ser., nos. I-23. Lahore, I $868-87$.

Complete set: I.L.

Nos. I-2I, I $868-83$ (nos. I9, 20 wanting) : G.S.I.

_ Indian Medical Journal (see No. Ioog).

III5. — Punjab Historical Society.

Journal. Vols. I-. Calcutta, I9II- : A.S.B., I.L.

Lucknow.-Archaeological Survey of India, North-Western Provinces and Oudh (see No. III3).

IIr6. Madras.-Agri-Horticultural Society.

Proceedings. New ser., vol. 4, I885-87. Madras, I888:

I.L. I894-. [Madras, I894]- (issues of 1897, '99-1901 wanting, issues of $1894-96,98$ defective) : I.L.

M.o. = Mieteorological Office, Alipore.

M.Ph. = Medical College, Physiological Laboratory.

P.C. = Presidency College.

P.o. = Patent Office

R.B.G. = Royal Botanic Garden.

S.C.C. $=$ Scottish Churches College
S.G. = Surgeon-General's Library.

S.I. = Survey of India.

S.T.M. = School of Tropical Medicine.

T.D. = Telegraph Department

X.C $=$ St. Xavier's College.

Z.s.1. = Zoological Survey of India 
III7. Madras.-Antiseptic. Ed. by T. M. Nair. Vol. 6. Madras, I009: S.T.M.

IIIS. - Archaeological Survey of India, Southern (Madras and Coorg) Circle.

(a) Annual (Progress) Report. I902,03-. Madras, [IgO3]-.

Complete sets : A.S.B., I.L.

Reps. for $1902 / 03-$ (Reps. for $1903 / 04-05 / 06$ wanting) : A.S.

(b) Annual (Progress) Report of the Government Epigraphist (Assistant Superintendent for Epigraphy). I890/9I-. Madras, [I89I]-.

Reps. for I890/9I, '97/98-I900/or, '06/07- : I.L.

Reps. for I $898,99-$ (Reps. for I902/03-'05 o6 wanting): A.S.

Reps. for I906/07-: A.S.B.

I I 9. - Archaeological Survey of Southern India.

(a) Reports. [Old series.] Vols. I-4. Madras, I882-86: A.S., A.S.B., I.L. [Vols. I and 2 are officially referred to as vols. 7 and 8 of the New Imperial series (No. I I $47(c)$ ) or as vols. 2 and 3 of the New series (infra). Vols. 3 and 4 are also officially referred to as Nos. I and 2 of the Old series.]

(b) Reports. [New series.] Vols. I-9. London, Madras, I887-97. [Vol. 2 is in two portions, the second being in three parts entitled vol. 2, pts. 1-3. Yols. I, 2 (Ist portion) and 2 (2nd portion) are officially referted to as vols. 6, 9 and to respectively of the New Imperial series (No. I I 47 (c)) ; vols. 6 , 7,8 and 9 are respectively vols. $15,17,21$ and 25 of that series. Vols. $I$ and 2 of the Old series (supra) are also some. times officially referred to as rols. 2 and 3 of the New series, the two portions of the work referred to above as vol. 2, being then styled vols. 4 and 5.]

Complete sets: A.S., I.L.

Vols. I-9, I 887-97 (vol. 2, ist portion wanting): A.S.B.

I $20 .-$ College of Engineering.

Professional Papers. Nos. I-. Madras, Igoo- : I.L.

II2I. - Department of (Land Records and) Agriculture, Madras.

Bulletin. Nos. I-. Madras, I889-.

Nos. I-, I889- (no. 23 wanting): B.S.I.

Nos. I-, I889- (nos. 3, 4, 8, 9, 10, I4, 2I, 36, 37 wanting) : I.L.

\footnotetext{
A.S. = Archaeological Section, Indian Museum.

A.S.B. = Asiatic Society of Bengal.

B.C. = Bishop's College.

B.S.I. = Botanical Survey of India.

C.E. = Chemical Examiner to the Govt. of Bengal.

C.U. = Calcutta University.
}

E.C. = Civil Engineering College, Sibpur.

G.S.I. = Geological Survey of India.

I L. = Imperial Library.

M.Ba. = Medical College, Bacteriological Laboratory

M.Bi. = Medical College, Biological Laboratory.

M.C. = Medical College (main library). 
I 22. Madras.-Fisheries Bureau.

Bulletin. Nos. I-. Madras, (I007)- : I.L., Z.S.I. [No. I was published in 1915; 110s. 2 and 3,1907 , '09, do not bear numbers or the title of the series.]

II23. - Government of Madras.

Selections from the Records of the Madras Government. Nos. I-IO. Madras, I854. [2nd ser.], nos. I-87. Madras, I855-66. [3rd ser.], nos. I-66. Madras, I $867-80$.

Nos. I-IO, I854; 2nd ser., nos. I-87, I $855-66$ (no. 6o wanting); 3rd ser., nos. I -66, I $867-80$ (no. 8 wanting) : I.L.

Nos. I-IO, I854; 2nd ser., nos. I-87, I $855-66$ (several nos. wanting); 3rd ser., nos. I -65, I $867-79$ : G.S.I.

II24. - Government Museum.

Bulletin. Vols. I-5. Madras, I894-I907: A.S.B., I.L., Z.S.I.

I 25. - Indian Mathematical (Club) Society.

Journal. Vols. I-. Madras, I9o0-.

Complete set : C.U.

Vols. 4-, I912-: P.C.

Vols. 6-, I9I4- : S.C.C.

Juggarow Observatory (see No. II52).

I126. — King Institute of Preventive Medicine.

(a) Annual Report on the work of the Bacteriologica? Section. rgo6-rgo8/og. Madras, Igo7-09: I.L. [Continued as (b).]

(b) Report on the working of the Micro-Biological Section. Igog--. Madras, IgIO- : I.L. [A continuation as $(a)$.]

(c) Work of the ..... Institute .... and Report on Vaccination in the Madras Presidency. Igo5/06-. Madras, Ig06- : I.L.

I 127. Literary Society (and Auxiliary of the Royal Asiatic Society).

(a) Journal of Literaturc and Science. Ed. by J. C. Morris. Vol. I, I833. Madras, I834 : A.S.B., C.U., G.S.I., R.B.G. [Continued as (b).]

(b) Madras Journal of Literature and Science. Ed. by J. C. Morris and (afterwards) R. Cole, C. P. Brown, the Committee of the ..... Society. Vols. 2-I7. Madras, I835-53. New series, rols. I-6 (=vols. I7 [bis]-20). Ed. by the Committee of the ...... Society. Madras,

M.o. = Meteorological Ofitice, Alipore.

M.Ph. = Medical College, Pbysiological Laboratory.

P.C. = Presidency College.

P.o. = Patent Office.

R.B.G $=$ Royal Botanic Garden.

S.C.C. $=$ Scottish Churches College.

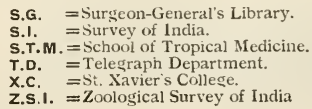


I 27. Madras.-Literary Society .. (Continued).

I857-6I. Third series, nos. I, 2. Madras, I864-66. 4 vols., I878-8I. Ed G. Oppert. Nungumbaukum, Madras, London, I87a-82. 4 vols., I886/87-I889/94. E,d. by R. H. C. Tufnell. Nungumbaukum, Madras, I,ondon, I887-94, [For vol. I, Ist ser., see $(a)$.]

Vol. 2- vol. for I889 94 , I835-94 (3rd ser., no. 2 defective): A.S.B.

Vol. 2- vol. for I889:94, I835-94 (many parts wanting) : I.L.

Vol. 2- vol. for IS8I, I835-82 : G.S.I.

Vol. 2- new ser., vol. 6, I835-6I : C.U., R.B.G.

II28. - Madras Monthly Journal of Medical Science Vols. I-7. Madras, I870-73.

Complete set: G.S.I.

Vol. I, I870 : A.S.B.

I129. - Madras Quarterly Journal of Medical Science. Ed by H. B. Montgomery, W. R. Cornish and others. Vols. I-I2. Madras, I860-68: G.S.I. General index to vols. I-I2 at end of vol. I2. [Continued as No. Ir 28.$]$

- Museum (see No. II24).

II30. - Observatories (Madras and Kodaikanal).

(a) Bulletin of the Kodaikanal Observatory. Nos. IMadras, I(j05-.

Complete sets : G.S.I., M.O.

Nos. I-, I905- (nos. 2, 4, I8 wanting): A.S.B.

Nos. I-, I g05- (nos. 3, 37, 43 wanting) : I.L.

(b) Magnetical Observations made at the Hon. E. I. Company's Observatory at Madras. 2 vols. I846-50, '5 I55. Madras, I 854, ' 84 .

Vol. for $\mathrm{I}_{4} 6-50$ : M.O.

Vol. for I85I-55: G.S.I.

(c) Memoirs of the Kodaikanal Observatory. Vols. I-. Madras, I909- : A.S.B., G.S.I., I.L.

(d) Meteorological Observations made at the Hon. E. I. Company's Observatory at Madras. 2 vols. I $846-55$. Madras, I854, '74: M O.

(e) Report: Kodaikanal and Madras Observatories. I899-. Madras, I9oo-

Reps. for I899- : G.S.I.

Reps. for I9OI-: I.L.

A.S. = Archaeological Section. indian Museum.

A.S.B. = Asiatic Society of Bengal.

B.C. = Bishop's College.

B.S.l. = Botanical Survey of India

C.E. = Chemical Examiner to the Govt. of Bengal.

c.U. = Calcutta University.
E.C. =Civil Engineering College, Sibpur.

G.S.I. = Geological Survey of India.

I.L. = Imperial Library.

M.Ba. = Medical College. Bacterioloøical Laboratory.

M.Bi. = Medical College. Biological Laboratory.

M.C. = Medical College (main library). 
II30. Madras.-Observatories (Madras and Kodaikanal) (Continued).

(f) Results of Astronomical Observations made at the Hon. E. I. Company's Observatory at Madras. By 'T. G. Taylor. Vols. I-5, I83I-39. Madras, I832-40 : B.C.

(g) Results of the Observations of the Fixed Stars made with the Meridian Circle at the Government Observatory: Madras. Vols. [I]-9, I862-87. Madras, I887-99.

Complete set: G.S.I.

Vols. I-8, I887-94 (vol. 2 wanting) : M.O.

_- Pasteur Institute of Southern India (see No. IIo8).

II3I. - South Indian Association.

Journal. Vols. I-, IgIo/II-. Madras, [IgII]-: A.S.B., I.L.

I 132. Mandalay.-Archaeological Survey of India, Burma Circle.

Report. I901/02-. Rangoon, [1902]-.

Complete set : I.L.

Reps. for I901/02- (Rep. for 1903/04 wanting): A.S.B.

Reps. for I901/02- (Reps. for 1904'05, '05/06 wanting) : A.S.

I 133. Muktesar.-Imperial Bacteriological Laboratory.

Annual Report of the Imperial Bacteriologist. 1895/96-.

Calcutta, I896- (Reps. for 1901/02-'05, 06, '07/08 wanting) : I.L. [Reps. for 1908/09-19rI are nos. I and 3 of No. $1055(b)$.]

I 134. Nagpur.-Department of Agriculture, Central Provinces.

(a) Bulletin. Nos. I-9. Nagpur, I895-I902. New series, nos. I-. Nagpur, I908-.

Nos. I-9, I895-I902; new ser., nos. I-, I908-: B.S.I.

Nos. 3-9, I90I-02 ; new ser., nos. 2-, I908- : I.L.

(b) Report on the Agricultural Stations in the Central Provinces. I905/06-. Nagpur, I906-: I.L. [A contin uation of $(c)$.]

(c) Report on the Department of Agriculture, the Civil Veterinary Department and the Nagpur Experimental Farm in the Central Provinces. I902/03-1904/05. Nagpur, I903-05: I.L. [Continned as (b).]

I 35. - Museum.

Records. I-. Nagpur, I9I6- : Z.S.I.

Nova Goa-see Goa.

M.o. = Meteorological Office, Alipore.

M.Ph. = Medical Colleđe, Physiological Laboratory

P.C. =Presidency College.

P.o. = Patent Ofice

R.B.G. = Royal Botanic Garden

S.C.C. $=$ Scottish Churches College.
S.G = Sur seon-General's Libraty.

S.I. = Survey of India.

S.T.M. = Schoot of Tropical Mecicins.

T.D. = Telegrapin Department

X.C =St. Xavier's Cullege.

Z.S.1. =Zoological survey of $1 \cdot$ dia 
Patna.-Bihar and Orissa Research Society (see No. 1025).

II36. - Department of Agriculture, Bihar and Orissa.

Agricultural Journal. Vols. I-. Patna, I9I3- : A.S.B., I.L.

II 37 Peradeniya.-Royal Botanic Gardens, Ceylon.

(a) Annals. Vols. I-. Colombo, I9oI-.

Complete set : R.B.G.

Vols. I-, IgOI- (vol. + defective) : A.S.B.

(b) Circulars and Agricultural Journal. Vols. I- Colombo, I897-: B.S.I.

(c) Tropical Agriculturist. Vols. I- Colombo, I88I-. Complete set: R.B.G.

Vols. I-, I $88 \mathrm{I}-$ (rol. 12 defective) : B.S.I.

II38. Peshawar.-Archaeological Survey of India, Frontier Circle.

(a) Annual Report. I906/07-. Peshawar, [1907]-: A.S., A.S.B., I.L.

(b) Report of the Archaeological Survey Work in the NorthWest Frontier Province and Baluchistan. I904/05. Peshawar, I905: A.S. [Continued as (a).]

II39. Pondicherry.-Observations Météorologiques : Etablissements francais de l'Inde. I905-. Pondicherry [1905]- I.L.

Ir40. Poona.-Ganeshkhind Botanical Garden.

Annual Report on the Experimental Work of the .... Garden. I906/07-. Bombay, 1907-.

Reps. for I906/07-I9IO'I I : B.S.I.

Reps. for IQI2/I3- : I.L.

II4I. Pusa.--Agricultural Research Institute.

(a) Agricultural Journal of India. Vols. I--. Calcutta, I906--, A.S.B., B.S.I., C.U., G.S.I., I.L., R.B.G., Z.S.I.

(b) Bulletin. Nos. I- Calcutta, I904-.

Complete set : B.S.I.

Nos. I-, I904- (no. 33 wanting): I.L.

Nos. 3-, I906- (nos. $6-8,10-12,18,25,30$ wanting): A.S.B.

(c) Memoirs of the Department of Agriculture in India. Bacteriological Series. Vols. I-. Calcutta, I912-: A.S.B., I.L.

A.S. = Archaeological Section, Indian M Iseum.

A.S.B. = Asialic Society of Bengal.

B.C. = Bishop's College.

B.S.I. = Botanical Survey of India.

C.E. = Chemical Examiner to the Govl of Bengal.

C.U. = Calculta University:
E.C. = Civil Engineering College, Sibpur

G.S.J = Geological Survey of India.

I.L. = Imperial Library.

M.Ba. = Medical College, Bacteriological Laborat ury

M.BI. = Medical College. Btological Laburalory.

M.C. = II dical College (man hibrary) 
II4I. Pusa.-Agricultural Research Institute (Continued).

Botanical Series. Vols. I-. Calcutta, I006-: A.S.B., B.S.I., C.U., G.S.I., I.L., R.B.G.

Chemical Series. Vols. I-. Calcutta, I906-: A.S.B., B.S.I., C.U., G.S.I., I.L., R.B.G.

- Entomological Series. Vols. I-. Calcutta, I006-: A.S.B., C.U., G.S.I., I.L., R.B.G., Z.S.I.

Veterinary Series. Vols. I-. Calcutta, I9I3- I.L.

(d) Report on the Progress of Agriculture in India. Igo7 o9-. Calcutta, Igog-: A.S.B.

Rangoon.-Archaeological Survey of India, Burma Circle (see No. II32)

II42. — Burma Research Society.

Journal. Vols. 6-. Rangoon, IgI6- (vol. 6 defective) : A.S.B.

Ir43. - Department of Agriculture, Burma.

Bulletin. Nos. I-. Rangoon, I909-: I.L.

Ir44. - Ethnographical Survey of India.-Burma.

[Memoirs.] Nos. 2-4. Rangoon, I909-IO: Z.S.I.

Roorkee.-Archaeological Survey of India, North-IVestern Provinces and Oudh (see No. III3).

Survey of India (see No. Iog8).

Ir45. - Thomason College.

Professional Papers on Indian Engineering. Vols. I-7. Roorkee, I863-70. 2nd series, vols. I-II. Roorkee, I872-82. 3rd series, vols. I-4. Roorkee, I883-86. Index to Ist and 2nd series.

Complete set and index: G.S.I.

Complete set: I.L.

Vols. I- 3rd ser., vol. 4, I863-86 (vol. 11, 2nd ser. wanting): A.S.B.

I 46. Shillong.-Department of (Land Records and) Agriculture, Assam.

Bulletin. Nos. I-. Shillong, I894-.

Complete set : B.S.I.

Nos. I-, I894 (nos. 9, IO, 12, I3, I5, I8-20 wanting: I.L.

I 47. Simla.-Archaeological Survey of India.

(a) Annual Report. I902,03-. Calcutta, I904-: A.S., A.S.B., I.I.

m.o. = Meteorological Office, Aitoore.

M.Ph = Medical College, Physiological Laboratory

P C. = Presidency College.

P. = Patent Ofnce.

R B.G = Roval Botanic Garder.

$\mathbf{S} \mathbf{C} \mathbf{C}=$ Scottish Churches College.
s.G. = Surgeon-Generai's Library

S.I. = Survey ot Incia.

S.T.M $=$ School of Tropical Medicine.

T.D. = Telegraph Departmen.

X.C. =St Xavier s College.

Z.s.I. = Zoological Survey of India. 
I 47. Simla.-Archaeological Survey of India (Coniinued).

(b) Anmual Report, Part I. I903/04-. Calcutta, I904-. Complete sets: A.S., I.L.

Reps. for I905'06-: A.S.B.

(c) Reports. (New Imperial Series.) Vols. [I]-. London, Calcutta, Bombay, Madras, Allahabad, I874-. [Under this title the Reports of the Archaeological Surveys of Northern, Southern and Western India (new series) are combined (see Nos. IOzO $(c)$, III3 $(g)$, III9 $(a, b)$ ), together with the first 2 vols. of No. $1046(a)$. The series was, however, not established until 1894 , with the result that the 13 vols. published before that date do not bear the vol. nos. or title of the series; they are nevertheless officially referred to as component parts of the series. From vol. I4 onwards most vols. bear two vol. nos., that of the local series in addition to that of the New Imperial Series.]

Complete sets : A.S., I.L.

Vols. 2-, I876- (vols. 7, 9, 20 wanting) : A.S.B.

II48. _ Board of Scientific Adrice for India.

Annual Report. I904 05-. Calcutta, I906-.

Complete sets : G.S.I., I.L., M.O., Z.S.I.

Reps. for I904 05-(Reps. for I909/IO, 'II I2 wanting): A.S.B.

I 49. - Committee for the Study of Malaria in India.

Paludism, being the transactions of the Committee .... Ed.

by S. P. James, S. R. Christophers and others. Nos.

I-5. Simla, I9IO-I2.

Complete sets : A.S.B., I.L., Z.S.I.

Nos. I-5, IgIO-I2 (no. 4 wanting): S.T.M.

II50. Government of India.

Selections from the Records of the Government of India. Nos. I-448. Calcutta, Simla, I853-I9II. [Nos. 9199, II5, I I6 not publisled.]

Nos. I-448. I853-I9I I (nos. I I , 446 wanting) : I.L.

Nos. I-443, I853-I9IO: G.S.I.

Imperial Department of Agriculture (see No. I I4I).

II5I. - Meteorological Department, Government of India.

(a) Cyclone Memoirs. Parts I-5. Calcutta, I888-93.

Complete sets : G.S.I., M.O.

Parts 2-5, I890-93: R.B.G.

A.S. =Archaeological Section. Indian Museum.

A.S B $=$ Asiatic Society of Bengal.

B.C. = Bishop $\mathrm{s}$ College

B.S.I. = Botanical Survey of India.

C.E. Chemical Examiner to the Govt. of Benga!

C.U = Calcutta University.
E.C. = Civil Engineering College. Sibpur: G.S.I. = veolosical Survey of India.

i L. = Imperial Library

M.Ba, = Medical College, Bacteriological Laborat $n$ y

M.Bi. = Medical College. Biological Laboratory.

M.C. = Medical College (main library). 
JAPAN, CHINA AND INDO-CHINESE COUNTRIES. [I I 5I-I I53

I I5 I. Simla.-Meteorological Department . . (Continued).

(b) Indian Meteorological Memoirs, being occasional discussions and compilations of meteorological data relating to India and the neighbouring countries. Vols. I-. Calcutta, I876-8I-.

Complete sets : I.L., M.O., R.B.G., X.C.

Vols. I-, I876-8I- (vol. I 3 wanting) : G.S.I., Z.S.I.

Vols. I-, I876-8I- (vol. I3 wanting, vols. I5, I9-21 defective): A.S.B.

(c) India Weather Review. I89I-. Calcutta, Simla, I892-. [Issued in monthly parts as the Monthly Weather Review (with Annual Summary). A continuation of $(f)$.]

Complete sets: M.O., X.C.

Vols. for I891-I902 : R.B.G.

Vols. for I 892 - : G.S.I.

Vols. for I892- (vols. for I893, '94 wanting): A.S.B.

Vols. for Igoo-: I.L.

(d) Meteorological Obscrvations recorded at six (seven) stations in India .... corrected and reduced. I879-94. Calcutta, I88I-95 : G.S.I., M.O. [Vols. for I889, '90 not published.]

(e) Rainfall of India. I89I-. Calcutta, I892-.

Complete sets: G.S.I., M.O.

Vols. for I89I- (vol. for Igon wanting) : R.B.G.

Vols. for I897- : X.c.

(f) Report on the Meteorology of India. By H. F. Blanford. I875-90. Calcutta, I877-92: G.S.I., M.O., R.B.G., X.C. [Continued as (c).]

- Monthly Weather Review (see No. I I.5I (c)).

— Paludism (sec No. II49).

II52. Vizagapatam.-Juggarow Observatory.

Results of Meteorological Observations. I872-94. Calcutta, Madras, I873-96.

Vols. for $1872-94$ (vols. for 1873, '76, '77, '9I-93 want. ing): A.S.B.

Vols. for I $880-89$ : X.C.

\section{JAPAN, CHINA AND INDO-CHINESE COUNTRIES.}

II53. Bangkok. - Natural History Society of Siam.

Journal. Vols. I-. Bangkok, I9I4-: Z.S.I.

M.o. = Meteorological Office, Mlipore

M.Ph. = Medical College, Physiological Laboratory.

P.C. = Presldency College.

P.o. = Patent Office.

R.B.G. = Royal Botanic Garden

S.C.C. $=$ Scottish Churches College
s.G. = Surgeon-General s Library

S.I. = Survey of Indla.

S.T.M.=School of Tropical Medicine

T.D. = Telegraph Department

X.c. =St. Xavier's College.

Z.S.I. = Zoological Survey of India. 
Ir54. Bangkok.-Royal Survey Department, Siam.

General Report on the Operations of the .... Department. I900/or一. Bangkok, I90r-(Rep. for 1904/05 wanting): S.I.

II55. - Siam Society.

Journal. Vols. I-. Bangkok, r904-: I.L.

I156. Hanoi.-Bulletin Economique de l'Indo-Chine. Nos. 2-42, = Ann. I-4. [Vols. I-3.] Saigon, I898-Igor. Nouv sér., nos. I-, = vols. 4-, Ann. r902-. Hanoi-Haiphong, 1902-.

No. 2- nouv. sér., 110. 69 (=vols. I-9), r898-1907 (no. 27 wanting): G.S.I.

Nour. sér., nos. I- (=vols. $4-)$, I902-: B.S.I.

- Commission Archéologique de 1'Indo-Chine (see No. 496).

Ir 57. - Ecole Française d'Extréme-Orient.

Bulletin. Tom. I-. Hanoi, rgor-.

Complete set: A.S.B.

Tom. I-, IgoI- (tom. I defective) : I.L.

—_Excursions et Reconnaissances (see No. I 165).

Ir58. - Service Géologique de 1'Indo-Chine.

(a) Bulletin. Vol. $\mathrm{x}-$. Hanoi-Haiphong, I9r3-: G.S.I.

(b) Mémoires. Vol. I, fasc. 2-. Hanoi-Haiphong, I9I2-: G.S.I.

I159. Kuala Lumpur.-Department of Agriculture, Federated Malay States.

Bulletin. Nos. I-. Kuala Lumpur, rgo9- (no. 8 wanting): B.S.I.

I160. - Federated Malay States Museums.

Journal. Vols. I-. Kuala Lumpur, I906-: Z.S.I.

I161. Kuraschiki.-Ōhara Institut für Landwirtschaftliche Forschungen.

Berichte. Bde. I-. Kuraschiki (prov. Okayama, Japan), I9I6-: Z.S.I.

I 162. Kyoto.-Entomological Society of Japan.

Entomological Magazine. Vols. I-. Kyoto, I915-: Z.S.I.

II63. Imperial University.

Acta Scholae Medicinalis Universitatis Imperialis in Kioto. Vol. I, fasc. 2-. Kioto, rgI6-: A.S.B.

A.S. = Archaeological Section, Indian Museum.

A.S.B. = Asiatic Society of Bengal

B.C. = Bishop's College.

B.S.1. = Botanical Survey of India

C.E. = Chemical Examiner to the Guvt. of Bengal

C.U. = Calcutta University
E.C. = Civil Engineering College, Sibpur G.S.I. = Geological Survey of India.

I.L. = Imperial Library

M.Ba. = Medical College, Bacteriological Laboratory M.Bi. = Medical College. Biological Laboratory.

M.C. = Medical College (main library) 
I 64. Macao.-Observatorio Meteorologico.

Boletim Meteorologico: Capitania do Porto de Macau. Anno I6-I9, I897-I900. Macau, I897-I900: X.C. [Continued as:] Boletim Meteorologico.... Anno 2029, Igor-Io. Nacau, I904-Io: X.C.

Saigon.-Builetin Economique de l'Indo-Chine (see No. I I56).

II65. Excursions et Reconnaissances, Cochinchine Française. Nos. I-I6. Saigon, IS79-83. Tom. 7-I5. Saigon, Hanoi, I $884-90$.

Nos. I-I6, I $879-83$ (nos. 3, 4 wanting); tom. 7-I5, I $884-90$ : R.B.G.

Tom. I3-I5, I887-90 (tom. i5 defective) : Z.S.I.

I I66. Sendai.--Tóhoku Imperial University.

(a) Science Reports. First series. Mathematics, Physics, Chemistry. Vols. I-. Sendai, I9I2-: A.S.B., G.S.I.

(b) Science Reports. Second series. Geology. Vols. I-. Sendai, I9I2- : A.S.B., G.S.I.

II67. - Tôhoku Mathematical Journal. Ed. by 'T. Hayashi. Vols. 6-. Sendai, I0I4-: C.U.

I 68 . Seoul.-Mineral Resources of Chōsen (Korea). Vols. I-. Keijo (Seou1), I913-: G.S.I. [Part 2 of vol. I is entitled "Bulletin on the Mineral Survey of Chösen.']

II60. Seoul.-Royal Asiatic Society.-Korea Branch.

Transactions. Vols. I-. Seoul, Yokohama, Igoo- (vol. 2 , pt. 2 wanting): A.S.B.

II70. Shanghai.-China Medical Journal. Vols. 24-. Shanghai I9IO- : A.S.B.

II7I. - Mémoires concernant l'Histoire Naturelle de l'Empire Chinois. Par des Pères de la Compagnie de Jésus. Tom. I-4. Changhai, I880-98: Z.S.I.

II72. - Royal Asiatic Society, (North) China Branch.

Journal. Vols I, 2. Shanghai, I859-60. New series, vols. I-. Shanghai, I864-.

Vol. I, no. 3, vol. 2 , no. I, I859-60; new ser., vols. I-32, I $864-98$ (vols. $10,27,29$ wanting) : G.S.I.

New ser., vols. 4-, I867-(vol, 12, 16, 17, 29, f1 wanting; vols. 2 I, 34 defective): A.S.B.

I 73. Singapore.-Botanic Gardens.

(a) Agricultural Bulletin of the Straits and Federated Malay

States. New (2nd) series, vols. I-IO. Singapore,

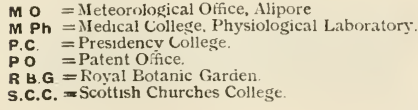


II73-II80] JAPAN, CHINA AND INDO-CHINESE COUNTRIES.

II73. Singapore.-Botanic Gardens (Continued).

I902-II. Third series, vol. I, pts. I-5. Singapore, I0I2. [Continued as (b).]

Complete set : B.S.I.

New ser., vols. I-9, I902-IO : R.B.G.

(b) Gardens' Bulletin, Straits Settlements. Third series, vol. I, pt. 6- Singapore, I9I2-: B.S.I.

II74. - Institute for Medical Research, Federated Malay States

Studies. Nos. I-. Singapore, London, IgoI-: A.S.B.

II75. - Journal of the Indian Archipelago and Eastern Asia. Ed. by J. R. Logan. Vols. I-9. Singapore, I847-55. New series, vols. I-3. Singapore, I856-59.

Vols. I- new ser., vol. 2, I847-58: A.S.B., I.L., R.B.G.

New ser., vol. 2 , no. 4, rol. 3, pt. I, I857, '59: G.S.I.

II76. Malay Medical Journal. Vols. 5-7. Singapore, rgo8-II (defective): A.S.B.

II77. — Royal Asiatic Society, Straits Branch.

(a) Journal. Nos. I-. Singapore, I878-. Index to nos. I-5o.

Complete set and index : G.S.I.

Nos. I, 35-48. I878, I 90 I-O 7 (nos. 36, 38, 40 wanting) : A.S.B.

(b) Notes and Queries. Nos I-4. Singapore, $1885-87$ : G.S.I.

I 78. Taiping.-Perak Museum.

Notes. Nos. I-3. Taiping, I893-94: G.S.I., Z.S.I. Vol. 2, pts. I-2. Taiping, I $897-98$ : G.S.I., Z.S.I.

Tokyo.-Annotationes Zoologicae Japonenses (see No. Ir87).

I 79. — Deutsche Gesellschaft für Natur- und Völkerkunde Ostasiens.

Mittheilungen. Bde. I-. Yokohama, Tokio, I873-.

Complete set : G.S.I.

Bde. I-, I873- (Bde. 3, 4, 8, II-I4 defective) : A.S.B.

- Geological Survey of Japan (see No. II83).

I $80 .-$ Imperial Academy.

(a) Memoirs. Sect. II. Vol. I, no. I. Tokyo, I913:

A.S.B.

(b) Proceedings. Vols. I-. 'Tokyo, I9I2-: A.S.B.

\footnotetext{
A.S. Archaeological Section, Indian Muscum.

A.S.B. = Asiatic Societv of Bengal.

B.C. = Bishop's College.

B.S.I. = Botanical Survey of India

C.E. =Chemical Examiner to the Govt. of Bengal

c.U. = Calcutta University.
}

E.C. = Civil Engincering College, Sibpur.

G.S.I. = Geological Survey of India

I L. = Imperial Library.

M Ba. = Medical College, Bacteriological Laboratory

M.Bi. = Medical College. Biological Laboralory.

M.C. Nedical College (main library). 
JAPAN, CHINA AND INDO-CHINESE COUNTRIES. [I I 8 I-I I 85

ri8r. Tokyo.-Imperial Bureau of Fisheries.

Report. Scientific Investigations. Vols. I, 2. Tokyo, I9I2-I3: Z.S.I.

Ir82. - Imperial Earthquake Investigation Committee.

(a) Bulletin. Vols. I- Tokio, I907-

Complete sets: G.S.I., M.O.

Nos. 3-24, I9o0-7 (no. 7 wanting): A.S.B.

(b) Publications of the .... Committee in foreign languages.

Nos. I-. Tokyo, I897- : G.S.I.

Ir83. - (Imperial) Geological Survey of Japan.

(a) Bulletin. Vols. I4-. Tokio. I90I- (vols. 15, 22 wanting) : G.S.I.

(b) Report of Progress. By B. S. Lyman. 2nd year. Tokei, I878: G.S.I. For I878/79. Tookei, I879: G.S.I. [A continuation of $(c)$.]

(c) Report of Progress .... Geological Survey of the Oil lands of Japan. By B. S. Lyman. Ist year. Tokei, I877: G.S.I. [Continued as $(b)$.]

I 84 . Imperial Museum.

Proceedings of the Department of Natural History. Vol. I, nos. I, 2. Tokyo, I904: Z.S.I.

Ir85. - Imperial University of Japan ('Teikoku Daigaku).

(a) Bulletin of the College of Agriculture. Vols. 2-8. Tokyo, I894-I909: B.S.I. [Continued as (b).]

(b) Journal of the College of Agriculture. Vols. I- Tokyo, Igog-.

Complete sets : B.S.I., R.B.G.

Vols. I-, I909- (vol. 2 wanting, vol. I defective): Z.S.I.

(c) Journal of the College of Engineering. Vol. 4. Tokyo, I906 : I.L.

(d) Journal of the College of Science. Vols. I- Tokyo, I887 (I886)-. General index, vols. I-25.

Complete sets : I.L., Z.S.I.

Vols. I-, I887- (rol. 34 wanting; vols. I 5, 29, 33, 35 defective): A.S.B.

Vols. 5-, I893- (vols. 5, 6 defective) and index: G.S.I.

(e) Memoirs of the Science Department. Vols.I-3, pt. I. Tokio, I879-80: G.S.I., Z.S.I. Nos. 4-ro. Tokio, I88I-83 : G.S.I., Z.S.I.

M.o. = Meteorological Office, Nlipore.

M.Ph. = Medical College, Physiological Laboratory:

P.C. = Presidency College

P.O. = Patent Orfice.

R.B.G = Royal Botanic Garden.

s.C.C. $=$ Scottish Churches College
S.G. = Sur seon-General's Library.

S.I. = Survey of India.

S.T.M. = School of Tropical Medicine.

T.D. = Telegraph Department.

X.c. =St. Xavier's College.

Z.s.l. = Zoological Survey of India. 
I 85. Tokyo.-Imperial University of Japan (Continued).

(f) Mitteilungen aus dem Medizinische Facultät der Kaiserlich-japanischen Universität. Vols. I-. Tokyo, I 892 -

Vols. I-, I892- (vols. 6,8 defective) : A.S.B.

Vols. 7-, Ig06-: I.L.

—_ Kaiserlich-japanische Universität (see No. II85).

II86. - Sei-i-Kwai.

Sei-i-Kwai Medical Journal. Vols. 30-. Tokyo, I9II(defective): A.S.B.

_- Seismological Society of Japan (see No. II89).

II87. - Zoological Society.

Annotationes Zoologicae Japonenses. Vols. I- Tokyo, I897- : Z.S.I.

ri88. Yokohama.-Asiatic Society of Japan.

Transactions. V'ols. I-, I872-. Yokohama, I874-.

General index to vols. I-23, I 895 .

Vols. I-, I874- (vols. I7, 28 wanting; vols. 16, I8, 29, $30,32-34,36$ defective) and index : A.S.B.

Vols. I-22, I874-94, and index: G.S.I.

Vols. 3-8, I875-80: R.B.G.

Vols. I 2, I3, I884-85 (defective) : Z.S.I.

- Deutsche Gesellschaft für Natur- und Völkerkunde Ostasiens (see No. I I 79).

I 89. - Seismological Society of Japan.

Transactions. Vols. I-20. Yokohama, Tokio, I880-95 : G.S.I.

MALAYSIA, PHILIPPINES, OCEANIA.

II9o. Batavia.-Bataviaasch Genootschap van Kunsten en Wetenschappen.

(a) Nederlandsch-Indisch Plakaatboek, I602-I8II. Door J. A. van der Chijs. Deel I-I7. Batavia,'s Hage, I 885-Igoo.

D1. I-I7, I 885-I900: A.S.B.

D1. I-II, I885-93: G.S.I.

A.S. = Archaeological Section, Indian IIuseum.

A.S.B. = Asiatic Society of Bengal.

B.C. = Bishop s College.

B.S.I. = Botanical Survey of Indma

C.E. = Chemical Examiner to the Govt. of Bengal.

c.U. = Calcutta University.
E.C. = Civil Engineering College, Sibpur.

G.S I. = Geological Survey of India.

I.L. = Imperial Library.

M.Ba. = Medical College, Bacteriological Laboratory

M.Bi. =Medical College. Biological Laboratory.

M.C. = liedical College (main library). 
I rgo. Batavia.-Bataviaasch Genootschap .. (Continued).

(b) Notulen van de Algemeene en Bestuurs-I'ergaderingen van het.... Genootschap. Deel I-. Batavia, I864-. Register op de Notulen, I867-78, I879; I889-98, I899.

D1. I-, I864- (D1. 23,3 I, 34, 38, 39 defective) and index : A.S.B.

D1. I-3I, I864-93 (D1. 7, 8, 23, 25, 31 defective) and index: G.S.I.

D1. I-6, I864-69: Z.S.I.

(c) Rapporten van de Commissic in Nederlandsch-Indië voor Ondhoidkundig Onderzoek op Java en Madoera. IgOI-. Batavia, I904- (vols. for 1905, '06, 'o8 wanting): A.S.B.

(d) Tijdschrift voor Indisch: Taal-, Land-en Volkenkunde, uitgegeven door het .... Genootschap. Deel (Jahrg.) I-. Batavia, I852-.

D1. I-, I $852-$ (D1. $3,30,36,39,42,45-47$ wanting) : A.S.B.

D1. I-37, I852-93 (D1. 3, 21, 22 wanting, D1. I, 6, I8, 3I, 36,37 defective) : G.S.I.

(e) Verhandelingen. Deel I- Batavia, I779-. 'Register op de 4I eerste Deelen', I779-I88I, at end of Deel $4 \mathrm{I}$.

D1. I-, I779- (D1. 43-45, 52, 54-56 defective): A.S.B.

D1. I-46, I 779-I89I (D1. 17,34 , 35 wanting) : G.S.I.

D1. I-I 5, I779-I883 (D1. wanting) : I.L.

D1. 9, IO, I3, I4, I825-33: R.B.G.

- Bijdragen tot de Taal-, Land-, en Volkenkunde van Nederlandsch-Indië (see No. 8or).

II9I. - Burgerlijk Geneeskundig Dienst in Nederlandsch-Indië(Civil Medical Service of Netherlands India).

Mededeelingen (Publications). Deel I b-, Batavia, I9I2- : Z.S.I.

Departement van Landbouw in Nederlandsch-Indië (see No. I I99).

I 192. - Dienst der Pestbestrijding.

Verslag. I9I5-. Batavia, I9I5-: Z.S.I.

Jaarboek van het Mijnwesen in Nederlandsch Oost-Indië (see No. 784).

\footnotetext{
M. $\mathbf{O}=$ Meteorological Office, Alipore.

M.Ph. = Medical College, Physiological Laboratory.

P.c. = Presidency College

P.O. = Patent Office.

R.B.G = Royal Botanic Garden.

s c.C. $=$ Scottish Churches Collegc.
}

S.G. = Surgeon-General's Library.

S.I. = Survey of India.

S.T.M. = School of Tropical Medicine

T.D. = Telegraph Department.

X.C. =St. Xavier's College.

Z.s.l = Zoological Survey of India. 
Batavia.-Koninklijk Instituut voor de Taal-, Land-, en Volkenkunde van Nederlandsch-Indië (see No. 8or).

I 93. — Koninklijk Magnetisch en Meteorologisch Observatorium.

(a) Observations made at the (Royal) Magnetical and Meteorological Observatory at Batavia. Vols. I-23, I866Igoo. Batavia, I87I-I902.

Vols. I-23, I87 I-I902: X.C.

Vols. I-22, I87I-I900: M.O.

Yols. I-6, I 87 I-85: A.S.B.

(b) Regenwaarnemingen in Nederlandsch-Indië. Jaarg. I-, I879-. Batavia, I880-.

Jaarg. I-, I880- (J Jaarg. I3-I9 wanting): X.C.

Jaarg. I-32, I880-I9I2: M.O.

I 194. — Koninklijke Natuurkundige Vereeniging in NederlandschIndië.

(a) Natuurkundig Tijdschrift voor Nederlandsch-Indië. Jaarg. (Deel) I-. Batavia, 's Gravenhage, Weltevreden, Amsterdam, I850-. Alphabetisch Register op Deel 3I-50.

Complete set and index : Z.S.I.

Jaarg. I-, I850-(Jaarg. 2, 3, D1. 14-17, 20, 21, 25, 27, 33 wanting): A.S.B.

Jaarg. 2-, I $85 \mathrm{I}-$ : R.B.G.

Deel $34-$, I $874-$ : G.S.I

(b) Verhandelingen. (Acta Societatis Scientiarum IndoNeérlandicae). Deel (vol.) I+8. Batavia. I856-60

Complete set : Z.S.I.

D1. I-7, I $856-60$ : R.B.G.

D1. 5,6, I $858-59$ : A.S.B.

__ Magnetical and Meteorological Observatory (see No. I I93).

Natuurkundig Tijdschrift voor Nederlandsch-Indië (see No. II94 $(a)$ ).

Regenwaarnemingen in Nederlandsch-Indië (see No. II93 (b)). Societas Scientiarum Indo-Neérlandica (see No. I I94 (b)).

I I95. - Teysmannia. Onder redact. van J . van Breda de Haan (and others). Deel I4-. Batavia, I903-(D1. I4 defective) : B.S.I.

Tijdschrift voor Indische Taal-, Land-en Volkenkunde (see No I Igo $(d))$.

A.S. = Archaeological Section, Indian Museum.

A.S.B. = Asiatic Society of Bengal.

B.C. = Bishop s College.

B.S.I. = Botanical Survey of India.

C.E. = Chemical Examiner to the Govt of Bengal.

c. $U$ Calculta University.
E.c. = Civil Engineering College, Sibpur.

G.S.I. = Geological Survey of India.

1. L. = Imperial Library.

M. Ba, = Medical College, Bacteriological Laboratory

M Bi. = Medical College. Biological Laboratory

M.C. = Medical College (main library). 
II96. Batavia.-Tijdschrift voor Neêrlands-Indië. $6^{\text {de }}$ Jaarg., Deel I-4. Bataria, I844: A.S.B. $7^{\text {de }}$ Jaarg., Deel I, 2. Batavia, I845 : A.S.B.

II97. - Visscherij-Station te Batavia.

Mededeelingen. Nos. I-9. Buitenzorg, Batavia, I908-I2 (nos. 3,4 wanting): Z.S.I.

I I98. Bencoolen.-Malayan Miscellanies. Yols I, 2. Bencoolen, I8202 I : R.B.G.

Buitenzorg.-Département de l'Agriculture aux Indes Néerlandaises (see No. I I99).

I I 99. - Departement van Landbouw in Nederlandsch-Indië.

(a) Bulletin du Département de l'Agriculture aux Indes Néerlandaises. Nos. I-. Buitenzorg, I906-.

Complete set: B.S.I.

Nos. I-, I906- (nos. I 5, 22, 45 wanting) : A.S.B.

Nos. 8, 20, I907, '08 : Z.S.I.

Nos. II-, I907- : R.B.G.

(b) Jaarboek van het Departement.... I905-. Batavia, Igo6-. [For earlier vols. see $(g)$.]

Complete sets : B.S.I., R.B.G.

Vols. for Igo9- (vol. for I9I3 wanting): A.S.B.

(c) Korte Berichten voor Landbouw, Nijverheid en Handel. Jaarg. I-. [Buitenzorg], IgIO-(Jaarg. I defective) : B.S.I.

(d) Mededeelingen uitgaande van het Departement.... Nos. I-. Batavia, I905- : B.S.I., R.B.G.

(e) Mededeelingen uit 'sLands Plantentuin. Nos. 4-75. Batavia, I887-I904. [Continued as (d).]

Nos. 4-75, I887-I 904 : R.B.G.

Nos. 9-75, I89I-I904 B.S.I.

(f) Mededeelingen van het Proefstation voor Thee. Nos. I-, Igo7-. Buitenzorg, Igo8- : B.S.I.

(g) Verslag omtrent den Staat van 'sLands Plantentuin te Buitenzorg. I885-I904. Batavia, I886--I905. [Continued as $(b)$.]

Vols. for I885-I904: R.B.G.

Vuls. for I900-04 : B.S.I.

I200. - Departement van Landbouw.-Instituut ronr Plantenziekten en Cultures.

Mededeclingen van het Laboratorium voor Plantenziekten No. $23,24,26$. Batavia, I9I6 : Z.S.I.

M o. = Meteorological Office, Alipore

M.Ph. = Medical College, Physiological Laboratory.

P.C. $=$ Presidency College.

P.o. = Patent Office.

R.B.G. = Roval Botanic Garden.

S.C.C. = Scuttish Churches College.
S.G. = Surgeon-Generai's Library.

S I. = Survey of India.

S.T.M $=$ School of Tropical Medicine

T.D. = Telegraph Department.

X.c. $=$ St. Xavier $\mathrm{s}$ College.

Z.s.I. =Zoological Survey of India 
I20I. Buitenzorg.-Institut Botanique de (l'état de) Buitenzorg.

(a) Bulletin. Nos. I-22. Buitenzorg, I898-I905.

Complete set: B.S.I.

Nos. I-22, I80 R-I905 (110. I6 wanting) : R.B.G.

(b) Observations Météorologiques. Igor-II. Buitenzorg, I902-I 2 .

Vols. for IgOI-II ; M.O.

Vols. for I 901 , 'O2: B.S.I.

_- Institut voor Plantenziekten en Cultures (see No. I20o).

I202. - Instituts Scientifiques de Buitenzorg "sLands Plantentuin.'

Contributions à la Faune des Indes Néerlandaises. Dir. par J. C. Koningsberger. Vol. I, fasc. 2,-. BuitenZorg, I9I5- : Z.S.I.

I203. - Jardin Botanique

(a) Annales. Vols. I-. Batavia, Leide, I876-. [Vols. I6-, I899-, are also styled " $2^{\text {me }}$ sér., vols. I-."]

Complete set: R.B.G.

Vols. I7-, I90I- (vol. 20 defective): A.S.B.

(b) Bulletin. $2^{\text {me }}$ sér., nos. I-. Buitenzorg, I9I I-.

Complete set: A.S.B.

Nos. I - I9II- (nos. I6, I8 wanting): R.B.G.

(c) Icones Bogorienses. Vols. I-. Leide, I897-: A.S.B. R.B.G.

__ Proefstation voor Thee (see No. I I99 $(\dot{f}))$.

I204. Honolulu.-Bernice Pauahi Bishop Museum of Polynesian Ethnology and Natural History.

(a) Memoirs. Vols. I-. Honolulu, I899-.

Complete set : Z.S.I.

Vol. I, nos. I, 4, I899, I902: A.S.B.

(b) Occasional Papers. Vols. I-. Honolulu, I898-, A.S.B., Z.S.I.

1205. - Board of Agriculture and Forestry.

(a) Hawaiian Forester and Agriculturist: a monthly magazine of forestry, entomology and agriculture. Vols. IHonolulu, I904- : B.S.I.

(b) Report of the Board of Commissioners. 2-5, I905-08. Honolulu, I go6-o9: B.S.I.

A.s = Archaeological Section, Indian Museum.

A.S.B. = Asiatic Society of Bengal.

B.C. = Bishop s College.

B.S.I. = Botanical Survey of India.

C.E. = Chemical Examiner to the Govt. of Bengal.

c.U. = Calcutta University.
E.c. =Civil Engineering College, Sibfur.

G.S.I. = Geological Survey of India.

I.L. = Imperial Library.

M.Ba. = Medical Coltese, Bacleriological Laboratory

M.Bi. = Medical College. B!ological Laboraiory:

M.C. = Medical College (man library). 
I206. Honolulu.-Board of Agriculture and Forestry.-Hawaii Agricultural Experiment Station.

(a) Annual Report. Igo6-. Honolulu, WVashington, I $907-$ : B.S.I.

(b) Bulletin. Vols I-. Honolulu, Washington, I903-: B.S.I.

(c) Press Bulletin. Nos. I-. Honolulu, I903- (no. 6 wanting): B.S.I.

Hawaiian Forester and Agriculturist (see No. $1205(a)$ ).

I207. - Hawaiian Sugar Planters' Association.

Hawaiian Planters' Monthly. Vols. 23-28. Honolulu, I904-09: B.S.I.

I208. - Hawaiian Sugar Planters' Association-Experiment Station.

(a) Bulletin. Agricultural and Chemical series. Nos.I-. Honolulu, I905- (no. I2 wanting): B.S.I.

(b) Bulletin. Entomological series. Nos. I- Honolulu. I905-.

Complete set : Z.S.I.

Nos. I-, I905- (no. 2-5 wanting): B.S.I.

(c) Bulletin. Pathological and Physiological series. Nos. I-. Honolulu, I905-.

Complete set : B.S.I.

Nos. 2: 4, 5, 1905-06: Z.S.I.

1209. Kuching.-Sarawak Museum.

Journal. Tols. I-. Singapore, IgI I- : A.S.B., Z.S.I.

I2IO. Malang.-Proefstation.

Mededeelingen. Nos. I-. Malang, Soerabaia, IgI I- : R.B.G.

I2 I I. Manila.-Bureau of Agriculture, Philippine Is.

(a) Farmers' Bulletin. Nos. I-. Manila, Igo2- : B.S.I.

(b) Philippine Agricultural Review. Vols. I-. Manila, rgo8-.

Complete sets : B.S.I., R.B.G.

Vol. 6-, I9I3- (vol. 6, nos. I-6 wanting) : A.S.B.

(c) Press Bulletin. Nos. 8-. Manila, I906-: B.S.I.

I2I 2. - Bureau of Forestry, Philippine Is.

(a) Bulletin. Nos. I-. Manila, I903- : B.S.I.

(b) Circular. Nos. I-. Manila, I906-: B.S.I.

M.o. = Meteorological Office, Alipore.

M.Ph. = Medical College, Physiological Laboratory.

P.C. = Presidency College.

P.o. = Patent Office.

R.B.G, = Royal Botanic Garden.

S.C.C. = Scottish Churches Collegi.
S.G. = Surgeon-General's Library.

S.I. = Survey of India.

S.T.M. $=$ School of Tropical Medicine.

T.D. = Telegraph Department.

X.C. =St. Xavier's College.

Z.S.I. $=$ Zoological Survey of India. 
I2I3. Manila.-Bureau of Government Laboratories, Philippine Is.

[Publications of the] Biological Laboratory. Nos. I-36. Manila, I903-06. [Continued as No. 1214(a).]

Nos. I -35, I903-05 (nos. 3, 6, 7, 9-12, 17, 30 wanting) : A.S.B.

Nos. 5, 22, 34, 36, I903-06 : Z.S.I.

I254. - Bureau of Science, Philippine Is.

(a) Philippine Journal of Science. Vol. I. Manila, I906:

B.S.I., G.S.I., R.B.G., Z.S.I. [Continued as $(b),(c),(d)$ and $(e)$.]

(b) - Sect. A. General Science (afterwards Chemical and Geological Science and the Industries). Vols.2-. Manila, I907-. [The change in title begins with vol. 5.]

Complete set : B.S.I.

Vols. 2-, I907- (vol. 5 defective): A.S.B.

Vols. 2-5, I907-IO: Z.S.I.

Vols. 3-, Igo8- : G.S.I.

(c) Sect. B. Medical Science. Vols. 2-. Manila, I907-.

Complete set: M.Ba.

Vols. 2-, I907- (vols, 2, 4 defective) : A.S.B.

(d) - Sect. C. Botany. Vols. 2-. Manila, I907-. A.S.B., B.S.I., R.B.G.

(e) Sect. D. Ethnology, Anthropology and General Biology. Vols. 5--. Manila I9IO- : A.S.B., Z.S.I.

Contents and index, vols. I-IO : Z.S.I.

I2I5. — Weather Bureau, Philippine Is.

Annual Report (of the Director). I903-. Manila, Ig04-: M.O.

I2I6. Weather Bureau.-Central Observatory.

(.Monthly) Bulletin. I903-. Manila, I903-.

Vols. for I903- : M.O.

Vols. for I904- (vols. for $1907-09$ wanting): X.C.

I217. - Ethnological Survey of the Philippine Is.

Publications. Vols. I-4, Manila, I904-05 (vol. 3 wanting : vols. 2,4 defective): Z.S.I.

I2IS. - Leaflets of Philippine Botany. Ed. by A. D. E. Elmer. Vols. I-. Manila, Igo6- : B.S.I., R.B.G

12I9. - Medical Society.

Bulletin. Ed. by W. E. Musgrave. Vols. 2-4. Manila, I9IO-I2 (vols. 2,3 defective): A.S.B.

A.S. = Archaeological Section. Indian Museum.

A.S.B. = Asiatic Society of Bengal.

B.c. = Bishop's College.

B.S.I. = Botanical Survey of India.

C.E. = Chemical Examuner to the Govt. of Bengal.

c.u. = Calcutta University.
E.C. = Civil Eugineering College, Sibpur.

G.S.I. = Geolowical Survey of India.

I.L. = Imperial Library.

M.Ba. = Medical College. Bacteriological Laboratory.

M.Bi. = Medical College. Biological Laboratory.

M.C. = Medical College (main library). 
I220. Manila.-Mining Bureau.

Bulletin. Nos. I-5. Manila, I902-05 (no. 3 wanting) : G.S.I.

I22 r. - Observatorio (Meteorológico) de Manila.

(a) Bulletin Mensual. Año I894-96. Manila, I894-96: X.C.

(b) Magnetismo Terrestre en Filipinas. r893-95. Manila, I893-95: X.C.

— Philippine Agricultural Review (see No. I2I I (b)).

1222. - Philippine Agriculturist and Forester. Vols. I-. Manila, IgII-: B.S.I.

Philippine Journal of Science (see No. IzI4).

1223. Pekalongan.-Proefstation voor Suikerriet in IVest-Java "Kagok."

(a) Bulletin. Nos.6-Io. Tegal, Igo3-06: B.S.I.

(b) Mededeelingen. Nos. 66-I00. Soerabaia, I903-07: B.S.I.

(c) Verslag. I902-06. Tegal, I903-07: B.S.I.

I 224 Salatiga.-Algemeen-Proefstation.

Verslag omtrent den Staat van het .... Proefstation I97708. [Samarang, I907-08] : B.S.I.

I225. Surabaya.-Proefstation Oost-Java.

Mededeelingen. $3^{\text {de }}$ serie, no. 48 . Soerabaia, I9o3: B.S.I. $4^{\text {de }}$ serie, nos. I-39. Soerabaia, I903-08: B.S.I.

I226. - Proefstation voor de Java-Suikerindustrie.

(a) Jaaverslag. I907-. Soerabaia, I908: B.S.I.

(b) Mededeelingen. Deel I-. [Soerabaia], I907-: B.S.I.

\section{ALSTRALASTA.}

I227. Adelaide.-Department of Agriculture, South Australia.

(a) Journal. Vols. I-I4. Adelaide, I897-I9II : B.S.I.

(b) Report. I902/03-. Adelaide, I903-: B.S.I.

— Philosophical Society (see No. I230 (c)).

mo = Meteorological Office, Alipore

M Ph $=$ Medical College. Physiological Jaboratory.

P.C = Presidency College.

PO = Patent Ofice

$\mathbf{R} \mathbf{b} \mathbf{G}=$ Roval Botanic Garcien.

S.C.C. $=$ Scottish Churches College.
S.G. = Surgeon-Generai's Library.

S.I. = Survey of India.

S.T.M. = School of Tropical Medicine.

T.D. = Telegraph Department.

X.C. =St. Xavier $\mathrm{s}$ College.

Z.S.I. = Zoological Survey of Incia. 
I228. Adelaide.-Royal Agricultural and Horticultural Society of South Australia.

Proceedings. I 88 I -8 3. Adelaide, I 88 I-83 : R.B.G.

I229. - Royal Geographical Society of Australasia: South Australian Branch.

Proceedings. Vols. 2-. Adelaide, I890-: A.S.B.

I230. Royal Society of South Australia.

(a) Memoirs. Vols. I-. Adelaide, [I899]- : G.S.I., Z.S.I.

(b) Transactions and Proceedings and Report. Vols. 3-. Adelaide, I88o-. [A continuation of $(c)$.]

Vols. 3-, I880- (vols. 5, 6, 34 wanting): G.S.I.

Vols. 9-, I 886 - (pt. 2, vol. 3I wanting): Z.S.I.

Vols. II, I7, I889, '93: A.S.B.

(c) Transactions and Proceedings and Report of the Philosophical Society of Adelaide. Vols. I, 2. Adelaide, I $878-79$ : G.S.I. [Continued as (b).]

Index to $(c)$ and $(b)$, vols. I-24, I $877-$ I900: G.S.I.

I23I. Brisbane.-Department of Agriculture, Queensland.

(a) Annual Report. I890/9I-I900'or. Brisbane, I89IIOOI.

Reps. for I890 9I-I900/OI: A.S.B.

Reps. for I $898 / 99-$ I900/OI : B.S.I.

(b) Botany Bulletin. Nos. 8-I6. Brisbane, I893-I903 (no. Io wanting): A.S.B.

(c) Bulletin. Nos. 4-25. Brisbane, 1890-93 (nos. 5, II, I2, 15, I6 wanting): A.S.B. $2^{\text {nd }}$ ser., nos. I, 5, 7, IO, I3. Brisbane, I893-97: A.S.B.

(d) Queensland Agricultural Journal. Vols. I-3I. Brisbane, I897-I9I3. New ser., vols. I-. Brisbane, I9I 4 -

Complete set: B.S.I.

Vols. I-IO, I897-I902: A.S.B.

I232. — Department of Mines, Queensland.

Queensland Government Mining Journal. Vols. 6-. Brisbane, I905- (vol. 6 defective) : G.S.I.

Department of Mines.-Geological Survey of Queensland (see No. I233).

1233. - Geological Survey of Queensland.

(a) Bulletin. Nos. I-I8. Brisbane, I895-I902 (nos. 7, I2 wanting): G.S.I.

A. =Archaeological Section, Indian Museun.

A.S.B. = Asiatic Society of Bengal

B.C. = Bishop s College.

B.S.I. = Botanical Survey of India.

C.E. Chemical Examiner to the Govt. of Bengal.

C.U. = Calcutta University:
E.C. = Civil Engineering College, Sibpur.

G.S.1. = Geological Survey of Indiat.

I.L. = Imperial Library.

M.Ba. = Medical College, Bacteriological Laboratory

M. Bi. = Medical College. Biological Laboratory.

M.C. = Medical College (main library). 
I233. Brisbane.-Geological Survey of Queensland (Continued).

(b) Publication (Report). New ser., vols. $\mathrm{I}-$, = Nos.

I77-. Brisbane, I905- (defective) : G.S.I.

- Queensland Agricultural Journal (see No. I23I (d)).

- Queensland Geographical Journal (see No. I235 (c)).

- Queensland Government Mining Journal (see No. 1232).

I234. - Queensland Museum.

(a) Annals. Nos. I-. Brisbane, I89I-.

Nos. I-, I89I- (no. 2 wanting): Z.S.I.

Nos. I-, I89I- (nos. 3,6 wanting): A.S.B.

Nos. I-5, I89I-I900 (no. 4 wanting): G.S.I.

(b) Memoirs. Vols. I-. Brisbane, I9I2-: G.S.I., Z.S.I.

I235. (Royal) Geographical Society of Australasia: Queensland Branch.

(a) Proceedings. Vol. I, I885-86. Brisbane, I886: G.S.I. [Continued as $(b)$.]

(b) Proceedings and Transactions. Vols. 2-I4, I886-99. Brisbane, I886-99: G.S.I. [Continued as (c).]

(c) Queensland Geographical Journal. New series. Vols. I5-, I899-. Brisbane, [I900]-: G.S.I.

1236. Brisbane.- Rnyal Society of Queensland.

Proceedings. Vols. I-, I884-. Brisbane, I884-. Index to vols. I-I5.

Vols. I-, I 884 - (vols. 20,24 wanting): A.S.B.

Vols. I-, I884-(vols. $2,3,7,8$ wanting) and index:

G.S.I.

1237. Christchurch.-Canterbury Museum.

Records. Vols. I-. Christchurch, I907-: Z.S.I.

1238. Geelong.-Field Naturalists' Club.

Geelong Naturalist: Journal of the Gordon College Field Naturalists' and Science Association. Vols. I-3. Geelong, I89I-94 (defective); Z.S.I. [Continued as:] Geelong Naturalist: Journal of the ... Chib. Vols. 4-6. Geelong, I894-98 (defective): Z.S.I. [Continued as :] Geelong Naturalist: Quarterly Journal of the ... Club. $2^{\text {nd }}$ ser., vols. I-3, part 3. Geelong, I904-07: G.S.I., Z.S.I.

I239. Hobart.-Department of Agriculture, Tasmania.

Agricultural Gazette of Tasmania. Vols. 12-. Hobart, I $904-$ : B.S.I.

\footnotetext{
M.o. = Meteorological Office, Aipore.

M.Ph = Medical College. Physiological Laborator

P C. = Presidencv Colleg:

Po. = Patent Ofince

R B.G = Royal Botanic Garcien.

S C C $=$ Scottish Churches College.
}

S.G. =Surgeon-Generai's Library

S.G. = Survev or Incia.

S.T. $M=$ School of Tronical Medicine.

T.D. = Telegraph Deparmen:

X.C. =St Xavier 5 College

Z.s.1. =Zoological Survey of India. 
1240. Hobart.-Department of Mines, Tasmania.

Report of the Secretary for Mines. I899/I900-. Tasmania, I900- (Reps. for I90I, 'o7-09 wanting): G.S.I. No. I 24I).

1241. Geological Survey of Tasmania.

(a) Bulletin. Nos. I-. Hobart, I907- (no. 13 wanting): G.S.I.

(b) Record. Nos. I-. Hobart, I9I3-: G.S.I.

1242. - Magnetical and Meteorological Observatory.

Obscrvations made at the .... Observatory at Hobarton in van Diemen Island, and by the Antarctic Naval Expedition. Printed under the superintendence of Lieut.Col. E. Sabine. Vols. I-3, I84I-46. I,ondon, I85053 : A.S.B.

1243. - Royal Society of Tasmania.

(Monthly Notices of) Papers and Proceedings (and Report) of the ... Society ... I 873 -. Tasmania, Hobart, I $874-$ (vols. for 1892-97, I900-07 wanting): G.S.I.

I244. - Royal Society of van Diemen's Land.

Papers and Proceedings. Vol. I. Hobart Town: I85I : A.S.B.

I245. Melbourne.-Australasian Institute of Mining Engineers.

Transactions. Vols. I-I6. Sydney, Melbourne. I894-I9I2 (vol. 7 wanting): G.S.I. New ser., nos. 6-. Melbourne, I9I2-: G.S.I. [Vol. 10 includes 'New ser., 110s, I-5'.] Index, I894-I9I4 : G.S.I.

- Australian Medical Journal (see No. I247).

1246. - Australian Mining Standard and Financial Review. Vols. 3I-48. Melbourne, IgO4-I2 (vol. 48 defective) : G.S.I.

I247. British Medical Association: Victorian Branch.

Journal of the ....Association ... A Astralian Medical Joumal. New ser. vols. I-. Melbourne, I9I2-: S.T.M.

I248. - Department of Agriculture, Victoria.

(a) Guide to Growers. Nos. 7-48. Melbourne, I893-I90 I (110s, 12, I8, 28 wanting) : B.S.I.

A.S. = Archaeological Section, Indian Museum

A.S.B. = Asiatic Society of Bengal.

B.C. = Bishop s College.

B.S.I. = Botanical Survey of India

C.E. = Chemical Examiner to the Guyt. of Bengal.

c. $U$ Calcutta Unversity:
E.c. = Civil Engineering College, Sibpur.

G.S.I. = Geological Survey of India.

I. L. = Imperial Ltbrary

M. Ba. = Medical College, Bacteriological Laboralory

M Bi. = Iledical College. Biological Laboratory

M. C. = Iledical College (main library). 
I248. Melbourne.-Department of Agriculture, Victoria (Continued).

(b) Journal. Vols. I-. Melbourne, I902-.

Complete set: B.S.I.

Vols. I, 2, I 902-03 (defective) : Z.S.I.

Vol. 6, Igo8: R.B.G.

1249. - Department of Mines, Victoria.

Annual Report of the Secretary for Mines (and Water Supply). I884-. Melbourne, I885- (Reps. for I888, I906 wanting): G.S.I.

Department of Mines.-Geological Survey of Victoria (see No. I25I).

$125^{\circ}-$ Geological Society of Australasia.

Transactions. Ed. by R. T. Litton. Vol. I, parts I-6. Melbourne, London. I886-92 : G.S.I.

125I. - Geological Survey of Victoria.

(a) Bulletins. Nos. I-. Melbourne, Igo3- (no. 2I wanting) : G.S.I.

(b) Memoirs. Nos. I-. Melbourne, Igo3-: G.S.I.

(c) Prodromus of the Palaeontology of Victoria, etc. By F. McCoy. Decades I-6. Melbourne, I874-79: G.S.I.

(d) Progress Report. No. 9. Melbourne, I898: G.S.I.

(e) Records. Vols. I-. Melbourne, Igo2-: G.S.I.

(f) Report of Progress. Nos. [I]-7. Melbourne, I878-84: G.S.I.

1252. - National Museum.

Memoirs. Nos. I-. Melbourne, Igo6-: G.S.I., Z.S.I.

- Philosophical Institute of Victoria (see No. I255 (e)).

- Prodromus of the Palaeontology of Victoria (see No. I25I (c)).

I253. - Prodromus of the Zoology of Victoria, etc. Ed. by F. McCoy. Decades I-20 (=vols. I, 2). Melbourne, London, I878-9o.

Complete sets : G.S.I., Z.S.I.

Dec. I-20. I878-90 (Dec. 19 wanting) : A.S.B.

I254. - Royal Geographical Society of Australasia.

Victorian Geographical Journal. Vols. 26-3o. Melbourne, Igo8-I3: G.S.I.

1255. - Royal Society of Victoria (Philosophical Institute of Victoria).

(a) Proceedings. New ser., vols. I-. Melbourne, r889-.

M.O. = Meteorological Otifice, Alipore.

M.Ph. = Medical Collese, Physiological Laboratory.

P.C. = Presidency College.

P.O. = Patent Oifice.

R.B.G $=$ Roval Botanic Garden

s.c.c. $=$ Scottish Churches College

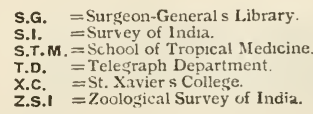

S.G. = Surgeon-General s Library

S.I. = Survey of India.

T.D. = Telegraph Department.

Z.S.I = Zoological Survey of India. 
I255. Melbourne.-Royal Society of Victoria (Continued).

[For earlier series see $(d)$.]

Complete set: G.S.I.

Vols. I-, I889- (vols. I1, I2 defective) : A.S.B.

Vols. II-, I898- (vols. I1, 12 defective) : Z.S.I.

(b) Transactions. Vol. 5. Melbourne, I860 [I86I]. [A continuation of $(e)$, continued as $(d)$.

(c) Transactions. Vols. I- Melbourne, I888-. [For earlier series see $(d),(e)$ and $(b)$.]

Complete sets : A.S.B., G.S.I.

Vol. 5, I909: Z.S.I.

(d) Transactions and Proceedings. Vols. 6-24. Melbourne, I865-88. [Continued as $(a)$ and $(c)$.]

Complete set : G.S.I.

Vols. 9-24, I 868-88 (vol.15 wanting): A.S.B.

(e) Transactions of the Philosophical Institute of Victoria. Vols. 2-4. Melbourne, I858-6o: G.S.I. [Continued as (b).]

I256. - University.

Collected Papers from the Science Laboratories. Vols. I, 2. Melbourne, I006-09: Z.S.I.

- Victorian Geographical Journal (see No. 1254).

I 257. Perth.-Department of Agriculture, Western Australia.

Journal. Nos. I-I8. Perth, I899-I909: B.S.I.

I258. - Geological Survey of Western Australia.

(a) Annual Progress Report. I897-. Perth, I898- (Rep. for Igol wanting): G.S.I.

(b) Bulletin. Nos. I-. Ferth, I898-: G.S.I.

I259. - Western Australian Museum and Art Gallery.

Records. Vols. I-. Perth, IgIO-: Z.S.I.

1260. Sydney.-Australasian Association for the Advancement of Science.

Report. Vols. I-I2, I888-I909. Sydney, I889-I9Io.

Vols. I-I 2, I889-I9IO (vols. 2, 5, IO wanting): A.S.B.

Vols. 5-I2, I 894-I9I0 (vols. 6, 8, Io wanting): G.S.I.

Australasian Institute of Mining Engineers (see No. 1245).

I261. - Australasian Medical Gazette. Vols. 25-35. Sydney, I906-

I4: C.U. [Continued as No. 1270.]

A.S. = Archaeological Section, Indian Museum.

A.S.B. = Asiatic Society of Bengal.

B.C. = Bishop s College.

B.S.1. = Botanical Survey of India

C.E. = Chemical Examiner to the Govt. of Bengal.

C.U. = Calcutta University.
E.C. = Civil Engineering College, Sibpur.

G.S.l = Geolosical Survey of India.

i.L. = Imperial Library

M.Ba. = Medical College, Bacteriological Laboratory

M.Bi. = Medical College. Btological Laboratory.

M.C. = Medical College (nam library). 
1262. Sydney.-Australian Museum.

(a) Memoir. [Nos.] I-. Sydney, I887-. [No. I is a reprint.]

Complete set: G.S.I.

Nos. 3-, I 889- (pt. 9, no. 3 wanting) : Z.S.I.

Nos. 4-, I899-: A.S.B.

(b) Records. Vols. I-. Sydney I890-.

Complete sets : G.S.I., Z.S.I.

Vols. 6-, I905- (no. I, vol. 8 wanting): A.S.B.

- Australian Naturalist (see No. I27 I (a)).

- Australian Zoologist (see No. 1274).

Biological Results of the Fishing Experiments carried out by the F.I.S. 'Endeavour' (see No. I265).

I263. - Department of Agriculture, New South Wales.

Agricultural Gazette of New South Wales. Vols. I-

Sydney, I890-. Author and subject index, vols. I-20, I 890-I909.

Complete set and index : B.S.I.

Complete set : R.B.G.

Vols. I6-, I905- : I.L.

1264. Department of Mines (and Agriculture), New South Wales. Annual Report. I876-. Sydney, I 877- (Rep. for 1897 wanting): G.S.I.

1265. Department of Trade and Customs, Commonwealth of Australia.-Fisheries.

Zoological Results of the Fishing Experiments carried out by the F.I.S. 'Endeavour.' Vols. I-3. Sydney, I9III5: Z.S.I. [Continued as:] Biological Results ...... Vols. 4-. Sydney, I9I6-: Z.S.I.

I266. E Entomological Society of New South Wales.

Transactions. Vols. I, 2. Sydney and London, (I862) I866-73.

Complete set: Z.S.I.

Vol. 2, I873: G.S.I.

1267. - Geological Survey of New South Wales.

(a) Memoirs. Ethnological Series. Nos. I-. Sydney, I899-: G.S.I.

(b) Memoirs. Geology. Nos. 2-. Sydney, I9oI-: G.S.I.

(c) Memoirs. Palaeontology. Nos. I-. Sydney, I888-: G.S.I.

o $=$ Meteorolosical Office, Alipore.

M.Ph. = Medical College. Physiological Laboratory

P.C. = Presidency College.

P.o. = Patent O.fice.

R.B.G. = Roval Botanic Garden

S.C.C. $=$ Sciottish Churches College.
S.G. = Surgeon-Generai's Library.

S I. = Survey of India.

S.T.M. = School of Tropical Medicine

T.D. = Telegraph Department.

X.C. =St. Xavier s College.

z.s.I. = Zoningical Survey of India 
I267. Sydney.-Geological Survey of New South Wales (Continued).

(d) Mineral Resources. Nos. I-. Sydney, I898- (no. 14 wanting, no. 18 defective): G.S.I.

(e) Records. Vols. I-. Sydney, I889-: G.S.I.

I268. - Linnean Society of New South Wales.

Proceedings. Vols. I-Io. Sydney, I $877-86$. Author index, vols. I-IO. 2nd ser., vols. I-IO [ = II-20], Sydney, I886-96. Vols. 2I-. Sydney, I896-.

Complete set and index to vols. I-IO : G.S.I.

Vols. 7-, I883- (2nd ser., vol. 2 [12] and vol. 32 defective): A.S.B.

2nd ser., vol. I [II]-, I887-: Z.S.I.

I269. Malaysian Essays. By J. E. Tenison-Woods. Nos. I, 2. Sydney, $[n . d$.$] : G.S.I.$

r270. - Medical Journal of Australia. Vols. I-. Sydney, I9I4- : C.U. [For earlier series see No. I261.]

I271. - New South Wales Naturalists' Club.

(a) Australian Naturalist. Journal of the ... Club. Vol. I, parts I-3. Sydney, I906: Z.S.I.

(b) Memoirs. Nos. I, 2. Sydney, I903-04: Z.S.I.

__ Philosophical Society of New South Wales (see No. I273(c)).

1272. - Royal Anthropological Society of Australasia.

Journal of the .... Society. Science of Man. New ser., vols. 2-. Sydney, I899-(rol. 8 wanting, vols. 2, 6, 7. 9-12 defective) : A.S.B.

I273. - Royal Society of New South Wales.

(a) Journal and Proceedings. Vols. I0-, I876-. Sydney, I877-. [A continuation of $(b)$.]

Complete set: G.S.I.

Vols. I0-, 1877 - (rol. 43 wanting): A.S.B.

(b) Transactions (and Proceedings). Vols. [2]-9, I868-75. Sydney, I860-76: G.S.I. [A continuation of $(c)$, continued as $(a)$. 1

(c) Transactions of the Philosophical Society of New South Wales. [Vol. I.] I862-65. Sydney, I866: G.S.I. [Continued as $(b)$.

1274. - Royal Zoological Society of New South Wales.

Australian Zoologist. Vols. I-. Sydney, I9I4-: Z.S.I.

A.S. =Archaeological Section, Indian Museum.

A.S B $=\Lambda$ siatic Society of 13engal.

B.C. = Bishop s College.

B.S.I. = Botanical Survey of India.

C.E. = Chemical Examiner to the Govt, of Bengat

C.U = Calcutta University.
E.c. = Civil Engineering College, Sibpur. G.S.l. = vieolosical Survey of India.

IL. = Imperial Library.

M. Ba. = Medical College, Bacteriological Laboratory. M.BI. = Medical College. Biological Laboratory.

M.c. = Medical College (nau library). 
I275. Sydney.-Technological Museum, New South Wales.

[Publications.] Technical Education Series. Nos. 7 Sydney, I892-: B.S.I.

Zoological Results of the Fishing Experiments carried out by the F.I.S. 'Endeavour' (see No. I265).

I276. Wellington.-Colonial Museum and Geological Survey of New Zealand.

Meteorological Report. I869-84. Wellington, I87 I-85: G.S.I.

1277. - Department of Agriculture, New Zealand.

(a) Anmual Report. Igo3-. Wellington, I903- : B.S.I.

(b) Bulletin. New ser, vols. I-. Wellington, rgro-: B.S.I.

(c) Bulletin. Dairy Division. Nos. 8-. Wellington, I $906-$ (no. 9 wanting) : B.S.I.

(d) Bulletin. Division of Biology and Horticulture. Nos. I-. Wellington, I904-: B.S.I.

(e) Bulletin. Division of Veterinary Science. Nos. IWellington, I903-(nos. $2-5$ wanting) : B.S.I.

I278. - Geological Survey of New Zealand.

(a) Bulletin. (New ser.) Nos. I-. Wellington, Igo6-: G.S.I.

(b) Palaeontological Bulletin. Nos. I-. Wellington, I9I3- : G.S.I.

(c) Reports of Geological Explorations. I870,7I-'87,88. Wellington, I87 I-88 (Rep. for I88 1 wanting): G.S.I. Index, I866-85 : G.S.I.

1279. L Lands and Survey Department.

Report on the Surveys of New Zealand. I876/77-. Wellington, I877- (Reps. for $1877 / 78$, '79/80-' $83 / 84,{ }^{\prime} 85 / 86$, '86/87, '91/92, 1905/06, '08/09, 'Io/I I wanting): S.I.

I280. - New Zealand Institute.

Transactions and Proceedings. Vols. I-40. Wellington, I 869-I9o8. Index to vols. I-I7. [Continued as:] Transactions. Vols. 4I-. Wellington, Igog-.

Vols. I-, I $869-$ ( vol. 20 wanting) and index: G.S.I.

Vols. IO-, I $878-$ (vol. 20 wanting) and index : A.S.B.

Vols. 2-6, 38, I870-74, I906: Z.S.I.

New Zealand Journal of Science and Technology (see No. I606).

M.O. = Meteorological Office, Alipore.

M.Ph. = Medical College, Physiological I aboratory.

P.C. = Presidency College.

P.O. = Patent Office.

R.B.G = Royal Botanic Garden

s.C. $C=$ Scottish Churches College.
S.G. =Surgeon-General's Library:

S.I. =Survey of India.

S.T.M. = School of Tropical Medicine.

T.D. = Telegraph Department.

X.c. =St. Xavier's College.

Z.s.I. =Zoological Survey of India. 
128I. Wellington.-Papers and Reports relating to Minerals and Mining.

I 894 - Wellington, I894- (vols. for I900-04 wanting): G.S.I.

[A continuation of No. 1283.$]$

1282. Polynesian Society.

Journal. Vols. I-. Wellington, I8g2- (no. 2, vol. I, wanting): A.S.B.

I283. - Reports on the Mining Industry of Nere Zealand. I88792. Wellington, I887-92 : G.S.I. [Continued as No. I28ז.]

\section{AMERICA.}

CANADA.

1284. Halifax, N.S.-Department of Mines, Nova Scotia.

Report. Igor, '02. Halifax, I902, '03: G.S.I.

1285. - Nova Scotian Institute of (Natural) Science.

Proceedings and Transactions. Vol. 7, pt. 4. Halifax, N.S., I8go. Vols. $8-(=$ new ser., vols. I-). Halifax, N.S., I89I-.

Vol. 7, pt. 4, and vols. 8-, I89I- : G.S.I., Z.S.I.

Vols. 8 -, I 89 I - (vols. IO, I2 defective) : A.S.B.

I286. Hamilton.-(Scientific) Association.

Journal and Proceedings. Parts I-, I882/83-. Hamilton, I884-.

Parts I-, I884- (pts. for I886-90 wanting) : G.S.I.

Parts I-, I884- (pts. for I886/90, I903/04 wanting):

A.S.B.

1287. London, Ont.-Canadian Entomologist. Ed. by W. Saunders. Vols. 22-. London, Ont., I890-: Z.S.I.

Montreal.-Geological (and Natural History) Survey of Canada (see No. 1293).

— Royal Society of Canada (see No. 1296).

I288. Ottawa.-Canadian Mining Institute.

(a) Bulletin. Nos. 20-. Montreal, I9I2-: G.S.I.

(b) Journal. Vols. 3-. Ottawa, Igoo-: G.S.I. General index, vols. I-IO, I898-I907 : G.S.I

A.S. = Archaeological Section. Indian Museum

A.S.B. = Asiatic Society of Bensal

B.C. Bishop's College.

B.S.l = Botanical Survey of Inclia.

C.E. = Chemical Examiner to the Govt. of Bengal.

C.U. = Calcutta Universitv.
E.C. =Civil Engineering College, Sibpur G.S.I. = Geological Survey of India.

I.L. = Imperial Library

M.Ba. = Medical College, Bacteriological Laboratory

M.Bi. = Medical College. Biological Laboratory.

M.C. = Medical College (main library) 
I289. Ottawa.-Contributions to Canadian Biology: being studies from the Marine Biological Stations of Canada. I906-1o. Ottawa, IOI2: Z.S.I.

Contributions to Canadian Palaeontolog'' (see No. I293 (b)).

I290. - Department of Agriculture, Canada.

Reports. Experimental Farms. I887-. Ottawa, I888-. Reps. for 1887 - : B.S.I. Reps. for I898- : R.B.G.

I29I. - Department of Agriculture.-Division of Entomology.

(a) (Entomological) Bulletin. Nos. I-. Ottawa, IgII-: Z.S.I.

(b) Entomological Circular. Nos. I-. Ottawa, I9r3-: Z.S.I.

I292. - Department of Mines, Canada.

(a) Anmual Report. I904. Ottawa, I905: G.S.I. [Continued as:] Annual Report on the Mineral Industries of Canada. I905. Ottawa, I907: G.S.I. [Continued as:] Anmual Report on the Mineral Production of Canada. I906-. Ottawa, I909-: G.S.I.

(b) Bulletin. Nos. I-. Ottawa, I909- (nos. 7, 8 wanting): G.S.I.

(c) Summary Report of the Geological Survey Branch. I907-. Ottawa, I908-: G.S.I.

(d) Summary Report of the Mines Branch. I907/08-. Ottawa, I908- (Reps. for I9ng-10 wanting) : G.S.I.

I293. - Geological Survey of Canada (Exploration Géologique du Canada; Geological and Natural History Survey of Canada).

(a) Annual Report. New series, vols. I-I6, I885-1904. Montreal, I886-I906: G.S.I. General index, I885I906: G.S.I. [A continuation of $(i)$.]

(b) Contributions to Canadian Palaeontology. Vols. I-. Ottawa, I885-: G.S.I.

(c) Figures and Descriptions of Canadian Organic Remains. Decades I-4. Montreal, I $858-65$ : G.S.I.

(d) Memoir. Biological Series. Yos. I-. Ottawa, IgIO- : G.S.I.

(e) Memoir. Geological Series. Nos. [I]-. Ottawa, I910-(nos. 3, 19-21, 34, 63, 68, 72 wanting): G.S.I.

(f) Memoir. Topographical Series. No. I. Ottawa, I9I0: G.S.I. $[(d),(e)$ and $(f)$, together with an 'Anthro-

M.o. = Meteorological Office, Alipore.

M.Ph. = Medical College, Physiological Laboratory.

P.C. = Presidency College.

P.o. = Patent Office.

R.B.G. = Royal Botanic Garden

s.c.c. $=$ Scottish Churches College.
S.G. = Surgeon-General's Library.

S.I. = Survey of India.

S.T.M. = School of Tropical Medicine

T.D. = Telegraph Deparlment.

X.C. =St. Xavier s College.

Z.s.I. = Zoological Survey of India. 
I293. Ottawa.-Geological Survey of Canada (Continued).

pological Series' (wanting), also bear numbers as a combined series of Memoirs.]

(g) Mezozoic Fossils. Vol. I, parts I-3. Montreal, I87684 : G.S.I.

(h) Palaeozoic Fossils. Vols. I-3. Montreal, Ottawa, I86I-I006: G.S.I.

(i) Report of Progress (Rapport de Progrès). I844, '48/49, '52/53-58, '63-70/7I, '72/73-84. Montreal, Quebec, Toronto, I $846-84$ : G.S.I. [Continued as (a).]

(i) Report of Progress from its commencement to $\mathrm{I} 863$. Montreal, I863: G.S.I.

General index to $(j)$, and to $(i)$, I $863-84$ : G.S.I.

Geological Survey of Canada.-Department of Mines (see No. I292).

I294. - Geological Survey of Canada.-Victoria Memorial Museum.

(a) Bulletin. No. I. Ottawa, I9I3: G.S.I., A.S.B. [Continued as (b).]

(b) Museum Bulletin. Nos.2-. Ottawa, I9I4-.

Nos. 2-, I9I4- (110. I9 wanting): A.S.B.

Nos. 2-, igI 4-(nos. 6, 9, I6 wanting) : G.S.I.

I295. - Inland Revenue Department, Canada.

Bulletin of the Laboratory of the ... Depariment. Nos. I267. Ottawa, I887-I9I3 (many nos. wanting): B.S.I.

I296. — Royal Society of Canada.

Proceedings and Transactions (Mémoires et Comptes rendus).

Vols. ('Tom.) I-I2, I882-94. Montreal, Ottawa, I88395. General index at end of vol. I2. 2nd ser., vols. I-I2, Ottawa, I895-I9o6. 3rd ser., vols. I-. Ottawa. I907-.

Vols. I-, I $883-$ (2nd ser., vols. 9, Io and 3 rd ser., rol. I wanting): G.S.I.

Vols. I- 2nd ser., vol. II, I883-I906: A.S.B.

Victoria Memorial Museum (see No. I294).

I 297. Quebec.-Department of Colonization, Mines and Fisheries (Ministère de la Colonisation des Mines et des Pêcheries).

Report on Mining Operations in the Province of Quebec (Rapport sur les Opérations. Minières dans la Province de Qliébec). I9II-. Quebec, I9I2-: G.S.I.

A.5. Archaeological Section, Indian Museum.

A.S.B. = Asialic Societv of Bengal.

B.C. = Bishop s College.

B.S.l. = Botanical Survey of India.

C.E. = Chemical Examiner to the Govt. of Bengal.

C.U. = Calcutla University.
E C. = Civil Engineering College, Sibpur. G S.I. = Geolosical Survey of India.

I L. = Imperial Library

M Ba. = Medical Collere, Bacteriological Laboratory

M.Bi. = Medical College. Biological Laboratory.

M.C. I Medical College (main Iibrary). 
I298. Quebec.-Literary and Historical Society.

Transactions. New ser., parts 20-25. Quebec, I89r-I905: G.S.I.

1299. Toronto.-Bureau of Mines.

Report. Nos. 6-15, Toronto, I897-rgo6 (nos. 8, 9, 11 wanting): G.S.I. [Continued as:] Annual Report. Nos. I6-. Toronto, rgo7- : G.S.I.

I300. Canadian Institute.

(a) Anmual (Archacological) Report. Nos. [I]-7, I886/87I893/94. Toronto, I888-94: G.S.I. [The sixth Report is erroneously styled 'Fifth.']

(b) Canadian Journal: a Repertory of Industry, Science, and $A$ rt. Ed. by H. Y. Hind. Vols. I-3. Toronto, I852-55: G.S.I. New ser., vols. I-II. Toronto, I85668: G.S.I. [Continued as:] Canadian Journal of Science, Literature and History. Vols. I2-I5. Toronto, (I868) I870-78: G.S.I. [Continued as (c).]

(c) Proceedings. Vols. I-7. Toronto, I884-90: A.S.B., G.S.I. [Continned as $(d)$. For earlier series see (b).] New ser., vols. I, 2. Toronto, I897-I904 (defective) : G.S.I.

(d) Transactions. Vols. I-. Toronto, I89o-: A.S.B., G.S.I. [For earlier series see $(b)$ and $(c)$.]

General index to Publications, I852-I9I2 : G.S.I.

_L Canadian Journal (see No. I $300(b)$ ).

I зог. - Canadian Mining Journal. Vols. 30-. Toronto, rgog-: G.S.I.

Department of Agriculture, Ontario (see No. I305).

I302. - Entomological Society of Ontario.

Annual Report. Nos. [5]-, I874-. Toronto, I875(nos. 19-24, 26, 33, 34, 4I, 42 wanting): Z.S.I. [Reports for I $874-83$ do not bear numbers.]

1303. - Fruit Growers' Association of Ontario.

Report. I875-78. Toronto, I876-78: Z.S.I.

I304. - Magnetical and Meteorological Observatory.

Observations made at the ...... Observatory. Vols. I-3, I840-48. London, I845-57 : A.S.B.

I305. - Ontario Department of Agriculture.

Bulletin. Nos. I36--. Toronto, I904-(nos. I37-142, 146, 152, 157, 160 wanting): B.S.I.

M.O. = Meteorological Office, Alipore.

M.Ph. = Medical College, Physiological Laboratory.

P.C. = Presidency College.

P.O. = Patent Otfice

R.B.G. = Royal Botanic Garden.

S.C.C. $=$ Scottish Churches College
S.G = Surceon-General's Library.

S.I. = Survey of India.

S.T.M. = Schwol of Tropical Medicine.

T.D. = Telegraph Department

X.C =St. Xivier's Cullege.

Z.s.I. Zoological Survey of India 
I306. Toronto.--Ontario Department of Agriculture.-Bureau of Industries.

(a) Annual Report. I903-. 'Toronto, I904-: B.S.I.

(b) Crop Bulletin. Nos. 85-. Toronto, I904- : B.S.I.

I307. - Public Health Journal. Vols. 3-. Toronto, I9I2- : S.T.M.

I308. University.

(a) Studies: Biological series. Nos.3-. Toronto, I902-: Z.S.I.

(b) - Geological series. Nos. 3-. Toronto, I905(No. 4 wanting) : Z.S.I.

(c) : Pathological series. No. I. Toronto, Igo6: Z.S.I.

(d) - : Physiological series. Nos. I-. Toronto, IgOO- : Z.S.I.

UNITED STATES.

Alabama, State of (see No. I3I4).

I309. Albany.-New York State Museum of Natural History.

(a) Annual Report of the Regents of the University (of the State of New York) on the condition of the State Cabinet of Natural History. Nos. 20-23. Albany, 1867-73 [I872]: G.S.I. [Continued as $(b)$.]

(b) Annual Report on the .... Museum .... by the Regents of the University of the State of New York. Nos. 24-Albany, I872- (nos. 40, 42 wanting): G.S.I.

(c) Bulletin. Nos. I- Albany, I887-.

Nos. I-IO, I887-90: G.S.I.

No. 86, I905: nos. I24-, I908-(nos. 129, 145, 146, $148-154,157$ wanting) : Z.S.I.

(d) Memoir. [No.] 1s, part 2-. Albany, Igo8-: Z.S.I.

(e) Report (being the) Annual Report of the Education Department. Vols. I-. Albany, Ig05-. G.S.I.

Index to publications, I837-I902: G.S.I.

I3I0. - New York State Museum of Natural History.-State Entomologist.

Annual Report on the Injurious and other Insects of the State of New York. Nos. I-I4. Albany, I882-98: Z.S.I.

A.S. Archaeological Section. Indian Museum.

A.S.B. = Asiatic Society of Bengal

B.e. = Bishop s Collegुe

B.S.1. = Botanical Survey of India.

C.E. = Chemical Examiner to the Govt. of Bengat.

c. $U$. Catculta University.
E.C. = Civil Engineering College, Sibpur.

G.S.I. = Geological Survey of India.

I.L. = Imperial Library

M.Ba. = Medical Colterge, Bacteriological Laboratory

M.B1. = Medical College. Biological Laboratory.

M.C. = Medical College (man library). 
I3I I. Ames.-Iowa State College of Agriculture and the Mechanic Arts.-Agricultural Experiment Station.

(a) Bulletin. Nos. I-. Ames, I888- (nos. 7, 9, 23-26, 60, $68,72,85$ wanting): B.S.I.

(b) Research Bulletin. Nos. I-. Ames, IgII- : B.S.I.

I3I2. Amherst.-Massachusetts Agricultural College.-Hatch Experiment Station.

(a) Annual Report. Nos. 5-. I893-. Amherst, I893- : B.S.I.

(b) Bulletin. Nos. 84-II4. Amherst, I902-07 : B.S.I.

Ann Arbor.-Michigan Academy of Science (see No. I387).

I3I3. Atlanta.-Georgia Experiment Station.

Bulletin and Annual Report. Nos. 3, 4. Atlanta, I89396 : B.S.I.

I3I4. Auburn.-Alabama Polytechnic Institute--Agricultural Experiment Station.

Bulletin. Nos. IOI-. Montgomery. Opelika, I899-: B.S.I.

I3I5. Augusta.-Commissioner of Fisheries of the State of Maine.

Report. Nos. I-4: I867-70. Augusta, I869-70: G.S.I., Z.S.I.

I3I6. Austin.-Texas Academy of Science.

Transactions. Tol. I0, I907. Austin, I908: Z.S.I.

I3I7. University of Texas.

Bulletin. Nos. 246, 307, 365 (= Scientific series, nos. 23, 35, 29). Austin. I9I2-I4 : G.S.I. Bulletin. No. I7. Austin, I9I5: G.S.I.

I3I8. Baltimore.-American Chemical Journal. Ed. by I. Remsen. Vols. I-. Baltimore, I879-. Indexes to vols. I-50, I879-I9I3.

Complete set and inderes : G.S.I.

Vols. I-, I 879 - (vols. I3, 32 defective) and indexes to vols. I-20 : A.S.B.

- American Journal of Mathematics (see No. I320 (a)).

- American Journal of Physiology (see No. I328).

I3I9. - John Hopkins Hospital.

Bulletin. Vols. 7-I3. Baltimore, I896-I902 (vols. 8, I2, 13 defective): S.G.

M.o. = Meteorological Office, Alipore.

M.Ph. = Medical College, Physiological Laboratory:

P.c. = Presidency College.

P.O. = Patent Office.

R.B.G. = Royal Botanic Garden.

S.C.C. $=$ Scottish Churches College.
S.G. = Surgeon-General's Library.

S.I. = Survey of India.

S.T.M. = School of Tropical Medicine,

T.D. = Telegraph Department.

X.c. =St. Xavier's College.

Z.S.I. = Zoological Survey of India. 
I320. Baltimore.-John Hopkins University.

(a) American Journal of Mathematics. Vols. I-- Balt. imore, I878-. Indexes to vols. I-Io and II-20.

Complete set: C.U.

Vols. 2-, I879-- : I.L., P.C.

Vols. 2-, I879- (vol. is defective) and indexes: A.S.B.

(b) Annual Report. I879-86. Baltimore, I879-86.

Vols. for I $879-86$ : G.S.I.

Vols. for I880-86 (vol. for I88I wanting) : A.S.B.

(c) Circulars. Vols. I-22 (= nos. I-I64), I879-I903. Baltimore, (I879) I882-I903: G.S.I. New Series, vols. $23-(=$ nos. I66- $)$, I904-. Baltimore, I904(vols. 23-25 defective) : A.S.B.

(d) Studies in Historical and Political Science. Vols. 3-. Baltimore, I885- (vols. II, 23 defective): A.S.B.

I32I. - John Hopkins University.--Biological Laboratory.

(a) Chesapeake Zoölogical Laboratory. Scientific Results. Conducted by W. K. Brooks. [Vol. I.] Session 1878 . Baltimore, I879. [Continued as]: Studies from the Biological Laboratory. Ed. by N. Martin and W. K. Brooks. Vols. 2-5. Baltimore, I883-93.

Vols. I-5, I879-93 (pt. I, vol. 2 wanting): Z.S.I.

Vols. 3-5, I884-93: A.S.B., G.S.I.

(b) Memoirs from the Biological Laboratory. Eid. by W. K. Brooks. Vol. 4, nos. I-4. Baltimore, I808-I900: A.S.B.

1322. - Journal of Biological Chemistry. Vols. I--. New York, Baltimore, Ig05-.

Complete set: M.Ph.

Vols. 24-, I9I6- : P.C.

- Journal of Experimental Zoology (see No. I471).

I323. - Maryland Geological Survey.

[General Reports.] Vols. I-9. Baltimore, I897-I9II: G.S.I.

I324. - Maryland Weather Service.

[Reports.] Vols. I-3. Baltimore, I899-I9IO: G.S.I.

National Academy of Sciences of the United States of America (see No. I5I7).

Berkeley.-Endocrinology (see No. I395).

— Mining and Scientific Press (see No. I493).

A.S. = Archaeological Section. Indian Museum

A.S.B. $=$ Asiatic Society of Bengal.

B.C. = Bishop s College

B.S.I. = Botanical Survey of Jndia

C.E. = Chemical Examiner to the Govt. of Bengal.

c.U. = Calcutta University.
E.c. = Civil Engineering College, Sibpur.

G.S.I. = Geological Survey of India.

I.L. = Imperial Library

M. Ba = Iredical College. Bacteriological Laboratory.

M.Bi. = Aledical College. Biological Laboratory.

M.c. = Medical College (main library). 
I 325. Berkeley.-University of California.

(a) Bulletin of the Department of Geology (see (f)).

(b) Lick Observatory Bulletin. Vols. 6-. Berkeley, I9Io(vol. 7 defective): P.C.

(c) Memoirs. Vols. I-. Berkeley, 1908-.

Complete set: G.S.I.

Vol. 2, I910: R.B.G.

(d) Publications in American Archaeology and Ethnology. Vols. I-. Berkeley, I9o3- (vol. 6 wanting; vols. 4 , 7 defectlve): A.S.B.

(e) Publications in Botany. Vols. I-. Berkeley. I902-. Complete set : R.B.G.

Vol. 3-, I907- : G.S.I.

(f) Publications in Geology (Bulletin of the Department of Geology). Vols. I-. Berkeley, I893-: G.S.I.

(g) Publications in Zoology. Vols. I-. Berkeley, Igo2-: A.S.B., Z.S.I.

I326. - University of California.-Agricultural Experiment Station.

Bulletin. Nos. 78-. Sacramento, I888-(Nos. 80, 81, 83$87,91,93,96,97$, IOI, I03, I05, 107 wanting): B.S.I,

1327. Boston.-American Academy of Arts and Sciences.

(a) Memoirs. Vols. I-4. Boston, I785-I 821 . New ser., vols. I-. Boston, Cambridge, I833-.

Conıplete set: G.S.I.

Vols. I-4, I785-I $82 \mathrm{I}$, and new ser., vols. 3, 5. 8, I 848 , '55, '6r : A.S.B.

Vol. I, I785; new ser., vols. I2, I3, I902-08 : R.B.G.

(b) Proceedings. Vols. I-8. Boston and Cambridge, I848-73. New ser., vols. I-23 (=vols. 9-3I). Boston and Cambridge, I874-96. Vols. 32-. Boston, I897-.

Vols. I-, I $848-$ (rol. 46 wanting) : G.S.I.

Vol. I, I848, and new ser., vols. I-4 (=9-I2), I874$77 ;$ A.S.B.

New ser., vols. $20-(=28-)$, I893- : R.B.G.

American Association for the Advancement of Science (see No. I499).

— American Naturalist (see No. I465).

I 328. - American Oriental Society.

Journal. Tols. I-. Boston and New Haven, I849(vols. 20, 3I, 32 defective): A.S.B.

M. O. = Meteorological Ofince. Aiipore

M.Ph $=$ Medical College. Physiological Laboratory

P C. = Presidencv College.

PO. = Patent Ofincs.

R $\mathbf{B} \cdot \mathbf{G}=$ Roval Botanic Garcien.

S C C $=$ Scottish Churches College.

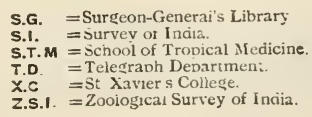

S.G. = Surgeon-Generai's Library

S.I. = survey or India.

T.D = Teleqraph Departmen:

Z.S.I. = Zoological Survey of Inciia. 
1329. Boston.-American Physiological Society.

American Journal of Physiology. Edited for the ... Society by H. P. Bowditch (and others). Vols. I-. Boston, I898-.

Complete set: P.C.

Vols. 22-, I908-(rols. 31, 32 wanting, vol. 35 defective): M.Ph.

— Boston Journal of Natural History (see No. I334 (a)).

1330. - Boston Medical and Surgical Journal. Vols. 158-. Boston. I908-: : A.S.B.

I33I. - Iron and Steel Magazine (successor to the Metallographist). Ed. by A. Sauveur. Vols. 7-II. Boston and Cambridge. I904-06 : G.S.I. [For eariier vols, see No. 1333.]

- Journal of Morphology (see No. 1472).

1332. - Massachusetts Horticultural Society.

Transactions. I899-- Boston, I899- : R.B.G.

I333. - Metallographist. Ed. by A. Sauveur. Vols. I-6. Boston, I898--I903 : G.S.I. [Continued as No. I33I.]

1334. - Society of Natural History.

(a) Boston Journal of Natural History, containing Papers, etc., read to the ...... Society. Vols. I-7, I834-63. Boston, I837-63: A.S.B., G.S.I. [Continued as (b).]

(b) Memoirs read before the .... Society .... being a new series of the Boston Journal. Vols. I-. Boston. I866-.

Complete set: G.S.I.

Vols. I-7, I866-I9I 2 (vol. 6 wanting; rols. I, 5 defective) : A.S.B.

Vols. 3, 4, I878-94 : R.B.G.

Vol. 7 , I912 : Z.S.I.

(c) Occasional Papers. Vols. I-. Boston, I869-.

Vols. I-6, I 869-I90I (vol. 5 wanting): G.S.I.

Vols. 2-, I875- (vols. 5,6 wanting) : A.S.B.

(d) Proceedings. Vols. $\mathrm{I}-\mathrm{I} 84 \mathrm{I}-$. Boston, $\mathrm{I} 844-$.

Complete set: G.S.I.

Vols. 2-, I848- (vol. 31 defective) : A.S.B.

Vols. 9, I3-28, I862-63, '69-99 : R.B.G.

Vols. I9-, I878- : Z.S.I.

A.S. = Archaeological Section, Indian Museum.,

A.S.B. = Asiatic Society of Bengal.

B.C. = Bishop's College.

B.S.I. = Botanical Survey of India.

C.E. = Chemical Fxaminer to the Govt. of Bengal.

c.U. Calcutta University.
E.C. = Civil Engineerins College, Sibpur

G.S.I. = Geologtcal Survey of India

I L. = Imperial Library.

M Ba. = Medical Colleqe, Bacteriological Laboratory

M.Bi. = Medical College. Biological Laboratory.

M.C. = Medical College (nain library). 
I335. Boston.-State Board of Agriculture, Massachusetts.

Anmul Report on the Injurious and Beneficial Insects of Massachusetts. By A. S. Packard. Vols. I, 2. Boston, I87I-72: G.S.I.

I336. Boulder.-University of Colorado.

Studies. Editor, F. Ramaley. Vols. 3-. Boulder, I905- : R.B.G.

I337. Brooklyn.-Institute of Arts and Sciences.-Museum.

(a) Brooklyn Museum Quarterly. Vols I-. Brookiyn, I9I4- : Z.S.I. [A continuation of (c).]

(b) Cold Spring Harbor Monographs. Nos. I-7. Brook1yn, I903-09: Z.S.I.

(c) Museum Neres. Vols. I-6. Brooklyn. I905-I I (defective): Z.S.I. [Vols. 7,8 wanting; continued as $(a)$.]

(d) Science Bulletin. Vols. I-. New York, Igor-.

Complete set : Z.S.I.

Vols. 2-, I9I3- : A.S.B.

I338. Buffalo.- Society of Natural Sciences.

Bulletin. Vols. I-7. Buffalo, x874-I9oI.

Vols. I-6, I 874-99 : G.S.I.

Vols. 5-7, no. I, I897-I90I : A.S.B.

I339. Burlington.-Geological Survey, State of Vermont.

Annual Report on the Geology of the State of Iermont. By C. B. Adams. Nos. I-3. Burlington, I 845-47 : G.S.I.

California, State of (see Nos. I325, I326, I484, I485, I492).

Cambridge, Mass.-American Academy of Arts and Sciences (see No. I327).

American Association for the Advancement of Science (see No. I489).

r340. - Entomological Club.

Psyche: organ of the Cambridge .... Club. Vols. I, I7,

I9. Cambridge, I877, I9I0, I9I2 (all defective): Z.S.I.

I34I. - Harvard College.-Museum of Comparative Zoology.

(a) Annual Report. I862-. Cambridge, r873-.

Reps. for 1862 - : G.S.I.

Reps. for I89I/92-I9I2/I3: Z.S.I.

M.O. = Meteorological Ofíce, Alipore.

M.Ph. = Medical Collere, Physiological Laboratory.

P.C. = Presidency College.

P.O. = Patent Olfice

R.B.G = Royal Botanic Garden.

S.C.C. $=$ Scottish Churches College.

S.G = Surgeon-General's Library

S.I. = Survey of India.

S.T.M. = School of Tropical Meciicinc.

T.D. = Telegraph Department.

X.C =St. Xavier's College.

Z.s.l. = Zoological survey of India 
I34I. Cambridge.-Harvard College--Museum .. (Continued).

(b) Bulletin. Vols. I-. Cambridge, I863-.

Complete set: G.S.I.

Vols. 2, 3, I $870-76$ (defective) : A.S.B.

Vols. 4,5 , I4-I6, 23--, I878-79, I 888-95, I892(vols. 5 , I6 defective) : Z.S.I.

(c) Illustrated Catalogue of the Museum. Nos. I-8. Cam. bridge, I $864-75$. [Continued as $(d)$, see note thereunder.]

Complete set: G.S.I.

Nos I-4, I865-7I; No. 8, I874-75: Z.S.I.

(d) Memoirs. Nos. 9, Io. Cambridge, I876. [A continuation of $(c)$. These numbers, together with those given under (c), were afterwards collected into volumes: Vol. $\mathrm{\tau}=$ Nos. 1 and 2 ; Vol. $2=$ Nos. $3-6$ and 9 ; Vol. $3=$ No. 7 ; Vol. 4 $=$ Nos. 8 and ro. $]$ Vols. 5-. Cambridge, 1877 -

Nos. 9, Io and vols. 5-, I8g6- (rols. 20, 30 wanting) : G.S.I.

Nos. 9, Io and vol. 5, no. I, I $876-77$ : A.S.B.

Vols. 5, 6, 7, I4, I7, I877-94 (all defective); vols. I 8 -, I $895^{---}$: Z.S.I.

I342. - Harvard College.-Astronomical Observatory.

Annals. Vols. I I-. Cambridge, I879)-(rols. It, 18 want ing) : M.O.

- Psyche (see No. I340).

1343. Carlisle.-Military Surgeon. Vols. 22-. Carlisle, Washington, Igo8- : S.T.M.

I344. Charlotte.-Charlotte Medical Journal. Vols. 57-. Charlotte, Igo8- : S.T.M.

I345. Charlottesville.-Virginia Geological Survey.

Bulletin. Nos. I-. Charlottesville, I005-: G.S.I.

I346. Chicago.-Academy of Sciences.

Bulletin. Vols. 2, 3. Chicago, I895-I9I I (pt. 1, vol. 2, wanting); Z.S.I.

I347. Academy of Sciences.-Geological and Natural History Survey.

Bulletin. Nos. I-7. Chicago, I896-I909: Z.S.I.

Nos. I-7, I 896-I909: Z.S.I.

Nos. I-4, pt. I, I 896-I 900 : G.S.I.

I348. Acetylene Journal. Vols. 7-I4. Chicago, I9O5-I3 (vol. it wanting; vols. $7,12-14$ defective) : I.L.

A.S. = Archaeological Section, indian Museum

A.S.8. = Asiatic Society of Bengal

B.C. = Bishop's College

B.S.1. = Botanical Survey of India

C.E. Chemical Examiner to the Govt. of Bengal.

C.U. = Calcutta University
E.C. Civil Engineering College, Sibpu G.S.I. = Geologtcal Survey of India.

I.L. = Imperial Library.

M. Ba. = Medical College, Bacteriological Laburatory

M.Bi. = Medical College. Biological Laboratory.

M.C. = Iedical College (main library) 
I349. Chicago.-American Antiquarian (and Oriental) Journal. Ed. by S. D. Peet. Vols. 5-I5. Chicago, Salem, I883-I9Io (vols. I9. 2 I-27 wanting, vols. I6-I $8,20,28-32$ defective) : A.S.B.

1350. - American Medical Association.

Journal. Vols. 50-. Chicago, I9o8- : S.T.M.

- Astrophysical Journal (see No. 1356).

- Botanical Gazette (see No. I364).

I35I. - Field Museum of Natural History (Field Columbian Museum).

(a) Publications. Anthropological series. Vols. I-. Chicago, I895-

Complete set: Z.S.I.

Vols. I-, I895- (rols. 6, 7 defective) : A.S.B.

(b) - Botanical series. Vols. I-. Chicago, I895-.

Complete set: Z.S.I.

Vols. I-, I895- (vols. 2, 3 defective) : A.S.B.

(c) Geological series. Vols. I-. Chicago, I895-.

Complete sets: A.S.B., Z.S.I.

Vols. I-, I895- (vol. I defective) : G.S.I.

(d) Ornithological series. Vols. I-. Chicago, I8g6- : A.S.B., Z.S.I.

(e) - Report series. Vols. I-. Chicago, I895-.

Complete sets : A.S.B., Z.S.I.

Vols. I-, I895- (vol. I defective) : G.S.I.

(f) Z Zoological series. Vols. I-. Chicago, I895-.

Complete sets : A.S.B., Z.S.I.

Vol. I, I895-99 (defective) : G.S.I.

I352. Journal of Geology : a semi-quarterly Magazine of Geology and related Sciences. Editors T. C. Chamberlain, R. D. Salisbury and others. Vols. I- Chicago, I893-.

Complete set : G.S.I.

Vols. 5-I7, I897-I909 (no. 2, vol. 9 wanting): A.S.B.

I353. - Journal of Infectious Diseases. Ed. by I. Hektoen and E. O. Jordan. Vols. I6-. Chicago, I9I5-: M.Ba.

I354. - Ophthalmology. Ed. by H. V. WVürdemann. Vols. 3-. Seattle, Chicago, Igo6- (vols. 3, 5 defective) : S.T.M.

I355. - Wilson Bulletin: a quarterly journal of ornithology. Vols. I4-. Chicago, Seattle, I902- (no. 38, vol. I4 wanting): A.S.B.

M.o. = Meteorological Office, Alipore.

M.Ph. = Medical College. Physiological Laboratory.

P.C. = Presidency College.

P.o. = Patent Office

R.B.G. = Royal Botanic Garden

S.C.C. $=$ Scottish Churches College.

s.G. =Surgeon-General's Library.

S.I. = Survey of India

S.T.M. = School of Tropical Medicine

T.D. = Telegraph Department.

X.C. =St. Xavier s College.

Z.S.I. = Zoological Survey of India. 
1356. Chicago.-University.

Astrophysical Journal: an international review of spectroscopy and astronomical physics. Vols. I-. Chicago, I895-. General index to vols. I-25, I895-1907.

Vols. $1-36, \mathrm{r} 895^{-1912}$, and index : X.c.

Vols. 2I-, I905-: P.C.

1357. Cincinnati.-Lloyd Library of Botany, Pharmacy and Materia Medica.

(a) Bulletin. Mycological series. Nos. I-. Cincinnati, 1902- : B.S.I.

(b) - Pharmacy series. Nos. I-. Cincinnati. I902-: B.S.I.

(c) - Reproduction series. Nos. I-. Cincinnati, 1900- : B.S.I.

(d) Mycological writings of Mr. C. G. Lloyd. Letters. Nos. I-28. Cincinnati, I904-I0: B.S.I.

(e) - Mycological Notes. Nos. I-36. Cincinnati, I898-r910: B.S.I.

(f) - Mycological Notes, Polyporoid Issue. Nos. I-3. Cincinnati, I908-IO: B.S.I.

1358. - Society of Natural History.

Journal. Vols. I-. Cincinnati, I878-.

Complete set: G.S.I.

Vol. I6, no. I, I893; vols. I7-, I894-: Z.S.I.

I359. University.

Terrestrial Magnetism: an international quarterly journal.

Vols. I-3. Chicago, Cincinnati, I 896-98: M.o. [Continued as:] Terrestrial Magnetism and Atmospheric Electricity... Vols. 4-. Cincinnati, I899-: M.o.

1360. Claremont.-Pomona College.

(a) Journal of Economic Botany, as applied to subtropical Horticulture. Vols. I--. Claremont, I9II-: B.S.I., R.B.G.

(b) Journal of Entomology (and Zoology). Vols. I-. Claremont, I909- : Z.S.I.

Cleveland.-American Antiquarian and Oriental Journal (see No. I349).

Colorado, State of (see No. 1336).

I361. Columbus.-Geological Survey of Ohio.

(a) Bulletin. Ser. 4. Nos. I-I7. Columbus, I903-12 (nos. 7, 9 wanting) : G.S.I.

\footnotetext{
A.S. Archaeological Section. Indian Museum.

A.S.B = Asiatic Society of Bengal.

B.C. = Bi.hop s College.

B.C. = Botanical Survey of Incia.

C.E. = Chemical Examiner to the Govt of Bengal.

C.U = Calcutta University.
}

E.C. = Civil Engineering College, Sibpur.

G.S.1. = Geological Survey of India.

M. B. = Imperial Library,

M Bi. = Medical College. Biological Laboratory

M.C. = Medical College (main library). 
136r. Columbus.-Geological Survey of Ohio (Continued).

(b) Report. Vols. I-6. Columbus, $1873-88$ (vol. 3 defective ; rols. 4,5 wanting): G.S.I.

(c) Report of Progress (Berichte des Fortschritts). I869, '70. Columbus, I870, '7I : G.S.I. [Continned as (b).]

1362. - Ohio Academy of Science and Ohio State University Scientific Society.

Ohio Journal of Science : official organ of the ... Academy... and ... Society. Vols. 16, no. 5-. Columbus, I916-: A.S.B.

1363. - Ohio State Board of Agriculture.

Annual Report. 2nd ser., nos. 27-32, I872-77. Columbus, I $873-78$ (no. 30 wanting): G.S.I.

Connecticut, State of (see Nos. I4II, I4I2, I5OI).

I364 Crawfordsville.-Botanical Gazette. Ed. by J. M. and M. S. Coulter (and afterwards) C. R. Barnes and J. C. Arthur. Vols. 2-Crawfordsville and Logansport, I878-- : R.B.G. [For vol. I see No. $\left.{ }^{3} 75.\right]$ Author and subject index to vols. I-IO, I875-85: R.B.G.

I365. Davenport.-Academy of Natural Sciences.

Proceedings. Vols. I-, I867一. Davenport, 1876-.

Vol. I, I876: A.S.B.

Vols. 4-, I 886 - : Z.S.I.

Vols. I, 2, $1876-77$ : G.S.I.

1366. Denver.-Ophthalmic Literature. Ed. by E. Jackson. Vols. I, 2. Denver, I9II-I2 (defective) : S.T.M.

1367. Des Moines.-Iowa Geological Survey.

(Annual) Report, with accompanying papers. Vols. I-, I892-. Des Moines, I893-.

Complete set: G.S.I.

Vols. I2-20, I902-IO (vols. 15, I9 wanting) : R.B.G.

I368. Detroit.-Therapentic Gazelte, incorporating Medicine and the Medical Age. 3 rd ser., vols. $24-(=$ vols. $32-)$. Detroit, I908- : S.T.M.

I369. Durham, N. H.--New Hampshire College of Agriculture and Mechanic Arts.-Agricultural Experiment Station.

Bulletin. Nos. 16-. Durham, I892- (nos. 43, 47, 49, 51 , $52,54,55,73,9^{6}, 9^{8}, 99$ wanting) : B.S.I.

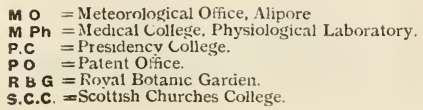

S.C.C. $=$ Scottish Churches College.

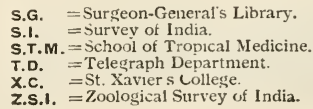


Easton.-American Chemical Society (see No. 1417).

- American Institute of Mining Engineers (see No. I42I).

I370. - American Leather Chemists Association.

Journal. Vols. I-. Easton, Igo6- (vol. I defective): B.S.I.

Chemical Abstracts (see No. I4I7 (a)).

I37I. - Journal of Analytical Chemistry. Ed. by E. Hart. Vols. I, 2. Easton, I $887-88$ : G.S.I.

1372. Flagstaff. -Lowell Observatory.

Bulletin. Vols. I-. Flagstaff, I903-: I.L.

1373. Frankfort, Ky.-Geological Survey of Kentucky.

Report. By D. D. Owen. I854-57. Frankfort, I856-57 : G.S.I.

1374. Geneva, N.Y.-New York Agricultural Experiment Station.

(a) Annual Report of the Board of Control. I892-94. Albany, I $893-95$ : B.S.I.

(b) Bulletin. Nos. 143-286. Geneva, I898-I907 (nos. 145, $148,153,160,165,216,231-8$ wanting) : B.S.I.

Georgia, State of (see No. I313).

I375. Hanover, Ind.-Botanical Bulletin. [Ed. by J. M. Coulter.] Vol. I. Hanover, 1875-76: G.S.I. [For subsequent rols. see No. 1364.1

I376. Harrisburg.-Geological Survey of Pennsylvania.

(a) Annual Report. I885-87. Harrisburg, 1886-89: A.S.B.

(b) Reports of Progress. A-Z. Harrisburg, $1875^{-85}$ : G.S.I.

Illinois, State of (see Nos. I496, I505. I506, I509, I500).

Indiana, State of (see No. I377-1379).

1377. Indianapolis.-Department of Creology and Natural Resources of Indiana.

Anmial Report. Nos. 22-27, I897-1902. Indianapolis, I $898-$ I 903 .

Nos. 22-26, I 898-I903: G.S.I.

Nos. $22-27,1898-1903$ (110. 25 wanting) : A.S.B.

A.S = Archaeological Section, Indian Museum.

A.S.B. = Astatic Society of Bengal.

B.C. = Bishop: Collegse.

B.S.I. = Botanical Survey of India.

C.E. = Chemical Examiner to the Gurt of Bengal.

c.U. = Calcutla University:
E.C. = Civil Engineering College. Sibpur

G.S.1. = Geological Survey of India.

i.L. = Imperial Library.

M.Ba. = Medtcal Coltere. Bacteriological Laboratory

M.Bi. = Medical College. Bılogical Laboraiory,

M.C. = Medical College (main library). 
I378. Indianapolis.--Geological Survey of Indiana.

Annual Report. By E. T. Cox. Nos. I-IO, I869-78. Indianapolis, I869-79: G.S.I.

1379. - Indiana Academy of Science.

Proceedings. I894-. Indianapolis, I895-.

Vols. for I $894-1908$ : G.S.I., Z.S.I.

Vols. for I895- (vols. for 1896 , 1902, 'O4, '10 wanting): A.S.B.

Iowa, State of (see Nos. I3I I, I367).

Ithaca.-American Physical Society (see No. I382).

1380. Cornell University.-Agricultural Experiment Station.

(a) Annual Report. I892-I90I. Albany, I893-I902 (rols. for 1893 , '96 wanting) : B.S.I.

(b) Bulletin. Nos. 7-. Ithaca, I889-(many nos. wanting) : B.S.I.

I38r. - New York State Weather Bureau.

Report. I889-98. Ithaca, I889-98: M.O.

1382. - Physical Review: a journal of experimental and theoretical physics. Ed. by E. L. Nichols, E. Merritt and F. Bedell. Vols. I-35. New York, Lancaster and Ithaca, I894-I9I2. Ser. 2, vols. I-. Lancaster and Ithaca, I9I3-. [Vols. I6-35 conducted with the co-operation of the American Physical Society; ser. 2, vols. 1-, conducted by the Society.]

Complete set: P.C.

Vols. 22-, I906-: E.C.

1383. Jefferson City.-Missouri Bureau of Geology and Mines.

(a) Annual Report of the Geological Survey of Missouri. Nos. I. 2. By G. C. Swallow. Jefferson City, I855: G.S.I.

(b) Biennial Report of the State Geologist. I901/02-. Jefferson City, I903- : G.S.I.

(c) [Publications.] Missouri Geological Survey. Vols. 4. 6, 7, I3. Jefferson City, I894-I900: G.S.I. [Continued as:] [Publications.] Missouri Bureau of Geology and Mines. Ser. 2, vols. I-. Jefferson City. I903-: G.S.I.

(d) Report of the Geological Survey of the State of Missouri, including Field Work of $\mathrm{I} 873-74$. By G. C. Broadhead. Jefferson City, I874 : G.S.I.

M.o. = Meteorological Office, Alipore.

M.Ph. = Medical College, Physiological I aboratory.

P.C. = Presidency College.

P.O. = Patent O.fice.

R.B.G = Royal Botanic Garden.

S.C.C. = Scottish Churches College.
S.G. = Surgcon-General s Library.

S.I. = Survey of India.

S.T.M. = School of Tropical Medicine

T.D. = Telegraph Department

x.c. =St. Navier s College.

z.s.I. = Zoological Survey of India. 
I384. Jefferson City.-Missouri State Board of Agriculture.

Annual Report on.the Noxious, Beneficial and other Insects of the State of Missouri. By C. V. Riley. Nos. I-9. Jefferson City, I869-77: Z.S.I. Supplement and general index to Nos. I-9, i 88I : Z.S.I. [The supplement and index form Bulletin 6 of No. 1535.]

Kansas, State of (see Nos. I390, I39I).

Kentucky, State of (see Nos. 1373, I392).

I385. Kingston, R. I. - Rhode I. College of Agriculture and Mechanic Arts.-Agricultural Experiment Station of the Rhode I. State College.

(a) Bulletin. Nos. 6-. Kingston, 1890- (nos. 7-15, 17$22,25-28,30,60$ wanting) : B.S.I.

(b) Report. I893-. Kingston, I894- (Reps. for 1893, 1903/04 defective) : B.S.I.

Lancaster.-American Mathematical Society (see No. I424).

- American Physical Society (see No. 1382).

1386. - Economic Geology, with which is incorporated the American Geologist. Vols. I-. Lancaster, I906-: G.S.I. [For earlier series see No. I4 4 I.]

- Journal of Morphology (see No. 1472).

- Physical Review (see No. 1382).

I387. Lansing.-Michigan Academy of Science.

Report. Nos. I-. I894-99-. Lansing, I900-.

Complete set: A.S.B.

No. 7-, I905- : R.B.G.

1388. - Michigan State Agricultural College-Experiment Station.

(a) Bulletin. Nos. 212-. I ansing, I904-: B.S.I.

(b) Special Bulletin. Nos. 25-. Lansing, I904-: B.S.I.

I389. Las Cruces.--New Mexicn College of Agriculture and Mechanic Arts.-Agricultural Experiment Station.

Bulletin. Nos. 8I-. I as Cruces, I9I2-: Z.S.I.

1390. Lawrence.--University of Kansas.

(a) Kansas University Quarterly. Vols. I-IO. Lawrence. I893-I9or. [Continued as $(b)$.]

Complete set: G.S.I.

Vols. I-IO, I893-I9OI (no. 3, vol. 10, wanting) : A.S.B.

Vols. I-IO, I893-I9OI (vols. I, 2, 5-8 defective) : Z.S.I.

\footnotetext{
A.S. = Archaeological Section, Indian Muscum

A.S.B. = Asiatic Sociely of Bengal.

B.C. = Bishop s College.

B.S.I. = Botanical Survey of India

C.E. = Chemical Examiner to the Guvt. of Bengal

c.U. = Calculta University.
}
E.C. = Cuvil Engineering College, Sibpur.
G.S.I = Gcological Survey of India.
I.L. = Imperial Library.
M.Ba. = Medical College, Bacterioloyical Laboralory
M.Bi. = Medical College. Bıological Labotatory.
M.C. = Medical College (nam library)


I390. Lawrence.-University of Kansas (Contimued).

(b) Kansas University Science Bulletin. Vols. I-. Lawrence, I902-: A.S.B., G.S.I., Z.S.I.

I391. University.—Geological Survey of Kansas.

(a) Anmual Bulletin on Mineral Resources of Kansas. I897-I903. Lawrence, I898-I904 (vols. for I899-Igor wanting) : G.S.I.

(b) [Publications.] Vols. I-9. Topeka, I896-Igo8.

Vols. I-9, I896-I908 (vol. 7 wanting) : G.S.I.

Vols. 8, 9, 1904-08 : A.S.B.

1392. Lexington.-State College of Kentucky.-Agricultural Experiment Station.

(a) Annual Report. Nos. 19-, I906-. Lexington, I907- : B.S.I.

(b) Bulletin. Nos. 2-. Lexingtori, 1886- (nos. 3-5, 7, 8, 10, 88,98, т05-107, 134 wanting) : B.S.I.

1393. Lincoln, Neb.-University of Nebraska.

(a) Studies from the Zoological Laboratory. Vols. 4(=Nos. 6I-). Lincoln, I905-: Z.S.I.

(b) University Studies. Vols. Io-. Lincoln, I910-: A.S.B.

1394. - University of Nebraska.-Agricultural Experiment Station.

Bulletin. Nos. 85-. Lincoln, I904-(nos. 86, 88, 91, 92, 97, 98,103, IO6, Ir $5-118$ wanting) : B.S.I.

I395. Los Angeles.-Association for the Study of Internal Secretions.

Endocrinology, Bulletin of the Association.. Vols. I-. [Los Angeles?], I9I7- : S.T.M.

I396. Madison.-Geological and Natural History Survey of the State of Wisconsin.

(a) Bulletin. Nos. I-. Madison, I898-.

Nos. I, 2, I 898 : Z.S.I.

Nos. I0-, I903-- (nos. II-I4, I9, 29-32 wanting): G.S.I.

I397. - University of Wisconsin.-Agricultural Experiment Station. Annual Report. Nos. II-I3. I894-96. Madison, I895-97 : B.S.I.

1398. W Wisconsin Academy of Sciences, Arts and Letters.

Transactions. Vols. 3-. Madison, I876-.

Vols. 3-, I876-: Z.S.I.

Vols. 3-I5, I 876-Ig07 (pt. 2, vol. I4 wanting) : A.S.B.

Vols. 8. II-I3, pt. I. I892, '98-I90I : R.B.G.

Vols. II, I5, pt. 2-, I898, I907-: G.S.I.

м o. = Meteorological Office, Alipore.

M.Ph. = Medical College. Physiological Laboratory.

P.C. = Presidency College.

P.O. = Patent Oifice.

R.B.G. = Royal Botanic Garden.

S.C.C. = Scuttish Churches College
S.G. = Surgeon-General's Library.

S.I. = Survey of Iuclia.

S.T.M. = School of Tropical Medicine

T.D. = Telesraph Department.

X.c. =St. Xavier s Collese.

Z.S.I. = Zoological Survey of Indiat 
Maine, State of (see Nos. I3I5, I458).

Maryland, State of (see Nos. I323, I324).

Massachusetts, State of (see Nos. I3I2, I332. I335).

Medford see Tufts College.

Michigan, State of (see Nos. 1387, I388).

I399. Milwaukee.-Public Museum.

Bulletin. Nos. I-. Milwaukee, Igro-: Z.S.I.

I400. - Wisconsin Natural History Society.

(a) Bulletin. New ser., vols. I-. Milwaukee, I900-.

Complete set: R.B.G.

Vols. I-, I900- (pt. I, vol. 2, wanting) : Z.S.I.

(b) Occasional Papers. Vol. I, no. 3 ; vols. 2, 3. Milwaukee, I 890 , I $892-96$ : Z.S.I.

I40I. Minneapolis-American Geologist: a monthly journal of geology and allied sciences. Vols. 9-36. Minneapolis, I892-I905: G.S.I. Index to vols. I-36 at end of vol. 36. [Afterwards incorporated with No. 1386.]

I402. - Geological and Natural History Survey of Minnesota.

(a) Anmual Reports. Nos. I-24, $1872-98$. Minneapolis, St. Paul, $1876-99$ (nos. $1-3$ are of the 2 nd edition; no. 14 wanting): G.S.I.

(b) Bulletin. Nos. I, 4, 5, 7, 8, Io. St. Paul, I887-94: G.S.I.

(c) Final Reports. Geology of Minnesota. Vols. I-6. Minneapolis, St. Paul, I884-IgoI : G.S.I.

(d) Minnesota Botanical Studies. Vol. I (=Bullctin, Bot. Series, no. 9, and Reports, Bot. Series, no. 2). Ed. by C. Macmillan. Minneapolis, I $894-98$ : R.B.G. Ser. 2, Parts I-. Minneapolis, I $898-$ : R.B.G.

(c) Reports of the Survey. Botanical Series. No. I. Miuneapolis, I892 : R.B.G. [For no. 2 see $(d)$.]

r403. - Minnesota Academy of Natural Sciences.

Bulletin. Vols. I-3; vol. 4, no. I, pts. I, 2, and no. 3 . Minneapolis, I 874-I9I0: G.S.I.

_ Minnesota Botanical Studies (sec No. $1402(d)$ ).

1404. University of Minnesota.-Geological Survey.

Bulletin. Nos. II-. Minneapolis, I9I4- : G.S.I.

A.s. = Archaeological Section, Indian Museum.

A.S.B. $=$ A siatic Society of Bengal.

B.C. = Bishop s College.

B.S.I. = Botanical Survey of India.

C.E. = Chemical Examiner to the Govt. of Bengal.

c.U. = Calcutta University.
E.C. = Civil Enøineering College. Sibpur.

G.S I. = Geolorical Survey of India.

I.L. = Impertal Library

M.Ba. = Medical Collere, Bacterioloøical Laboratory

M.Ba. = Medical College. Biological Laboratory.

M.C. = Miedical College (main library). 
I405. Minneapolis.-University of Minnesota.-Minnesota School of Mines.-Experiment Station.

Bulletin. Nos.3-. Minneapolis, I9I5-: G.S.I.

Minnesota, State of (see Nos. 1402-I405).

1406. Missoula.--University of Montana.

Bulletin. Biological Series. Nos. I, 3, 6, I0, II, I4. Missoula, I90I-0S: Z.S.I.

Missouri, State of (see Nos. I383, I384, I 488 ).

Montana, State of (see No. I406).

Montgomery.-Alabama Agricultural Experiment Station (see No. I3I4).

Mount Hamilton.-Lick Observatory (see No. I325 (b)).

I407. Mount Weather.-Observatory.

Bulletin. Vols. I-. Washington, I908-: M.O.

I408. Nashville.-Geological Survey of the State of Tennessee.

(a) Bulletin. Nos. 3-5. Nashville, I9IO-II : G.S.I.

(b) Geological Report to the .... General Assembly. Nos. 5, 6, I839, I84I. Nashville, I840-4I : G.S I.

Nebraska, State of (see Nos. I393, I394).

I409. New Brunswick.-New Jersey Agricultural Experiment Station.

(a) Annual Report. 1902-. Trenton, I903- : B.S.I.

(b) Bulletin. Nos. 156-. New Brunswick, I902(nos. $165_{5}-167,204$ wanting) : B.S.I.

(c) Report of the Entomological Department. I8g6-I9iI. Trenton, Somerville, Paterson, I897-I9I2 (Reps. for I899, 1906, '07.' 'og wanting): Z.S.I.

New Hampshire, State of (see No. 1369).

I4Io. New Haven.-American Journal of Science, more especially of Mineralogy, Geology an.t other branches of Natural History..... Conducted by B. Silliman. Vol. I. New York, I8I9. [Continued as:] American Journal of Science and Arts. Conducted by Silliman Sr. and Jr., Vols. 2-50. New Haven, I 820-45 [47]. [Vol. 50 is a general index to vols. I-49.] 2nd series. Conducted by Silliman and Dana. Vols. I-50. New Haven, I846-70. 3rd series. Editors... Dana, Silliman, and others. Vols. I-I8. New Haven, I871-79. [Continued as:] American Joumal of

M.o. = Meteorological Office, Alipors

M.Ph. = Medical College. Physiological Laboratory.

P.C. = Presidency College.

P.o. = Patent O.fice

R.B.G = Royal Botanic Garden

s.C.C. $=$ Scottish Churches College
S.G. = Surgeon-General's Library.

s.l. = Survey of India.

S.T.M. = School of Tropical Medicine.

T.D. = Telegraph Department.

X.C. =St. Xavier's College.

Z.S.I. = Zoological Survey of India. 
I4Io. New Haven.-American Journal of Science (Continued).

Science. 3rd series. Editors... Dana, Silliman, and others. Vols. I9-50. New Haven, I880-95. 4th series. Editors... Dana, and others. Vols. I-. New Haven, I896-. [In the second and succeeding series there are general indexes at the end of every tenth volume.]

Complete set: G.S.I.

2nd ser., vol. I5- 3rd ser., vol. 6, I853-73 : R.B.G. 2nd ser., vol. I9- $4^{\text {th }}$ ser., vol. I6, I855-I903 (3rd ser., vol. 6, wanting) : A.S.B.

2nd ser., vols. $23-29$, I $857^{-59}$ (vols. 25,26 wanting : E.C.

2nd ser., vol. $4 \mathrm{I}$ - 3rd ser.. vol. 6, I866-73: Z.S.I.

American Oriental Society (see No. I328).

I4II. - Connecticut Academy of Arts and Sciences.

(a) Memoirs. Vols. 2-. New Haven, Igro-: A.S.B., Z.S.I.

(b) Transactions. Vols. I-. New Haven, I866-.

Complete set : Z.S.I.

Vols. I-, I 866- (vol. 13 defective) : A.S.B.

Vols. I-, I 866 - (vols. I4, I7 defective) : G.S.I.

Vols. I-, I 866 - (vols. I5, I7 wanting; vol. 16 defective): M.O.

(c) Memoirs. Vols. 4-. New Haven, I9I5- : G.S.I.

I4I2. - Connecticut Agricultural Experiment Station.

Annual Report. I884-. New Haven, Hartford, I885B.S.I.

I4I3. Yale University.

Studies from the Yale P'sychological Laboratory. Ed. by E. W. Scripture. I 892 93. New Haven, I893: A.S.B. Vols. 4-IO. New Haven, I8g6-igo2 (vol. 8 wanting): A.S.B.

I4r4. - Yale University.-Astronomical Observatory.

Transactions. Vols. I-. New Haven, [I887]- (rol. 2 defective): A.S.B.

New Jersey, State of (see No. I409, I502).

New Mexico, State of (see No. I380).

I4I5. New Orleans.-New Orleans Medical and Surgical Journal. Vols. 69-. New Orleans, I9I6- : S.T.M.

A.S. Archaeological Section. Indian Museum

A.S B $=$ Asiatic Society of Bengal.

B.C. = Bishop s College.

B.S.I. = Botanical Survey of India

C.E. = Chemical Examiner to the Govt. of Benga!

C.U =Calcutta Unversity.
E.c. = Civil Engineering College, Sibpur

G.S.I. = ireological Survey of India.

IL. = Imperial Library.

M. $\mathbf{B a}_{2}=$ Medical Collese, Bacteriological Laboratory:

M.B1. = Medical College. Biological Liboratory:

M.C. = Sedical College (main library). 
I4I6. New York.-Academy of Sciences.

(a) Amnals. [2nd ser.] Vols. I- New York, I879 (I877)- (vols. 5, 6 defective): G.S.I. [For earlier series see No. 1446].

(b) Memoirs. No. I. pt. I : vol. 2, pts. I-4. New York, I895, IS99-I905: G.S.I.

(c) Transactions. Vols. I-I6. New York, I88I-98: G.S.I.

- Agricultural Experiment Station (see No. I380).

I4I7. - American Chemical Society.

(a) Chemical Abstracts. Vols. 4-. Easton, Igro-: E.C.

(b) Journal. Vols. 23-. Easton, I gor-.

$$
\begin{aligned}
& \text { Vols. } 23-\text { I I90I-: G.S.I. } \\
& \text { Vols. } 31-\text { I I909-: E.C. } \\
& \text { Vols. } 36-\text { I0I4-: P.C. }
\end{aligned}
$$

I4I8. American Chemist. Edited by C. F. and IV. H. Chandler. Vols. 2, 3. New York. I8 $72-3$ : C.E.

I4I9. - American Fithnological Society.

Transactions. Vol.2. New York. I848: A.S.B.

1420. - American Geographical Society.

(a) Bulletin (formerly Journal). Vols. 42-47. New York, I9IO-I5: G.S.I. [Continued as (b).]

(b) Geographical Reriere. Vols. I-. New York, I9I6-: G.S.I.

I42I. - American Institute of Mining Engineers.

(a) Bulletin. Nos.37-. New York, I9I0-(nos. 90, 108I 10 wanting): G.S.I.

(b) Transactions. Vols. I-. Philadelphia, Easton, New York, IS73- : G.S.I. Contents and index to vols. I25 : G.S.I. General alphabetical and analytical index, vols. I-35. 36-40 : G.S.I.

- American Joumal of Science (see No. I4Io).

1422. - American Journal of Surgery: a magazine of practical surgery $i n$ all its branches. Vols. 22-. New York. I908- : S.T.M.

I423. - American Journal of the Medical Sciences. Vols. 79-. Philadelphia and New York, I880-(vols. 83, 84, 91-94, 96-98, Io3, IOg wanting): S.G.

M.o. = Meteorological Oftice, Alipore.

M.Ph. = Medical Collese. Physiological Laboratort:

P.C. = Presidency College

P.o. = Patent Office.

R.B.G. = Royal Botanic Garden.

S.C.C. = Scottish Churches College.
S.G. = surseon-General's Library:

S.I. = Survey of India.

S.T.M. = School of Tropical Medicine.

T.D. = Telegraph Department.

X.C. = St. Xavier's College.

Z.S I. = Zooiogical Survey of India. 
I424. New York.-American Mathematical Society.

(a) Bulletin. 2nd ser., vols. I-- Lancaster and New York, I895 (I894) - : P.C. General indexes, vols. I20: P.C.

(b) Transactions. Vols. I-. Lancaster and New York, Igoo-.

Complete set: C.U.

Vols. II-, IgIO- : P.C.

1425. - American Metrological Society.

Proceedings. Vols. I, 2, I873-79. New York, I880 : G.S.I.

I426. - American Museum of Natural History.

(a) Annual Report. I88o-. New York, I880-.

Reps. for I880-: Z.S.I.

Reps. for I887/58- : A.S.B.

Reps. for I 896 - (Rep. for 1937 wanting): G.S.I.

(b) Anthropological Papers. Vols. I-. New York, I 907 - : Z.S.I.

(c) American Museum Journal (a popular record of the progress of the .... Museum). Vols. I-. New York, IgOO-: Z.S.I.

(d) Bulletin. Vols. I- New York, I88I-.

Vols. I-, I $88 \mathrm{I}$ - (pt. 2, vol. 25 wanting): Z.S.I.

Vols. I-, I $88 \mathrm{I}-$ (vols. 15,25 wanting, vols. 17,18 defective): A.S.B.

Vols. 2-, IS87- (vol. 25 defective) : G.S.I.

(c) Memoirs. Vols. I-I4. [New York.] I893-I9o8.

New ser., vols. I-. New York, I9I2-.

Vols. I-I4 and new ser., vols. I-, I893- (old ser., vols. $2,6,7$ wanting, remainder mostly defective): Z.S.I.

Vols. I-I4 and new ser., vols. I-, I893- (old ser., vols. $2,5,7,8$ wanting, vols. $3,4,6,9$ defective): A.S.B.

Vol. I, I893-I903; vol. 3, pt. 3, I904; vol. 9, I90509 ; new ser., vols. I-, I9I2-: G.S.I.

List of papers published in the Bulletin and Memoirs, I88I-I902 : Z.S.I.

American Naturalist (see No. 1465 ).

I427. - American Society of Civil Engineers.

(a) Proceedings. Vols. I9-. New York, I893-.

Vols. I9-, I893- : I.L.

Vols. 27-3I, I9OI-05: E.C.

\footnotetext{
A.S. = Archaeological Section. indian Museum

A.S.B. = Asiatic Society of Ben'sal.

B.C. = Bi<hop $\mathrm{s}$ College.

B.S.I. = Botanical survey of Inda.

C. E. = Chemcal Examiner to the Govt. of Bengal.

c.U. = Calcutta Unversity.
}

E.c. = Civil Engineering College. Sibpur. G.S.I. = reolosical Survey of India.

I.L. = Imperiai Library.

M.Ba. = Medical Colleøe. Bacteriological Laboratory

M. Bi. = Medical College. Biological Laboratory.

M.C. = Medical College (man library). 
1427. New York.-American Society of Civil Engineers (Continued).

(b) Transactions. Vols. $2 \mathrm{I}$-. New York, I889-. Indexes to vols. I-27.

Vols. 2I-, I889- (vols. 24, 26 wanting) and indexes :

I.L.

Vols. 56-6I, 1906-08: E.C.

I428. - Annals of Surgerv. Ed. by L. S. Pilcher and others. Vols. I-52. St. Louis, Philadelphia and New York. I885-I9Io.

Vols. I-44 I 885-I906: S.G.

Vols. I7-52, I893-I910: A.S.B.

1429. - Archives of Oth'halmologv. Ed. by H. Knapp and others. Vols. I7-35. New York. I888-I906: S.G. General indexes to vols. I5-28: S.G.

I430. - Botanical Garden.

(a) Bulletin. Vols. I-. [Lancaster] I896-.

Complete sets : B.S.I., R.B.G.

Vol. 2-, I9OI-: A.S.B.

(b) Journal. Vols. I-. Lancaster, I900-: B.S.I.

- Chemical Abstracts (see No. I4I (a)).

1431. - Coal Age: devnted to Coal Mining and Coke Manufacture. Vols. I-. New York, I9II- : G S.I.

I432 - Columbia University.-Biochemical Association.

Binchemical Bulletin. Vols. I-, nos. I-, I9II-. New York, I9I2-: C.U. •

I433. - Dietetic and Hygienic Gazette. Vols. 27-29. New York, I9I I-I3 (defective) : S.T.M.

1434. — Electrical World. Vols. 9-. New York, I887-.

Vols. 9-, I887- : T.D.

Vols. 55--, I910- : E.C.

1435. Electrochemical and Metallurgical Intustry, with which is incorporated Iron and Steel Magazine. Vols. 4-7. New York, I 906-09 (vols. 4, 5 defective) : G.S.I. [For continuation see No. I450.]

I436. Engineering and Mining Journal. Vols. 73-. New York, I902-.

Vols. 73-, I902- (vols. 74, 75 defective) : G.S.I.

Vols. 79-, I905-(vol. 79 defective) : I.L.

Vols. $82-$, I906- (vol. 87 wanting) : E.C.

M.O. = Meteorological Office. Altpore.

M.Fh = Medical Collerse, Physiological Laboratory.

P.C. = Presiciency College.

P.O. = Patent O. ice

R B.G, = Royal Bo:anic Garden.

S.C.C. $=$ Scottish Churches College.
S.G = Surdeon-General's Library.

S.I. = Survey or India.

S.T.M. = icnux) of Topical Medicine.

T D. = Teiegrapi Depar:ment.

X.C = Si. Xiviers villese.

Z.S.I Zootogical Survey or India

[ 227 ] 
I437. New York.-Engineering Magazine. Vols. I4-. New York and London, I897-.

Vols. I4-, I897- (vols. 26, 30 wanting) : E.C.

Vols. 3I-34, I906-07 (defective) : I.L.

I438. - Engineering News. Vols. 53-. New York, I905- (vol. 53 defective) : I.L.

I439. - Geological Society of America.

Bulletin. Vols. I-. New York, I89o- : G.S.I. Index, vols. I-IO : G.S.I.

I440. - Index Medicus: a monthly classified record of the medical literature of the world. Vols. I-2I. New York, I878-99: C.U. 2nd ser., vols. I-4. New York, I903-06: C.U.

I44I. India Rubber World. Ed. by H. C. Pearson. Vols. 23-. New York, I900- : B.S.I.

1442. - Iron Agc. Vols. 77-. New York, Igo6- (vols. 85-88 want ${ }^{-}$ ing; remainder mostly defective) : I.L.

- Journal of Biological Chemistry (see No. 1322).

I443. - Journal of Experimental Medicine. Ed. by S. Flexner and E. L. Opie. Vols. Io-. New York, Igo8-: M.Ba.

I444. - Journal of Indiustrial and Engineering Chemistry. Vols. I-. New York, Igog- (vol. 2 wanting): E.C.

I445. - Journal of Physical Chemistry. Ed. by W. D. Bancroft and others. Vols. I-. New York, I8g6-.

Complete set: P.C.

Vols. IO-, Igo6-: E.C.

I446. - Lyceum of Natural History:

(a) Annals. Vols. I-II. New York. I\$24-76: A.S.B., G.S.I. (Continued as No. $1 \neq 16(a)$ ).

(b) Proceedings. Ser. I and 2. New York, I870-74: G.S.I.

I447. - Machinery. I9I4-. New York. I9I4- : E.C.

I448. - Metallurgical and Chemical Engineerng. Vols. 8-. New York. I9IO-: E.C., G.S.I. [For earlier vols. see No. 1435.]

I449. - Meteorological Observatory.

Report (of the Director). I874-. New York, I876-: M.o.

- Mineral Industry (see No. 227).

1450. - Mining Magazine: an international monthly review of current progress in mining and metallurgy. Vols. IO-I3. New York, 1904-06 (vols. 12, 13 defective) : G.S.I.

A.S. Archaeological Section. Inclian Museum.

A.S.B. = Asiatic Society of Bengal.

B.c. = Bishop s College.

B.S.I. = Botanical Survey of India

C.E. Chemical Examiner to the Govt. of Bengal.

c.u. $=$ Calcutta University.
E.C. = Civil Enџineering College, Sibpur.

G.S I. = Geological Survey of India.

I.L. = Imperial Library.

M.Ba. = Medical College, Bacteriological Laboratory

M.Bi. = Medical College. Biological Laboratory.

M.c. = licdical College (main library). 
145I. New York.-New York State Journal of Medicine. Vols. 8-. Brooklyn, New York rgo8-: S.T.M.

- Physical Review (sce No. 1382).

1452. - Scientific American. Vols. 20-. New York, I869-.

Vols. 20-103, I869-I9Io (vols. 21-41, 43, 45, 92, 93, 100, roI wanting): E.C.

Vols. $38-$, I 878 - : I.L.

Vols $40-$, I $879-$ (vol. 87 defective) : X.C.

Vols. 66-, IS92-: G.S.I.

1453. - Scientific American Supplement. Vols. 15-. New York, I 883 -

Vols. I5-, I883- (vols. 59, 60, 65, 66 wanting) : E.C.

Vols. 2I-, I886- (vols. 27-32 wanting) : X.C.

Vols. 33-, I892- : G.S.I.

- State Museum of Natural History (see No. I 309).

I454. - Torrey Botanical Club.

Bulletin. Ed. by W. H. Leggett. Vols. 13-24. New York, I886-97 (vols. 17,20 wanting): R.B.G.

I455. - Zoological Society.

Zoologia: Scientific Contributions of the Nerw York.... Society. Vols. I-. New York, I907-.

Complete set : Z.S.I.

Vols. I-, I907- (vol. I defective) : A.S.B.

New York, State of (see Nos. I309, I3Io, 1374).

1456. Newport.-Natural History Society.

Proceedings. Nos. I-9. Newport, I883-I900 (nos. 5, 6 wanting): A.S.B.

North Carolina, State of (see Nos. I48I, I 482 ).

1457. Northfield.-Astronomy and Astro-physics. Ed. by W. W. Payne and G. E. Hale. Vols. II-I3. Northfield, I892-94: A.S.B.

Ohio, State of (see Nos. I361-1363).

Oklahoma, State of (see No. 1500).

1458. Orono.-University of Maine.-Agricultural Experiment Station. Bulletin. Nos. 176-. Orono, rgro-.

Nos. $176-$, I910 - (no. 189 wanting) : B.S.I.

Nos. I90-, I9I I - (many nos, wanting): A.S.B.

Pennsylvania, State of (see Nos. I376, I467, I468, I475-I477, I 498, I 499).

M.O. = Neteorological Office, Aliporc.

M.Ph. = Medical Collese. Physiological 1 aboratory.

P.C. = Presidency College

P.O. = Patent O.fice.

R.B.G = Roval Butanic Garden

s.C.C. $=$ Scottish Churches College.
S.G. = Surgeon-Generai's Library.

S.l. = Survey of India.

S.T.M. = School of Tropical Medicise.

T.D. = Telegraph Department.

X.c. =St. Xavier's College.

z.s.l. = Zoological Survey of Indiz. 
I459. Philadelphia.-Academy of Natural Sciences.

(a) Journal. Vols. I-8. Philadelphia, I8I7-42. Ser. 2, vols. I-. Philadelphia, I847-.

Complete sets : A.S.B., G.S.I.

Vols. I-6, ISI7-29: R.B.G.

Ser. 2, vols. II-. IS99- (vol. 1 I defective) : Z.S.I.

(b) Proceedings. Vols. I-, I $84 \mathrm{I}$-. Philadelphia, I843-.

[Vols, 9-52 do not bear vol. nos.]

Complete sets : A.S.B., G.S.I., R.B.G.

Vols. for $\mathrm{I}^{8} 88_{3}-$ : Z.S.I.

I460. - Academy of Natural Sciences.-Conchological Section.

American Joumal of Conchology. (Published by the ....

Academy ...) Ed. by G. IV. Tryon. Vols. I-7. Philadelphia, I $865-72$ : G.S.I. TVols. $3-7$ were published by the Academy.]

I46I. - Academy of Natural Sciences.-Entomological Section.

Entomological News and Proceedings of the .... Section of the Academy.... Vols. I-. Philadelphia, I890-: Z.S.I.

I462. - American Academy of Political and Social Science.

Annals. Vols. 23-. Philadelphia, Igo4- (vol. 24-26, 71, 72 wanting) : A.S.B.

American Association for the Adrancement of Science (see No. 1489 ).

I463. - American Entomological Society.

Transactions. Vols. 42-. Philadelphia, I9I6-: Z.S.I.

- American Institute of Mining Engineers (see No. I42I).

- American Journal of Conchology (see No. I46o).

1464. - American Joumal of Pharmacy. Ser. 4, vols. 45-47. Philadelphia, I893-95: C.E.

- American Journal of the Medical Sciences (see No. I423).

I465. - American Naluralist. Ed. by A. S. Packard Jr., E. S. Morse, R. H. Ward, E. D. Cope and others. Vols. I-. Salem, New York, Boston, Philadelphia, IS68-.

Vols. I-, I868- (vols. 10-39 wanting; vols. 8, 40 defective): A.S.B.

Vols. I-4I, I868-I907 (vols. IO, 11 wanting, vols. 40, $4^{\text {I }}$ defertive): G.S.I.

Vols. 36-, I002 (vol. 36 defective) : Z.S.I.

\footnotetext{
A.S = Archaeological Section. Indian Museum.

A.S B. = Astatic suciety ot Bengal.

B.C = Bishos Cisllege.

B.S I. = Eotanical Survey of India

C.E. = Chemcal Examiner to the Govt of 13engal

c.U. = Calcutta Universty
}

E.c. = Civil En sineerung Collese, Sibpur.

G.S.I. = Geolosical survey o: India.

I L. = Imoerial Librart.

M. Ba. = Medical Colle ie, Bacteriological Lahoratory

M.Bi. = Medical Collese. Biological Laburaiory.

M.C. = Medicil College (maul library). 
1466. Philadelphia.-American Philosophical Society.

(a) Early Proceedings ..... compiled from manuscript minutes of Meetings from $\mathrm{I}_{744}$ to $\mathrm{I}_{3} 38$. Philadelphia, I 884 : A.S.B.

(b) Proceedings. Vols. I-, I838-. Philadelphia, I840-. General index to vols. I-50, I838-I9II.

Vols. I-, I $840-$ (vols. 42, 43 wanting, vol. I, 2, 4, 7 de fective) : G.S.I.

Vols. I-, I $840-$ (vols. $6,7,14,40$ wanting; vols. II, 4 I, $43,45,47$ defective) and index: A.S.B.

Vols. $43-$, Igo4-, and index: Z.S.I.

(c) Transactions. Visls. I-6. Philadelphia, I789, I786I809 [vol. 1 of 2 nd ed.]. General index to vols. $\mathrm{I}-4$ at end of vol. 4 , and to vols. 5, 6 at end of vol. 6 . New ser., vols. I-. Philadelphia. I8 8 -. Index to vols. I-4 at end of vol. 4 .

Complete set: G.S.I.

Vo:s. I-, I786- (new ser., vol. 15 wanting): A.S.B.

New ser., vols. I6-, I89o- : Z.S.I.

Subject Register to $(b)$ and (c), I77I-I889: G.S.I.

- Annals of Surgery (see No. I428).

_ Entomological Neres (see No. I46r).

I467. Franklin Institute of the State of Pennsylvania.

(a) Franklin Journal and American Mechanics' Magazine. Vols. 5. 6. Philadelphia, I828: G.S.I. [Continued as (b).]

(b) Journal. New ser., vols. 3-26 $(=7-30)$. Philadelphia, I829-40. Third ser., vols. I-IOo (=3I-I30). Philadelphia, I84I-90. [Whole series.] Vols. I3I-. Philadelphia, I89I-.

Complete set: G.S.I.

Vols. I 45一, I898- (defective) : A.S.B.

Vols. I62-, I906- (vols. 167 , 168 wanting): E.C.

I468. - Geological Society of Pennsylvania.

Transactions. Vol. I. Philadelphia. I834-35.

Vol. I, I834-35: A.S.B.

Vol. I, pt. I, I834: G.S.I.

I469. - Henry Phipps Institute for the Study, Treatment and Prevention of Tuberculosis.

Annual Report. 1907 08. Philadelphia, I909: A.S.B.

\footnotetext{
Mo. = Meteorological Office, Alipore.

M.Ph = Medical Collere. Physiological Laboratory

P.C. = Presidency College.

P.o. = Patent O.fice.

R B.G = Roval Botanic Garder.

S.C C. $=$ Scintish Churches College.
}

S.G. = Surgeon-General's Library.

S I. = Survey of India.

S T. $M=$ schoul of Tropical Medicine

T. D. = Telegranh Depariment.

X.c. =St. Xavier s Collese.

z.s.I. = Zoological survey of India 
1470. Philadelphia.- Journal of Comparative Medicine and Surgery. Ed. by W. A. Conklin and R. S. Huidekoper. Vols. 9-I2. Philadelphia, I888--9I (vol. I2 defective): A.S.B.

I47I. Journal of Experimental Zoology. Ed. by R. G. Harrison and others. Vols. I-. Baltimore, Philadelphia, Igo4-: Z.S.I. [Vols. 5-, Igo8-, published by the Wistar Institute of Anatomy and Biology.]

I472. - Journal of Morphology. Ed. by C. O. Whitman, E. P. Ellis Jr., J. S. Kingsley and others. Vols. I-. Boston, Lancaster, Philadelphia, I887-: Z.S.I. [Vols. I9-, I908-, published by the Wistar Institute of Anatomy and Biology.]

I473. - Medical Neres and Abstract. Ed. by I. Minis Hays. Vols. 38, 39. Philadelphia I880-8I : S.G.

1474. - Monthly American Joumal of Geology and Natural Science. [Ed by G. W. Featherstonhaugh.] Vol. I. nos. 2,3,5. Philadelphia, I83I : A.S.B.

- Museum of Science and Art (see No. 1477).

I475. - Pennsylvania Museum and School of Industrial Art.

(a) Annual Report. Nos. 4-, I879-. Philadelphia, I 880 - : B.S.I.

(b) Bulletin of the...Musenm. Nos. I-. Philadelphia. I903- (pts. I- 3 of no. I wanting): B.S.I.

I476. University of Pennsylvania.

(a) Contributions from the Botanical Laboratory. Vols. I-. Philadelphia, I892-：A.S.B. [Reprinted from Transactions and Proceedings of the Botanical Socicty of Pennsylvania.]

(b) Contributions from the Zoological Laboratory. I898-. Philadelphia, I898- (vol. for 1900 wanting): A.S.B. [Vols. for 1903- are styled 'vols. I0-'.] [Reprinted from various scientific publications.]

(c) Medical Bulletin. Vols. 21, 22. Philadelphia. I9o9 (no. 1, vol. 22, wanting): A.S.B.

I477. - University of Pennsylvania.--Museum of Science and Art.

(a) Bulletin. Department of Archreology and Palaeontology. Nos. I-4. Philadelphia, I897-98: Z.S.I. Vol. 2, nos. I, 2, 4 ; vol. 3 , no. 4 . Philadelphia, I899-I902 : Z.S.I.

(b) Museum Journal. Vols. I-. Philadelphia, I9Io(110. 4, vol. I, wanting): A.S.B.

\footnotetext{
A.S. = Archaeological Section. Indian Museum.

A.S.B. = Asia:ic Society of Bengal.

B C. = Bishop s College.

B.S.I. = Botanical Survey of India

C.E. = Chemical Examiner to the Govt of Bengal.

c.U. = Calcutta University.
}

E.C. = Civil Engineerung College, Silppur.

G.S.1 = Geological Survey of India.

I.L. = Imperial Library

M.Ba. = Mcilical Collere, Bacteriolosical Laboratory

M.CI. = IIedical College. Biological Laboratory.

M.C. = Medical College (maul library). 
I478. Philadelphia.-IVagner Free Institute of Science.

Transactions. Vols. I-6. Philadelphia, I887-I9o3.

Vols. I-6, I887-I903 (vol. 3 defective) : G.S.I.

Vol. I, I 887 : Z.S.I. I 472 ).

I 479. Pittsburgh.- Carnegie IIseum.

(a) Amnals. Vols. I-. Lancaster, Pittsburgh, I9oI-.

Complete set: Z.S.I.

Vols. I-8, no. I, I90I-II : G.S.I.

(b) Memoirs. Vols. I-. Pittsburgh, I90I- : Z.S.I.

I480. Portland.-Society of Natural History.

Proceedings. V'ols. I-. Portland, I862-.

Vol. I ; vol. 2. pts. I-5, I862-I90I : G.S.I.

Vol. 2, pt. 3-, I895-(vol. 2, pts. 6-8 wanting) : Z.S.I.

I48I. Raleigh.- North Carolina College of Agriculture and Mechanic Arts.-Agricultural Experiment Station.

Bulletin. Nos. I86-. Raleigh, I903- (nos. 190-193 wanting): B.S.I.

I482. - North Carolina Department of Agriculture.

Bulletin. Nos. 25一. Raleigh, I904- : B.S.I.

Rhode Island, State of (see No. 1385 ;.

I483. Rochester, N.Y.-Academy of Science.

Proceedings. Vols. I-, I889-. Rochester, I89I (I890)- : G.S.I.

I484. Sacramento.-California State Agricultural Society.

Transactions. I882-93. Sacramento, I883-94 (vols. for I883, '87-9I wanting): R.B.G.

I 485. California State Mining Bureau.

Anmual Report of the State Mineralogist. Nos. 3-Io. Sacramento, I883-90: (no. 5 wanting): G.S.I. [Continued as:] Report of the .... Mineralogist. Nos. II, I2. Sacramento, I893-94 : G.S.I.

University of California.-Agricultural Experiment Station (see No. I326).

M O = Meteornlogical Office, Aipore

$\mathbf{M} \mathbf{P h}=$ Medical College. Physiological Laboratory.

P.C = Presidency College.

PO = Patent O.fice.

$\mathbf{R} \mathbf{b} \mathbf{G}=$ Koval Botanic Garden.

S.C.C. $=$ Scottish Churches College

30
S.G. =Surgeon-Generais Library.

S.I. = survev or India.

S.T.M. = School of Tronical Medicine.

T.D. = Telegraph Departmeni.

X.C. =St. Xavier s Lollesse.

Z.S.I. = Zoological Survey of India. 
I486. St. Louis.-Academy of Science.

Transactions. Vols. 6-. St. Louis, I805-.

Vols. 6-. 1895-(vols. 8, 9, 12 defective) : A.S.B.

Vols. 6-I7, I895-1907 : Z.S.I.

I487. - Annals of Ophthalmo'ogy: a quarter'y journal and review of ophthalmic science. Vols. II-. St. Louis, IgO2-(vols. 12, I417 defective): S.T.M.

- Annals of Surgery (see No. 1428).

I488. - Missouri Botanical Garden

(a) Annals. Vols. I-. Concord, I9I4- : A.S.B., R.B.G.

(b) Annual Report. Nos. [I]-, I890-. St. I,ouis, I890-. Complete set: R.B.G.

Nos. 8-, 1897-: A.S.B.

Nos. II-, I900- : B.S.I.

St. Pau1.-Geological and Natural History Survey of Minnesota (see No. I402).

Salem.-American Antiquarian and Oriental Journal (see No. I349).

I489. - American Association for the Advancement of Science.

(a) Memoirs. [No.] I. Salem, I875: G.S.I.

(b) Proceedings. Meetings I-49. Philadelphia, Salem. etc., I849-I900: G.S.I.

I490. Essex Institute.

(a) Bulletin. Vols. I-30, I869-98. Salem. I870-98

Complete sets : G.S.I., Z.S.I.

Vols. I-25, I870-93: A.S.B.

(b) Proceedings (Communications). Vols. I-6, I848-70. Salem, I856-71. [Continued as (a).]

Complete set : G.S.I.

Vol. 6, I870-7I . Z.S.I.

I491. - Peabody Academy of Science.

(a) Annual Report. Nos. I-I9, I869-86. Salem, I864-87.

Nos. I-I9, I869-87 (no. I8 wanting): G.S.I.

Nos. I-4, I869-72: A.S.B.

(b) Memoirs. Vol. I, nos. I-6; vol. 2. Salem, I869-8I, I 886 : G.S.I.

A.S. = Archaeological Section. Indian Museum

A.S B. A Asiatic Society of Bengal.

B.C. = Bishop s Collerfe.

B.S.I. = Botanical Survey of India.

C.E. = Chemical Exammer to the Govt. of Bengal

c.U = Calcutta University
E.C. = Civil Enermeering College, Sibpur.

G.S.I. = ireolosical Survey of Incia.

i L. = Imperial Library.

M. Ba $=$ Medical Collese. Bacteriolorical Laboratory

M.Bl. = Medical College. Biological Laboratory.

M.c. = Medical College (main liorary). 
1492. San Francisco.-California Academy of Sciences.

(a) Bulletin. Vols. I, 2 (=Nos. I-8). San Francisco. I $884-87$ : A.S.B., G.S.I.

(b) Memoirs. Vols. I-4. San Francisco, I868-I904.

Complete set: G.S.I.

Vol. I, pt. 2, I868; vol. 2. nos. I-5, I888-96: A.S.B.

Vol. 3, I903: Z.S.I.

(c) Occasional Papers. Nos. I-8. San Francisco, I8goIgoI : A.S.B., G.S.I.

(d) Proceedings. Vols. I-7, I854-76. San Francisco, I87377 (vol. 1 of 2 nd ed., vols. 2,3 wanting): A.S.B., G.S.I. [Resumed as (a) and afterwards continued as:] Proceedings. Ser 2, vols. I-6, I 888-06. San Francisco, I889-97 : A.S.B., G.S.I. [Continued in sections as follows:] Ser. 3. Botany. Vols. I, 2. San Francisco, I897-I904: A.S.B., G.S.I. Geology. Vols. I-2, no. I. San Francisco, I897-I904: A.S.B., G.S.I. Mathematical-Physical. Vol. I. San Francisco, I898-I903: A.S.B., G.S.I. Zoology. Vols. I-3. San Francisco, Igoo (I897)Igo4: A.S.B., G.S.I. [Continued as :] Ser. 4, vols. I-. San Francisco, I907- : A.S.B., G.S.I., Z.S.I.

1493. - Mining and Scientific Press. Vols. 93-. Berkeley, San Francisco, I906- : G.S.I.

1494. Scranton.-Colliery Engineer. Vols. 33, no. 8-. Scranton, I9r3-. [A continuation of No. I495.]

Vols. 33, no. 8-, I9I3- : E.C.

Vol. 33, no. 8 - vol 34 , no. 7 . I9I3-I 4 : G.S.I.

I495. Wines and Minerals. Vols. 27-33, no. 7. Scranton, I9o6I3. [Continued as No. 1494.]

Vols. 27-33, no. 7, I906-I3 : E.C.

Vols. 27-33, no. 7, Ig06-I3 (pt. + vol. 31, wanting) : G.S.I.

Seattle.-Ophthalmology (see No. I354).

Springfield.-Geological Survey of Illinois (see No. I505).

I496. - Illinois State Museum of Natural History. Bulletin. Nos. 2-I2. Springfield, I884-97 : G.S.I.

I497. Stanford University.-Leland Stanford Junior University.

Publications. University series. Nos. I-. Stanford University, I908-: Z.S.I.

M.O. = Meteorological Office, Alipore.

M.Ph. = Medical Collere. Physiological Laboratory:

P.C. = Presidency College.

P.O. = Patent O.fice

R.B.G. = Roval Botanic Garden

S.C.C. = Scottish Churches College.
S.G. = Surgeon-General s Library

S.l. = Survey of India.

S.T.M. = School of Tropical Medicine

T.D. = Telegraph Department

X.c. =St. Xavier s College.

Z.S.I = Zoological Survey of India. 
1498. State College.-Pennsylvania State College.

Report. Departments of Instruction. I888-94. State College, I889-95 (Rep. for 1893 wanting) : B.S.I. [Issued and bound up with No. $1499(b) .1$

1499. - Pennsylvania State College.-Agricultural Experiment Station.

(a) Bulletin. Nos. 42-. State College, I898- (nos. 43-67 wanting) : B.S.I.

(b) Report. I888-94. State College, I889-95 (Rep. for 1893 wanting) : B.S.I. [Issued and bound up with No. 1498.]

1500. Stillwater.-Oklahoma Agricultural and Mechanical College.Agricultural Experiment Station.

Bulletin. Nos. 45-89. Stillwater, I900-Io (nos. 46, 47, 4964 warting): B.S.I.

I50r. Storrs.-Agricultural College.-Connecticut Agricultural Experiment Station.

(a) Annual Report. Nos. I-20, I888-1907. Hartford and Middletown, I889-1908 (nos. 11, I5, I6, I9 wanting) : B.S.I.

(b) Bulletin. Nos. I-5I. Storrs, I880-I908 (nos. 2, 3, го, I I, I5-I9 wanting) : B.S.I.

Tennessee, State of (see No. I40S).

Texas, State of (see Nos. 13I6, I3I7).

Topeka.-Geological Survey of Kansas (see No. I39r).

1502. Trenton.-Geological Survey of New Jersey.

(a) Annual Report of the State Geologist. I900-09. Trenton, IgoI-Io: G.S.I. [Continued in $(b)$.

(b) Bulletin. Vols. I-. Trenton, IgII-: G.S.I.

(c) Final Report of the State Geologist. Vols. 5, 6. Trenton, I902-04: G.S.I.

1503. - Natural History Society.

Journal. Vol. I. Trenton, I886-88 : A.S.B.

I504. Tufts College.-Tufts College.

Studies. Vols. I-. Tufts College [Medford. Mass.], I894-.

Complete set: Z.S.I.

Vols. I-, I894-- (rols. I, 3 defective) : A.S.B.

A.S. = Archaeolosical Section. Indian Mureum

A.S.B = Asiattc Society of Benga:

B.C. = Bishop s College.

B.S.1 = Botanical Survev of India

C.E. = Chemical Examiner to the Govt of Bensal.

c. $\boldsymbol{U}=$ Calcutta University
E C. = Civil Engineering College, Sibnur.

G.S.I. = Geolosical Survey of Indsa.

1.L. = Imperial Library.

M.Ba. = Medical Collese, Bacteriological Laburatory

M Bi. = Medical Collere. Biolosical Laboratory

M.C. = Medical College (main librar: $)$. 
I505. Urbana.-Geological Survey of Illinois.

(a) Bulletin. Nos. 4, 9. Urbana, I907, I908: G.S.I.

(b) [Reports] Geology iand Palacontology). Vols. I-8. [Springfield], I866-90: G.S.I.

1506. - Illinois State Laboratory of Natural History.

Bulletin. Vols. 5-. Urbana, I897-.

Vols. 5-, I 897 - : A.S.B.

Vols. 5, 7. I897-I90I, I904-07 (rol. 5 defective) : Z.S.I.

I507. - Journal of Parasitology: a quarterly journal devoted to medical zoology. Ed. by H. B. Ward. Vols. 3-. Urbana, I9r6-:

M.Ba.

I5c8. - University of Illinois.

Illinois Biological Monographs. Vols. I-. Urbana, I9I4-: Z.S.I.

I509. - University of Illinois.-Agricultural Experiment Station.

(a) Bulletin. Nos. 79-. Urbana, I902-: B.S.I.

(b) Circular. Nos. 82-. Urbana, I904-: B.S.I.

Vermont, State of (see No. 1339).

Virginia, State of (see No. I345).

I510. Washington.-Academy of Sciences.

(a) Journal. Vols. I-. Baltimore, I9II- (no. 3, vol. 2 wanting): A.S.B.

(b) Proceedings. Vols. I-I2. Washington, I899-I910.

Vols. I-I2, I899-I9IO: A.S.B.

Vol. 8, I906-07 : G.S.I.

American Association for the Advancement of Science (see No. $\left.14^{8} 9\right)$.

I5II. - American Forestry. I9I3-. Washington. I9I3- : E.C.

I512. - American Genetic Association.

Journal of Heredity: a monthly publication devoted to plant breeding, animal breeding and eugenics. Organ of the ...... Association. Vols. 5-. Washington, I9I4- (rols. 5, 6 defective): B.S.I.

I513. - Anthropological Society.

Transactions. Vol. 3, I883-85. Washington, I 885 : A.S.B.

Astrophysical Observatory (see No I520).

\footnotetext{
m.o. = Meteorological Office, Ainore

M.Ph = Medical College. Physiological Laboratory

P C. = Presidency Colles 6 .

P o. = Patent Ofrice.

R B. G = Roval Botanic Garder

$\mathbf{S} \mathbf{C} \mathbf{C}=$ Scottish Churches College
}

S.G. = Surgeon-Generai's Library
S.I. = Survey or India.
S.T. $\mathbf{M}=$ School of Troptcal Medicine.
T.D. = Telegraph Departmeni.
X.C. = St Xavier S College.
Z.S.I. = Zoological Survey of India.

[ 237 ] 
I5I4. Washington.-Biological Society.

Proceedings. Vols. I3-. Washington, I899- (vols. 16IS wanting; vols. $13,22-24$ defective) : A.S.B.

Contributions to North American Ethnology (see No. I536).

I5I5. Geological Exploration of the Fortieth Parallel.

Report. Vols. I-7. Washington, I870-80 : G.S.I.

I5I6. - Government Hospital for the Insane.

Bulletin. Nos.2-. Washington, I9Io-: I.L.

- Insect Life (see No. I53I (c)).

- Journal of Agricultural Research (sce No. I529(e)).

- Journal of Heredity (see No. I512).

- Military Surgeon (see No. I343).

I5I7. National Academy of Sciences.

(a) Memoirs. Vols. 2-. Washington, I884-.

Vol. 2-, I884- (vol. 9 wanting, vol. 8 defective) : G.S.I.

Vols. 6, 7, I893-95 : Z.S.I.

(b) Proceedings. Vol. I, pt. 2. Washington, I884: G.S.I.

(c) Proceedings. Vols. I-. Baltimore. I9I5-: A.S.B., G.S.I., Z.S.I.

(d) Report. I866, '67, '83, '84. Washington, I867-85: G.S.I.

— National Herbarium (see No. I522 (b)).

- National Museum (see No. I522).

- North American Fauna (see No. I530 (b)).

I5I8. Philosophical Society.

Bulletin. Vols. I-I5. Washington, I874-I9Io. Index to vols. I-IO at end of vol. IO. [Vols. I IO were also issued in vols. 20,25 , and 33 of No. I5I9 (c).]

Vols. I-I 5, I874-I 1 IO (vols. 7 wanting; vol. I5 defective) : A.S.B.

Vols. I-I2, IS74-95: G.S.I.

I5I9. - Smithsonian Institution.

(a) Anmual Report of the Board of Regents. I854-. Washington, I855-. [From r884 issued in two vols. annually, the second being No. $1522(d)$.

$$
\text { Reps. for I } 854-\text { : G.S.I. }
$$

A.S. = Irchaeological Scction. Indian Museum., A.S.B. = Isiatic Societ $y$ of IBengal.

B.C. = Li:hops Collerse

B.S I. = Botanical Survev of India

C.E. = Chemical Examiner to the Govt. of Bengal.

C.U. = Calcutta University.
E C. = Civil Encineering College. Sibpur.

G S I. = Geolosical Survey of Incia

I L. = Inoerial Librarv.

M Ba. = Me hical Colle:ec, Bacteriolosical Laboratory

M. $\mathbf{E l}=$ IIe iscal Collere. Biolosical L.aboratory.

M.C. = Medical College (main Library). 
I5I9. Washington.-Smithsonian Institution (Continued).

Reps. for 1854 - (Reps. for 1855 , '60, '66 wanting) : A.S.B.

Reps. for 1868 - (Reps. for $1871,{ }^{\prime} 76$ wanting) : Z.S.I.

Reps. for I869-95: M.O.

Reps. for I880-82: S.G.

Reps. for $1884-94$ (Reps. for 1885 , '86 wanting) : B.S.I.

Reps. for I888- (Rep. for 1906 wanting) : S.I.

Reps for $1893-$ : X.C.

(b) Smithsonian Contributions to Knowledge. Vols. IWashington, I848-.

Complete set : G.S.I.

Vols. I-, I848- (vols. 3, 4, Io wanting): A.S.B.

(c) Smithsonian Miscellaneous Collections. Vols. I-

Washington, I860-.

Complete set : G.S.I.

Vols. I-, r860-(vols. 6, 7, 34, 35, 37-39 wanting; vols. 44,46 defective): A.S.B.

Vols. I-9, I $860-69$ : Z.S.I.

I520. - Smithsonian Institution.-Astrophysical Observatory.

Annals. Vols. I, 2. Washington, I900-08.

Vol. I, I900: I.L.

Vol. 2, I 908 : G.S.I.

r52r. - Smithsonian Institution.-Bureau of American Ethnology.

(a) Annual Report. Nos. I-, I879-. Washington, I88r-.

Complete sets : A.S.B., Z.S.I.

Nos. I-3, I88I-84 : G.S.I.

(b) Bulletin. Nos. 25-. Washington, r903-.

Nos. 25一, I903- (nos. 3I, 36, 46 wanting) : Z.S.I.

Nos. 25一, I903-(nos. 3I, 36, 46, 49 wanting; no. 40 defective) : A.S.B.

1522. - Smithsonian Institution.-United States National Museum.

(a) Bulletin. Nos. I-. Washington, I875-.

Complete set : Z.S.I.

Nos. I-, I $875-$ (nos. $28,32,49,78$, 80 wanting) : G.S.I.

Nos. 16 - $1882-$ (defective): I.L.

Nos. 33 -, I889-(nos. $40,70,78,80$ wanting; no. 39 defective): A.S.B.

(b) Contributions from the U.S. National Herbarium. Vols. I-. Washington, I890-. [Vols, I-7, I890-92 were

M.O. = Meteorological Office, Alipore.

M.Ph. = Medical College. Physiological Laboratory.

P.C. = Presidency College.

PO = Patent $O$ ice

R.B.G. = Roval Botanic Garden.

S.B.G. = Scottish Churches College.
S.G. = Surgeon-General's Library.

S.G. = Survev of India.

S.T.M. = School of Tropical Medicine

T D. = Telegraph Department.

X.c. =St. Xivier s College.

Z.S.l. = Zoological Survey of India. 
I522. Washington.-Smithsonian Institution .. (Continued).

published by the U. S. Dept. of Agriculture, Division of Botany.]

Complete set: R.B.G.

Vols. 9-. I905- : G.S.I.

Vols. 9-, I 90 - (vol. Io defective) : A.S.B.

(c) Proceedings. Vols. I-. Washington, I879-. [Vols. 1-t were also issued as vols. I9 and 22 of No. 15 19 (c).]

Complete set : Z.S.I.

Vols. IO-, I888- : A.S.B., G.S.I.

Vols. II-, I889-(defective) : I.L.

(d) Report. I884-. Washington, I885-[Issued as annual volumes supplemental to No. $1519(a)$, with which prior to I 884 they were incorporated. 1

Complete sets : A.S.B., Z.S.I.

Reps. for I888- : G.S.I.

Reps. for $1889-$ : R.B.G.

Reps. for I 89495 - : I.L.

(e) Special Bulletin. Nos. I-. Washington, I892-.

Complete set: Z.S.I.

No. 4, I900-I5 : G.S.I.

I524. United States Army.-Engineer Department.

(a) Report of the Geological Exploration of the Fortieth Parallel (see No. I5I5).

(b) Report of the United States Geographical Surveys West of the One Hundredth Meridian (see No. I537).

I525. United States Bureau of Fisheries.

(a) Bulletin of the U.S. Fish Commission. Vols. 8-23, I888-I903. W'ashington, I890-I906: Z.S.I. [Continued as:] Bulletin of the Bureau of Fisheries. Vols. 24-, I904-. Washington, I905- : Z.S.I.

(b) Documents. Nos.609-. Mashington, I907-: I.L.

(c) Report of the Commissioner of Fish and Fisheries. Parts 2-29, I872-I903. Washington, I874-I905. [Continued as:] Report of the Burean of Fisheries. I 904. Washington, I905. [Continued as:] Report of the Commissioner of Fisheries ... and special papers. Igo5-. Washington. Ino6-.

Parts $2-29$, for $1872-1903$ (pts. $3,4,6,18,23$, for $187+$ 76, ' 78 , '91'92, '96 97 wanting); rols. for 1904-: Z.S.I.

Vols. for I908 - (vol. for 1909 wanting): A.S.B.

A.S. = Archaeological Section, Indian Museum.

A.S.B. = Asiatic Sociely of Bengal

B.C. = Bishop's College.

B.S.I. = Botancal Survey of India.

C.E. Chemical Examiner to the Govt. of Bengal.

c.u. = Calcutta University
E.c. = Civil Engineering College, Sibpur G.S.I = Geological Survey of India.

I.L =Imperial Library.

M.Ba. = Medical College, Bacteriological Lavoraloty

M.Bl. = I Iedical College. Biological Laboratory.

M.c. = Medical College (main library) 
I526. Washington. - United States Bureau of Mines.

(a) Bulletin. Nos.3-. Washington, I9ro-.

Nos. 3-, I9IO- (defective) : I.L.

Nos. $53,64,77$, I9I3-I4 : G.S.I.

(b) Technical Paper. Nos. 5, 8, 10, 15, I6, 32, 100, I25-- Washington, I9I 2 - : G.S.I

I527. United States Bureau of Standards.

(a) Bulletin. Vols. 2-. Washington, rgo6- (defective) : I.L.

(b) Circular. Nos. 2-. Washington, I9ro-- (defective) : I.L.

(c) Technologic Papers. Nos. 2-. Washington, I9I2(defective): I.L.

I528. United States Coast and Geodetic Survey.

(a) Geodesy. Special Publication. Nos. I0, I2-I4, I8, 27 , 30, 31, 35-. Washington, I912-: G.S.I.

(b) [Annual] Report of the Superintendent of the U.S. Coast Survey, etc. I849-77. Washington, I850-80. [Continued as :] Report of the Superintendsnt of the U.S. Coast and Geodetic Survey, etc. I878-. Washington, I88I-.

Reps. for I849-62, I882/83, '95/96, I902/03 : G.S.I.

Reps. for I869-, I872-(Reps. for 1904, 'o6 wanting) :

S.I.

I529. United States Department of Agriculture.

(a) Crop Reporter. Nos. 5-. Washington, I903-: B.S.I.

(b) Bulletin (Professional Papers). Nos. I-. Washington, I9I3- (defective): I.L.

(c) Bulletin of the Mount Weather Observatory (see No. 1407).

(d) Farmers' Bulletin. Nos. I-I84. Washington, I880I9O3 (no. 183 wanting): B.S.I.

(e) Journal of Agricultural Research. Vols. I-. Washington, I9I3-.

Complete sets : B.S.I., I.L., Z.S.I.

Vols. 6-, I9I6- (vols. 6, 8 defective); A.S.B.

(f) Monthly Reports. I866-76. Washington, I867-77.

Reps. for I $866-76$ (Reps. for 1868 , '7I wanting) : A.S.B. Reps. for $1871-76$ : G.S.I.

(g) Report of the Commissioner of Agriculture. I866-85. Washington, I867-85. [Continued as:] Report of the

M.o. = Meteorological Office, Alipore.

M.Ph. = Medical College, Physiological Laooratory

P.c. = Presidency College.

P.O. = Patent Office

R.B.G. = Royal Botanic Garden

s.c.C. $=$ Scottish Churches College.
S.G. =Surgeon-General's Library.

s.I. = Survey of India

S.T.M. $=$ School of Tropical Medicine.

T.D. = Telegraph Department.

X.c. = St. Xavier s College.

Z.s.I. = Zoological Survey of India. 
I529. Washington.-United States Department of Agriculture (Continued).

Secretary of Agriculture. I889-93. Washington, I889-94. [Continued as $(h)$.]

Reps. for I 866-93 (Reps. for 1867, ' 69 , '70, '74, '76, '84, '86-89, '92 wanting) : A.S.B.

Reps. for $1868-83$ (Rep. for 1874 wanting) : G.S.I.

(h) Yearbook of the U.S. Department of Agriculture. I894-. Washington, I895-.

Complete sets : B.S.I., R.B.G.

Vols for I896-rgIo (vols. for I902, 'O3, 'O8 wanting): A.S.B.

Vols. for $1896-99$ : G.S.I.

Vols. for I9II-: Z.S.I.

I530. - United States Department of Agriculture.-Division of Biological Survey (Division of Ornithology and Mammalogy).

(a) Bulletin. Nos. I-44. Washington, I889-I9I2.

Nos. I-I 4, I889-I900 (nos. 2, 7 wanting): G.S.I.

Nos. 9-44, I898-I9I2 (nos. 15-31, 34, 43 wanting): Z.S.I.

(b) North American Fauna. Nos. I-. Washington, I889-. [Nos. 6, 9 not published.]

Nos. I-, I 889 - (nos. I2, 24-26, 35, 39 wanting) : Z.S.I.

Nos. I-, I $889-$ - (defective) : I.L.

Nos. I-22, I889-I902: A.S.B., G.S.I.

- United States Department of Agriculture.-Division of Botany (see No. $1522(b)$ ).

I53I. - United States Department of Agriculture.-Division of Entomology.

(a) Bulletin. Nos. I, 5, 3-16, 20-30. Washington, I88393: Z.S.I. New ser., nos. I-, I895- (nos. $23,52,65-67$, 7I-74, 76-78, 81, 84, 86-88, 103 wanting): Z.S.I.

(b) [Bulletin.] Technical series. Nos. I-. Washington, I895- (nos. I I, 25, 26 wanting) : Z.S.I.

(c) Insect Life. Periodical Bulletin. Vols. I-7. Washington, I888-95 : B.S.I., Z.S.I. General index, I888-95: Z.S.I.

United States Department of Agriculture.-Entomological Commission (see No. 1535).

\footnotetext{
A.S. = Archaeological Section, Indian Museum.

A.S.B. = Asiatic Society of Bengal.

B.C. = Bishop's College.

B.S.I. = Botanical Survey of India.

C.E. = Chemical Examiner to the Govt, of Bengal.

c.U. = Calcutta University.
}

E.C. = Civil Engineering College, Sibpur.

G.S.I. = Geolosical Survey of India.

I.L. = Imperial Library.

M.Ba. = Medical College, Bacteriological Laboratory

M.Bi. = Medical Cnllege. Biological Laboratory.

M.C. = Medical College (main library) 
I532. Washington.--United States Department of Agriculture.-Experiment Stations.

Experiment Station Records. Vols. 2-. Washington, r890-.

Vols. 2-, I890- : R.B.G.

Vols. 2-, I $890-$ (vols. 2, 3, 5 defective) : B.S.I.

I533. - United States Department of Agriculture.-Weather Bureau.

(a) Monthly Weather Review. Vols. [5]-, I877-. Washington, I877-[Vols. 12-, I884-, bear vol. nos.]

Vols. 5-, I877-: M.O.

Vol. I0-I4, I88I-86: A.S.B.

(b) Report of the Chief of the .... Bureau. IS9I 92I906/07. Washington, I893-I908 (Reps. for $1892 / 93$, '95-98 wanting) : M.O.

I534. - United States Department of Nary.-Bureau of Medicine and Surgery.

United States Naval Bulletin. Vols. 4-. Washington, I9ro- (defective) : I.L.

I535. - United States Entomological Commission.

(a) (Annual) Report. Nos. I-4. Washington, I878-85.

Nos. I-4, $1878-85$ : Z.S.I.

No. I, I878: G.S.I.

(b) Bulletin. Nos. I-7. Washington, I877-8I.

Nos. I-7, I877-8I : Z.S.I. .

Nos. I, 2, I877 : G.S.I.

_- United States Fish Commission (see No. I525).

I536. - United States Geographical and Geological Survey of the Rocky Mountain Region.

Contributions to North American Ethnology. Vols. I-9. Washington, I877-93.

Vols. I-5, I $877-82$ (vol. 4 wanting) : G.S.I.

Vols. 5-9, $1882-93$ (vol. 8 wanting): Z.S.I.

I537. - United States Geographical Surveys IVest of the One Hundredth Meridian.

Report. Vols. 2-7. Washington, I874-8I : G.S.I.

1538. United States Geological and Geographical Survey of the Territories.

(a) Annual Report. Nos. I-I 2, I867-78. Washington, I 87 I -83 : G.S.I.

M O = Meteorological Office, Alipore

$\mathbf{M}$ Ph $=$ Medical College. Physiological Laboratory

P.C = Prestdency Collesie.

P O Patent Office.

$\mathbf{R} \mathbf{B} \mathbf{G}=$ Royal Botanic Garden.

S.C.C. = Scottish Churches College.
S.G. = Surgeon-General's Library

S.1. = Survey of India.

S.T.M. = School of Tropical Medicine.

T.D. = Telegraph Departmeni

X.C. $=$ St. Xavier s college.

Z.S.I. Z Zoological Survey of India. 
1538. Washington.-UUnited States Geological and Geographical Survey of the Territories (Continued).

(b) Bulletin. Vols. I-6. Washington, 1874-82.

Complete set : G.S.I.

Vols. 2-6, $1876-82$ : A.S.B.

(c) Miscellaneous Publications. Nos. I-I2. Washington, I873-80 : G.S.I.

(d) Report. Vols. I-3, 5-I2. Washington, I873-84. [Vol. 4 was not published.]

Complete set: G.S.I.

Vols. 5, 6, II, I2, I873-79: Z.S.I.

United States Genlogical and Geographical Survey of the

Territories.-Entomological Commission (see No. 1535).

United States Geological Exploration of the Fortieth Parallel (see No. I5 I5)

I539. - United States Geological Survey.

(a) Annual Report. Nos. I-, I879/80-. Washington, I880- : A.S.B., G.S.I.

(b) Bulletins. Nos. I-. Washington, I883-. [Nos. I-54 form vols. I-8.]

Complete set: G.S.I.

Nos. 3-, I 884 - (nos. 177,205 wanting) : A.S.B.

Nos. 67一, I890- (defective) : I.L.

(c) Mineral Resources of the United States. I882-93, I $900-$ - Washington, I883-94, I90I- : A.S.B., G.S.I. [The issues for $1894-99$ were published as part of $(a)$.]

(d) Monographs. Vols. I-. Washington, (1890) I882-.

Complete set : G.S.I.

Vols. I-, I882- (pt. 1, vol. 32 wanting): A.S.B.

(e) Professional Papers. Nos. I-. Washington, I902-.

Complete set : G.S.I.

Nos. I-, I902- (nos. 74,76 wanting): A.S.B.

(f) Water Supply (and Irrigation) Papers. Nos. 65-.

Washingto1, I902-.

Nos. 65一, 1902- : G.S.I.

Nos. 66-, 1902- (defective) : A.S.B.

United States Geological Survey of the Territories (see

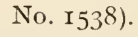

— United States Mint (see No. 1544).

\footnotetext{
A.S. = Archaeological Section. Indian Museum

A.S. B = Asialic Sociely of Bengal.

B.C. = Bishop s College.

B.S.I. = Bolanical Survey of Indua.

C.E. = Chemical Examiner to the Govt. of Bengal

C. $\boldsymbol{U}=$ Calcutta University
}

E.C. = Civil Engineering College, Sibpur.

G.S.I. = Geological Survey of India.

I.L. = Imperial Library.

M. $\mathbf{8 1}$. = Medical College. Biological Laboratory

M.C. = Medical College (main library). 
Washington.-United States National Herbarium (see No. I522(b)).

_- United States National Museum (see No. I522).

— United States Naval Bulletin (see No. I5.34).

I540. United States Naval Observatory.

(a) Magnetic Observations at the .... Observatory. I88889. Washington, I8go: X.C.

(b) Publications of the .... Observatory. 2nd'ser., vol. 5 . Washington, I903: X.C.

I54I. - United States Patent Office.

Office Gazette. Nos. 74-. Washington, I896- : P.O.

1542. - United States Public Health and Marine Hospital Service.

(a) Public Health Bulletin. Nos. 32-. Washington, I 9 IO- (defective) : I.L.

(b) Studies upon Leprosy. Nos. 4-. Washington, I909-: I.L.

I543. United States Public Health and Marine Hospital Service.Hygienic Laboratory.

Bulletin. Nos. 37-. Washington. I908- (110s. 67, 69, 73, $75-78,80,81$ wanting): A.S.B.

1544. - United States Treasury Department.

(a) Annual Report of the Director of the Mint to the Secretary of the Treasury. I885-94. Washington, I885-94: G.S.I.

(b) Report of the Director of the Mint upon the Production of Precious Metals in the United States. I886-92. Washington, I887-93: G.S.I.

1545. United States War Department.

(a) Monthly Weather Review (see No. I533(a)).

(b) Reports of Explorations and Surveys to ascertain the most practicable and economical Route for a Railroad from the Mississippi River to the Pacific Ocean. Vols. I-I2. IVashington, I855-60 : G.S.I.

Wisconsin, State of (see Nos. I396-I398, I400).

I546. Woods Hole.-Marine Biological Laboratory.

Biological Bulletin. Vols. 3-. Washington, I902- : Z.S.I.

M.o. = Meteorological Office, Alipore.

M.Ph. = Medical College, Physiological Laboratory.

P.C. = Presidency College.

P.o. = Patent Office

R. B.G = Royal Botanic Garden

S.C.C. $=$ Scottish Churches College.
s.G. = Surgeon-General's Library.

s.l. = Survey of India.

S.T.M. = School of Tropical Mecitcine.

T.D. = Telegraph Department.

X.C =St. Xavier's College.

Z.S.I. =Zonlogical Survey of India 


\section{SOUTH AND CENTRAL AMERICA AND WEST INDIES.}

I547. Bridgetown.-Imperial Department of Agriculture for the West Indies.

(a) Agricultural News. A fortnightly Review of the ...... Departmint..... Nos. I-. Bridgetown, I902-: B.S.I., I.L., R.B.G.

(b) [Bulletin.] Pamphlet series. Nos.3-. Bridgetown, I900- (no. 4 wanting) : B.S.I.

(c) West Indian Bulletin. Journal of the .... Department .... Nos. I-. Barbados, I899-: B.S.I., R.B.G.

I548. Buenos Ayres.-Ministerio de Agricultura.

Anales. Sección Geología, Mineralogía y Minería. Tom. I- Buenos Aires, I906- (defective, tom. 2, 3 wanting): G.S.I.

I549. — Ministerio de Agricultura.-Dirrección General de Minas, Geología e Hidrología.

Boletin. Ser. B, Geología. No I-7. Buenos Aires, I9I3 : G.S.I.

I550. — Museo Nacional (Museo Público).

(a) Anales. Tom. I-3. Buenos Aires, I864-90. Ser. 2, tom. I-4 $(=4-7)$. Buenos Aires, I895-1902. Ser. 3, tom. I- $(=8-)$. Buenos Aires, I902-. Indexes, tom. $\mathrm{I}-20$.

Tom. I-, I864- (ser. 3, tom. 2 wanting) : A.S.B.

Tom. I-3, I864-90; ser. 2, tom. I-3, I 895-99; ser. 3, tom. 2, 7, 24-, I903, '05, 'I3-(tom. 2, 7 defective) and indexes: G.S.I.

Ser. 2 , tom. I-3 ; ser. 3, tom. 4-6 and 8, I895-Igo6 : Z.S.I.

(b) Comunicaciones. Public. por C. Berg. Tom. I. Buenos Aires, I898-I90I : G.S.I., Z.S.I.

I55I. - Sociedad Química Argentina.

Anales. Tom. I-. Buenos Aires, I9I3- : G.S.I.

I552 Caracas. - Museo Nacionale.

Gaceta. Tom. I-. Caracas, rgI2- (tom. 2 defective): Z.S.I.

I553. Cordova.-Academia Nacional de Ciencias.

(a) Actas. Tom. I. Buenos Aires, I875: A.S.B. Tonı. 5. Buenos Aires, I883-86 : G.S.I.

A.S. =Archaeological Section, Indian M useum.

A.S.B. $=$ Asiatic Society of Bengal.

B.c. $=$ Bishop s College.

B.S.I. = Botanical Survey of India

C.E. = Chemical Examiner to the Govt. of Bengal.

c.U. = Calcutta University.
E.C. = Civil Engineering College, Sibpur.

G.S.1 = Geological Survey of India.

l.L. = Imperial Library

M.Ba. = Medical College, Bacteriological Laboratory

M.B1. = Mledical College, Biological Laboratory.

M.C. = Medical College (man library). 
SOUTH AND CENTRAL AMERICA AND WEST INDIES. [I553-I562

1553. Cordova.-Academia Nacional de Ciencias (Continued).

(b) Boletin. Tom. I-18. Buenos Aires, I874-1906.

Tom. I-I8, I874-I906 (tom. 2-5 wanting; tom. II, I3, 15, I8 defective) : A.S.B.

Tom. 6-, I884-(tom. I8, I9 defective) : G.S.I.

1554. Georgetown.-Board of Agriculture of British Guiana.

Journal. Vols. I-. Demarara, I907- : B.S.I.

1555. Havana.-Estacion Experimental Agronomica de Cuba.

(a) Boletin: Estacion Central Agronomica de Cuba. Nos. I-I8. Habana, I905-09: B.S.I. [Continued as:] Boletin: Estacion Experimental .... Nos. 19-. Habana, I9Io- : B.S.I.

(b) Circular. Nos. I0-. Habana, 1905-(nos. II-19, 21-22 wanting) : B.S.I.

I556. — Prensa Medica. An. [4]-7. Habana, I9I3-16 (defective) : S.T.M.

I557. - Secretaria de Agricultura. Comercio y Trabajo (de Agricultura, Industria y Comercio).

Boletin Correspondiente al Mes. Nov., I904-March, I906. Habana, I905-06. [Continued as :] Boletin Oficial. Vols. I-. Habana, I906-.

Complete set : M.o.

Vols. I-, I906- (rols. I-+ defective) : B.S.I.

I558. - Universidad.

Revista de la Facultad de Letras y Ciencias. Vols. I-Habana. I905-.

Vols. I-, I905-(vols. I, 2, 9-I I defective): Z.S.I.

Vols. I-, I905- (vols. II, I2 wanting: vols. I, 6 , IO, I4 defective): A.S.B.

I559. 'Vida Nueva': Revista de Medicina y Ciencias auxiliares. Año 8-. Habana, IgI6-: S.T.M.

1560. Kingston.-Department of Agriculture, Jamaica.

Bulletin. New ser., vols. I-. Kingston, I9II-- : R.B.G.

156r. - Department of Public Gardens and Plantations, Jamaica.

Bulletin of the Botanical Department. New ser., vols. I-8. Kingston, I894-I90I : R.B.G.

I562. — Institute of Jamaica.

Journal. Vols. I, nos. 3-8; vols. 2, nos. I and 3-5. Kingston, $1892-97$ : Z.S.I.

M o. = Meteorological Office, Alipore

M.Ph. = Medical College. Physiological Laboratory.

P.C. = Presidency College.

P. = Patent Otfice.

R.B.G = Royal Botanic Garden

S.C C. $=$ Scuttısh Churches College.
S.G. = Surgeon-Generai's Library:

S 1. = Survey of India.

S.T.M. $=$ School of Tropical Medicine

T.D. = Telegraph Denartment.

X.c. =St. Xavier s College.

z.S.I. = Zoological Survey of India 
1563. Kingston.-Jamaica Agricultural Society.

Journal. Vols. 6-. Kingston, I902- : B.S.I.

1564. La Plata.-Universidad Nacional.-Museo de la Plata.

(a) Anales. Sección Antropológia. Pt. I. La Plata, I896: G.S.I.

(b) - Sección Botánica. Pt. I. La Plata, I902: G.S.I.

(c) Sección de Arqueología. Pt. 2, 3. La Plata, I892 : G.S.I.

(d) - Sección de Geológica e Mineralógica. Pt. I-3. Ira Plata, I892-I900: G.S.I.

(e) Sección de Historia General. Pt. I. La Plata, I892 : G.S.I.

(f) - Sección de Paleontológica (Paleontología Argentina). Pt. I-3. La Plata, I89I-I903: G.S.I.

(g) Sección de Zoológica. Pt. I-3. La Plata, I8939.5 : G.S.I.

(h) - Segunda Serie, Tom. I. Buenos Aires, I907-08: A.S.B., G.S.I.

(i) Revista. Tom. I-. I,a Plata, Buenos Aires, I890-.

Tom. I-, I890 - (tom 8 wanting, tom. 9, ro defective) : G.S.I.

Tom. II-, I904-: A.S.B.

I565. Lima.-Cuerpo de Ingenieros di Minas del Perú.

Boletin. Nos. I-. Lima, I902-.

Nos. $\mathrm{i}$-, 1902- (nos. 3, 4, 11, 15, 20, 21, 24, 54, 55 wanting): A.S.B.

Nos. 8I-, I9r5-: G.S.I.

I566. Merida.-Sección Meteorológica del Estado de Yucatán.

Boletin Mensuel. Año I905/06-. Mérida di Yucatán, I906- (Año IgI2/ I3 defective) : M.O.

Mexico.-Comisión Geológica de .Iéxico (sce No. 1567 ).

_ Estación Agricola Central (see No. 1569).

I567. - Instituto Geológico de México.

(a) Anales. Num. I-. México, IgI7-: G.S.I.

(b) Boletin de la Comisión Geológica de México. No. I. México, I895: G.S.I. [Continued as :] Boletín del Instituto .... Nos. 2-. México, I895-: G.S.I. [Nos. 18,19 not published.]

(c) Parergones. Tom. I-. México, I905-(tom I, 3 defective) : G.S.I.

\footnotetext{
A.S. A Archaeological Section. Indian Museum.

A.S.B. = Asiatic Society of Bengal.

B.C. = Bishop s College.

B.S.I. = Botanical Survey of India.

C.E. = Chemical Examiner to the Govt. of Bengal.

c.U. = Calcutta University.
}

\footnotetext{
E.c. =Civil Engineering College, Sibpur.

G.S I. = Geological Survey of Incia.

I.L. = Imperial Library.

M.Ba. = Medical College, Bacteriological Laboratory

M.Bi. = Medical Collegre. Biological Laboratory.

M.C. = Medical College (main library).
} 
SOUTH AND CENTRAL, AMERICA AND WEST INDIES. [I568-I 575

I568. Mexico.-Ministerio de Fomento.

(a) Informes y Documentos relativos á Comercio Interior y Exterior, Agricultura, Minería é Industrias. Nos. 4666. México, I889-90: A.S.B.

(b) Memoria presentada al Congreso de la Union. I87785. México, I885-87 : A.S.B.

I569. - Ministerio de Fomento.-Estación Agricola Central.

Boletin. Nos. I-32. México, I908-09 (nos. 8-18, 27-3I wanting) : B.S.I.

I570. - Museo Nacional.

(a) Anales. Segunda Época, tom. I-. México, 1903-.

Complete set : I.L.

Tom. I-5, 1903-08 : Z.S.I.

(b) Boletin. Segunda Época, tom. I-. México, I903-.

Complete set : I.L.

Tom. I, I903-04 : Z.S.I.

I571. - Museo Nacional de Arqueológia, Historia é Etnológia.

(a) Anales. Tom. 3-5. México, I9II-I3 (defective) : A.S.

(b) Boletín. I9II-I3. México, I9II-I3 (defective) : A.S.

I572. — Sociedad Científica "Antonio Alzate."

Memorias ( $y$ Revista). Tom. I-. México, I887-(tom. $24 \cdot 26$ wanting; tom. $7-9,11,18,19,20,22,27$ defective) : A.S.B.

I573. - Sociedad Geológica Mexicana.

Boletin. Tom. I-. México, I905- : G.S.I.

I574. Montevideo.-Museo Nacional.

(a) Anales. Vol. I-7. Montevideo, 1894-I9II. Ser. 2, vol. I-. Montevideo, I904-.

Vol. I-6, I894-I908; ser. 2, vol. I-, I904-: Z.S.I.

Vol I-4, I894-I9OI (defective): A.S.B.

Vol. 5-7, 1903-07 (vol. 7 defective), ser. 2, vol. I, I904-II (defective) : G.S.I.

(b) Anales. Sección Histórico-Filosófica. Tom. I, 2, entr.

I. Montevideo, I904-05.

Complete set: G.S.I.

Tom. I, rg04: Z.S.I.

I575. Ouro Preto.-Escola de Minas.

Annaes. No. I. 3. Rio de Janeiro, I88I, ' 84 : G.S.I.

M.o. = Meteorological Office, Alipore.

M.Ph. = Medical College, Physiological Laboratory.

P.C. = Presidency College.

P.o. = Patent Office.

R.B.G $=$ Royal Botanic Garden.

s. C.C. $=$ Scottish Churches College.
S.G. = Surgeon-General's Library.

S.I. = Survey of India.

S.T.M. = School of Tropical Medicine.

T.D. = Telegraph Department.

X.C. =St. Xavier's College.

Z.S.I. $=$ Zoological Survey of India. 
1576. Para.-Museu Goeldi (Paraense) de Historia Natural e Ethnologia.

(a) Boletin. Vol. 1-. Pará, 1894-.

Complete set : G.S.I.

Vol. 2-, I898- : Z.S.I.

Vol. 2-7, I898-I9IO: A.S.B.

(b) Memorias. [Nos.] 3, 4. Rio de Janeiro, Pará, I902, '05: Z.S.I.

1577. Paramaribo.-Departement van den Landbouw, Suriname.

(a) Bulletin. Inspectie van den Landbouw in West-Indië.

Nos. I-I 4. Paramaribo, I904-08 : B.S.I. [Continued as :] Bulletin. Departement van den Landboure, Suriname. Nos. I5-. Paramaribo, I9o9-: B.S.I.

(b) Terslag. Inspectie van den Landboww in West-Indië. Jaar I904-7. Paramaribo, I905-08: B.S.I. [Continued as:] Terslag. Departement van den Landbouw, Suriname. Jaar I908-. Paramaribo, I909-: B.S.I.

1578. Port of Spain.-Board of Agriculture, Trinidad.

(a) Bulletin of Miscellaneous Information. Vols. 2-. Port of Spain, I895- (vols. 2, 3 defective) : B.S.I.

(b) Circular. Nos. I-. Port of Spain, I9II- (nos. 7-II wanting) : B.S.I.

1579. Scientific Association of Trinidad.

Proceedings. Parts I-9 (=vols. I, 2. No. I). Port-ofSpain, I $866-73$ (pt. 8 wanting): G.S.I.

1580. Rio de Janeiro.-Instituto Oswaldo Cruz.

Memorias. Tom. I-. Rio de Janeiro, I909-: Z.S.I.

1581. - Museu Botanico do Amazonas

Vellosia : Contribuições do Museu ... I885-88. (Segunda ediçao.) Vol. I-4. Rio de Janeiro, I89I-92: R.B.G.

1582. - Museu Nacional.

Archivos. Vols. 2-8. Rin de Janeiro, I877-92. [Continued as :] Revista. Vol. I (= vol. 9). Rio de Janeiro, I896. [Continued as :] Archivos. Vol. IO-I5. Rio de Janeiro, I899-I909.

Vol. 2-I5, I877-1909 (vols. 3, 4, 6, I2 wanting) : Z.S.I.

Vol. 4-9, I 88I-96 (vol. 6 wanting): A.S.B.

Vol. 8-I I, I892-I90I : G.S.I.

I583. - Observatorio (Nacional).

(a) Annuario. Ann. I3-. Rio de Janeiro, I896-.

Ann. I3-, I896- (Ann. 30-32 wanting) : G.S.I.

Ann. 2I-, I905-(Ann. 25-27 wanting): A.S.B

A.S. = Archaeological Section, Indian Museum.

A.S B = Asiattc Society of Bengal.

B.C. = Bishop s College.

B.S.I. = Botanical Survey of India

C.E. = Chemical Examiner to the Guvt. of Bengat

C. $U=C$ alcutta University.
E.C. = Civil Engineering College, Sibpur.

G.S.I. = Ueological Survey of 1ndia.

I L. = Imperial Library

M.Ba, = Medical Collese, Bacteriological Laburatory.

M.Bi. = Mledical College. Biological Laboratory.

M.C. = Mledical College (main library). 
SOUTH AND CENTRAL AMERICA AND WEST INDIES. [I583-I592

I583 Rio de Jaṇeiro.-Observatorio (Nacional) (Continued).

(b) Boletim (Mensal). I900-og. Rio de Janerio, I90o-I2 (vol. for Igo6 defective): G.S.I.

1584. - Sociedad Nacional de Agricultura.

Lavoura : Boletim da Sociedade..... Nos. 9-. Rio de Janeiro, I905- : B.S.I.

- Vellosia (see No. I58I).

I585. San José.-Instituto Fisico-geográfico Nacional de Costa Rica.

Anales del Instituto .....y del Museo Nacional de Costa. Rica. Tom. 3, 4, I890-9I. San José, I892-93 : Z.S.I.

I586. - Ministerio de Fomento, Costa Rica.

Boletin de Fomento. Año I-. San José, I9I I - (defect ive) : R.B.G.

I587. - Museo Nacional de Costa Rica.

Anales. Tom. I, I887. San José, I888: Z.S.I. [Subsequently continued in conjunction with No. 1585.]

I588. São Paulo.-Serviço Meteorologico.

Boletim : Commissão Geographica c Geologica do Provincia (Estado) de São Paulo. Nos. I-2I. São Paulo, I889Igo6 (nos. IO-I5 wanting): G.S.I. [Continued as:] Boletim: Observatorio de S. Paulo. Ser. $2^{\mathrm{a}}$, Nos. I-. São Paulo, I907-(nos. 2, 5, I2-16 wanting): G.S.I.

I589. - Museu Paulista.

Revista. Vols. I-4. São Paulo, I895-I90o : Z.S.I.

I590. Santiago.-Deutscher Wissenschaftlicher Verein.

Verhandlungen. Bde. I-6. Santiago, Valparaiso, I885I9I2 (Bde. 1, 2, 5, 6 defective): A.S.B. Index to Bde. I-4 at end of $\mathrm{Bd} .4$.

I59I. - Société Scientifique du Chili.

Actes. Tom. I-I9. Santiago, I892-I909.

Tom. I-I9, I892-I909 (tom. I4 wanting; tonn. 9, I I-I3 defective) : Z.S.I.

Tom. 2-4, I892-94: A.S.B.

Valparaiso.-Deutscher Wissenschaftlicher Verein (see No. I 590 ). I592. — Museo.

Revista Chilena de Historia Natural. Vol. 2-IO, no. I. Valparaiso, I898-I906 (vol. 2, 3 defective) : Z.S.I.

m.o. = Meteorological Ofrice, Aiipore.

M.Ph = Medical College. Physiological Laboratory

P c. = Presidency College.

P O. = Patent Office.

R B.G = Roval Botanic Garcier

S C C $\approx$ Scottish Churches College.
S.G. = Surgeon-Gencrai's Library

S.G. = Survey of India.

S.T. $M=$ School of Tropical Medicine.

T.D. = Telegraph Denartmen:

X.c. =St Xavier s College

Z.s.I. = Zoological Survey of India. 


\section{INTERNATTONAL.}

Académie Internationale de Géographie Botanique (see No. 448).

Association Internationale des Botanistes (see No. 688).

Commission Internationale de Magnétisme Terrestre (see No. 792).

1593. Conférence Générale de 1'Association Géodésique Internationale (Allgemeine Conferenz der Internationalen Erdmessung).

Comptes Rendus (Verhandlungen). Conférences I2-, I898-. Berlin, etc., I899- (Rep. of Conference I3 wanting): M.O.

I594. Congrès Géologique International.

Comptes Rendus. Session I-, I $878-$. Paris, etc., I $880-$ : G.S.I.

I595. Congrès International d'Entomologie.

(a) Procès-verbaux (Proceedings). Ist meeting-. Bruxelles, etc., I9I2- : Z.S.I.

(b) Mémoires (Transactions). Ist meeting-. Bruxelles, etc., IgII-: Z.S.I. [One vol. of $(a)$ and one of $(b)$ are issued for each meeting, the former being styled 'Vol. I,' and the latter 'Vol. 2.']

I596. Congrès International de Zoologie.

Résumés des Communications. gth meeting. Sér. I-3, Monaco, I9I3: Z.S.I.

Conseil Permanent International pour l'Exploration de la Mer (see No. 422).

I597. International Catalogue of Scientific Literature. [Notices of literature for 1900-; in continuation of No. $298(b)$.

Anatomy. Vols. I-. London, I903-.

Complete sets : A.S.B., C.U., I.L., Z.S.I.

Vols. I-3, I903-05: S.G.

Vols. 5-, I907-(vols. 8, 9 wanting): P.C.

Anthropology. Vols. I-. London, I903-.

Complete sets : A.S.B., C.U., I.L., Z.S.I.

Vols. 5-, I907- (vol. 8 wanting) : P.C.

Astronomy. Vols. [-. I,ondon. ז902-: A.S.B., C.U., I.L., M.O., P.C.

Racteriology. Vols. I-. London, I902-.

Complete sets : A.S.B., C.U., I.L., Z.S.I.

Vols. I-3, I $902-05$ : S.G.

\footnotetext{
A.5. - Archaeological Section, Indian Museum. A.S.B. = Asiatic Society of Bengal.

8.C. = Bishop's College.

B.I. = Botanical Survey of India.

C.E. = Chemical Examiner to the Guvt of Bengal.

C.U. = Calcutia University:
}

E.C. = Civil Engineering College, Sibpur.

G.S.I. = Geological Survey of India.

I L. = Imperial Library

M Ba. = Medical College, Bacteriological Laboratory

M. Bi. = Medical College. Biological Laboralory.

M.C. = Medical College (main library). 
I597. International Catalogue of Scientific Literature (Continued).

Botany. Vols I-. London, I9I2-: A.S.B., B.S.I., C.U., I.L., P.C., R.B.G.

Chemistry. Vols. I-. London, I902-: A.S.B., B.S.I., C.U., G.S.I., I.L., M.O., P.C.

General Biology. Vols. I-. London, I903-.

Complete sets : A.S.B., B.S.I., C.U., I.L., R.B.G., Z.S.I.

Vols. I-3, I903-05: S.G.

Vols. 5-, I907-: P.C.

Geography. Vols. I-. London, I903-.

Complete sets : A.S.B., C.U., G.S.I., I.L., M.O., S.I., Z.S.I. Vols. 5-, 1906-: P.C.

Geology. Vols. I-. London, I903- : A.S.B., C.U., G.S.I., I.L., M.O., P.C.

Wathematics. Vols. I-. London, Igo2-: A.S.B., C.U., I.L., M.O., P.C.

Mechanics. Vols. I-. London. 1902- : A.S.B., C.U., I.L., M.O., P.C.

Meteorology. Vols. I-. London, I902-: A.S.B., C.U., I.L., M.O., P.C.

Mineralogy. Vols. I-. London, I903-: A.S.B., C.U., G.S.I., I.L., M.O., P.C.

Palaeontology. Vols. I-. London, I903-

Complete sets : A.S.B., C.U., G.S.I., I.L., Z.S.I.

Vols. 4-, 1906- (vols. 5, 9 wanting): P.C.

Physics. Vols. I-. London, I902- : A.S.B., B.S.I., C.U., I.L., M.O., P.C.

Physiology. Vols. I-. London. I902-.

Complete sets: A.S.B., C.U., I.L., Z.S.I.

Vols. I-3, I902-05: S.G.

Vols. 5-, r907- (vol. 8 wanting): P.C.

Zoology. Vols. I-. London, I904-: A.S.B., C.U., I.L., P.C., Z.S.I. [For earlier series see No. $327(d)$.]

International Engineering Congress (see No. I84).

International Institute of Agriculture (see No. 862).

Internationale Gletschercommission (see No. 6r2).

Internationale Kommission für Wissenchaftliche Luftschiffahrt (see No. 763).

Internationale Mitteilungen für Bodenkunde (see No. 589).

M. $=$ Meteorological Office, Alipore.

M.Ph. = Medical College, Physiological Laboralory:

P.C. = Presidency College.

P.o. = Patent Office

R.B.G = Royal Botanic Garden

S.C.C. $=$ Scottish Churches College.
S.G. = Surgeon-Generals Library.

S.l. = Survey of India.

S.T.M. = School of Tropical Medicine.

T.D. = Telegraph Department

X.c. = St. Xavier's College.

Z.S.l = Zoological Survey of Indiz. 
Internationale Monalsschrift für Anatomie und Hisiologie (see No. 7I7).

Internationale Revue des gesamten Hydrobiologie und Hydrographie (see No. 718).

Internationaler Entomologischer Verein (see No. 653).

Internationales Archiv fïr Ethnographie (see No. 803).

Revue Coloniale Internationale (see No. 790).

\section{ADDENDA.}

\section{GREAT BRITAIN AND IRELAND.}

1598. London.-Annals of British Geology. By J. F. Blake. I890-93. London, I89I-95: G.S.I.

I599. - Anmual Report of the Progress of Chemistry. By J Liebig and H. Kopp. Ed. by A. W. Hofmann and others. Vols. I-3, 1847-49. London, I849-52 : G.S.I. [See also No. $\operatorname{Iog}(a)$.]

I60o. Manchester.-Egyptian and Oriental Society.

Journal. I9II-. Manchester, I9II-: A.S.B.

\section{AUSTRIA-HUNGARY.}

r6or. Vienna.-Archiv fïr Practische Geologie. Herausg von F. Pošepný. Bde. I, 2. Wien, Freiberg in Sachsen, I880, '95 : G.S.I.

\section{GERMANY.}

1602. Berlin.-Beiträge zur Mineralogischen und Geognostischen Kenntniss der Mark Brandenburg. Von K. F. Klöden. Stücke I-7. Berlin, I828-34 (Stück 3 wanting) : G.S.I.

r6o3. Casse1.-Palaeontologische Mittheilungen. Herausg von A. Oppel (and afterwards) K. A. Zittel. Bde. I-3. Stuttgart, Cassel, I $862-84$ : G.S.I.

I604. Leipsig.-Bericht über die Fortschritte der Eisenhütten-Technik. Von A. K. Kerpely. Jahrg. [I]-5, I864-68. I,eipzig, I86670 : G.S.I.

A.S. = Archaeological Section, Indian Museum.

A.S.B. = Asiatic Society of Bengal.

B.C. = Bishop's College.

B.S.I. = Botanical Survey of India

C.E. Chemical Examiner to the Govt. of Bengal

c.U. = Calcutta University
E.C. = Civil Engineering College, Sibpur.

G.S.I. = Geological Survey of India.

I.L. = Imperial Library.

M.Ba. = Medical College, Bacteriological Laboratory

M.BI. = Medical Collesse. Biological Laboratory.

M.C. = Medical College (main library) 


\section{INDIA.}

I605. Bangalore.-Indian Institute of Science.

Journal. Vols. I-. Bangalore, I9I4- : Z.S.I.

\section{AUSTRALASIA.}

1606. Wellington.-New Zealand Board of Science and Art.

New Zealand Journal of Science and Technology.' Vols. I-. Wellington, I9ז8- : Z.S.I.

\section{SOUTH AMERICA.}

I607. Pinheiro.-Escola Superior de Agricultura e Medicina Veterinaria.

Archivos. Vol. I-. Pinheiro (Est. do Rio), I9I7-: Z.S.I.

M. o. = Meteorological Office, Alipore

M.Ph. = Medical College, Physiological Laboratory

P.C. = Presidency College

P.O. = Patent Office

R.B.G. = Royal Botanic Garden

s.c.c. $=$ Scottish Churches College
S.G. = Surgeon-General's Library.

s.I. = Survey of India

S.T.M. $=$ School of Tropical Medicine.

T.D. = Telegraph Department.

X.c. =St, Xavier s College.

Z.s.1. = Zoological Survey of India. 



\section{INDEX}

\section{OF NAMES OF TOWNS AND OF ABBREVIATED TITLES OF INSTITU. TIONS AND PUBLICATIONS.}

Titles of publications are printed in italics, those of institutions, etc., in roman, and names of towns in heavy type.

\section{A}

Aarb. Bergen Mus. $878(a)$.

Aarb. Nordisk Oldkynd. og Hist. $425(a)$.

Aarsber. Bergen Mus. $878(b)$.

Abbild. Beschr. Foss. Pflanz.-Reste. $601(a)$.

Abbild. Naturh. Gegenstände. 664.

Abh. Bayer. Akad. Wiss. $750(a-c)$.

Abh. Ber. K. Zool. Anthr. Mus. Dresden. $645(a)$.

Abh. Ber. Mus. Nat.-Hiematk. Magdeburg. 744.

Abh Ber. Ver. Naturk. Cassel. 637 (a).

Abh. Deut. Naturw.-Med. Ver. Böhm.

'Lotos' 362 (a).

Abh. Geb. Naturwiss. 68I (a).

Abh. Geol. Specialkarte Els.-Lothr. $765(a)$.

Abh. Geol. Specialkarte Preussen etc. 601 (c). Abh. Hallisch. Naturf. Ges. $672(b)$.

Abh. Kais.-Kön. Geogr. Ges. Wien. $374(a)$.

Abh. Kais.-Kön. Geol. Reichsanst. Wien. $375(a)$.

Abh. Kais.-Kön. Zool.-bot.Ges. Wien. 381 (a).

Abh. Kön. Akad. Wiss. Berlin. $600(a-c)$.

Abh. Kön. Bayer. Akad. Wiss. $750(a-c)$.

Abh. Kön. Ges. Wiss. Göttingen. $666(a-c)$.

Abh. Kön. Preuss, Akad. Wiss. $600(a-c)$.

Abh. Kön. Preuss. Geol. Landesanst. 60 I (b).

Abh. Kön. Sächs. Ges. Wiss. $724(a, b)$.

Abh. Kunde Morgenländes. $712(a)$.

Abh. Naturf. Ges. Halle. $672(a)$.

Abh. Naturf. Ges. Ziurich. 96 i (a).

Abh. Naturh. Ges. Nürnberg. 757.

Abh. Naturw.-Med. Ver. 'Lotos.' $362(a)$.

Abh. Naturw. Ver. Hamburg. 68 I (a).

Abh. Naturw. Ver. Magdeburg. 744.

Abh. Naturw. Ver. Sachs. Thüring. $673(a)$. Abh. Mus Nat.-Hiematk. Magdeburg. 744. $A b h$. Schles. Ges. Vaterl. Cultur. $624(a, b)$. Abh. Schweiz. Paläont. Ges. 952.

Abh. Senckenb. Naturf. Ges. $655(a)$.

Abh. Ver. Naturk. Cassel. $637(a)$.

Abh. Ver. Naturw. Unterhalt. 682.

Abh. Zool.-bot. Ver. Wien. 38 I (b).

Abh. Zool. Mus. Dresden. $645(a)$.

Abs. Papers Roy. Soc. London. $298($ a).

Abs. Proc. L'pool Geol. Soc. 64.

Abs. Ris. Meteor. Obs. Calcutta. $1098(a)$.

Acad. Archéol. Belgique. 395.

Acad. Caes. Leop.-Car. Germ. Nat. Cur. 669.
Acad. Groningana. 794.

A.cad. Imp. Sci. St. Pétersbourg. 900-905.

Acad. Internat. Géogr. Botanique. 448.

Acad. Malgache. 963 .

Acad. Nac. Cienc. Cordova. I 553.

Acad. Nat. Hongr. Sci. 355.

Acad. Nat. Sci. Davenport. I 365 .

Acad. Nat. Sci. Philadelphia. I459-1461.

Acad. Polytechn. Porto. 926.

Acad. Real Sci. Lisboa. 918.

Acad. Roy. Inscript. Belles-Lettres Paris. 468.

Acad. Roy. Metz. 746.

Acad. Roy. Sci. Belgique. 398.

Acad. Roy. Sci. Bruxelles. 397.

Acad. Roy. Sci. France. 501.

Acad. Roy. Sci. Turin. 87 I $(a)$.

Acad. Sci. Belgique. 398.

Acad. Sci. Bordeaux. 434.

Acad. Sci. Bruxelles. 397.

Acad. Sci. Caen. 437.

Acad. Sci. Chicago. I 346.

Acad. Sci. Dijon. 444.

Acad. Sci. France. 50 .

Acad. Sci. Imp. Petropolit. 900.

Acad. Sci. Inst. Bononiensis. 8I 5 .

Acad. Sci. Lyon. $45 \mathrm{I}$.

Acad. Sci. New York. I4I6.

Acad. Sci. Philadelphia. I459-I46I.

Acad. Sci. Rochester. 1483.

Acad. Sci. St. Louis. I486.

Acad. Sci. Turin. 8 7I $(d)$.

Acad. Sci. Washington. I 510 .

Acad. Sci. Wisconsin. 1398.

Academy 70.

Accad. Gioen. Sci. Nat. 819.

Accad. Sci. Acireale. 812 .

Accad. Sci. Fis. Mat. Napoli. 840.

Accad. Sci Ist. Bologna. 815.

Accad. Sci. Lett. Arti Zelanti. 8I2.

Account Oper. Gt. Trig. Surv. Ind. $1098(b)$.

Acetylene. 7 I.

Acetylene Journ. $\quad 1348$.

Acetylene Lighting Welding Journ. 72.

Acireale. $8 \mathrm{I} 2$.

Acta Acad. Sci. Imp. Petropolit. $900($ a).

Acta Horti Petropolit. 908.

Acta Mathematica. 929.

Acta Nova Reg. Soc. Med. Havniensis. 429.

Acta Physico-Medica. 669 (a).

Acta Saec. Acad. Groningana. 794.

Acta Schol. Med. Univ. Imp. Kioto. 1163. 
Acta Soc. Faun. Flor. Fennica. $893($ a). Acta Soc. Sci. Fennica. $889(a)$ Acta Soc. Sci. Ind. Neer. I $194(b)$. Actas Acad. Nac. Cienc. Cordova. I553 (a). Actes Acad. Sci. Bordeaux. $434(a, b)$. Actes Soc. Acad. Ind.-Chin. 544 (a). Actes Soc. Linn. Bordeaux. 436 (a). Actes Soc. Sci. Chili. I591.

Adansonia. 469.

Adelaide. I227-1230.

Admm. Rep. Mavine Surv India. $1084(a)$. Athandl. Uppsats. Sver. Geul. Undersök. 935 (a).

A gric. Bull. Bengal. $1056(a)$.

Agric. Bull. Straits Fed. Malay States. (a).

Agric. Coloniale. 822.

Agric. Exp Stat. Connecticut. I 50r.

Agric. Exp. Stat Cornell Univ. I380.

Agric Exp. Stat. Illinois. I 509.

Agric. Exp Stat. Kentucky. I 392.

Agric. Exp. Stat. Maine. I 458 .

Agric. Exp. Stat. Michigan. I 388.

Agric. Exp. Stat. Nebraska. I 394.

Agric. Exp. Stat. Pennsylvania. 1499.

Agric. Exp. Stat. Rhode I. I 385.

Agric. Exp. Stat. Univ. California. 1326.

Agric. Exp. Stat Wisconsin. I 397.

Agric. Dep. Brit. E. Africa. 987.

A gric. Gaz. N.S. Wales. 1263.

Agric. Gaz. Tasmania. I239.

Agric. Horticult. Soc. India. 1044.

Agric. Horticult. Soc. W. India. 1026.

Agric. Journ. Bihar Orissa. I 36.

Agric. Journ. Brit. E. Africa. 987 (a).

Agric. Journ. C. of Good Hope. $972(a)$.

Agric. Journ. India. II4I (a).

Agric. Journ. Mozambique Co. 966.

Agric. Journ. R. Bot. Gard. Ceylon. I1 $37(b)$.

Agric. Journ Union S. Africa. 994.

A gric. Ledger. $\quad 1045$.

A gric. News Barbados. I 547 (a).

Agric Pratique Pays Chauds. 505.

Agric. Research Inst. Ind. I I4I.

Agri-Hort. Soc. Bombay. 1027.

Agri-Hort Soc. Madras. I I 16.

Agronomie Tropicale. $42 \mathrm{I}$.

Alabaina Polytechn. Inst. I3 I4.

Alabama Agric. Exp. Stat. I3I4.

Albany. I309, I 3 ro.

Albany Mus. 984 .

Algemeen-Proefst. Salatiga. I224.

Allahabad. IO06-IOI5.

Allahabad Univ. ror 5 .

Allgem. Conf. Internat. Erdmessung. I 593. Allgem. Schweiz. Ges. gesamt. Naturw. 957. Almanach K. Acad. Wiss. Wien. $372(a)$.

Amer. Acad. Arts Sci. I 327.

Amer. Acad. Polit. Social Sci. 1462.

Amer. Antiq. (Orient.) Journ. I 349.

Amer. Assoc. Adv. Sci. 1489.

Amer. Chem. Journ. I 318.

Amer. Chem. Soc. 1417.

Amer. Chemist. 1418.

Amer. Entomol. Soc. 1463 .

Amer. Ethnol. Soc. 1419.
Amer. Forestry. I5II.

Amer. Genetic Assoc. I 5 I 2.

Amer. Geogr. Soc. 1420.

Amer. Geologist. I40r.

Amer. Inst. Mining Eng. I421.

Amer. Journ. Conchology. I460.

Amer. Journ. Mathematics. I $320(a)$.

Amer. Journ. Med. Sci. 1423.

Amer. Journ. Pharmacy. 1464.

Amer. Journ. Physiol. 1329.

Amer. Journ. Sci (Arts). I4lo.

Amer. Journ. Surgery. 1422.

Amer. Leather Chem. Assoc. I 370.

Amer. Machnnist. 73.

Amer. Math. Soc. I424.

Amer. Med. Assoc. 1350.

Amer. Metrol. Soc. 1425.

Amer. Mus. Journ. I426 (c).

Amer. Mus. Nat. Hist. 1426.

Amer. Naturalist. 1465.

Amer. Orient. Soc. I 328.

Amer. Phil. Soc. 1466.

Amer Phys. Soc. I382.

Amer. Physiol. Soc. I329.

Amer. Soc. Civil Eng. I427.

Ames. I3II.

Amberst. I3I2.

Amiens. 432.

Amsterdam. 784-791.

An. Biuroului Geol. Bucuresci. 392.

An. Inst. Fis.-geogr. Costa Rica. I585.

An. Inst. Geol. México. I567 (a).

An. Inst. Geol. României. $39 \mathrm{I}$.

An. Junta Ciènc. Nat. Barcelona. 917.

An. Minist. Agr. Buenos Aires. 1548.

An. Mus. Geol. Paleont. Bucuresci. 393.

An. Mus. La Plata. $1564(a-h)$

An. Mus. Nac. Arqueol. Hist. Etnol. I57 I (a).

An. Mus. Nac. Buenos Aires. I $550(a)$.

An. Mus. Nac. Costa Rica. 1587.

An. Mus. Nac. México. I $570(a)$.

An. Mus. Nac. Montevideo. $1574(a, b)$.

An. Mus. Públ. Buenos Aires. $1550(a)$.

An. Soc. Quim. Argentina. 1551.

An Zürcherisch. Jugend. $96 \mathrm{I}(b)$.

Analyst. 74 .

Analyt. Labs. Southall Bros. \& Barclay. 2.

Ancient Egypt, 75.

Angers. 433 .

Ann. Acad. Arch. Belgique. 395.

Ann. Acad. Groningana. 794.

Ann. Acad. Roy. Sci. etc. Belgique. 398 (a).

Ann. Agriculture. 77.

Ann. Amer. Acad. Polit. Social Sci. 1462.

Ann. Archaeol. Rep. Canad. Inst. I $300(a)$.

Ann. Astron. Observ. Harvard. I342.

Ann. Astrophys. Observ. Washington. I 520.

Ann. Biol. Lacustre. 399.

Ann. Bot. 78.

Ann. Bot. Roma. 86r.

Ann. Bot. Ziurnch. 959.

Ann. Brit Geol. 1598.

Ann. Bull. Miner. Resources Kansas. I39I

(a).

Ann. Carnegie Mus. 1479 (a). 
Ann. Chem. Pharmacie. $726(a, d)$.

Ann. Chim. 470.

Ann. Chim. Physique. $47 \mathrm{I}$.

Ann. Club Alpin Franc. 494.

Ann. Conserv. Jard. Bot. Genève. 949.

Ann. Durban Mus. 982.

Ann. Ecole Polytechn. Delft. 793.

Ann. Esc. Minas Ouro Preto. I 75.

Ann. Fac. Sci. Warseille. 462.

Ann. Falsifications. $47 j$.

Ann. Géogr. 472.

Ann. Géol. Minéral. Russ. 9I4.

Ann. Géol. Paléontol. 850.

Ann. Géol. Universal. 432.

Ann. Hist.-nat. Mus. Vat. Hungar. $353(a)$.

Ann. Hyg. Publique etc. 480.

Ann. Inst. Bot.-Géol. Colon. Marseille. 463.

Ann. Inst. Colon. Marseille. 463.

Ann. Inst. Wétéor. Vorvége. 883 (b).

Ann. Inst. Océanogr. $502(a)$.

Ann. Inst. Pasteur. 503 (a).

Ann. Jard. Bot. Buitenzorg. $1203(a)$.

Ann K. K. Naturhist. Hofmus. Wien. 37\%.

Ann. K. Sternw. Mïnchen. 75I (a).

Ann. Lyceum Nat. Hist. N. York. $1446(a)$. Ann. Mag. Nat. Hist. 76.

Ann. Malucol. 473.

Ann. Hatem. pura applic 834 .

Ann. Mines. 476 .

Ann. Missouri Bot. Garden. $1433(a)$.

Ann. Mus. Bot. Lugduno-Batavi. 303.

Ann. Mus. Civ. Stor. Nat. Genova. 832.

Ann. Mus. Colon. Marseille. 464.

Ann. Mus. Congo. 403.

Ann. Mus. Guimet. $\quad 524(a-c)$.

Ann. Mus. Hist. Nat. Belgique. $f_{0}+(a)$.

Ann. Mus. Hist. Nat. Marseille. $46_{5}$.

Ann. Mus. Hist. Nat. Paris. $525(a)$.

Ann. Mus. Roy. Hist. Nat. Belg. ${ }_{404}$ (a).

Ann. Mus. Zool. Napoli. $8+2$.

Ann. Mus. Zool. St. Pétersbourg. 905 (a)

Ann . Mycol. Berlin. 572.

Ann. Nat. Hist. 79.

Ann. Natal Gov. Wus. 990.

Ann. New York Acad. Sci. IfI6 (a).

Ann. New York Lyceum Nat. Hist. I446(a).

Ann. Obseri. Nac. Rio de Janeiro. $1533(a)$.

Ann. Observ. R yy Bruxelles. 405.

Ann. Ophthalmslogy. I +87 .

Ann. Paléontsl. 474 .

Ann. Pharm cie. 726 (b).

Ann. Philosophy. 80.

Ann. Physik. 7or $(a, b)$.

Ann. Physik Chem. Jor $(c, d)$.

Ann. Physik Physikal. Chem. jor (c).

Ann. Progr. Rep. Arch. Suro. Ind. Madras etc. I I $3(a)$.

[III3 $(a-f)$.

Ann. Progr. Res Arch. Suro. Ind. N. Circle. Ann. Progr. Rep. Arch. Suro. Ind. S. Circle. I I $8(a)$.

Ann. Progr. Rep. Arch. Surv. Ind. Punjab etc. III3 $(a-f)$.

Ann. Progr. Rep. Geol. Surv. W. Austral. $1258(a)$.

Aun. Progr. Rep. Gov. Epigraphist Madras. I I $18(b)$.
Ann. Progr. Rep Suber. Arch. Surv. Ind. N.

Circle. I I $3(a-f)$.

Ann. Queensland.Mus. I23f (a).

Ann. R. Accad. Sci. Torino. 87 I (a).

Ann. R. Ist. Bot. Torino. 873.

Ann. R. Scuola Sup. Agric. Portici. 860 (a).

Ann. R. Staz. Bacol. Padova. 847.

Ann. Rep.Agr Deb. Brit. E. Afr. 987 (b).

Ann. Rep. Amer. Mus. Nat. Hist. $1426(a)$.

Ann. Rep. Analyt. Labs. Southall Bros. etc.

2.

Ann. Rep. Arch. Dep. Hyderabad. II II $(a)$.

Ann. Rep. Arch. Dep. Mysore. ror6 (a).

Ann. Rep. Arch. Suro. Ceylon. I $100(a)$.

Ann. Rep. Arch. Suro. Ind. IIt7 (a).

Ann. Rep. Arch. Surv. Ind. E. Circle. I04\%.

Ann. Reb. Arch. Suri. Ind. Front. Circle. I I $33(a)$.

$[(a)$.

Ann. Rep. Arch. Surv. Ind. Madras etc. I I 8

Ann. Rep. Arch. Surv. Ind. S. Circle. II 8 (a).

Ann. Rep. Arch. Surv. Mysore. Ior6 (b).

Ann. Rep. Assist. Super. Epigrashy. Madras. I I $8(b)$.

Ann. Rep. Bact. Sect. King Inst. Med. I I26 (a).

Ann. Rep. Board Sii. Advice Ind. I 48.

Ann. Rep. Bur. Amer. Ethnol. Ij2I.

Ann. Rep. Bur. Indust. Ontario. I306 (a).

Ann. Rep. Cambridge Observ. I2 (a).

Ann. Rep. Canad. Inst. I $300(a)$.

Ann. Rep. Connecticut Agr. Exp. Stat. I+12, I jor $(a)$.

Ann. Rep. Cornell Univ. Agr. Exp. Stat. I3 $30(a)$.

Ann. Rep. Dep.Agric. N. Zealand. т277(a). Ann. Rep. Dep. Agric. Queensl. I 23 I (a).

Ann. Rep. Dep. Archaeol. Hyderabad. III I (a).

Ann. Rep. Dep. Geol. Nat. Resources Indiana. I 377 .

Ann. Rep. Dep. Mines Canada. $1292(a)$. Aun. Rep. Dep. Mines N.S. Wales. I 264. Ann. Rep. Direct. U.S. Mint. I543(a).

Ann. Rep. Ent. Soc. Ontario. I302.

Ann. Rep. Ganeshk. Bot. Gard. II 40.

Ann. Rep. Geol. Comm. C. of Good Hope. 973. Ann. Rep. Geol. Nat. Hist. Surv. Canada. $1293(a)$.

Ann. Rep. Geol Nat. Hist. Suro. Minnesota. $1402(a)$.

Ann. Red. Geol. Suro. Canada. I 293 (a).

Ann. Rep. Geol. Surv. India. I06I (a).

Ann. Rep. Geol. Surv. Indiana. I 378.

Ann. Rep. Geol. Surv. Inwa. I 367.

Ann. Rep. Geol. Suro. Missouri. ${ }_{13} 3(a)$. Ann. Rep. Geol. Suro. Pennsylv. ז $376(a)$. Ann. Rep. Geol. Suro. S. Africa. 99j.

Anu. Rep. Geol. Vermont. I 337 .

Ann. Rep. Georgia Exp. Stat. I3I3.

Ann. Rep. Gov Epigraphist .Hadras. It 8 (b).

Ann. Rep. Hatch Exp. Siat. I312.

Ann. Rep. Hawaii. Agr. Exp. Stat, $1206(a)$.

Ann. Rep. Henry Phipps Inst. Study Tuberculosis. 1469 . 
Ann. Rep. Imp. Bact. Muktesar. II 33 . Ann. Rep. Imp. Inst., Ind. Sect. I73 (a). Ann. Rep. Injur. Benef. Insects Mass. 1335. Ann. Rep. Injur. Insects N. York. 1310. Ann Rep. Iowa Geol. Surv. I 367.

Ann. Rep. John Hopkins Univ. I $320(b)$. Ann. Rep. Kentucky Agr. Exp. Stat. 1392 (a).

Ann. Rep. L'pool Mar. Biol. Comm. $67(a)$. Ann. Rep. Marine Biol. Assoc. W. Scotland. $55(a)$.

Ann. Rep. Miner. Indust. Canada. $1292(a)$. Ann. Rep. Miner. Prod. Canada. I $292(a)$. Ann. Rep. Missouri Bot. Gard. $1488(b)$. Ann. Rep. MissouriGeol. Surv. I $383(a)$. Ann. Rep. Mus. Comp. Zool. Havvard. I34I (a).

Ann. Rep. N. Jersey Agr. Exp. Stat. I409 (a).

Ann. Rep. N.Y. Agric. Exp. Stat. I 374 (a). Ann. Rep. N.Y. State Mus. Nat. Hist. I 309 $(a, b)$.

Ann. Rep. Noxious Insects Missouri. 1384. Ann. Rep. Ohio State Board Agric. I 363. Ann. Rep. Peabody Acad. Sci. I49I (a). Ann. Rep. Pennsylv. Mus. etc. I475 (a). Ann. Rep. Philipp. Weather Bur. 1215. Ann. Rep. Progress Chemistry. IO9 (a), 1599. Ann. Rep. Rhodesia Mus. 967.

Ann. Rep. Secr. Mines Victoria. 1249.

Ann. Rep. Smithson. Inst. I $519(a)$. Ann. Rep. State Geol. N. Jersey. ${ }_{1502}(a)$. Ann. Rep. State Mineral. Calnfornia. 1485. Ann. Rep. Super. U.S. Coast Geod. Surv. I $528(b)$.

Ann. Rep. U.S. Ent. Comm. I $535(a)$. Ann. Rep. U.S. Geol. Geogr. Surv. Territ. I 538 (a)

Ann. Rep. U.S. Geol. Surv. I $539(a)$.

Ann. Rep. Yorkshive Phil. Soc. 349.

Ann. Rep. Weather Bur. Philipp. Is. I2I 5.

Ann. Rep. Wisconsin Agr. Exp. Stat. 1397. Ann. Roy. Bot. Gard. Calcutta. 1096. Ann. Roy. Bot. Gard. Ceylon. I I $37(a)$. Ann. Sci. Acad. Polytechn. Porto. 926. Ann. Sci. Géol. 477.

Ann. Sci. Géol. ou Arch. Géol. 478.

Ann. Sci. Nat. 479.

Ann. Sci. Phys. Nat. etc. 457 (b).

Ann. Scottish Nat. Hist. 30.

Ann. Stor. Nat. Bologna. 813.

Ann. Soc. Agric. Sci. Indust. Lyon. 457.

Ann. Soc. Ent. Belgique. $408(a)$.

Ann. Soc. Ent. France. $555(a)$.

Ann. Soc. Géol. Belgique. 4 I $7(a)$.

Ann. Soc. Géol. Nord France. $450(a)$.

Ann. Soc. Linn. Lyon. 460.

Ann. Soc. Malacol. Belgique. 4 II $(a)$.

Ann. Soc. Roy. Zool. Mal. Belg. 4I I (a).

Ann. Soc. Sci. Bruxelles. 4I2 (a).

Ann. South Afric. Mus. 977.

Ann. Surgery. 1428.

Ann. Télégraph. $48 \mathrm{I}$.

Ann. Transvaal Mus. 998.

Ann. Trop. Med. Parasitol. $68(a)$.

Ann. Univ. Lyon. 46 I.
Ann. Wiener Mus. Naturgesch. 389.

Annot. Zool. Japon. $\quad 1187$.

Antananarivo. 963-965.

Anthropol. Ges. Wien. 367 .

Anthropol. Inst. Gt. Brit. 280.

Anthropol. Pap. Amer. Mus. Nat. Hist. ${ }_{1426}$ (b).

Anthropol. Rev. 8I (a).

Anthropol. Soc. Bombay. IO28.

Anthropol. Soc. London. 81 .

Anthropol. Soc. Washington. 1513 .

Anthropologie. 483 .

Anthropos. 368 .

Antiq. Soc. Cambridge. 6.

Antiseptic. III7.

Antwerp. $395,396$.

Anvers see Antwerp.

Apeldoorn. 792 .

Aquila. 354 .

Arb. Biol. Stat. Wolga-Fluss. 913.

Arb. Bot. Inst. Würzburg. $78 \mathrm{I}(b)$.

Arb. Zool. Inst. Graz. 360.

Arb. Zool. Inst. Wien. 380.

Arb. Zool.-zootom. Inst. Würzburg. 781 (a).

Arch. Anat. Entwicklungsgesch. $702(b)$.

Arch. Anat. Physiol. $7 \mathrm{O} 2(c)$.

Arch. Anat. Physiol. Wiss. Med. $702(a)$.

Arch. Antropol. Etnol. 830.

Arch. Biolngie. 4I5.

Arch. Botanik. 703 .

Arch. Botanique. 484.

Arch. Com. Naturw. Landesdurcht. Böhm. $36 \mathrm{I}$.

Arch. Entomol. 487.

Arch. Esc. Super. Agric. etc. Pinheiro. 1607. Arch. gesammt. Physiol. Mensch. Thiere. 617.

Arch. Inst. Bot. Liége. 419.

Arch. Kunde Oesterreich. Gesch.-Quellen. 372 (b).

Arch. Mikroscop. Anat. 618.

Arch. Mus. Hist. Nat. Lyon. $456(a)$.

Arch. Mus. Hist. Nat. Paris. $525(b)$.

Arch. Mus, Hist. Nat. Toulouse. 568.

Arch. Mus. Lyon. $456(a)$.

Arch. Mus. Nac. Rio de Janeiro. I 582.

Arch. Mus. Paris. 525 (b).

Arch. Mus. Teyler. 792.

Arch. Mus. Toulouse. 568.

Arch. Naturgesch. 573.

Arch. Natuurk. Liv-Ehst-Kurlands. 887 (a).

Arih. Néerland. Sci. Exactes Nat. 796.

Arch. Ophthalmology. 1429.

Arch. Parasitol. 485.

Arch. Path. Anat. etc. Berlin. 574.

Arch. Pharm. $580(a)$.

Arch. Phvsiol. $702(d)$.

Arch. Physiol. Mensch. Thieve. 617.

Arch. Pract. Geol. I601.

Arch. Protistenkunde. 687.

Arch. Schiffs-Tropen-Hygiene. 705.

Arch. Sci. Phys. Nat. 945 (b).

Arch. Slaves Biol. 488.

Arch. Suisses Anthrop. Gén. 948.

Arch. Surgery. 82.

Arch. Systemat. Naturgesch. 704. 
Arch. Ver. Freunde. Naturg. Meklenburg. 753.

Arch. Zool. Anat. Fisiol. 831 .

Arch. Zool. Exp. Gén. 486.

Archaeol. Dep. Mysore. IoI6.

Archaeol. Dep. Hyderabad. IIII.

Archaeol. Soc. Hyderabad. III2.

Archaeol. Surv. Ceylon. I 100.

Archaeol. Surv. Ind. 1046, 1147.

Archaeol. Surv. Ind. Bengal Circle. 1047

Archaeol. Surv. Ind. Burma Circle. 1132.

Archaeol. Surv. Ind. E. Circle. I047.

Archaeol. Surv. Ind. Front. Circle. I I 38.

Archaeol. Surv. Ind. Madras Coorg. I 118.

Archaeol. Surv. Ind. N. Circle. III3.

Archaeol. Surv. Ind. S. Circle. II 18.

Archaeol. Surv. Ind IV. Circle. IO29.

Archaeol. Surv. Mysore. IoI6 (b).

Archaeol. Surv N.W. Prov. Ind. III3.

Archaeol. Surv. Punjab etc. III3.

Archaeol. Surv. S. India. I 119.

Archaeol. Surv. W. India. 1030.

Archaeologia. 3I I (a).

Ark. Bot. 932 (a).

Ark. Kemi Mineral. Geol. $932(b)$.

Ark. Mat. Astron. Fys. 932 (c).

Ark. Zool. 932 (d).

Arkeolog. Drutvžo Zagreb. 390.

Arsb. K. Svensk. Vetensk.-Akad. 932 (e).

Arsb. Sver. Geol. Undersökning. 935 (b).

Arskr. K. Univ. Upsala. 938 (a).

Artizan. 83.

Ashmolean Nat. Hist. Soc. 340.

$A$ siat. Researches. $1048(a-c)$.

Asiat. Soc. Bengal. 1048.

Asiat. Soc. Japan. I 88.

Assoc. Franç. Avanc. Sci. 489.

Assoc. Internat. Botanistes. 688.

Assoc. Lyonnaise Amis Sci. Nat. $45^{2}$.

Assoc. Sci. France. 489.

Assoc. Study Internal Secretions. I 395.

Astron. Astro-physics. 1457.

Astron. Beob. Prag. 363 (a).

Astron. Magn. Meteor. Beob. Prag. 363 (b).

Astron. Nachr. 697.

Astron. Obs. Cambridge. I2 (b).

Astron. Obs. Roy. Observ. Edinb. 45.

Astron. Observ. Harvard. I 342.

Astron. Observ. Yale. I4I4.

Astron. Soc. India. 1049.

Astron. Soc. London. 283.

Astronomie. 490.

Astrophys. Observ. Washington. I 520.

Astrophys. Journ. 1356.

Athenaeum. 84

Atlanta. I 313.

Atti Acad. Gioen. Sci. Nat. 819.

Atti Accad. Sci. etc. Acireale. $812(a-e)$.

Atti Accad.Sci. Fis. Mat. Napoli. 840 (a).

Atti Adunanze I.R. Ist. Veneto. 877 (a).

Atti Fond. Sci. Cagnola. 835.

Atti I.R. Ist. Veneto. 877 (a).

Atti Mus.Civ. Stor. Nat. Milano. 838 (a).

Atti Mus. Civ. Stor. Nat. Trieste. 365.
Atti R. Accad. Lincei. $864(a-d)$.

Atti R. Accad. Sci. etc. Acireale. $812(a-e)$.

Atti R. Accad. Sci. To ino. 87 I (b).

Atti R. Accad. Sci. Fis, Mat. Napoli. $840^{\circ}(a)$.

Atti R. Ist. Incorrag. $84 \mathrm{I}$.

AttiR. Ist. Veneto. $877(a)$.

Atti Riun. Scienz. Ita!. 858.

Atti Soc. Geol. Milano. $838(a)$.

Atti Soc. Ital. Progr. Sci. 868 (a).

Atti Soc. Ital. Sci. Nat. 838 (a).

Atti Soc. Rom. Antropol. 869.

Atti Soc. Tosc. Sci. Nat. $859(a, b)$.

Auburn. I3I4.

Augsberg. $57 \mathrm{I}$.

Augusta. I 3 I 5 .

Auserlesene Med.-chīr.-anat.-chym, Abh. 758.

Ausland. 767.

Austin. I316,1317.

Australas. Assoc. Adv. Sci. 1260.

Australas. Inst. Mining Eng. 1245.

Australas. Med. Gaz. I261.

Australian Med. Journ. I247.

Australian Mining Standard Financ. Rev. 1246.

Australian Mus. 1262.

Australian Naturalist. $1271(a)$.

Australian Zoologist. $\quad 1274$.

\section{B}

Baessler Arch. 603.

Baessler Inst. 603 .

Baltimore. $1318-1324$.

Bangalore. 1016-1024.

Bangkok. II 53.

Bankipore. 1025 .

Barcelona. 916, 917.

Basle. 939.

Batavia. I190-I 197.

Bataviaasch Genoot. Kunst. Wet. I I90.

Baumgarten's Jahresb. 723 .

Beira. 966.

Beitr. Biol. Pflanzen. 623.

Beitr. Geogn. Kennt. Erzgebirges. 657.

Beitr. Geol. Erforsch. Deut. Schutzgeb. 601

(d).

Beitr. Geol. Karte Schweiz. 958.

Beitr. Geophysik. 764.

Beitr. Kennt. Erzgebirges. 657.

Beitr. Kennt. Russ. Reiches etc. $900(b)$.

Beitr. Miner. Geogn. Kennt. Mark Brandenburg. 1602.

Beitr. Naturk. Preussens. $700(a)$.

Beitr. Paläont. Oesterreich-Ungarns etc. 369.

Beitr. Wiss. Bot. 706.

Belfast. I.

Belfast Nat. Hist. Phil. Soc. I.

Belgique Horticole. $4 \mathrm{I} 6$.

Bencoolen. I 198 .

Bengal Econom. Assoc. 1050.

Bengal Econom. Joum. 1050.

Beob. Bal ons Drachen etc. $763(a)$.

Ber. Deut. Chem. Ges. 576.

Ber. Fortschr. Eisenhutten-Techn. 1604.

Ber. Ges. Freunde Naturwiss. Wien. 37 I (a).

Ber. K. Sächs. Ges. Wiss. $724(c, d)$. 
Ber. K. Zool. Anthr. Mus. Dresden. 645 (a). Ber. Land-Forstwirtsch. Deut.-Ost-Afrika. $980(a)$.

Ber. Mitt. Ges. Freunde Naturw. Wien. $37 \mathrm{I}$ (a).

Ber. Naturf. Ges. Halle. $672(a)$.

Ber. Naturf. Ges. Freiburg. 659 (a).

Ber. Oberhess. Ges. Nat.-Heilk. $660(a-c)$.

Ber. Ohara Inst. Landwirt. Forsch. II6I.

Ber. Senckenb. Naturf. Ges. $655(b)$.

Ber. Sitz. Naturf. Ges. Halle. 672 (c).

Ber. Ver. Naturk. Cassel. $637(b)$

Ber Verh. Ges. Beförd. Naturwiss. $659(b)$.

Ber. Verh. K. Sächs. Ges. Wiss. $724(c, d)$.

Ber. Verh. Naturt. Ges. Basel. $939(a)$.

Ber. Verh. Naturf. Ges. Freiburg. $659(b)$.

Ber. Verh. Naturf. Ges. Zürich. 96I (c).

Bergen. 878,879 .

Bergen Mus. 878 .

Bergen Mus. Aarb. $878(a)$.

Bergen Mus. Aarsber. $878(b)$.

Berkeley. I325, I326.

Berlin. 572-616.

Berliner Ent. Zeitsch. 584.

Berlinisch. Ges. $588(a, d)$.

Berne. 940-943.

Bernice Pauahi Bishop Mus. I 204.

Berolinum see Berlin.

Beschäft. Berlin. Ges. 588 (a).

Bianconi's Repert. Italicum. 816 .

Bibliogr. Geologica. 400.

Bibliogr. Zoologica. 707.

Biblioth. Etudes Miss. Guimet. $524(b)$.

Biblioth. Univ. (Rev. Suisse). $945(a, b)$.

Biblioth. Zoologica. 762 .

Bidr. Kännen. Finlands Nat. Folk. $889(b)$.

Bienn. Rep. State Geol. Missouri. ${ }_{1} 383(b)$.

Bih. Handl. K. Svensk. Vet.-Akad. $932(f)$.

Bihar Orissa Research Soc. IO25.

Bijdr. Dierkunde. 788. (a).

Bijdr. Taal-Land-Volkenk. Ned.-Ind. 801

Biochem. Assoc. Columbia Univ. I432.

Biochem. Bull. I432.

Biochem. Journ. 7.

Biol. Anstalt Helgoland. 698.

Biol. Bull. Woods Hole. I 546.

Biol. Centralblatt. 708.

Biol. Lab. John Hopkins Univ. 1321 .

Biol. Lab. Philipp. Is. I 213.

Biol. Results Fish. Exp. 'Endeavour.' 1265.

Biol. Soc. Liverpool. 62 .

Biol. Soc. Washington. 1514 .

Biol. Stat. Port Erin. 67 .

Biol. Stat. Wolga-Fluss. 913.

Biometrika. 8.

Birmingham. 2, 3 .

Birmingham Nat. Hist. Phil. Soc. 3.

Blumenbach's Abbild. Naturh. Gegenstände. 664.

Board Agric. Brit. Guiana. I554.

Board Agric. Forest. Hawaii. 1205.

Board Agric. London. 85 .

Board Agric. Trinidad. I 578 .

Board Sci. Advice India. I 488.

Board Trade. 86.

Bol. Acad. Nac. Cienc. Cordova. $1553(b)$.
Bol. Comis. Geol. México. I $567(b)$.

Bol. Commiss. Geogr. Geol. São Paulo. I 588.

Bol. Cuerpo Ing. Minas Perú. 1565.

Bol. Direcc. Gen. Minas Argentina. I 549.

Bol. Est. Agric. Centr. México. I 569.

Bol. Est. Exp. Agron. Cuba. 1555 (a).

Bol. Fomento Costa Rica. 1586.

Bol. Inst. Geol. México. I $567(b)$.

Bol. Mens. Secc. Meteor. Est. Yucatán. I 566.

Bol. Meteor. Macau. I 64.

Bol. Minist. Agr. Argentina. I 549.

Bol. Mus. Goeldi. I $576(a)$.

Bol. Mus. Nac. Arqueol. Hist. Etnol. I57 I (b).

Bol. Mus. Nac. México. I $570(b)$.

Bol. Mus. Paraense. $1576(a)$.

Bol. Observ. Rio de Janeiro. I $583(b)$.

Bol. Observ. São Paulo. I 588.

Bol. R. Soc. Geogr. Madrid. $924(a)$.

Bol. Secr. Agr. Comerc. Trab. Cuba. 1557.

Bol. Soc. Geogr. Lisboa. 920

Bol. Soc. Geogr. Madrid. $924(a)$.

Bol. Soc. Geol. Mexicana.' 1573.

Bol. Soc. Nac. Agr. Rio de Janeiro. I 584 .

Boll. Bimestr. R. Com. Talassogr. Ital. 876.

Boll. Comit. Talassogr. $868(b)$.

Boll. Lab. Zool. Gen. Agr. Portici. $860(b)$.

Boll. Mens. Bachicolt. 846.

Boll. Meteor. Osserv. Astron. Torino. $872(a)$. Boll. Mus. Zool. Anat. Comp. Genova. 833. Boll. Mus. Zool. Anat. Comp. Torino. 875.

Boll. $R$ Comit. Geol. Ital. $865(a)$.

Boll. Soc. Adriat. Sci. Nat. 366.

Boll. Soc. Afric. Ital. 844 .

Boll. Soc. Geol. Ital. 867.

Boll. Soc. Sismol. Ital. 839.

Bologna. 813-818.

Bombay. IC26-1043.

Bombay Lit. Soc. $\mathrm{IO}_{3} 8$.

Bombay Nat. Hist. Soc. 1040.

Bombay Observ. IO4I.

Bonn. 617-622.

Bononia see Bologna.

Bononiensis Sci. Art. Acad. 815.

Bonplandia. 684 .

Bordeaux. 434.

Bosnisch-Herzegovin. Landesmus. 364.

Boston. $1327^{-1} 335$.

Boston Journ. Nat. Hist. $133+(a)$.

Boston Med. Surg. Journ. I330.

Boston Soc. Nat. Hist. 1334.

Bot. Abh. Geb. Morphol. Physiol. 619.

Bot. Bull. I 1375.

Bot. Bull. Dep. Agric. Queensl. I23I (b).

Bot. Cabinet. 87.

Bot. Centralblatt. 632

Bot. Gard. New York. I430.

Bot. Gard. Singapore. I 73 .

Bot. Gazette. I 364 .

Bot. Inst. Würzburg. $78_{1}$ (b).

Bot. Jahrb. Syst. Pflanzengesch. etc. 709.

Bot. Jahresber. 575 .

Bot. Kab. i Bot. Sad. 915.

Bot. Lit.-Blatter Fortsch. Pflanzenk. $760(a)$.

Bot. Mag. 88.

Bot. Mag. Zürich. 960. 
Bot. Miscellany. 89.

Bot. Mitt. a. d. Tropen. 689.

Bot. Mus. Berlin. 602 .

Bot. Notiser. 928.

Bot. Publ. Univ. California. I $325(e)$.

Bot. Register. 90.

Bot. Repository. 92.

Bot. Soc. London. 91.

Bot. Staatsinst. Hamburg. 675 .

Bot. Surv. India. IO5I.

Bot. Zeitun . 7 Io.

Bot. Zeitung Rescens. Abh. etc. $760(b)$.

Boulder. $\quad 1336$.

Brain. 93.

Braithwaite's Retrospect Med. 278.

Braunschweig see Brunswick.

Breslau. 623-626.

Bridgetown. I 547 .

Brief Sketch Met. Unit. Prov. etc. 1005.

Brisbane. I23 I-I236.

Bristol. 4-5.

Bristol Med.-chirurg. Soc. 4.

Bristol Med.-chirurg. Journ. 4.

Bristol Naturalists' Soc. 5.

Brit. Assoc. Adv. Sci. 96.

Brit. Astron. A soc. 97.

Brit. Colonial Druggist. 94.

Brit. Ecological Soc. 98.

Brit. Economic Assoc. 287.

Brit. Flower Garden. 99.

Brut. Foreign Med.-chir. Rev. 95.

Brit. Guiana Board Agric. I 554 .

Brit. Journ. Opthalmo!. IOO.

Brit. Journ. Tuberculosis. IOI.

Brit. Med. Assoc. IO2.

Brit. Med. Assoc., Victorian Branch. 1247.

Brit. Med. Journ. IO2.

Brit. Ornithologists' Union. 103.

Brit. Pharmaceut. Conf. IO4.

Brookiyn. I 337 .

Brooklyn Mus. I 337 .

Brooklyn Mus. Quart. I 337 (a).

Brunswick 627-63I.

Brussels. $397-412$.

Bucuresti see Bukharest.

Budapest. $\quad 350-358$.

Buenos Ayres. I $548-155$ I.

Buffalo. I 338 .

Buffalo Soc. Nat. Sci. 1338.

Builder. I05.

Buitenzorg. I I99-I203.

Bukharest. 39I-394.

Bull. Acad. Imp. Sci. St. Pétersbourg. 900 $(c, d)$.

Bull. Acad. Internat. Géogr. Bot. 448 (a).

Bull. Acad. Malgache. 963.

Bull. Acad. Nat. Hongr. Sci. $355($ a).

Bull. Acad. Roy. Sci. Belgique. $398(b-d)$.

Bull. Acad. Roy. Sci. Bruxelles. 397 (a).

Bull. Acad. Sci. Chicago. I 346.

Bull. Agric. Congo Belge. 401.

Bull. Agric. Exp. Stat. Rhode I. I $385($ a).

Bull. Agric. Research Inst. Ind. I I4I (b).

Bull. Alabama .Agr. Exp. Stat. I3I4.

Bull. Amer. Geogr. Soc. $1420(a)$.

Bull. Amer. Inst. Mining. Eng. I42I (a).
Bull. Amer. Math. Soc. $1424(a)$.

Bull. Amer. Mus. Nat. Hist. $1426(d)$.

Bull. Astron. Paris. 530.

Bull. Bengal Fish. Dep. I057.

Bull. Buffalo Soc. Nat. Sci. $\quad$ I 338.

Bull. Bot. Dep. Jamaica. I561.

Bull. Bur. Agric. Intell. etc. $862(a)$.

Bull. Bur. Amer. Ethnol. I 52 I.

Bull. Bur. Econ. Social Intell. $862(b)$.

Bull. Bur. Fish. Madras. I 122.

Bull. Bur. Fish. U.S.A. I $525(a)$.

Bull. Bur. Forestry Philipp. Is. I2I $2(a)$.

Bull. Calcutta Math. Soc. I085.

Bull. Calcutta Microsc. Soc. 1093.

Bull. California Acad. Sci. I $492(a)$.

Bull. California Agr. Exp. Stat. 1326.

Bull. Canad. Mining Inst. 1288 (a).

Bull. Carte Géol. France. 543.

Bull. Centr. Observ. Manula. I2I6.

Bull. Chicago Acad. Sci. I 346.

Bull. College Agric. Tokyo. II $85(a)$.

Bull. Com. Asie Franc. 495.

Bull. Com. Géol. St. Pétersbourg. $907(a)$

Butl. Comm. Archéol. Indo-Chine. 496.

Bull. Comm. Cent. Sism. Perm. St. Pétersbourg. 9OI $(a)$.

Bull. Comm. Géol. Finland. $892(a)$.

Bull. Comm. Sism. Perm. St. Pétersb'g. $90 \mathrm{I}$ (a).

Bull. Connecticut Agr. Exp. Stat. I 50 O (b).

Bull. Cornell Univ. Agr. Exp. Stat. I $380(b)$.

Bull. Dep. Agric. Assam. II 66.

Buil. Dep. Agric. Bengal. $\operatorname{I056}(a, b)$.

Bull. Dep. A gric. Bombay. IO3I.

Bull. Dep. Agric. Burma. I 143 .

Bull. Dep. Agric Cent. Prov. Ind. II 34 (a).

Bull. Dep. Agric. Fed. Malay States, I 59.

Bull. Dép. Agric. Ind. Néerl. I I99(a).

Bull. Dep. Agric. Jamaica. I 560.

Bull. Dep. Agric. Madras. I 21.

Bull. Dep. Agric. Mysore. $1017(a)$.

Bull. Dep. Agric. N. Zealand. ${ }_{1277}(b-e)$.

Bull. Dep. Agric. Ontario. I 305.

Bull. Dep. Agric. Queensland. I23I (c).

Bull. Dep. Agric. Unit. Prov. I006.

Bull. Dep. Fisheries Bengal etc. I057.

Bull. Dep. Geol. Univ. California. I $325(f)$.

Bull. Dep. Land Rec. Agr. Assam. I 46.

Bull. Dep. Land Rec. Agr. Bengal. ${ }_{1056}$ $(a, b)$.

Bull. Dep. Land Rec. Agr. Bombay. IO3I.

Bull. Dep. Land Rec. Agr. Madras. I I I

Bull. Dep. Land Rec. Agr. Unit. Prov. 1006.

Bull. Dep. Landb. Suriname. $1577(a)$.

Bull. Dep. Mines Canada. I $292(b)$.

Bull. Div. Entom. Dept. Agr. Canada. I29I (a).

Bull. Earthq. Invest. Comm. I I $82(a)$.

Bull. Ecole Franç. Extr.-Orient. II 57.

Bull. Econom. Indo-chine. I 56.

Bull. Econom. Madagascar. 964.

Bull. Entom. Research. I72 (a).

Bull. Essex. Inst. $1490(a)$.

Bull. Exp. Stat. Hawaii. I $208(a, b)$.

Bull. Exp. Stat. Minnesota School Mines. 1405. 
Bull. Fish. Bur. Madras. II 22.

Bull. Gén. Univ. Annonces Nouvelles Sci. Paris. $5_{6} 6_{3}(a)$.

Bull. Geol. Dep. Mysore. Iorg (a).

Bull. Geol. Inst Upsala. $938(b)$.

Bull. Geol. Nat. Hist. Surv. Chicago. I 347.

Bull. Geol. Nat. Hist. Surv. Minnesota. 1402 (b).

Bull. Geol. Nat. Hist. Surv. Wisconsin. I 396.

Bull. Geol. Surv. Illinois. I $505(a)$.

Bull. Geol. Surv. Japan. I I83 (a).

Bull. Geol. Surv. Minnesota. 1404.

Bull. Geol. Surv. New Jersey. I $502(b)$.

Bull. Geol. Surv. New Zealand. I278(a).

Bull. Geol. Surv. Ohio. I 36 I $(a)$.

Bull. Geol. Surv. Queensland. I233 (a).

Bull. Geol Surv. Tasmania. I24I (a).

Bull. Geol. Surv. Tennessee. 1408.

Bull. Geol. Surv. Victoria. I25 I (a).

Bull. Geol. Surv. Virginia. I 345.

Bull. Geol. Surv. W. Austral. I $258(b)$.

Bull. Geol. Surv. Wisconsin. I 396.

Bull. Georgia Exp. Stat. I3 I 3.

Bull. Gov. Hospital Insane. I 5 I6.

Bull. Gov. Mus. Madras. I 124.

Bull. Hatch Exp. Stat. I3I 2.

Bull. Hawaii Agr. Exp. Stat. I 206 (b).

Bull. Herb. Boissier. 946.

Bull. Hist. Nat. Bordeaux. $436(b)$.

Bull. Hyg. Publique. 492.

Bull. Illinois Agr. Exp. Stat. I $509(a)$.

Bull. Illinois Geol. Surv. I $505(a)$.

Bull. Illinois State Lab. Nat. Hist. I 506.

Bull. Illinois State Mus. Nat. Hist. 1496.

Bull. Imp. Earthq. Invest. Comm. I $182(a)$.

Bull. Imp. Geol. Surv. Japan. I I83 (a).

Bull. Imp. Inst. I $73(b)$.

Bull. Ind. Ass. Cult. Sci. IO70 (a).

Bull. Insectol. Agric. 548.

Bull. Inspect. Landb. Suriname. I $577(a)$.

Bull. Inst. Bot. Buitenzorg. I 201 (a).

Bull. Inst. Egypt. 968.

Bull. Inst. Océanog. Monaco. 466.

Bull. Inst. Pasteur. $503(b)$.

Bull. Iowa Agric. Exp. Stat. I 3 I I (a).

Bull. Jard. Bot. Bruxelles. 402.

Bull. Jard. Bot. Buitenzorg. I 203 (b).

Bull. John Hopkins Hosp. I 319.

Bull. John Ryland Libr. 33 I.

Bull. Kentucky.Agv. Exp. Stat. I $392(b)$.

Bull. Kodaikanal Observ. II $30(a)$.

Bull. Kolon. Mus. Haarlem. 799 (a).

Bull. Kön. Bayer. Akad. Wiss. $750(d)$.

Bull. Lab. Inl. Rev. Dep. Canada. I 295.

Bull. Lick Observ. I 325 (b).

Bull. Lloyd Libr. Bot. etc. I $357(a-c)$.

Bull. Lowell Observ. I 372.

Bull. Madras Fish. Bur. II 22.

Bull. Madras Mus. I I 24.

Bull. Maine Agr. Exp. Stat. 1458.

Bull. Manila Med. Soc. I 219.

Bull. Math. Soc. Calcutta. I085.

Bull. Med Soc. Manila. I 219.

Bull. Mém. Soc. Anthropol. Paris, 55 I (b).

Bull. Mens. Bur. Cent. Météor. Paris. 493(a).

Bull. Mens. Observ. Meteor. Manila. I22I (a).
Bull. Mens. Soc. Linn. Paris. 558.

Bull. Meteor. Osserv. Colleg. Romano. 863.

Bull. Meteor. R. Osserv. Palermo. 854.

Bull. Michigan Agric. Exp. Stat. $1388(a)$.

Bull. Microsc. Soc. Calcutta. I093.

Bull. Miner. Surv. Chōsen. I I68.

Bull. Mining Bur. Philipp. Is. I 220.

Bull. Minnesota Acad. Nat. Sci. 1403.

Bull. Misc. Inform. Board. Agr. Trinidad. I $578(a)$.

Bull. Misc. Inform. Kew. $284(a, b)$.

Bull. Mount Weather Observ. I 407.

Bull. Mus. Caucase. IOOI (a).

Bull. Mus. Comp. Zool. Harvard. I34I (b).

Bull. Mus. Hist. Nat. Belgique. $404(b)$.

Bull. Mus. Hist. Nat. Paris, 525 (c).

Bull. Mus. Madras. I 24.

Bull. Mus. Océanog. Monaco. 466.

Bull. Mus. Paris. $525(c)$.

Bull. Mus. Roy. Hist. Nat. Belg. $404(b)$.

Bull. Mus. Sci. Art. Pennsylvania. $\mathrm{I} 477(a)$.

Bull. Mus. Zool. Anat. Comp. Torino. 875 .

Bull. Nebraska Agr. Exp. Stat. I 394.

Bull. New Hamps. Agr Exp. Stat. I 369.

Bull. New Jersey Agr. Exp. Stat. 1409 (b).

Bull. New Mexico Agr. Exp. Stat I 389.

Bull. New York Agr. Exp. Stat. I $374(b)$.

Bull. New York Bot. Gard. $1430(a)$.

Bull. New York State Mus. Nat. Hist. I 309 (c).

Bull. North Carolina Agr. Exp. Stat I48I.

Bull. North Carolina Dep. Agric. 1482.

Bull. Obs. Météor. Observ. St. Louis. 6r.

Bull. Observ. Ebre. 927.

Bull. Olito Geol. Surv. i 36 I (a).

Bull. Oklahoma Agr. Exp. Stat. I 500.

Bull. Ontario Dep. Agric. I 305.

Bull. Orient. Inst. Vladivostok. IOC3.

Butl. Ort. Bot. Napoli. 843.

Bull. Pasteur Inst. S. India. I 108.

Bull. Pennsylv.Agr. Exp. Stat. 1469 (a).

Bull. Pennsylv. Mus. I $475(b)$.

Bull. Pennsylv. Mus. Sci. Art. 1477 (a).

Bull. Phil. Soc. Washington. 1518.

Bull. Pop. Piscicult. etc. 570.

Bull. Proefst. Suik. W.-Java 'Kagok.' I 223 (a).

Bull. Public Mus. Milwaukee. I 399.

Bull. R. Mus. Commerc. Torino. 874.

Bull. R. Ort. Bot. Palermo. 852.

Bull. Roy. Bot. Gard. Kew. $284(a, b)$.

Bull. Sci. Agric. Econ. Paris. $563(e)$.

Bull. Sci. Géogr. Econ. Publ. etc. $563(\mathrm{~g})$.

Bull. Sci. Hist. Antiq. Philol. $563(h)$.

Bull. Sci. Math. 49I.

Bull. Sci. Math. Astron. etc. $563(b)$.

Bull Sci. Méd. Paris. $5^{6} 3(d)$.

Bull. Sci. Militaires Paris. $563(i)$.

Bull. Sci. Nat. Géol. Paris. 563 (c).

Bull. Sci. Paris. 562.

Bull. Sci. Soc. Philom. Paris. 562.

Bull. Sci. Technol. Paris. $563(f)$.

Bull. serv. Carte Géol. France. 543.

Bull. Serv. Géol. Indo-chine. I $5^{8}(a)$.

Bull. Sleeping Sickness Bur. 309 (a).

Bull. Soc. Acad. Indo-chin. $544(b)$. 
Bull. Soc, Afric. Ital., Sez. Fiorent. 826. Bull. Soc. Anthropol. Lyon. $45^{8}$. Bull. Soc. Anthropol. Paris. $55 \mathrm{I}(a, b)$. Bull. Soc. Astron. France. 546. Bull. Soc. Belge Etudes Colon. 406.

Bull. Soc. Belge Géogr. 409.

Bull. Soc. Belge Géol. Paléont. etc. 407 (a). Bull. Soc. Bot. France. 547.

Bull. Soc. Bot. Ital. 828 .

Bull. Soc. Chim. France. 549.

Bull. Soc. Ent. Belgique, $408(b)$.

Bull. Soc. Ent. France 555 (b).

Bull. Soc. Ent. Ital. 829.

Bull. Soc. Ent. Suisse. 942.

Bull. Soc. Etudes Colon. Belge. 406.

Bull. Soc. Etudes Sci. Angers. 433.

Bull. Soc. Fribourg. Sci. Nat. 944.

Bull. Soc. Géogr. Commerc. Bordeaux. 435.

Bull. Soc. Géogr. Commerc. Havre. 446.

Bull. Soc. Géogr. Finlande. 894.

Bull. Soc. Géogr. Lyon. 459.

Bull. Soc. Géogr. Paris. $553(a)$.

Bull. Soc. Géol. France. 557 (a).

Bull. Soc. Géol. Normand. 447.

Bull. Soc. Hongr. Géogr. $35 \mathrm{I}$.

Bull. Soc Imp. Nat. Mnscou. 897 (a).

Bull. Soc. Linn. Nord France. 432.

Bull. Soc. Linn. Normand. $440(a)$.

Bull. Soc. Linn. Paris. $55^{8}$.

Bull. Soc. Math. France. 559.

Bull. Soc. Minéral. France. 560.

Bull. Soc. Nat. Sci. Buffalo. 1338.

Bull. Soc. Neuchat. Sci. Nat. $956(a)$.

Bull. Soc. Path. Exotique. 554.

Bull. Soc. Philom. Paris. 562.

Bull. Soc. Portug. Sci. Nat. 92 I (a).

Bull. Soc. Roy. Belge Géogr. 409.

Bull. Soc. Roy. Bot. Belgique. 4 IO.

Bull. Soc. Roy. Géogr. Anvers. 396.

Bull. Soc. Sci. Méd. Ouest. 566.

Bull. Soc. Sci. Nat. Neuchatel. $956(a)$.

Bull. Soc. Sci. Nat. Ouest France. 467.

Bull. Soc. Vaud. Sci. Nat. 955.

Bull. Soc. Zool. France. $\quad 565(a)$.

Bull. Stat. Agronom. Mauritius. $992(a)$.

Bull. Stat. Pisc. Hydrobiol. Toulouse. 570.

Bull. Torrey Bot. Club. 1454.

Bull. U.S. Bur. Fisheries. I 525 (a).

Bull. U.S. Bur. Mines. I $526(a)$.

Bull. U.S. Bur. Standards. I $527(a)$.

Bull. U.S. Dep. Agr. I $529(b)$.

Bull. U.S. Dep. Agr., Div. Biol. Surv. I 530 (a).

Bull. U.S. Dep. Agr., Div. Entomol. I53I $(a, b)$.

Bull. U.S. Dep.Agr., Div. Orn. Mamm. I 530 (a).

Bull. U.S. Ent. Comm. I 535 (b).

Bull. U.S. Fish. Comm. I $525(a)$.

Bull. U.S. Geol. Geogr. Surv. Territ. I $538(b)$.

Bull. U.S. Geol. Surv. I 539 (b).

Bull U.S. Hygienic Lab. I 543 .

Bull. U S. Nat. Mus. I 522.

Bull. Univ. Montana. 1406.

Bull. Univ. Sci. Indust. $563(a)$.

Bull. Univ. Texas. 1317.
Bull. Victoria Memor. Mus. $1294(a)$.

Bull. Virginia Geol. Surv. I 345.

Bull. Washington Phil. Soc. I 518.

Bull. Wisconsin. Nat. Hist Soc. $1400(a)$.

Bull. Yellow Fever Bur. 68 (b).

Buluwayo. 967.

Bur. Agric. Philipp. Is. I 2 I I.

Bur. Amer. Ethnology. I 52 I.

Bur. Cent. Météor. Paris. 493.

Bur. Fisheries Tokyo. I 18 I.

Bur. Forestry Philipp. Is. 1212.

Bur. Govt. Labs. Philipp. Is. I213.

Bur. Indust. Ontario. 1306.

Bur. Mines Toronto. I 299.

Bur. Sci. Philipp. Is. I 214 .

Burgerlijk Geneesk. Dienst Ned.-Ind. I I9I .

Burlington. I 339 .

Burma Research Soc. II 42.

Butll. Club Montan. Barcelona. 9I6.

\section{C}

Caen. 437-440.

Cairo. 968-97 I

Calcutta. I044-1099.

Calcutta Journ. Med. 1052.

Calcutta Journ. Nat. Hist. I053.

Calcutta Math. Soc. I085.

Calcutta Med. Club. 1087.

Calcutta Med. Journ. 1087.

Calcutta Med. News. I054.

Calcutta Microsc. Soc. I093.

California Acad. Sci. 1492.

California Agr. Exp. Stat. I326.

California State. Agr. Soc. 1484.

California State Mining Bur. $1_{485}$.

California Univ. I325, I 326.

Cambridge. 6-I5.

Cambridge, Mass. I $340-1342$.

Cambridge Antiq. Soc. 6.

Cambridge Ent. Club. I 340.

Cambridge Observatory. I2.

Cambridge Phil. Soc. 14.

Cambridge Univ. 5 .

Canad. Entomologist. 1287.

Canad. Inst. I 300.

Canad. Journ. $1300(b)$.

Canad. Mining Inst. I 288.

Canad. Mining Journ. 1301.

Canterbury Mus. 1237.

Cape Town. 972-979.

Caracas. I 552 .

Caract. Magnét. chaque jour Mois. 792.

Carlisle. I 343.

Carnegie Mus. I479.

Carnegie Schol. Memoirs. $188($ a).

Cassel. 632-637.

Cassier's Engin. Monthly. I06.

Cat. Sci. Papers Roy. Soc. London. 298 (b).

Central Observ. Manila. I2I6.

Centralbl. Bakt. Parasitenk. etc. 690.

Centralbl. Miner. Geol. Palaeont. 768.

Ceylon Administ. Reps. IIO2.

Ceylon Antiq Lit. Register. IIOI.

Ceylon Marine Biol. Reps. I IO3 $(a)$.

Challenger Soc. IO7. 
Charlesworth's Mag. 208.

Charlotte. I 344.

Charlotte Med. Journ. 1344.

Charlottesville. I 345.

Chateauroux. 44I.

Chem. Abstracts. I4I7 (a).

Chem. Central-Blatt. 676.

Chem. News. 108.

Chem. Soc. 109.

Chem. Trade Journ. 329.

Chem. World. I IO.

Chemist. II 1.

Chemist \& Druggist. I 12.

Cherbourg. 442 .

Chesapeake Zoöl. Lab. I32I (a).

Chicago. 1346-1356.

Chicago Acad. Sci. I 346.

Chicago Univ. I 356.

China Med. Journ. I I 70.

Christchurch. I 237 .

Christiania. 880-885.

Cincinnati. I357-1359.

Cincinnati Soc. Nat. Hist. 1358.

Cincinnati Univ. I 359.

Circ. Agr. Journ. R. Bot. Gard. Ceylon. II 37 (b).

Circ. Board Agr. Trinidad. I $578(b)$.

Circ. Bur. Forestry Philipp. Is. I21 $2(b)$.

Curc. Est. Exp. Agron. Cuba. I $555(b)$.

Circ. Illinois Agr. Exp. Stat. 1509 (b).

Circ. John Hopkins Univ. $1320($ c).

Circ. Roy. Bot. Gard. Ceylon. II $37(b)$.

Circ. U.S. Bur. Standards. $1527(b)$.

Circ. W. India Committee. $320(a)$.

Circolo Matem. Palermo. 851.

Cistula Entomologica. I I 3.

Civ. Engin. Archit. Journ. II4.

Civ. Med. Service Neth. Ind. I I9I.

Civ. Vet. Dep. India. I055.

Claremont. I 360 .

Clinical Excerpts. II 5.

Clinical Journ. I I6.

Clinical Soc. I 17.

Clinical Studies. 32.

Club Alpin Français. 494.

Club Montanyenc Barcelona. 9I6.

Coal Age. 143 I.

Cold Spring Harb. Monogr. $1337(b)$.

Coll. Pap. Sci. Labs. Melbourne Univ. I256.

College Agric. Tokyo. $1185(a, b)$.

College Engin. Madras. I 120.

College Engin. Tokyo. $1185(c)$.

College Sci. Tokyo. I $85(d)$.

Collegio Romano. 863.

Colliery Engin. 1494.

Colliery Guardian. I 8 .

Colombo. I IOO-I 107 .

Colombo Mus. II05.

Colombo Observ. 1104.

Colonial Mus. N. Zealand. I 276.

Colonial Reports. 173 (c).

Colorado Univ. 1336.

Columbia Univ. I 432 .

Columbus. $1361-1363$.

Comis. Geol. México. I $567(b)$.

Comitato Talassografico. $868(b)$.
Comité Asie Française. 495.

Comité Géol. St. Pétersbourg. 907.

Comité Naturw. I,andesdurch. Böhmens. 361 .

Comment. Acad. Sci.Bononiensis. 8I 5 (a).

Comment. Acad. Sci. Imp. Petropol. $900(e)$.

Commerc. litt. ad rei med. sci. nat. etc. 754.

Commiss. Archéol. Indo-Chine. 476.

Commiss. Arckeol. Ind. Portugueza. I I IO.

Commiss. Cent. Sism. Perm. St. Pétersb'g. 901.

Commiss. Départ. Météor. Rhône. 453.

Commiss. Fish. Maine. I 3 I 5.

Commiss. Gèogr. Geol. São Paulo. I 588.

Commiss. Géol. Finland. 892.

Commiss. Internat. Aérost. Sci. 763 .

Commiss. Internat. Magn. Terrestre. 792.

Commiss. Mediterr. Fever. $298(k)$.

Commiss. Ned.-Ind. Oudheidk. Onderz. I 190 (c).

Commiss. Serv. Geol. Portugal. 919.

Commiss. Sci. Arch. Orenburg. 899.

Commiss. Sism. Perm. St. Pétersbourg. 901.

Commiss. Vervaard. Geol. Beschrijv. Kaart Ned. 795.

Committee Study Malaria India. I149.

Companhia Moçambique. 966.

Companion Bot. Mag. I I9.

Commun. Direç. Trab. Geol. Portugal. 919.

Commun. Comm. Sevv. Geol. Portugal. 919.

Commun. Comm. Trab. Geol. Portugal. 919.

Commun. Essex 1nst. I $490(b)$.

Commun. Millport Marine Biol. Stat. $55(b)$.

Commun. Mus. Nac. Buenos Aires. $1550(b)$.

Compt. Rend. Acad. Sci. Paris. 501 (a).

Compt. Rend. Assoc. Lyonn. Amis Sci. Nat. 452 .

Compt. Rend. Comm. Sism. Perm. St. Pétersb'g. $901(b)$.

Compt. Rend. Conf. Gén. Assoc. Géod. Internat. I 593 .

Compt. Rend. Congr. Géol. Internat. I 594.

Compt. Rend. Soc. Biol. Paris. 552.

Compt. Rend. Soc. Géngr. Paris. $553(b)$.

Compt. Rend. Soc. Ent. Belgique. $408(b)$.

Compt. Rend. Soc. Fribourg. Sci. Nat. 944.

Compt. Rend. Soc. Phys. Hist. Nat. Genève. $953(a)$.

Concarneau. 443.

Conch. Mitt. etc. 633.

Conch. Soc. Gt. Brit. Ireland. 120.

Concrete Construct. Eng. I 21 .

Contér. Biblioth. Vulgarisation. $524(d)$.

Confér. Gén. Assoc. Géod. Internat. I 593.

Congr. Géol. Internat. I 594.

Congr. Internat. Entomol. I595.

Congr. Internat. Zool. I 596.

Connaiss. des Temps. $50 \mathrm{I}(b)$.

Connecticut Acad. Arts Sci. I4II.

Connecticut Agric. Exp. Stats. I4I2, 1501.

Conseil Perm. Internat. Explor. Mer. 422.

Contrib. Bot. Lab. Pennsylv. Univ. $1476(a)$.

Contrib. Canad. Biol. 1289.

Contrib. Canad. Palaeont. I 293 (b).

Contrib. Faune Ind. Néerland. 1202.

Contrib. North Amer. Ethnol. I 536. 
Contrib. U.S Nat. Herbarium. I522(b).

Contrib. Zool. Lab. Pennsylv. Univ. $1476(b)$.

Coonoor. I 108.

Copenbagen. $422-431$.

Cordova. I 553 .

Cork. I6.

Cork Cuvierian Soc. 22.

Cornell Univ. 1380 .

Corpus Inscript. Indicarum. $1046(a)$.

Corres.-Bl. Ent. Ver. 'Iris'' $641(a)$.

Corres.-Bl. Naturh. Ver. Preuss, Rheinl. etc. $620(a)$.

Cosmos. 523,870 .

Cotteswold Naturalists' Field Club. 58

Country Folklore. I5O (a).

Crawfordsville. 1364 .

Crop Bult. Ontario. $1306(b)$.

Crop Reporter. U.S.A. I $529(a)$.

Cuerpo Ing. Minas Perú. I 565 .

Cullercoats. I 7 .

Cunningham Memoirs. 28 (a).

Curtis's Bot. Mag. 122.

Cuvierian Soc. Cult. Sci. 16.

Cyclone Memoirs. II5I (a).

Cyprus Journ. 1004.

\section{$\mathrm{D}$}

Danish Biol. Stat. 423.

Dantzig. 638 .

Dar-es-Salam. 980.

Darmstadt. 639.

Davenport. 1365 .

Davenport Acad. Nat. Sci. 1365.

Dehra Dun. I I09.

Delft. 793.

Denkschr. Allg. Schweiz. Ges. Naturw. 957 (a).

Denkschr. K. Acad. Wiss. Wien. $372(c)$.

Denkschr. K. Bayer. Akad. Wiss. $750(e)$.

Denkschr. K. Bayer. Bot. Ges. $760(c)$.

Denkschr. Med.-naturwiss. Ges. Jena. $693($ a).

Denver. I 366.

Dep. Agric. Assam. I 146.

Dep. Agric. Bengal. I056.

Dep. Agric. Bihar Orissa. I 136.

Dep. Agric. Bombay. IO3I.

Dep. Agric. Brit. E. Africa. 987.

Dep. Agric. Burma. I 43 .

Dep. Agric. Canada. I290, 1291.

Dep. Agric. C. of Gnod Hope. 972.

Dep. Agric. Cent. Prov. Ind. 1134.

Dep. Agric. Fed. Malay States. I 59.

Dép. Agric. Ind. Néerl. I 199.

Dep. Agric. India. II4I.

Dep. Agric. Jamaica. I 560 .

Dep. Agric. Madras. II 1 .

Dep. Agric. Mysore. IOI7.

Dep. Agric. Natal. 988.

Dep. Agric. New South Wales. 1263.

Dep. Agric New Zealand. 1277.

Dep. Agric. Ontario. I305.

Dep. Agric. Queensland. I23I.

Dep. Agric. Rhodesia. IOOO.

Dep. Agric. S. Australia. 1227.

Dep. Agric. Tasmania. 1239 .
Dep. Agric, Techn. Instr. Ireland. I 8.

Dep. Agric. Transvaal. 993.

Dep. Agric. Union S. Africa. 994.

Dep. Agric. United Prov. 1006.

Dep. Agric. Victoria. 1248.

Dep. Agric. W. Australia. 1257.

Dep. Agric. W. Indies. I 547.

Dep. Archaeol. Hyderabad. II II.

Dep. Colon. Mines Fis' 1 . Quebec. 1297.

Dep. Fish. Bengal (Bihar Orissa). 1057.

Dep. Econ. Allahabad Univ. IOI5.

Dep. Geol. Nat. Resources Indiana. 1377.

Dep. Geol. Univ. California. I $325(f)$.

Dep. Land Rec. Agr. Assam. I i 46.

Dep. Land Rec. Agr. Bengal. 1056.

Dep. Land Rec. Agr. Bombay. IO3I.

Dep. Land Rec. Agr. Madras. II 21.

Dep. Land Rec. Agr. Unit. Prov. 1006.

Dep. Landbouw Ned.-Ind. 1199.

Dep. Landbouw Suriname. 1577.

Dep. Mines Agric. N. S. Wales. 1264.

Dep. Mines Canada. 1292.

Dep. Mines India. I058.

Dep. Mines N. S. Wales. 1264.

Dep. Mines Nova Scotia. I284.

Dep. Mines Queensland. I232.

Dep. Mines Tasmania. 1240.

Dep. Mines Transvaal. 997.

Dep. Mines Union S. Africa. 995.

Dep. Mines Victoria. I 249.

Dep. Public Gardens Jamaica. I56I.

Dep. Trade Customs Australia. 1265.

Des Moines. I 367 .

Detroit. 1368.

Deutsch. Ap thekerverein. 580.

Deutsch. Arch. Physiol. $702(e)$.

Deutsch. Bunsen Ges. Physik. Chem. 668.

Deutsch. Chem. Ges. 576.

Deutsch. Eletr.-Chem. Ges. 668.

Deutsch. Ent. Ges. 577.

Deutsch. Ent. Nat.-Bibliothek. $581(a)$.

Deutsch. Ent. Nat.-Mus. $5^{81}$.

Deutsch. Ent. Zeitsch. 577, 584, 641.

Deutsch. Geol. Ges. 578 .

Deutsch. Ges. Mech. Optik. 579.

Deutsch. Ges. Nat.-Volkerk. Ostasiens. I 179.

Deutsch. Malakozool. Ges. 651 .

Deutsch. Math. Vereinigung. 711 .

Deutsch. Mechan.-Zeitung. $579(a)$.

Deutsch. Meteorol. Ges. 376.

Deutsch. Mineral. Ges. 691.

Deutsch. Morgenländ. Ges. 712.

Deutsch. Naturw.-Med. Ver. Böhm. ' Lotos.' 362 .

Deutsch. Ornithol.-Ges. 771.

Deutsch. Ornithol.-Ver. 771.

Deutsch. Wiss. Ver. Santiago. I 590.

Deutsch. Zool. Ges. 7โ3.

Deut chlands Insecten. 759.

Devon Assoc. Adv. Sci. Lit. Art. 345.

Dienst Pestbestrijding. I 192.

Dietetic Hyg. Gaz. 1433.

Dijon. 444 .

Dinglers Polytechn. Journ. 572.

Direcc. Gen. Minas Argentina. I 549.

Dissert. Misc. Dieces rel. to Asia. 123. 
Documents U.S. Bur. Fisheries. I $525(b)$. Donovan's Nat's. Repository. 239.

Dorpat. 887.

Dove Marine Lab. I7.

Dresden. 640- 48 .

Drude's Ann. Physik. 701 (b).

Dublin. I 8-28.

Dublin Journ. Med. Sci. 19.

Dublin Med. Journ. 20.

Dublin Nat. Mus. Sci. Art. 21.

Dublin Nat. Hist. Soc. 23.

Dublin Univ. Zool. Assoc. 22.

Dundee. 29.

Durban. $98 \mathrm{I}-983$.

Durban Mus. 982 .

Durham, N. H. I369.

Dusseldorf. 649.

\section{E}

Early Proc. Amer. Phil. Soc. $1466(a)$.

Earthq. Invest. Comm. Japan. I 82.

East India Assoc. I24.

Easton. I37O, I37 I.

Eclogae Geol. Helvetiae. 943.

Ecole Franç. Extr.-Orient. I I 57.

Ecole Polytechn. Delft. 793.

Ecole Polytechn. Paris. 497.

Ecole Supér. Pharm. Paris. 498.

Econ. Geol. I 386.

Econ. Journ. 287.

Econ. Review. $\quad 125$.

Econ. Proc. Roy. Dublin Soc. $25(a)$.

Edinburgh. 3O-5I.

Edinb. Bot. Soc. 31,

Edinb. Geol. Soc. 3\%.

Edinb. Journ. Sci. 33.

Edinb. Med. Journ. 34.

Edinb. New Phil. Journ.

Edinb. Phıl. Journ. 36.

Edinb. Roy. Observ. 45.

Edinb. Roy. Phys. Soc. 46.

Edinb. Roy. Soc. 49.

Edward's Bot. Register. I 26.

Egypt Explor. Fund. 127.

Egypt Geol. Surv. 97 I.

Electr. Engineering. I 28.

Electr. Engineering Suppl.

129

Electr. Review. I 30.

Electr. World. I 434.

Electrician. I3I.

Electro-chem. Metallurg. Industry. 1435.

Electro-chemist, Metallurgist etc. 132.

Embryol. Inst. K.-K. Univ. Wien. 378 .

Endocrinology. I 395.

Eng. Journ. 1059.

Eng. Mag. I35, I437.

Eng. Mining Journ. 1436.

Eng. News. $143^{8}$.

Eng. Rev. I36.

Engineer. 133.

Engineering. I 34 .

Engler's Bot. Jahrbücher. 709.

English Mechanic \&o World Sci. 137.

Entomol. Annual. I4I.

Entomol. Ber. $802(a)$.
Entomol. Bull. Dep. Agr. Canada. I291 (a). Entomol. Bull. Dep. Agr. Mysore. Ior7 (b). Entomol. Circ. Dep. Ag . Canada. 129 I (b). Entomol. Fören. Stockholm. 930.

Entomol. Mag. 138.

Entomol. Mag. Kyoto. I I62.

Entomol. Mitt. $58 \mathrm{I}$.

Entomol. Month. Mag. I42.

Entomol. Nachr. 583.

Entomol. News. 1461.

Entomol. Record. I43.

Entomol. Research Comm. I72 (a).

Entomol. Rundschau. 769.

Entomol. Soc. Japan. II62.

Entomol. Soc. London. 139.

Entomol. Soc. N. S. Wales. I 266.

Entomol. Soc. Ontario. I $3 \mathrm{O} 2$.

Entomol. Tidskrift. 930.

Entomol. Ver. Berlin. 584.

Entomol. Ver. 'Iris.' 641.

Entomol. Ver. Stettin. 76I

Entomol. Zeitsch. 653.

Entomol. Zeitung. 76I (a).

Entomologist. $\quad$ I 40.

Ephemerides. $669(b)$.

Epidemiol. Soc. I44.

Epigraph. Carnatica. Io16 (c).

Epigraph. Indica. $1046(b)$.

Epigraph. Indo-Moslemica. $1046(c)$.

Epigraph. Zeylanica. I IOO $(b)$.

Erdbeben-Commiss. Wien. $372(e)$.

Ergebn. Anat. Entwicklungsgesch. 778.

Erlangen. 650 .

Ertesit. Magyar Tudomán. Akad. 355 (b).

Escola Minas Ouro Preto. I 575 .

Escola Super. Agric. etc. Pinheiro. I607.

Essex Inst. 1490.

Est. Agric. Centr. Mexico. I 569.

Est. Centr. Agron. Cuba. I 555.

Est. Exp. Agron. Cuba. I 555 .

Ethnogr. Surv. Ind. I 144.

Ethnol. Soc. London. 145.

Ethnol. Surv. Mysore. IOI8.

Ethnol. Surv. Philipp. Is. 1217.

Etudes Entomol. 888.

Evans' Analyt. Notes. 63.

Evolution Comm. Roy. Soc. London. 298 $(m)$.

Excurs, Reconn. Cochinchine Franc. $\quad 1165$.

Exper. Stat. Hawaii Sug. Plant. Assoc. I 208.

Exper. Stat. Rec. I 532.

Explor. Géol. Canada. 1293.

Exposé Electr. 499.

Extra Bull. Kolon. Mus. Haarlem. 799 (b). Extracts Narr. Reps. Surv. India. $1098(c)$. Extraits Proc.-Verb. Soc. Philom. Paris. 562. Ezhegh. Gheol. Mineral. Ross. 914.

\section{F.}

Facult. Sci. Marseille. $\$ 62$.

Facult. Sci. Rennes. 567.

Faraday Soc. 146 .

Farmers' Bull. Manila. I2I I $(a)$.

Farmers' Bull. Philipp. Is. I2II (a).

Farmers' Bull. U.S.A. I $529(d)$. 


\section{INDEX.}

Faun. Flor. G. von Neapel. 845 (a).

Faun. Insect. German. Initia. 755.

Faun. Russ. Pays Limitrophes. 905 (b).

Fed. Inst. Mining Eng. 336.

Fed. Malay States Museums. I I6o.

Fennia. 894.

Field Columbian Mus. Chicago. I 35 I .

Field Mus. Nat. Hist. Chicago. I35I.

Fig. Descr. Canad. Org. Remains. ${ }_{1293}($ c).

Final Rep. Geol. Minnesota. I402 (c).

Final Rep. State Gecl. N. Jersey. I 502 (c).

Finska Vetensk-Soc. 889.

Firenze see Florence.

Fisheries Bur. Madras. I 122.

Fisheries Dep. Australia. I 265.

Fisheries, Ireland, Sci. Invest. I8.

Fishery Board Scotland. 52.

Flagstaff. I 372 .

Flora. $760(d)$.

Floral Cab. Mag. Exotic Bot. I47.

Floral Mag. I48.

Flore Serres Jard. Europe. 500.

Florence. $82 \mathrm{I}-830$.

Földrajzi Közlemények. $35 \mathrm{I}$.

Földtani Közlöny. $352(a)$.

Folia Therapeutica. I49.

Folklore. I5o (d).

Folklore Journal. I5o (b).

Folklore Record. I $50(c)$.

Folklore Soc. I 50 :

Fondaz. Sci. Cagnola. 835 .

Forest Bull. I IO9 $(a, b)$.

Forest Leaflets. I IO9 (c).

Forest Pamphlets. I IOO (d).

Forest Research Inst. Dehra Dun. I 109.

Förhandl. Geol. Fören. Stockholm. 931.

Förhandl. Vidensk.-Selsk. Christiania. 885 (a).

Fornuännen. 934.

Fortschr. Miner. Krystallogr. Petrogr. 691,

Francofurtum see Frankfurt on the Main.

Frankfort, Ky. 1373 .

Frankfort on the Oder. 656 .

Frankfurt on the Main. $65 \mathrm{I}-655$.

Franklin Inst. 1467 .

Franklin Journ. $1467(a)$.

Freiberg. 657,658 .

Freiburg in Breisgau. 659.

Frelon. 44 .

Fribourg, 944.

Fruit Growers' Assoc. Ontario. 1303.

\section{G}

Gac. Mus. Nac. Caracas. I552.

Gaea Norvegica. $88 \mathrm{i}$

Ganeshkhind Bot. Gard. I I 40.

Gardener. I 5 I.

Gardener's Chronicle. I 52.

Gardener's Mag. I53, 1060.

Gardens' Bull. Straits Settlements. I I73 (b).

Garten-Blumenzeitung. 677.

Gartenflora. 585 .

Geelong. 1238 .

Geelong Field Nat's. Club. 1238 .

Geelong Naturalist. $123^{8}$.
Gegenbaur's Morphol. Jahrb. 728.

Gelehrte Anz. Mïnchen. $750(f)$.

Gemeinniitz. Bl. Beförd. Bergbaues etc. 652. Gen. Rep. Geol. Surv. India. I06I (b).

Gen. Rep. Oper. Gt. Trig. Surv. Ind. $1098(d)$.

Gen. Rep. Oper. Marine Surv. Ind. $1084(b)$.

Gen. Rep. Oper. Roy. Survey Dep. Siam. I I 54 .

Gen. Rep. Oper. Surv. India. $\operatorname{Iog} 8(e)$.

Gen. Rep. Rev. Surv. Oper. Ind. I098 (f).

Gen. Rep. Topogr. Surv. Oper. Ind. Iog $8(g)$.

Geneva. 945-953.

Geneva, N.Y. I 374 .

Genf see Geneva.

Genoa. 83 I-833.

Geodät. Arb. Norway. 882 (a).

Geodesy. $\quad 1528(a)$.

Geogn.-Paläont. Beitr. 748.

Geogr. Abhandl. 599.

Geogr. Anstalt Gotha. 663.

Geogr. Anz. 66r.

Geogr. Fören. Finland. 89I.

Geogr. Ges. Lübeck. 743.

Geogr. Ges. München. 749.

Geogr. Inst. Berlin. 599.

Geogr. Jahrb. 662.

Geogr. Journal. $288(a)$.

Geogr. Mag. 154.

Geogr. Rev. I420 (b).

Geogr. Soc. Austral., Queensl. Branch. 1235.

Geogr. Soc. Bombay. 1032.

Géographie. $553(\mathrm{c})$.

Geol. Centralblatt. 714.

Geol. Comm. C. of Good Hope. 973.

Geol. Dept. Mysore. Iorg.

Geol. Explor. Fortieth Parallel. I5 I5.

Geol. Fören. Stockholm. 931.

Geol. Ges. Wien. 370.

Geol. Inst. Upsala. $938(b)$.

Geol. Komitet S.-Peterburgh. 907

Geol. Knmm. Finland. 892 .

Geol. Kommiss. Schweiz. 958.

Geol. Lit. Geol. Soc. London. I $57(d)$.

Geol. Mag. 155.

Geol.-mijubouwk. Genoot. Ned, etc. 800.

Geol. Mining Soc. Manchester. 330.

Geol. Minnesota. $1402(c)$.

Geol. Mitt. Budapest. $352(a)$.

Geol. Nat. Hist. Surv. Canada. I 293.

Geol. Nat. Hist. Surv. Chicago. I 347.

Geol. Nat. Hist. Surv. Minnesota. 1402.

Geol. Nat. Hist. Surv. Wisconsin. 1396.

Geol. Palaeont. Abhandl. Jena. 694.

Geol. Palaeont. Illinois. I $505(b)$.

Geol. Publ. Univ. California. $1325(f)$.

Geol. Record. I 56.

Geol. Reichs-Mus. Leiden. 806.

Geol. Rep. Tennessee. I408.

Geol. Soc. America. 1439.

Geol. Soc. Australasia. I250.

Geol. Soc. Dublin. $27(a)$.

Geol. Soc. Edinburgh. 37.

Geol. Soc. Glasgow. 53.

Geol. Soc. Liverpool. 64 .

Geol. Soc. London. 157 .

Geol. Soc. Manchester. 330. 
Geol. Soc. Pennsylvania. I468

Geol. Soc. S. Africa. 985.

Geol. Surv. Canada. I 293.

Geol. Surv. Egypt. 971.

Geol. Surv. Gt. Britain. I 58 .

Geol. Surv. Illinois. I 505 .

Geol. Surv. India. I06I.

Geol. Surv. Indiana. I 378.

Geol. Surv. Japan. I 183.

Geol. Surv. Kansas. I39r.

Geol. Surv. Kentucky. I 373.

Geol. Surv. Iowa. I 367.

Geol. Surv. Minnesota. I 404, r.405.

Geol. Surv. Missouri. I 383 .

Geol. Surv. Natal Zululand. 989.

Geol. Surv. New Jersey. I $5 \mathrm{O} 2$.

Geol. Surv. New South Wales. I 267.

Geol. Surv. New Zealand. 1278 .

Geol. Surv. Ohio. I36I.

Geol. Surv. Oil lands Japan. I I $83(c)$.

Geol. Surv. Pennsylvania. I 376.

Geol. Surv. Queensland. 1233 .

Geol. Surv. S. Africa. 995.

Geol. Surv. Tasmania. I 24 I.

Geol. Surv. Tennessee. I 408.

Geol. Surv. Trausvaal. 997.

Geol. Surv. Vermont. I 339.

Geol. Surv. Victoria. I25 I.

Geol. Surv. Virginia. I 345.

Geol. Surv. W. Australia. I 258 .

Seol. Surv. Wisconsin. I 396.

Geologist. I 59, I60.

Geologist's Assoc. London. I6I.

Georgetown, I 554 .

Georgia Exp. Stat. I3I3.

Geotekn. Meddel. Geol. Komm. Finland. 892 (b).

Germar's Mag. Entomol. 67I.

Germar's Zeitsch. Entomol. 734.

Ges. Anthrop. Ethnol. Urgesch. Berlin. 586.

Ges. Beförd. gesammt. Naturw. Marburg. 745.

Ges. Beförd. Naturwiss. $659(b)$.

Ges. Bot. Zool. Dresden. 640.

Ges. Erdk. Berlin. 587 .

Ges. Erdk. Leipzig. 71 5.

Ges. Freunde Naturwiss. Wien. 37 I.

Ges. gesammte Mineral. St. Petersburg. 9Io.

Ges. 'Iris' Dresden. 64I.

Ges. Naturf. Freunde Berlin. 588.

Ghent. 413 .

Giessen. 660.

Gilbert's Ann. Physik. 7OI (a).

Giorn. Bot. Ital. 821.

Giorn. I.R. Ist. Lomb. Sci. etc. $837(a)$.

Giorn. Sci. Nat. Econom. 854.

Grorn. Soc. Asiat. Ital. 827.

Glacialists' Assoc. I62.

Glacialists' Mag. I62.

Glasgow. 52-56.

Glasgow Geol. Soc. 53.

Glasgow Med. Journ. 54.

Glasgow Phil. Soc. 57.

Glasgow Roy. Phil. Suc. 57.

Gleanings in Science. 1062.

Gloucester. $\quad 58$.
Goa. I I IO.

Gordon Memor. College Khartoum. 986.

Gotha. $661-663$.

Göttingen 664-667.

Göttingische Gelehrte Anz. 666 (d).

Gouv. Gén. Madagascar. 964.

Gov. Bengal. I063.

Gov. Bombay. 1033 .

Gov. India. I 50.

Gov. Madras. II 23.

Gov. Mus. Madras. โI 24.

Gov. N.W. Prov. India. 1007.

Gov. Punjab. IIIf.

Grahamstown. 984 .

Grant College Med. Soc. I034.

Gray's Spicilegia Zoologica. 3I5.

Gray's Zool. Misc. 325.

Graz. $359,360$.

Great Trig. Surv. India. $\operatorname{Ing} 8(b, d, k)$.

Greenwich. 59.

Grenoble. 445.

Grevillea. 163.

Gröningen. 794 .

Guide to Growers, Victoria. $\mathrm{I} 248(a)$.

Guy's Hospital. I64.

Guy's Hospital Gaz. I64.

Gypsy Lore Soc. 38 .

\section{$\mathrm{H}$}

Haarlem. 795-799.

Habana see Havana.

Hague. $800-802$.

Haidinger's Naturwiss. Abhandl.

Half-yearly Abs. Med. Sci. ${ }_{16}$.

37 I $(b)$

Halifax, N.S. 1284.

Halle. 668-674.

Hallisch. Naturf. Ges. $672(b)$.

Hamburg. 675-683.

Hamburgisch. Wiss. Anst. $675(b), 678$.

Hamilton. I 286.

Hamilton (Sci.) Assoc. I 286.

Handl. K. Svensk. Vetensk.-Akad. $932(\mathrm{~g})$.

Hanoi. I I 56-I I $5^{8}$.

Hanover. $684,685$.

Hanover, Ind. I 375 .

Hanstein's Bot. Abhandl. 6 I9.

Harrisburg. I 376.

Harvard Coll. I 34I, I 342.

Hatch Exp. Stat. Mass. I3I2.

Havana. I 555-I 559.

Havnia see Copenhagen.

Havre. $446,447$.

Hawaii Agric. Exp. Stat. I 206.

Hawaii Forest. Agricult. $\mathrm{I}_{205}$ (a).

Hawaii Planters Monthly. I 207.

Hawaii Sugar Planters' Assoc. I207.

Haye see Hague.

Heart. 166.

Hedwigia. 642.

Heidelburg. 686.

Helios. 656 .

Helsingfors. 888-895.

Henry Phipps Inst. Study Tuberculosis. I 469 .

Herbier Boissier. 946 . 
Herbier Paris. 526.

Highland Agric. Soc. Scotland. 39.

Highland Soc. Scotland. 39.

Hist. Acad. Roy. Inscript. Belles-Lettres. 468.

Hist. Mem. Acad. Real Sci. Lisboa. 918 (a).

Hobart. I239-I 244 .

Hollandsche Maatsch. Wetensch. 796.

Honolulu I 204-I 208 .

Hooker's Icones Plantarm. 167.

Hooker's Journ. Bot. I68.

Hope Dep. Zool. Oxford. 342.

Hope Reports. 342.

Hovae Soc. Ent. Ross. 9 I I $(a)$.

Horticult. Soc. London. 289.

Hortus Petropolitanus. 908.

Hospital. 169.

Humboldt. 770.

Hyderabad. IIII, III2.

Hyderabad Archaeol. Dep. II II.

Hyderabad Archaeol. Series. II II (b).

Hyderabad Archaeol. Soc. III2.

\section{I}

Ibis. 103.

Icones Bogorienses. I 203 (c).

Icones Plantarum. I70.

Illiger's Mag. Insektenk. 629.

Illinois Agric. Exp. Stat. I 509.

Illinois Biol. Mon. I 508 .

Illinois State Geol. Surv. I 505.

Illinois State Lab. Nat. Hist. 1506.

Jllinois State Mus. Nat. Hist. 1496.

Illinois Univ. I 508, I 509.

Illustr. Cat. Mus. Harvard. I34I (c).

Illustr. Horticole. 413.

Iliustr. Med. News. $17 \mathrm{I}$.

Illustr. Official Journ. Patent Office. $254($ a).

Illustr. Zool. R I.M. Investigator. IO80(a).

Imp. Acad. Tokyo. I 180 .

Imp. Akad. Nauk S.-Peterburgh. 900-905.

Imp. Bact. Lab. Muktesar. I 33 .

Imp. Bur. Entomol. I72.

Imp. Bur. Fisheries Tokyo. I I8I.

Imp Customs Dep. India. IO83.

Imp. Dep. Agric. W. Indies. I 547.

Inp. Geol. Surv. Japan. II 83 .

Imp. Inst. 173 .

Imp. Inst. Journ. $\quad$ I73 $(d)$.

Imp. Earthq. Invest. Comm. Japan. II82.

Imp. Mus. Japan. I 184.

Imp Mus. Tokyo. II 84 .

Imp. Nikitsk. Sad. 915.

Imp. R. Ist. Regno Lomb.-Veneto. 836.

Imp. Russ. Geogr. Obshch. 909.

Imp S. Peterburgh. Bot. Sad. 908.

Imp. S.-Peterburgh. Mineral. Obshch. 9Io.

Imp S.-Peterburgh. Obshch. Estestvo sp. 912.

Imp. Univ. J apan. I I 85.

Imp. Univ. Kyoto. I 163 .

Ind. Agriculturist. 1067.

Ind. Ann. Mag. Nat. Sci. 1035.

Ind Ann. Med. Sci. Io69.

Ind. Antiquary. I036.

Ind. Assoc. Cult. Sci 1070.
Ind. East. Eng. 1068.

Ind. Economist. I07 I.

Ind. Engineer. I072.

Ind. Engineering. 1073.

Ind. Engineers' Assoc. 1074.

Ind. Forest Rec. I I $\log (f)$.

Ind. Forest Mem. I IO9 (e).

Ind. Forester. 1008.

Ind. Gardening. I075.

Ind. Inst. Sci. 1605.

Ind. Journ. Economics. IOI 5.

Ind. Journ. Med. Phys. Sci. ${ }^{1064 .}$

Ind. Journ. Med. Sci. I065.

Ind. Journ. Med Research. IO79 (a).

Ind. Lancet. 1076.

Ind. Math. Soc. I 125.

Ind. Med. Gaz. I077.

Ind. Med. Journ. I009.

Ind. Med. Rec. Io78.

Ind. Med. Service. IO79.

Ind. Meteor. Mem. II5I (b).

Ind. Mus. Io80.

Ind. Mus. Notes. Ioso (b).

Ind. Notes Queries. Ioro.

Ind. Planting Gardening. I08I.

Ind. Rev. Journ. For. Sci. Arts. 1066.

Ind. Rubher World. I44I.

Ind. Tea Assoc. I082.

Ind. Telegraphist. IOI I.

Ind. Textile Journ. IO37.

Ind. Weather Rev. II I I (c).

Index Medicus. I440.

Indiana Acad. Sci. I 379.

Indiana Geol. Surv. 1378.

Indianapolis. $\quad 1377-1379$.

Industries and Iron. I75.

Inform. Doc. Comerc. Agr. México. 1568 (a).

Inland Customs Dep. India. I083.

Inland Rev. Dep. Canada. I 295.

Insect Life. I53 I (c).

Insecta. 567.

Insecta Transvaal. I76.

Insecten-Belustigung. 756.

Insekten-Borse. 716

Inspect. Landbouw Suriname. 1577.

Inst. Arts Sci. Brooklyn. 1337.

Inst. Bot. Buitenzorg. I2OI.

Inst. Bot.-Géol. Colon. Marseille. 463.

Inst. Bot. Liége. 419.

Inst. Civil Engineers. I 78 .

Inst. Colonial Marseille. 463 .

Inst. Egyptien. 968.

Inst. Electr. Engineers. I 79.

Inst. Fis.-Geogr. Nac. Costa Rica. I 585.

Inst. France. 5OI.

Inst. Géol Louvain. 420.

Inst. Geol. México. 1567.

Inst. Geol. României. 391 .

Inst. Jamaica. I 562 .

Inst. Plantenziekt. Cultures. I 200.

Inst. Mechan. Engineers. 180.

Inst. Météor. Norvége. $883(b)$

Inst. Med. Research Fed. Malay States. I 74.

Inst. Mining Eing. 336.

Inst. Mining Metallurgy. I8I.

Inst. Nat. Genèvois. 947. 
Inst. Océanogr. Monaco. 466.

Inst. Océanogr. Paris. 502.

Inst. Oswaldo Cruz. 1580.

Inst. Pasteur. 503.

Inst. Patent Agents. I 77 .

Inst. Petrol. Technologists. 182.

Inst. Sci. Buitenzorg. 1202.

Inst. Suisse Anthrop. Générale. 948.

Intellectual Observer. 183.

Interméd. Mathématiciens. 504.

Internat. Arch. Ethnogr. 803.

Internat. Cat. Sci. Lit. I 597.

Internat. Congr. Entomol. I 595.

Internat. Engin. Congress. 184.

Internat. Entomol. Ver. 653.

Internat. Gletschercommission. 6I2.

Internat. Inst. Agric. 862.

Internat. Koumm. Wiss. Luftschiffahrt. 763 .

Internat. Mitt. Bodenkunde. 589.

Internat. Monats. Anat. Physiol. 717.

Internat. Monats. Anat. Histol. 717.

Internat. Rev. Hydrobiol. Hydrogr. 7:8.

Internat. Sugar Journ. 185.

Iowa Agric. Exp. Stat. 13II.

Inwa Geol. Surv. I 367 .

Iowa State Coll. Agr. Mech. Arts. I3II.

Iris. $64 \mathrm{r}(b)$.

Iron. 186.

Iron Age. I 442.

Iron Coal Trades Rev. $\quad 187$

Iron Steel Inst. 188.

Iron Steel Mag. I33I.

Isis (Encyclopäd. Zeit.). 719.

Ist. Agric. Colon. Ital. 822.

Ist. Bot. Pisa. 857.

Ist. Geol. Padova. 848 ,

Ithaca. I380-1 382.

İvyest. Imp. Akad. Nauk. $900(c)$.

Izvvest. Imp. Russ. Geogr. Obshch. 909.

Izvyest. Vostochn. Inst. IOO3.

\section{$\mathrm{J}$}

Jaarb. Dep. Landb. Ned.-Ind. II $99(b)$.

Jaarb. K. Akad. Wetensch. Amsterdam. 789 (a).

Jaarb. Mijnwesen Ned. Oost-Ind. 784.

Jaarversl. Proefst. Java-Suikerindust. 1226 (a).

Jacquin's Misc. Austriaca. 383.

Jahrb. Astron. Geophys. 720.

Jahrb. Berg-Huttenwesen K. Sachsen. 658.

Jahrb. Chem. 627.

Jahrh. Deut. Malakozo l. Ges. 65I (a).

Jahrb. Fortschr. Math. 590.

Jahrb. Gewächskunde. 591.

Jahrb. Hamburgisch. Wiss. Anst. $675(b)$, 678.

Jahrb. Kais.-Kön. Cent. Anst. Met. Erdmagn. 373.

Jahrb. Kais.-Kön. Geol. Reichsanst. Wien. $375(b)$.

Jahrb. Kön. Bayer. Akad. Wiss. $750(\mathrm{~g})$.

Jahrb. Kön. Bot. Garten Berlin. 602 (a).

Jahrb. Kön. Preuss. Geol. Landesanst. $601(e)$.

Jahrb. Miner. Geogn. Geol. etc. 686.
Jahrb. Mus. Völkerk. Leipzig. $733($ a). Jahrb. Nassauisch. Ver. Naturk. $779(a)$. Jahrb. Norweg. Meteor. Inst. $883(a)$. Jahrb. Oesterr. Alpen-ver. 384. Jahrb. Radioact. Electronik. $72 \mathrm{I}$. Jahrb. Schweiz. Alpenclub. 94I. Jahrb. Städt. Mus. Völkerk. Leipzig. 733 (a). Jahrb. Ver. Naturk. Herzogth. Nassau. 779 (b).

Jahresb. Bot. Staatsinst. Hamburg. 675 (a). Jahresb. Deut. Math. Ver. 7 II.

Jahresb. Fortschr. Anat. Physiol. 722.

Jahresb. Fortschr. Anat. Entwicklungsges. 692.

Jahresb. Fortschr. Bot. $932(h)$.

Jahresb. Fortschr. Chemie etc. 628.

Jahresb. Fortschr. Gesamtgeb. Agr.-Chem. 592.

Jahresb. Fortschr. Lehre Path. Mikroorg. 723. Jahresb. Geb. Pflanzenkr. 594.

Jahresb. Kōn. Ungar. Geol. Reichsanst. 352 (b).

Jahresb. Leist. Fortschr. Med. 593.

Jahresb. Naturhist. Ver. 'Lotos.' $362(b)$.

Jahresb. Naturw. Ver. Sachs. Thüring. 673 $(b)$.

Jahresb. Newer. Liest. Geb. Pflanzenkr. 594. Jahresb. Pharm. $580(b)$.

Jahresb. Schles. Ges. Vaterl. Cultur. $624(c)$. Jahresb. Ungar. Geol. Ges. $352(b)$.

Jahresb. Ver. Erdk. Dresden. 747.

Jahresb. Ver. Naturw. Braunschweig. 63I.

Jahresh. Ver. Schles. Insektenk. 625 (a).

Jahresh. Ver. Vaterl. Naturk. Württ. 773.

Jamaica Agric. Soc. I 563 .

Jard. Bot. Bruxelles. 402.

Jard. Bot. Buitenzorg. I 203.

Jard. Bot. Genève. 949.

Jard. Bot. Tiflis. 1002 .

Jard. Colon. Franç. 505.

Jefferson City. 1383,1384 .

Jena. 687-696.

Jenaisch. Denkschr. $693($ a).

Jenaisch. Zeitsch. (Med.) Naturwiss. 693 (b).

Jersey. 60.

Johannesburg. 985 .

John Hopkins Hosp. I 3 I9.

John Hopkins Univ. I 320.

John Hopkins Univ. Circ. $1320($ c).

John Ryland Library. 33I.

Jones' Dissertations etc. 123.

Journ. Acad. Nat. Sci. Philadelphia. I459(a).

Journ. Acad. Sci. Washington. I $510(a)$.

Journ. Agric. 189.

Journ. Agric. Hort. Soc. India. $1044(a, b)$.

Journ. Agric. Research. I529 (e).

Journ. Agric. Sci. 9.

Journ. Agric. Tropicale. 506.

Journ. Amer. Chem. Soc. I4I7 (b).

Journ. Amer. Leathe Chem. Assoc. 1370.

Journ. Amer. Med. Assoc. I 1350.

Journ. Amer. Orient. Soc. 1328.

Journ. Analyt. Chem. I37I.

Journ. Anat. Physiol, 190.

Journ. Anat. Physiol. norm. path. Homme Anim. 510. 
Journ. Anthropol. 8I (b).

Journ. Anthropol. Inst. Gt. Brit. $280(a)$.

Journ. Anthropol. Soc. Bambay. I028.

Journ. Archaeol. Soc. Hyderabad. III2.

Journ. Asiat. Soc. Bengal. $1048(d, e)$.

Journ. Asiatique. $545(a)$.

Journ. Astron. Soc. Ind. 1049.

Journ. Bengal Econ. Assoc. I050.

Journ. Bihar Orissa Research Soc. I025.

Journ. Biol. Chem. I322.

Journ. Board Agriculture. 85.

Journ. Board Agr. Brit. Guiana. I 554.

Journ. Board Trade. 86.

Journ Bombay Anthropol. Soc. I 28 .

Journ. Bombay Nat. Hist. Soc. I040.

Journ. Botanik. 665.

Journ. Botanique. 507.

Journ. Bot. Appl. Agric. Pharm. etc. 508.

Journ. Bot. Néerland. 785 .

Journ. Botany. I91, I92.

Journ. Brit. Astronom. Assoc. 97.

Journ. Brit. Med. Ass., Victorian Branch. 1247.

Journ. Burma Research Soc. II 42 .

Journ. Canad. Mining Inst. I288 (b).

Journ. Chem. Soc. Iog (b).

Journ. Cincinnati Soc. Nat. Hist. $135^{8}$.

Journ. College Agric. Tokyc. I I $85(b)$.

Journ. College Eng. Tokyo. I I $85(c)$.

Journ. College Sci. Tokyo. II $55(d)$.

Journ. Comp. Med. Surgery. 1470.

Journ. Conchology. I20.

Journ. Conchyliol. 509.

Journ. Dep. Agric. S. Austral. 1227 (a).

Journ. Dep. Agric. Victoria. I $248(b)$.

Journ. Dep. Agric. W. Austral. 1257.

Journ. Dublin Feol. Soc. $27(a)$.

Journ. East India Assoc. I24.

Journ. Ecole Polytechn. Paris. 497.

Joum. Ecology. 98.

Journ. Econ. Biol. 193.

Journ. Econ. Bot. I360 (a).

Journ. Economistes. 5 I4.

Journ. Egypt. Orient. Soc. Manchester. I600. Journ. Ent. I94, 930.

Journ. Ent. Pomona Coll. I $360(b)$.

Journ. Ent. Zool. I $360(b)$.

Journ. Ethnol. Soc. I45 ( $a_{\text {; }}$.

Journ. Exp. Med. I443.

Journ. Exp. Zool. I47I.

Journ. Fed. Malay States Mus. I 160.

Journ. Franklin Inst. I467 (b).

Journ. Genetics. Io.

Journ. Genl. I 352 .

Journ. Geol. Soc. Dublin. 27 (a).

Journ. Gypsy Lore Soc. 38.

Journ. Hamilton (Sci.) Assoc. 1286.

Journ. Heredity. I5 12.

Journ. Horticult. Soc. London. 289 (a).

Journ. Hyderabad Arch. Soc. III2.

Journ. Hygiene. II.

Journ. Imp. Dep. Agr. W. Indies. 1547 (c).

Journ. Imp. Inst. $\quad$ I $73($ d).

Journ. Ind. Archipel. E. Asia. I175.

Journ. Ind. Inst. Sci. 1605.

Journ. Ind. Math. Soc. I 125.
Journ. Industr. Eng. Chemistry. I444. Journ. Infect. Diseases. I353.

Journ. Inst. Electr. Engineers. 179.

Journ. Inst. Jamaica. I 562.

Journ. Inst. Petrol. Technologists. I 82.

Journ. Iron Steel Inst. $188(b)$.

Journ. Jamaica Agric. Soc. I 563 .

Journ. Linn. Soc. London. $203(a, b)$.

Journ. Lit. Sci. Madras. I 127 (a).

Journ. London School Trop. Med. 304.

Journ. Manchester Egypt. Orient. Soc. 1600.

Journ. Marine Biol. Assoc. 346.

Journ. Marine Zool. Microsc. 60.

Journ. Math. Pures Appliq. $5 \mathrm{II}$.

Journ. Med. Graduates Coll. 220.

Journ. Mental Sci. 225.

Journ. Microsc. Soc. London. $294($ a).

Journ. Mineralog. Soc. London. 228.

Journ. Morphol. 1472.

Journ. Mus. Goddefroy. 679.

Journ. Nat. Hist. Soc. Siam. I 53.

Journ. New York Bot. Gard. I430(b).

Journ. Numism. Soc. London. 296.

Journ. Ornithologie. 634.

Journ. Parasitology. 1507.

Journ. Path. Bact. 40.

Journ. Phys, Chemistry. 1445.

Journ. Physiol. 195.

Journ. Physique. 512 .

Journ. Polyn. Soc. I282.

Journ. Pract. Diet. Bact. Ther. 196.

Journ. Proc. Agr. Hort. Soc. India. 1044 (b).

Journ. Proc. Asiat. Soc. Bengal. $1048(e)$.

Journ. Proc. Hamilton (Sci.) Assoc. 1286.

Journ. Proc. Roy. Snc. N.S.W. $1273($ a).

Journ. Proc. (Sci.) Assoc. Hamilton. I 286.

Journ. Punjab Hist. Soc. III 5.

Journ. Quekett .Microsc. Club. 275.

Journ. Rein. Angew. Math. 595.

Journ. Roy. Agric. Soc. England. 279.

Journ. Roy. Anthropol. Inst. 280 (a).

Journ. Roy. Anthropol. Soc. Australasia. 1272 .

Journ. Roy. Army Med. Corps. 281.

Journ. Roy. Asiat. Soc. Bombay. 1043.

Journ. Roy. Asiat. Soc. Ceylon. I $106(a)$.

Journ. Roy. Asiat. Soc. China. II72.

Journ. Roy. Asiat. Soc. Gt. Brit. $282($ a).

Journ. Roy. Asiat. Soc. N. China. II 72.

Journ. Roy. Asiat. Soc. Siraits. I I77 (a).

Journ. Roy. Colonial Inst. $286(a)$.

Journ. Roy. Dublin Soc. $25(b)$.

Journ. Roy. Geogr. Soc. Ireland. 26.

Journ. Roy. Geogr. Soc. London. $288(b)$.

Journ. Roy. Geol. Soc. Ireland. $27(b)$.

Journ. Roy. Horticult. Soc. London 289 (a).

Journ. Roy. Inst. Brit. Archit. $290(a, b)$.

Journ. Roy. Inst. Gt. Brit. 29 I (a).

Journ. Roy. Microsc. Soc. London. 294 (a).

Journ. Roy. Naval Med. Service. 295.

Journ. Roy. Numism. Soc. London. 296.

Journ. Rov. Sanitary Inst. 297.

Journ. Roy. Scott. Soc. Arts. 48 (a).

Journ. Roy. Soc. Arts London. 299 (a).

Journ. Roy. Soc. N.S. Wales. $1273(a)$.

Journ. Roy. Statist. Soc. London. 30I. 
Journ. Sarawak Mus. I 209.

Journ. Savans. 515.

Journ. School Trop. Med. London. 304.

Journ. Sci. Arts. 291 (b).

Journ. Sci. Math. Phys. Nat. Lisboa. $918(b)$.

Journ. Siam Soc. II 55.

Journ. Social Sci. 197.

Journ. Soc. Arts London. $299(a)$.

Journ. Soc. Chem. Indust. London.

Journ. Soc. Finno-Ougr. 895 (a).

Journ. Soc. Nat. Hist. Cincinnati.

Journ. Soc. Telegraph Eng. 313.

Journ. South Ind. Assoc. 1131.

Journ. Statist. Soc. London. 301.

Journ. Télégr. 940.

Journ. Travel Nat. Hist. 198.

Journ. Trenton Nat. Hist. Soc. I 503.

Journ. Trop. Med. Hyg. 199.

Journ. Trop. Vet. Sci. $\operatorname{I0} 55(a)$.

Journ. Unit. Prov. Hist. Soc. IOI4.

Journ. Vacc. Therapy. 200.

Journ. Washington Acad. Sci. I 5 Io $(a)$.

Journ. Zool. 513.

Journ. Zool. Research. 201.

Juggarow Observ. II 52 .

Junta Ciènc. Nat. Barcelona. 917.

Jurjew see Dorpat.

Just's Bot. Jahresb. 596.

Jusius Licbig's Ann. Chem. (Pharm). 726 $(c, d)$.

Justus Perthes' Geogr. Anst. 663.

\section{K}

Kais. Akad. Wiss. Wien. 372.

Kais. Gouv. Deutsch-Ost-Afrika. 980.

Kais.-Kön. Cent.-Anst. Meteor. Erdmagn. Wien. 373.

Kais.-Köı. Geogr. Ges. Wien. 374.

Kais.-Kön. Geol. Reichsanst. Wien. 375.

Kais.-Kön. Naturhist. Hofmus. Wien. 377

Kais.-Kön. Oesterreich. Ges. Meteor. 376.

Kais.-Kön. Sternwarte Prag. 363 .

Kais.-Kön. Zool.-bot. Ges. Wien. 381 .

Kais. Leop.-Car. Deut. Akad. Naturf. 669.

Kais. Marine. 597.

Kais. Mineral. Ges. St. Petersburg. 910.

Kais.-Wilhelms-Univ. Strassburg. 764 .

Kala Azar Bull. $309(b)$.

Kali. 670.

Kansas Geol. Surv. I 391 .

Kansas Univ. I 390.

Kansas Univ. Quart. I $390(a)$.

Kansas Univ. Sci. Bull. I $390(b)$.

Kartblad Sver. Geol. Undersökning. 935 (c).

Kassel see Cassel.

Kaukasisch. Mus. 1001 .

Kentucky Agr. Exp. Stat. I 392.

Kentucky Geol. Surv. 1373.

Kew Bulletin. $284(a, b)$.

Kew Gardens. 284.

Khartoum. 986.

Khedivial Agric. Soc. 969.

Kiel. 697-699.

Kiev. 896.

King Inst. Prev, Med. I 126.

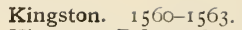

Kingston, R.I. I 385 .

Kioto see Kyoto.

Kjöbenhavn see Copenhagen.

Kodaikanal Observ. I I 30 .

Kolloid-Chem. Beihefte. 643.

Kolloid-Zeitsch. $643(b)$.

Koloniaal Inst. Amsterdam. 786.

Koloniaal Mus. Haarlem. 799.

Kolonial Wirtsch. Kom. 598.

Komm. Geol. Landesuntersuch. Els.-Lothr. 765 .

Komm. Wiss. Untersuch. Deut. Meere. 698.

Kön. Akad. Wiss. Berlin. 600.

Kön. Bayer. Akad. Wiss. 750.

Kön. Bayer. Bot. Ges. Regensburg. 760.

Kön. Bot. Garten Berlin. 602.

Kön. Ethnogr. Mus. Dresden. 645.

Kön. Fried.-Wilh.-Univ, Berlin. 599.

Kön. Ges. Wiss. Göttingen. 666.

Kön. Jul.-Maximil.-Univ. Würzburg. 781 .

Kön. Min.-Geol. Prähist. Mus. Dresden. 644 .

Kön. Mus. Völkerk. Berlin. 603.

Kön. Phys.-Oekon. Ges. Konigsberg. 700.

Kön. Preuss. Akad. Wiss. 600.

Kön. Preuss. Geol. Landesanst. Gor.

Kön. Sächs. Ges. Wiss. 724 .

Kön. Schwedisch. Akad. Wiss. $932(h)$.

Kön Sternwarte München. 75I.

Kön. Ungar. Geol. Reichsanst. 352.

Kön. Ungar. Naturwiss. Ges. 356.

Kön. Zool. Anthr.-Ethn. Mus. Dresden. 645.

Kön. Zool. Mus. Dresden. 645.

Kongel. Dansk Vidensk. Selsk. 424.

Kongel. Nordisk Oldskr.-Selsk. 425.

Kongel. Norske Vidensk.-Selskab. 886.

Kongel. Norske Vidensk.-Selsk. Skrift. 886.

Kongl. Humanist. Vet.-Samfund. 936.

Kongl. Svensk. Vetensk.-Akad. 932.

Kongl. Univ. Upsala. 938.

Kongl. Vetensk.-Soc. Upsala. 937.

Kongl. Vitterh. Hist. Antikv. A kad. 934.

Konigsberg. 700 .

Koniuk. Akad. Wet. Amsterdam. 789.

Konink. Inst. Taal-I,and-Volkenk. Ned.-Ind. 801 .

Konink. Magn. Meteor. Obs. Batavia. I I93.

Konink. Natuurk. Ver. Ned.-Ind. II94.

Konink. Ned. Inst. Wet. etc. 787.

Konink. Zool. Gen. 'Natura Artis Magistra.' 788 .

Korte Ber. Landbouw etc. II 99 (c).

Kosmos. 725.

Kristiania see Christiania.

Kuala Lumpur. II 59, I I60.

Kuching. 1209.

Kuraschiki. I 6 I.

Kyoto. I 162, I 163 .

L

Lab. Etudes Soie. 454.

Lab. Géol. Grenoble. 445 .

Lab. Zool. Gen. Agrar. Portici. $860(b)$.

Lab. Zool. Pnysiol. Marit. Concarneau. 443.

Lahore. III3-III5. 
Lancash. Sea-Fisheries I,ab. 65 .

Lancaster. I 386.

Lancet. $2 \mathrm{O} 2$

Lands Surv. Dep. N. Zealand. I 279.

Lansing. 1387,1388 .

La Plata. I 564.

Las Cruces. I 389 .

Laurence. I 390, I 39 I.

Lausanne. 955 .

Lavori R. Orto Bot. Fiorent. 824.

Lavoura. I 584 .

Leach's Zool. Misc. 326.

Leaflets Philippine Botany. 1218.

Lefnadst. K. Svensk. Vet.-Akad. 932 (i).

Leiden see Leyden.

Leipzig. $70 \mathrm{I}-742$.

Leland Stanford Junior Univ. I497.

Le Mans. 448.

Leopoldina. 659 (c).

Leyden. 803-809.

Leyden Mus. 807.

Lexington. I 392 .

Lick Observ. I $325(b)$.

Lick Observ. Bull. I $325(b)$.

Liebig's Ann. Chem. (Pharm.). $726(c, d)$.

Liége. 4I5-4I9.

Lille. 449,450 .

Lima. 1565 .

Lincoln, Neb. I 393, I 394 .

Linn. Soc. London. 203.

Linn. Soc. N. S. Wales. $\quad 1268$.

Linnaea. 604.

Linnea Entomol. 76 I (b).

Lipsia see Leipzig.

Lisbon. 918-921.

Lit.-Blätter Reine Angew. Bot. $760(e)$.

Lit. Gazette etc. 204.

Lit. Hist. Soc, Quebec. I 298.

Lit. Phil. Soc. Liverpool. 66.

Lit. Phil. Soc. Manchester. 332.

Lit, Sci. Inst. Kilkenny. 22.

Lit. Soc. Bombay. 1038.

Lit. Soc. Madras. I I 27.

Liverpool. 62-69.

L'pool Biol. Soc. 62 .

L'pool Geol. Soc. 64.

L'pool Lit. Phil. Soc. 66.

L'pool Marine Biol. Committee. 67.

L'pool Mar. Biol. Comm. Mem. $67(b)$.

L'pool School Trop. Med. 68.

Lloyd Libr. Bot. Pharm. etc. 1357.

London. 70-328.

London, Ont. T 287 .

London Bot. Soc. 9I.

London Edinb. Dublin Phil. Mag. 205.

London Edinb. Phil. Mag. 205.

London Geol. Journ. 206.

London Journ. Bot. 207.

London School Trop. Med. 304.

London Soc. Antiquaries. 3 II.

Los Angeles. I 395 .

Lotos. $362(c-e)$.

Loudon's Magazine. 208.

Louvain. 420.

Lowell Observ. I 372 .

Lubeck. 743 .
Lugdunum Batavorum see Leyden.

Lund. 928 .

Lyceum Nat. Hist. New York. I446.

Lyons. $45 \mathrm{I}-46 \mathrm{I}$.

\section{M}

Macao. I I64.

Machinery. I 147.

Madison. I 396-I 398.

Madras. II I 8-I I 3 I.

Madras Agri-Hort. Soc. II I6.

Madras Journ. Lit. Sci. II27 (b).

Madras Lit. Soc. I I 27

Madras Month. Journ. Med. Sci. I 128.

Madras Mus. I 124.

Madras Observ. I I 30 .

Madras Quart. Journ. Med. Sci. II 29.

Madrid. 922-924.

Mag. Bot. Zürich. 960.

Mag. Conchyliol. 5 I6.

Mag. Entomol. 671

Mag. Ges. Naturf. Freunde Berlin. $588(b)$.

Mag. Insektenkunde 629.

Mag. Nat. Hist. 208.

Mag. Sci. and School Arts. 209.

Mag. Zool. 5 I 7 .

Mag. Zool. Anat. Comp. Palaeont. 5 I8.

Mag. Zool. Bot. 4I.

Magdeburg. 744 .

Magn. Meteor. Beob. Prag. $363(d)$.

Magn. Meteor. Obs. Bombay. IO4I.

Magn. Meteor. Observ. Batavia. II93.

Magn. Meteor Observ. St. Helena. 999.

Magn. Meteor. Observ. Tasmania. I 242.

Magn. Meteor. Observ. Toronto. I 304.

Magn. Obs. Madras. I I $30(b)$.

Magn. Obs. U.S. Naval Observ. I $540(a)$.

Magn. Terrestre Filipinas. I22I (b).

Ilagyar Bot. Lapok. 350.

Magyar Földr. Társaság. 351 .

Magyar Kir. Földt. Intez. 352.

Magyar Nemzeti Múzeum. 353.

Magyar Ornithol. Központ. 354.

Magyar Tudományos Akad. 355.

Maine Agric. Exp. Stat. I458.

Malacol. Soc. London. 2 I I

Malacol. Zoob. Mag. 2 Io.

Malakol. Blatter. 635.

Malang. I2IO.

Malaria Comm. Roy. Soc. London. $298(n)$.

Malay Med. Journ. I 176.

Malayan Miscellanies. I 198.

Malaysian Essays. $\quad \mathbf{2} 69$.

Malpighia. 820 .

Man. $280(b)$.

Manchester. 329-335.

Manchester Egypt. Orient. Soc. I600.

Manchester Geol. Soc. 330.

Manchester Lit. Phil. Soc. 332.

Manchester Mus. 335 .

Mandalay. I 132 .

Manila. I2 I I-I 222.

Manila Med. Soc. 1219.

Marburg. 745 .

Marconigraph. 212. 
Marine Biol. Assoc. W. Scotland. 55. Marine Biol. Assoc. United Kingdom. 346. Marine Biol. Comm. L'pool. 67. Marine Biol. Lab. Ceylon. I IO3. Marine Biol. Lab. Woods Hole. I 546. Marine Biol. Rep. C. of Good Hope. 979. Marine Invest. S. Africa. $972(b)$.

Marine Survey India. 1084.

Marseilles. 462-465.

Martens' Conch. Mitt. 633.

Maryland Geol. Surv. I 323.

Maryland Weather Serr. 1324.

Massachusetts. Agr. College. I 312.

Massachusetts Hort. Soc. I 332.

Massachusetts State Board Agr. 1335 .

Material. Geol. Russlands. 9 Io $(a)$.

Material. Gheol. Rossī. $910(a)$.

Matériaux Carte Géol. Suisse. $95^{8}$.

Matériaux Hist. Posit. Phil. Honime. 519.

Matériaux Hist. Primit. Nat. Homme. 520.

Matériaux Paléont. Suisse. 950.

Math. Ann. 727.

Math. Gazette. 213.

Math. Naturwiss. Ber. Ungarn. 356.

Math. Soc. Calcutta. 1085.

Math. Soc. London. 214.

Matières Grasses. 521.

Mauritius see Port Louis.

Mechanic's Mag. 215.

Meckel's Arch. Anat. Physiol. 702 (c).

Meddel. Finsk. Vet.-Soc. Förhandl. $889(c)$.

Meddel. Geogr. För. Finland. 89I $(a)$.

Meddel. Nobel Inst. $933(b)$.

Meddel. Soc. Faun. Flor. Fennica. $893(b)$.

Meded. Burg. Geneesk. Dienst Ned-Ind. II9I.

Meded. Dept. Landb. Ned.-Ind. I I99 (d).

Meded. Kolon. Inst. Amsterdam. $786(a),(b)$.

Meded. Lab. Plantenziekt. I 200.

Meded. Proefst. Java-Suikerindust. $1226(b)$

Meded. Proefst. Malang. I 210.

Meded. Proefst. Oost-Java. 1225.

Meded. Proefst. Suik. W.-Java 'Kagok' 1223 (b).

Meded. Proefst. Thee Buitenarg. I $199\left(\begin{array}{l}f \\ )\end{array}\right)$.

Meded. 'sLands Plant. Buitenzorg. I I99 (e).

Meded. Transvaal Mus. 998.

Meded. Vischerij Stat. Batavia. 1197.

Medic. Ann. Practit. Index. 217.

Medic. Ann. Synopt Index. 218.

Medic. Bull. Univ. Pennsylvania. $1476(c)$.

Medic.-chirurg. Soc. Bristol. 4.

Medic-chirurg. Transactions. 292.

Medic. Chronicle. 333

Medic. Critic Psychol. Joum. 219.

Medic. Graduates College. 220.

Medic. Journ. Australia. 1270.

Medic. Journ. S. Africa. 98 $\mathrm{I}$.

Medic.-naturwiss. Ges. Jena. 693.

Medic. News Abstract. 1473.

Medic. Officer. $22 \mathbf{t}$.

Medic. Phys. Soc. Bombay. 1039.

Medic. Phys. Soc. Calcutta. 1086.

Medic. Press Circular. 222.

Medic. Psychol. Assoc. Gt. Brit. 225.

Medic. Record. 1089.

Medic. Reporter. 1090.
Medic. Rev. 223.

Medic. Service Neth. Ind. II9I.

Medic. Soc. Manila. I219.

Medic. Surg. Rev. of Revs. 216.

Medic.-Surg. Journ. Tropics. 1091.

Medic. Times Gazette. 224.

Mediz. Fac. Kais.-japan. Univ. I I $85(f)$.

Mélanges Phil. Math. $87 \mathrm{I}(\mathrm{c})$.

Melbourne. I245-1256.

Melbourne Univ. I 256.

Mém. Acad. Imp. Sci.St. Pétersbourg. 900 $(f, i, j)$.

Mcm. Acad. Real Sci. Lisboa. 9I8 $(a, c)$.

Mém. Acad. Roy. Sci. Belgique. $398(e-j)$.

Iém. Acad. Roy. Sci. Bruxelles. $397(b-c)$.

Mém. Acad. Roy. Sci. Paris. $501(c-h)$.

Mém. Acad. Roy. Sci. Turin. 87 I $(d)$.

Mém. Acad. Roy. Metz, 746.

Mém. Acad. Sci. etc. Dijon. 444.

Mém. Acad. Sci. France. 501 i $(c-h)$.

Mém. Acad. Sci. etc. Lyon. 45 I $(a-d)$.

Mém. Acad. Sci. Paris. $501(c-h)$.

Mém. Acad. Sci etc. Turin. 87I $(d)$.

Mem. Accad. Sci. etc. Acireale. $812(a, c, d)$.

Mem. Accad. Sci. Ist. Bologna. $815(c-e)$.

Mem Accad. Sci. Torino. 871 (e).

Mém. Agric. Paris. 564.

Mém. Agric. Econ. vurale etc. 564.

Mem. Amer. Acad. Arts Sci. $\mathrm{I} 327(a)$.

Mem. Amer. Assoc. Adv. Sci. I489 (a).

Mem. Amer. Mus. Nat. Hist. 1426 (e).

Mem. Anthropol. Soc. 8 I (c).

Mem. Asiat. Soc. Bengal. $1048(f)$.

Mem. Astron. Soc. London. $283(a)$.

Mem. Austral. Mus. $1262(a)$.

Men. Bernice Pauahi Mus. $\mathrm{I}_{204}(a)$.

Mem. Biol. Lab. John Hopkins Univ. I $321(b)$.

Mem. Boston Nat. Hist. Soc. I $334(b)$.

Mem. Califomia Acad. Sci. I $492(b)$.

Mem. Carnegie Mus. I 479 (b).

Mem. Civ. Vet. Dept. India. $1055(b)$.

Mem. Challenger Soc. IO7.

Mem. Colombo Mus. I Io5 (a).

Mém. Com. Géol. St. Pétersbourg. 907 (b).

Mém. Compt. Rend. Soc. Roy. Canada. I 296.

Mém. concern. Hist. Nat. Emp. Chinois. II7I.

Mém. Congr. Internat. Ent. $1595(b)$.

Mem. Connecticut Acad. Arts Sci. I4I I (a).

Mém. Cour. Acad. Roy. Sci. Belgique. 398 $(i, j)$.

Mém. Cour. Acad. Roy. Sci. Bruxelles. 397 $(c, d)$.

Men. Dep. Agric. India. II4I (c).

Mem. Descriz. Carta Geol. Ital. $865(b), 866$.

Mem. Egypt Explor. Fund. 127.

Mém. Entomol. $802(b)$.

Mem. Geol. Dep. Mysore. 1019 (b).

Mem. Geol. Surv. Canada. $1293(d-f)$.

Mem. Geol. Surv. Gt. Britain. 158.

Mem. Geol. Surv. India. I06I (d).

Mem. Geol. Surv. N.S. Wales. I $267(a-c)$.

Mem. Geol. Surv. Transvaal. $997(a)$.

Mem. Geol. Surv. Victoria. I25I (b).

Mém. Hist. Nat. Empire Chinois. I17 I.

Mem. Imp. Acad. Tokyo. II $80(a)$.

Mem. Imp. R. Ist. Regno Lomb.-Veneto. 836. 
Mem. Imp. R. Ist. Veneto. $877(b)$. Mem. Ind. Mus $1099(a)$.

Mém. Inst. Géol. Louvain. 420.

Mèm. Inst. Nat. Genèvois. 947.

Mem. Inst. Oswaldo Cruz. I 580.

Mem. Ist. Geol. Padova. 848.

Mem. Kodaikanal Observ. I I $30(c)$.

Mem. L'pool. Mar. Biol. Comm. $67(b)$.

Mem. Manch. Lit, Phil. Soc. $332(a)$.

Mem. Minist. Fomento México. I $568(b)$.

Mém. Mus. Caucase. IOOI (b).

Mem. Mus. Civ. Stor. Nat. Milano. $838(b)$.

Mem. Mus. Colombo. I IO5 (a).

Mem. Mus. Comp. Zool. Harvard. I34 I (d).

Mem. Mus. Econ. Geology. 158.

Mem. Mus. Goeldi. i $576(b)$.

Mém. Mus. Hist. Nat. Belgique. $404(c)$.

Mém. Mus. Hist. Nat. Paris. $525(d)$.

Mem. Mus, Paraense. I $576(b)$.

Mém. Mus. Paris. $525(d)$.

Mém. Mus. Roy. d'Hist. Nat. Belg. $f 04$ (c).

Mem. Nat. Acad. Sci. Washington. I $517(a)$.

Mem. Nat. Mus. Melbourne. 1252.

Mem. New South Wales Nats'. Club. I27 I (b).

Mem. New York Acad Sci. I4I6 (b).

Mem. New York State.Mus. Nat. Hist. I 309 (d).

Mém. Paléont. Soc. Géol. France. $557(\mathrm{c})$.

Mem. Peabody Acad. Sci. 149I (b).

Mem. Queensland Mus. I234 (b).

Mem. R. Acad. Cienc. Madrid. $922(a)$.

Mem R. Accad. Lincei. $864(a)$.

Mem. R. Accad. Sci. etc. Acireale. 812 (a, $c, d)$.

Mem. R. Accad. Sci. Ist. Bologna. 8 I $5(c-e)$.

Mem. R. Accad. Sci. Torino. 87 I (e).

Mem. R. Ist. Lomb. Sci. etc. $837(b, c)$.

Mem. R. Ist. Veneto. $877(b)$.

Mem. Rev. Soc. Cient. Ant. Alzate. I 572.

Mem. Roy. Astron. Soc. London. $283($ a).

Mem. Roy. Soc. S. Austral. I230 (a).

Mém. Savans Acad. Roy. Sci. France. 501 $(f)$.

Mém. Serv. Géol. Indo-Chine. I I $58(b)$.

Mém. serv. Descript. Géol. France. 522.

Mem. Sci. Dept. Univ. Japan. I $85(e)$.

Mém. Soc. Anthropol. Paris. $55 \mathrm{I}(c)$.

Mém. Soc. Belge Géol. Paléont. etc. 407 (a).

Mém. Soc. Biol. Paris. 552.

Mem. Soc. Bot. Ital. 823.

Mem. Soc. Cient. Ant. Alzate. I 572.

Mém. Soc. Ent. Pays-Bas. $802(b)$.

Mém. Soc. Ent. Belgique. 408 (c).

Mém. Soc. Finno-Ougr. $895(b)$.

Mém. Soc. Géol. Belgique. 4I7 (b).

Hém. Soc. Géol. France. $557(b, c)$.

Mém. Soc. Géol. Nord France. $450(b)$.

Mém. Soc. Hist. Nat. Strasbourg. 766.

Mém. Soc. Imp Nat. Moscou. 897 (b).

Mém. Soc. Imp. Sci. etc. Cherbourg. 442.

Mem. Soc. Ital. Sci. Nat. $838(b)$.

Mém. Soc. Linn. Calvados. 439.

Mém. Soc. Linn. Normand. 440 (b).

Mém. Soc. Mus. Hist. Nat. Strasbourg. 766.

Mém. Soc. Nat. Kiev. 896.

Mém. Soc. Nat. Nouv. Russie. 898.
Wém. Soc. Nat. Sci. etc. Cherbourg. 442.

Mem. Soc. Neuchat. Sci. Nat. $956(b)$.

Mém. Soc. Paléont. Suisse. 952.

Mém. Soc. Phys. Hist. Nat. Genève. 953 (b).

Mém. Soc. Portug. Sci. Nat. 921 (b).

Mém. Soc. Roy. Antiq. Nord. $425(b)$.

Mém. Soc. Roy. Canada. I 296.

Mém. Soc. Roy. Sci. Liége. 418.

Mém. Soc. Sci. Nat. Math. Cherbourg. 442.

Mém. Soc. Sci. Nat. Neuchatel. $956(b)$.

Mém. Soc. Sci. Nat. Strasbourg. 766.

Mem. Soc. Spettrosc. Ital. 853.

Mem. Soc. Tosc. Sci. Nat. $859(a)$.

Mém. Soc. Zool. France. $565(b)$.

Mem. Univ. California. I325 (c).

Mem. Wernerian Nat. Hist. Soc. $5 \mathrm{I}$.

Merck's Ann. Rep. etc. 639.

Merida. I $; 66$.

Messenger of Mathematics. 226.

Metallographist. I 333 .

Metallurg. Chem. Eng. 1450.

Meteor. Aarbog Christiania. $883(b)$.

Meteor. Dep. Bengal. I092.

Meteor. Ceylon. $1 \mathrm{IO} 4$ (a).

Meteor. Dep. India. I $15 \mathrm{I}$.

Meteor. Dep. Mysore. IO2O.

Meteor. Iakttag. Sverige. $932(j)$.

Meteor. Jahrb. Finland. 890.

Météor. Lyonnaise. 455.

Meteor. Magn. Beob. Mïnchen. 75 I (b).

Meteor. Mysore. IOzO (a).

Meteor. Obs. Ind. I I 5 I $(d)$.

Meteor. Obs. Madras. I I $30(d)$.

Meteor. Observ. New York. I440.

Meteor. Office Colombo. I IO4.

Meteor. Record. 293 (a).

Meteor. Register Calcutta. Ic98 (h).

Meteor. Rep. New Zealand. 1276.

Meteor. Reporter Bengal. Iog2.

Meteor. Soc. London. $293(b)$.

Meteor. United Prov. etc. 1005.

Meteor. Zeitschrift. 376.

Meteor. Zentralanst. Finland. 890.

Metz. 746.

Mexico. I 567-I 573 .

Mezozoic Fossils. I293 (g).

Michigan Acad. Sci. $13^{87 .}$

Michigan Agric. Exp. Stat. I 388.

Michigan State Agric. College. I 388.

Microsc. Soc. Calcutta. I093.

Microsc. Soc. London. 294.

Milan. $834-838$.

Military Surgeon. I 343.

Millport Marine Biol. Stat. $55(b)$.

Miltitz. 747.

Milwaukee. I 399, I400.

Mind. 229.

Mineral Industry. 227.

Mineral Resources Chösen (Korea). I I68.

Mineral Resources N.S. Wales. I267 (d).

Mineral Resources United States. 539 (c).

Mineral Surv. Chōsen (Korea). I I68.

Mineralog.-Geol. Inst. Hamburg. 678 .

Mineralog. Mag. 228.

Mineralog. Mitt. 382.

Mineralog. Petrogr. Witt. 382. 
Mineralog. Soc. London. 228.

Mineralog. Surv. Ceylon. IIO2 $(a)$.

Mines Dep. Transvaal. 997.

Mines Dep. Union S. Africa. 995

Mines Minerals. 1495.

Mining Bur. Philipp. Is. I 220.

Mining Geol. Inst. India. I094.

Mining Journ. 230.

Mining Mag. 231 , 1450.

Mining Manual. 232.

Mining Sci. Press. I493.

Mining World Eng. Record. 233

Minist. Agric. Argentina. I 548, I 549.

Minist. Colon. Mines Pêch. Quebec. 1297.

Minist. Fomento Costa Rica. I 586.

Minist. Fomento México. 1568, 1569.

Minist. Lucrărilor ū Publice. $3 y^{2}$.

Minneapolis. I40I-1405.

Minnesota Acad. Nat. Sci. 1403.

Minnesota Bot. Studies. $1402(d)$.

Minnesota School Mines Exp. Stat. I405.

Minutes Proc. Inst. Civ. Engineers. I 78 (a).

Misc. Austriaca. 383.

Misc. Curiosa Medico-physica. $669(d)$.

Misc. Phil-math. Soc. Taurinensis. $87 \mathrm{I}(f)$. Misc. Publ. U.S. Geol. Surv. Territ. I $538(c)$.

Missoula. 1406.

Missouri Bot. Garden. 1488.

Missouri Bur. Geol. Mines. ${ }_{3} 83$.

Missouri Geol. Surv. I383.

Missouri State Board Agric. 1384.

Mitt. Anthropol. Ges. Wien. 367.

Mitt. Bot. Staatsinst. Hamburg. $675(b)$.

Mitt. Deut. Ges. Nat.-Volkerk. Ostasiens. I I 79.

Mitt. Embryol. Inst. Wien. 378.

Mitt. Erdb.-Comm. Wien. $372(e)$.

Mitt. Geol. Ges. Wien. 370.

Mitt. Geogr. Ges. München. 749.

Mitt. Geogr. Ges. Naturh. Mus. Lübeck. 743.

Mitt. Ges. Erdk. Leipzig. 7 I $5(a)$.

Mitt. Ges. Freunde Naturwiss. Wien. $37 \mathrm{I}(a)$.

Mitt. Jahrb. K. Ungar. Geol. Reichsanst. $35^{2}$ (c).

Mitt. Kais.-Kön. Geogr. Ges. IVien. $374(b)$.

Mitt. Kaukas. Mus. IOOI (c).

Mitt. Komm. Geol. Landesunters. Els.-Lothr. $765(b)$.

Mitt. Kön. Min.-Geol. Prähist. Mus. Dresden. 644 .

Mitt. Kön. Ungar. Geol. Reichsanst. $352(c)$.

Mitt. Kön. Zool. Mus. Dresden. $645(b)$.

Mitt. Justus Perthes' Geogr. Anst. $66_{3}(a, b)$.

Mitt. Med. Fac. Kais.-japan. Univ. II $85(f)$.

Mitt. Miner.-Geol. Inst. Hamburg. 678.

Mitt. Mus. Naturk. Berlin. 605.

Mitt. Naturf. Ges. Halle. $672(d)$.

Mitt. Naturf. Ges. Zürich. $961(d)$.

Mitt. Naturh. Mus. Hamburg. 680.

Mitt. Naturwiss. Ver. Steiermark. 359.

Mitt. Ornith. Ver. Wien. 386.

Mitt. Perthes' Geogr. Anst, $663(a, b)$.

Mitt. Schweiz. Entomol. Ges. 942.

Mitt. Schweiz. Geol. Ges. 943.

Mitt. Ungar. Geol. Ges. $352(c)$.

Mitt. Ver. Erdk. Leipzig. 7 I s (b).
Mitt. Zool. Mus. Berlin. $6 \mathrm{I} 6$.

Mitt. Zool. Samml. Mus. Naturk. Berlin. 605 .

Mitt. Zool. Stat. Neapel. $845(b)$.

Modena. 839.

Moltschulsky's Etudes Entomol. 888.

Monaco. 466.

Monatl.-herausg. Insect.-Belust. 756.

Monatsb. K. Preuss. Akad. Wiss. $600(d)$.

Monde des Plantes. $448(b)$.

Mondes. 523.

Monit. Jard. Bot. Tiflis. 1002.

Monogr. Agric. Co-op. etc. $862(\mathrm{c})$.

Monogr. Palaeontogr. Soc. 253.

Monogr. U.S. Geol. Surv. I $539(d)$.

Montana Univ. 1406.

Montevideo. I 574 .

Month. Amer. Journ. Geol. Nat. Sci. 1474. Month. Bull. Centr. Observ. Manila. 1216. Month. Internat. Journ. Anat. Hist. 717. Month. Microsc. Journ. 294 (b).

Month. Notices Pap. Proc. Roy. Soc. Tasmania. 1243.

Month. Notices (Roy) Astron. Soc. Eondon. $283(b)$.

Month. Numismat. Circular. 234.

Month. Rec. Geography. 288 (c).

Month. Rep. U.S. Dept. Agric. I $529(f)$.

Month. Weather Rev. India. II I (c).

Month. Weather Rev. U.S.A. I $533(a)$.

Morphol. Jahrb. 728.

Moscow. 897.

Mount Weather. I407.

Mount Weather Observ. I407.

Mozambique Company. 966.

Muktesar. 1133.

Müller's Arch. Anat. Physiol. etc. 702 (a).

München see Munich.

Munich. 748-752.

Mus. Anthr. Ethnogr. St. Petersbourg. 903.

Mus. Assoc. 236.

Mus. Bot. Amazonas. I 581 .

Mus. Bot. Leiden. 808.

Mus. Bot. St. Pétersbourg. 902 .

Mus. Bull. Dublin. 21.

Mus. Bull. Ottawa. $1294(b)$.

Mus. Calicase. IOOI.

Mus. Civ. Stor. Nat. Genova. 832.

Mus. Civ. Stor. Nat. Milano. $838(a, b)$.

Mus. Civ. Stor. Nat. Trieste. 365.

Mus. Colombo. I IO5.

Mus. Colonial Marseille. 464.

Mus. Commerc. Torino. 874 .

Mus. Comp. Zool. Harvard. I 34I.

Mus. Congo. 403.

Mus. Fed. Malay States. II60.

Mus. Geol. Paleont. Bucuresci. 393.

Mus. Géol. Pierre le Grand. 904.

Mus. Goddefroy. 679.

Mus. Goeldi Hist. Nat. Ethnol. I 576.

Mus. Guimet. 524 .

Mus. Hist. Nat. Genève. 951 .

Mus. Hist. Nat. Belgique. 404.

Mus. Hist. Nat. Lyon. 456.

Mus. Hist. Nat. Marseille. 465.

Mus. Hist. Nat. Paris. 525. 
Mus. Hist. Nat. Toulouse. 568.

Mus. Journ. 236.

Mus. Journ. (Philadelphia). $\quad$ 1477 (b).

Mus. La Plata. 1564 .

Mus. Nac. Arqueol. Hist. Etnol. I57 I

Mus. Nac. Buenos Aires. I 550.

Mus. Nac. Caracas. 1552.

Mus. Nac. Costa Rica. I 585, I 587.

Mus. Nac. México. 1570.

Mus. Nac. Moutevideo. I 574.

Mus. Nac. Rio de Janeiro. I 582 .

Mus. Nation. Hungaricum. 353.

Mus. Natur- Hiematk. Magdeburg. $74+$.

Mus. Naturkunde Berlin. 605.

Mus. News. I $337($ c).

Mus Océanogr. Monaco. 466.

Mus. Paraense. I 576.

Mus. Paulista. I 589 .

Mus. Públ. Buenos Aires. 1550.

Mus. Roy. Ethnogr. Leyde. $805(a)$.

Mus. Roy. Hist. Nat. Belgique. 404.

Mus. Rusticum Commerciale. 235.

Mus. Sci. Art Pennsylvania. 1477.

Mus. Senckenbergianum. 655 (c).

Mus Stor. Nat. Genova. 832.

Mus. Stor. Nat. Trieste. 365 .

Mus. Teyler. 797.

Mus. Tokyo. 1 I 84.

Mus. Valparaiso. I 592.

Mus. Zool. Anat. Comp. Genova. 833.

Mus. Zool. Anat. Comp. Torino. 875.

Mus. Zool. Dundee. 29.

Mus. Zool. Harvard. I 341 .

Mus. Zool. Napoli. 842 .

Mus. Zool. St. Pétersbourg. 905.

Museumskunde. 606.

Mycol. Bull. Dep. Agr. Mysore. 1017 (c).

Mycol. Writings C. G. Lloyd. I $357(d-f)$.

Mysore Econ. Conf. IO2I.

Mysore Econ. Journ. IO2I.

Mysore Meteor. Mem. $1020(b)$.

Mythic Soc. 1022 .

\section{$\mathrm{N}$}

Nachr.-Bl. Deut. Malakozool. Ges. 65 I (b). Nachr. K. Gess. Wiss. Göttingen. $666(e-h)$.

Nageli's Beitr. Wiss. Bot. 706.

Nagpur. I I 34.

Nagpur Mus. I I 35.

Nairobi. 987.

Nantes. 467.

Naples. $840-845$.

Nashville. 1408 .

Nassauisch. Ver. Naturk. 779.

Natal Agric. Journ. (Min. Rec.). 988 (a).

Natal Govt. Museum. 990.

Nation. Acad. Sci. Washington. I 517.

Nation. Ass. Prom. Social Sci. 237.

Nation. Mus. Melbourne. 1252.

Nation. Mus. Sci. Art Dublin. 21.

Natural Hist. Phil. Soc. Belfast. I.

Natural Hist. Phil. Soc. B'ham. 3.

Natural Hist. Review. 22.

Natural Hist. Soc. Newport. 1456.

Natural Hist. Soc. Northumberland etc. 337 .
Natural Hist. Soc. Trenton. I 503.

Natural Hist. Soc. Siam. II 53.

Natural Hist. Trans. Northumb. Durham. 337.

Natural Scicnce. 238.

Naturaliste. 527.

Naturalist's Repository. 239

Naturalists' Soc. Bristol. 5.

Naturc. 240.

Nature, Paris. 528

Naturforsch. Ges. Basel. 939.

Naturforsch. Ges. Danzig. 638.

Naturforsch. Ges. Dorpat. 887

Naturforsch. Ges. Freiburg. 659.

Naturforsch. Ges. Halle. 172.

Naturforsch. Ges. Zürich. 961.

Naturhist. Foren. Copenhagen. 427

Naturhist. Ges. Nïrnberg. 757.

Naturhist. Mus. Hamburg. 680.

Naturhist. Mus. Liibeck. 743.

Naturhist.-Selsk. Copenhagen. 426.

Naturhist. Tidsschrift. 428.

Naturhist. Ver. 'Lotos.' 362.

Naturhist. Ver. Preuss. Rheinl. etc. 620.

Naturwiss. Abh. Wien. 37 I (b).

Naturwiss. Ges. 'Isis.' 646.

Naturwiss.-Med. Ver. 'Lotos' 362.

Naturwiss. Rundschau. 630.

Naturwiss. Ver. Frankfurt. 656.

Naturwiss. Ver. Hamburg. 681.

Naturwiss. Ver. Magdeburg. 744

Naturwiss. Ver. Reg.-Bez. Frankfurt. $6 ; 6$.

Naturwiss. Ver. Sachs. Thüring. 673.

Naturwiss. Ver. Steiermark. 359.

Natuurk. Tijdschr. Ned.-Ind. II94 (a).

Naumannia. 77 I.

Nautical Almanac. 24I.

Nebraska Agric. Exp. Stat. I 394.

Nebraska Univ. I 393, I 394.

Nederl. Bot. Vereenig. 8 Io.

Nederl. Dierk. Vereenig. 811.

Nederl. Ent. Vereenig. 802.

Nederl.-Ind. Plakaatboek. II $90(a)$.

Nederl. Kruidk. Archief. 8 In $(a)$.

Nederl. Maatsch. Bevord. Nijverh. 798.

Nederl. Tijdschr. Dierkunde. $788(b)$.

Neubrandenburg. 753.

Neuchatel. 956.

Neue Ann. F. Sternw. Mïnchen. 75 I (c).

Neue Denk. Allg. Schweiz. Ges. Naturw. $957(b)$.

Neue Entdeck. Pflanzenkunde. 729.

Neue Gross. Schrift. Oekon. Soc. Leipzig. $730(a)$.

Neue Phil. Abh. K. Bayer. Akad. Wiss. $750(h)$.

Neue Schrift. Ges. Naturt. Freunde Berlin. $588(c)$.

Neue Schrift. Naturf. Ges. Halle. $672(e)$.

Neue Zool. Ges. Frankfurt. 654 .

Neues Jahrb. Miner. Geogn. Geol. Petrefaktenk. 772 .

Neues Jahrb. Miner. Geol. Paläont. 772.

Neves Mag. Bot. 962.

Neujahrsbl. Naturf. Ges. Zürich. 96 I (e).

New Brunswick. I409. 
New Hamps. Agric. Exp. Stat. I 369 . New Hamps. Coll. Agr. Mech. Arts. I 369.

New Haven. I410-14I4.

New Jersey Agric. Exp. Stat. I409.

New Mexico Agric. Exp. Stat. I 389.

New Mexico College Agric. etc. I 389.

New Orleans. I4 I5.

New Orleans Med. Surg. Journ. I4I 5.

New Phytologist. 242.

New South Wales Nats'. Club. I27 I.

New York. I4I6-1455.

New York Acad. Sci. I416.

New York Agric. Exp. Stat 1374 .

New York Bot. Gard. 1430.

New York Lyceum Nat. Hist. I 446.

New York Meteor. Observ. I448.

New York State Journ. Ned. I45 I.

New York State Mus. Nat. Hist. 1309.

New York State Weather Bur. 138 I.

New York Zool. Soc. I455.

New Zealand Board Sci. Art. I606.

New Zealand Inst. 1280.

New Zealand Journ. Sci. Technol. 1606.

Newcastle-upon-Tyne. 336-339.

Newport. I456.

Newport Nat. Hist. Soc. I 456.

Nicosia. IOO4.

Niederl. Arch. Zool. 804.

Niederrh. Ges. Nat.-Heilkunde. 621.

Nieuwe Verh. K. Ned. Inst. Wetensch. $787(a)$.

Nimeguen. 810.

Nobel Inst. 933.

Nordiske Fortsminder. 425 (c).

Norimberga see Nuremburg.

Norsk Gradmaalingskommiss. 882.

Norsk Meteor. Aarbog. 883 (c).

Norsk Meteor. Inst. 883

North Amer. Fauna. I $530(b)$.

North Carolina Agric. Exp. Stat. 1481 .

North Carolina College Agric., etc. I48I.

North Carolina Dept. Agric. I482.

North Engl. Inst. Min. (Mechan.) Eng. 338.

North Ind. Notes Queries. IoI 2.

Northfield. 1457 .

Norweg. Commiss. Europ. Gradmessung. 882 .

Norweg. Meteor. Inst. 883 .

Notes Econ. Entom. $1080(d)$.

Notes Leyden Mus. 807 (a).

Notes Manchester Mus. 335.

Notes Perak Mus. 1178.

Notes Quevies, London. 243.

Notes Queries, Singapore. II $77(b)$.

Notes Roy. Bot. Gard. Edinb. 44.

Notes Roy. Zool. Mus. Netherlands. $807(b)$.

Notices Anthropol. Leyde. $805(a)$.

Notices Roy. Inst. Gt.-Brit. $29 \mathrm{I}($ (c).

Notulae Systematicae. 526.

Notulen Batav. Genootsch. I $190(b)$.

Nouv. Ann. Math. 529.

Nouv. Ann. Mus. Hist. Nat. Paris. $525(e)$.

Nouv. Arch. Mus. Hist. Nat. Paris. $525(f)$.
Nouv. Bull. Sci. Paris. 562.

Nouv. Journ. Asiatique. $545(b)$.

Nouv. Mém. Acad. Roy. Sci. Bruxelles. $397(f)$.

Nouv. Mém. Soc. Belge Géol. Paléont. etc. $407(b)$.

Nouv. Mém. Soc. Helvét. Sii. Nat. 957 (b).

Nouv. Mém. Soc. Imp. Nat. Moscou. $897(c)$.

Nova Acta Acad. Sri. Imp. Petropol.

Nova Acta Physico-Medica. 669 (e).

Nova Acta Reg. Soc. Upsal. 937.

Nova Goa see Goa.

Nova Scotian Inst. (Nat.) Sci. 1285.

Novi Comment. Acad. Sci. Bononiensis. $815(f)$.

Novi Comment. Acad. Sci. Imp. Petrop. $900(h)$.

Novitates Zoologicae. 348.

Novoross. Obshch. Estestvoisp. 898.

Numismat. Chronicle. 244, 296.

Numismat. Journ. 245.

Numismat. Soc. London. 296.

Nunquam Otiosus. $6,0$.

Nuov. Ann. Sci. Nat. 8I4.

Nuov. Giorn. Bot. Ital. 823.

Nuremburg. 754-758.

Nürnberg see Nuremburg.

$\lceil 424(a)$

Nye Samling Skrivt. K. Dansk. Vit Selsk.

Nyelv. Fiozlemények. 355 (d).

Nyelv. Rég. Magyar Codexek. 355 (c).

Nyt Mag. Naturvidensk. 884.

\section{O}

Oberhess. Ges. Nat.-Heilkunde. 660.

Obs. Ascens. Internat. Simult. etc. $763(a)$

Obs. Magn. Met. Observ. Batavia. I I93 (a).

Obs. Magn. Met. Observ. Bombay. Io41.

Obs. Mag. Met. Observ. C. of Good Hope. 974.

Obs. Magn. Meteor. Observ. Hobarton. 1242 Obs. Magn. Meteor. Observ. Toronto. I 1304.

Obs. Météor. Etabl. Franc. Inde. I I 39.

Obs. Meteor. Inst, Bot. Buitenzorg. I 201 (b).

Obs. Meteor. Ona. 925.

Obs. Météor. Pondicherry. II 39.

Obs. Meteor. Suédoises. $932(j)$.

Obs. Roy. Magn. Met. Observ. Batavia I $193($ a).

Obs. St. Helena Obșerv. 999.

Observ. Bombay. IO4I,

Observ. Cambridge. I2.

Observ. Colombo. I IO4.

Observ. Ebre. 927.

Observ. Kodaikanal. I I 30.

Observ. Madras. II 30 .

Observ, Manila. I22I.

Observ. Meteor. Macau. II64.

Observ. Meteor. Manila. I 22 I.

Observ. Philipp. Is. I $2 \mathrm{I}$.

Observ. Nac. Rio de Janeiro. ${ }_{15} 83$.

Observ. Paris. 530.

Observ. Rio de Janeiro. $\quad{ }^{5} 83$.

Observ. Roy. Bruxelles. 405.

Observ. Roy. Madagascar. 965. 
Observ. St. Louis. 6I.

Observ. São Paulo. I 588.

Observ. Stoneyhurst College. 347.

Observatory. 246.

Obshch. Estestroisp. Kiev. 896.

Occas. Pap. Bernice Pauahi Mus. $1204(b)$.

Occas. Pap. Boston Nat. Hist. Soc. $1334(c)$.

Occas. Pap. California Acad. Sci. 1492 (c).

occas. Pap. Wisconsin Nat. Hist. Soc. $1400(b)$.

Ocean Highway's. 247.

Odessa. 898

Oekonom. Soc. Leipzig. 730.

Oesterr. Alpen.-Verein. 384.

Oesterr. Geschichts-Quellen. $372(f)$.

Oesterr. Ges. Meteor. 376.

Oesterr. Monats. Orient. $3^{8} 5$.

Official Journ. Patent Office. $254(b)$.

Ofvers. Finsk. Vet.-Soc. Förhandl. $889(\mathrm{c})$.

Ofvers. Förhandl. K. Svensk. Vet.-Akad. $932(k)$.

Ohara Inst. Landwirtsch. Forsch. II6I.

Ohio Acad. Sci. 1362 .

Ohio Geol. Surv. I36r.

Ohio Journ. Sci. I 362 .

Ohio State Board Agric. I 363.

Ohio State Univ. Sci. Soc. 1362.

Oklahoma Agric. Exp. Stat. I 500.

Oklahoma Agr.c. Mech. College. I 500.

Ona. 925 .

Ontario Dep. Agric. 1305.

Ophthalmic Literature. I 366.

Opthalmic Review. 248.

Ophthalmology. I 354 .

Ophthalmoscope. 249.

Oporto. 926.

Opuscules Entrmol. $53 \mathrm{I}$.

Orchid Review. 250.

Ordnance Surv. Gt. Brit. 25 I.

Orenburg 899.

Orient. Archiv. 731.

Orient. Inst. K.-K. Univ. WVien. 379.

Orient. Inst. Vladivostok. $\mathrm{IOO}_{3}$.

Orient. Miscellany. 1095.

Orient. Mus. Wien. 385.

Orientalist. 1042.

Ornithol. Miscellany. 252.

Ornithol. Ver. Wien. 386.

Orono. I 458 .

Ort. Bot. Fiorentino. 824 .

Ort. Bot. Napoli. 843 .

Ort. Bot. Palermo. 852 .

Osserv. Astron. Torino. 872 .

Osserv. Collegio Romano. 863.

Osserv. Meteor. Torino. $872(b)$.

Otchet Imp. Russ. Geogr. Obshch. 909.

Ottawa. I288-1296.

Ouro Preto. I 575.

Orers. Forh. K. Dansk. Vidensk. Selsk. $424(b)$.

Owens College Manchester. 334.

Oxford. $340-34^{2}$.

\section{$\mathrm{P}$}

Padua. 846-849.

Palaeont. Abhandl. Jena. 694.
Palaeont. Bull. Geol. Surv. N. Zealand. $1278(b)$.

Palaeont. Indica. 106 I (c).

Palaeont. Mitt. 1603 .

Palaentographia Italica. $856(a)$.

Palaeontographica. 636

Palaeontographical Soc. 253.

Palaeozoic Fossils. $1293(h)$.

Palermo. 850-854.

Pallas' Spicilegia Zoologica. 609.

Paludism. II 49.

Pamphlets W. India Committee. $320(b)$.

Panzer's Faun. Insect. German. Init. 755.

Pap. Mech. Roy. Soc. Arts. $299(b)$.

Pap. Proc. Roy. Soc. Van Diemen's I. 1244.

Pap. Proc. Roy. Soc. Tasmania. I243.

Pap. Rep. Miner. Mining N. Zealand. 1281.

Para. 1576.

Paramaribo. I 577 .

Parasitology. I3.

Parergones Inst. Geol. México. I 567 (c).

Paris. $468-565$.

Parma. 855 .

Pasteur Inst. S. India. I 108.

Patent Office London. 254

Patent Office U.S.A. I 54I.

Patna. 1136.

Paxton's Mag. Botany. 255.

Peabody Acad. Sci. I491.

Pekalongan. 1223.

Penck's Geogr. Abhandl. 599.

Pennsylv. Agric. Exp. Stat. I 499.

Pennsylv. Geol. Surv. 1376.

Pennsylv. Mus. School Industr. Art. I 475 .

Pennsylv. Mus. Sci. Art. 1477 .

Pennsylv. State College. I 498, I 499.

Pennsylv. Univ. 1476, 1477.

Penzance. 343.

Peradeniya. I 37.

Perak Museuni. I 78

Perfum. Essent. Oil Record. 256.

Perth. 344.

Perth (Australia). I 257-1 259.

Perthes' Geogr. Anstalt. 663.

Peshawar. II 38 .

Petermanns' Mittheilungen. 663 (c).

Petites Nouv. Entomol. 532.

Petrograd. 900-912.

Petroleum Indust. Techn. Rev. 257.

Petroleum Review. 257.

Petropolis see Petrograd.

Pflanzer. $980(b)$.

Pfluger's Archiv. Physiol. Mensch. Thieve 622.

Pharmaceut. Journal. 259.

Pharmaceut. Journ. and Pharmacist. 258.

Pharmaceut. Soc. 259.

Phil. Inst. Victoria. $1255(e)$.

Phil. Mag. 260.

Phil. Soc. Adelaide. $1230($ c).

Phil. Soc. Cambridge. 14.

Phil. Soc. Glasgow. 57.

Phil. Soc. N. S. Wales. $1273(c)$.

Phil. Soc. Washington. I 5 I8.

Phil. Trans. $298(d-g)$.

Philadelphia. I459-1478. 
Philippine Agric. Forester. I222.

Philippine Agric. Rev. I2 I I (b).

Philippine Journ. Sci. I2 I4 $(a-e)$.

Philippine Weather Bur. I2I 5 .

Phys. Rev. I 382.

Phys. Soc. London. $26 \mathrm{I}$.

Physik-Med. Ges. Würzburg. 782 .

Physik.-med. Soc. Erlangen. 650.

Physik.-ökonom. Ges. Konigsberg. 700.

Physik. Zeitsch. 732.

Physiogr. Foren. Christiania. 884.

Phytologist. 262.

Pietermaritzburg. 988-99o.

Pinheiro. 1607.

Pisa. $856-859$.

Pittsburgh. 1479 .

Planter's Chron. 1023.

Plymouth. 345,346 .

Poggendorff's Ann. Physik Chem. 701 (c).

Polyclinic. 220.

Polynesian Soc. 1282.

Pomona College. I 360.

Pondicherry. I I 39.

Pontif. Univ. Gregoriana. 863.

Poona. I I 40 .

Port Erin. Biol. Stat. 67.

Port of Spain. I 578 .

Portici. 860.

Portland. I 480 .

Portland Soc. Nat. Hist. I 480.

Porto see Oporto.

Post Office Electr. Eng. Journ. 263.

Pract. Mechanic's Journ. $\quad 56$.

Practitioner. 264.

Prag. $36 \mathrm{I}-363$.

Prensa Medica. $\quad 1556$.

Prescriber. 42.

Press Bull. Bur. Agr. Philipp. Is. I2II (c).

Press Bull. Hawaii Agr. Exp. Stat. I 206 (c).

Pretoria. 993-999.

Prix Nobel. $933(a)$

Prize Ess. Trans. Highl. Agr. Soc. Scotland. $39(a)$.

Proc. Acad. Nat. Sci. Davenport. 1365.

Proc. Acad. Nat. Sci. Philadelphia. I $459(b)$.

Proc. Acad. Sci. Washington. $1510(b)$.

Proc. Agri-Hort. Soc. Madras. 1116.

Proc. Amer. Acad. Arts Sci. $1327(b)$.

Proc. Amer. Assoc. Adv. Sci. ${ }^{4} 489(b)$.

Proc. Amer. Metrol. Soc. 1425.

Proc. Amer. Phil. Soc. $1466(b)$.

Proc. Amer. Soc. Civil Eng. $1427(a)$.

Proc. Antiq. Soc. Cambridge. 6.

Proc. Ashmolean Nat. Hist. Soc. 340.

Proc. Asiat. Soc. Bengal. $1048(\mathrm{~g})$.

Proc. Belfast Nat. Hist. Phil. Soc. I.

Proc. Biol. Soc. L'pool. 62.

Proc. Biol. Soc. Washington. 1514.

Proc. Birming. Nat. Hist. Phil. Soc. 3.

Proc. Boston Nat. Hist. Soc. $1334(d)$.

Proc. Bot. Soc. London. 91.

Proc. Bristol Nats'. Soc. 5.

Proc. California Acad. Sci. $1492(d)$.

Jroc. Cambridge Antiq. Soc. 6.

Proc. Cambridge Phil. Soc. It (a).

Proc. Canad. Inst. $1300(c)$.
Proc. Cotteswold Nats.' Field Club. 58. Proc. Davenport Acad. Nat. Sci. ${ }_{13} 6_{5}$. Proc. Dublin Nat. Hist. Soc. 23.

Proc. Essex Inst. $1490(b)$.

Proc. Geogr. Soc. Australasia. 1229, $1235(a)$.

Proc. Geol. Soc. L'pool. $64(b)$.

Proc. Geol. Soc. London. I $57(a)$.

Prac. Geologist's Assoc. London. I6I.

Proc. Glasgow (Roy.) Phil. Soc. 57.

- Proc. Imp. Acad. Tokyo. I I $80(b)$.

Proc. Imp. Mus. Japan. II 84.

Proc. Indian Ass. Cult. Sci. $1070(b)$.

Proc. Indiana Acad. Sci. 1379.

Proc. Inst. Civ. Engineers. $178(a)$.

Proc. Inst. Mechan. Engineers. I80.

Proc. Internat. Congr. Entomol. I $595($ a).

Proc. Internat. Engin. Congr. 184.

Proc. Linn. Soc. N.S. Wales. I 268.

Proc. Lit. Phil. Soc. Manch. $332(c)$.

Proc. L'poo! Biol. Soc. 62.

Proc. L'pool Geol. Soc. $64(b)$.

Proc. L'pool Lit. Phil. Soc. 66.

Proc. London Bot. Soc. 91.

Proc. London Soc. Antiq. 3 I I (b).

Proc. Madras Agri-Hort. Soc. 1 i I6.

Proc. Malacol. Soc. London. 211.

Proc. Manch. Lit. Phil. Soc. $332(c)$.

Proc. Math. Soc. London. 214

Proc. Nat. Acad. Sci. Washington. $1517(b, c)$.

Proc. Nat. Hist. Dcp. Mus. Tokyo. II 84 .

Proc. Nat. Hist. Phil. Soc. Birmingham. 3.

Proc. Naturalists' Soc. Bristol. 5.

Proc. New York Lyceum Nat. Hist. $1446(b)$.

Proc. Newport Nat. Hist. Soc. 1456.

Proc. Nova Scotian Inst. Sci. 1285.

Proc. Phys. Soc. London. 26 I.

Proc. Portland Soc. Nat. Hist. 1480.

Proc. Rochester Acad. Sci. 1483.

Proc. Roy. Agri-Hort. Soc. S. Austral. 1228.

Proc. Roy. Asiat. Soc. Ceylon. $1106(b)$.

Proc. Roy. Colonial Inst. 286.

Proc. Roy. Dublin Soc. $25(a, c)$.

Proc. Roy. Geogr. Soc. London. $288(a, c)$.

Proc. Roy. Inst. Brit. Arch. 290 (c).

Proc. Roy. Inst. Gt. Brit. $291(c)$.

Proc. Roy. Irish Acad. $28(b-h)$.

Proc. Roy. Phys. Soc. Edinb. 46.

Proc. Roy. Soc. Canada. 1296.

Proc. Roy. Soc. Edinb. 49 (a)

Proc. Roy. Soc. London. $298(h-j)$.

Proc. Roy. Soc. Med. London. 300.

Proc. Roy. Soc. N.S. Wales. $1273(a, b)$.

Proc. Roy. Soc. Oucensland. 1236.

Proc. Roy. Soc. Tasmania. 1243.

Proc. Roy. Soc. Victoria. 1255.

Proc. Sci. Assoc. Trinidad. 1579.

Proc. Sect. Sci. Amsterdam. $789(b)$.

Proc. Soc. Antiq. London. 3I I (b).

Proc. Trans. Geogr. Soc. Australasia. I 235 (b).

Proc. Trans. Nova Scotian Inst. Sci. 1285.

Proc. Trans. Roy. Soc. Canada. 1296.

Proc. U.S. Nat. Mus. $1522(c)$.

Proc. Washington Acad. Sci. 15 IO $(b)$.

Proc. Zool. Soc. London. $327(a)$.

Proc.-Verb. Congr. Internat. Ent. I 595 (a). 
Proc. Verb. Soc. Malacol. Belgique. 4I I (b). Proc.-Verb. Soc. Roy. Zool. MIal. Belg. 4 II (b).

Proc.-Verb. Soc. Tosc. Sci. Nat. $859(b)$.

Prof.-Verb. Soc. Vaud. Sci. Nat. 955.

Prodr. Palacont. Tictoria. I25I (c).

Prodr. Zool. Fictoria. I 253.

Progr. Rep. Arch. Surv. Ind. W. Circle. I029.

Progr. Rep. Arch. Surv. Punjab etc. III3 $(a-f)$.

Progr. Rep. Arch. Surv. Unit. Prov. Ind. I I $3(a-f)$.

Progr. Rep.Arch. Surv. W. India. I030 (a)

Progr. Rep. Geol. Surv. I'ictoria. I25I (d).

Progressive Medicine. 265.

Progressus Rei Botanicac. 688

Proefst. Java-Suikerindustrie. I 226.

Proefst. Malang. I2IO.

Proefst. Oost-Java. I 225.

Proefst. Salatiga. I224.

Proefst. Suikerriet W.-Java 'Kagok.' I223.

Proefst. Thee Buitenzorg. I I99 $(f)$.

Prof. Papers College Eng. Madras. II 20.

Prof. Papers Ind. Eng. I I 45.

Prof. Papers Surv. Ind. I098 (i).

Prof. Papers U.S. Dep. Agric. I $529(b)$.

Prof. Papers U.S. Geol. Surv. I $539(e)$.

Prometheus. 607.

Psyche. I 340.

Public Health. 266.

Public Health Bull. I 542 (a).

Public Health Journ. I 307.

Public Mus, Milwaukee. I 399.

Publicat. Junta Ciènc. Nat. Barcelona. 917 $(b-d)$.

Publicat. Amer. Arch. Ethnol. I $325(d)$

Publicat. Bot. Univ. California. I $325(e)$.

Publicat. Circonstance. 422.

Publicat. Civ. Med. Service Neth. Ind. IIgr.

Publicat. Ethnol. Surv. Philipp. Is. I2I7.

Publicat. Field Colnmbian Mus. Chicago. I35 I $(a-f)$.

Publicat. Field Mus. Nat. Hist. Chicago. I 35 I $(a-f)$.

Publicat. Geol. Surv. Queensland. $1233(b)$.

Publicat. Geol. Univ. California. I $325(f)$.

Publicat. Imp. Earthq. Invest. Comm. II8z (b).

Publicat. Mus. Anthr. Ethnogr. St. Pétersb'g. 903.

Publicat. Stanford Univ. I497.

Publicat. U.S. Naval Observ. I 540 (b).

Publicat. Univ. California. I $325(d-g)$.

Publicat. Zool. Univ. California. I325 (g).

Publikat. K. Ethn. Mus. Dresden. 645 (c).

Punjab Hist. Soc. I I I5.

Punjab Notes Quevies, IOI3.

Pusa. I I 4 I.

Quarry. 267.

Quart. Journ. Agriculture. 268.

Quart. Journ. Chem. Soc. IOg (c).

Quart. Journ. Conchology. 269.

Quart. Journ. Dep. Agric. Bengal. 1056.
Quart. Journ. Exp. Physiol. 270.

Quart. Journ. Geol. Soc. London. I 57 (b).

Quart. Journ. Ind. Tea Assoc. 1082.

Quart. Journ. Lit. Sci. Arts. 29 I (d).

Quart. Journ. Medicine. 34 I.

Quart. Journ. Meteor. Soc. London. $293(b)$

Quart. Journ. Microsc. Sci. 27 I.

Quart. Journ. Mythic Soc. IO22.

Quart. Journ. Public Health. 272.

Quart. Journ. Pure Applied Math. 273

Quart. Joum. Sci. 274.

Quart. Journ. Sci. Lit. Arts. 29I (e-f).

Quart. Journ. Univ. Inst. Comm. Research

Tropics. 69.

Quart. Journ. Vet. Sci. India. $\mathrm{IO} 24$.

Quart. Notes Gcol. Surv. Ind. I06I (e).

Quart. Rec. Roy. Bot. Soc. London. 285.

Quebec. I297, I298.

Quekett Microsc. Club. 275.

Oueensland Agric. Journ. I23I (d).

Queensland Geogr. Journ. I235 (c).

Queensland Gov. Mining Journ. I232.

Queensland Mus. I234.

\section{$\mathrm{R}$}

Rabot. Volzhsk. Biol. Stantz. 9I3.

Radium Radioact. Radiations. 533.

Rainfall India. I I I I (e).

Raleigh. I48I, I482.

Rangoon. II $42-$ I I 44

Rapp. Acad. Sci. Caen. 437.

Rapp. Ann. Stat. Agron. Mauritius. $992($ b).

Rapp. Comm. Ned.-Ind. Oudheidk. Onder: I $190(c)$.

Rapp. Gén. Acad. Sci. Caen. 437.

Rapp. Géol. Assoc. Franc. Avanc. Sci. 489.

Rapp. Lab. Etudes Soie. 454.

Rapp. Mus. Hist. Nat. Lyon. $456(b)$.

Rapp. Opér. Minières Quebec. I 297.

Rapp. Progr. Expl. Géol. Canada. I293 (i).

Rapp. Trav. Mus. Hist. Nat. Lyon. $456(b)$.

Rapp. Trav. Soc. Philom. Paris. 562.

Ratisbon. 759,760 .

Real. Acad. Cienc. Madrid. 922

Real. Accad. Lincei Roma. 864.

Real. Accad. Sci. etc. Acireale. 812.

Real. Accad. Sci. Fis. Mat. Napoli. 840.

Real. Accad. Sci. Ist. Bologna. 8I 5.

Real. Accad. Sci. Lett. Arti Zelanti. 8 I 2.

Real. Accad. Sci. Torino. 871.

Real. Comit. Geol. Ital. 865.

Real. Comit. Talassogr. Ital. 876.

Real. Ist. Bot. Torino. 873 .

Real. Ist. Incorrag. Napoli. 84 I.

Real. Ist. Lombardo Sci. etc. 837.

Real. Ist. Tecn. Palermo. $\$ 54$.

Real. Ist. Veneto Sci. etc. 877.

Real. Mus. Commerc. Torino. 874

Real. Ort. Bot. Fiorentino. 824 .

Real. Ort. Bot. Palermo. 852 .

Real. Osserv. Palermo. 854 .

Real. Osserv. Astron. Torino. 872.

Real. Scuola Super. Agric. Portici. 860.

Real. Soc. Geogr. Madrid. 924.

Real. Staz. Bacologia Padova. 847. 
Real. Staz. Entomol. Agraria. 825 . Real. Ufficio Geol. Ital. 866.

Rec. Albany Mus. 984.

Rec. Austral. Mus. $1262(b)$.

Rec. Bot. Surv. Ind. I05I.

Rec. Canterbury Mus. 1237.

Rec. Geol. Dep. Mysore. IOI9 (c).

Rec. Geol. Surv. Ind. I06 I $(f)$.

Rec. Geol. Surv. N.S.IT. $1267(e)$.

Rec. Geol. Surv. Tasmania. I24I (b).

Rec. Geol. Surv. Victoria. I25I (e).

Rec. Ind. Mus. 1099 (b).

Rec. Nagpur Mus. I 35.

Rec. Surv. Ind. $\operatorname{Iog} 8(i)$.

Rec. West. Austral. Mus. 1259.

Rec. Zool. Literature. $327(b)$.

Recreations Agric. Nat.-Hist. etc. 276.

Recueil Actes Acad. Sci. Bordeaux. 434 (c).

Receuil Ophtalmologie. 534.

Recueil Périod. Soc. Géol. Suisse. 943.

Recueil Piéces Acad. (Roy.) Sci. France. 5 OI $(i)$.

Recueil Trav. Bot. Néerland. $810(b)$.

Recueil Voy. et Mém. Soc. Géogr. Paris. 553 (d).

Redia. 825 .

Reduction Greenwich Meteor. Obs. $59($ a).

Refugium Botanicum. 277.

Regensburg see Ratisbon.

Regenwarn. Ned.-Ind. II93 (b).

Régi Magyar Költök Tára. 355 (e).

Regia Soc. Med. Havniensis. 429.

Regia Soc. Upsaliensis. 937.

Regia Univ. Genova. 833.

Regia Univ. Napoli. 842,843 .

Regia Univ. Padova. 848 .

Regia Univ. Pisa. 856,857 .

Regia Univ. Torino. 875 .

Rend. Accad. Sci. etc. Acireale. 812 $(a-e)$.

Rend. Accad. Sci. Fis. Mat. Napoli. $840(b)$.

Rend. Accad. Sci. Ist. Bologna. 8I5 $(g-i)$.

Rend. Circ. Mat. Palermo. 851.

Rend. R. Accad. Lincei. $864(b, c, e)$.

Rend. R. Accad. Sci. etc. Acireale. $812(a-e)$.

Rend. R. Accad. Sci. Fis. Mat. Napoli. 840 (b).

Rend. R. Accad. Sci. Ist. Bologna. 8 I 5 (g-i). Rend. R. Ist. Lomb. Sci. etc. $837(d)$.

Rend. Sess. Accad. Sci. Bologna. 815 (g-i).

Rend. Soc. Agrar. Bologna. 814.

Rennes. 566,567 .

Rep. Agric. Exp. Stat. Rhode I. $\mathrm{I}_{385}(b)$.

Rep. Agri-Hort. Soc. Bombay. 1027.

Rep. Agric. Stat. Cent. Prov. Ind. II $34(b)$.

Rep. Arch. Dep. Mysore. IoI6 (d).

Rep. Arch. Surv. Ind. I046 (d), I I $47(a, b)$.

Rep. Arch. Surv. Ind. Burma Circle. II 32.

Rep. Arch. Surv. Ind. Front. Circle. I1 38 $(a, b)$.

Rep. Arch. Surv. Ind. N. Circle. I I $3(a-f)$.

Rep. Arch. Surv. Ind. N.-IV. Frontier. I I $3^{8}$ (b).

Rcp. Arch. Surv. Ind. S. Circle. III $8(a)$.

Rep. Arch. Surv. N. India. III3 $(g)$.

Rep. Arch. Surv. S. India. I I $9(a, b)$.

Rep. Arch. Surv. H. India. IOzO $(b, c)$.
Rep. Ashmolean Nat. Hist. Soc. 340.

Rep. Australas. Assoc. Adv. Sci. 1260.

Rep. Board Agric. Forest. Hawaii. ${ }_{2} 205$ (b).

Rep. Brit. Assoc. Adv. Sci. 96.

Rep. Bur. Fisheries. I525 (c).

Rep. Bur. Mines Toronto. I 299.

Rep. California State Mining Bur. 1485 .

Rep. Chief Insp. Mines India. I058.

Rep. Chief U.S. Weather Bur. I $533(b)$.

Rep. Colombo Observ. I IO4 (b).

Rep. Comm. Agric. U.S.A. I $529(\mathrm{~g})$.

Rep. Comm. Control S. Afric. Locust Bur. 976.

Rep. Conm. Fish. Maine. I3I5.

Rep. Comm. Mediterr. Fever. $298(k)$.

Rep. Cuvierian Soc. Cult. Sci. I6.

Rep. Danish Biol. Stat. 423.

Rep. Dep. Agric. Cent. Prov. Ind. II $34(c)$.

Rep. Dep. Agric. S. Austral. 1227 (b).

Rep. Dep. Mines Nova Scotia. I284.

Rep. Direct. Meteor. Obs. N. York. I 449.

Rep. Direct. U.S. Mint. I543 $(a, b)$.

Rep. Dove Marine Lab. I7.

Rep. Ent. Dep. N. Jersey Agr. Exp. Stat. I409 (c).

Rep. Essent. Oils Synth. Perf. 747.

Rep. Evolution Committee. $298(\mathrm{~m})$.

Rep. Exp. Farms Canada. I290.

Rep. Explor. Surv. Mississippi R. to Pacific. I $545(b)$.

Rep. Fruit Growers' Ass. Ontario. 1303.

Rep. Geol. Explor. Fortieth Parallel. I5I5.

Rep. Geol. Explor. N. Zealand. I278 (c).

Rep. Geol. Nat. Hist. Surv. Canada. 1293 $(i, j)$.

Rep. Geol. Nat. Hist. Surv. Minnesota. 1402 (e).

Rep. Geol. Surv. Canada. $\mathrm{I} 293(i, j)$.

Rep. Geol. Surv. India. Iобі $(a, b)$.

Rep. Geol. Surv. Iowa. I 367.

Rep. Geol. Surv. Japan. I $83(b, c)$.

Rep. Geol. Surv. Kentucky. 1373.

Rep. Geol Surv. Missoutri. I $383(d)$.

Rep. Geol, Surv. Natal Zululand. 989

Rep. Geol. Surv. Ohio. I 36 I $(b, c)$.

Rep. Geol. Surv. Pennsylv. I $376(a, b)$.

Rep. Geol. Surv. Queensland. I233 (b).

Rep. Geol. Surv. Transvaal. $997(b)$.

Rep. Geol. Surv. W. Austral. I258 (a).

Rep. Gov. Entomol. Natal. $988(b)$.

Rep. Gov. Epigraphist Madras. I I $8(b)$.

Rep. Hawaii Agric. Exp. Stat. $1206(a)$.

Rep. Imp. Bur. Fisheries Tokyo. IISI.

Rep. Imp. Customs Dep. India. ${ }_{1083 .}$

Rep. Imp. Inst. I $73(e)$.

Rep. Ind. Ass. Cult. Sci. I070 (c).

Rep. Inl. Customs Dep. India. Io83.

Rep. Inspect. Mines India. I058.

Rcp. Iowa Geol. Surv. I 367 .

Rep. Kodaikanal Madras Observs. I I $30(e)$.

Rep. Lanc. Sea-Fisheries Lab. 65.

Rep. Malaria Committee. $298(n)$.

Rep. Marine Biol. Ceylon. $\mathrm{IO}_{1}(b)$.

Rep. Marine Biol. Lab. Ceylon. I $103(b)$.

Rep. Marine Surv. India. 1084.

Rep. Meteor. Ceylon. IIO4 $(b, c)$. 
Rep. Meteor. India. I I5 I $(f)$.

Rep. Meteor. Observ. N. York. I 449. Rep. Meteor. Reporter Bengal. I092. Rep. Michigan Acad. Sci. $\quad 1387$. Rep. Micro.-Biol. Sect. King Inst. Med. I 26 Rep. Mining Indust. N. Zealand. 1283. Rep. Mining Oper. Quebec. 1297. Rep. Nat. Acad. Sci. Washington. I $517($ d). Rep. Norweg. Fish. Marine Invest. 879. Rep. Norweg. Marine Invest. $878(\mathrm{c})$. Rep. New York Meteor. Observ. I 448. Rep. New York State Mus. Nat. Hist. 1309 (e).

Rep. New York State Weather Bur. I38I.

Rep. Ohio Geol. Surv. I $361(b, c)$.

Rep. Patent Cases. 254 (c).

Rep. Pennsylv. Agr. Exp. Stai. I499(b).

Rep. Pennsylv. State College. 1498.

Rep. Product. Precious Metals U.S.A. I 543 (b).

Rep. Progr. Agric. India. II4I (d).

Rep. Progr. Geol. Surv. Canada. $1293(i, j)$. Rep. Progr. Geol. Surv. Japan. I $183(b, c)$. Rep. Progr. Geol. Surv. Ohio. I36I (c).

Rep. Progr. Geol. Surv. Pennsylv. I $376(b)$. Rep. Progr. Geol. Surv. Victoria. 125 I (f).

Rep. Progr. Imp. Geol. Surv. Japan. II $8_{3}$ (b).

Rep. Progr. Ordnance Surv. Gt. Brit. 251.

Rep. Rainfall Reg. Mysore. IOzO (c).

Rep. Roy. Soc. Tasmania. 1243.

Rep. Roy. Surv. Dep. Siam. II 54.

Rep. St. Thomas's Hospital. 302.

Rep. Schimmel \& Co. 747.

Rep. Secret. Mines Tasmania. I 240.

Rep. Sleeping Sickness Commission. $298(l)$.

Rep. Soc. Study Disease Children. 3 IO.

Rep. State Mining Eng. S. Africa. 996.

Rep. Super. U.S. Coast Geod. Surv. I $528(b)$.

Rep. Suvv. N. Zealand. I279.

Rep. Thomson Yates Labs. 318.

Rep. U.S. Bur. Fisheries. I $525(c)$.

Rep. U.S. Coast Geod. Surv. I $528(b)$.

Rep. U.S. Commiss. Agric. I $529(\mathrm{~g})$.

Rep. U.S. Commiss. Fish Fisheries. 1525 (c).

Rep. U.S. Entomol. Commiss. I535(a).

Rep. U.S. Geogr. Surv. W. Iooth Mer. I 537.

Rep. U.S. Geol. Geogr. Surv. Territ. I $538(d)$.

Rep. U.S. Nat. Mus. I 522 (d).

Pep. Wellcome Research Labs. 986.

Repert. Chem. Theils Mineral. 608.

Repert. Exp.-Phys., Phys. Techn.etc. 752. Repert. Italicum. 816.

Repert. Physik. 752 .

Research Bull. Iowa Agr. Exp. St. I 3 I I (b). Results Astron. Obs. Madras. I $30(f)$.

Results Magn. Meteor. Obs. Greenwich. (b).

Results Meteor. Magn. Obs. Stoneyhurst. 347. Results Meteor. Obs. Juggarow Observ. I 52. Results Meteor. Obs. Vizagapatam. II 52. Results Obs. Fixed Stars Madras. I $30(\mathrm{~g})$. Results Photo-heliogr. Obs. Greenwich. 59 (c). Résumé Gén. Temps Europe. $493(b)$.

Résumés Commun. Congr. Internat. Zool. I 596.
Retrospect Medicine. 278.

Rev. Agric. Maurice, Réunion, Madagascar. 991.

Rev. Archéol. 535.

Rev. Applied Entom. $172(b)$.

Rev. Biol. Nord France. 449.

Rev. Botaniquc. 569.

Rev. Chilena Hist. Nat. I 592.

Rev. Coloniale Internat. 790.

Rev. Comm. Arch. Ind. Portugueza. I IIs.

Rev. Cours Sci. France Etranger. 537.

Rei:. Cultures Coloniales. 538 .

Rev. Crit. Paléozool. 536.

Rev. Eaux Forêts. 539.

Rev. Entomologie. 438.

Rev. Fac. Letras Cienc. Univ. Habana. I 558.

Rev. Gén. Techn. Piscicult. etc. 570

Rev. Geogr. Colon. Mercant. $924(b)$.

Rev. Hist. Religions. $524(c)$.

Rev. Mag. Zool. Pure Appiiq. 540.

Rev. Mineral. Cristallogr. Ital. 849.

Rev. Mus. La Plata. I564 (i).

Rev. Mus. Paulista. I 589.

Rev. Neurol. Psychiatry. 43.

Rev. Pétrole. 394.

Rev. Progr. Cienc. Exact. Fis. Nat. 923.

Rev. Quest. Sci. Bruxelles. $412(b)$.

Rev. R. Acad. Cienc. Madrid. 922 (b).

Rev. Russe Entom,l. 9 II (b).

Rev. Sci. (France Etranger). 54I.

Rev. Sci. Nat. Appliq. 56r.

Rev. Soc. Cient. Ant. Alzate. I572.

Rev. Univ. Mines Métallurgie. 542.

Rev. Zoologique. 550.

Rhode I. College Agric. etc. I385.

Rhodesia Agric. Journ. I000.

Rhodesia Mus. 967.

Ric. Lav. Ist. Bot. Pisa. 857.

Rijks Ethnogr. Mus. Leiden. 805.

Rijks Geol. Mıs. Leiden. 806.

Rijks Mus. Natuurl. Hist. Leiden. 807.

Rijks-Univ. Leidell. 808.

Rio de Janeiro. $1580-1584$.

Riv. Antropologia. 869.

Riv. Ital. Paleont. 855.

Rochester. ${ }_{4} 483$.

Rochester Acad. Sci. I483.

Rome. 86I-869.

Römer's Arch. Bot. 703.

Romisch Kais. Akad. Naturf. $75^{8}$.

Roorkee. I 45 .

Rosenhof's Insect.-Belustigung. 757.

Rotterdam. SII.

Rovartani Lapok. 357.

Rowley's Ornith. Miscellany. $25^{2}$.

Roy. Acad Med. Ireland. 24.

Roy. Agric. Hort. Soc. S. Australia. 1228.

Roy. Agric. Soc. England. 279.

Roy. Anthrop. Inst. Gt. Brit. 280.

Roy. Anthrop. Soc. Australasia. I272.

Roy. Army Med. Corps. 281.

Roy. Asiat. Soc. Bombay. IO43.

Roy. Asiat. Soc. Ceylon. I 106.

Roy. Asiat. Soc. China. I 72 .

Roy. Asiat. Soc. Gt. Brit. 282

Roy. Asiat. Soc. Korea. I 169. 
Roy. Asiat. Soc. N. China. II72.

Roy. Asiat. Soc. Straits. II 77.

Roy. Astronom. Soc. London. 283.

Roy. Bot. Gard. Calcutta. 1096.

Roy. Bot. Gard. Ceylon. II 37 .

Roy. Bot. Gard. Edinb. 44.

Roy. Bot. Gard. Kew. 284.

Roy. Bot. Soc. London. 285.

Roy. Colonial Inst. 286.

Roy. Dublin Soc. 25.

Roy. Econ. Soc. I,oudon. 287.

Roy. Geogr. Soc. Australas. I 254 .

Roy. Geogr. Soc. Australas., Queensl. Branch. I 235 .

Roy. Geogr. Soc. Australas., S. Austral. Branch. I 229.

Roy. Geogr. Soc. Ireland. 26.

Roy. Geogr. Soc. London. 288.

Roy. Geol. Soc. Cornivall. 343.

Roy. Geol. Soc. Ireland. 27.

Roy. Horticult. Soc. London. 289.

Roy. Inst. Brit. Architects. 290.

Roy. Inst. Gt. Britain. $29 \mathrm{I}$.

Roy. Irish. Acad. 28.

Roy. Magn. Meteor. Observ. Batavia. I 193.

Roy. Med. Chirurg. Soc. London. 292.

Roy. Meteor. Soc. London. 293.

Roy. Microsc. Soc. London. 294.

Roy. Naval Med. Service. 295.

Roy. Numismatic Soc. London. 296.

Roy. Observ. Cape Town. 974.

Roy. Observ. Edinb. 45.

Roy. Observ. Greenwich. 59.

Roy. Phil. Soc. Glasgow. 57.

Roy. Phys. Soc. Edinb. 46.

Roy. Sanitary Inst. 297.

Roy. Scott. Geogr. Soc. 47.

Roy. Scott. Soc. Arts. 48 .

Roy. Soc. Arts London. 299.

Roy. Soc. Canada. I $2 y 6$.

Roy. Soc. Edinburgh. 49.

Roy. Soc. London. 298.

Roy. Soc. Med. London. 300.

Roy. Soc. N. S. Wales. 1273.

Roy. Soc. Queensland. 1236.

Roy. Soc. S. Africa. 975.

Roy. Soc. S. Australia. 1230.

Roy. Soc. Tasmania. I 243.

Roy. Soc. Van Diemen's I. I 244 .

Roy. Soc. Victoria. I255.

Roy. Statist. Soc. London. 301.

Roy. Survey Dep. Siam. I I 54 .

Roy. Zool. Mus. Netherlands. $807(b)$.

Roy. Zool. Soc. N. S. Wales. I 274 .

Russ. Entomol. Obshch. 9 II.

Russ. Entomol. Obozryenie. 9 II (b).

Russ.-Kais. Mineral. Ges. 9 Io.

\section{$\mathrm{S}$}

Sacramento. 1484,1485 .

Saigon. I 65.

St. Helena. 999.

St. Louis. I486-1488.

St. Louis Acad. Sci. I486.

St. Petersburg see Petrograd.
St. Thomas's Hospital. 302.

Salatiga. I 224 .

Salem. I489-I49I.

Salisbury. I000.

Samml. Geol. Reichs-Mus. Leiden. 806 $(a-c)$.

San Francisco. I492, 1493.

San José. I 585-1587.

Sanitary Rec. 303 .

Santiago. I 590, I 59 I.

São Paulo. I 588, I 589 .

Sarajevo. 364

Saratow. 9I3.

Sarawak Mus. I 209.

Saunder's Refugium Botanicum. 277.

Sbornik Muzeya. 903.

Schaufuss' Zool. Mitt. 640.

Schimmel \& Co. 747.

Schimper's Bot. Mitt. Tropen. 689.

Schles. Ges. Vaterland. Cultur. 624.

Schneider's Zool. Beitr. 626.

School Trop. Med. Liverpool. 68.

School Trop. Med. London. 304.

Schrader's Journ. Bot. 665.

Schrift. Berlin. Ges. $588(d)$.

Schrift. Kais. Miner Ges. St. Petersburg. 9 IO $(b)$.

Schrift. Kön. Phys.-Oekon. Ges. Konigsberg. $700(b)$.

Schrift. Leipzig. Oekon. Soc. $730(b)$.

Schrift. Naturf. Ges. Danzig. 638.

Schrift. Naturf. Ges. Dorpat. $887(b)$

Schrift. Oekon. Soc. Leipzig. $730(b)$.

Schrift. Phys. Oekon. Ges. Konigsberg. 7 7no

(b).

Schweiz. Alpenclub. 94 .

Schweiz. Entomol. Ges. $94^{2}$

Schweiz. Paläont. Ges. 952.

Schweiz. Geol. Ges. 943.

Sci. Abstracts. 305 .

Sci. American. $\quad$ I452.

Sci. American Suppl. I 453.

Sci. Assoc. Hamilton. I 286.

Sci. Assoc. Trinidad. 1579.

Sci. Bull. Brooklyn Mus. I 337 (d).

Sci. Invest. Fisheries Ireland. I8.

Sci. Invest. Fishery Board Scotland. 52.

Sci. Invest. Imp. Bur. Fish. Tokyo. I I I.

Sci. Mem. 303.

Sci. Mem. Med. Off. Army Ind. $1079(b)$.

Sci. Mem. Off. Med. San. Deps. Ind. 1079 (c).

Sci. Proc. Roy. Dublin Soc. 25 (c).

Sci. Progress. 306.

Sci. Progress in 20th Cent. 307.

Sci. Reps. Tôhoku Imp. Univ. I I66 $(a, b)$.

Sci. Results Chesapeake Zoöl. Lab. I32 I (a).

Sci. Trans. Roy. Dublin Soc. $25(d)$.

Scienz. Ital. 858 .

Scott. Geogr. Mag. 47.

Scott. Naturalist. 50, 344 .

Scranton. I494, I495.

Séance Publ. Acad. Sci. Bordeaux. $43+(d)$. Séance Publ. Soc. Linn. Normand. 440 (c).

Séances Soc. Franc. Physique. 556.

Secc. Meteor. Est. Yucatán. I 566. 
Secr. Agr. Comerc. Trab. Cuba. I 557.

Sect. Agr. Industr. Conmerc. Cuba. 1557.

Seeman's Journ. Bot. I92.

Sei-i-Kwai Med. Journ. I 186.

Seismol. Soc. Japan. I I 89.

Sel. Publ. Corresp. Gov. N.-W. Prov. I007 $(a)$.

Sel. Publ. Corresp. Punjab Administr. I114 (a).

Sel. Rec. Bengal Gov. 1063.

Sel. Rec. Bombay Gov. 1033.

Sel. Rec. Gov. India. II 50.

Sel. Rec. Gov. N.-W. Prov. $\operatorname{IOO}(a, b)$.

Se?. Rec. Gov. Punjab. III4 $(a, b)$.

Sel. Rec. Madras Gov. II 23.

Scmi-ann. Rep. Esscut. Oils Synth. Perf. 747.

Semi-ann. Rep. Schimmel \& Co. 747.

Senckenb. Naturf. Ges. 655.

Sendai. I I66, I I67.

Seoul. I I68, I I69.

Service Carte Géol. France. 543.

Service Géol. Indo-Chine. I I 58.

Service IIeteor. São Paulo. I 588.

Serviz. Agrar. Eritrea Somalia.

's Gravenhage see Hague.

Shanghai. II 70-I I 72 .

Shillong. I 46 .

Siam Soc. I I 55 .

Simla. I I 47-I I 5 I.

Singapore. II73-II77.

Sitzungsb. Abh. Naturw. Ges. 'Isis.' 646(b).

Sitzungsb. Deut. Naturw.-Med. Ver. 'Lotos.' $362(f)$.

Sitzungsb. Ges. Bcförd. Naturw. Marburg. 745 .

Sitzungsb. Ges. Naturf. Freunde Berlin. $588(e)$

Situngst. K. Acad. Wiss. W'ien. $372(g, h)$ Sitzungsb. K. Bayer. Akad. W'iss. $750(i-k)$. Sitzungsb. K. Preuss. Akad. Wiss. $600(e)$.

Sitzungsb. Med.-naturwiss. Ges. Jena. 693(c).

Sitzungsb. Naturf. Ges. Dorpat. $887(c)$.

Sitzungsb. Naturw. Ges. 'Isis.' 646.

Sitzungsb. Naturw.-Med. Ver. 'Lotos,' $362(f)$.

Sitzungsb. Niederrh. Ges. Nat. Heilk. 621.

Sitzungsb. Physik.-med. Soc. Erlangen. 650.

Sitzungsb. Ver. Naturw. Unterhalt. 682.

Sitzungsb. Zool.-bot. Ver. Wien. 38 I (b)

Skand. Litt.-selskab. 430.

Skrift. K. Dansk. Vidensk. Sclsk. $424(c)$.

Skrift. K. Humanist. T'et.-Samtund. 936.

Skrift. K. Norske Vidensk. Selsk. 886.

Skrift. Skand. Litt.-Selskab. 430.

Skrift. Vidensk.-Selsk. Christiania. $885(b, c)$

Skrivt. K. Dansk. Vidensk. Selsk. $424(d)$.

Skrivt. Naturh.-Selsk. Copenhagen. 426.

Sleeping Sickness Bureau. 300 .

Sleeping Sickness Commission. 298 (l).

Smithson. Contrib. Knowledge. I 5 I9 (b).

Smithson. Inst. I5 I9-1522.

Smithson. Misc. Coll. I $519(c)$.

Soc. Acad. Indo-Chinoise. 544.

Soc. Adriat. Sci. Nat. 366.

Soc. Afric. Ital. 826,844 .

Soc. Agraria Bologna. 8 I4.
Soc. Agric. Départ. Scine. $\quad 564$

Soc. Agric. Insectol. 548.

Soc. Agric. Sci. Indust. Lyon. 457 .

Soc. Anthropol. Lyon. +58 .

Soc. Anthropol. aris. $55 \mathrm{I}$.

Soc. Antiquaries London. 3 I I.

Soc. Arts London. 299.

Soc. Asiat. Ital. 827.

Soc. Asiatique. 545.

Soc. Astron. France. 546.

Soc. Belge Etudes Coloniales. 106.

Soc. Belge Géogr. 409.

Soc. Belge Géol. Paléontol. Hydrol. 407.

Soc. Biol. Paris. 552.

Soc. Bot. France. 547,569 .

Soc. Bot. Ital. 828 .

Soc. Centr. Apicult. Insectol. 548 .

Soc. Chem. Industry London. 3 I2.

Soc. Chim. France. 549.

Soc. Cient. Antonio Alzate. I 572.

Soc. Cuvierienne. 550 .

Soc. Encouragement Arts etc. 297 (c).

Soc. Entomol. Belgique. 408.

Soc. Entomol. France. 555 .

Soc. Entomol. Pays-Bas. $802(b)$.

Soc. Entomol. Ital. 829.

Soc. Entomol. Russe. 9II.

Soc. Entomol. Suisse. 942.

Soc. Etudes Agric. Trop. 42 I.

Soc. Ettudes Coloniales Belge. 406.

Soc. Etudes Sci. Angers. 433.

Soc. Faun. Flor. Fennica. 893.

Soc. Finland. Géogr. 89 I.

Soc. Finno-Ougrienne. 895.

Soc. Franc. Botanique. 569.

Soc. Franç. Entomol. $43^{8}$.

Soc. Franç. Physique. 556.

Soc. Fribourg. Sci. Nat. 944.

Soc. Géogr. Commerciale Bordeaux. 435.

Soc. Géogr. Commerciale Havre. 446.

Soc. Géogr. Finlande. 894.

Soc. Geogr. Lisboa. 920.

Soc. Géogr. Lyoll. 459.

Soc. Geogr. Madrid. 924.

Soc. Géogr. Paris. 553.

Soc. Géol. Belgique. 4I 7 .

Soc. Géol. France. 557.

Soc. Geol. Mexicana. I 573.

Soc. Geol. Milano. $838(a)$.

Soc. Géol. Nord France. 450.

Soc. Géol. Normandie. 447.

Soc. Geol. Ital. 867.

Soc. Géol. Suisse. 943 .

Soc. Helvét. Sci. Nat. 957.

Soc. Hongroise Géogr. $35 \mathrm{I}$.

Soc. Imp. Nat. Moscow. 897.

Soc. Imp. Nat. St. Pétersbourg. 912

Soc. Imp. Sci. Nat. Math. Cherbourg. +42 .

Soc. Indo-Chinoise. 544.

Soc. Ital. Antropol. Etnol. 830

Soc. Ital. Progr. Sci. 868.

Soc. Ital. Sci. Nat. 838 .

Soc. Linn. Bordeaux. 436.

Soc. Linn. Calvados. 439.

Soc. Linn. Lyon. 460.

Soc. Linn. Nord France. $\$ 32$ 
Soc. Linn. Normandie. $44^{\circ}$.

Soc. Linn. Paris. $55^{8}$.

Soc. Malacol. Belgique. $41 \mathrm{I}$.

Soc. Math. France. 559.

Soc. Minéral. France. 560.

Soc. Mus. Hist. Nat. Strasbourg. 766.

Soc. Nac. Agr. Rio de Janeiro. I 584.

Soc. Nat. Acclimat. France. $56 r$.

Soc. Nat. Sci. Nat. Math. Cherbourg. 442.

Soc. Nat. Hist. Boston. 1334.

Soc. Nat. Hist. Cincinnati. 1358.

Soc. Nat. Kiev. 896.

Soc. Nat. Nouv. Russie. 898

Soc. Nat.'Sci. Buffalo. 1338.

Soc. Neuchatel. Sci. Nat. 956.

Soc. Paléont. Suisse. 952.

Soc. Path. Exotique. 554.

Soc. Philomat. Paris. 562.

Soc. Phys. Hist. Nat. Genève. 953.

Soc. Portug. Sci. Nat. 921.

Soc. Privata Taurinensis. $871(f)$.

Soc. Prop. Conn. Sci. Indust. Paris. 563.

Soc. Quím. Argentina. 1.55 .

Soc. Sci. Bruxelles. 4 I 2.

Soc. Sci. Chili. I 591 .

Soc. Sci. Fennica. $889(a)$.

Soc. Sci. Indo-Néer. I I94 (b).

Soc. Sci. Méd. Ouest. 566.

Soc. Sci Nat. Econom. Palermo. 854.

Soc. Sci. Nat. Math. Cherbourg. $44^{2}$.

Soc. Sci. Nat. Neuchatel. 956.

Soc. Sci. Nat. Ouest France. 467 .

Soc. Sci. Nat. Strasbourg. 766.

Soc. Sismol. Ital. 839.

Soc. Spettrosc. Ital. 853 .

Soc. Study Disease Children. 310.

Soc. Reale Napoli. 840,

Soc. Ronnana Antropol. 869.

Soc. Roy. Agric. Paris. 564.

Soc. Roy. Antiq. Nord. 425 .

Soc. Roy. Belge Géogr. 409.

Soc. Roy. Bot. Belgique. 4 ro.

Soc. Roy. Canada. 1296.

Soc. Roy. Centr. Agric. Paris. 564.

Soc. Roy. Géogr. Anvers. 396.

Soc. Roy. Sci. Liége. 418.

Soc. Roy. Zool. Malacol. Belgique. $4^{\text {I I }}$

Soc. Telegraph Eng. 313.

Soc. Tosc. Sci. Nat. 859.

Soc. Trop. Med. Hygiene. 3 I 4.

Soc. Vaud. Sci. Nat. 955.

Soc. Zool. France. 565 .

Soerabaia see Surabaya.

South Afric. Centr. Locust Bur. 976.

South Afric. Mus. 977.

South Afric. Phil. Soc. 978.

South Ind. Assoc. II 3 I.

Southall Bros. \& Barclay, Analyt. Labs. 2. Special Bull. Michigan Agr. Exp. Stat. $1388(b)$.

Special Bull. U.S. Nat. Mus. $1522(e)$.

Special Rep. Rhodesia Mus. 967.

Spengel's Zool. Jahrb. 696.

Spicilegia Zoologica. 3 35,607.

Spolia Zeylanica. I $105(b)$.

Sprengel's Neue Entdeckungen. 729.
Springfield. 1496.

Städt. Mus. Völkerk. Leipzig. 733

Stahl und Eisen. 649.

Stainton's Entomol. Annual. I4I.

Stanford University. I 497.

State Board Agric. Massachusetts. I 335.

State College. I 498, I 499.

State College Kentucky. I 392.

State Entomologist New York. I310.

Station Agronom. Mauritius. 992.

Station Entomol. Fac. Sci. Rennes. 567.

Station Pisc. Hydrobiol. Toulouse. 570.

Statist. Soc. London. $30 \mathrm{I}$.

Steiermark see Graz.

Stettin. $76 \mathrm{I}$.

Stillwater. I 500 .

Stockholm. 929-935.

Storrs. I 501 .

Strasburg. $762-767$.

Stray Feathers. I097.

Studi Ric. Chem. Agrar. $856(b)$.

Studies Biol. Lab. John Hopkins Univ. $1321(a)$.

Studies Biol. Manchester. 334.

Studies Hist. Polit. Sci. $1320(d)$.

Studies Inst. Med. Research Fed. Malay States. I 74 .

Studies Leprosy. I $542(b)$.

Studies Morphol. Lab. Cambridge. 15.

Studies Mus. Zool. Dundee. 29.

Studies Toronto Univ. $1308(a-d)$.

Studies Tufts College. 1504.

Studies Univ. Colorado. I 336.

Studies I Iniv. Toronto. I $308(a-d)$.

Studies Yale Psychol. Lab. 1413.

Studies Zool. Lab. Univ. Nebraska. I $393($ a).

Stuttgart. $767-775$.

Sugar Cane. 185.

Summ. Rep. Geol. Surv. Canada. I 202 (c).

Summ. Rep. Mines Branch Canada. I $292(d)$.

Suppl. Pap. Roy. Geogr. Soc. London. $288(d)$.

Survey Dep. Egypt. 970.

Survey Dep. Papers Egypt. 970.

Survey Dep. Siam. I 54 .

Survey India. I098.

Sveriges Geol. Undersökning. 935.

Swainson's Zool. Illustr. 323.

Sweet's Brit. Flower Garden. 99.

Sydney. I 260-1 275.

Sydow's Ann. Mycol. 572.

Symbolae Antillanae. 610.

Synops. Res. Gt. Trig. Surv. Ind. $1098(k)$.

\section{$\mathrm{T}$}

Tab. Reiseber. Meteor. Schiffstagebïch. 597.

Taiping. I 78 .

Tananarive see Antananarivo.

Taprobanian. I107.

Techn. Papers U.S. Bur. Mines. $1526(b)$.

Techn. Papers U.S. Bur. Standards. I $527(c)$.

Technol. Mus. N.S.Wales. I 275.

Teikoku Daigaku. 1185.

Telegr. Journ. \& Electr. Rev. 3I6.

Természetrajzi Fiizetek. $353(b)$. 
Terrestr. Magnetism. 1359

Teschendorf. 776 .

Teutschland Geogn.-geol. Dargestellt. 777.

Texas Acad. Sci. I 3 I 7 .

Tersmannia. 1195.

Therapeut. Gazette. 1368.

Therapist. $3 \mathrm{I} 7$.

Thomason College Roorkee. 1 I 45.

Thomson Yates (\& Johnson) Labs.

Tidssk. Naturvidenskaberne. 43I.

Tiflis. 1001,1002 .

Tiflissk. Bot. Sad. IOU2.

Tijdschr. Entomol. $802(c)$.

Tijdschr. Ind. Taal-Land-Volkenk. $1190(d)$.

Tijdschr. Natumrl. Geschied. Physiol. 791.

Tijdschr. Ned. Dierk. Vereenig. SII.

Tijdschr. Ned. Maatsch. Bevord. Nipverh. 798.

Tijdschr. Neerl.-Ind. I I96.

Tijdschr. Plantenziekten. 414

Tohoku Imp. Univ. I I 66.

Tóhoku Math. Journ. I I6-.

\section{Tokyo. i179-1187.}

Torino see Turin.

Toronto. I 299-1 308 .

Toronto Univ. 1308.

Torrey Bot. Club. $\quad 1+54$

Tortosa. 927 .

Toulouse. $568-570$.

T'oung Pao. 809 .

Trans. Agric. Hort. Soc. India. 1044 (c).

Trans. Agric. Hort. Soc. W. India. 1026.

Trans. Amer. Ent. Soc. 1463.

Trans. Amer. Ethnol. Soc. 1419.

Trans. Amer. Inst. Mining Eng. $1421(b)$.

Trans. Amer. Math. Soc. I424 (b).

Trans. Amer. Phil. Soc. I466(e).

Trans. Amer. Soc. Civil Eng. $1427(b)$.

Trans. Anthropol. Soc. Washington. 1513.

Trans. Asiat. Soc. Japan. 1188.

Trans. Astron. Observ Yale. I414.

Trans. Australas. Inst. Mining Eng. 124.5.

Trans. Belfast Nat. Hist. Phil. Soc. 22.

Trans. Biol. Soc. L'pool. 62.

Trans. Bombay Geogr. Soc. 1032.

Trans. Bombay Lit. Soc. 1038.

Trans. Bombay Med. Phys. Soc. 1039.

Trans. Bot. Soc. Edinb. $31(a)$.

Trans, Brit. Pharmacent. Conf. I04.

Trans. California State Agr. Soc. 1484.

Trans. Camb. Phil. Soc. ${ }_{14}(b)$.

Trans. Canad. Inst. $1300(d)$.

Trans. Clinical Soc. II 7 .

Trans. Connecticut Acad. Arts Sci. I4 I I (b).

Trans. Cork Cuvierian Soc. 22.

Trans. Devon Assoc. Adv. Sci. etc. 345.

Trans. Dublin Nat. Hist Soc. 22.

Trans. Dublin Unir. Zool. Assoc. 22.

Trans. Edinb. Bot. Soc. 31 (a).

Trans. Edinb. Geol. Soc. 37.

Trans. Entomol. Soc. I 39.

Trans. Entomol. Soc. N. S. Wales. I 266.

Trans. Epidemiol. Soc. 144.

Trans. Ethnol. Soc. $145(b)$.

Trans. Faraday Soc. 146.

Trans. Fed. Inst. Mining Eng. 336.
Trans. Folklore Sioc. I $50(d)$.

Trans. Geogr. Soc. Bombay. 1032.

Trans. Geol. Mining Soc. Manch. 330.

Trans. Geol. Soc. Australasia. 1250.

Trans Geol. Soc. Edinburgh. 37.

Trans. Geol. Soc. London. I57 (c).

Trans. Geol. Soc. Manchester. 330.

Trans. Geol. Soc. Pennsylvania. 1468.

Trans. Geol. Soc. S. Africa. 985.

Trans. Grant College .Med. Soc. 1034.

Trans. Highl. Agr. Soc. Scotland. $39(b)$.

Trans. Horticult. Soc. London. 289 (b).

Trans. Ind. Eng. Assoc. 1074.

Trans. Inst. Civ. Engineers. $178(b)$.

Trans. Inst. Mining Eng. 336.

Trans. Inst. Mining Metallurgy. $18 \mathrm{I}$.

Trans. Inst. Patent Agents. 177.

Trans. Internat. Congr. Ent. I $595(b)$.

Trans. Iron Steel Inst. $188(\mathrm{c})$.

Trans. Glasgow Geol. Soc. 53.

Trans. Linn. Soc. London. $203(c-e)$.

Trans. Lit. Hist. Soc. Quebec. 1298.

Trans. Lit. Sci. Inst. Kilkenny. 22.

Trans. Lit. Soc. Bombay. 1038.

Trans. L'pool Biol. Soc. 62.

Trans. Manch. Geol. Soc. 330.

Trans. Manch. Geol. Mining Soc. 330.

Trans. Massach. Hort. Soc. 1332.

Trans. Med. Phys. Soc. Bombay. 1039.

Trans. Med. Phys. Soc. Calcutta. 1086.

Trans. Microsc. Soc. London. $294(c)$.

Trans. Mining Geol. Inst. India. I094.

Trans. Nation. Ass. Prom. Social Sci. 237.

Trans. New York Acad. Sci. $1416(c)$.

Trans. New Zealand Inst. 1280.

Trans. North Engl. Inst. Min. (Mechan.) Eng. 338.

Trans. Nova Scotian Inst. Sci. 1285.

Trans. Pharmaceut. Soc. 250.

Trans. Phil. Inst. Victoria. $1255(e)$.

Trans. Phil. Soc. Adclaide. $1230(c)$.

Trans, Phil. Soc. N.S. Wales. $1273(c)$.

Trans. Proc. Edinb. Bot. Soc. 3 I (b).

Trans. Proc. Geol. Soc. S. Africa. 985.

Trans. Proc. Rep. Phil. Soc. Adelaide. I $230(c)$.

Trans. Proc. Rep. Roy. Soc. S. Austral. $1230(b)$.

Trans. Proc. Roy. Soc. N.S. Wales. $1273(b)$.

Trans. Proc. N. Zealand Inst. I 280.

Trans. Ouebec Lit. Hist. Soc. 1298

Trans. Roy. Acad. Med. Ireland. 24.

Trans. Roy. Asiat. Soc. Gt. Brit. $282(b)$.

Trans. Roy. Asiat. Soc. Forea. I 69.

Trans. Roy. Geol. Soc. Cornwall. 343.

Trans. Roy. Inst. Brit. Archit. $290($ d).

Trans. Roy. Irish Acad. $28(i-m)$.

Trans. Roy. Microsc. Soc. London. 294 (b).

Trans. Roy. Scott. Soc. Arts. $48(b)$.

Trans. Roy. Soc. Canada. 1296.

Trans. Roy. Soc. Edinb. $49(b)$.

Trans. Roy. Soc. N.S. Wales. $1273(b)$.

Trans. Roy. Soc. S. Africa. 975.

Trans Roy. Soc. S. Austral. I230 (b).

Trans. Roy. Soc. Victoria. $1255(b-d)$.

Trans. St. Louis Acad. Sci. I 486. 
Trans. Scismol. Soc. Japan. 1189.

Trans. Soc. Arts London. 299 (c).

Trans. Soc. Trop. Med. Higiene. $314(a)$.

Trans. South Afric. Phil. Soc. 978.

Trans. Texas Acad. Sit. 1317.

Trans. Tyneside Nats'. Field Club. 339.

Trans. Wagner Free Inst. Sci. I 478.

Trans. Washington Anthropol. Soc. I513.

Trans. Wisconsin Acad. Sci. etc. 1398.

Trans. Zool. Soc. London. 327 (c).

Trans. R. Accad. Lincei. $864(d)$.

Transvaal Agric. Journ. (193.

Transuaal Med. Journ. 983.

Transvaal Mus. 998.

Trav. Comm. Sci. Arch. Orenburg. 899.

Trav. Lah. Géol. Grenoble. 445.

Trav. Lab. Wat. Méd. Paris. 498.

Traz. Mus. Bot. St. Pétersbourg. 902.

Trav. Mus. Géol. Pierrele Grand. 904.

Trav. Sect. Géol. St. Pétersbourg. 906.

Trai. Sci. Lab. Zool. etc. Concarneau. 443.

Trav. Soc. Imp. Nat. St. Pétershourg $9 \mathrm{I} 2$.

Trenton. $1502,1503$.

Trento1 Nat Hist. Soc. $1: 0,3$.

Trieste. 365,366 .

Tring. $34^{8}$.

Tring Museum. 348 .

Trinidad Board Agric. 1578 .

Trondhjem. 886 .

Tropenpflanzer. $598(a, b)$.

Tropical Agriculturist. 1137 (c).

Tropical Diseases Bull. 174.

Tropical Diseases Bur. 174.

Tropical Life. 319

Trud. Gheol. Chasti S.-Peterburgh. 906.

Trud. Gheol. Komit. S.-Peterburgh. $907(b)$.

Trud. Imp. S.-Peterl. Bot. Sad. 908.

Trud. Imp. S.-Petcrb. Ohshch. Estestvoisp. 912.

Trud. Russ. Entomol. Obshch. 911 (a).

Trud. Ti issk. Bot. Sad. 1002.

Trud. Uchen. Arkhion. Lom. Orenburg. 899.

Tschermak's Miner. Petrogr. Mitt. 382.

Tufts College. 1504 .

Tufts College Studies, 1504.

Turin. $870-875$.

Tyneside Nats.' Fie!d Club. 3,39

\section{$\mathrm{U}$}

Uccle. 421.

Uchen. Arkhiv11. Kom. Orenburg. 899.

Uebers. Arb. Terand. Schles. Ges. Vaterl. Cultur. $624(d)$.

Ungar. Akad. Wiss. 356,358

Ungar. Bot. Blätter. 350.

Ungar. Geol. Ges. 352.

Ungar. Nat. Mus. 353.

Ungar, Reoue. 358 .

United Empire. 286.

United Prov. Hist. Soc. 1014.

United States Bur. Fisheries. 1,25

U.S. Bur. Med. Surg. 1534.

U.S. Bur. Mines. I 526.

U.S. Bur. Standards. 1527 .

U.S. Coast reod. Surv. 1528.
U.s. Dep. Agric. 1529 .

U.S. Dep. Agric., Div. Biol. Surv. I 530 .

U.S. Dep. Agric, Div. Bot. I $\$ 22(b)$.

U.S. Dep. Agric., Div. Entomol. I531.

U.S. Dep. Agric, Div. Or1. Mamm. 1530.

U.S. Dep. Agric., Entomol. Commiss. 1; 35 .

U.S. Dep. Agric. Exp. Stats. 1532.

U.S. Dep. Agric. Weather Bur. I533.

U.S. Dep. Navy. 1534 .

U.S. Entomol. Commiss. 1535

U.S. Fish Commission. I 525 .

U.S. Geogr. Surv. Rocky Mt. Reg. 1536.

U.S. Geogr. Surv. West 1ooth Mer. 1537.

U.S. Geol. Explor. Fortieth Parallel. 1517.

i.S. Geol. Geogr. Surv. Territ. 1538.

U.S. Geol. Geogr. Surv. Territ., Ent. Comm. 1535 .

U.S. Geol. Surv. 1539.

U.S. Hygienic Lab. 1543 .

U.S. Mint. 1543 .

U.S. Nat. Acad. Sci. 1516.

U.S. Nat. Herbarium. $1522(b)$

U.S. Nat. Mus. 1522 .

U.S. Naval Bull. 1534.

U.S. Naval Observ. $1=40$.

U.S. Patent Office. 1541 .

U.S. Patent Office Gaz. I 541 .

U.S. Public Health Service. 1542 .

U.S. Treasury Dep. I543.

U.S. Weather Bur. 1533 .

U.S. War Dep. 1544.

Univ. California. $1325,1326$.

Univ. Chicago. 1356.

Univ. Cincinnati. 1359.

Univ. Coll. Dundee. 29

Univ. Colorado. I 336.

Univ. Genova. 833 .

Univ. Grenoble 445 .

Univ. Habana. 1558

Univ. Illinois. I 508,1509 .

Univ. Imp. Kioto. 1 i 63.

Univ. Inst. Commerc. Research Tropics. 69.

Univ. Kansas. 1390.

Univ. La Plata. I 564.

Univ. Liége. 419.

Univ. Louvain. 420 .

Univ. Lyon 461 .

Univ. Maine. $14 \div 8$.

Univ. Melbourue. $\quad 1256$.

Univ. Minnesota. Ifo4.

[niv. Montana. I 4 or.

Univ. Nac. La Plata. 1564 .

Univ. Napoli. $x_{42}, 843$.

Univ. Nebraska. $1393,1394$.

Univ. Padova. $84^{8}$.

Univ. Pennsylvania. 1476,1477 .

Univ. Pisa. $8=6.857$.

Univ. Studies Nebraska. $1393(b)$.

Univ. Texas. 1317

Iniv. Torino. 875 .

Univ. Toronto. 1308.

Univ. Wisconsin. I 397 .

Upsala. $936-938$.

Urbana. 1505-1509.

Urban's Sumbolae Antillanae. 610. 


\section{V}

Valparaiso. I 592.

Vandstundsobserv. Norwat. $\$ 82(b)$.

Vellosia. 1581 .

Venice, 876,877 .

leveenig. Kolon. Inst. Amsterdam. $780(c)$.

Verein Erdk. Dresden. 647.

Verein Erdk. Leipzig. 715.

Verein Freunde Naturg. Meklenburg. 753.

Verein Naturk Cassel. 637.

Verein Naturk. Herzogth. Nassau. $779(b)$.

Verein Naturiv. Braunschweig. 631.

Verein Naturw. Unterhalt. Hamburg. 682.

Verein Schles. Insektenk. 625.

Verein Vaterl. Naturk. Wiirttemburg. 773.

Verh. Allg. Conf. Internat. Erdmessung. I 593 .

Verh. Batav, Genootsch. $1190(e)$.

Verh. Comm. Vervaard. Geol. Beschriju. Kaart Ned. 795.

Verh. Deutsch. Wiss. Ver. Santiago. I 590.

Verh. Deutsch. Zool. Ges. 713.

Verh. Geol-mijnbowk. Genoot. Ned. 800 $(a, b)$.

Verh. Ges. Erdk. Berlin. 587 (a).

Verh. Ges. gesammte Miner. St. Petersburg. 9 IO $(c)$.

Verh. Kais. Miner. Ges. St. Petersburg. 910 (c).

Verh. Liais.-Kön. Geol. Reichsanst. Wien. $375(c)$.

Verh. Fais.Kön. Zool.-bot. Ges. Wien $381(b)$.

Verh. Kais. Leop.-Car. Deut. Ahad. Naturf. $669(e)$.

Verh. Kon. Akad. Wetensch. Amsterdam. $789(c-f)$.

Verh. Hon. Natuurk. Ver. Ned.-Ind. 1194 (b).

Verh. Kon. Ned. Inst. Wetensch. 787 (b).

Verh. Naturf. Ges. Basel. $939(b)$.

Verh. Naturh. Ter. Preuss. Rheinl. $620(b)$.

Verh. Naturwiss. Ver. Hamburg. 68 I (b).

Verh. Russ.-Kais. Hiner. Ges. 9 Io (c).

Verh. Ver. Naturw. Unterhalt. 682.

Verh. Zool.-bot. Ver. Wien. 381 (b).

Vermont Geol. Surv. 133 .

Veröffentl. Siädt. Mus. Völkerk. Leipzig. $733(b)$.

Veröffentl. Internat. Komm. Wiss, Luftschiff. $763(b)$.

Versl. Dep. Landb. Suriname. $1577(b)$.

Versl. Dienst Pestbestrijding. I 192.

I'ersl. Inspect. Landb. Suriname. $1577(b)$.

Versl. K. Akad. IVetensch. Amsterdam. $789(g-1)$.

Versl. Meded. I. Akad. Wet. Amsterdam. $78,(g, h)$.

Versl. Meded. Ned. Bot. Veveenig. $810(c)$.

Versl. Profst. Suik. IW-Java 'Kagok.' $1223(c)$.

Versl. Rijks Ethnogr. Mus. Leiden. $805(b)$.

Versl. Staat Algem.-Proefst. Salatiga. 1224 .

Versl. Staat 'sLands Plant. Buitenzorg. I I09 $(g)$.
Fetensk. Meddel. Geogr. För. Finland $891(b)$.

Veterinary Dep. India. I055.

Victoria Memor. Mus. 1294.

Victorian Geogr. Journ. 1254.

Vida Nueva. I 559.

Vidensk. Meddel. Naturh. Foren. Copenhagen. 427.

Vidensk.-Selsk. Christiania. 885

Vidensk.-Selsk. Forhandl. 885 (a)

Vidensk.-Selsk. Skrift. $88 ;(b)$.

Vienna. $367-38 \mathrm{c}$.

Vienna Oriental Journ. 370

I'ierteljahrsschr. Naturf. Ges. Ziirich. $g(j)$ (f).

Viestn. Arkeol. Drǔtvo Zagreb. 390

Vindobona sec Vienna.

Virchow's Arch. Path. etc. 574

Virginia Geol. Surv. I34.5.

Visscherij Stat. Batavia $119 \%$

Vizagapatam. 1152 .

Vladivostok. 1003.

Volzhsk. Biol. Stantz. (1) 3

Vostoch11. Inst. IO()3.

\section{W}

Wagner Free Inst. Sci. I 478 .

Warsaw. 914.

Washington. 1510-1545.

Washington Acad. Sci. I5 IO.

Washington Anthropol. Soc. 1513 .

Washington Biol. Soc. I5 I4.

Washington Phil. Soc 1518.

Water Supply Papers. C.S.A. $1539(f)$.

Weather Bur. Philipp. Is. 1215.

Weather Bur. C.S.A. 1533.

Weimar. $7 \overrightarrow{7}$

Wellcome Research Labs, Khartoum. 9.6\%.

Wellington. $1276-1283$.

Wernerian Nat. Hist. Soc. ; I.

West. Austral. Mus. Art Gallery. 1259.

West India Committee. 320.

West Indian Bull. I 547 (c).

Wiedemann's tun. Physik. Chem gol (d).

Wiedemann's Zool. MI Iag. (ong.

Wien see Vienna.

Wiener Entomol. Monatsch. 387

Wiener Entomol. Zeitung. 388.

Wiener Mus. Naturgesch. 380

Wiesbaden. $77^{8}$.

Wilson Bull. 1355 .

Wireless World. 321

Wisconsin Acad. Sci. Arts. Letters. I 298.

Wisconsin Agric. Exp. Stat. 1397.

Wisconsin Geol. Nat. Hist. Surv. 1396.

Wisconsin Nat. Hist. Soc. 1400.

Wiss. Jahresb. Jorgenl. Studien. $712(b)$.

Wiss. Meeresuntersuch. Kiel. 698.

Wiss. .17itt. Bosnien Herzegovina. 364.

Wiss. Teröffentl. Ver. Erdk. Leipzig. 7 I $5(c)$.

Woods Hole. $154^{\circ}$.

Woods Hole Marine Biol. Lab. I 546.

Work King Inst. Prev. Hed. I $26(c)$.

Wurzburg. $78_{1-783}$

Wïrzburger Naturiss. Zeitsch. 782. 
Y

Vale Astronom. Observ. I4I4.

Yale Psychol. Lab. I4I3.

Yale Univ. I4I3, I4 I4.

Yalta. 915.

Yearbk. Khedivial Agric. Soc. gio.

Yearbk. Pharmacy. 104

Yearbk. Roy. Soc. London. $298($ o).

Yearbk. Soc. Trop. Med. Hyg. $314(b)$.

Yearbk. U.S. Dep. Agric. $1529(h)$.

Yearbk. Wireless Telegr. Teleph. 322.

Yellow Fever Bur. $68(b)$.

Yokohama. I I 88, I189.

York. 349.

Yorkshire Phil. Soc 349.

\section{Z}

Zagreb. 390 .

Zapiski. Imp. Akad. Nauk. $900(i, j)$.

Zapiski Imp. S.-Peterburgh. Min. Obshch. 9 IO $(c)$.

Zapiski Novoross. Obshch. Estestvoisp. 898.

Zapiski Obshch. Estestvoisp. Kiev. 896.

Zeitschr. Analyt. Chem. 780.

Zeitschr. Anorg. Chem. 683.

Zeitschr. Chem. Indust. Kolloid. 648.

Zeitschr. Deutsch. Geol. Ges. 578.

Zeitschr. Deutsch. Morgenl.Ges. 712 (c).

Zeitschr: Elektrochemie. 668.

Zeitschr. Elektrotechn. Elektrochem. 674.

Zeitschr. Entomol. 625 (b), 734.

Zeitschr. Ethnol. Berlin. 586.

Zeitschr. gesammt. Wissensch. $673($ c).

Zeitschr. Gesell. Erdk. Berlin. $587(b)$.

Zeitschr. Gletscherkunde. 612.

Zeitschr. Hyg. Infektionskr. 735 .

Zeitschr. Insektenbiol. 6I5.

Zeitschr. Instrumentenkunde. 6I 3.

Zeitschr. Kirystallogr. Miner. 736.

Zeitschr. Kunde Morgenländes. 667 .

Zeitschr. Malakozool. 685.

Zeitschr. Morphol. Anthropol. 774

Zeitschr. Naturwiss. $673(\mathrm{c})$.

Zeitschr. Parasitenkunde. 695.

Zeitschr. Physikal. Chem. Stoechiom. 73j.
Zeitschr. Physikal. Chem. Unterricht. 61 I. Zeitschr. Prakt. Geol. 614.

Zeitschr. Syst. Hymenopt. Dipterol. 776.

Zeitsçhr. Ungar. Geol. Ges. $352(a)$.

Zeitschr. Wiss. Insektenbiol. 615.

Zeitschr. Wiss. Mikrosk. Mikrosk. Techn. 738.

Zeitschr. Wiss. Zool. 739.

Zentralbl. Zool. Ally. Exper. Biol. 740.

Zool. Abh. II iener iius. Naturgesch. 389.

Zool. Ann. 783 .

Zool. Anz. 7t1.

Zool. Beitr. 626 .

Zool. Beob. 6:34 (b).

Zool.-bot. Ver. Wien. $38 \mathrm{I}$.

Zool. Centralblatt. 742 .

Zool. Garten. $654(a)$.

Zool. Ges. Dresden. 654

Zool. Illiustr. 323.

Zool. Inst. Graz. 360 .

Zool. Jahrbiicher. 696

Zool. Jahresb. $8_{45}(\mathrm{c})$.

Zool. Journ. 324.

Zool. Mag. 699.

Zool. Meded. Leiden. 807 (c).

Zool. Misc. 325, 326.

Zool. Mitt. Dresden. 640.

Zool. Mus. Berlin. 6 I6.

Zool. Inst. K.- K. Univ. Wien. 380.

Zool. Inst. Wurzburg. $781(a)$.

Zool. Record. 327 (d).

Zool. Results Fish. Exp. 'Endeavour.' 1265.

Zool. Publ. Univ. California. $1325(\mathrm{~g})$.

Zool. Soc. Japan. II 87.

Zool. Soc. London. 327

Zool. Soc. New York. 1455.

Zool. Soc Tokyo. II 87 .

Zool. Stat Neapel. 84 .

Zool. Stat. Trieste. 380 .

Zool. Surv. India. Iogy.

Zool. Zentralblatt. 742

Zool.-zootom. Inst. Wïrzburg. $78 \mathrm{I}(a)$

7oologia. 1455

Zoologica. 775

Zoologist. 328.

Zurich. 9:3-962. 


\section{DAY USE}

RETURN TO DESK FROM WHICH BORROWED

\section{LOAN DEPT.}

This book is due on the last date stamped below, or on the date to which renewed.

Renewed books are subject to immediate recall.

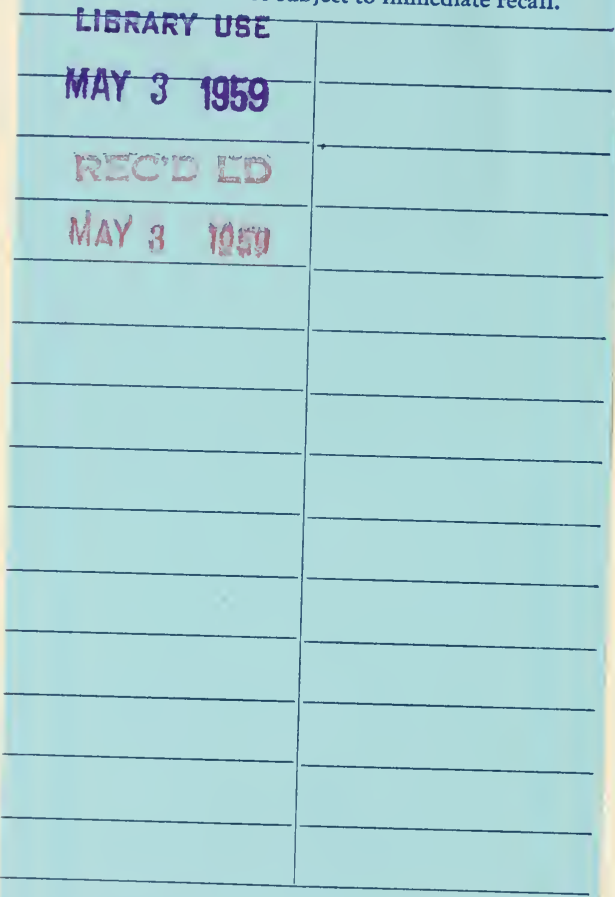

LD $21 \mathrm{~A}-50 m-9,{ }^{\prime} 58$

(6889s10)476B

General Library

University of California Berkeley 
yc 44057

\section{$39 \pm 164$}

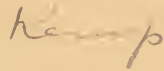

UNIVERSITY OF CALIFORNIA LIBRARY 


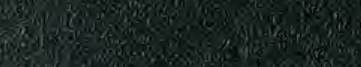

2.

(19) 5$)^{2}$

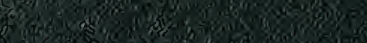

(x)

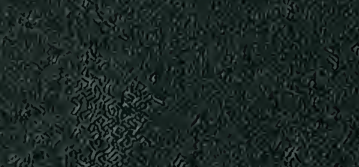

Hes

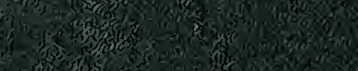

1.1.

sin

2ing

40

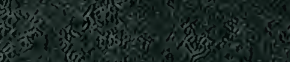

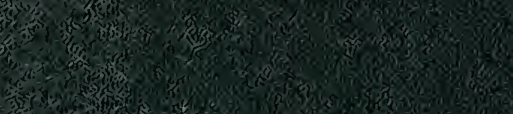

H.

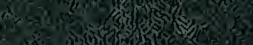

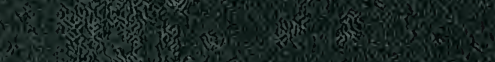

1.

(6)

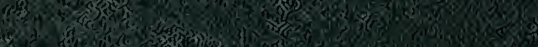

1. H

3
7

3.x.

4. (1.

S.

(3)

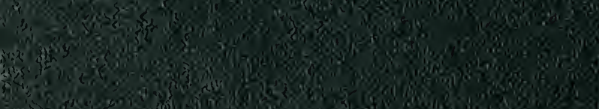

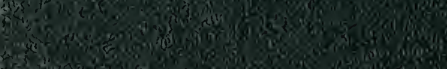

7. 